U.S. Coast Gvaid Oceanographic Repart

DEPARTMENT OF TRANSPORTATION

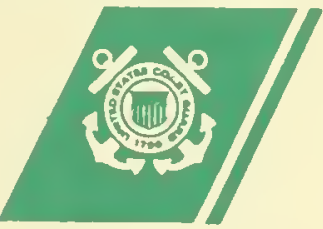

COAST

GUAR

\title{
OCEANOGRAPHIC OBSERVATIONS
}

\author{
NORTH ATLANTIC \\ OCEAN STATION DELTA
}

27 August 1967 - 25 August 1968

$6 c$

PLEASE RET $-R N$

Woods Hole Oceanngraphic !ns'itution

AILAS - GAZETTEER COLLECTION
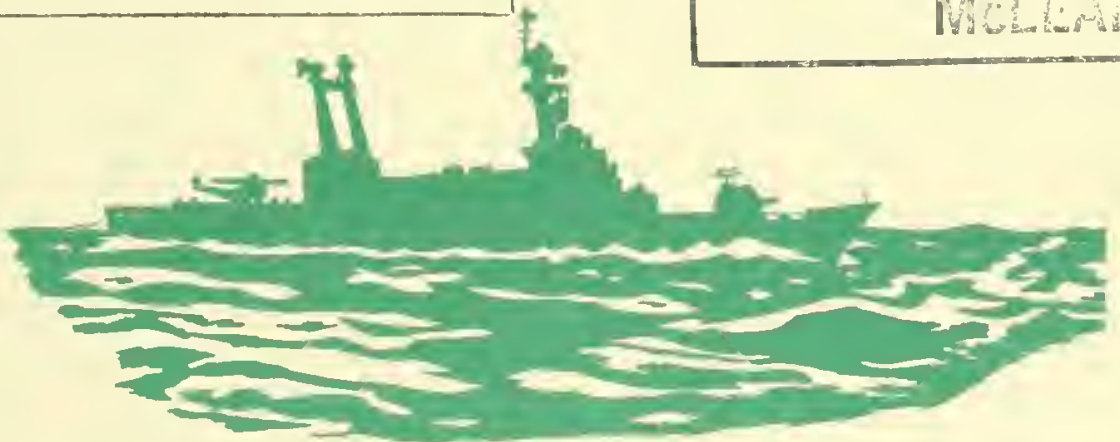

IINSTITUTION DATA LIBRARY

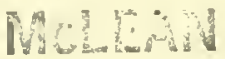

13

1 3

1658 



\title{
OCEANOGRAPHIC REPORT \\ No. CG 373-58
}

\section{NORTH ATLANTIC \\ OCEAN STATION DELTA \\ 27 August 1967 - 25 August 1968}

R. E. Hammond

\author{
United States Coast Guard \\ Oceanographic Unit \\ Washington, D.C.
}




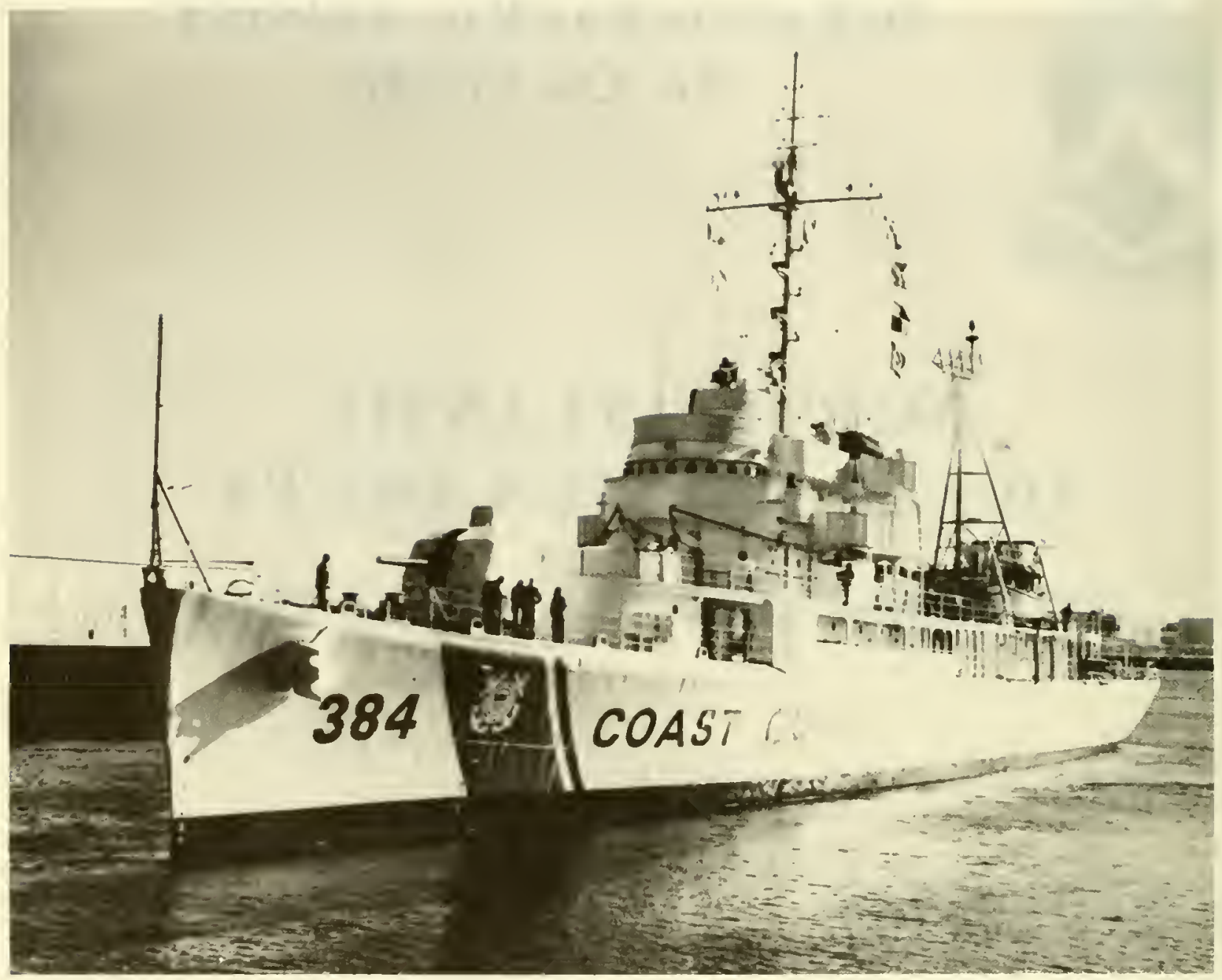

USCGC COOK INLET, a $311 \mathrm{Ft}$. High Endurance Cutter. 


\section{ABSTRACT}

The observed and interpolated temperature and salinity data plus the computed sigma-t, geopotential anomalies, and sound velocities are presented for 202 oceanographic stations occupied during thirteen Coast Guard cruises to Ocean Station DELTA (centered at $44^{\circ} 00^{\prime} \mathrm{N}, 41^{\circ} 00^{\prime} \mathrm{W}$ ) between 27 August 1967 and 25 August 1968. The surface layer $(300 \mathrm{~m})$ at OS DELTA is characterized by a complex structure because of the proximity of the North Atlantic Current and seasonal atmospheric variations.

Editor's Note: Reference to a product or comment with respect to it in this publication does not indicate, or permit any person to hold out by republication in whole or in part or otherwise, that the product has been endorsed, authorized, or approved by the Coast Guard. 



\section{TABLE OF CONTENTS}

Title Page

Abstract

Table of Contents

List of Illustrations

List of Tables

Introduction

Sampling Techniques

Analysis of Data

Northern Gulf Stream Extension

Contributing Water Masses

Seasonal Variations

Description of Observed Processes

References

Illustrations

Appendix A-Oceanographic Data

\section{LIST OF ILLUSTRATIONS}

Figure

Frontispiece: USCGC COOK INLET (WHEC-384)

1. Chart of world ocean stations and Standard Section A-3

2. Bathymetry of Ocean Station DELTA

3. Positions of USCGC INGHAM oceanographic stations, 27 August to 16 September 1967

4. Positions of USCGC CHINCOTEAGUE oceanographic stations, 22 September to 10 October 1967

5. Positions of USCGC ESCANABA oceanographic stations, 12 October to 3 November 1967

6. Positions of USCGC OWASCO oceanographic stations, 30 November to 15 December 1967

7. Positions of USCGC ABSECON oceanographic stations, 13 January to 30 January 1968

8. Positions of USCGC CASCO oceanographic stations, 28 February to 19 March 1968

9. Positions of USCGC ABSECON oceanographic stations, 20 March to 8 April 1968

10. Positions of USCGC COOK INLET oceanographic stations, 14 April to 5 May 1968

11. Positions of USCGC DALLAS oceanographic stations, 6 May to 28 May 1968

12. Positions of USCGC CASTLE ROCK oceanographic stations, 31 May to 19 June 1968

13. Positions of USCGC HAMILTON oceanographic stations, 6 July to 9 July 1968 
14. Positions of USCGC DALLAS oceanographic stations, 13 July to 4 August 1968

15. Positions of USCGC COOK INLET oceanographic stations, 5 August to 25 August 1968

16. Depth of $10^{\circ} \mathrm{C}$ isothermal surface

17. The oceanic Polar Front in the northern North Atlantic

18. Fluctuations of the North Atlantic Current

19. Temperature-Salinity diagram for period 27 August to 3 November 1967

20. Temperature-Salinity diagram, 30 November to 15 December 1967

21. Temperature-Salinity diagram, 13 January to 8 April 1968

22. Temperature-Salinity diagram, 14 April to 19 June 1968

20

23. Temperature-Salinity diagram, 6 July to 25 August 1968

21

24. Temperature versus time at selected depths at OS DELTA, 27 August 1967 to 25 August 1968

25a. Salinity versus time at selected depths at OS DELTA, 27 August 1967 to 5 May 1968

25b. Salinity versus time at selected depths at OS DELTA, 6 May to 25 August 1968

26. Envelope of temperature versus depth, 27 August to 16 September 1967

27. Envelope of temperature versus depth, 22 September to 10 October 1967

28. Envelope of temperature versus depth, 12 October to 3 November 1967

29. Envelope of temperature versus depth, 30 November to 15 December 1967

30. Envelope of temperature versus depth, 13 January to 30 January 1968

31. Envelope of temperature versus depth, 28 February to 19 March 1968

32. Envelope of temperature versus depth, 20 March to 8 April 1968

33. Envelope of temperature versus depth, 14 April to 5 May 1968

34. Envelope of temperature versus depth, 6 May to 28 May 1968

35. Envelope of temperature versus depth, 31 May to 19 June 1968

36. Envelope of temperature versus depth, 6 July to 9 July 1968

35

37. Envelope of temperature versus depth, 13 July to 4 August 1968

36

38. Envelope of temperature versus depth, 5 August to 25 August 1968

37

39. Envelope of salinity versus depth, 27 August to 16 September 1967

40. Envelope of salinity versus depth, 22 September to 10 October 1967

41. Envelope of salinity versus depth, 12 October to 3 November 1967

42. Envelope of salinity versus depth, 30 November to 15 December 1967

43. Envelope of salinity versus depth, 13 January to 30 January 1968

44. Envelope of salinity versus depth, 28 February to 19 March 1968

45. Envelope of salinity versus depth, 20 March to 8 April 1968

46. Envelope of salinity versus depth, 14 April to 5 May 1968

47. Envelope of salinity versus depth, 6 May to 28 May 1968

48. Envelope of salinity versus depth, 31 May to 19 June 1968

49. Envelope of salinity versus depth, 6 July to 9 July 1968

50. Envelope of salinity versus depth, 13 July to 4 August 1968

51. Envelope of salinity versus depth, 5 August to 25 August 1968

52. Comparison of temperature and salinity versus depth at OS DELTA, ECHO, CHARLIE, and Standard Section A-3, 31 January to 3 February 1968 
53. Comparison of temperature and salinity versus depth at OS DELTA, ECHO, CHARLIE, and Standard Section A-3, 6 August 1968

\section{LIST OF TABLES}

Tables

Page

1. Summary of oceanographic operations on Ocean Station DELTA, August 1967-August 1968

2. Description of water masses in the vicinity of Ocean Station DELTA

3. Summary of the variations of the predominant characteristics of salinity in the upper $800 \mathrm{~m}$ at Ocean Station DELTA, 27 August 1967 to 25 August 1968 



\title{
OCEANOGRAPHIC OBSERVATIONS NORTH ATLANTIC OCEAN STATION DELTA
}

\section{AUGUST 1967-25 AUGUST 1968}

\author{
by \\ ROBERT E. HAMMOND ${ }^{1}$
}

\section{INTRODUCTION}

Initial Coast Guard investigations on Ocean Station DELTA (OS DELTA), located at $44^{\circ} 00^{\prime} \mathrm{N}$, $41^{\circ} 00^{\prime} \mathrm{W}$, began during a one month cruise in April 1963 aboard the USCGC CASCO. Eighty oceanographic stations were taken to determine the spatial or temporal variations of temperature and salinity in the upper 800 meters. McGary and Morse (1964) concluded that the boundaries of several distinct water masses occur near OS DELTA and lead to large horizontal gradients in temperature and salinity distribution.

Experiments were conducted during five threeweek cruises by the U.S. Naval Oceanographic Office (June 1962-July 1963) to determine the vertical and horizontal variability of water properties in the immediate vicinity of OS DELTA. Analyses of these data were presented by Corton (1967) in which the water masses at OS DELTA were described as: (1) the surface water mass of Gulf Stream origin which varies in thickness from 475 meters to 800 meters, (2) an intermediate water mass of cold water of subarctic origin (with a small percentage of Mediterranean Water between 800 meters and 1000 meters) which extends to a depth of approximately 1750 meters, (3) a deep mass of North Atlantic Deep Water between 1750 meters and 2750 meters, and (4) a bottom water mass below 2750 meters, distinctive of Newfoundland Basin Water $\left(2.3^{\circ}-3.0^{\circ} \mathrm{C}, 34.90 \%\right.$ o- $34.96 \%$ o $)$.

The U.S. Coast Guard initiated a continuing series of oceanographic observations at OS DELTA in July 1966. Due to a limited number of deep sea reversing thermometers, these observations were restricted to alternate three-week

'U.S. Coast Guard Oceanographic Unit, Building 159-E, Navy Yard Annex, Washington, D.C. 20390. Presently with Commander, 17th Coast Guard District, Juneau, Alaska. cruises during the first twelve months. Husby (1969) reported on the data obtained from 136 oceanographic stations during the period from July 1966 to August 1967; he pointed out significant fluctuations in temperature and salinity caused by changes in the North Atlantic Current near OS DELTA.

This report describes conditions based on 202 oceanographic stations at OS DELTA from 27 August 1967 to 25 August 1968. A summary of oceanographic operations during that period is presented in Table 1 . Nansen casts were taken daily during the occupation of OS DELTA unless terminated by weather or other mission responsibilities. In addition, casts using a salinity-temperature-depth sensor system (STD) were conducted on OS DELTA for the first time (6 July to 9 July 1968) by USCGC HAMILTON.

\section{SAMPLING TECHNIQUES}

The location of OS DELTA in relation to the other ocean stations of the world and Standard Section A-3 is shown in figure 1 . The bathymetry in the vicinity of OS DELTA is shown in figure 2.

Ocean station vessels attempt to maintain their position within a ten mile square centered on the ocean station, although extenuating circumstances occasionally prevent this. The station position sheets (figs. 3-15) indicate those stations occupied outside the ten mile square.

The sampling program at OS DELTA consisted of daily Nansen bottle casts to a depth of 1,500 $\mathrm{m}$ when weather and other operations permitted. The desired sampling depths were surface, $25 \mathrm{~m}$, $50 \mathrm{~m}, 75 \mathrm{~m}, 100 \mathrm{~m}, 150 \mathrm{~m}, 200 \mathrm{~m}, 300 \mathrm{~m}, 400$ $\mathrm{m}, 500 \mathrm{~m} ; 600 \mathrm{~m}, 800 \mathrm{~m}, 1,000 \mathrm{~m}$, and 1,500 $\mathrm{m}$. However, the first three cruises during the period covered in this report did not sample at 
TABLE 1.-Summary of oceanographic operations on Ocean Station.

DELTA, August 1967-August 1968

\begin{tabular}{|c|c|c|c|c|c|c|c|c|}
\hline Vessel & Dates & $\begin{array}{c}\text { Casts } \\
\text { Shallow }\end{array}$ & Deep & $\begin{array}{l}\text { Average Max. } \\
\text { Sampling } \\
\text { Depth } \\
\text { Shallow Cast }\end{array}$ & $\begin{array}{l}\text { Range of Max } \\
\text { Sampling D } \\
\text { Shallow } \\
\text { Cast }\end{array}$ & $\begin{array}{l}\text { mum } \\
\text { pth } \\
\text { Deep } \\
\text { Cast }\end{array}$ & $\begin{array}{l}\text { NODC } \\
\text { Ref. } \\
\text { Number }\end{array}$ & $\begin{array}{c}\text { Appendix A } \\
\text { Table } \\
\text { Number }\end{array}$ \\
\hline USCGC INGHAM & $8 / 27-9 / 16 / 67$ & 18 & 1 & $1501 \mathrm{~m}$ & $1144-1866 \mathrm{~m}$ & $3321 \mathrm{~m}$ & $31-1158$ & I \\
\hline $\begin{array}{l}\text { USCGC } \\
\text { CHINCOTEAGUE }\end{array}$ & $9 / 22-10 / 10 / 67$ & 14 & - & $1462 \mathrm{~m}$ & $997-1621 \mathrm{~m}$ & - & $31-1171$ & II \\
\hline USCGC ESCANABA & $10 / 12 /-11 / 03 / 67$ & 22 & 1 & $1383 \mathrm{~m}$ & $883-1862 m$ & $4556 \mathrm{~m}$ & $31-1176$ & III \\
\hline USCGC OWASCO & $11 / 30-12 / 15 / 67$ & 10 & 1 & $1343 \mathrm{~m}$ & $992-1671 \mathrm{~m}$ & $4621 \mathrm{~m}$ & $31-1184$ & IV \\
\hline USCGC ABSECON & $1 / 13-1 / 30 / 68$ & 6 & 1 & $1500 \mathrm{~m}$ & $1115-1885 \mathrm{~m}$ & $4262 \mathrm{~m}$ & $31-1202$ & V \\
\hline USCGC CASCO & $2 / 28-3 / 19 / 68$ & 17 & 2 & $1475 \mathrm{~m}$ & $1100-1660 \mathrm{~m}$ & $\begin{array}{l}4324 \mathrm{~m} \\
3902 \mathrm{~m}\end{array}$ & $31-1210$ & VI \\
\hline USCGC ABSECON & $3 / 20-4 / 8 / 68$ & 9 & - & $1392 \mathrm{~m}$ & $1172-1604 \mathrm{~m}$ & - & $31-1238$ & VII \\
\hline USCGC COOK INLET & $4 / 14-5 / 5 / 68$ & 17 & 2 & $1572 \mathrm{~m}$ & $1368-1675 \mathrm{~m}$ & $\begin{array}{l}4454 \mathrm{~m} \\
4622 \mathrm{~m}\end{array}$ & $31-1241$ & VIII \\
\hline USCGC DALLAS & $5 / 6-5 / 28 / 68$ & 22 & 1 & $1482 \mathrm{~m}$ & $1253-1914 \mathrm{~m}$ & $4190 \mathrm{~m}$ & $31-1265$ & IX \\
\hline $\begin{array}{l}\text { USCGC CASTLE } \\
\text { ROCK }\end{array}$ & $5 / 31-6 / 19 / 68$ & 18 & 1 & $1474 \mathrm{~m}$ & $660-1786 \mathrm{~m}$ & $4446 m$ & $31-1269$ & $\mathrm{X}$ \\
\hline USCGC HAMILTON & $7 / 6-7 / 9 / 68$ & 13 & - & $1500 \mathrm{~m}$ & $1500 \mathrm{~m}$ & - & $31-8042$ & $\mathrm{XI}$ \\
\hline USCGC DALLAS & $7 / 13-8 / 4 / 68$ & 22 & 1 & $1417 \mathrm{~m}$ & $1283-1613 m$ & $4590 \mathrm{~m}$ & $31-1339$ & XII \\
\hline USCGC COOK INLET & $8 / 5-8 / 25 / 68$ & 14 & 1 & $1511 \mathrm{~m}$ & $1478-1652 \mathrm{~m}$ & $4211 \mathrm{~m}$ & $31-1316$ & XIII \\
\hline
\end{tabular}

$75 \mathrm{~m}$; instead they included a $1,250 \mathrm{~m}$ sample. For deep casts, the desired depths were $2,000 \mathrm{~m}$, $2,500 \mathrm{~m}, 3,000 \mathrm{~m}, 3,500 \mathrm{~m}, 4,000 \mathrm{~m}$, and 150 $\mathrm{m}$ and $50 \mathrm{~m}$ off the bottom. Attempts were made to complete at least one deep cast during each patrol.

A pair of protected deep sea reversing thermometers was fitted on each Nansen bottle. Four or five bottles at $200 \mathrm{~m}$ and below were each fitted with an unprotected thermometer for the thermometric determination of the sampling depths. Field observations of temperature were transmitted via radioteletype to the Coast Guard Oceanographic Unit for real time data processing and quality control. The reductions to in situ temperatures were accomplished with the use of a Digital Equipment Corporation PDP-5 computer.

Salinity samples were drawn from each Nansen bottle, and salinity values were determined at sea using an inductive salinometer. Duplicate samples were drawn from the top and bottom bottles of each cast and were analyzed by the Oceanographic Unit for quality control.

Processed temperature and salinity data for each cruise were submitted to the National Oceanographic Data Center (NODC), Washington, D.C. The interpolated temperatures, salinities, sigma-t values, geopotential anomalies, and sound velocities for the standard depths were computed by NODC and are listed in Tables I through XIII of Appendix A.

\section{ANALYSIS OF DATA}

OS DELTA provides an opportunity to investigate the surface $(600 \mathrm{~m})$ effects of a shifting current regime which separates the Subarctic Water mass to the north and the North Atlantic Central Water mass to the south. Prior to discussion of this system, a brief description of the influencing current and water masses is provided.

\section{Northern Gulf Stream Extension}

Just off the Tail of the Banks $\left(43^{\circ} \mathrm{N}, 51^{\circ} \mathrm{W}\right)$ the eastward flowing Gulf Stream develops large fluctuations in stream position as it migrates northeastward. Iselin (1936) defined these fluctuations as an extension of the Gulf Stream called 
the North Atlantic Current. He traced the position of the current by examining the $10^{\circ} \mathrm{C}$ isothermal surface (fig. 16). The steep gradients east of $50^{\circ} \mathrm{W}$ define the North Atlantic Current.

Dietrich (1964) continued this analysis and called the geographic location of the $10^{\circ} \mathrm{C}$ isotherm at $200 \mathrm{~m}$ the North Atlantic Current boundary (Polar Front). This boundary separates cold, lowsaline, subpolar water and warm, high-saline, subtropical water. The variations in the position of the front are shown in figure 17 (Dietrich, 1964). Fluctuations of the North Atlantic Current as determined by maximum cross current temperature gradients are shown in figure 18 (Fuglister and Worthington, 1951).

Information provided by Boisvert (1967) indicates that the North Atlantic Current is a rather sluggish and slow moving regime $(<1.0 \mathrm{kt})$ subject to the effects of shallow, wind driven surface movement known as North Atlantic Drift (Stommel, 1966).

Due to wide seasonal variations and the limited amount of available data for the North Atlantic Current, exact limits of temperature and salinity distribution cannot be clearly described. However, Corton (1967) indicates that a high salinity core $(35.6 \% 0-36.2 \%$ o $)$ exists at approximately $100 \mathrm{~m}$. Temperatures at this depth vary between $16^{\circ}$ and $17^{\circ} \mathrm{C}$ (Pub. No. 700, 1967).

\section{Contributing Water Masses}

The North Atlantic Current provides a dynamic boundary between two distinct water masses which are displaced and positioned along this boundary as a function of the current velocity and shape of developing meanders. The predominant water mass to the south has been designated North Atlantic Central Water (NACW) $\left(4^{\circ}-17^{\circ} \mathrm{C}\right.$,
$35.10 \%$ o-36.29\%o) (Sverdrup, et al. 1942, Defant, 1961). Corton (1967) has estimated this water to extend from the surface to approximately 600 $m$ in the vicinity of OS DELTA. Subarctic Water $\left(3^{\circ}-5^{\circ} \mathrm{C}, 34.7 \% 0-34.9 \%\right.$ ) to the north of this boundary is the dominant water mass from the surface to $1,750 \mathrm{~m}$ (Corton, 1967). However the observed characteristics at OS DELTA $\left(8^{\circ}-23^{\circ} \mathrm{C}\right.$, $35.10 \%$ - $36.70 \%$ ) indicate a regime whose physical properties are, at times, influenced by the North Atlantic Current. This influence was especially noticeable during the summer months when the wind mixed layer was at a minimum thickness.

The intermediate water at OS DELTA (600-1,$750 \mathrm{~m}$ ) was formed by the mixing of Subarctic Water with the more saline water transported by a northwestern fan of the Mediterranean effluent. The lower limit of this intermediate water mass can be identified by a salinity of $34.92 \%$ and has been estimated to be at a depth of $1,750 \mathrm{~m}$ in the vicinity of OS DELTA (Corton, 1967).

Table 2 summarizes and compares the various water masses found in the vicinity of OS DELTA. T-S diagrams of the data collected at OS DELTA emphasize these water masses and point out the effects of seasonal variation (figs. 19-23).

\section{Seasonal Variations}

The observations made at OS DELTA indicated the existence of a seasonal upper layer separated from the non-seasonal layer at about $300 \mathrm{~m}$ as can be seen from the vertical distribution of temperature (figs. 26-38). A permanent thermocline (approximately $1.5^{\circ} \mathrm{C} / 100 \mathrm{~m}$ ) existed from a depth of $300 \mathrm{~m}$ to $800 \mathrm{~m}$. The temperature then decreased from $6^{\circ} \mathrm{C}$ at $800 \mathrm{~m}$ through the

TABLE 2.-Description of water masses in the vicinity of Ocean Station DELTA.

\begin{tabular}{lcc} 
Water Type & $\begin{array}{c}\text { Temperature Range } \\
\left({ }^{\circ} \mathrm{C}\right)\end{array}$ & $\begin{array}{c}\text { Salinity Range } \\
(\% \circ)\end{array}$ \\
\hline $\begin{array}{l}\text { Subarctic* } \\
(\%)\end{array}$ & $3-5$ & $34.7-34.9$ \\
\hline $\begin{array}{l}\text { North Atlantic } \\
\text { Central* }\end{array}$ & $4-17$ & $35.1-36.3$ \\
\hline $\begin{array}{l}\text { North Atlantic } \\
\text { Deep* }\end{array}$ & $3-4$ & $34.9-35.0$ \\
\hline $\begin{array}{l}\text { North Atlantic } \\
\text { Bottom* }\end{array}$ & $1-3$ & $34.8-34.9$ \\
\hline OSD upper $800 \mathrm{~m}^{* *}$ & & \\
\hline
\end{tabular}


intermediate water mass to $4^{\circ} \mathrm{C} \pm 0.3 \mathrm{C}^{\circ}$. A seasonal isothermal layer was present from late October through early May (figs. 28-33), with a maximum depth of $300 \mathrm{~m}$ observed in early March. This isothermal feature was caused by the erosion and deepening of the seasonal thermocline which was influenced by increased convection due to cooling temperatures and wind mixing. A time-series plot is presented in figure 24 in support of the discussions of temporal variations in temperature.

The upper seasonal layer displayed maximum surface temperatures and stratification during the autumnal equinox; the minimum temperatures and the deepest thermocline erosion (isothermal layer) coincided with the vernal equinox.

The salinity structure (fig. 25) of the upper seasonal zone followed a trend similar to that of the temperature. An isohaline layer developed in December, with a maximum depth of $300 \mathrm{~m}$ occurring in early March, and was followed by the establishment of a halocline in May. This seasonal halocline blended into the permanent halocline which extended down to about $700 \mathrm{~m}$. The intermediate water $(600-1,750 \mathrm{~m})$ maintained a nearly constant salinity range of $34.92 \%$ $-35.00 \%$.

The vertical distribution of salinity for each cruise is shown in figures 39-51. The salinity maximum located at $100 \mathrm{~m}$ from August to December (figs. 39-42, 51 and Table 3 ) may have been an intrusion of the North Atlantic Current. The greater mixing forces that occurred during the months from January to May eroded this prominent feature into an isohaline layer (to approximately $300 \mathrm{~m}$ ) with only an occasional maximum at greater depths. This seems to indicate that the mixing processes were of sufficient magnitude to destroy any changes that could be brought about by intrusions from the North Atlantic Current.

Table 3 summarizes the variations of the predominant characteristics of salinity in the upper $800 \mathrm{~m}$ at OS DELTA.

\section{Description of Observed Processes}

Observations made in a limited geographical area over a relatively short period (one year) do not lend themselves to a comprehensive analysis of physical processes. However, it was possible to observe changes in physical properties at OS DELTA due to the variation in position and intensity of the North Atlantic Current.

Comparison of the physical properties of OS DELTA was made with those observed for Standard Section A-3 (SSA3) (Robe, 1971) in an attempt to determine the geographic relation between OS DELTA and the Polar Front. Only three dates were comparable (due to limited observations on the eastern end of SSA3): 4 November 1967, 3 February 1968, and 5 August 1968. During those times the Front was located 255,285 , and 200 nautical miles, respectively, west of OS DELTA.

It would be difficult to make an estimate of the Front's distance to the north of OS DELTA, due to a lack of data in that direction. However, at no time did the observed water characteristics indicate that the Front had traveled southward through OS DELTA. Passage of the Front or

TABLE 3.-Summary of the variations of the predominant characteristics of salinity in the upper 800 meters at Ocean Station DELTA, 27 August 1967 to 25 August 1968.

\begin{tabular}{|c|c|c|c|c|c|c|}
\hline $\begin{array}{c}\text { Inclusive } \\
\text { Cast Dates }\end{array}$ & $\begin{array}{c}\text { Surface } \\
\text { Salınity } \\
\text { Range }(\%)\end{array}$ & $\begin{array}{l}\text { Isohaline } \\
\text { Layer } \\
\text { Depth }(m)\end{array}$ & $\begin{array}{l}\text { Salinity M } \\
\text { Salinity } \\
(\% \circ)\end{array}$ & $\begin{array}{l}\text { Depth } \\
\text { (m) }\end{array}$ & $\begin{array}{l}\text { Salinity M } \\
\text { Salinity } \\
(\% 0)\end{array}$ & $\begin{array}{l}\text { Depth } \\
\text { (m) }\end{array}$ \\
\hline $8 / 27-9 / 16 / 67$ & $35.09-36.31$ & NONE & 36.54 & 100 & $35.00-35.05$ & $700-800$ \\
\hline $9 / 22-10 / 10 / 67$ & $34.35-36.20$ & NONE & $36.38,36.39$ & 50,100 & 34.72 & 570 \\
\hline $10 / 12-11 / 3 / 67$ & $35.00-36.14$ & 20 & 36.45 & $90-120$ & 34.77 & 540 \\
\hline $11 / 30-12 / 15 / 67$ & $35.72-35.90$ & 80 & 36.32 & 110 & $35.00,35.04$ & 760,810 \\
\hline $1 / 13-1 / 30 / 68$ & $35.87-36.13$ & 200 & 36.13 & $0-200$ & 34.97 & 780 \\
\hline $2 / 28-3 / 19 / 68$ & $35.79-36.02$ & 280 & $36.02,36.02$ & 0,300 & $35.00,34.97$ & 660,790 \\
\hline $3 / 20-4 / 8 / 68$ & $35.87-36.04$ & 100 & $36.03,36.04$ & 0,40 & 34.92 & 670 \\
\hline $4 / 14-5 / 5 / 68$ & $35.80-36.19$ & 100 & $36.17,36.19$ & 0,60 & $34.95,34.94$ & 660,770 \\
\hline $5 / 6-5 / 28 / 68$ & $35.80-36.11$ & $20-70$ & $36.11,36.13$ & 0,50 & 34.93 & 670 \\
\hline $5 / 31-6 / 19 / 68$ & $35.87-36.13$ & $70-100$ & 36.16 & 45 & 34.96 & $690-800$ \\
\hline $7 / 6-7 / 9 / 68^{*}$ & $33.26-35.48$ & NONE & $36.22,36.24$ & 30,70 & $\begin{array}{l}33.26 \\
34.81\end{array}$ & $\begin{array}{l}\text { Surface } \\
670\end{array}$ \\
\hline $7 / 13-8 / 4 / 68$ & $34.36-36.28$ & NONE & 36.36 & 90 & 34.66 & 550 \\
\hline $8 / 5-8 / 25 / 68$ & $34.15-36.03$ & NONE & 36.43 & 75 & 34.85 & 610 \\
\hline
\end{tabular}

*Casts during this period were taken for the first time at OSD with an STD. 
traverse of a large eddy would be marked by a sudden decrease of temperature and salinity due to the influx of Subarctic Water.

The influence of the North Atlantic Current, as it varies in strength and distance from OS DELTA, can be monitored by a change in the physical properties of the water in the vicinity of OS DELTA. Data gathered from stations occupied during the period covered by this report have been investigated for significant temporal variations as an indication of the influence of the North Atlantic Current.

Attention is directed to the USCGC HAMILTON cruise of 6-9 July 1968 (figs. 36 and 49) and specifically to stations 5,9 , and 13 , separated by intervals of 23.5 and 30.2 hours. Station five showed the reduced influence of North Atlantic Central Water (NACW), possibly due to an increase in intensity and / or a southeast movement of the North Atlantic Current. An apparent return of NACW was registered during occupation of station 13 .

Observations made during the cruise of the USCGC INGHAM (figs. 26 and 39) at stations 2,3 , and 4 showed temperature and salinity values indicative of NACW. Station 7, four days later, indicated temperature and salinity minima in the upper 800 meters as the Polar Front shifted position towards OS DELTA.

Similar distributions of properties were observed during the September 1967 cruise of USCGC CHINCOTEAGUE (figs. 27 and 40), May 1968 cruise of USCGC DALLAS (figs. 34 and 47), March 1968 cruise of USCGC ABSECON (figs. 32 and 45), and July 1968 cruise of USCGC DALLAS (figs. 37 and 50).

Due to relatively few "off station" casts, little can be evaluated concerning horizontal variations in physical properties. Separations were limited to 70 nautical miles in latitude with corresponding temperature and salinity differences of $1.0 \mathrm{C}^{\circ}$ and $0.5 \%$ in the upper $50 \mathrm{~m}$. Variations in temperature and salinity were not observed with longitudinal differences of up to 90 nautical miles.

The physical properties observed at OS DELTA and those of the surrounding water masses can best be compared by examining the observations made at OS DELTA, OS ECHO, OS CHARLIE, and selected stations along SSA3 (fig. 1). Observations at OS CHARLIE describe the water masses peculiar to the subarctic; properties observed at OS ECHO detail NACW characteristics, and observations along SSA3 provide insight into properties unique to the North Atlantic Current.
Comparison of temperature and salinity vertical distributions at the above locations was made for February and August 1968 (figs. 52 and 53).

Data from SSA3 and OS DELTA exhibit isohaline/isothermal layers down to about $100 \mathrm{~m}$ during February, with the greater depths displaying similar trends in their distributions. The nearly uniform distribution of Subarctic Water (especially at OS DELTA, OS CHARLIE, and SSA3) below $800 \mathrm{~m}$ becomes clear and is supported by the close coincidence of temperature and salinity vertical distributions.

During August, observations at SSA3 and OS DELTA showed similar characteristics of temperature and salinity versus depth, with the formation of definite thermoclines and haloclines down to $100 \mathrm{~m}$. As is typical of subarctic zones, salinity increases slightly with depth at OS DELTA and SSA3, but maximum salinities are observed at the surface of OS ECHO (subtropic location).

\section{REFERENCES}

Boisvert, W. E. (1967) Major currents in the North and South Atlantic Oceans between $64^{\circ} \mathrm{N}$ and $60^{\circ} \mathrm{S}$. U.S. Naval Oceanographic Office Technical Report No. 193, 92 pp.

Corton, E. L. (1967) Variations in the vertical water structure at OWS DELTA $\left(44^{\circ} \mathrm{N}, 41^{\circ} \mathrm{W}\right)$. U.S. Naval Oceanographic Office Informal Report No. 67-28, 24 pp.

Defant, A. (1961) Physical Oceanography Vol. I, The MacMillan Company, New York, 729 pp.

Dietrich, G. (1964) Oceanic Polar Front Survey in the North Atlantic. Research in Geophysics, MIT Press, Cambridge, 595 pp.

Fuglister, F. C. and L. V. Worthington (1951) Some results of a multiple ship survey of the Gulf Stream. Collected reprints WHOI, contribution No. $548,14 \mathrm{pp}$.

Husby, D. M. (1969) Oceanographic observations, North Atlantic Ocean Station DELTA, $44^{\circ} \mathrm{N}, 41^{\circ} \mathrm{W}$, July 1966-August 1967. U.S. Coast Guard Oceanographic Report No. 23, CG-373-23.

Iselin, C. O'D. (1936) A study of the circulation of the Western North Atlantic. Papers in Phys. Oceanog. and Met. MIT and WHOI, Vol. IV, No. 4.

McGary, J. W. and R. M. Morse (1964) Observations at North Atlantic Ocean Station DELTA, March-April 1963. U.S. Coast Guard Oceanographic Report No. 3, CG-373-3.

Robe, R. Q. (1971) Oceanographic observations North Atlantic Standard Monitoring Sections A-1, A-2, A-3, A-4, 19671968. U.S. Coast Guard Oceanographic Report No. 43, CG-373-43.

Stommel, H. (1966) The Gulf Stream, a physical and dynamical description. University of California Press, Berkely and Los Angeles, $248 \mathrm{pp}$.

Sverdrup, H. U., M. W. Johnson, and R. H. Fleming (1942) The Oceans: Their Chemistry, Physics and General Biology Prentice-Hall, New York, 1087 pp

U.S. Naval Oceanographic Office (1967) Oceanographic Atlas of the North Atlantic Ocean, Section II Physical Properties. Publication No. 700, pt. 2, 300 pp. 


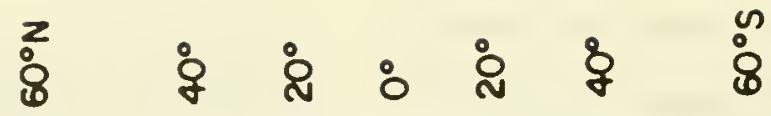

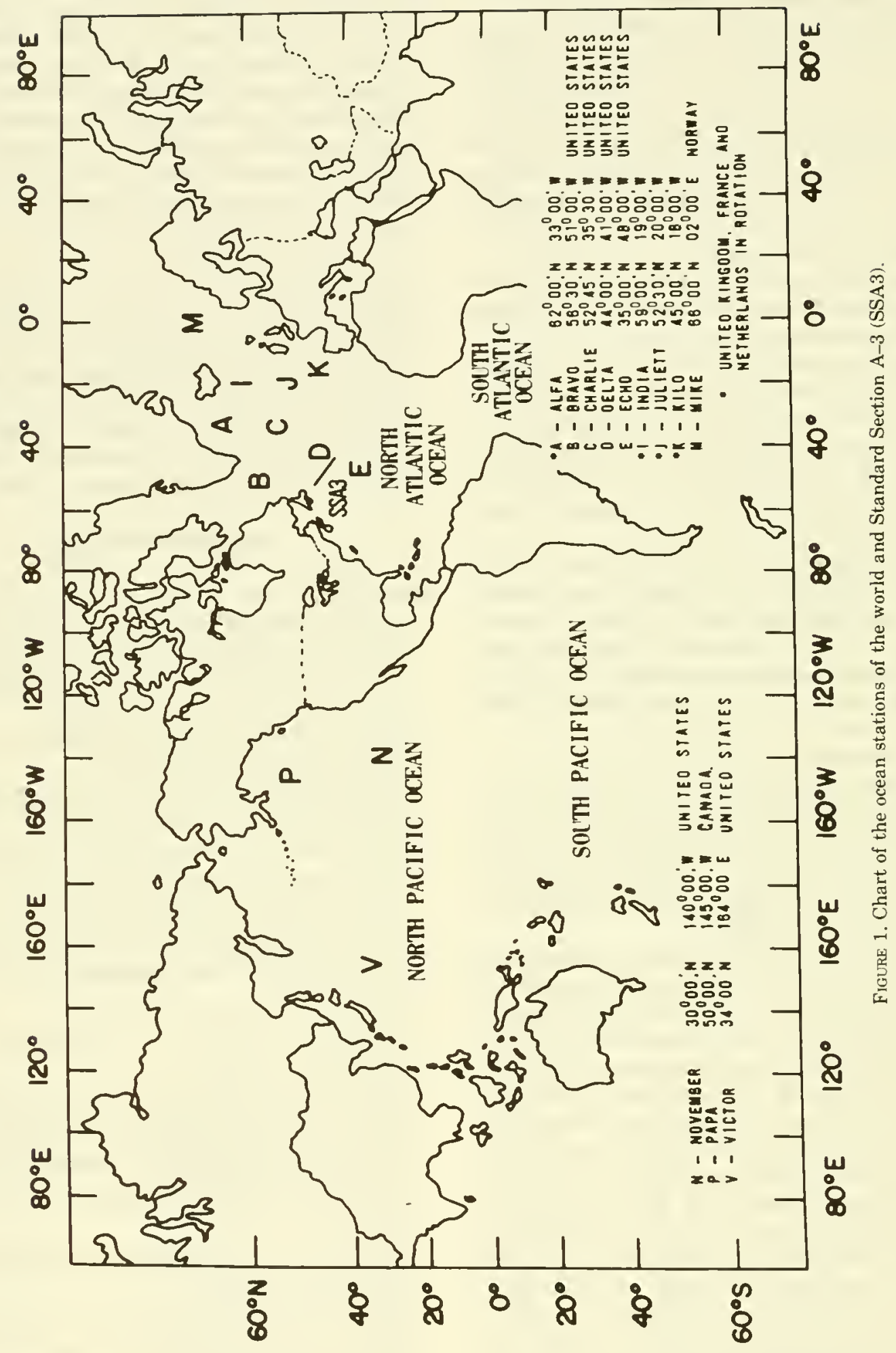




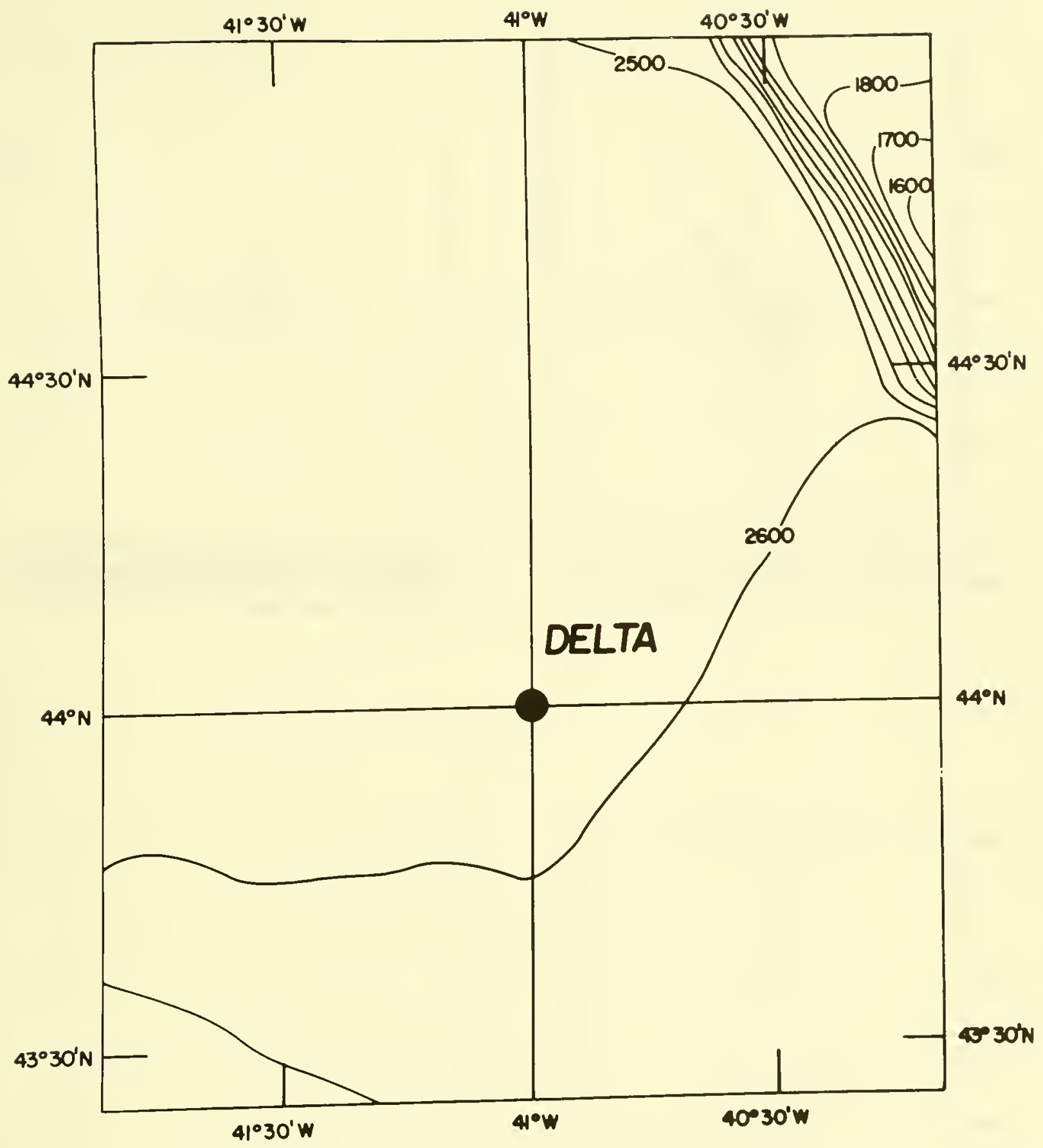

Figure 2. Bathymetry (in fathoms) of Ocean Station DELTA (from HO chart BC0508N). 

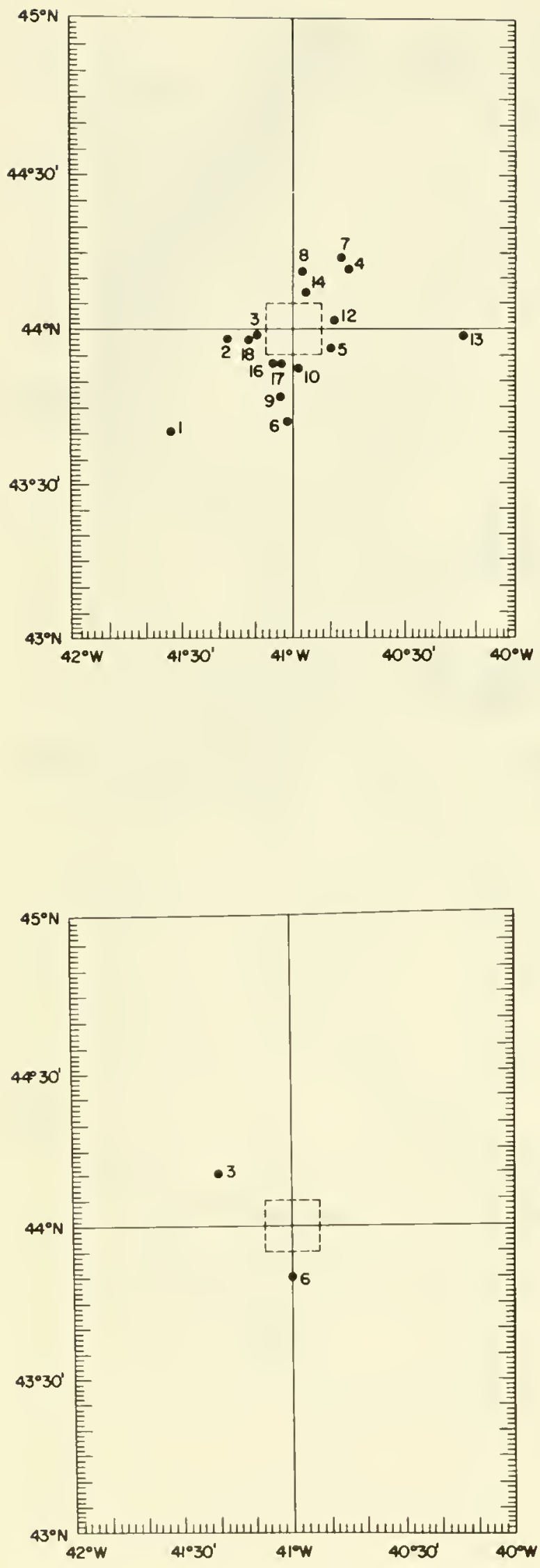

FIgURE 3. Positions of oceanographic stations taken by USCGC INGHAM at Ocean Station DELTA, 27 August-16 September 1967. Stations taken inside the 10-mile-square central grid are not shown.
Figure 4. Positions of oceanographic stations taken by USCGC CHINCOTEAGUE at Ocean Station DELTA, 22 September-10 October 1967. Stations taken inside the 10mile-square central grid are not shown. 

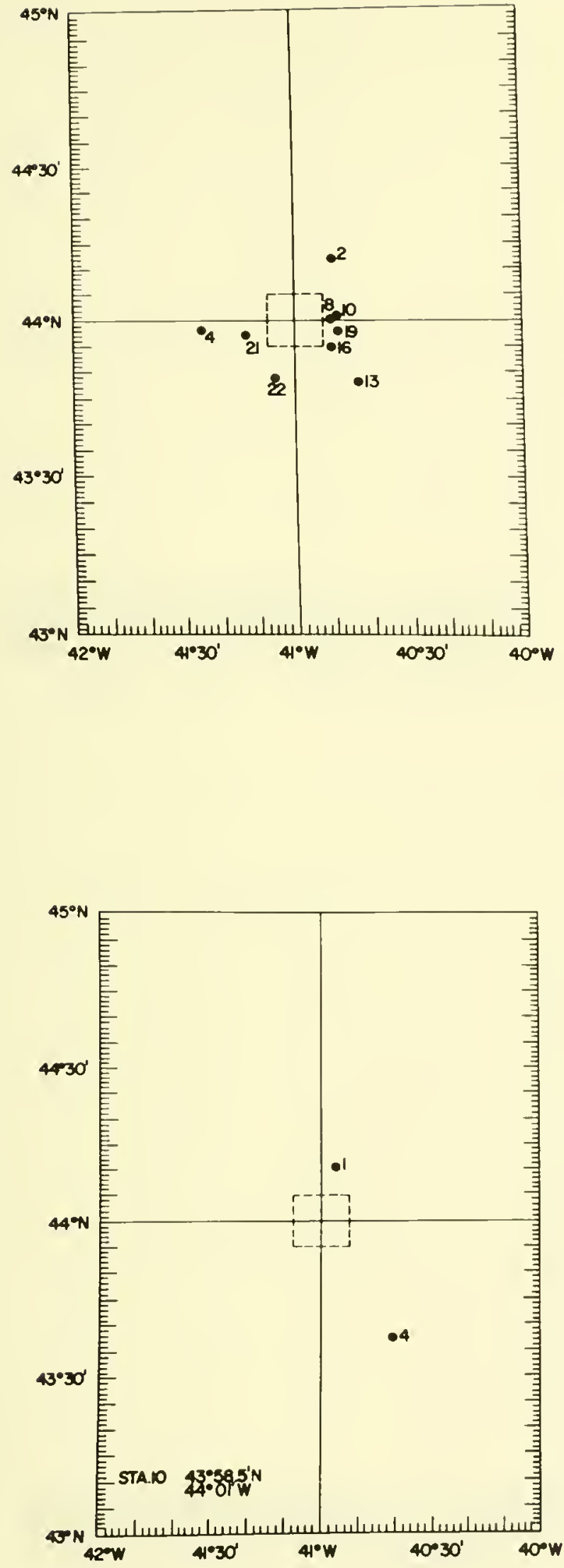

FlGURE 5. Positions of oceanographic stations taken by USCGC ESCANABA at Ocean Station DELTA, 12 October-3 November 1967. Stations taken inside the 10-mile-square central grid are not shown.
Figure 6. Positions of oceanographic stations taken by USCGC OWASCO at Ocean Station DELTA, 30 November-15 December 1967. Stations taken inside the 10-mile-square central grid are not shown. 


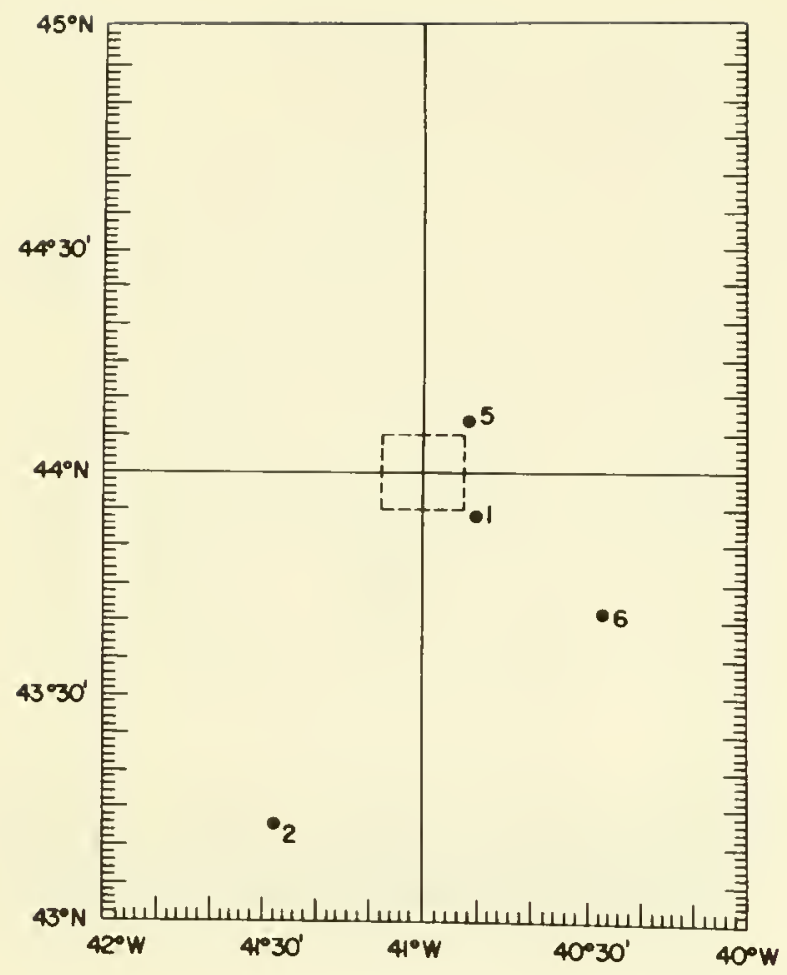

FIGURE 7. Positions of oceanographic stations taken by USCGC ABSECON at Ocean Station DELTA, 13 January-30 January 1968. Stations taken inside the 10-mile-square central grid are not shown.

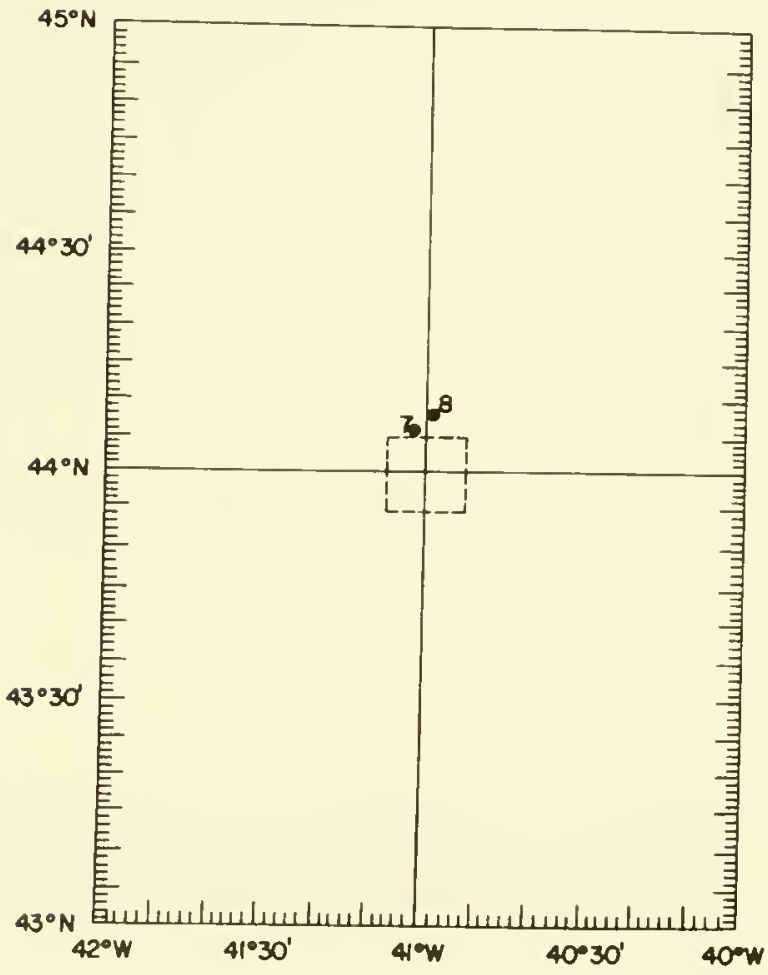

FIGURE 8. Positions of oceanographic stations taken by USCGC CASCO at Ocean Station DELTA, 28 February-19 March 1968. Stations taken inside the 10-mile-square central grid are not shown. 

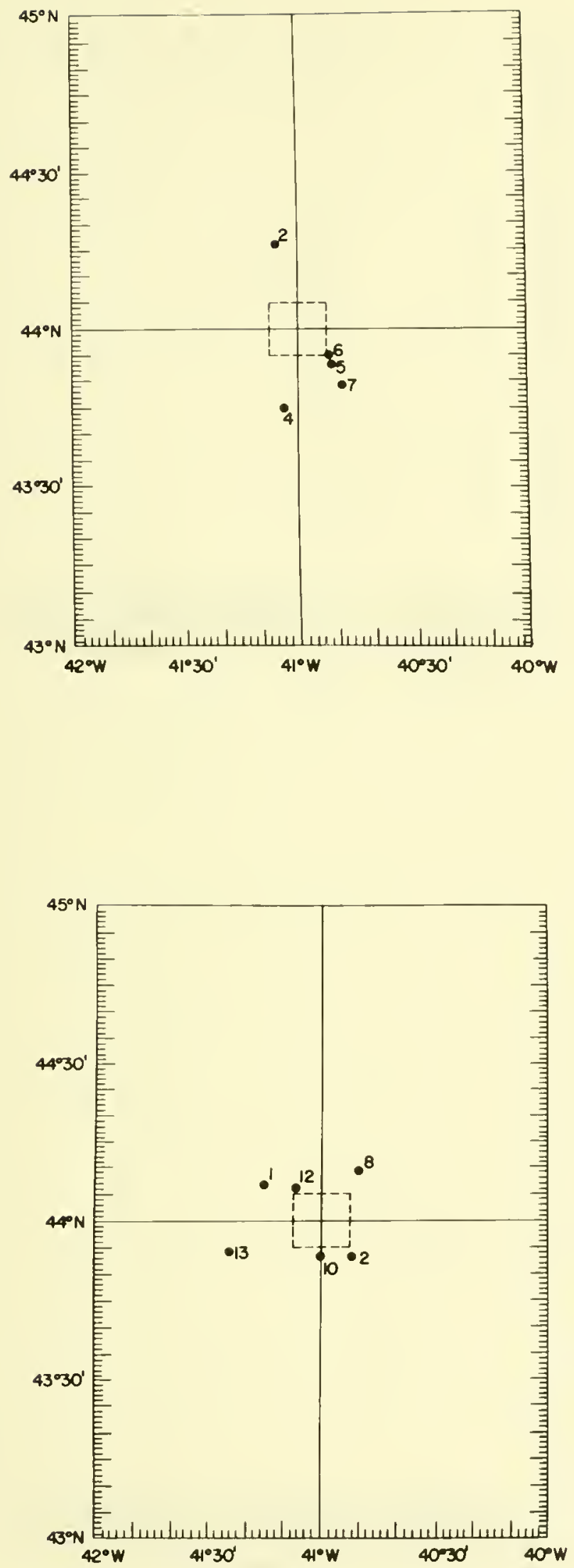

Figure 9. Positions of oceanographic stations taken by USCGC ABSECON at Ocean Station DELTA, 20 March-8 April 1968. Stations taken inside the 10-mile-square central grid are not shown.
Figure 10. Positions of oceanographic stations taken by USCGC COOK INLET at Ocean Station DELTA, 14 April-5 May 1968. Stations taken inside the 10-milesquare central grid are not shown. 

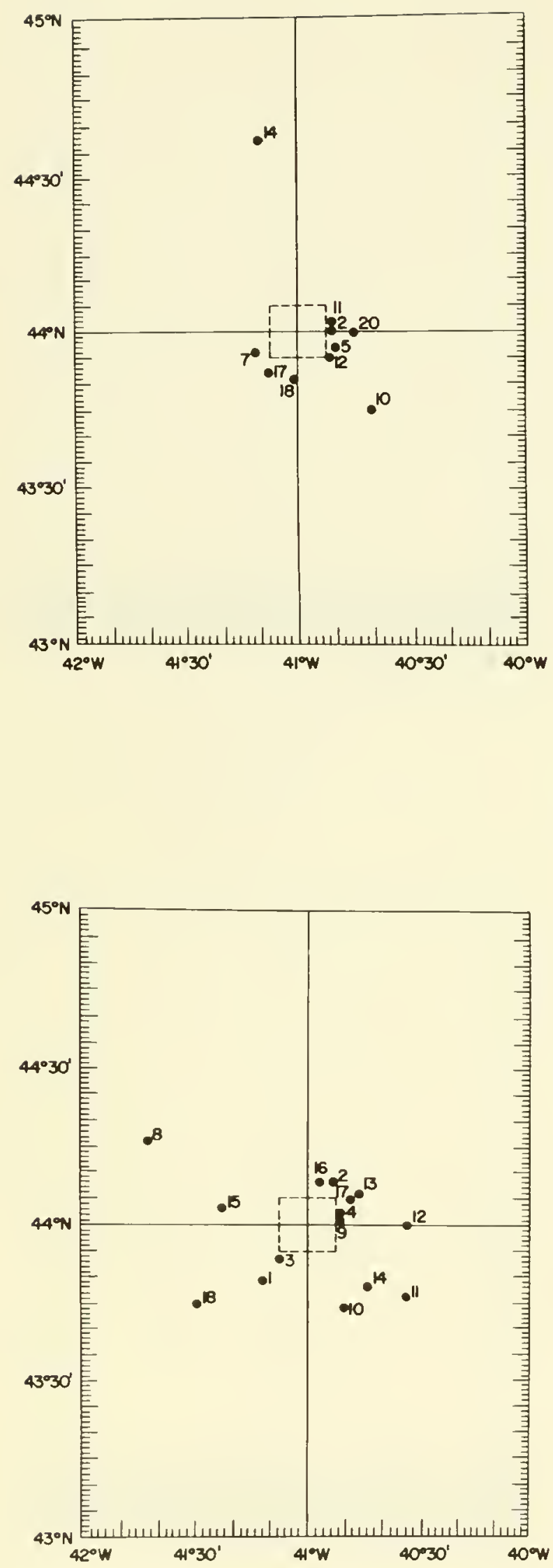

Figure 11. Positions of oceanographic stations taken by USCGC DALLAS at Ocean Station DELTA, 6 May-28 May 1968. Stations taken inside the 10-mile-square central grid are not shown.
Figure 12. Positions of oceanographic stations taken by USCGC CASTLE ROCK at Ocean Station DELTA, 31 May-19 June 1968. Stations taken inside the 10-milesquare central grid are not shown. 

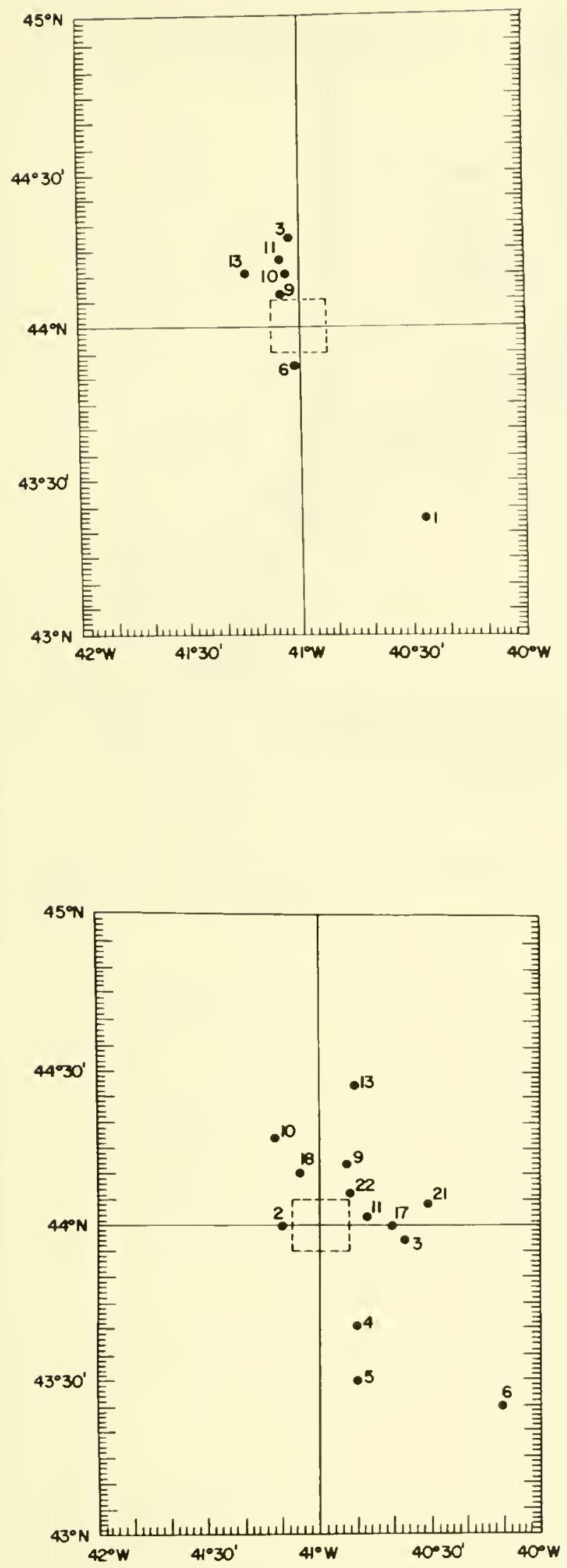

Flgure 13. Positions of oceanographic stations taken by USCGC HAMILTON at Ocean Station DELTA, 6 July-9 July 1968. Stations taken inside the 10-mile-square central grid are now shown.
Figure 14. Positions of oceanographic stations taken by USCGC DALLAS at Ocean Station DELTA, 13 July-4 August 1968. Stations taken inside the 10-mile-square central grid are not shown. 


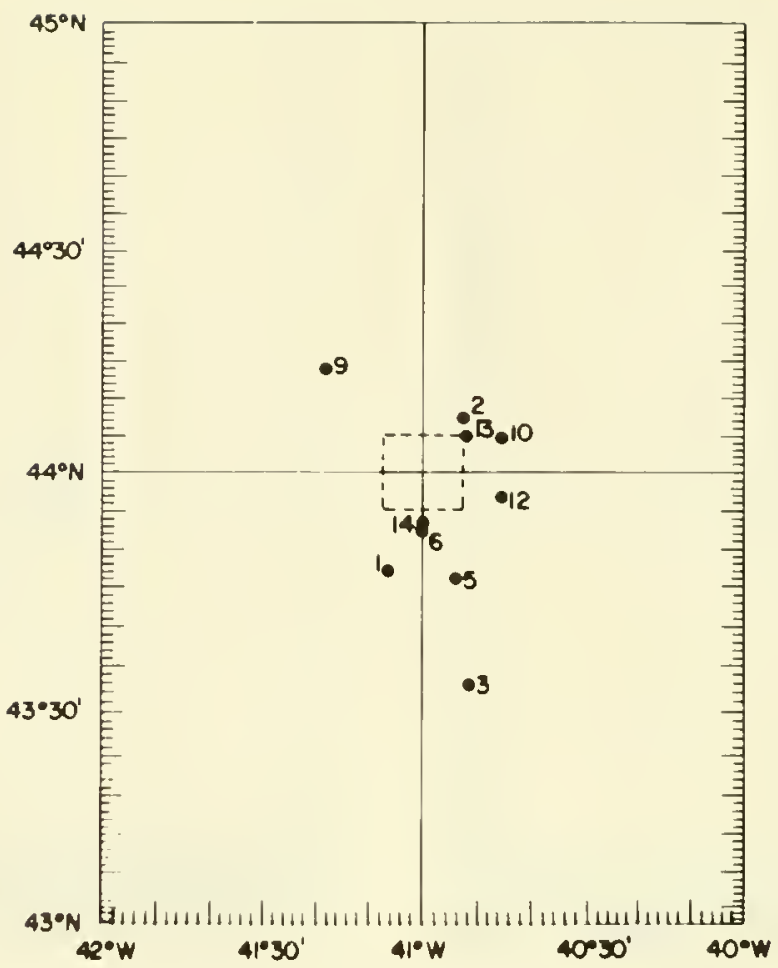

Figure 15. Positions of oceanographic stations taken by USCGC COOK INLET at Ocean Station DELTA, 5 August-25 August 1968. Stations taken inside the 10mile-square central grid are not shown. 


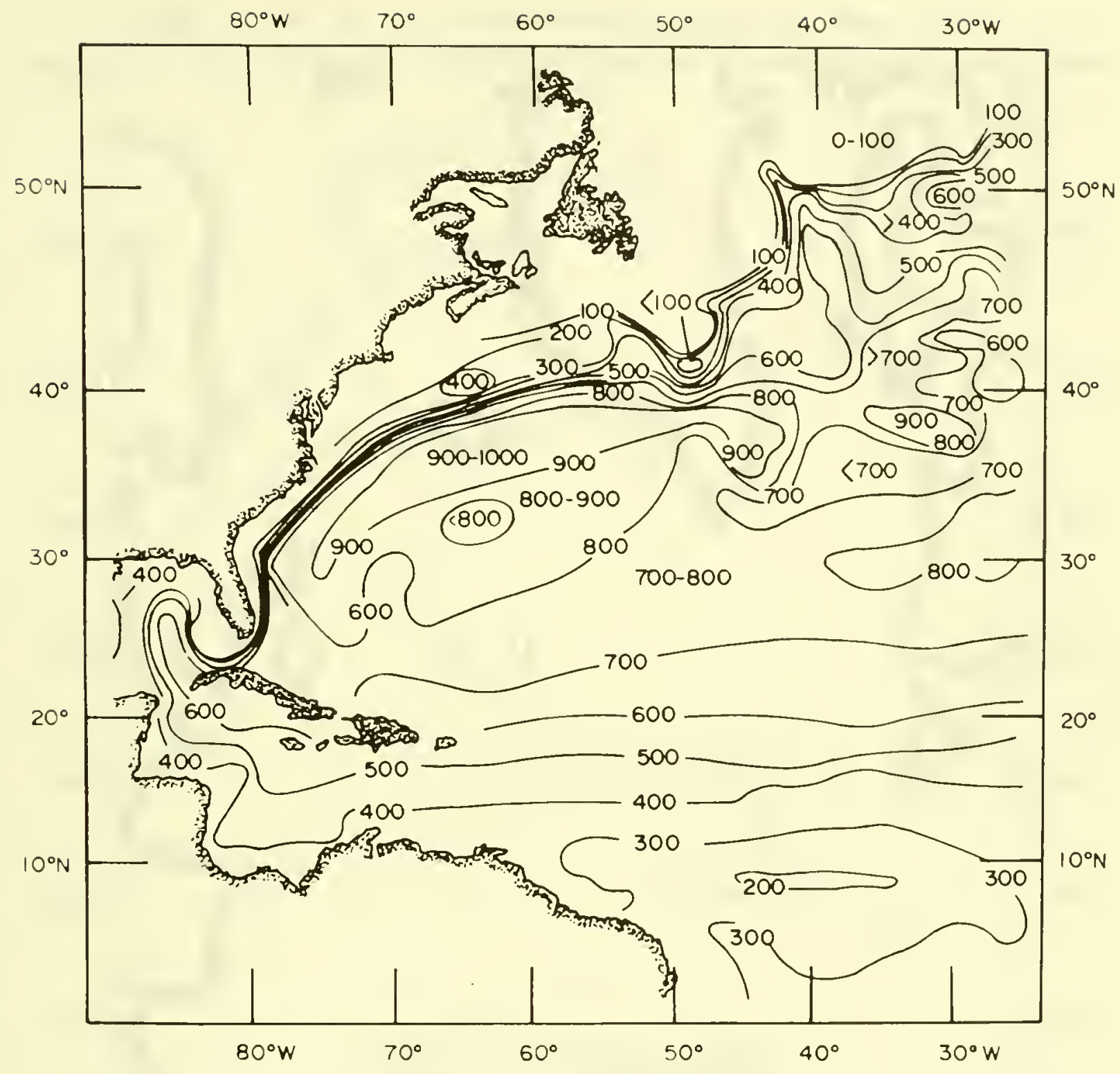

Figure 16. Depth of $10^{\circ} \mathrm{C}$ isothermal surface, according to Iselin (1936, fig. 47). Depths are given in meters. 


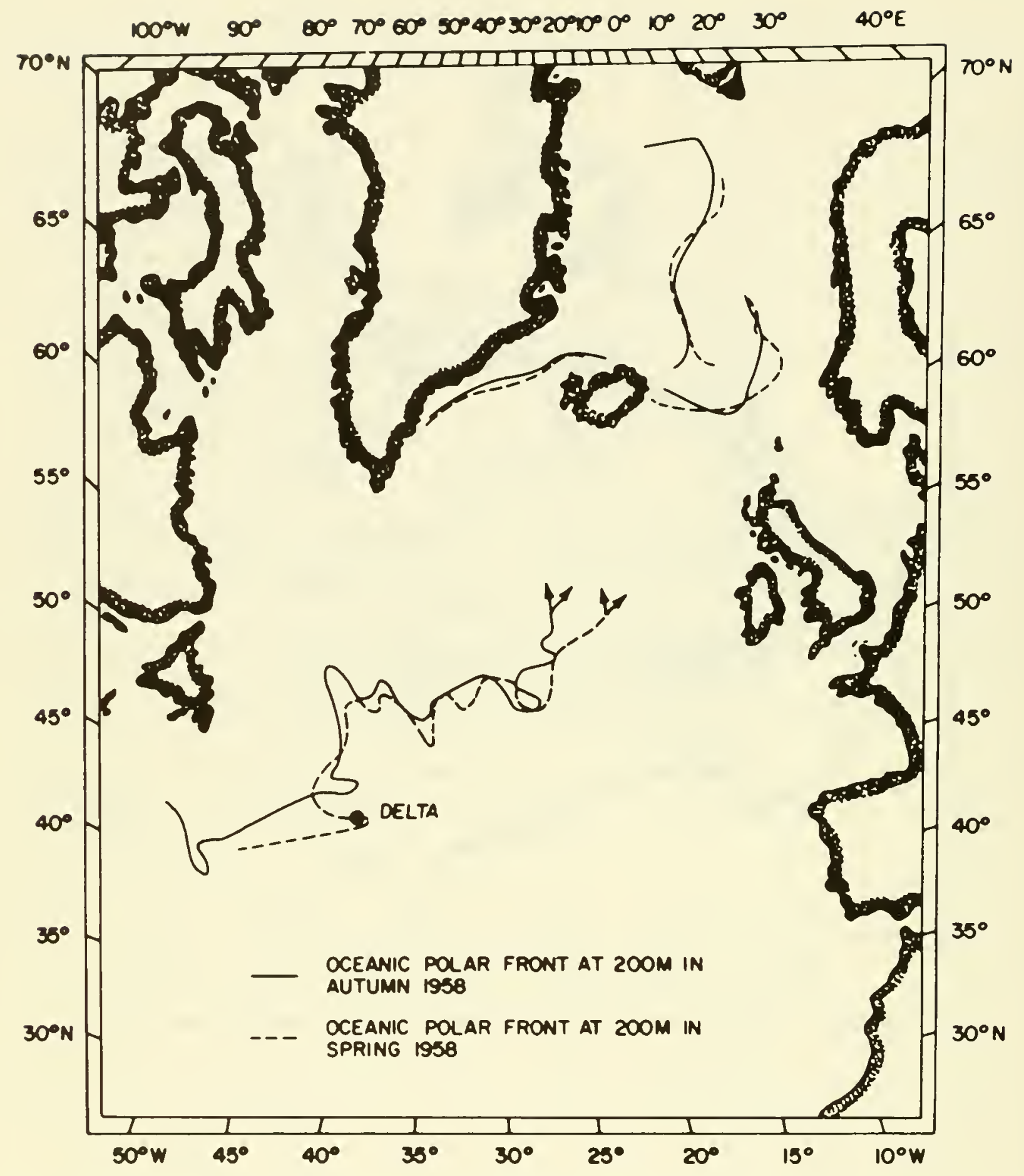

Figure 17. The Oceanic Polar Front in the North Atlantic at $200 \mathrm{~m}$ depth in autumn and spring 1958, according to Dietrich (1964, fig. 8). 


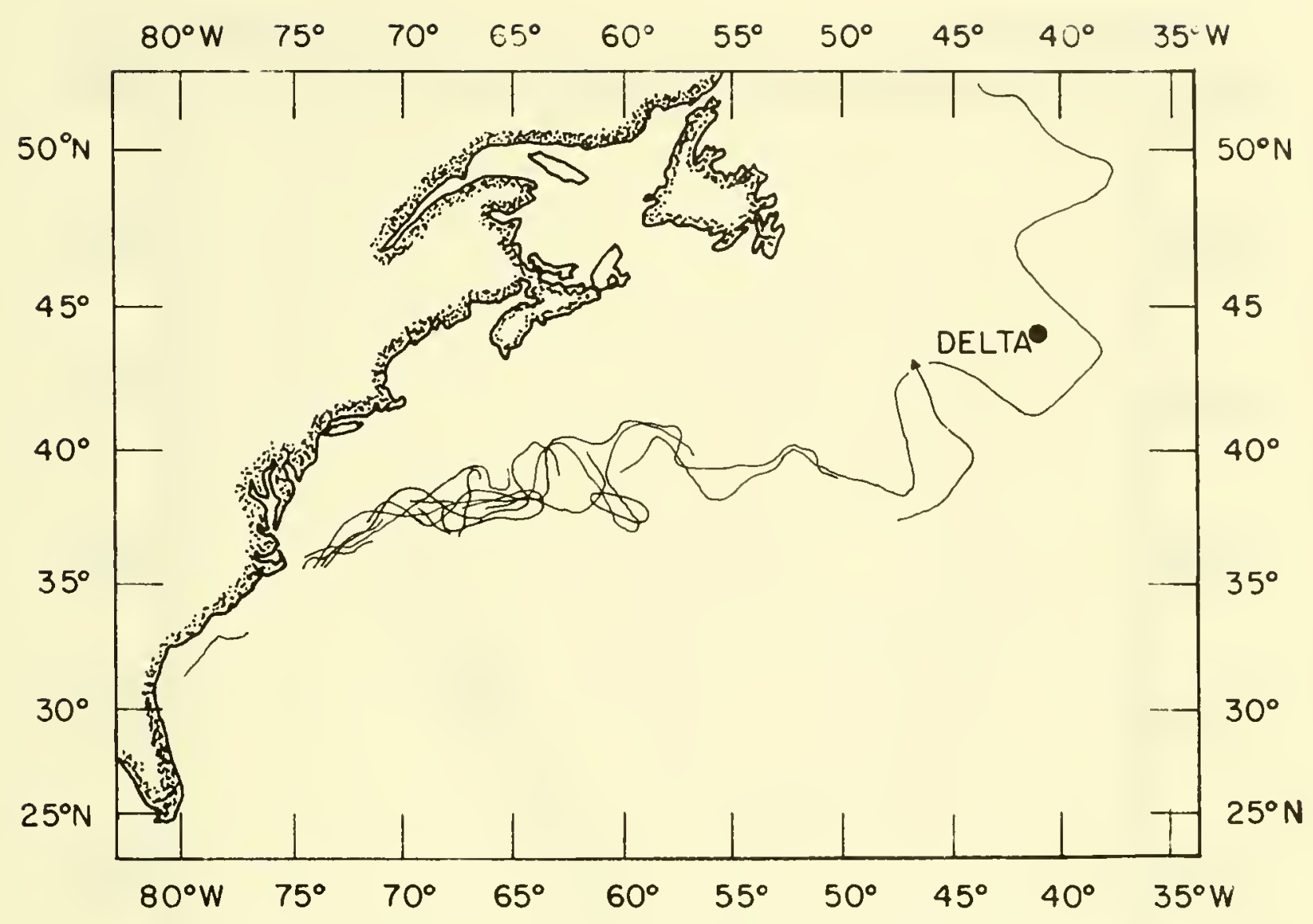

FIGURE 18. Fluctuations of North Atlantic current as determined by maximum cross current temperature gradients (after Fuglister and Worthington, 1951). 
TEMPERATURE $\left({ }^{\circ} \mathrm{C}\right)$

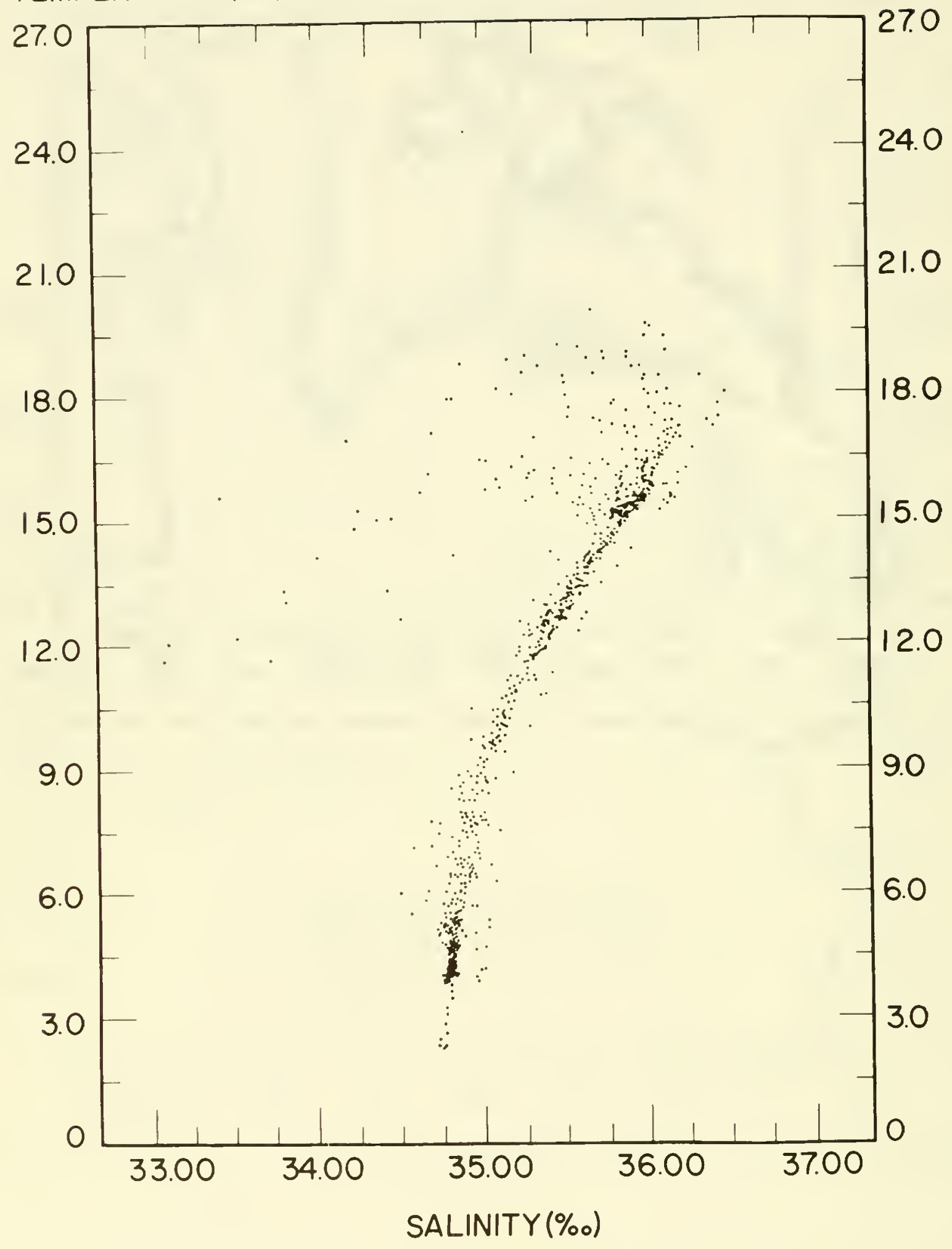

FIgURE 19. Temperature-Salinity diagram at Ocean Station DELTA for period 27 August to 3 November 1967. 
TEMPERATURE $\left({ }^{\circ} \mathrm{C}\right)$

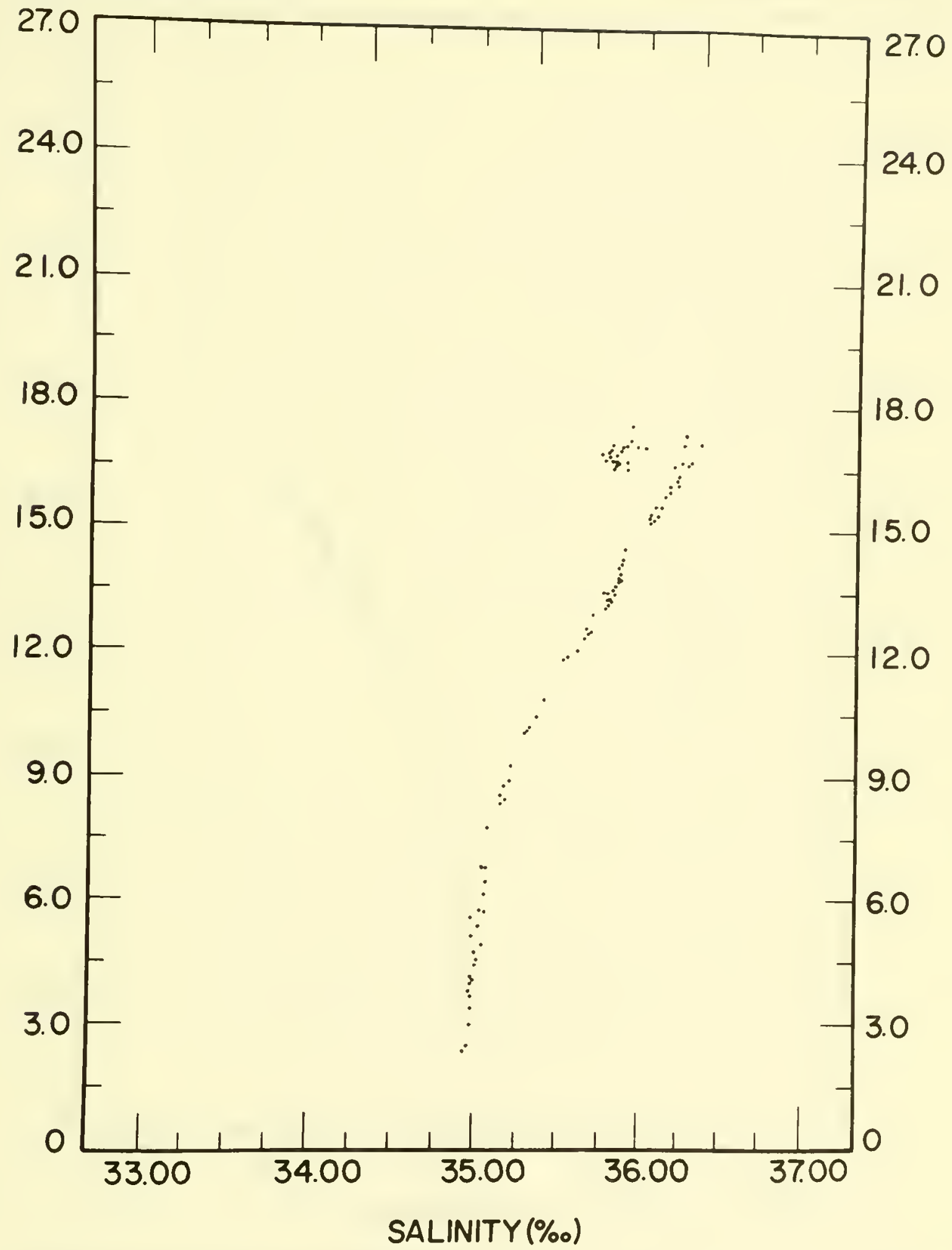

FIgURE 20. Temperature-Salinity diagram at Ocean Station DELTA for period 30 November to 15 December 1967. 


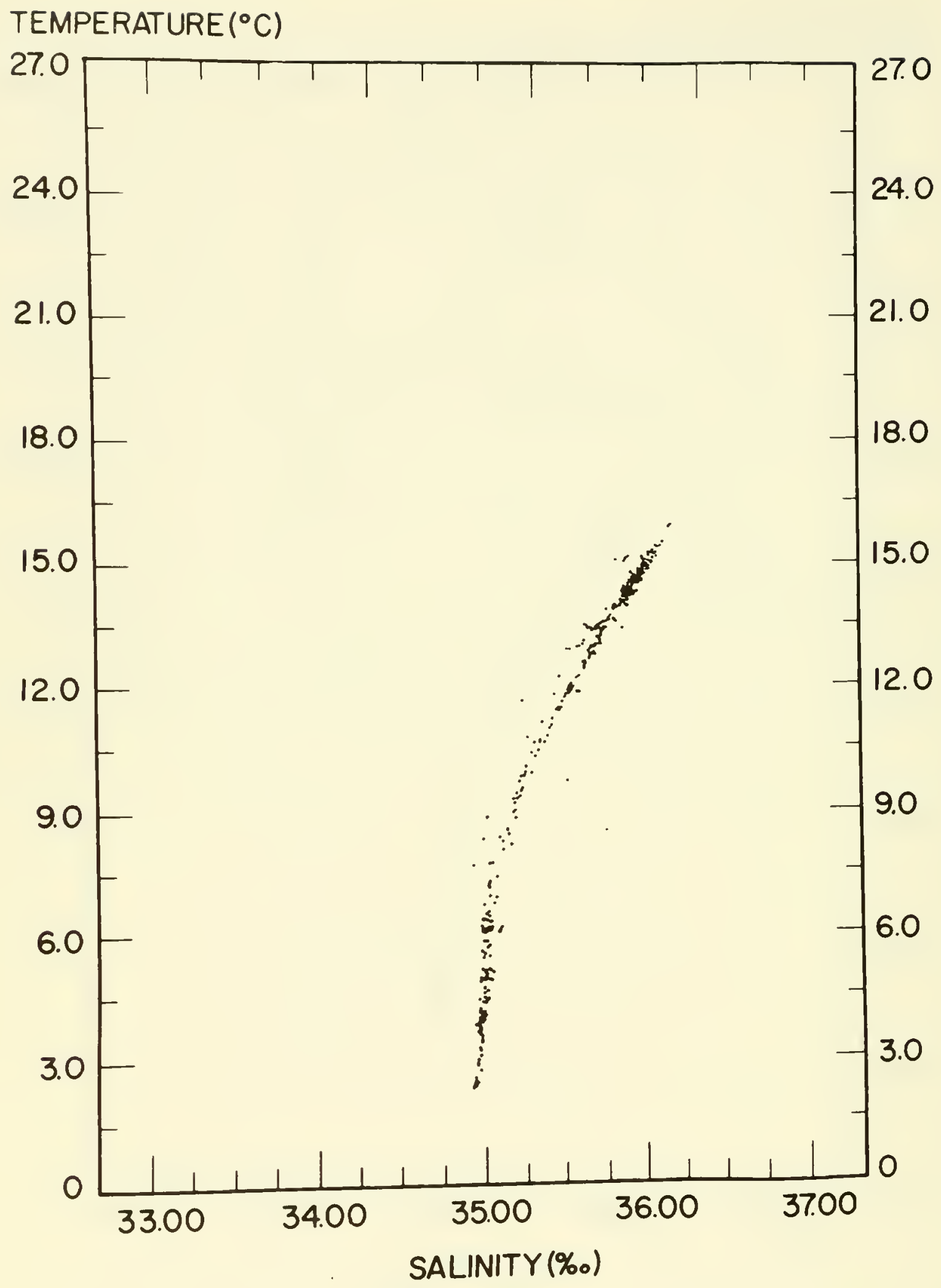

Figure 21. Temperature-Salinity diagram at Ocean Station DELTA for period 13 January to 8 April 1968. 
TEMPERATURE $\left({ }^{\circ} \mathrm{C}\right)$

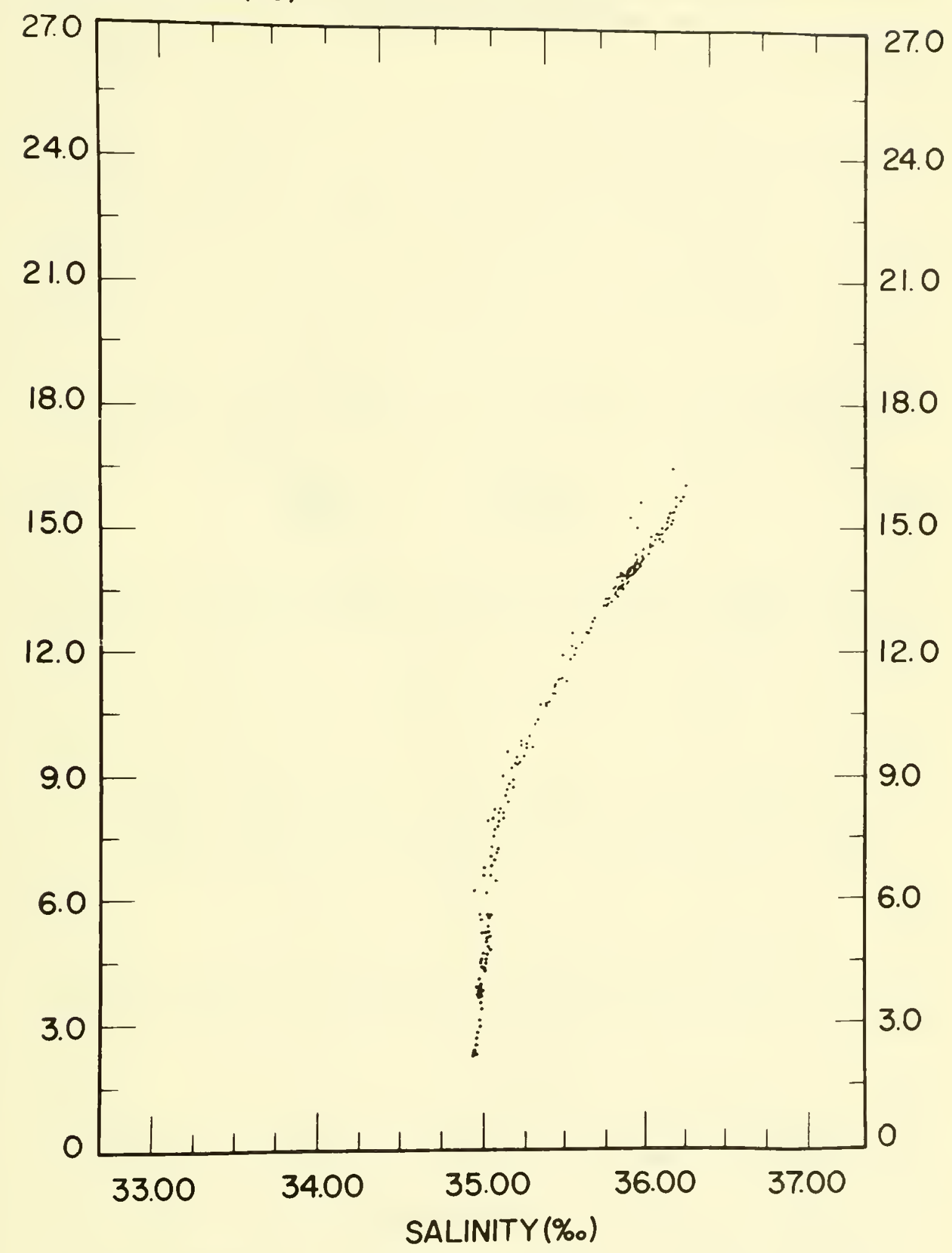

Figure 22. Temperature-Salinity diagram at Ocean Station DELTA for period 14 April to 19 June 1968. 
TEMPERATURE $\left({ }^{\circ} \mathrm{C}\right)$

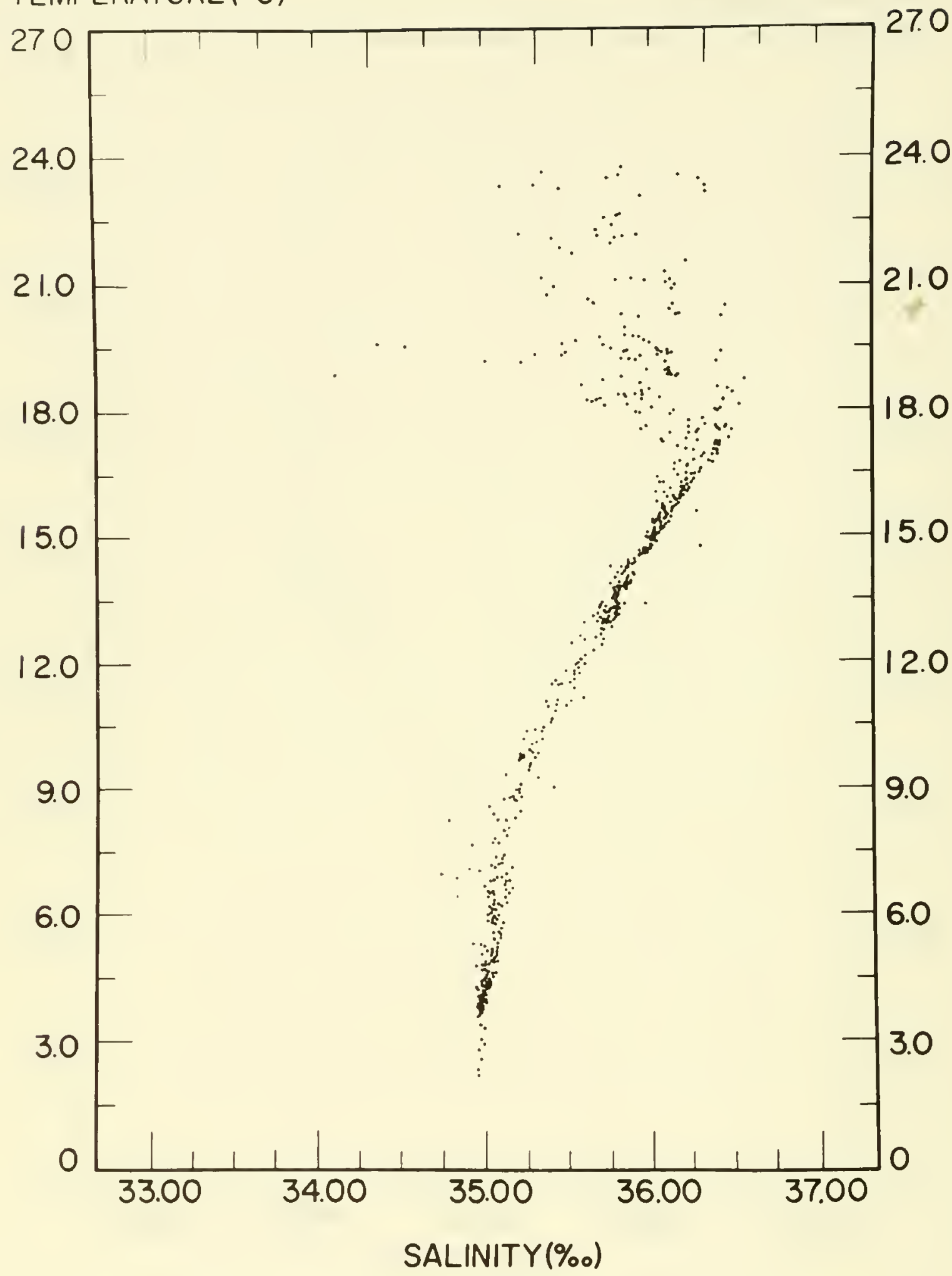

FIGURE 23. Temperature-Salinity diagram at Ocean Station DELTA for period 6 July to 25 August 1968 . 
TENPERAIURE 'C

TEMPERATURE 'C
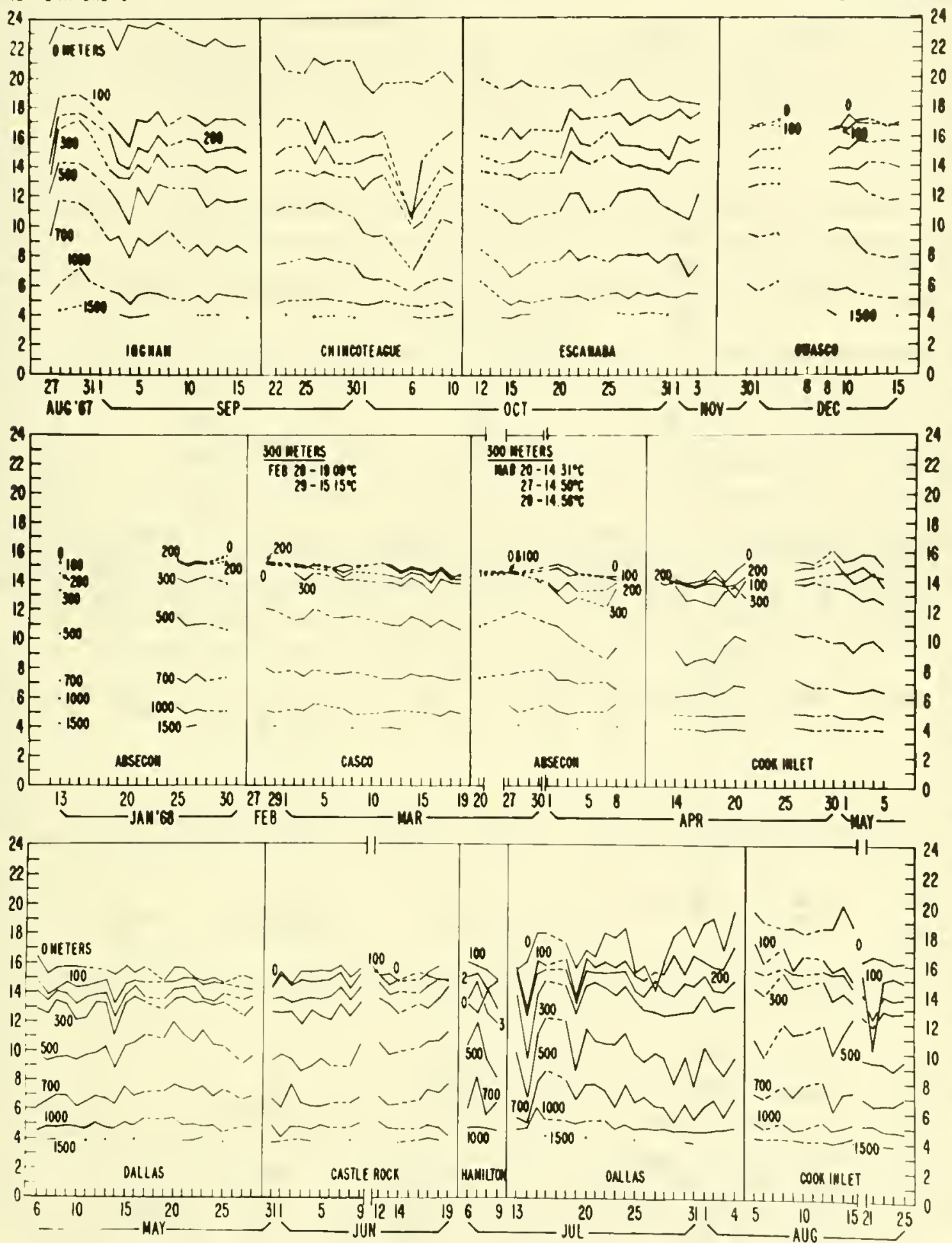

Flgure 24. Temperature versus time at selected depths at OS DELTA, 27 August 1967 to 25 August 1968. 


\section{SALINITY VERSUS TIME AT OCEAN STATION DELTA 27 AUGUST 1967 - 25 AUGUST 1968}

SALINITY \%

SALINITYY
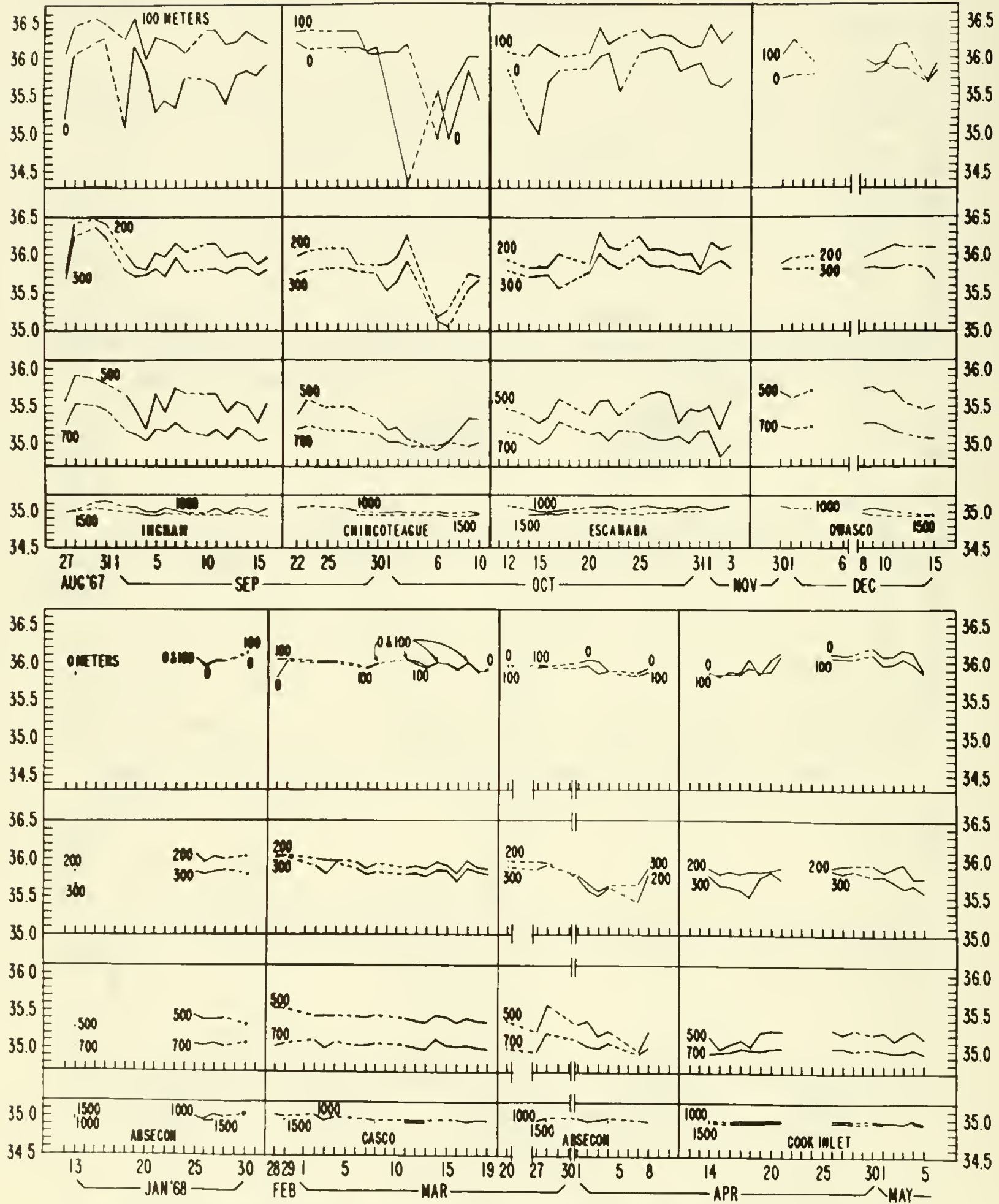

Figure 25a. Salinity versus time at selected depths at OS DELTA, 27 August 1967 to 5 May 1968. 


\section{SALINITY VERSUS TIME AT OCEAN STATION DELTA (CONT'D)
27 AUGUST 1967 - 25 AUGUST I968 SALINITY VERSUS TIME AT OCEAN STATION DELTA (CONT'D)
27 AUGUST 1967 - 25 AUGUST I968}

\section{SALINITY \%}

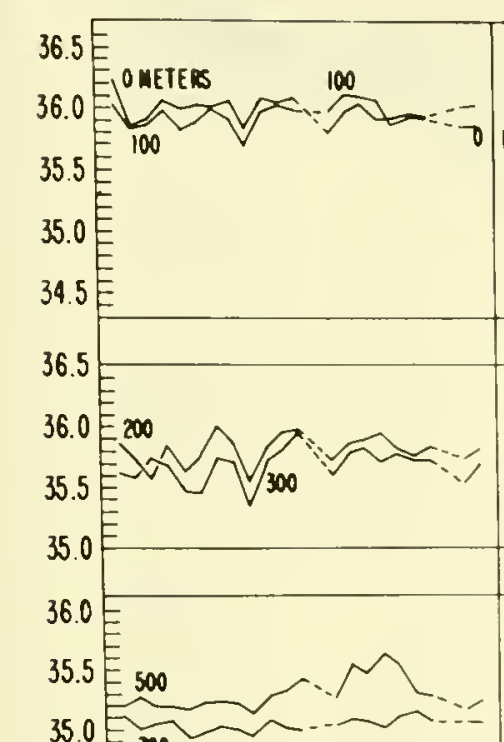

35.5 E

35.0

$34.5 \mathrm{E}$

\section{5}

36.0 E 200

\section{5}

35

36.0

\section{5}

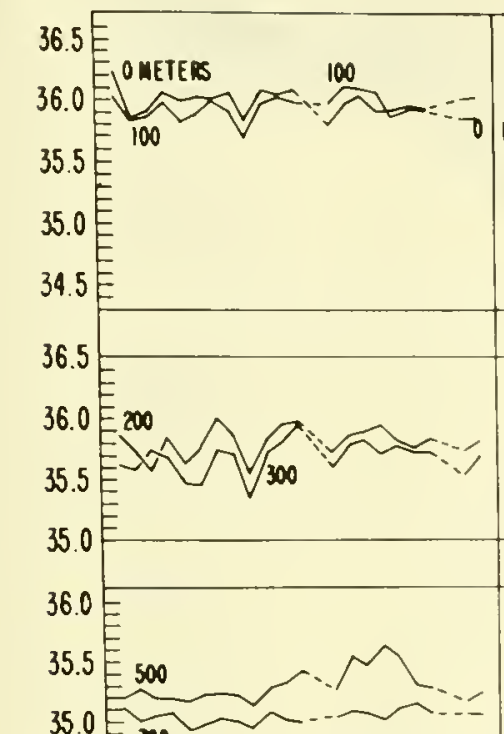

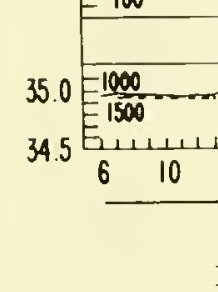
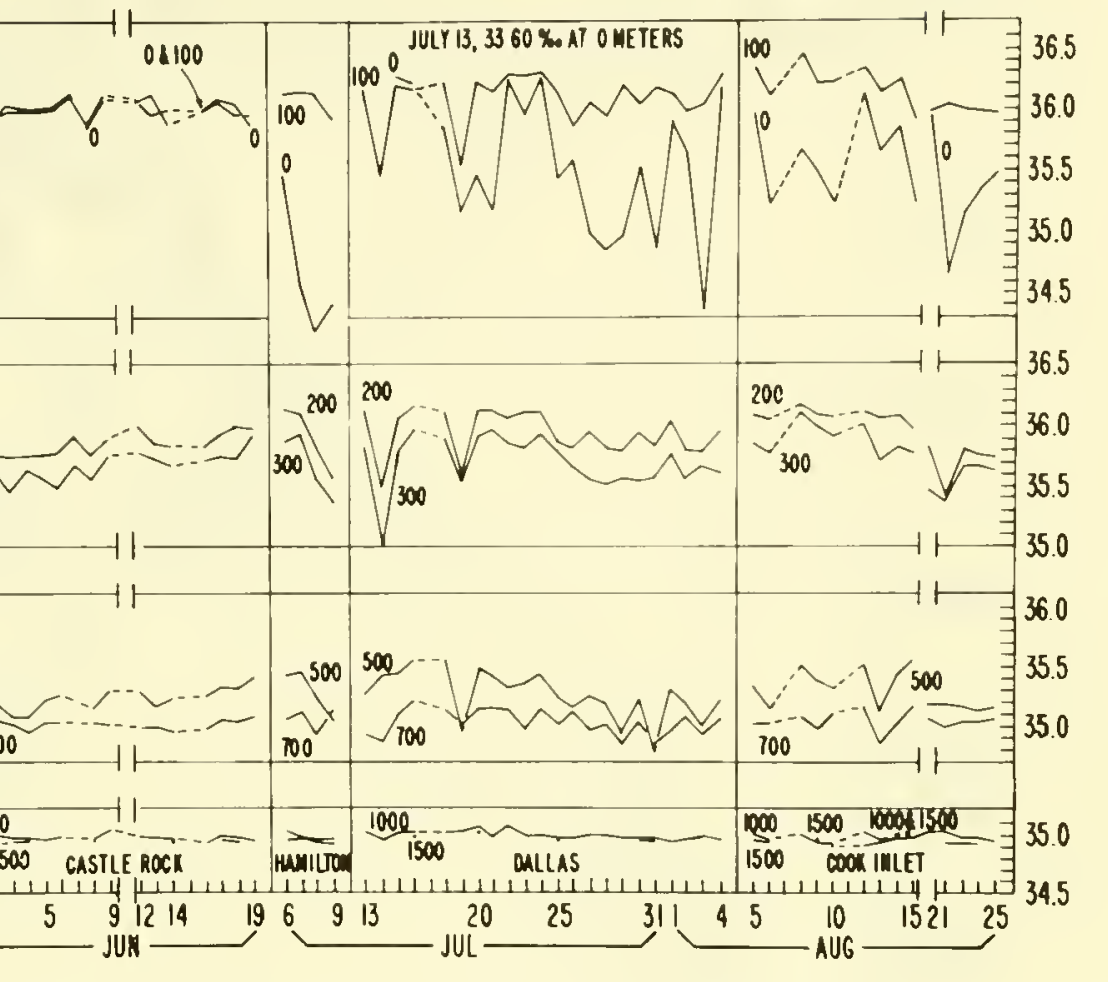

\section{SALINITY\%o}

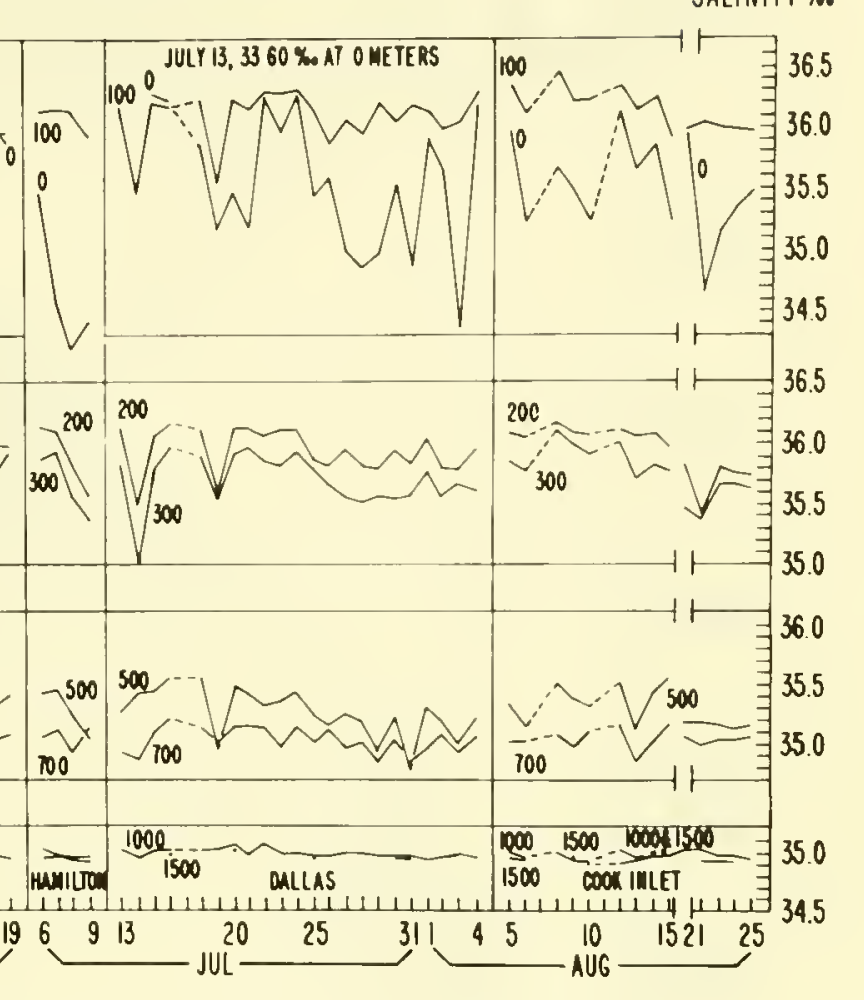

Figure 25b. Salinity versus time at selected depths at OS DELTA, 6 May to 25 August 1968.
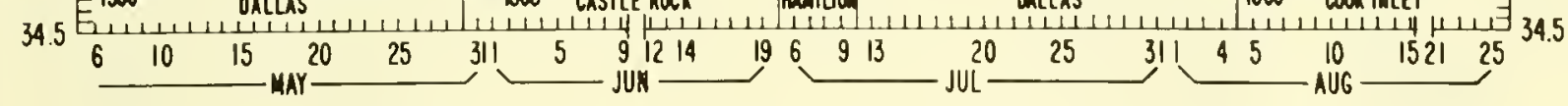

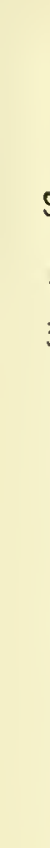
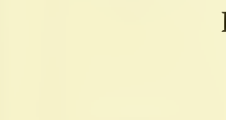

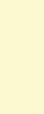




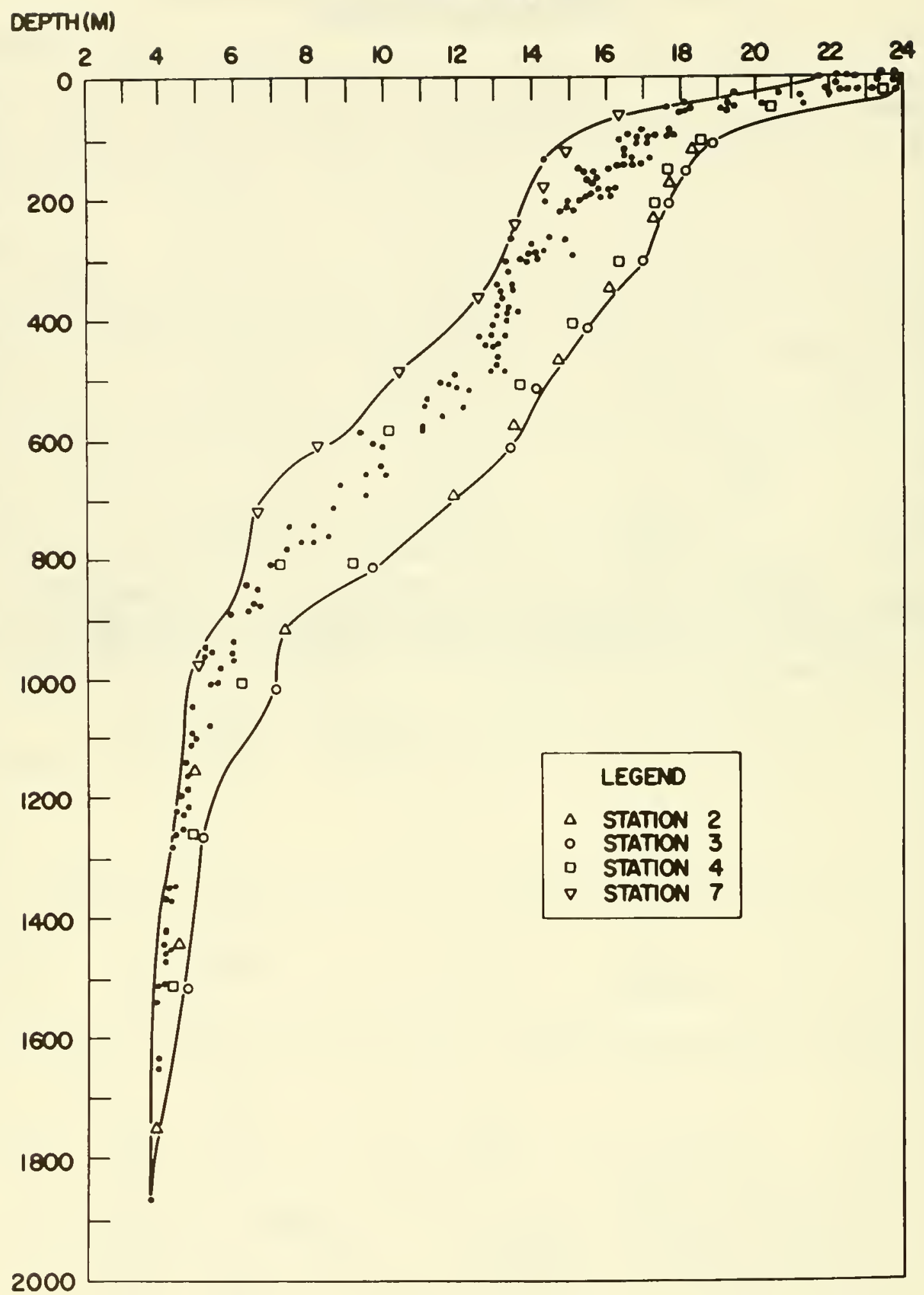

Figure 26. Envelope and plot of temperature $\left({ }^{\circ} \mathrm{C}\right)$ versus depth $(\mathrm{m})$ at Ocean Station DELTA, prepared from eighteen casts taken by USCGC INGHAM, 27 August-16 September 1967. 
TEMPERATURE $\left({ }^{\circ} \mathrm{C}\right)$

\section{DPTH (M)}

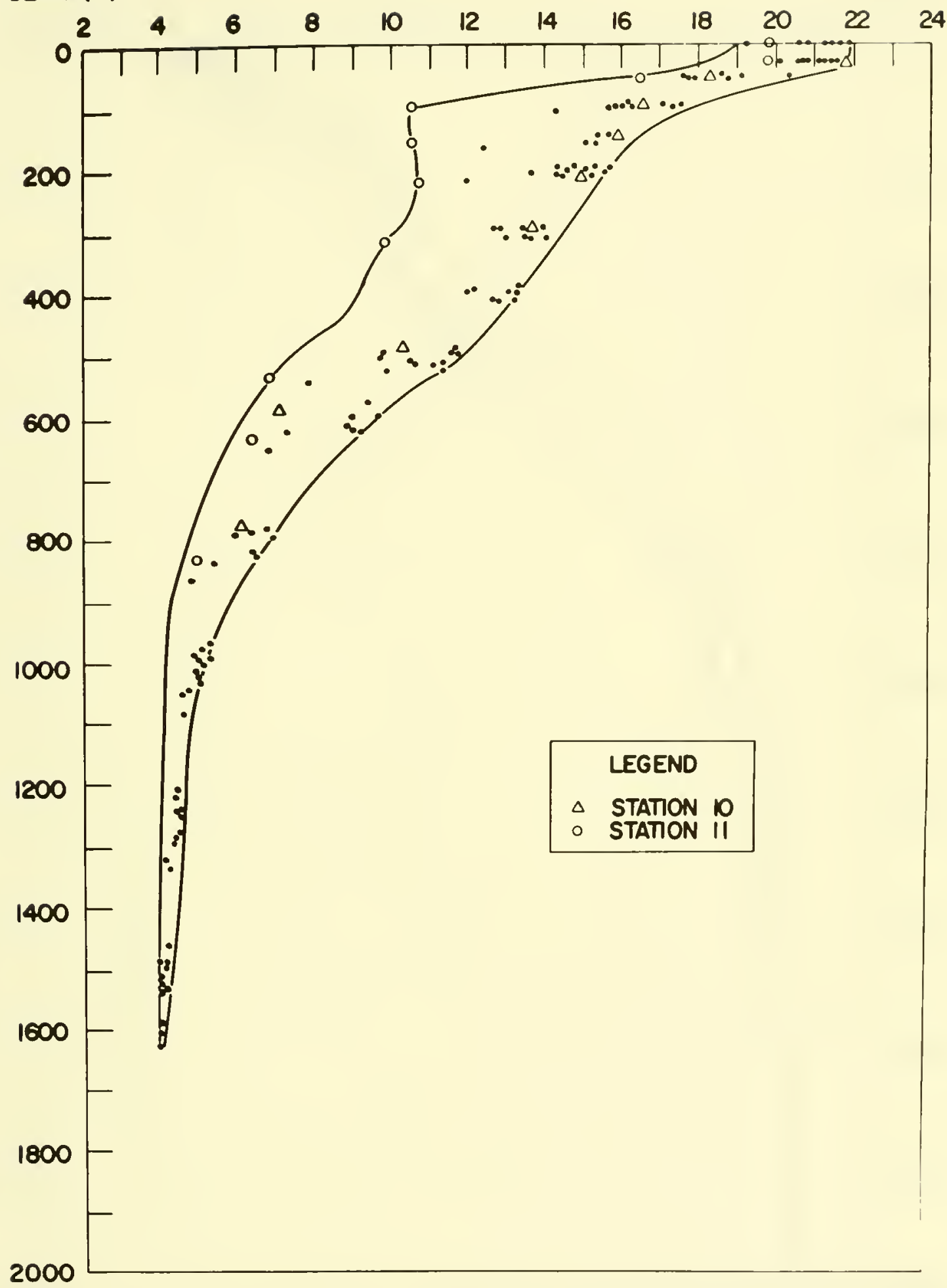

FIGURE 27. Envelope and plot of temperature $\left({ }^{\circ} \mathrm{C}\right)$ versus depth $(\mathrm{m})$ at Ocean Station DELTA, prepared from fourteen casts taken by IISCGC CHINCOTEAGUE, 22 September-10 October 1967. 
TEMPERATURE $\left({ }^{\circ} \mathrm{C}\right)$

\section{DEPTH(M)}

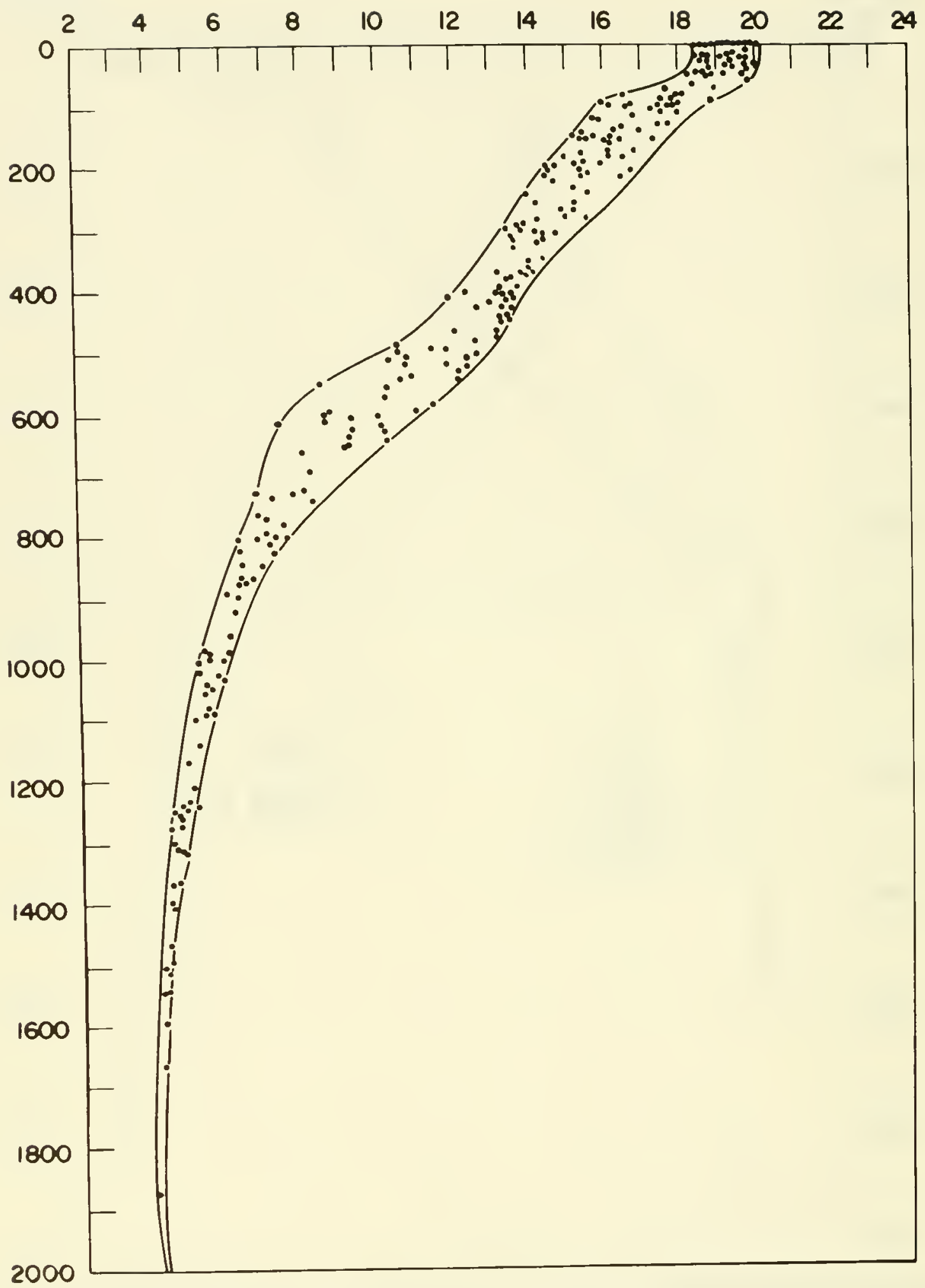

Figure, 28. Envelope and temperature $\left({ }^{\circ} \mathrm{C}\right)$ versus depth $(\mathrm{m})$ at Ocean Station DELTA, prepared from twenty-two casts taken by USCGC ESCANABA, 12 October-3 November 1967. 
TEMPERATURE $\left({ }^{\circ} \mathrm{C}\right)$

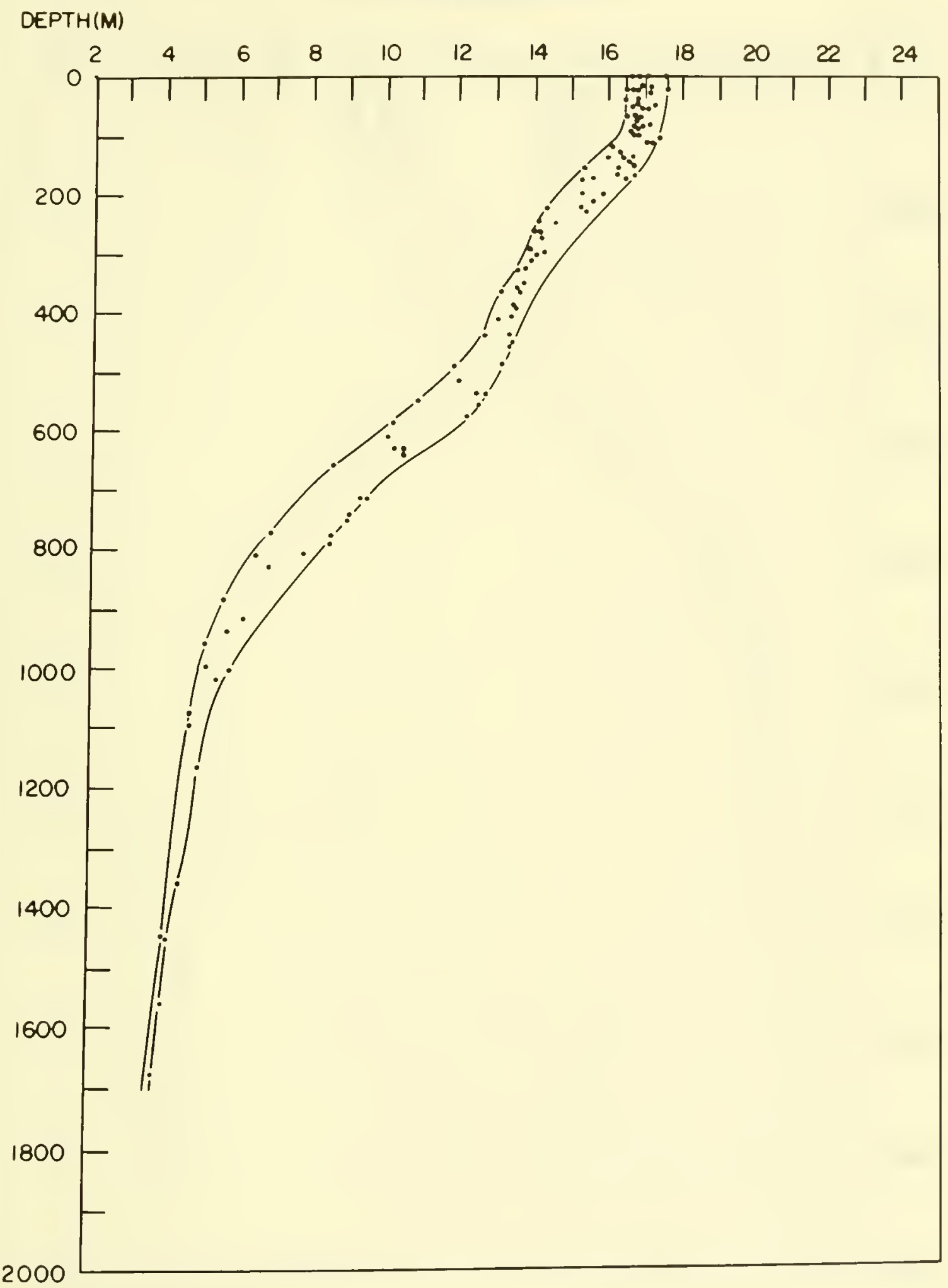

Figure 29. Envelope and plot of temperature $\left({ }^{\circ} \mathrm{C}\right)$ versus depth $(\mathrm{m})$ at Ocean Station DELTA, prepared from ten casts taken by USCGC OWASCO, 30 November-15 December 1967. 
TEMPERATURE $\left({ }^{\circ} \mathrm{C}\right)$

\section{DEPTH (M)}

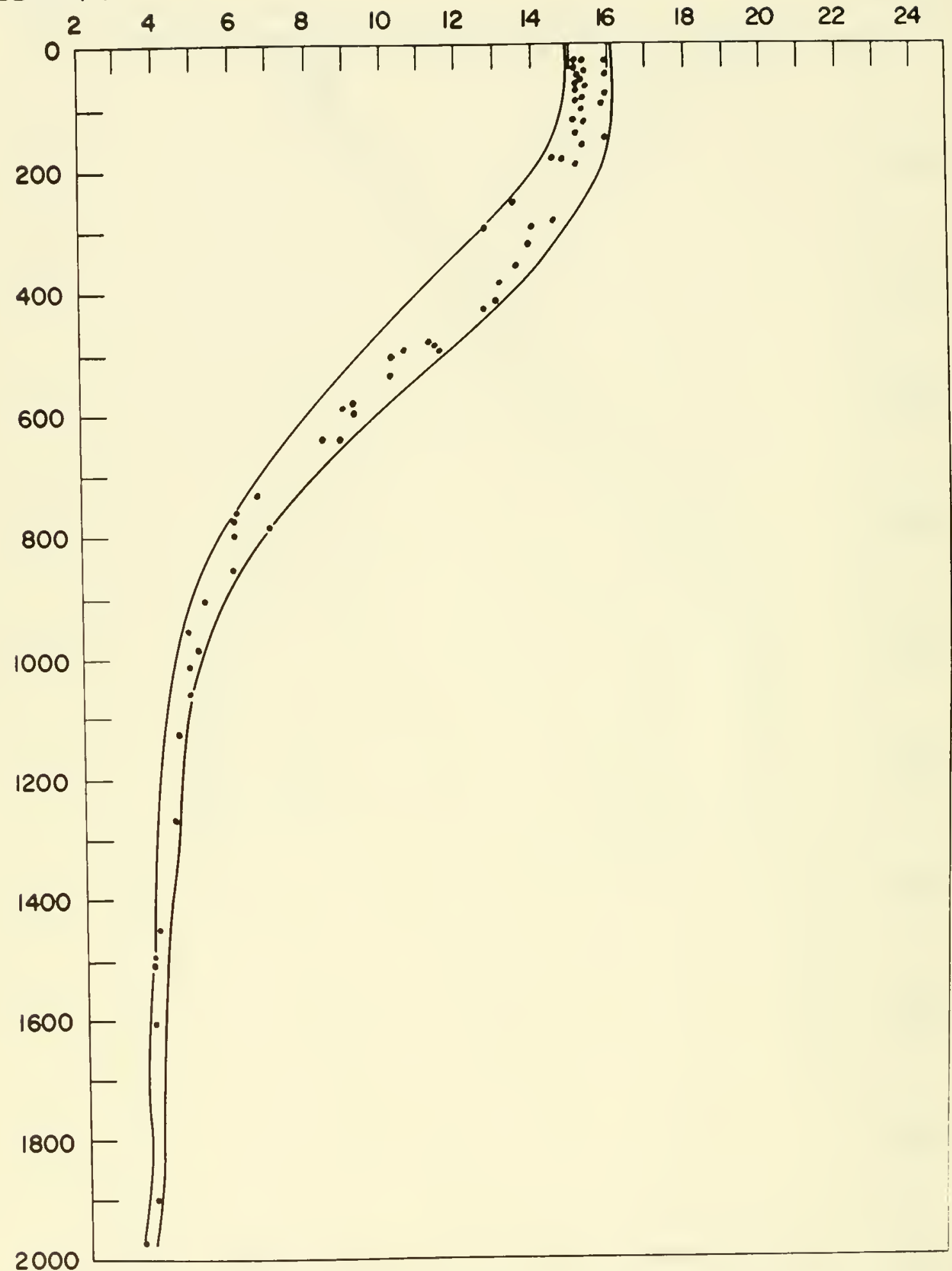

Figure 30. Envelope and plot of temperature $\left({ }^{\circ} \mathrm{C}\right)$ versus depth $(\mathrm{m})$ at Ocean Station DELTA, prepared from six casts taken by USCGC ABSECON, 13 January-30 January 1968. 
TEMPERATURE $\left({ }^{\circ} \mathrm{C}\right)$

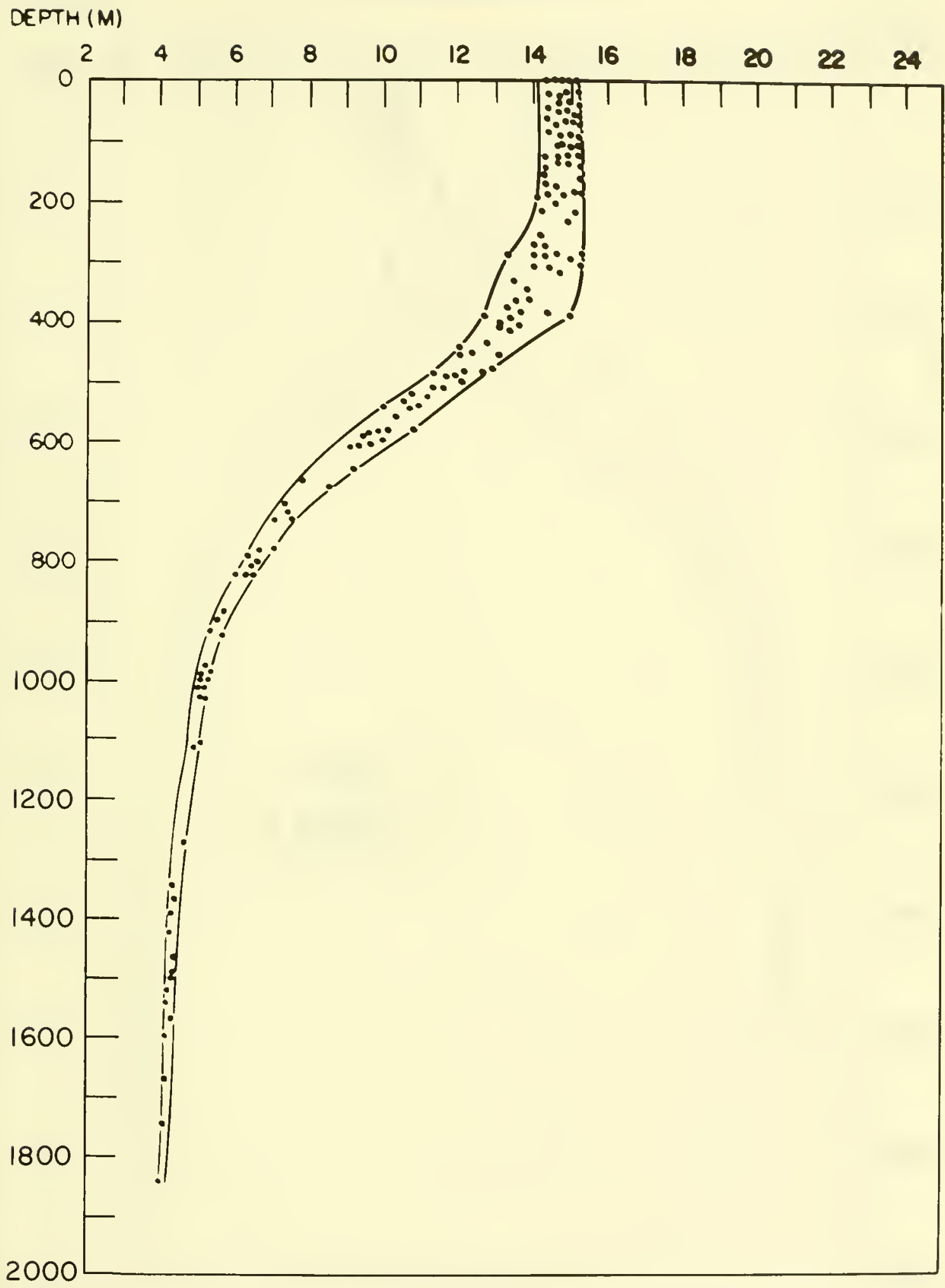

Figure 31. Envelope and plot of temperature $\left({ }^{\circ} \mathrm{C}\right)$ versus depth $(\mathrm{m})$ at Ocean Station DELTA, prepared from seventeen casts taken by USCGC CASCO, 28 February-19 March 1968. 
TEMPERATURE $\left({ }^{\circ} \mathrm{C}\right)$

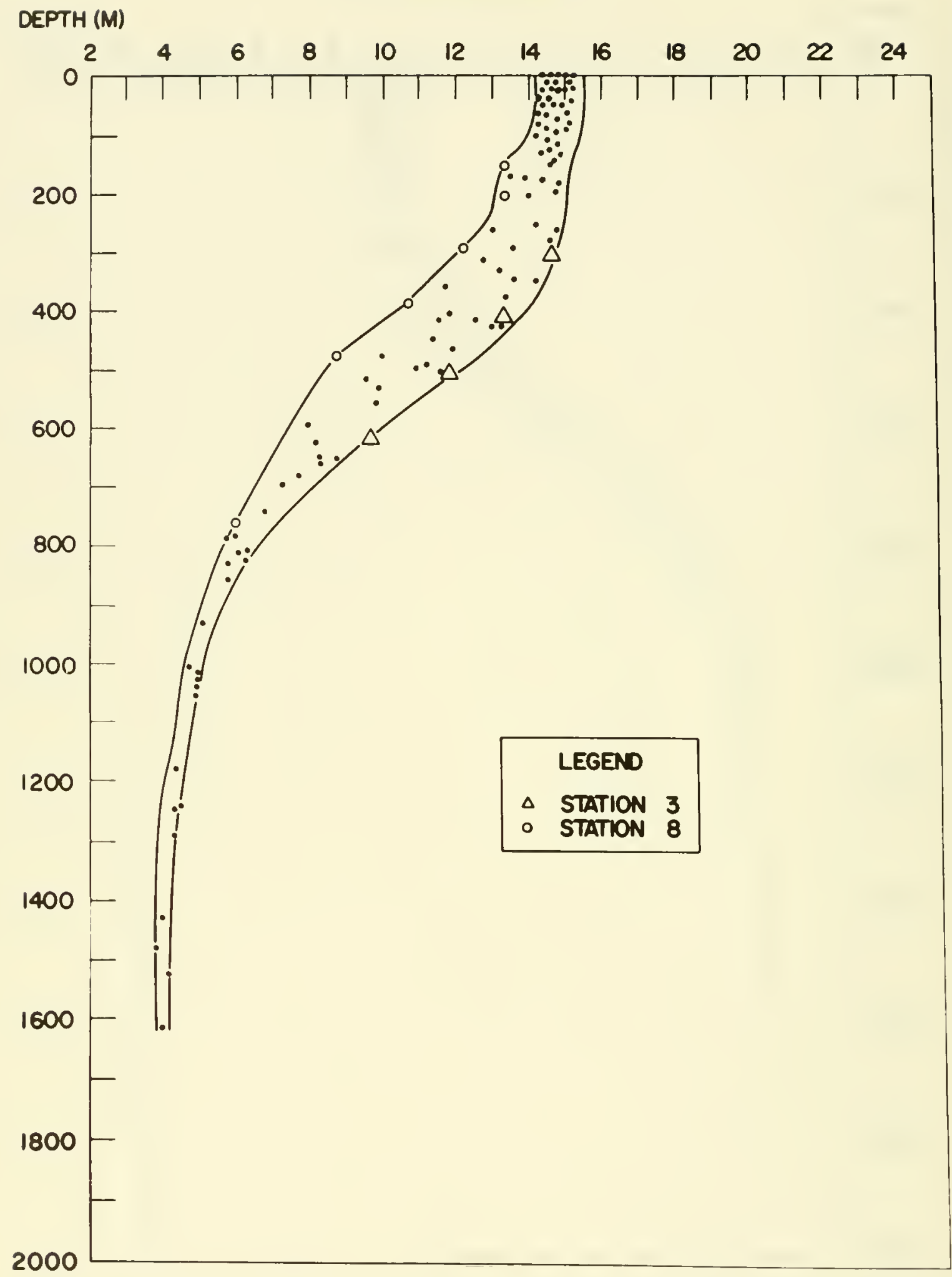

FIGURE 32. Envelope and plot of temperature $\left({ }^{\circ} \mathrm{C}\right)$ versus depth $(\mathrm{m})$ at Ocean Station DELTA, prepared from nine casts taken by USCGC ABSECON, 20 March-8 April 1968. 
TEMPERATURE $\left({ }^{\circ} \mathrm{C}\right)$

\section{DEPTH (M)}

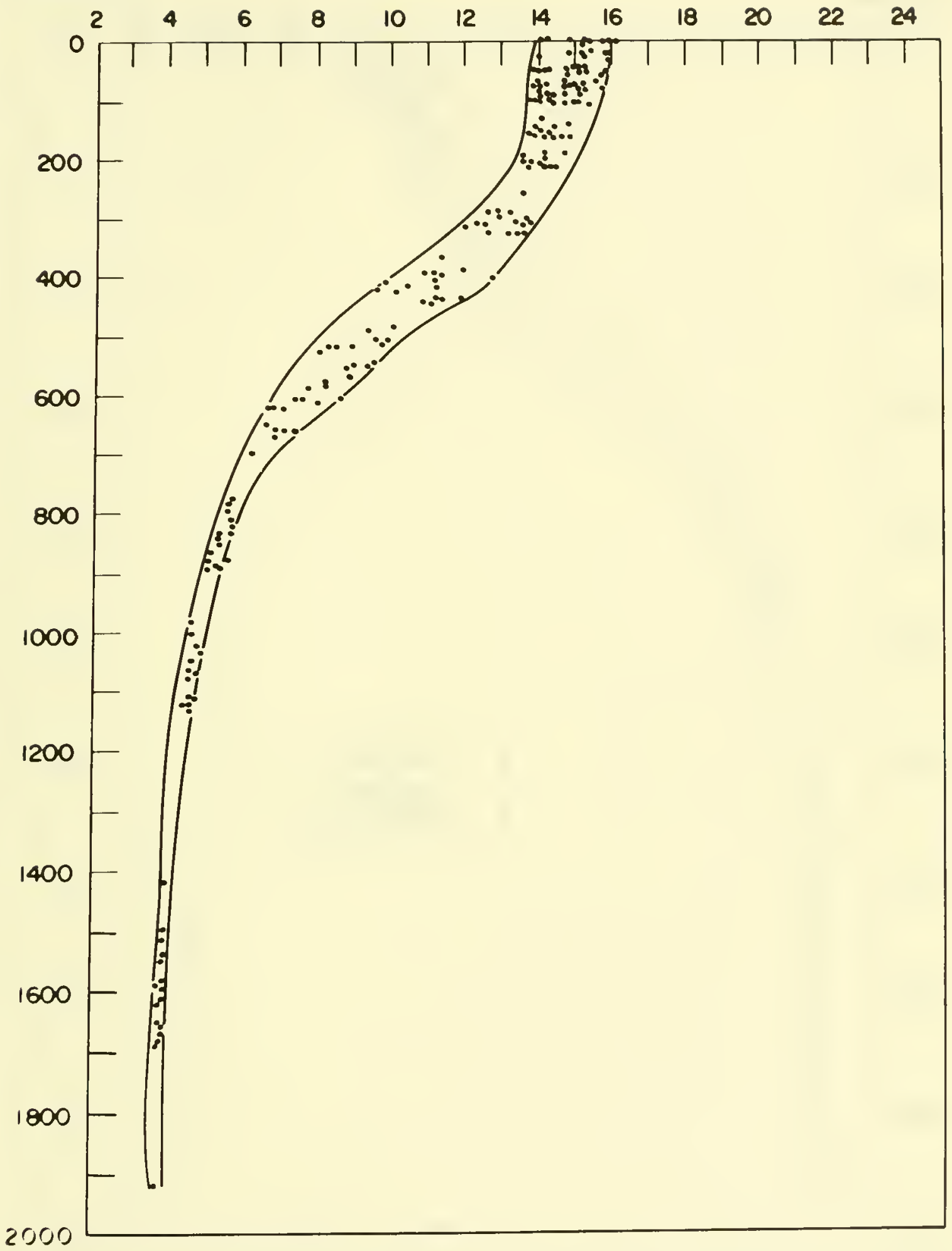

Figure 33. Envelope and plot of temperature $\left({ }^{\circ} \mathrm{C}\right)$ versus depth $(\mathrm{m})$ at Ocean Station DELTA, prepared from seventeen casts taken by USCGC COOK INLET, 14 April-5 May 1968. 
TEMPERATURE $\left({ }^{\circ} \mathrm{C}\right)$

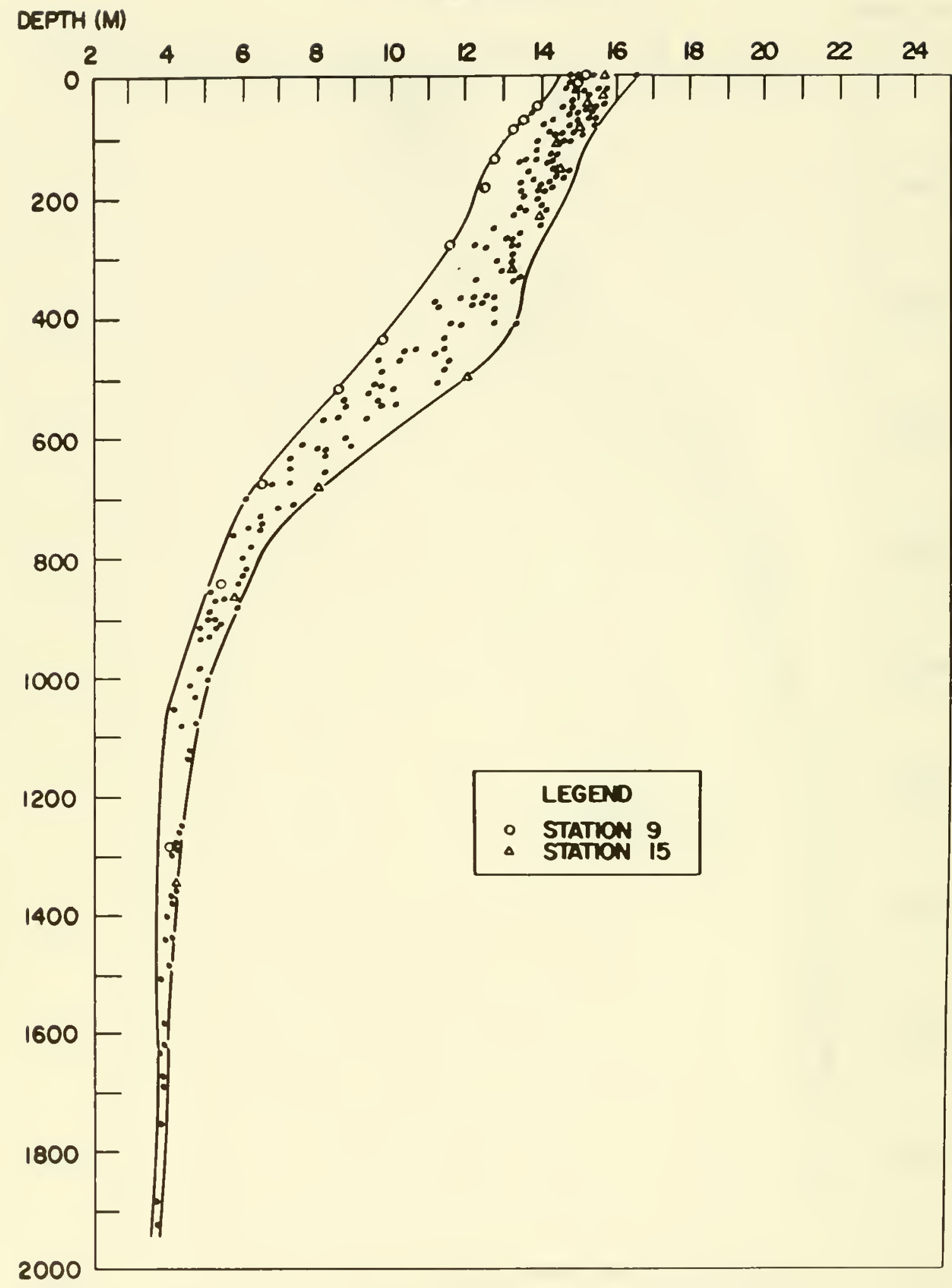

Figure 34. Envelope and plot of temperature $\left({ }^{\circ} \mathrm{C}\right)$ versus depth $(\mathrm{m})$ at Ocean Station DELTA, prepared from twenty-two casts taken by USCGC DALLAS, 6 May-28 May 1968. 


\section{DEPTH (M)}

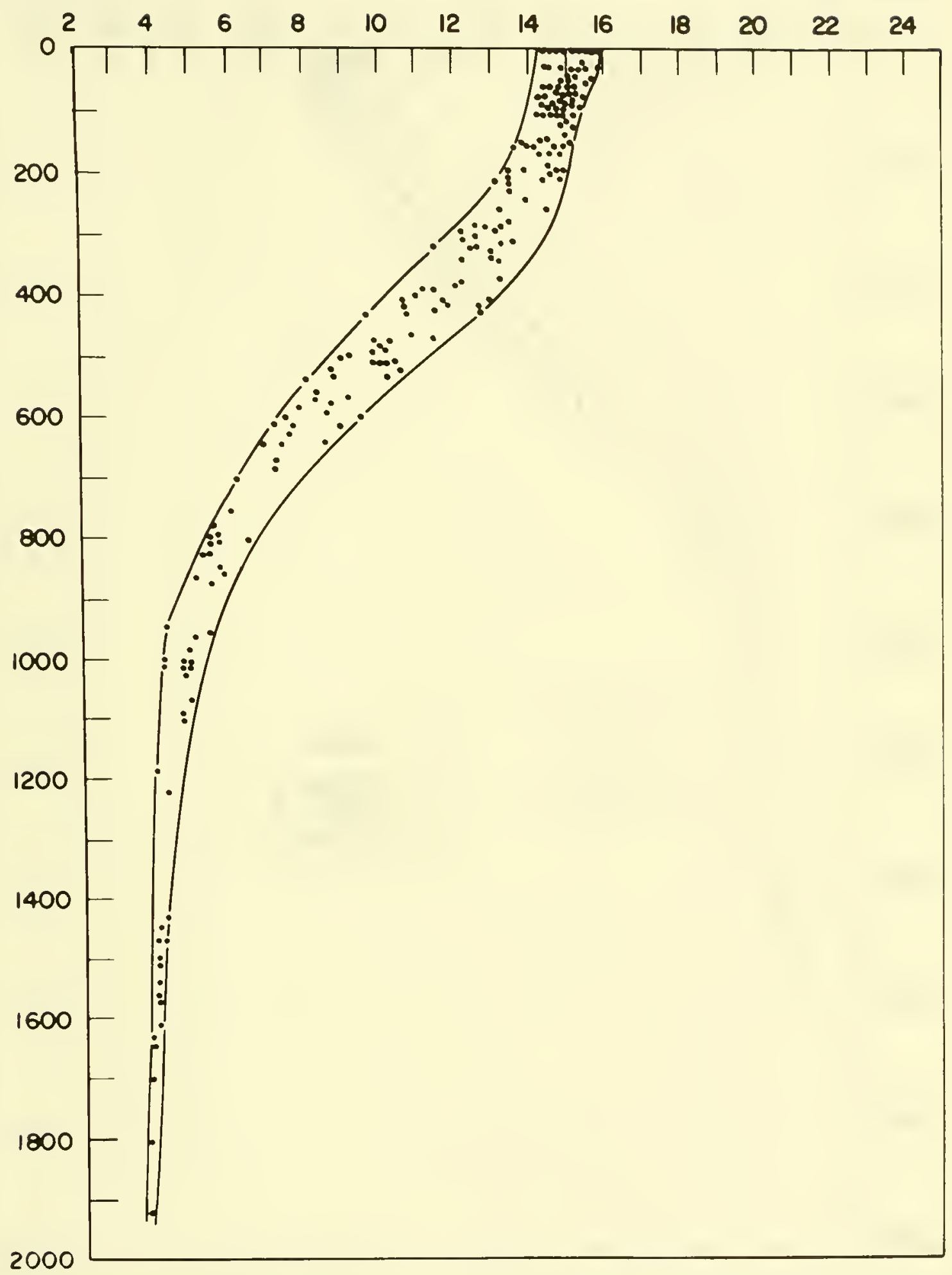

FIGURE 35. Envelope and plot of temperature $\left({ }^{\circ} \mathrm{C}\right)$ versus depth $(\mathrm{m})$ at Ocean Station DELTA, prepared from eighteen casts taken by USCGC CASTLE ROCK, 31 May-19 June 1968. 


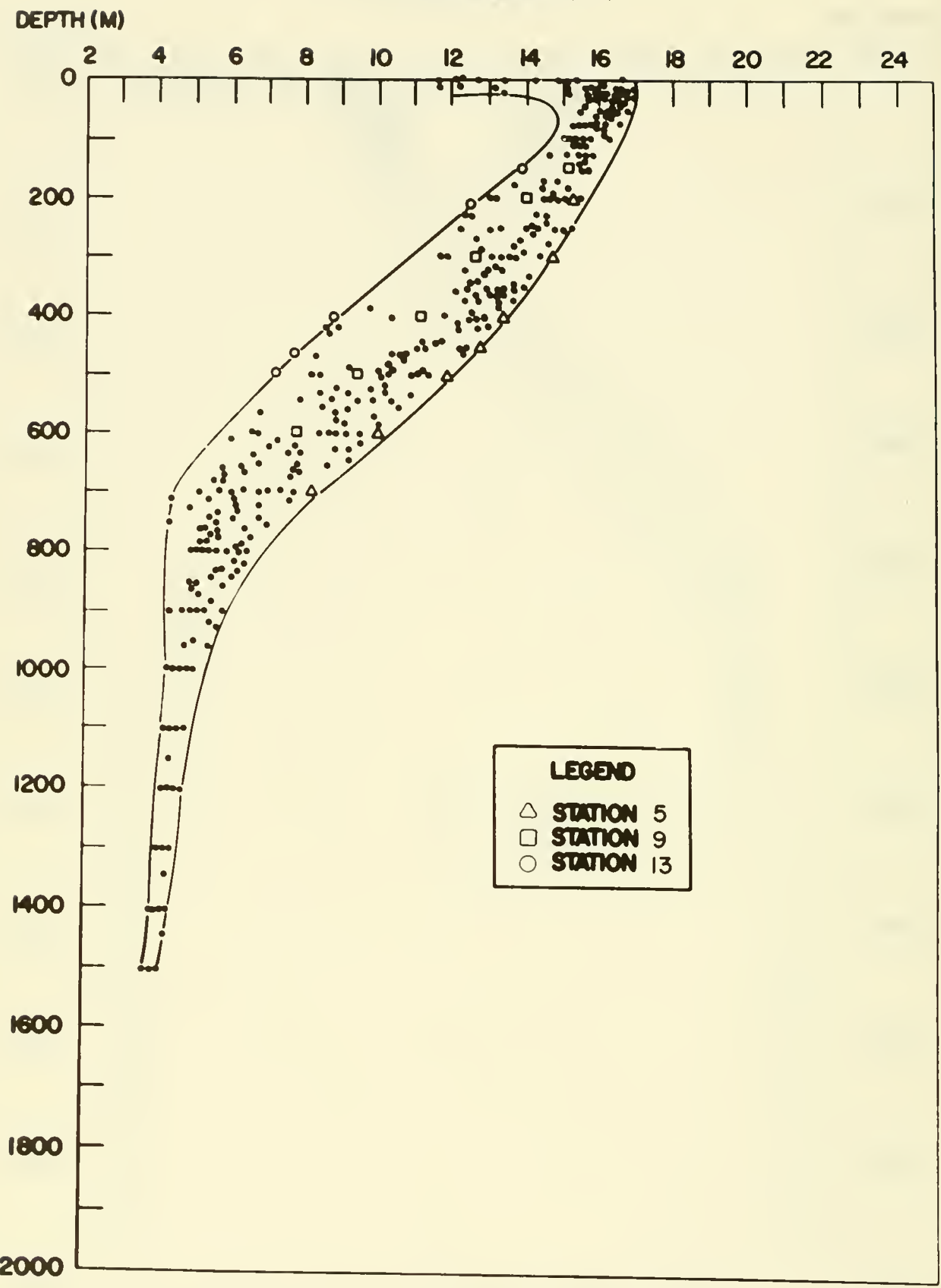

Figure 36. Envelope and plot of temperature $\left({ }^{\circ} \mathrm{C}\right)$ versus depth $(\mathrm{m})$ at Ocean Station DELTA, prepared from thirteen casts taken by USCGC HAMILTON, 6 July-9 July 1968. 
TEMPERATURE $\left({ }^{\circ} \mathrm{C}\right)$

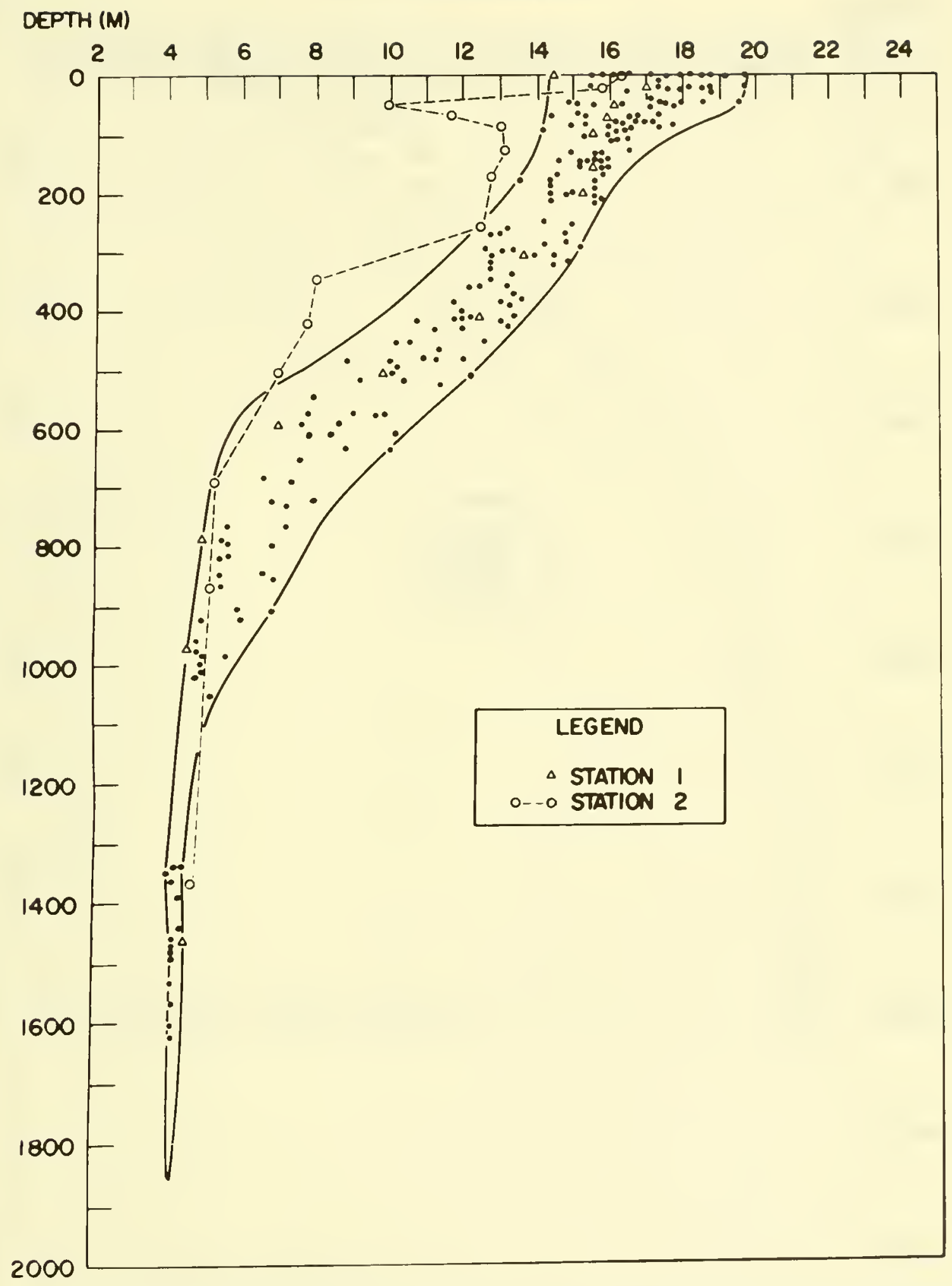

Figure 37. Envelope and plot of temperature $\left({ }^{\circ} \mathrm{C}\right)$ versus depth (m) at Ocean Station DELTA, prepared from twenty-two casts taken by USCGC DALLAS, 13 July-4 August 1968. Station number 2 is not included in the envelope due to its variance from the normal. 


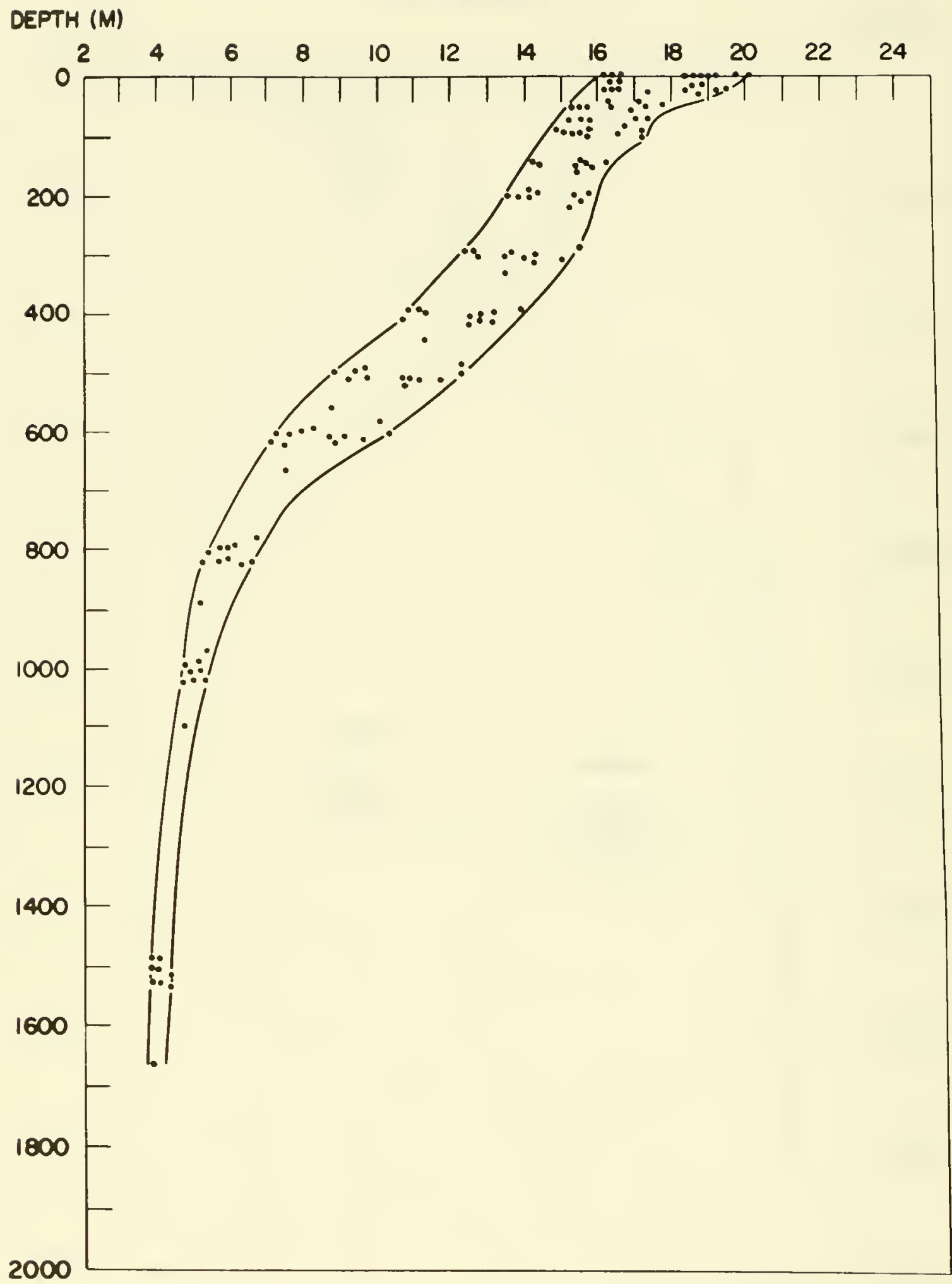

Figure 38. Envelope and plot of temperature $\left({ }^{\circ} \mathrm{C}\right)$ versus depth $(\mathrm{m})$ at Ocean Station DELTA, prepared from fourteen casts taken by USCGC COOK INLET, 5 August-25 August 1968. 


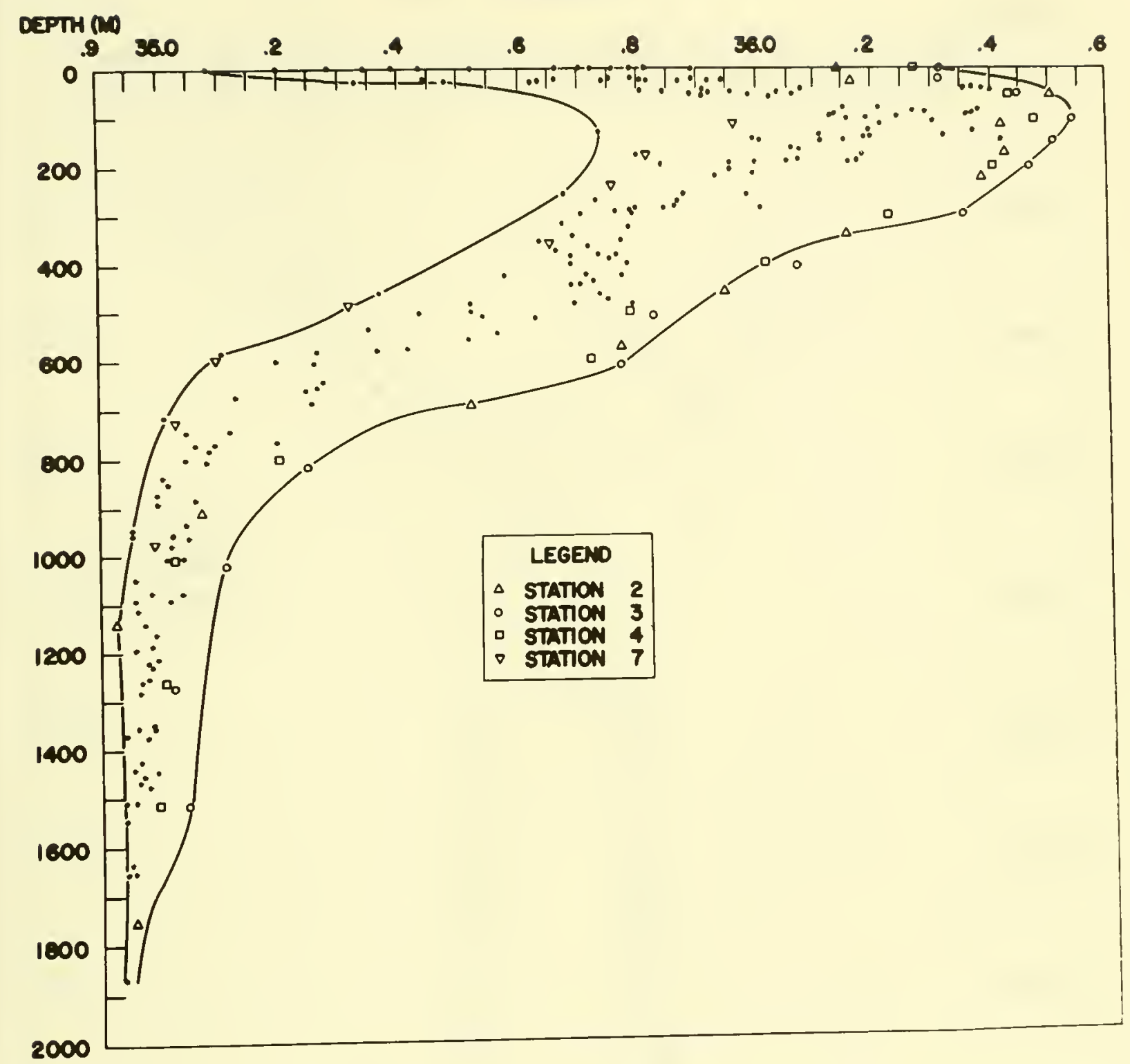

FIGURE 39. Envelope and plot of salinity $(\%)$ versus depth $(\mathrm{m})$ at Ocean Station DELTA, prepared from eighteen casts taken by USCGC INGHAM, 27 August-16 September 1967. 
DEPTH (M)

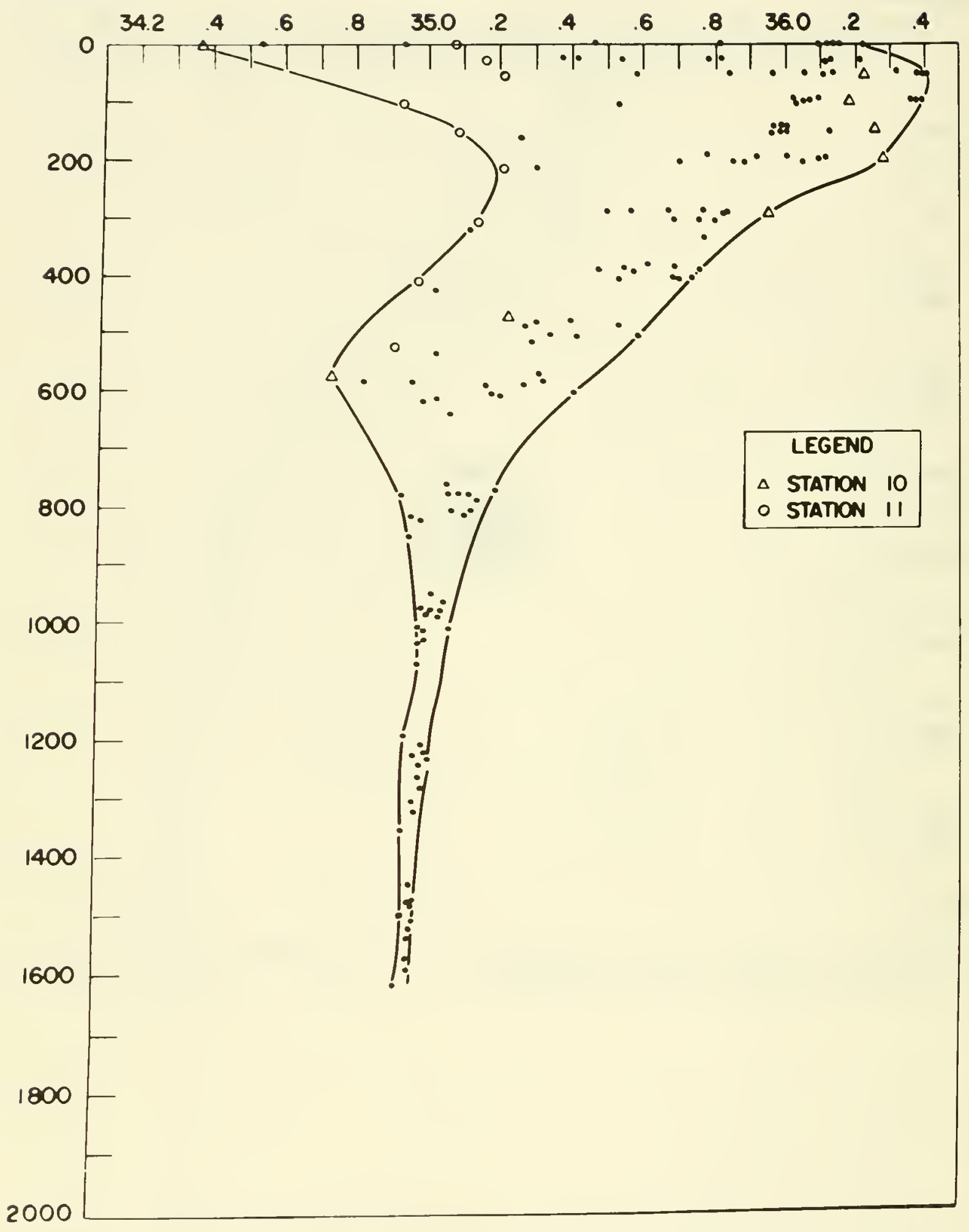

Figure 40. Envelope and plot of salinity $(\%)$ versus depth $(\mathrm{m})$ at Ocean Station DELTA, prepared from fourteen casts taken by USCGC CHINCOTEAGUE, 22 September-10 October 1967. 
SALNITY $(\%)$

\section{DEPTH (M)}

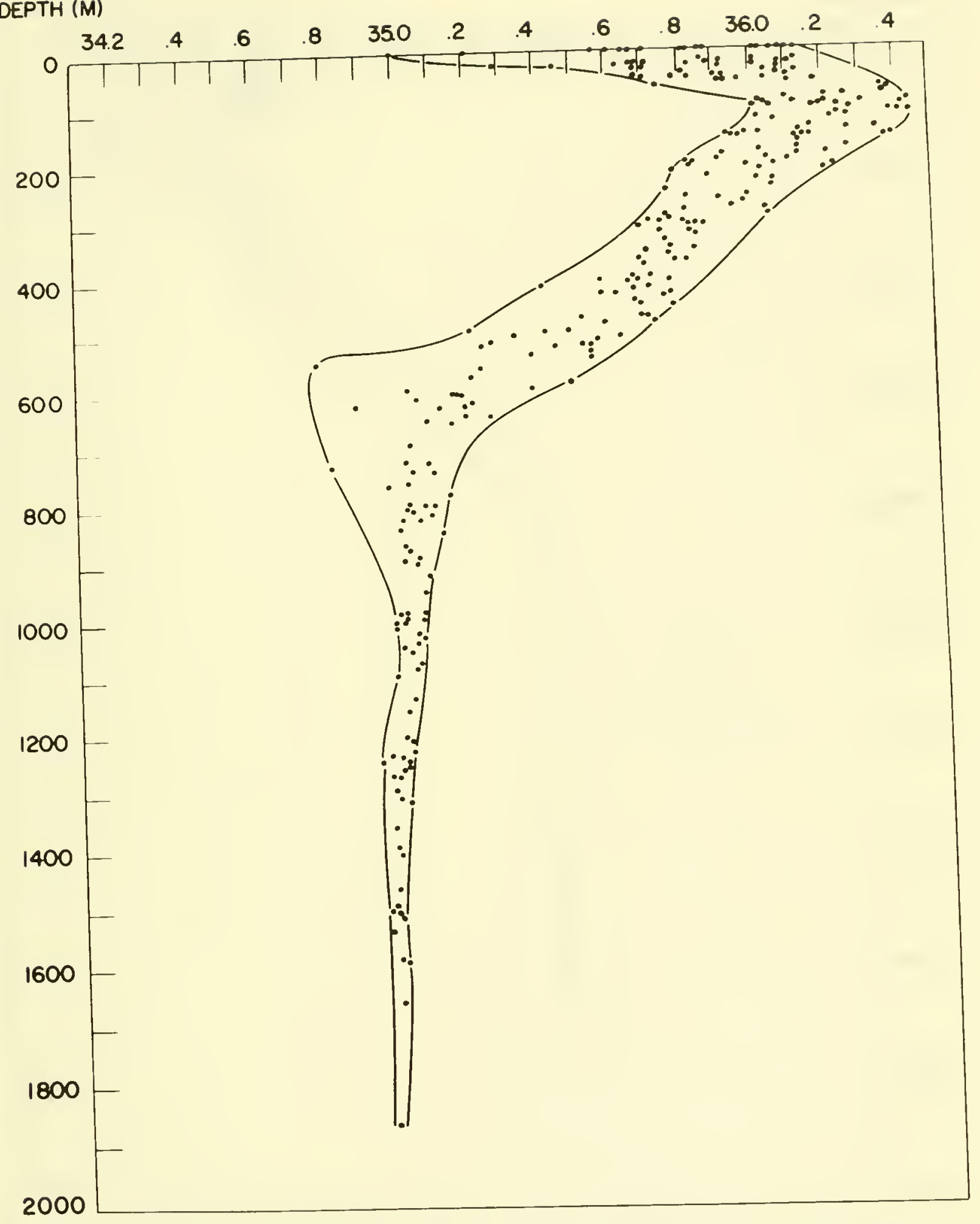

FIGURE 41. Envelope and plot of salinity $(\%)$ versus depth $(\mathrm{m})$ at Ocean Station DELTA, prepared from twenty-two casts taken by USCGC ESCANABA, 12 October-3 November 1967. 
SALINITY $(\%)$

DEPTH (M)

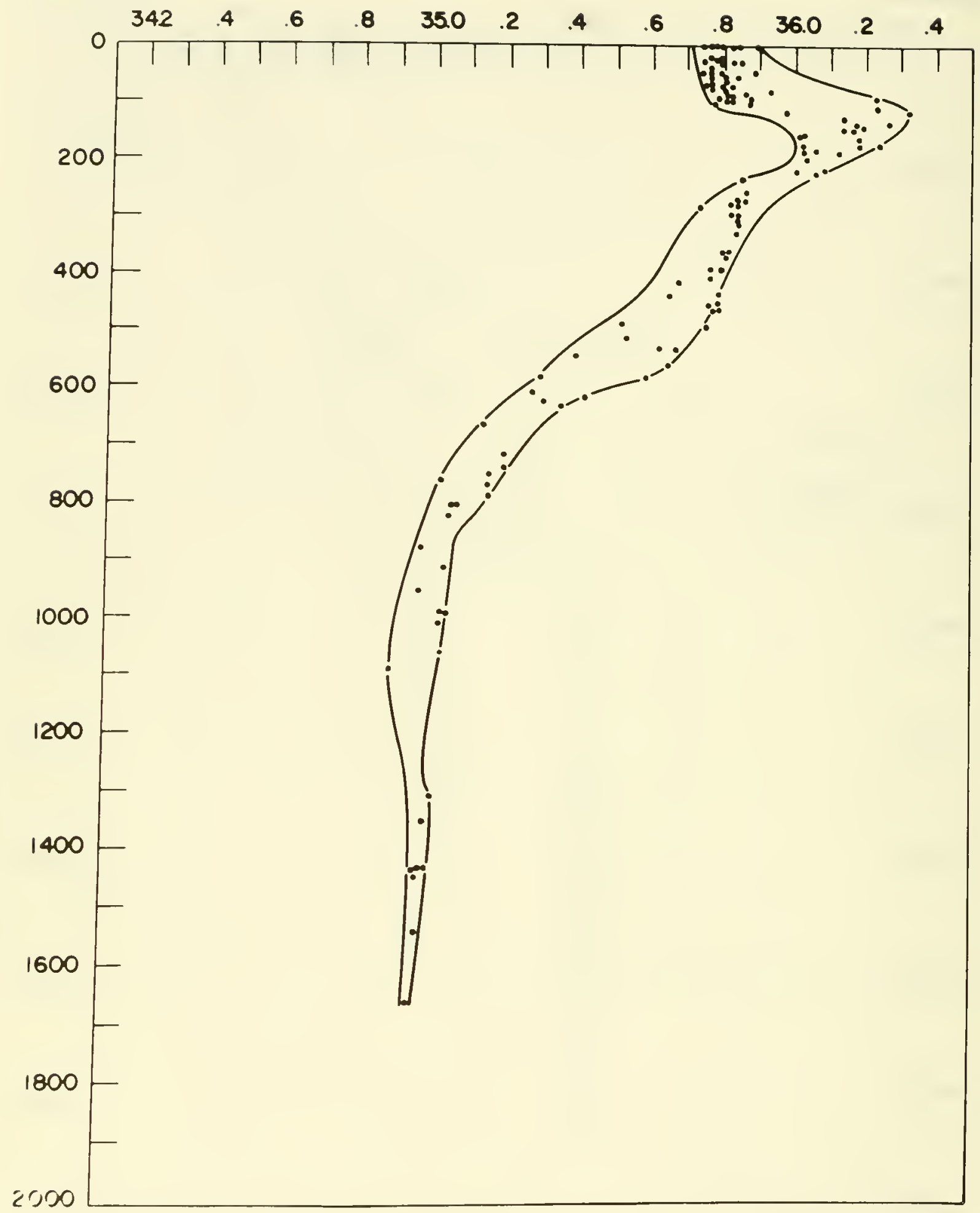

FIGURE 42. Envelope and plot of salinity (\%) versus depth $(\mathrm{m})$ at Ocean Station DELTA, prepared from ten casts taken by USCGC OWASCO, 30 November-15 December 1967. 


\section{DEPTH an}

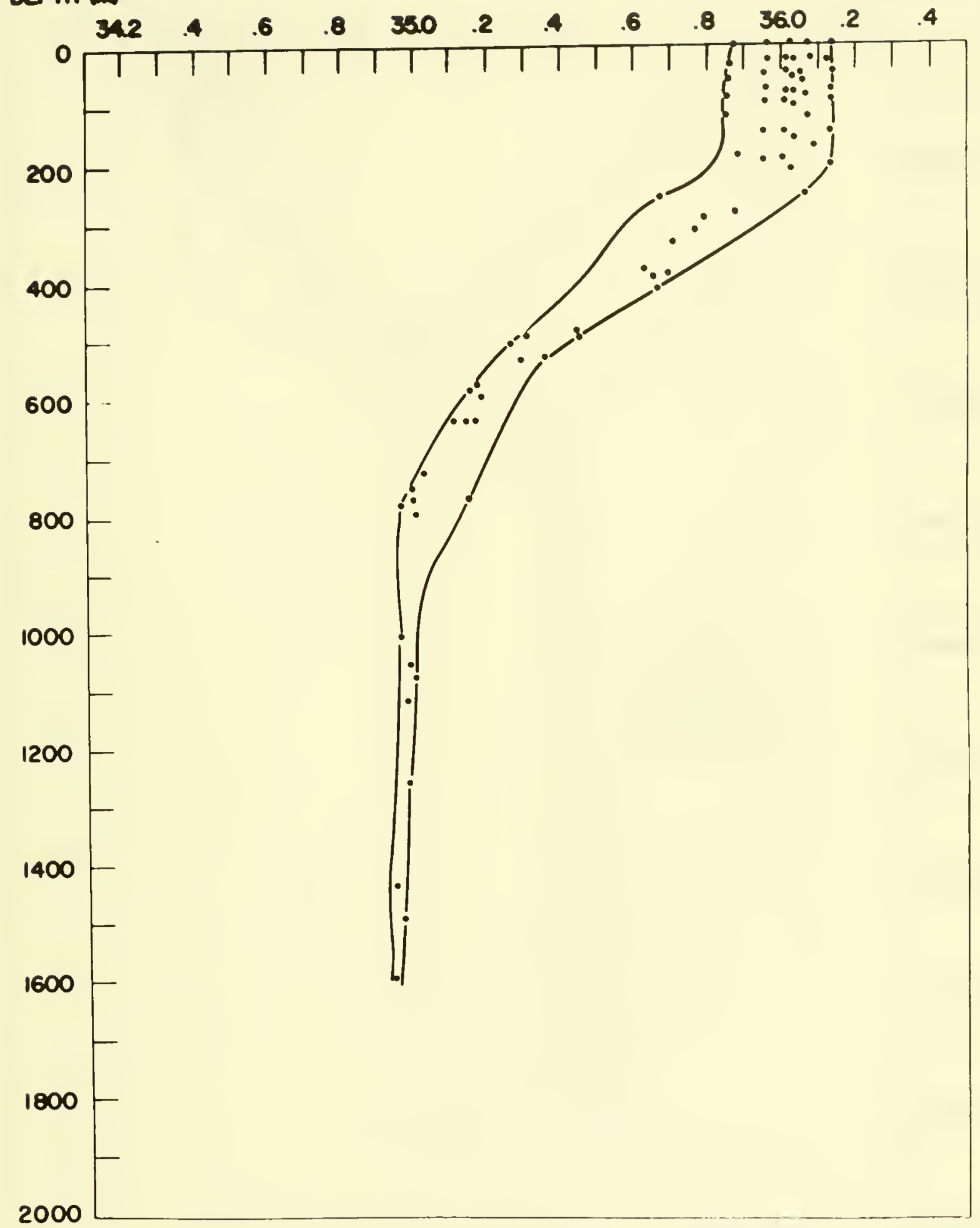

FIGURE 43. Envelope and plot of salinity $(\%)$ versus depth $(\mathrm{m})$ at Ocean Station DELTA, prepared from six casts taken by USCGC ABSECON, 13 January-30 January 1968. 
SALINTYY $(\%)$

DEPTH (M)

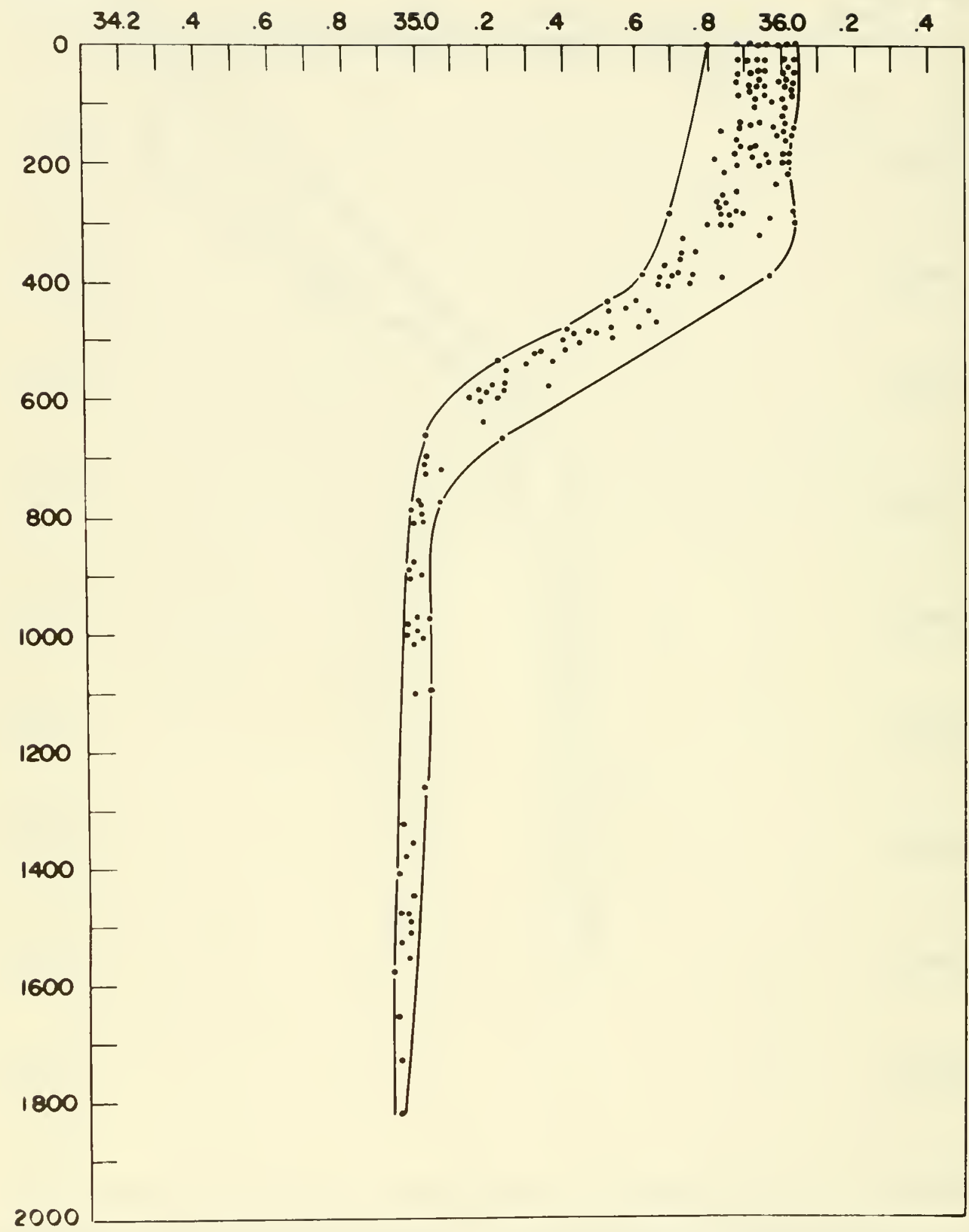

Figure 44. Envelope and plot of salinity (\%) versus depth $(\mathrm{m})$ at Ocean Station DELTA, prepared from seventeen casts taken by USCGC CASCO, 28 February-19 March 1968. 


\section{DEPTH (M)}

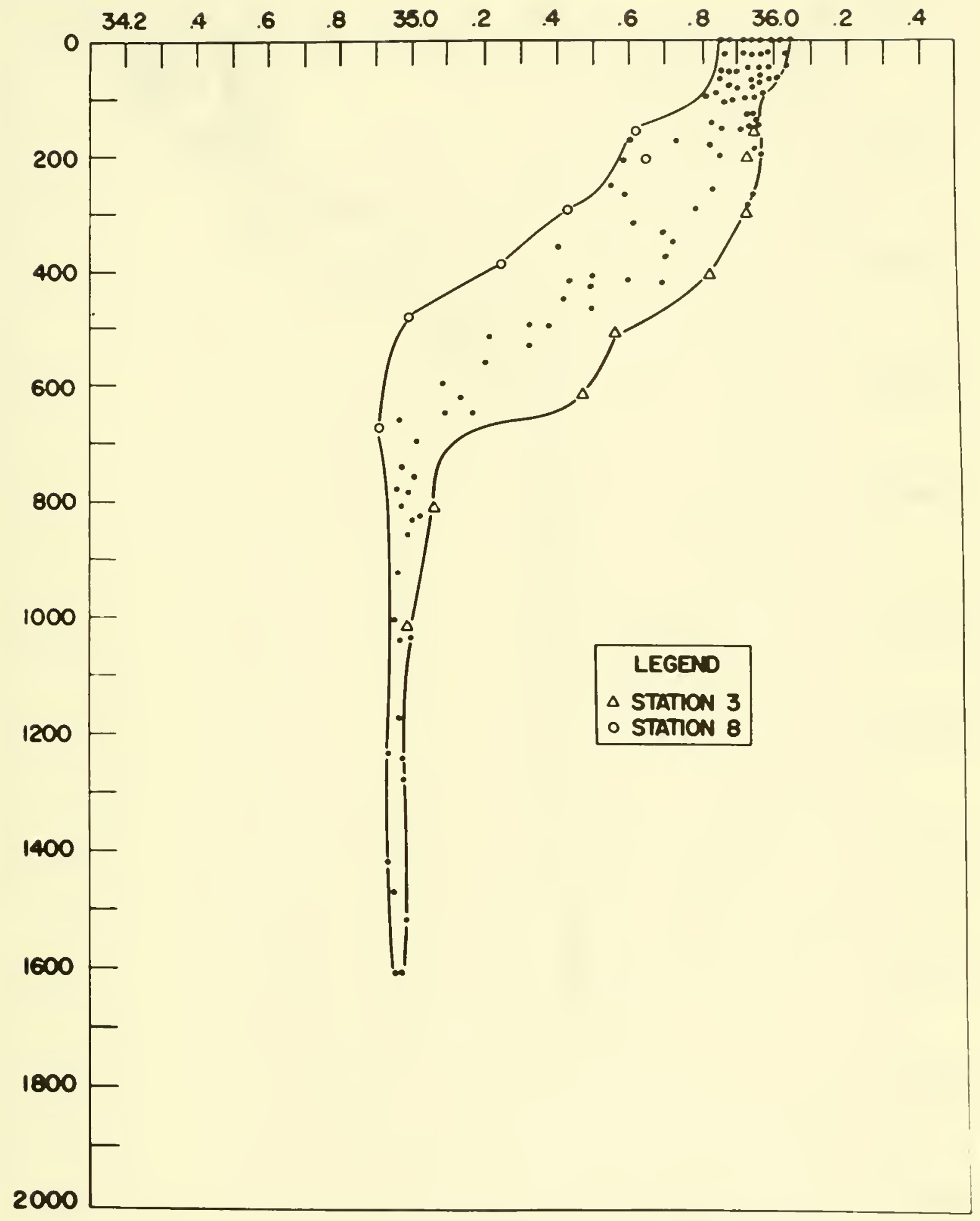

FIGURE 45. Envelope and plot of salinity $(\%)$ versus depth $(\mathrm{m})$ at Ocean Station DELTA, prepared from nine casts taken by USCGC ABSECON, 20 March-8 April 1968. 
SALINITY $\left(\%_{\infty}\right)$

DEPTH (M)

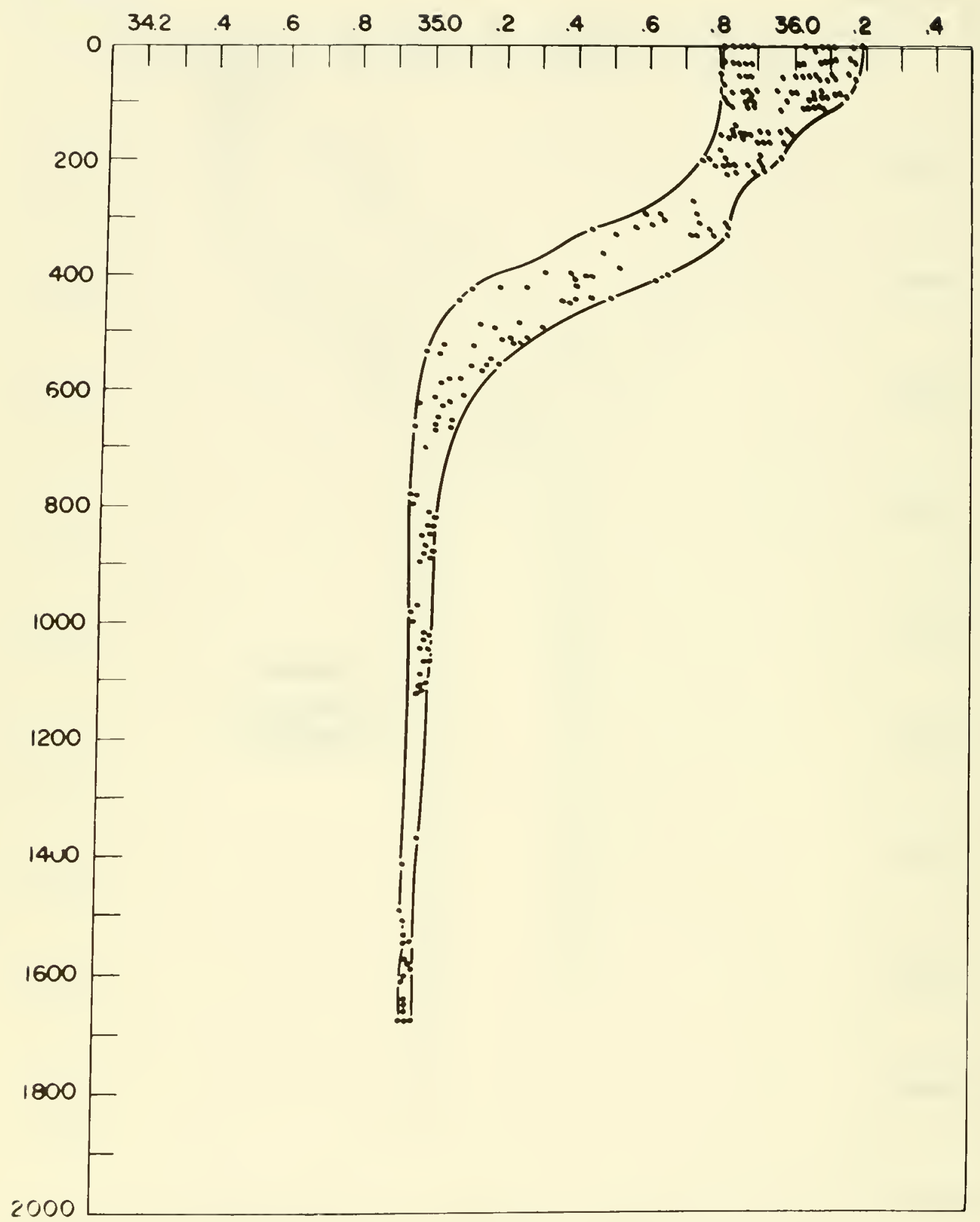

Figure 46. Envelope and plot of salinity $(\%)$ versus depth $(\mathrm{m})$ at Ocean Station DELTA, prepared from seventeen casts taken by USCGC COOK INLET, 14 April-5 May 1968. 
DEPTH (M)

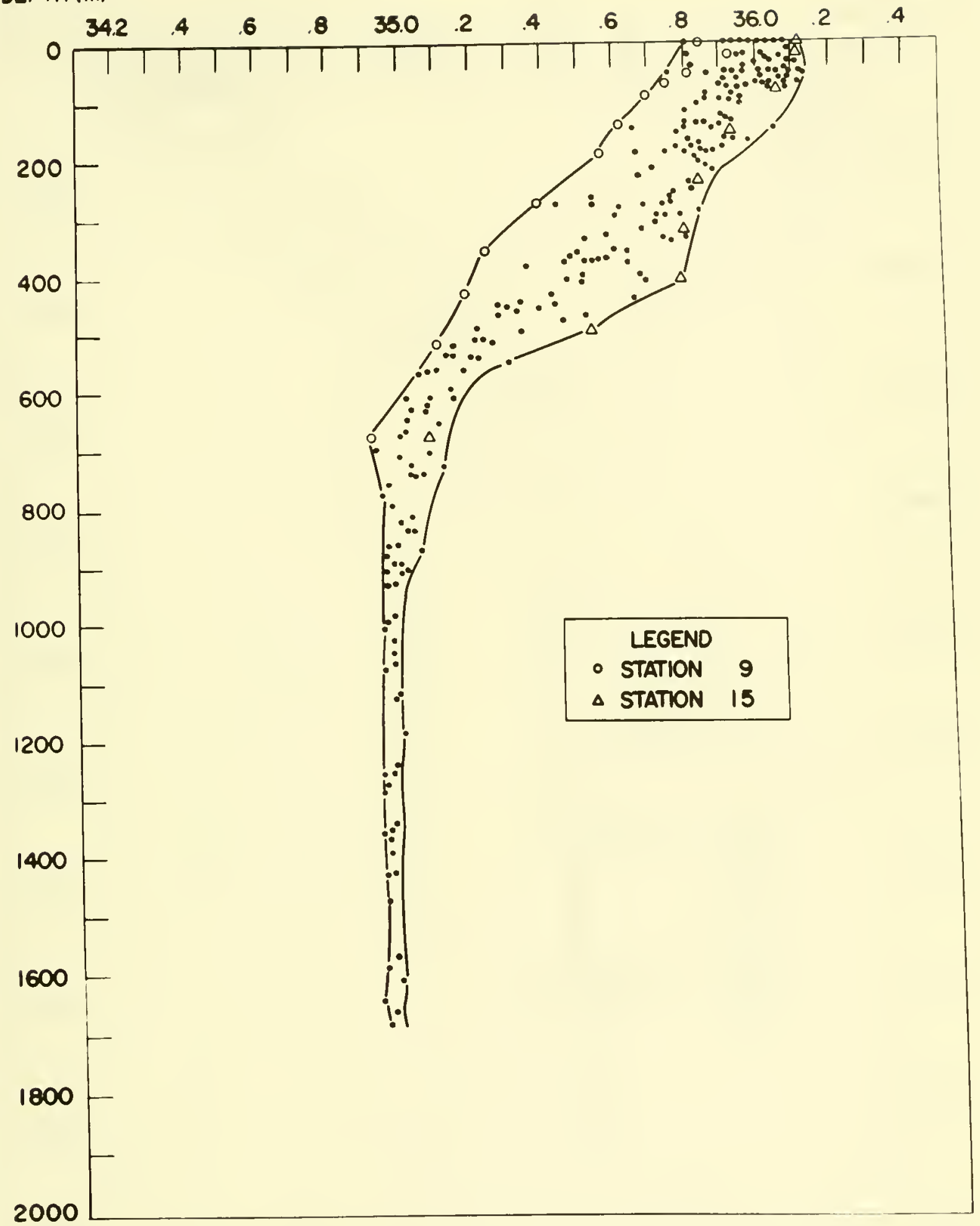

Figure 47. Envelope and plot of salinity $(\%)$ versus depth $(\mathrm{m})$ at Ocean Station DELTA, prepared from twenty-two casts taken by USCGC DALLAS, 6 May-28 May 1968. 


\section{SALINITY $(\%)$}

DEPTH (M)

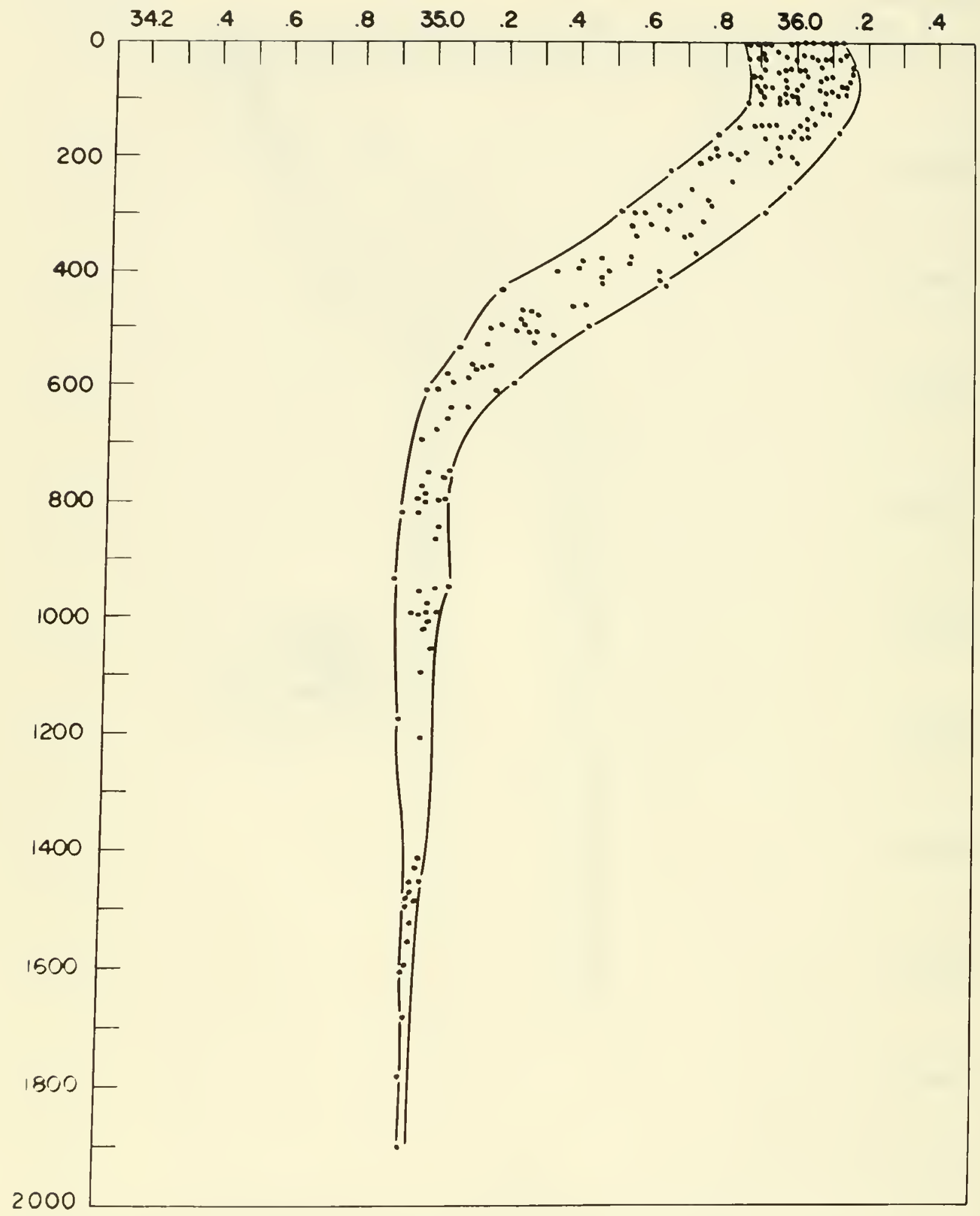

FIguRe 48. Envelope and plot of salinity $(\%)$ versus depth $(\mathrm{m})$ at Ocean Station DELTA, prepared from eighteen casts taken by USCGC CASTLE ROCK, 31 May-19 June 1968. 


\section{DEPTH (M)}

$\begin{array}{lllllllllllll}34.2 & .4 & .6 & .8 & 35.0 & .2 & .4 & .6 & .8 & 36.0 & .2 & .4\end{array}$

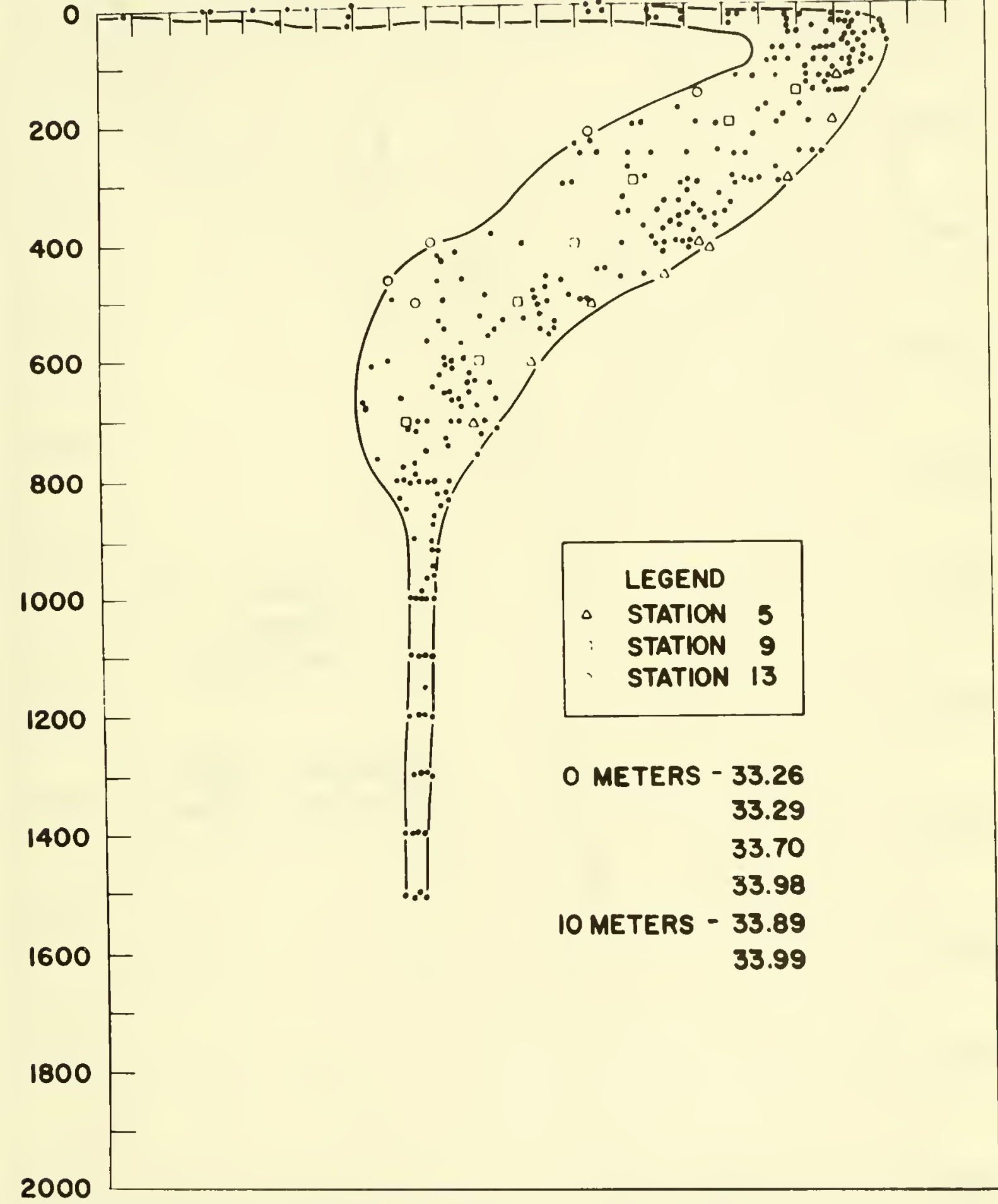

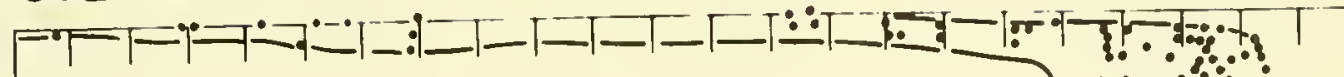

Figure 49. Envelope and plot of salinity $(\%)$ versus depth $(\mathrm{m})$ at Ocean Station DELTA, prepared from thirteen casts taken by USCGC HAMILTON, 6 July-9 July 1968 . 


\section{SALINITY (Yod}

\section{DEPTH (M)}

$\begin{array}{llllllllllll}34.2 & .4 & .6 & .8 & 350 & .2 & .4 & .6 & 8 & 360 & .2 & .4\end{array}$

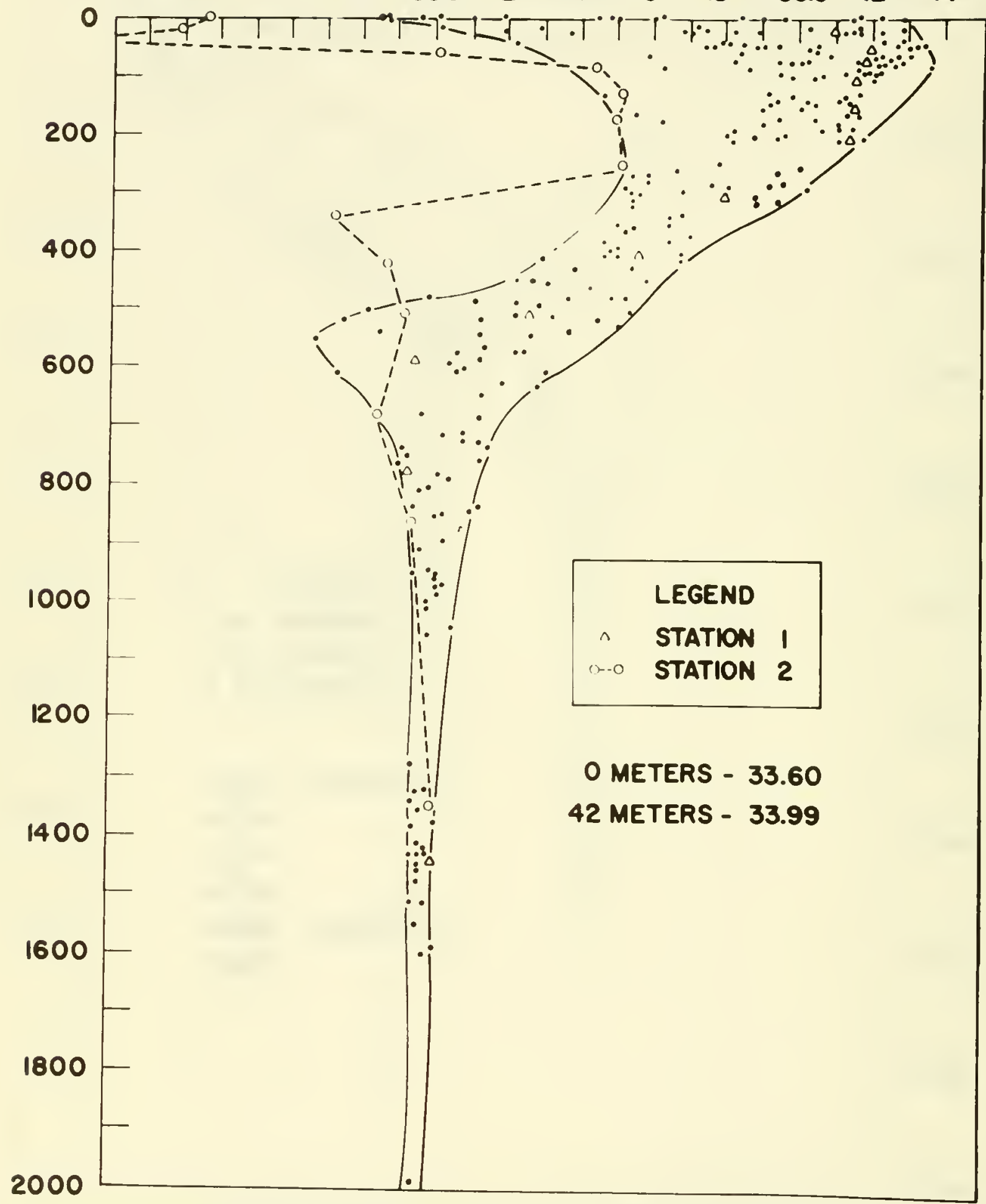

Figure 50. Envelope and plot of salinity $(\%)$ versus depth $(\mathrm{m})$ at Ocean Station DELTA, prepared from twenty-two casts taken by USCGC DALLAS, 13 July-4 August 1968. Station number 2 is not included in the envelope due to its variance from the normal. 


\section{SALINITY $(\% \circ)$}

DEPTH (M)

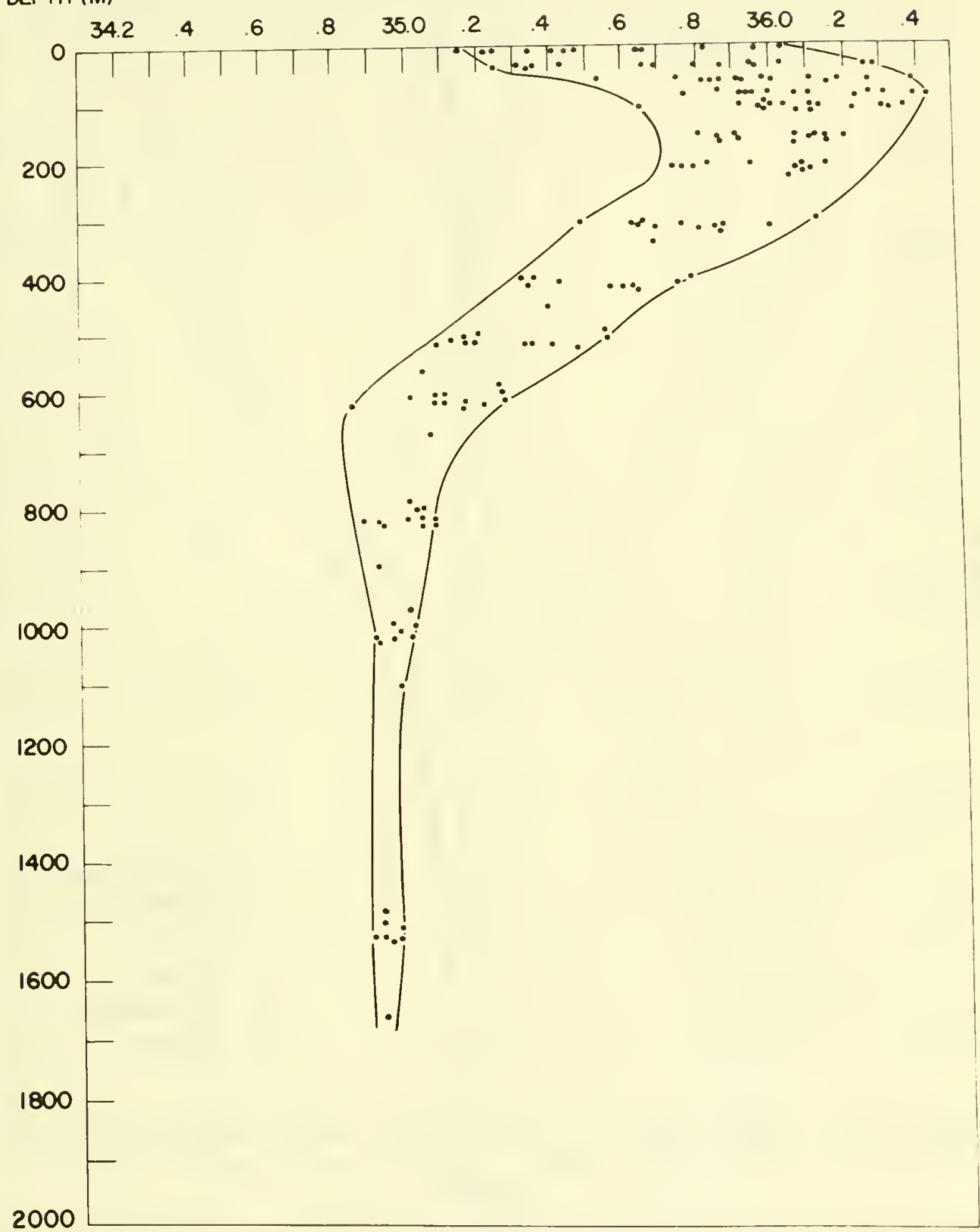

Figure 51. Envelope and plot of salinity $(\%)$ versus depth $(m)$ at Ocean Station DELTA, prepared from fourteen casts taken by USCGC COOK INLET, 5 August-25 August 1968. 
TEMPERATURE $\left({ }^{\circ} \mathrm{C}\right)$

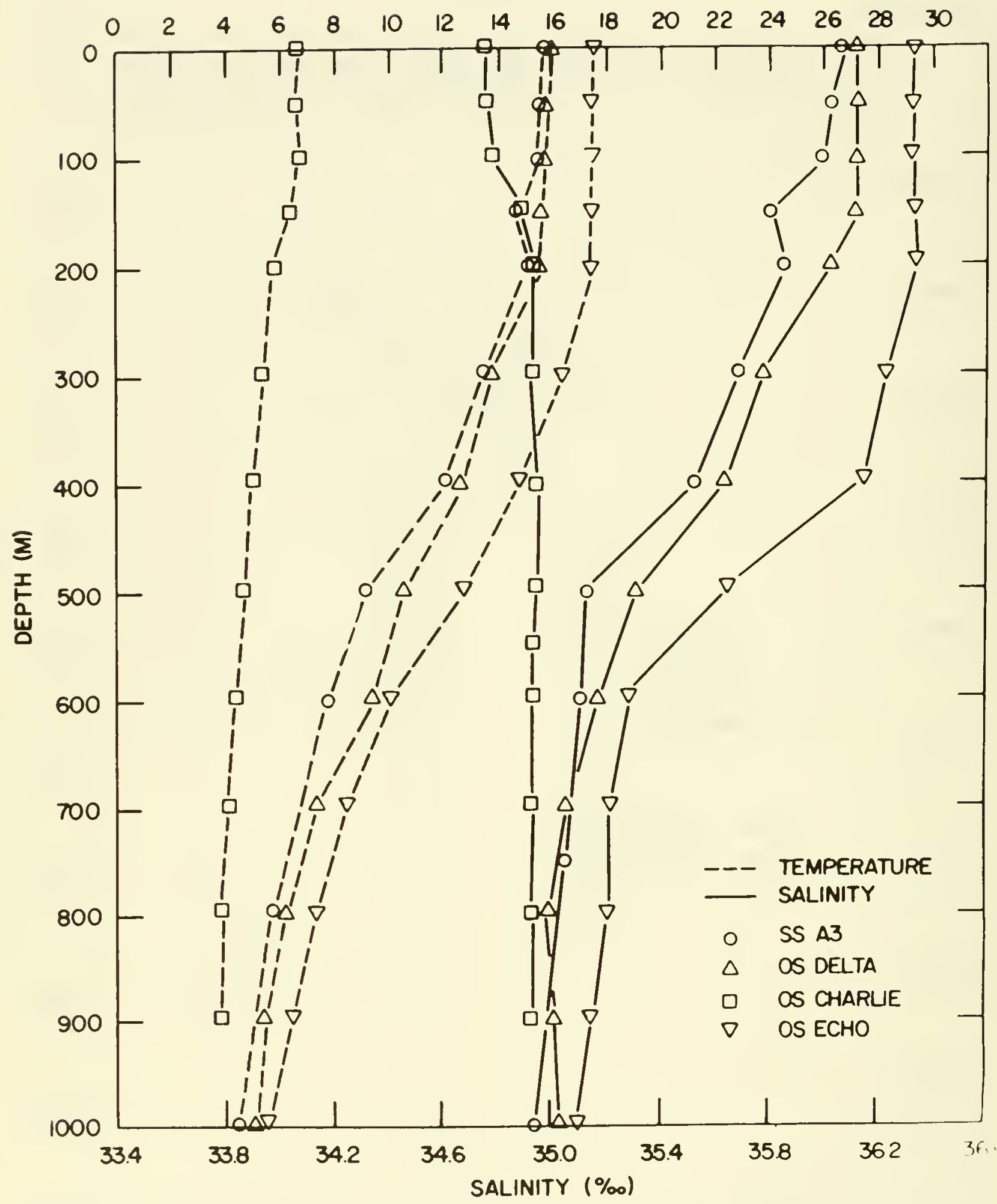

FIGURE 52. Temperature and salinity versus depth at OS DELTA, ECHO, CHARLIE, and Standard Section A-3 (station taken at $\left.43^{\circ} 40^{\prime} \mathrm{N}, 43^{\circ} 46^{\prime} \mathrm{W}\right), 31$ January to 3 February 1968. 
TEMPERATURE $\left({ }^{\circ} \mathrm{C}\right)$

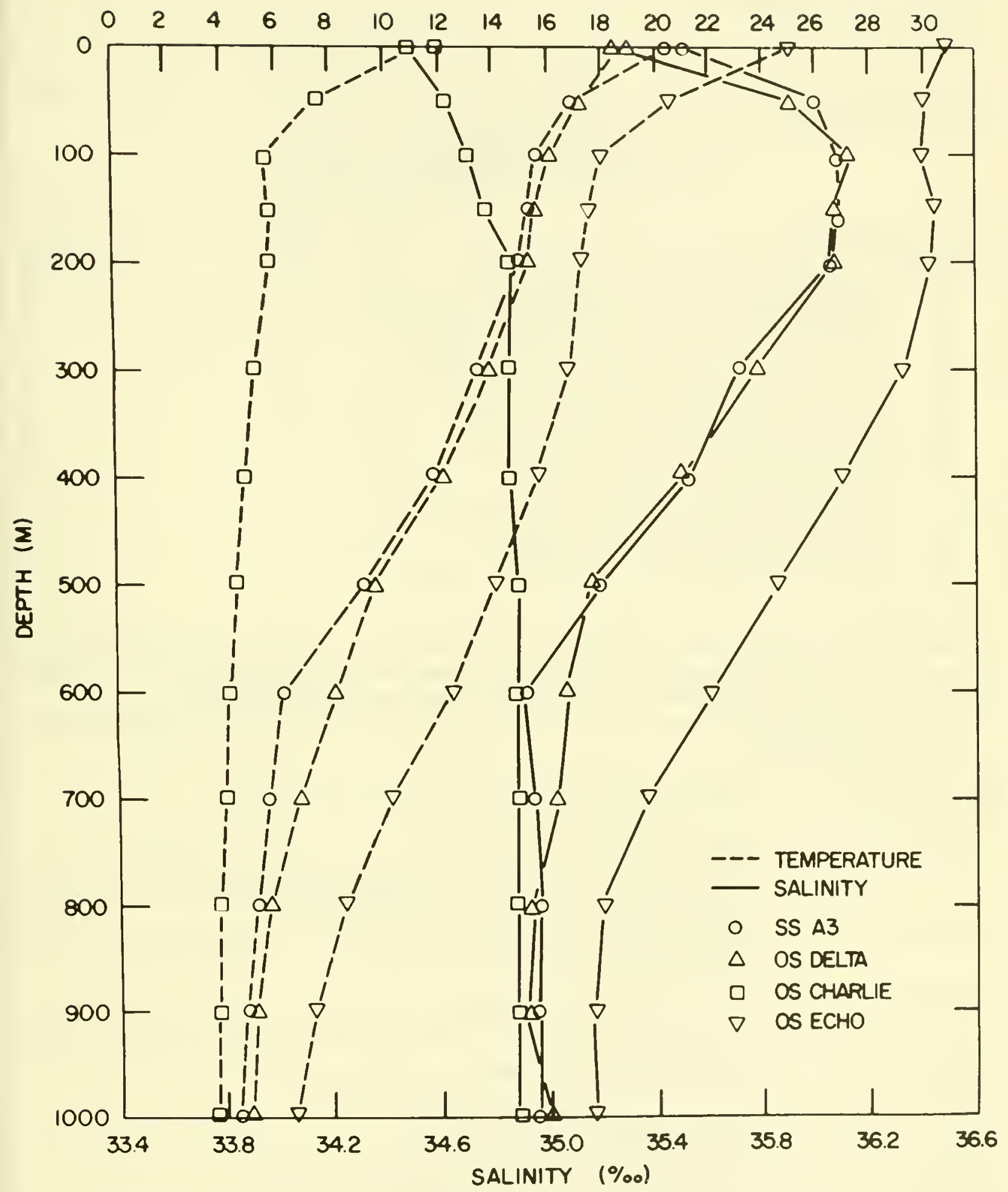

FIGURE 53. Temperature and salinity versus depth at OS DELTA, ECHO, CHARLIE, and Standard Section A-3 (station taken at $\left.43^{\circ} 54^{\prime} \mathrm{N}, 45^{\circ} 08^{\prime} \mathrm{W}\right), 6$ August 1968 . 


\section{APPENDIX A OCEANOGRAPHIC DATA}

\section{Cruises Listed}

Table

I. CGC INGHAM, 27 August to 16 September 1967

II. CGC CHINCOTEAGUE, 22 September to 10 October $1967 \ldots$

III. CGC ESCANABA, 12 October to 3 November $1967 \ldots \ldots \ldots$

IV. CGC OWASCO, 30 November to 15 December $1967 \ldots \ldots$

V. CGC ABSECON, 13 January to 30 January $1968 \ldots \ldots \ldots \ldots 1$

VI. CGC CASCO, 28 February to 19 March $1968 \ldots \ldots \ldots \ldots \ldots$

VII. CGC ABSECON, 20 March to 8 April $1968 \ldots \ldots \ldots \ldots$

VIII. CGC COOK INLET, 14 April to 5 May $1968 \ldots \ldots \ldots \ldots \ldots$

IX. CGC DALLAS, 6 May to 28 May $1968 \ldots \ldots \ldots \ldots$

X. CGC CASTLE ROCK, 31 May to 19 June $1968 \ldots \ldots \ldots \ldots \ldots$

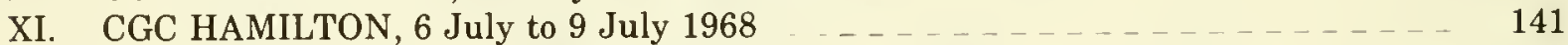

XII. CGC DALLAS, 13 July to 4 August $1968 \ldots \ldots \ldots \ldots$

XIII. CGC COOK INLET, 5 August to 25 August $1968 \ldots \ldots \ldots$

\section{Codes Utilized}

A complete description of the codes utilized in the tabulation of oceanographic station data can be found in National Oceanographic Data Center publication M-2, Processing Physical and Chemical Data from Oceanographic Stations. (Rev. August 1964, supplement issued May 1966.)

To facilitate use of the oceanographic station data listing, entry headings which are not self-explanatory are described below.

Depth to Bottom ....... Uncorrected sounding in meters.

Max. Depth of Samples ... Depth of deepest sample to nearest multiple of one hundred meters.

Wave observations

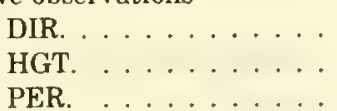

SEA .................

Weather Code .........

Cloud Code

Type ..........

Amount ........

Water

Color Code ........

Trans...........

Wind

Dir.

Speed

Barometer

Air Temp. ${ }^{\circ} \mathrm{C} \ldots \ldots$

Vis. Code

No. obs. depths
Rounded to nearest multiple of 10 degrees.

Increments of $1 / 2 \mathrm{~m}$. Sum of 5 meters plus increments of $1 / 2 \mathrm{~m}$ if 50 is added to direction.

If numerals 2 through 9 are entered, period in seconds is twice the numeric entry or $2 \mathrm{X}$ (numeric entry) +1. For other entries see WMO Code 3155.

Sea state according to WMO Code 3700

If preceded by X, weather according to WMO Code 4501. If a two-digit entry, weather according to WMO Code 4677.

Cloud type according to WMO Code 0500.

Cloud amount in eighths. Entry of the numeral 9 indicates cloud amount could not be estimated.

Color according to Forel-Ule scale.

Transparency in whole meters as determined by Secchi disc.

Rounded to nearest multiple of 10 degrees.

Letter $\mathrm{S}$ indicates wind speed in knots.

Barometric pressure given in tens, units and tenths of millibars.

Air temperature to tenths of a degree Celsius.

Visibility according to WMO Code 4300.

Number of observed levels associated with the station. 
Entered in hours and tenths of an hour GMT. For Nansen casts, indicates time of release of messenger applicable to the observational level. For STD casts, indicates the starting time of lowering the sensor.

Card type ......... OBS designates observed levels. STD indicates the values at this standard level were interpolated by a modified 3-point LaGrange formula.

Depth $(\mathrm{m}) \ldots \ldots \ldots$

Depth to nearest meter. A postscript $T$ indicates depth was obtained therometrically; $\mathrm{Z}$ indicates uncorrected "wire out" depth. Postscript Q indicates value was marked doubtful by originator; $P$ indicates value was considered doubtful by NODC. Postscripts $P$ and $Q$ retain this meaning throughout the following entries.

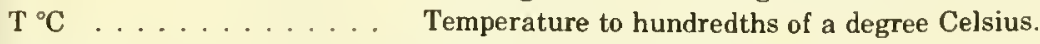

$\mathrm{S} \%$.............. Salinity in parts-per-thousand.

SIGMA-T ......... Entered to hundredths.

Specific-volume ...... Multiply entry by $10^{-7}$ to obtain specific-volume anomaly in cubic centimeters per gram.

$\mathrm{S} \Delta \mathrm{D}$ Dyn. $\mathrm{M} \times 10^{3} \ldots$ Multiply entry by $10^{-3}$ to obtain anomaly of dynamic depth in dynamic meters referenced to the sea surface.

Sound Velocity ....... Sound velocity according to Wilson's formula entered to tenths of a meter per second. 
TABLE I.-Observed and interpolated oceanographic data for stations taken by USCGC INGHAM at Ocean Station DELTA, 27 August-16 September 1967, prepared from NODC listing No. 31-1158 IN.

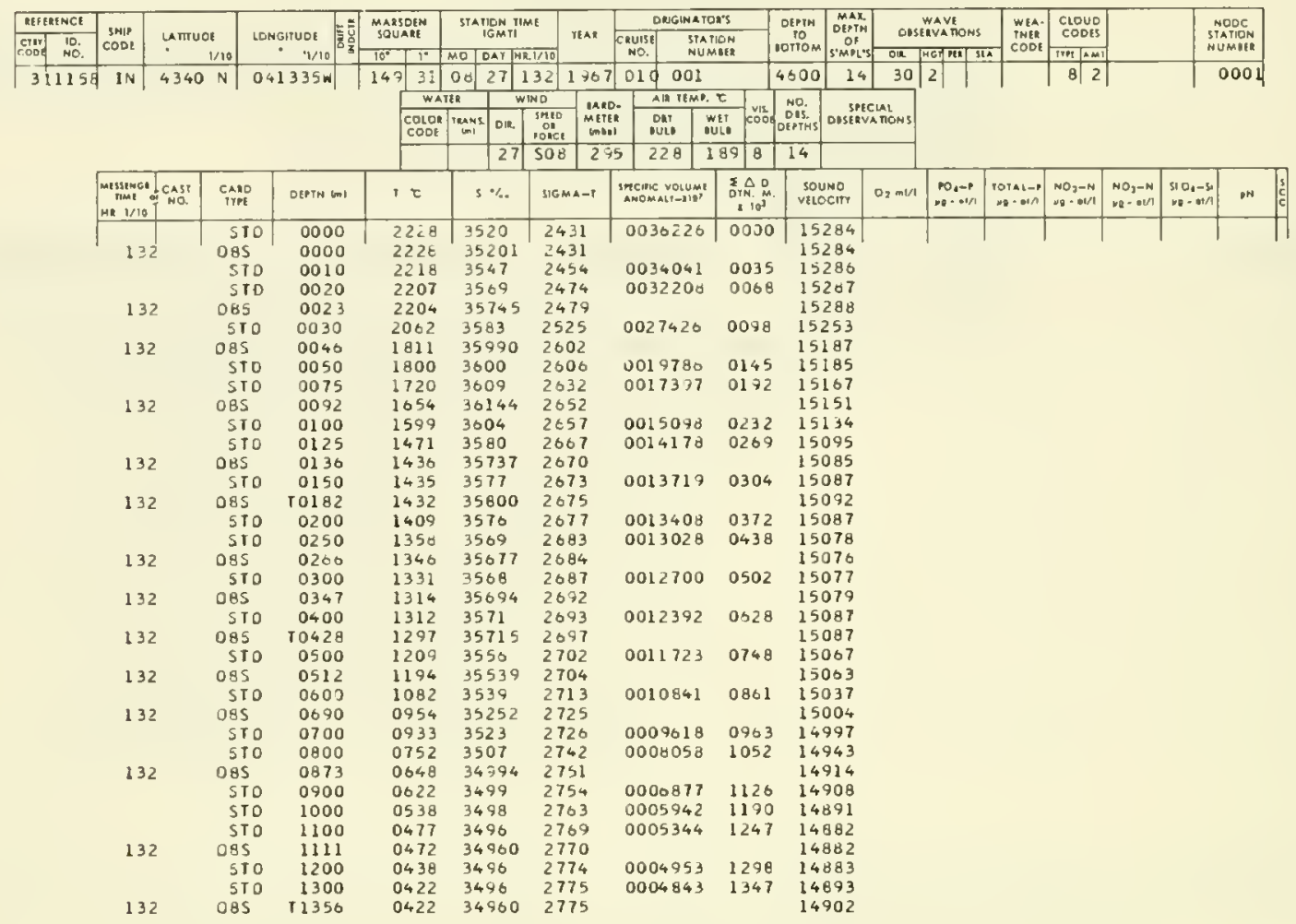

\begin{tabular}{|c|c|c|c|c|c|c|c|c|c|c|c|c|c|c|c|c|c|}
\hline AtL TENCE & \multirow{2}{*}{$\begin{array}{l}3 \mathrm{NNO} \\
\operatorname{CODE}\end{array}$} & \multirow{2}{*}{\multicolumn{2}{|c|}{$\begin{array}{c}\text { Lamude } \\
\quad 1 / 10 \\
\end{array}$}} & \multirow{2}{*}{$\begin{array}{c}\text { LONGITUDt } \\
\text { 1/10 }\end{array}$} & \multirow{2}{*}{$\begin{array}{c}\text { MARSOEN } \\
\text { SOUARE }\end{array}$} & \multirow{2}{*}{\multicolumn{2}{|c|}{$\begin{array}{l}\text { 3TAFION FIMT } \\
\text { IGMII }\end{array}$}} & \multicolumn{2}{|c|}{ DUEGINATOR'S } & \multirow{2}{*}{$\begin{array}{c}\text { Oeptm } \\
\text { To } \\
\text { cortom }\end{array}$} & \multirow{2}{*}{\begin{tabular}{|l|} 
MAR \\
DEPIN \\
OE \\
SHMPLS
\end{tabular}} & \multirow{2}{*}{$\begin{array}{l}\text { WaVt } \\
\text { Dosievanions }\end{array}$} & \multirow{2}{*}{$\mid \begin{array}{l}\text { WEA- } \\
\text { BHER } \\
\text { COOK }\end{array}$} & \multirow{2}{*}{ 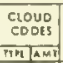 } & & \multirow{2}{*}{$\begin{array}{l}\text { NOOC } \\
\text { STAFS } \\
\text { NUMIER } \\
\text { NUM }\end{array}$} & \multirow{2}{*}{$\begin{array}{l}\text { NOOC } \\
\text { STAFS } \\
\text { NUMIER }\end{array}$} \\
\hline \begin{tabular}{l|l} 
cring & 10. \\
cood & No.
\end{tabular} & & & & & & & reAR & $\begin{array}{c}\text { CNuist } \\
\text { NO. }\end{array}$ & ION & & & & & & & & \\
\hline 315159 & IN & 4358 & $\mathrm{~N}$ & $04118 \mathrm{~W}$ & 14931 & \begin{tabular}{l|l|l}
08 & 28 \\
\end{tabular} & \begin{tabular}{|l|l|}
131 & 1967 \\
\end{tabular} & 010002 & & 4000 & 13 & $|2|-1$ & 60 & \begin{tabular}{|l|l|}
7 & 8 \\
\end{tabular} & & & 0002 \\
\hline & & & & & WAT & \begin{tabular}{l|l} 
IER & 1 \\
\end{tabular} & WINO & D. AE TEMP & & No. & & & & & & & \\
\hline & & & & & $\begin{array}{l}\text { COLOR } \\
\text { CODE }\end{array}$ & \begin{tabular}{|l|l|}
$\operatorname{len}_{n=1}$ & Dat \\
\end{tabular} & 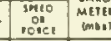 & \begin{tabular}{l|l} 
ont & W \\
Iut & is \\
\end{tabular} & coor & OOPT: & OISERVA TDNS & & & & & & \\
\hline & & & & & & 14 & 521 & 229 & 117 & 14 & & & & & & & \\
\hline & $\begin{array}{l}\text { Misings: } \\
\text { Thei } \\
\text { Me } 1 / 100\end{array}$ & $\begin{array}{l}\text { Cass? } \\
\text { I No. }\end{array}$ & Card & DEVTH Un) & $T t$ & s $\%$ & SIGMA-t & sincinc volumt & 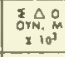 & $\begin{array}{l}\text { sou } \\
\text { vilo }\end{array}$ & $0,2=1 n$ & $\mid \begin{array}{ll}10 .-p \\
\cdots \cdots\end{array}$ & 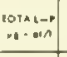 & $\mid$ & $\begin{array}{l}\text { NO, }, N \\
m \cdot \cdots / 1\end{array}$ & 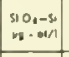 & 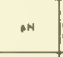 \\
\hline & & & STO & 0000 & 2364 & 3015 & 2463 & 0033143 & 0000 & 153 & 329 & & & & & & \\
\hline & 131 & & 085 & 0000 & 2304 & 36140 & 2463 & & & 153 & 29 & & & & & & \\
\hline & & & 510 & 0010 & 2298 & 3615 & 2483 & 0031246 & 0032 & 153 & 314 & & & & & & \\
\hline & & & STO & 0020 & $22 ? 0$ & 3610 & 2502 & 0029601 & 0003 & 153 & 303 & & & & & & \\
\hline & & & 510 & 0030 & 2177 & 3017 & 2519 & 0028005 & $00 \geqslant 1$ & 152 & 287 & & & & & & \\
\hline & 131 & & OBS & 0030 & & 35166 & & & & & & & & & & & \\
\hline & & & STO & 0050 & 2073 & 3644 & 2568 & 0023373 & 0143 & 152 & 200 & & & & & & \\
\hline & 131 & & OBS & 0058 & & 36508 & & & & & & & & & & & \\
\hline & & & STo & 0075 & 1964 & 3647 & 2600 & 0020477 & 0198 & 152 & 241 & & & & & & \\
\hline & & & 570 & 0100 & 1879 & 3644 & 2619 & $001868 ?$ & 0247 & 152 & 21 & & & & & & \\
\hline & 131 & & 085 & 0118 & 1634 & 36422 & 2629 & & & 152 & 211 & & & & & & \\
\hline & & & STO & 0125 & 1826 & 3042 & 2031 & $00: 7018$ & $02+2$ & 152 & 210 & & & & & & \\
\hline & & & STO & 0150 & 1799 & 3043 & 2038 & 0017040 & 0335 & 152 & 206 & & & & & & \\
\hline & 132 & & 085 & 0176 & 1776 & 36427 & 2645 & & & 152 & 203 & & & & & & \\
\hline & & & 510 & 0200 & 1758 & 3642 & 2048 & 0010286 & 0419 & 152 & 203 & & & & & & \\
\hline & 131 & & 085 & TO234 & 1732 & 36390 & 2652 & & & 152 & 200 & & & & & & \\
\hline & & & STO & 0256 & 1714 & 3636 & 2654 & 0015803 & 0499 & 151 & $1>7$ & & & & & & \\
\hline & & & STO & $030 C$ & 1659 & 3625 & 2059 & 0015501 & 0578 & 151 & 187 & & & & & & \\
\hline & 131 & & 085 & $034 \%$ & 1604 & 36157 & 2065 & & & 151 & 78 & & & & & & \\
\hline & & & $5 T 0$ & 0400 & 1548 & 3606 & 2670 & 0014773 & 0729 & 151 & 168 & & & & & & \\
\hline & 131 & & 085 & 0462 & 1478 & 35947 & 2677 & & & 151 & 154 & & & & & & \\
\hline & & & sto & 0500 & 1436 & 3550 & 2602 & $001 \$ 820$ & 0872 & 151 & 147 & & & & & & \\
\hline & 131 & & 085 & 10570 & 1340 & 35774 & 2092 & & & 151 & 28 & & & & & & \\
\hline & & & STO & 0600 & 1319 & 3572 & 2693 & 0012986 & 1006 & 151 & & & & & & & \\
\hline & 131 & & 085 & 0597 & 1184 & 35516 & 2704 & & & 150 & & & & & & & \\
\hline & & & STO & 0700 & 1176 & 3551 & 2705 & 0011954 & 1131 & 150 & 88 & & & & & & \\
\hline & & & STO & 0800 & 0938 & 3527 & 2729 & 0009013 & 1239 & 150 & & & & & & & \\
\hline & & & 510 & 0930 & 0747 & 3509 & 2744 & 0008031 & 1327 & 149 & 59 & & & & & & \\
\hline & 131 & & 085 & 0914 & 0726 & 35069 & 2746 & & & 1496 & & & & & & & \\
\hline & & & $5 T 0$ & 1000 & 0616 & 3500 & ¿ 755 & $000 \supset 850$ & 1401 & 149 & & & & & & & \\
\hline & & & STO & 1100 & $05 i 9$ & 3494 & 2763 & 0300368 & 1466 & 160 & 99 & & & & & & \\
\hline & 131 & & 085 & 1146 & 0486 & 34925 & 2765 & & & 148 & & & & & & & \\
\hline & & & STo & 1200 & 3479 & 3494 & 2707 & 0005041 & 1525 & $148^{\circ}$ & 99 & & & & & & \\
\hline & & & STO & 1300 & 3606 & 3457 & 2771 & 0005353 & 1580 & 147 & & & & & & & \\
\hline & & & ST0 & 1400 & 0451 & 3459 & 2774 & 0005107 & 1632 & 149 & & & & & & & \\
\hline & 131 & & OBS & 11447 & 0444 & $3459 c_{L}$ & 2775 & & & 1492 & 27 & & & & & & \\
\hline & & & STO & 1500 & 0435 & 3497 & 2776 & 0004990 & 1082 & 149 & 32 & & & & & & \\
\hline & & & STO & 1750 & 0350 & 3496 & 2779 & 0004826 & 1805 & 1475 & & & & & & & \\
\hline & 131 & & oss & 11750 & 0389 & 34954 & 2778 & & & 1495 & 15o & & & & & & \\
\hline
\end{tabular}


TABLE I.-Continued

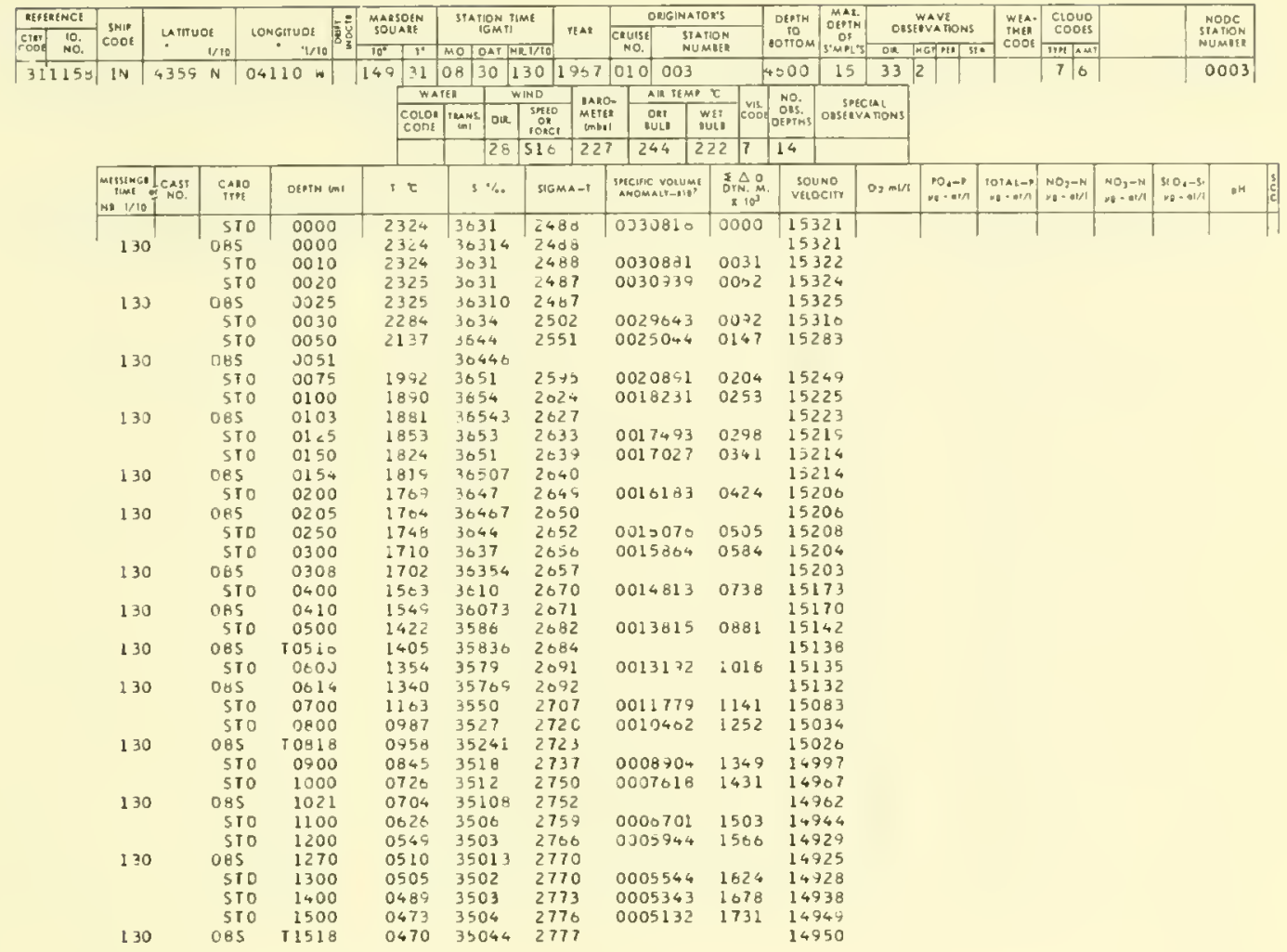

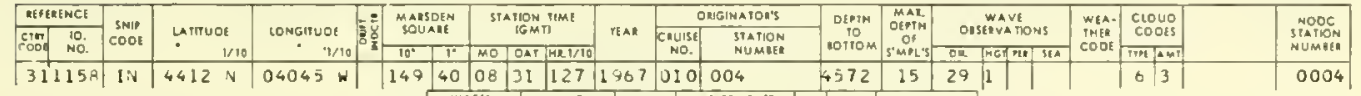

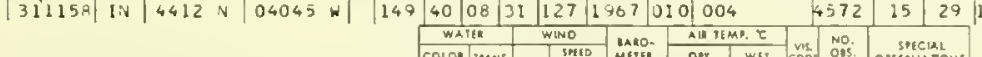

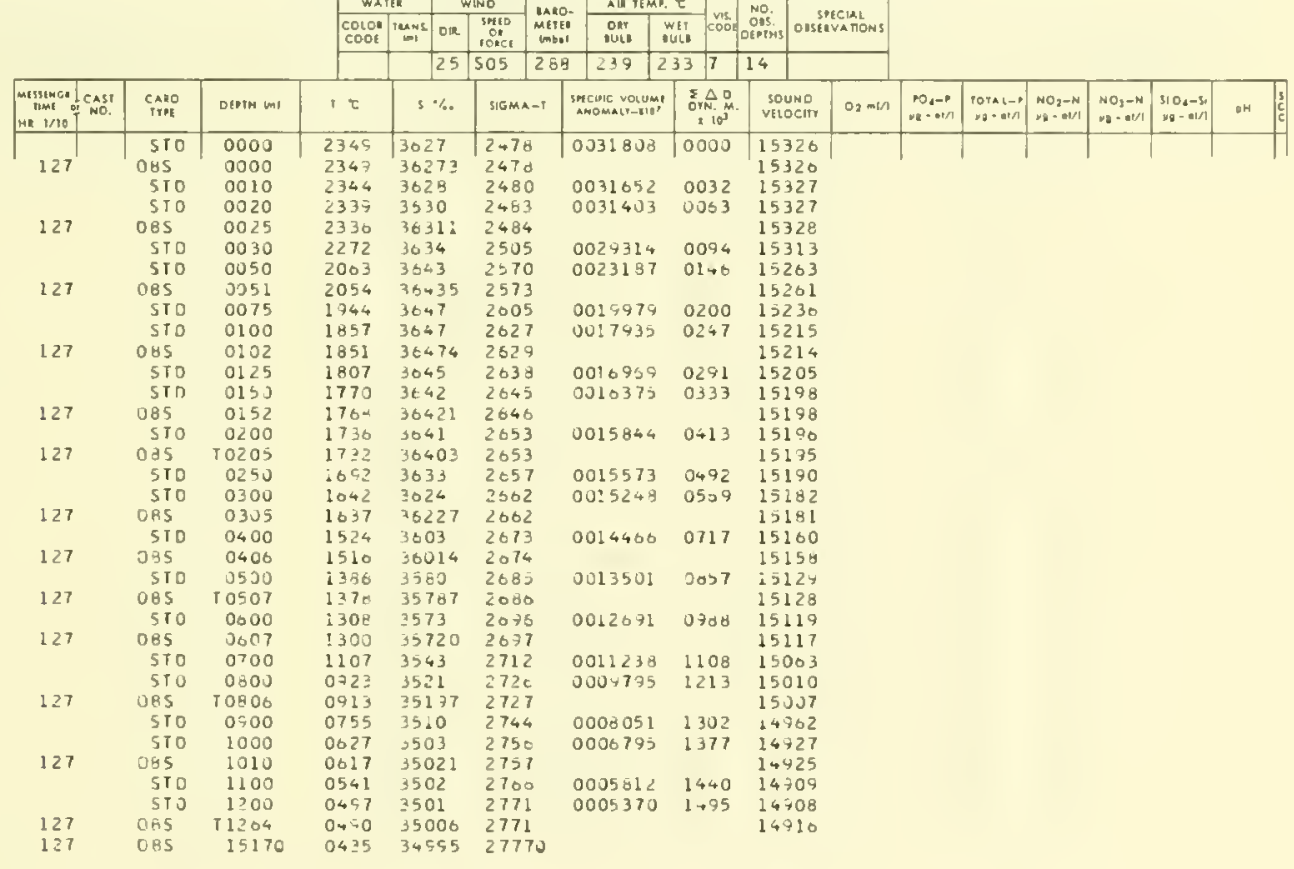


TABLE I.-Continued

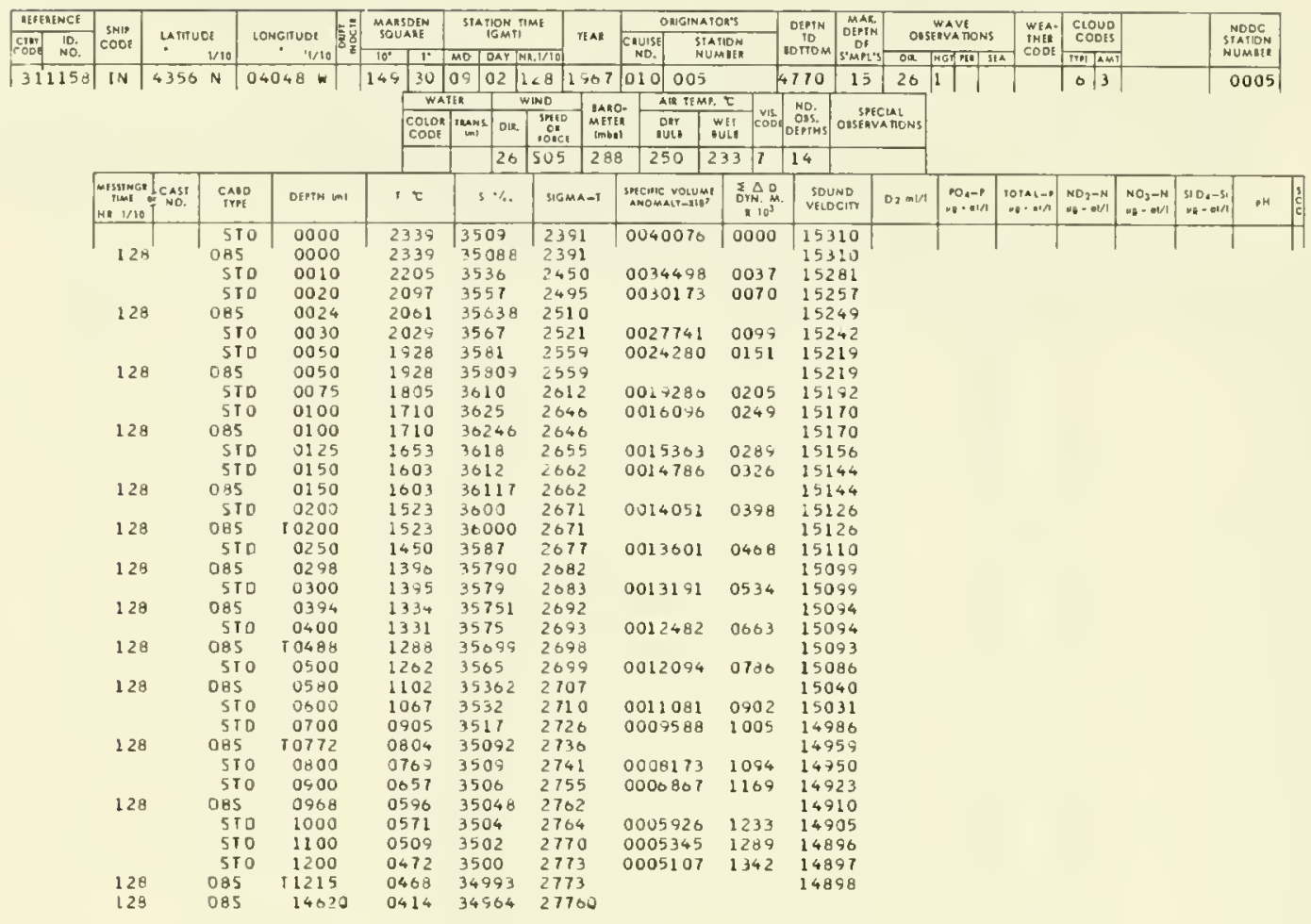

\begin{tabular}{|c|c|c|c|c|c|c|c|c|c|c|c|c|c|c|c|c|c|c|}
\hline DEFEHENGE & \multirow{2}{*}{$\begin{array}{c}S H I P \\
C O D\end{array}$} & \multirow{2}{*}{\multicolumn{2}{|c|}{$\begin{array}{l}\text { LATIUDE } \\
\text { W/10 }\end{array}$}} & \multirow{2}{*}{ LONGIIUDE } & \multirow{2}{*}{$\begin{array}{l}\text { MAASOEN } \\
\text { SDUARE }\end{array}$} & \multirow{2}{*}{ 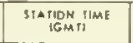 } & IIME & \multicolumn{2}{|c|}{ ONGINAFOA's } & \multirow{2}{*}{$\begin{array}{c}\text { DEP IM } \\
\text { IO } \\
\text { LOTIOM }\end{array}$} & \multirow{2}{*}{ 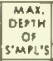 } & \multirow{2}{*}{\multicolumn{2}{|c|}{$\begin{array}{c}\text { Wave } \\
\text { OISEAvanons }\end{array}$}} & \multirow{2}{*}{$\begin{array}{l}\text { weat } \\
\text { iNhi } \\
\text { coOA }\end{array}$} & \multirow{2}{*}{ 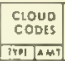 } & & & \multirow[b]{2}{*}{$\begin{array}{l}\text { NOOC } \\
\text { SIAFION } \\
\text { NUABEA }\end{array}$} \\
\hline 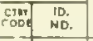 & & & & & & & YEAK & \begin{tabular}{|c|} 
CRUISE \\
NO.
\end{tabular} & $\begin{array}{l}\text { STAFION } \\
\text { NUMIER }\end{array}$ & & & & & & & & & \\
\hline 311158 & In & 4342 & $\mathrm{~N}$ & 04101 & \begin{tabular}{|l|l|}
149 & 31 \\
\end{tabular} & \begin{tabular}{|l|l|}
05 & 03 \\
\end{tabular} & $127 \mid 1967$ & \begin{tabular}{|l|l|}
010 & 000
\end{tabular} & & 4000 & 15 & 26 & \begin{tabular}{|l|l|l|}
2 & & \\
\end{tabular} & 18 & $8 / 6$ & & & 0006 \\
\hline & & & & & WA & itia & \begin{tabular}{l|l|} 
WIND & IMP \\
\end{tabular} & D. AIR TEMP. & & & & & & & & & & \\
\hline & & & & & $\begin{array}{l}\text { Colok } \\
\text { cobi }\end{array}$ & 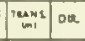 & 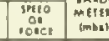 & \begin{tabular}{c|c} 
ORY \\
IULE
\end{tabular} & $\begin{array}{lll}\text { YEI } & \text { VII } \\
\text { UL } & \\
\end{array}$ & bo & ouserve & 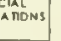 & & & & & & \\
\hline & & & & & & 21 & 506 & 233 & 227 & 14 & & & & & & & & \\
\hline & 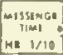 & Tcass & $\begin{array}{l}\text { CAND } \\
\text { IrNE }\end{array}$ & DETH $(m)$ & ir & $s \%$ & SIGMA-1 & 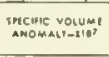 & 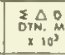 & & & $\mathrm{D}_{2} \mathrm{~m} / \mathrm{I}$ & 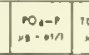 & 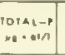 & $\mid \begin{array}{l}\text { No-ai/1 } \\
\mathrm{NO}^{2}-4\end{array}$ & $\begin{array}{l}\mathrm{NO}_{3}-\mathrm{N} \\
\mathrm{N}_{0}=\text { oit/1 }\end{array}$ & $\left|\begin{array}{ll}31 & 0,-51 \\
v a & -0.1 / 14\end{array}\right|$ & 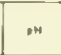 \\
\hline & & & 510 & 0000 & 2180 & 3552 & 2469 & 0032610 & 0000 & 152 & 275 & & & & & & & \\
\hline & 127 & & 085 & 0003 & 2180 & 35523 & 2465 & & & 152 & 275 & & & & & & & \\
\hline & & & 510 & 0010 & 2073 & 3551 & 2497 & 0029979 & 0031 & 152 & 249 & & & & & & & \\
\hline & & & 510 & 0020 & 1984 & 3549 & 2520 & 0027881 & 0000 & 152 & 226 & & & & & & & \\
\hline & 127 & & 085 & 0025 & 1966 & 35480 & 2529 & & & 152 & 216 & & & & & & & \\
\hline & & & $5 T D$ & 0030 & 1920 & 3550 & 2542 & 0025817 & $008 ?$ & 152 & 211 & & & & & & & \\
\hline & & & STO & 0050 & 1825 & 3584 & 2587 & 0021554 & 0134 & 151 & 190 & & & & & & & \\
\hline & 127 & & 085 & 0050 & 1825 & $35 \forall 41$ & 2587 & & & 151 & 190 & & & & & & & \\
\hline & & & STO & 0075 & 1721 & 3600 & 2025 & 0012074 & 9184 & 151 & 166 & & & & & & & \\
\hline & & & STO & 0100 & 1637 & 3615 & 2637 & $001511 \mathrm{i}$ & 0225 & 151 & 147 & & & & & & & \\
\hline & 127 & & 085 & $0: 01$ & 1634 & 30160 & 2058 & & & 151 & 140 & & & & & & & \\
\hline & & & $5 \mathrm{TO}$ & 0125 & $25 \varepsilon 0$ & 3600 & 2064 & 0014470 & 0202 & 151 & 133 & & & & & & & \\
\hline & & & $5 T 0$ & 0150 & 1528 & 9600 & 2670 & 0014026 & 0298 & 151 & 120 & & & & & & & \\
\hline & 127 & & 085 & 0150 & 1528 & 35597 & 2070 & & & 151 & 120 & & & & & & & \\
\hline & & & $5 \mathrm{TO}$ & 0200 & 1439 & $35 \varepsilon 5$ & 2677 & 0013399 & 0367 & 150 & 078 & & & & & & & \\
\hline & 127 & & 085 & 10202 & 1430 & 35840 & 2678 & & & 150 & 097 & & & & & & & \\
\hline & & & $5 T 0$ & 0250 & 1377 & 3575 & 2683 & 0012973 & 0433 & 150 & 985 & & & & & & & \\
\hline & & & $5 \mathrm{TD}$ & 0300 & 1339 & 3571 & 2688 & 0022041 & 0497 & 150 & 080 & & & & & & & \\
\hline & 127 & & 085 & 0302 & 1338 & 35708 & 2088 & & & 150 & & & & & & & & \\
\hline & & & $5 T 0$ & 0400 & 1335 & 3578 & 2694 & 00123.27 & 0621 & 150 & & & & & & & & \\
\hline & 127 & & 085 & 0402 & 1333 & 35782 & 2095 & & & 150 & & & & & & & & \\
\hline & & & 510 & 0500 & 1160 & 3545 & 2702 & 0011763 & 0742 & iso & & & & & & & & \\
\hline & 127 & & 085 & 10500 & 2158 & 35431 & 2702 & & & 150 & 47 & & & & & & & \\
\hline & & & $5 T O$ & 0600 & $0 \ngtr 82$ & 3520 & 2716 & 3010465 & 0853 & 149 & & & & & & & & \\
\hline & 127 & & 085 & 0603 & 0977 & 35153 & 2716 & & & 149 & 97 & & & & & & & \\
\hline & & & 510 & 0700 & $08+1$ & 3512 & 2733 & 0008910 & 0950 & 143 & & & & & & & & \\
\hline & & & STO & 0800 & 0722 & 3505 & 2744 & 0007785 & 1033 & 143 & & & & & & & & \\
\hline & 127 & & OBS & TOSO4 & 0718 & 35042 & 2745 & & & 149 & & & & & & & & \\
\hline & & & 550 & 0900 & 0620 & 3504 & 2757 & 0000506 & 1105 & 149 & 10 & & & & & & & \\
\hline & & & $5 T D$ & 1000 & 0550 & 3504 & 2707 & 0005035 & 1106 & 148 & & & & & & & & \\
\hline & 127 & & 085 & 1006 & 0540 & 35030 & 2707 & & & 148 & 396 & & & & & & & \\
\hline & & & 550 & 1100 & 0505 & 3501 & 2770 & 0005364 & 1221 & 148 & & & & & & & & \\
\hline & & & STD & 1200 & 0469 & 3493 & 2772 & 0005140 & 1274 & 145 & & & & & & & & \\
\hline & 127 & & 085 & $T 1258$ & 0452 & $3497 \%$ & 2773 & & & $14 d$ & & & & & & & & \\
\hline & & & STO & 1500 & 0441 & 3497 & 2774 & 0005017 & 1325 & 147 & & & & & & & & \\
\hline & & & $5 T 0$ & 1400 & 0420 & 3496 & 2775 & 0004912 & 1374 & 149 & & & & & & & & \\
\hline & & & D & 150.0 & 0400 & 3496 & 2777 & 0004820 & $\lcm{423}$ & 149 & & & & & & & & \\
\hline & 127 & & 085 & T1510 & 0.05 & 34957 & 2777 & & & 149 & & & & & & & & \\
\hline
\end{tabular}




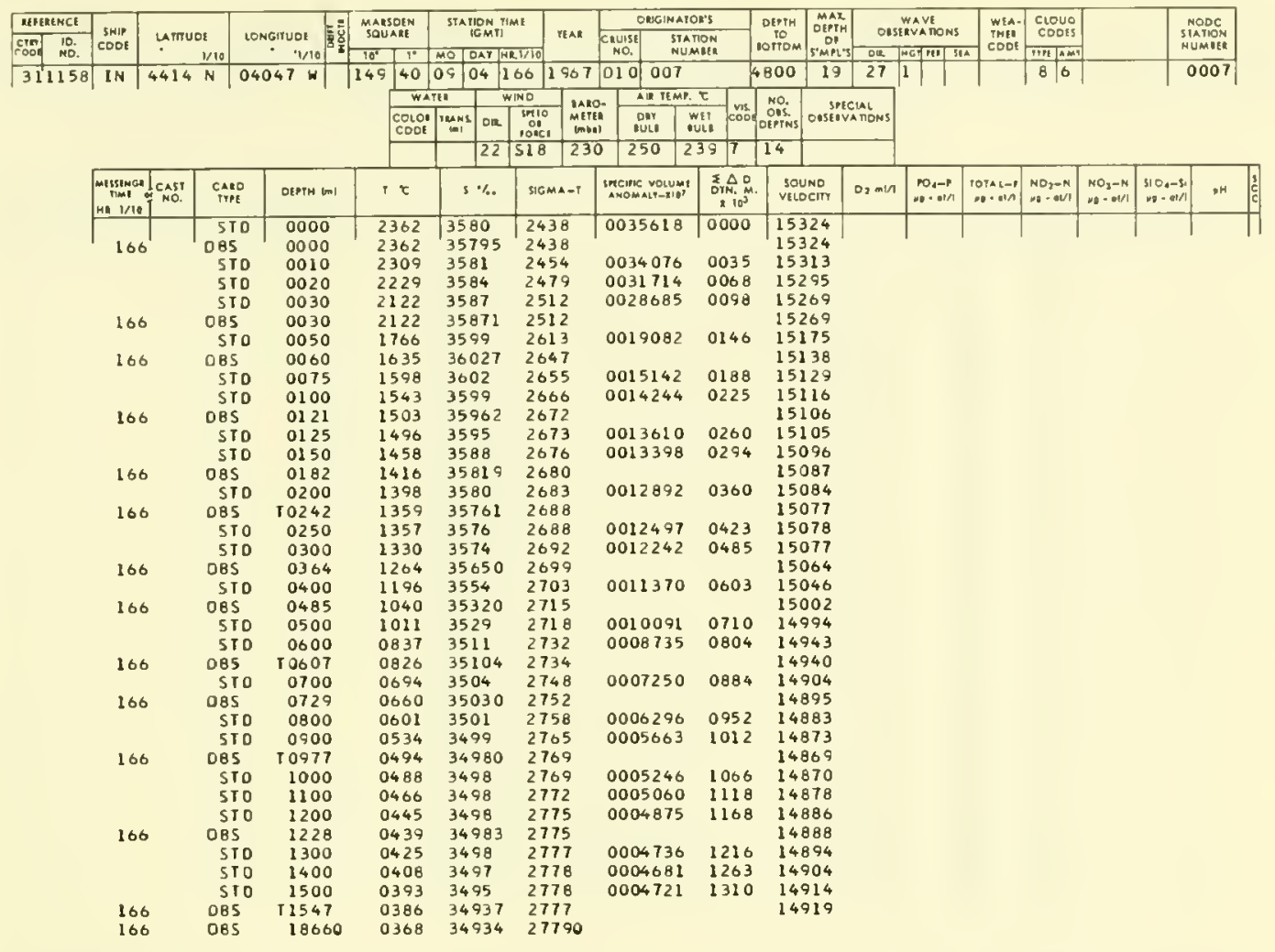

\begin{tabular}{|c|c|c|c|c|c|c|c|c|c|c|c|c|c|c|c|c|c|c|}
\hline \multirow{3}{*}{ 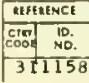 } & \multirow{3}{*}{\begin{tabular}{|c|} 
SMIF \\
coot \\
IN
\end{tabular}} & \multirow{2}{*}{\multicolumn{2}{|c|}{ untmod }} & \multirow{2}{*}{$\begin{array}{c}\text { LONGITUOR } \\
\text { '1/10 }\end{array}$} & \multirow{2}{*}{$\begin{array}{l}\text { MAasoter } \\
\text { SOUAKE }\end{array}$} & \multirow{2}{*}{$\begin{array}{l}\text { STARION TIME } \\
\text { IGM II }\end{array}$} & & \multicolumn{2}{|c|}{ T OMGINATON'S } & \multirow{2}{*}{$\begin{array}{c}\text { OEPH } \\
\text { TO } \\
\text { OOTOM M }\end{array}$} & \multirow{2}{*}{ 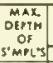 } & \multirow{2}{*}{\multicolumn{2}{|c|}{$\begin{array}{l}\text { WAVE } \\
\text { OHSEIYATIONS }\end{array}$}} & \multirow{2}{*}{$\begin{array}{c}\text { WIA. } \\
\text { THER } \\
\text { COOE }\end{array}$} & \multirow{2}{*}{$\begin{array}{l}\text { clouo } \\
\text { coots } \\
\text { TMM IAN }\end{array}$} & & & \multirow{2}{*}{ 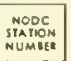 } \\
\hline & & & & & & & Rा:10 & \multirow{2}{*}{\begin{tabular}{|c|c|}
$\begin{array}{c}\text { CDUISE } \\
\text { No. } \\
010\end{array}$ & 0 \\
010 & 0
\end{tabular}} & $\begin{array}{l}\text { STAPIOA } \\
\text { MUMMEE }\end{array}$ & & & & & & & & & \\
\hline & & 4411 & $\mathrm{~N}$ & $4058 \mathrm{~W}$ & $\begin{array}{ll}149 & 40\end{array}$ & \begin{tabular}{l|l|}
05 & 05
\end{tabular} & \begin{tabular}{l|l}
129 & 1967
\end{tabular} & & & 4600 & 17 & 264 & $4=$ & & & & & 0008 \\
\hline & & & & & $W A$ & The & NINO & AIR TEMP. & & & & & & & & & & \\
\hline & & & & & $\begin{array}{l}\text { COlOE } \\
\text { COOE }\end{array}$ & $\lim _{m 1}$ DIR & \begin{tabular}{|l|}
$\sin 10$ \\
of \\
1001
\end{tabular} & 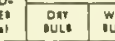 & Jit cood & Oons. & $\begin{array}{l}\text { sprc } \\
\text { oosenve }\end{array}$ & CATLNS & & & & & & \\
\hline & & & & & & 22 & 516 & 244 & 281 & 14 & & & & & & & & \\
\hline & $\begin{array}{l}\text { MISHNGR } \\
\text { Mant } \\
\text { MR } 1 / 10\end{array}$ & I CASET & cand & DEPTN Im & $1 \%$ & $5 \%$ & SIGMA-T & $\begin{array}{l}\text { SMCENC volume } \\
\text { ANOWAST-110" }\end{array}$ & 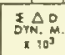 & & $\begin{array}{l}\text { UND } \\
\text { oerry }\end{array}$ & $0_{2}=1 / 1$ & 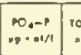 & 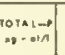 & 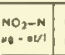 & $\begin{array}{l}N O_{3}-N \\
{ }_{n 10} \\
\cdots\end{array}$ & 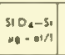 & $\rightarrow H$ \\
\hline & & & STO & 0000 & 2340 & 3529 & 2406 & 0038654 & 0000 & & 313 & & & & & & & \\
\hline & 129 & & 085 & 0000 & 2340 & 35289 & 2406 & & & & 313 & & & & & & & \\
\hline & & & STo & 0020 & 2246 & 3541 & 2442 & 0035240 & 0037 & & 292 & & & & & & & \\
\hline & & & STO & 0020 & 2150 & 3553 & 2478 & 0031847 & 0070 & & 271 & & & & & & & \\
\hline & 129 & & 085 & 0028 & 2072 & 35622 & 2506 & & & & 253 & & & & & & & \\
\hline & & & Sto & 0030 & 2047 & 3565 & 2515 & 0028344 & 0101 & & 247 & & & & & & & \\
\hline & & & $5 T 0$ & 0050 & 1840 & 3586 & 2585 & 0021774 & 0151 & & 195 & & & & & & & \\
\hline & 129 & & 085 & 0055 & 1801 & 35914 & 2599 & & & & 185 & & & & & & & \\
\hline & & & STO & 0075 & 1769 & 3613 & 2623 & 0018221 & 0201 & 15 & 182 & & & & & & & \\
\hline & & & STO & 0100 & 1723 & 3629 & 2646 & 0016148 & 0244 & & 174 & & & & & & & \\
\hline & 129 & & 085 & 0210 & 1703 & 36305 & 2653 & & & & 170 & & & & & & & \\
\hline & & & STO & 0125 & 1661 & 3622 & 2656 & 0015252 & 0283 & 15 & 159 & & & & & & & \\
\hline & & & STD & 0250 & 1603 & 3611 & 2661 & 0014837 & 0321 & & 144 & & & & & & & \\
\hline & 129 & & 085 & 0166 & 1573 & 36061 & 2604 & & & 15 & 137 & & & & & & & \\
\hline & & & STD & 0200 & 1538 & 3602 & 2669 & 0014229 & 0393 & 15 & 131 & & & & & & & \\
\hline & 129 & & 085 & T 0221 & 1515 & 35953 & 2672 & & & 15 & 127 & & & & & & & \\
\hline & & & STO & 0250 & 1473 & 3592 & 2676 & 0013720 & 0463 & 15 & 118 & & & & & & & \\
\hline & & & STO & 0300 & 1413 & 3582 & 2681 & $00: 3343$ & 0531 & 15 & 105 & & & & & & & \\
\hline & 129 & & DBS & 0330 & 1385 & 35787 & 2684 & & & 15 & 101 & & & & & & & \\
\hline & & & sro & 0400 & 1350 & 3578 & 2690 & 0012773 & 0661 & 15 & 103 & & & & & & & \\
\hline & 129 & & 085 & 0439 & 1329 & 35771 & 2695 & & & 15 & 100 & & & & & & & \\
\hline & & & 510 & 0500 & 1278 & 3567 & 2697 & 0012263 & 0786 & 150 & 092 & & & & & & & \\
\hline & 129 & & 085 & T 0548 & 1216 & 35562 & 2701 & & & 150 & 077 & & & & & & & \\
\hline & & & STO & 0600 & 1113 & 3540 & 2708 & 0011334 & 0904 & 150 & 048 & & & & & & & \\
\hline & 129 & & 085 & 0657 & 1007 & 35260 & 2716 & & & 150 & 018 & & & & & & & \\
\hline & & & 510 & 0700 & 0527 & 3519 & 2724 & 0009809 & 1010 & 14 & 995 & & & & & & & \\
\hline & & & 510 & 0800 & 0764 & 3507 & 2740 & 0008242 & 1100 & 14 & 948 & & & & & & & \\
\hline & 129 & & 085 & 0877 & 0662 & 35000 & 2749 & & & 14 & 920 & & & & & & & \\
\hline & & & 510 & 0900 & 0637 & 3459 & 2752 & 0007091 & 1177 & 14 & 914 & & & & & & & \\
\hline & & & Sio & 1000 & 0545 & 3497 & 2702 & 0006082 & 1243 & 140 & 293 & & & & & & & \\
\hline & 129 & & 085 & 1096 & 0479 & 34959 & 2769 & & & 14 & 882 & & & & & & & \\
\hline & & & 510 & 1100 & 0478 & 3496 & 2709 & 0005379 & 1300 & & 883 & & & & & & & \\
\hline & & & $5 \mathrm{IO}$ & 1200 & 0449 & 3495 & 2772 & 0005155 & 1353 & 14 & 887 & & & & & & & \\
\hline & & & SIO & 1300 & 0427 & 3494 & 2773 & 0005026 & 1404 & & 875 & & & & & & & \\
\hline & 129 & & 085 & 81371 & 0414 & 34939 & 2774 & & & 14 & 901 & & & & & & & \\
\hline & & & SIO & 1400 & 0410 & 3494 & 2775 & 0004927 & 1454 & 14 & 904 & & & & & & & \\
\hline & & & 510 & 1500 & 0398 & 3494 & 2776 & 0004860 & 1503 & & 916 & & & & & & & \\
\hline & 129 & & 085 & 11654 & 0391 & 34947 & 2777 & & & 149 & 939 & & & & & & & \\
\hline
\end{tabular}


TABLE I.-Continued

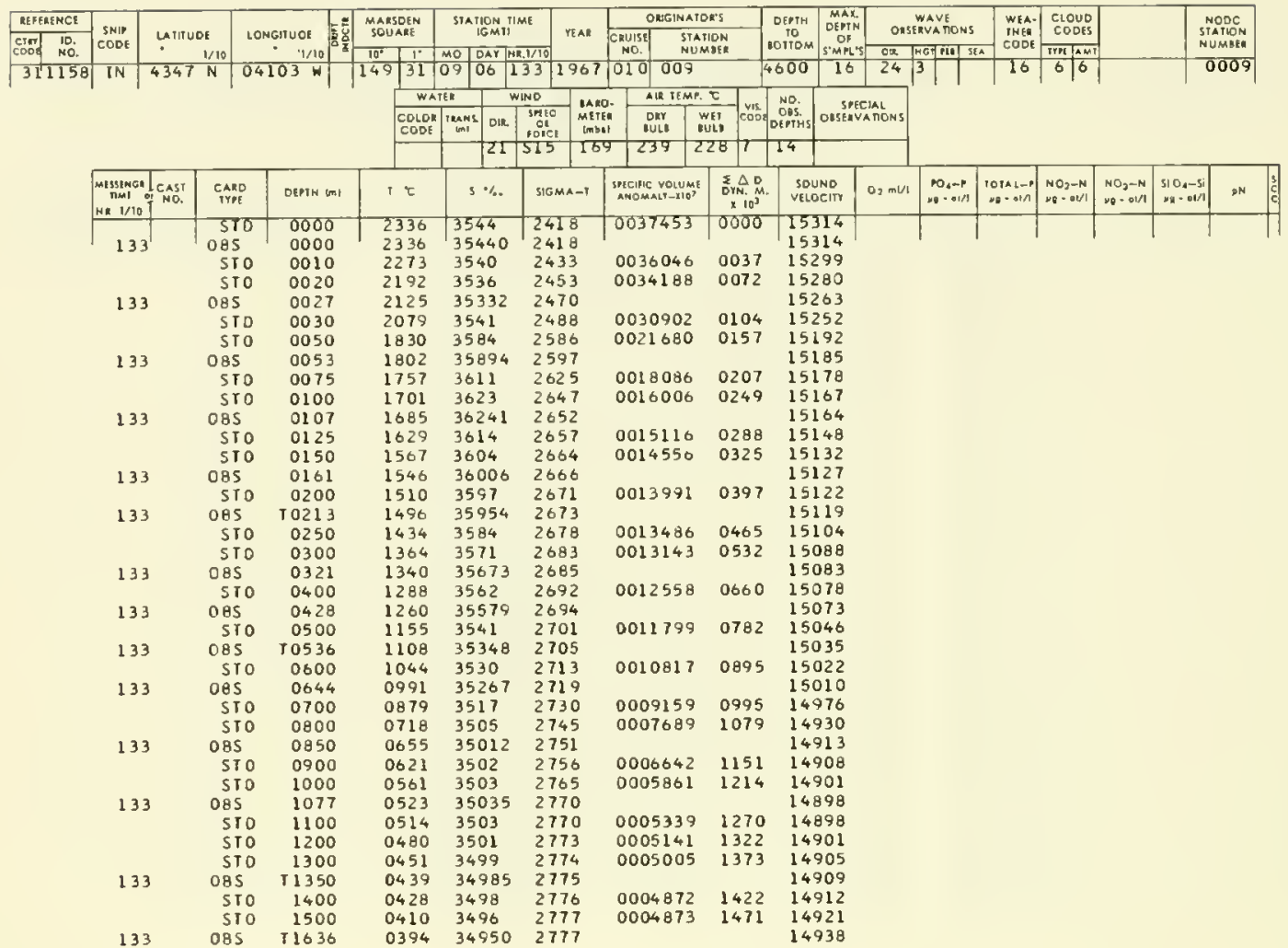

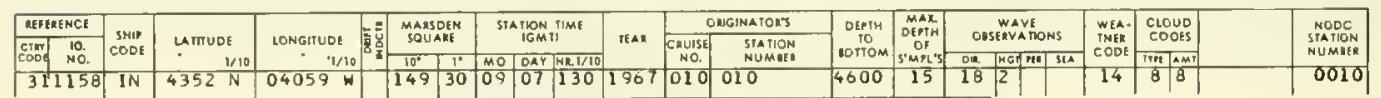

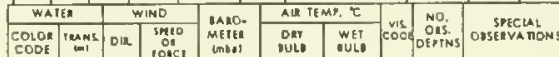

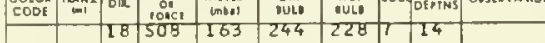

\begin{tabular}{|c|c|c|c|c|c|c|c|c|c|c|c|c|c|c|c|}
\hline 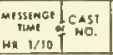 & $\begin{array}{l}\text { CARD } \\
\text { IYPE }\end{array}$ & DEPTK UnI & it & $3 \cdot \%$ & SIGMA-1 & 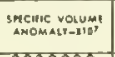 & 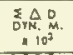 & $\begin{array}{l}\text { SOUNO } \\
\text { VELOCINY }\end{array}$ & $0,2 \mathrm{~m} / 1 / 1$ & 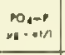 & 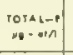 & $\begin{array}{l}\mathrm{NO}_{2}-\mathrm{N} \\
\mathrm{H}-0.1 / 1\end{array}$ & $\begin{array}{l}\mathrm{NO}_{1}-\mathrm{N} \\
\mathrm{HO}=\mathrm{N} / \mathrm{V} / 1\end{array}$ & 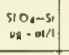 & $\mathrm{MN}$ \\
\hline \multirow{4}{*}{130} & डा० & 0000 & 2370 & 3535 & 2401 & 0039080 & 0000 & 15321 & & & & & & & \\
\hline & ogs & 0000 & 2370 & 35346 & 2401 & & & 15321 & & & & & & & \\
\hline & SIO & 0010 & 2375 & 3553 & 2414 & 0037944 & 0039 & 15326 & & & & & & & \\
\hline & STO & 0020 & 2381 & 3572 & 2426 & 0036765 & 0076 & 15331 & & & & & & & \\
\hline \multirow[t]{2}{*}{130} & OBS & 0024 & 2383 & 35809 & 2432 & & & 15333 & & & & & & & \\
\hline & STo & 0030 & 2205 & 3599 & 2475 & 0032195 & 0110 & 15312 & & & & & & & \\
\hline \multirow[t]{3}{*}{130} & OAS & 0049 & 2033 & 36403 & 2576 & & & 15255 & & & & & & & \\
\hline & SIO & 0050 & 2027 & 3640 & 2578 & 0022482 & 0165 & 15253 & & & & & & & \\
\hline & STO & 0075 & 1885 & 3634 & 2610 & 0019468 & 0217 & 15217 & & & & & & & \\
\hline \multirow[t]{3}{*}{130} & OBS & 0098 & 1786 & 36293 & 2031 & & & 15192 & & & & & & & \\
\hline & STo & 0100 & 1780 & 3629 & 2633 & 0027403 & 0264 & 15191 & & & & & & & \\
\hline & SIO & 0125 & 1718 & 3623 & 2643 & 0016480 & 0306 & 15176 & & & & & & & \\
\hline \multirow[t]{2}{*}{130} & oes & 0146 & 1676 & 36195 & 2651 & & & 15167 & & & & & & & \\
\hline & SIO & 0150 & 1671 & 3619 & 2651 & 0015779 & 0346 & 15166 & & & & & & & \\
\hline \multirow[t]{3}{*}{130} & 085 & 10195 & 1616 & 36161 & 2662 & & & 15156 & & & & & & & \\
\hline & SIO & 0200 & 1612 & 3616 & 2663 & 0014834 & 0423 & 15156 & & & & & & & \\
\hline & STO & 0250 & 1504 & 3009 & 2669 & 0014441 & 0496 & 15148 & & & & & & & \\
\hline \multirow[t]{2}{*}{130} & 085 & 0292 & 1515 & 36007 & 2673 & & & 15139 & & & & & & & \\
\hline & SIO & 0300 & 1499 & 3598 & 2675 & 0013987 & 0567 & 15135 & & & & & & & \\
\hline \multirow[t]{2}{*}{130} & 085 & 0388 & 1371 & 35761 & 2685 & & & 15105 & & & & & & & \\
\hline & STo & 0400 & 1366 & 3578 & 2688 & 0012957 & 0702 & 15106 & & & & & & & \\
\hline \multirow[t]{2}{*}{130} & 085 & T0484 & 1332 & 35795 & 2696 & & & 15109 & & & & & & & \\
\hline & STD & 0500 & 1290 & 3573 & 2700 & 0012064 & 0827 & 15096 & & & & & & & \\
\hline \multirow[t]{3}{*}{130} & 085 & 0581 & 1105 & 35470 & 2715 & & & 15043 & & & & & & & \\
\hline & STo & 0600 & 1079 & 3544 & 2717 & 0010422 & 0939 & 15036 & & & & & & & \\
\hline & STO & 0700 & 0939 & 3528 & 2729 & 0009354 & 1038 & 15000 & & & & & & & \\
\hline \multirow[t]{3}{*}{130} & OAS & 10764 & 0850 & 35191 & 2737 & & & 14976 & & & & & & & \\
\hline & STO & 0800 & 0789 & 3515 & 2743 & 0008045 & 1125 & 14959 & & & & & & & \\
\hline & STO & 0900 & 0646 & 3506 & 2750 & 0006707 & 1199 & 14918 & & & & & & & \\
\hline \multirow[t]{3}{*}{130} & 085 & 0954 & 0585 & 35018 & 2761 & & & 14903 & & & & & & & \\
\hline & STO & 1000 & 0552 & 3500 & 2763 & 0005957 & 1262 & 14897 & & & & & & & \\
\hline & STO & 1100 & 0493 & 3497 & 2768 & 0005497 & 1319 & 14889 & & & & & & & \\
\hline \multirow[t]{4}{*}{130} & 085 & T1196 & 0452 & 34950 & 2772 & & & 14888 & & & & & & & \\
\hline & STO & 1200 & 0452 & 3496 & 2772 & 0005163 & 1373 & 14888 & & & & & & & \\
\hline & STO & 1300 & 0441 & 3496 & 2773 & 0005084 & 1424 & 14901 & & & & & & & \\
\hline & STO & 1400 & 0430 & 3497 & 2775 & 0005002 & 1474 & 14913 & & & & & & & \\
\hline 130 & 085 & $T 1436$ & 0424 & 34969 & 2776 & & & 14920 & & & & & & & \\
\hline
\end{tabular}




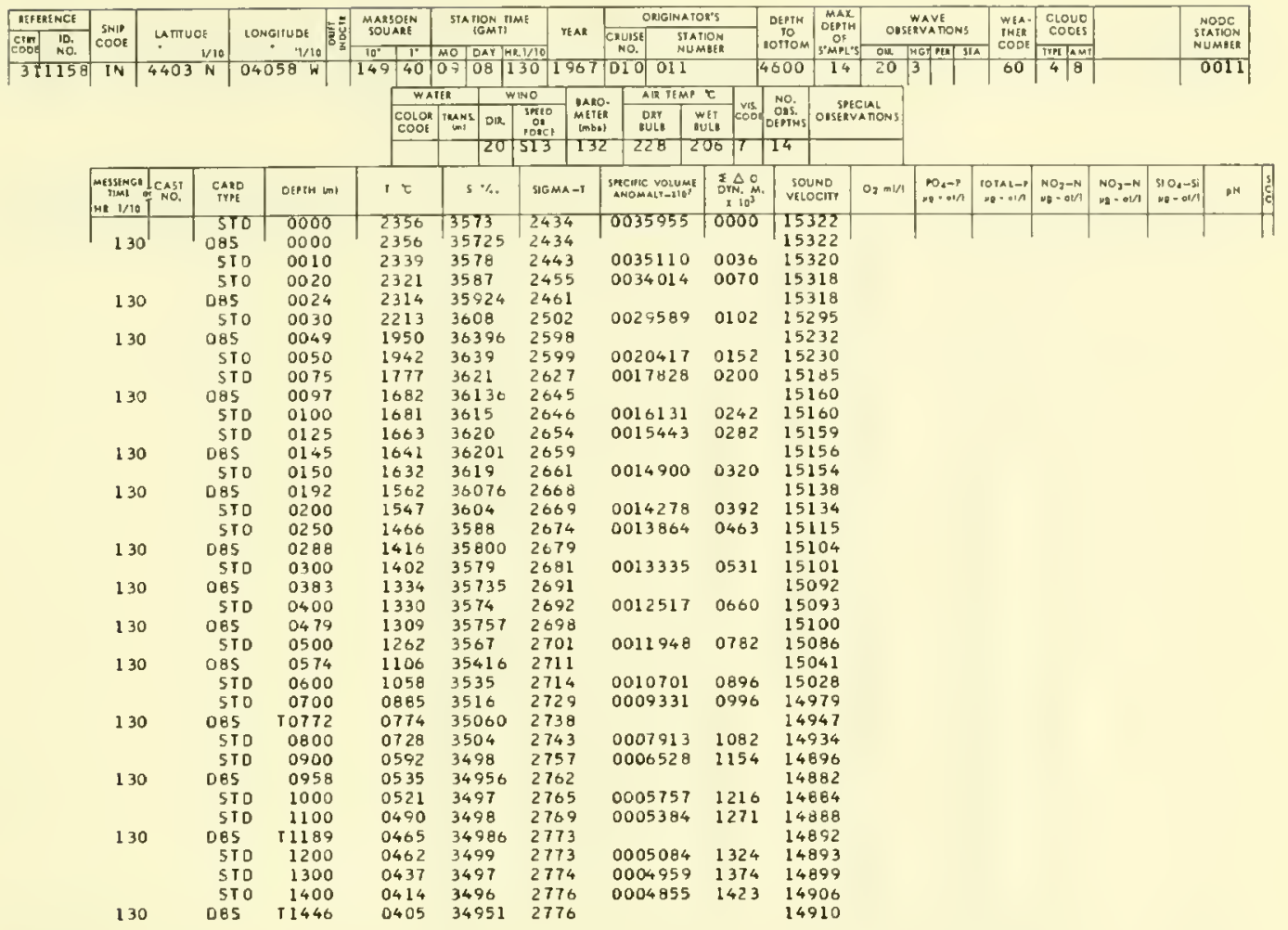

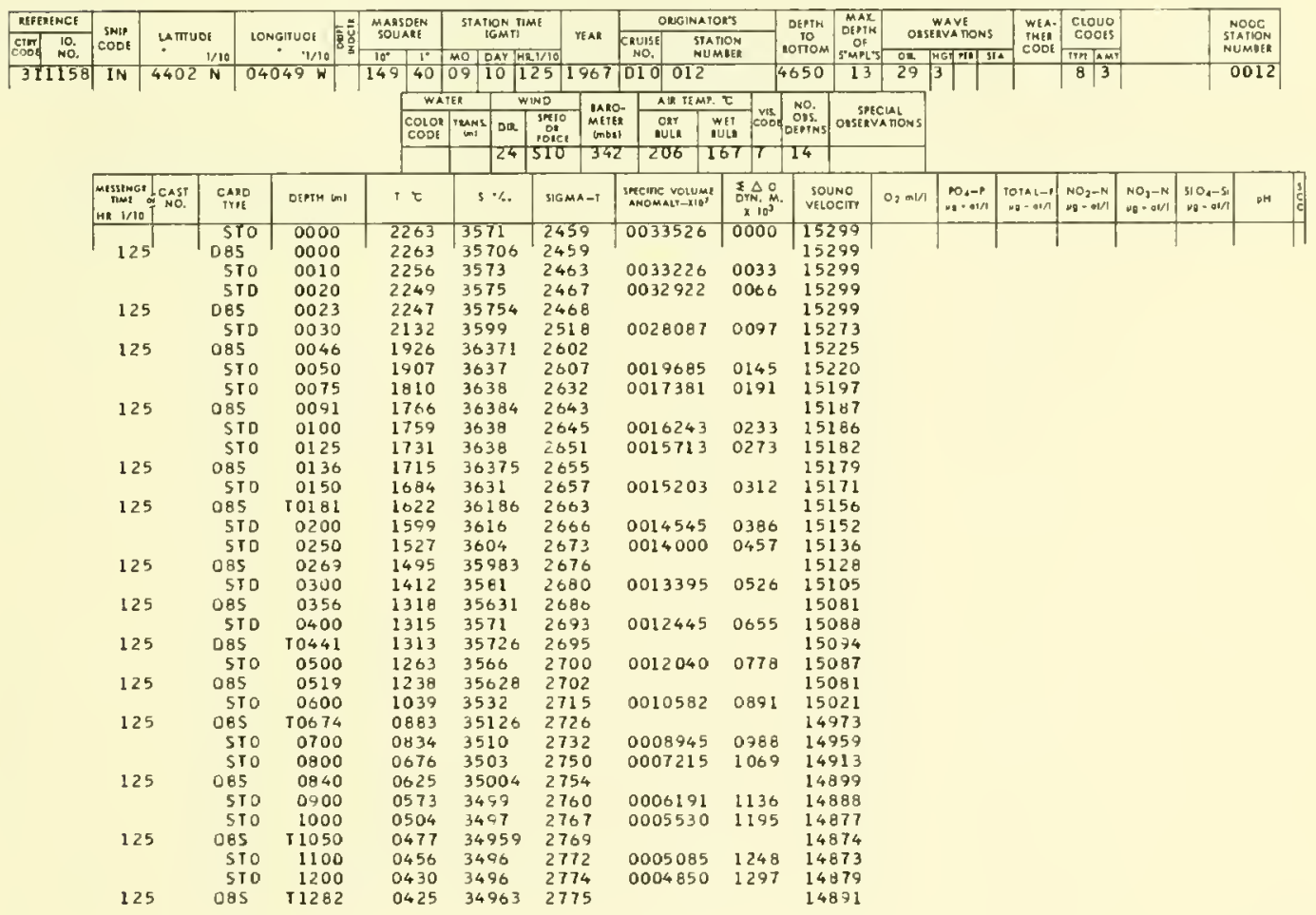


TABLE I.-Continued

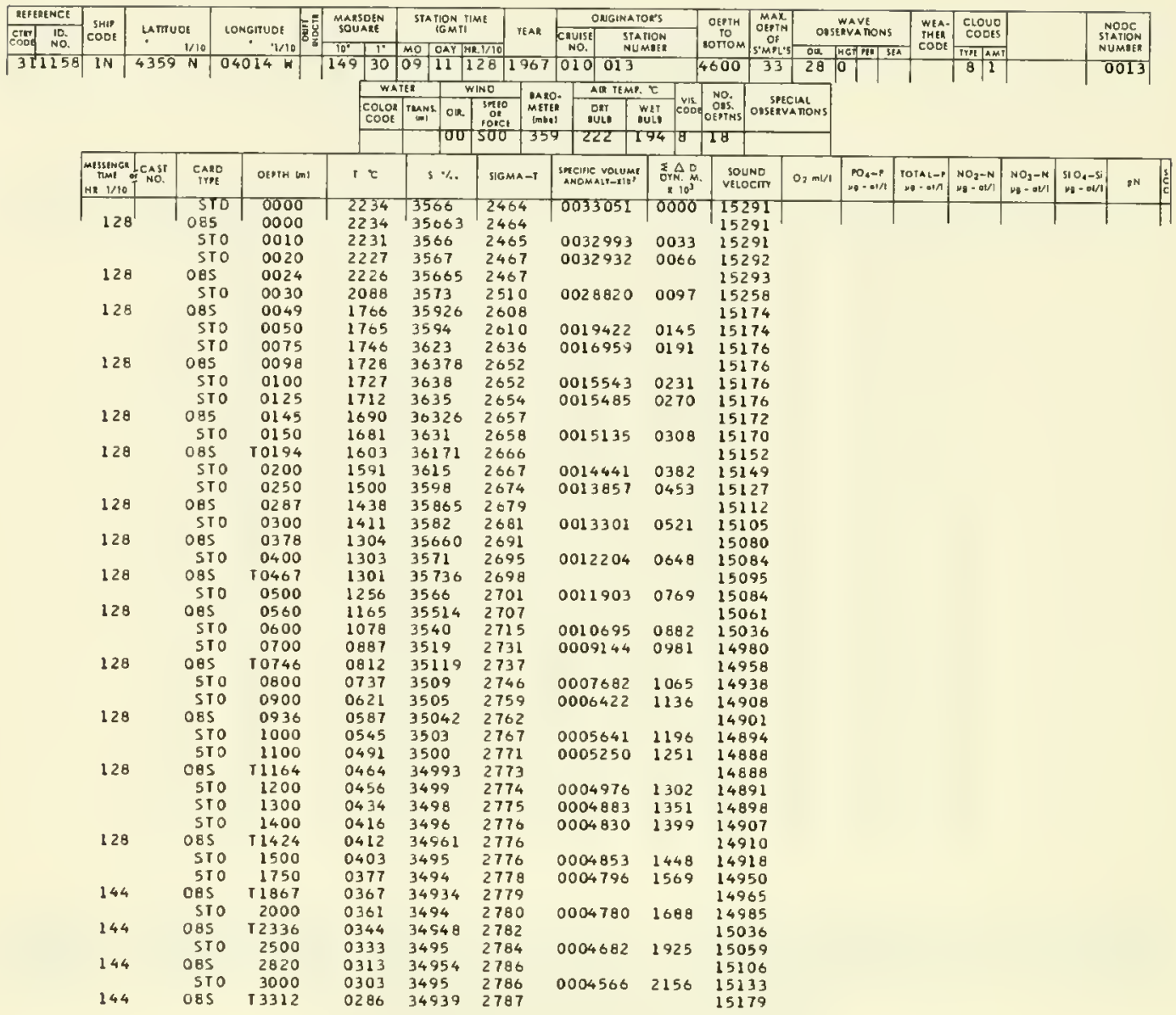




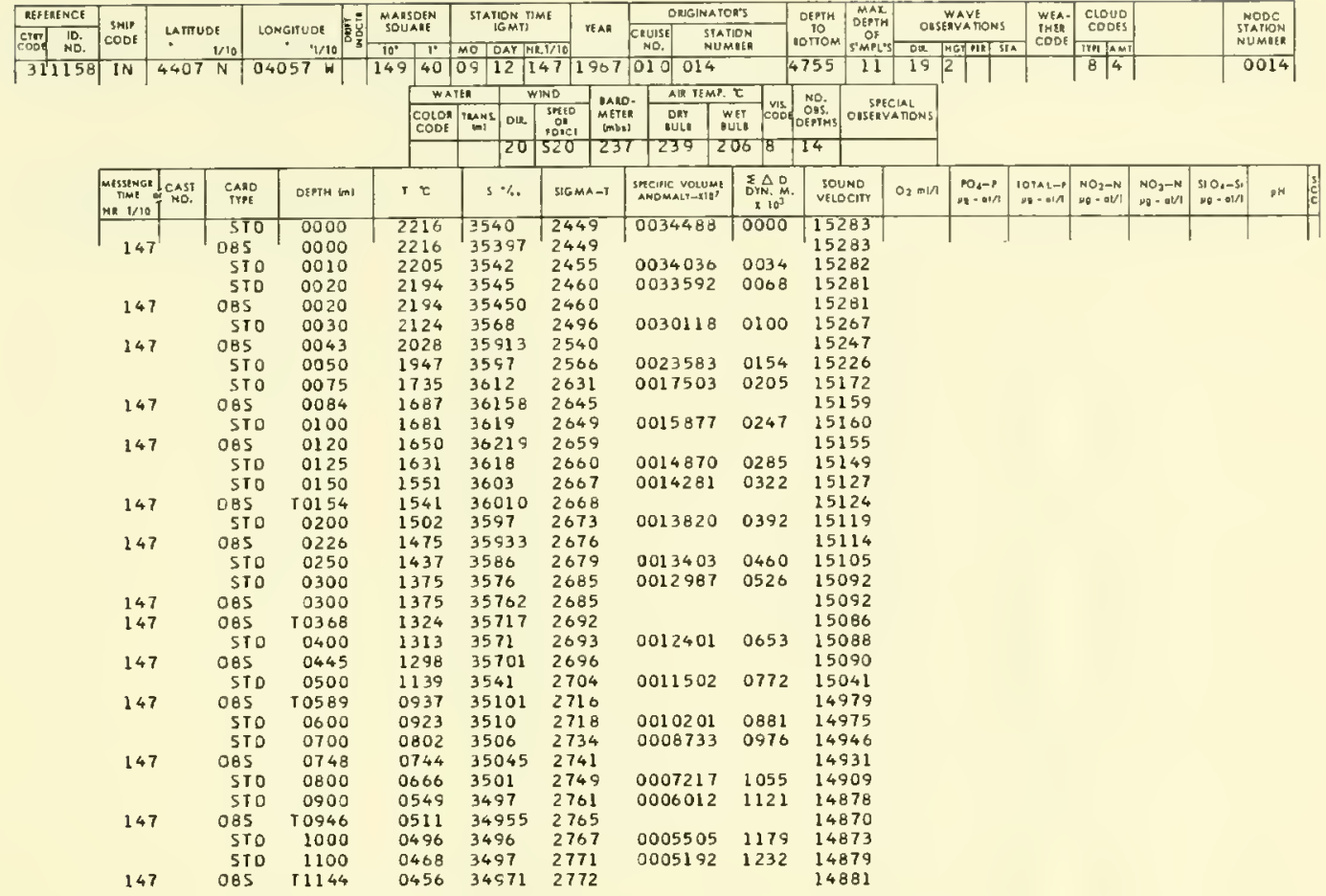

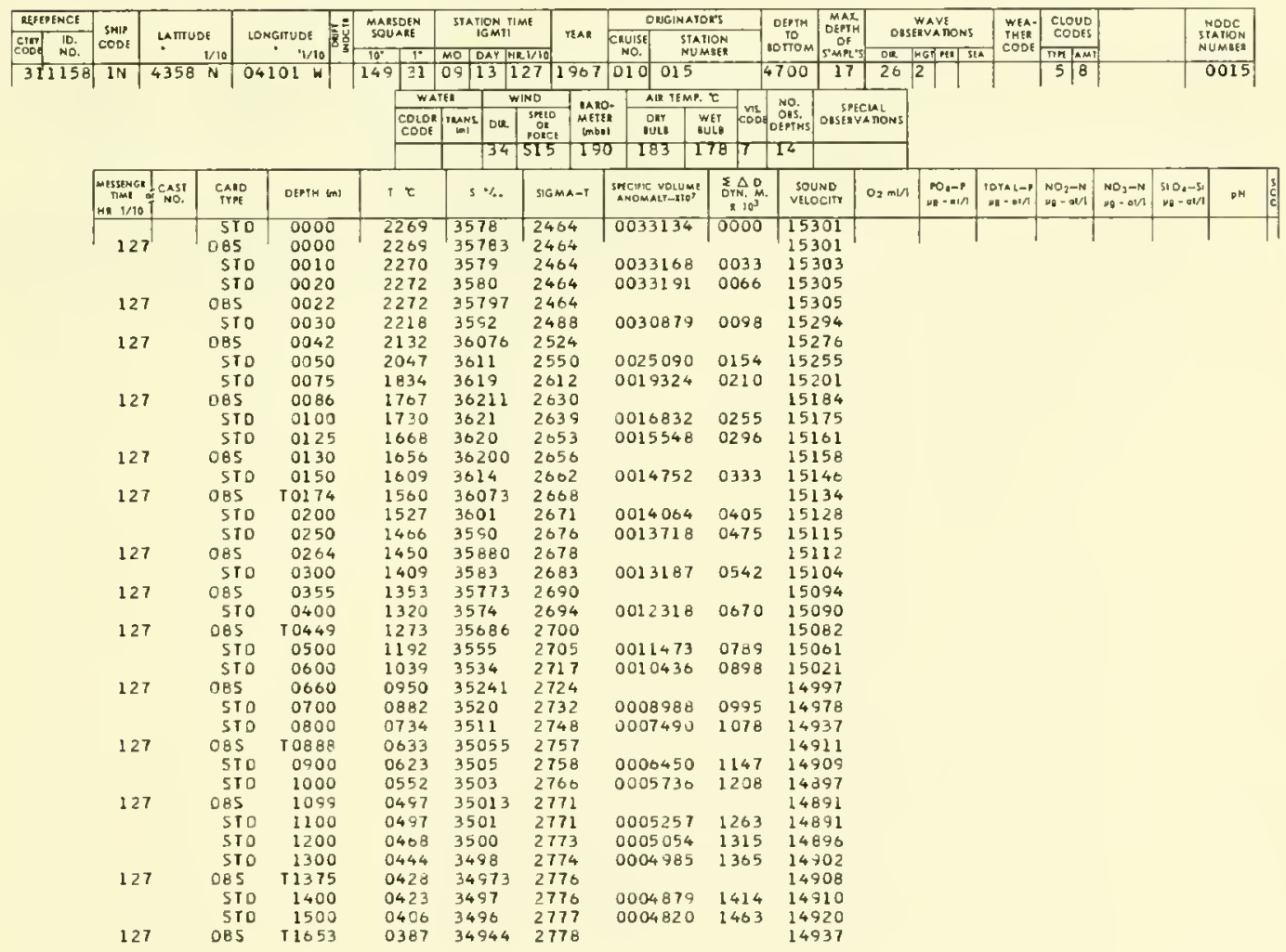


TaBle I.-Continued

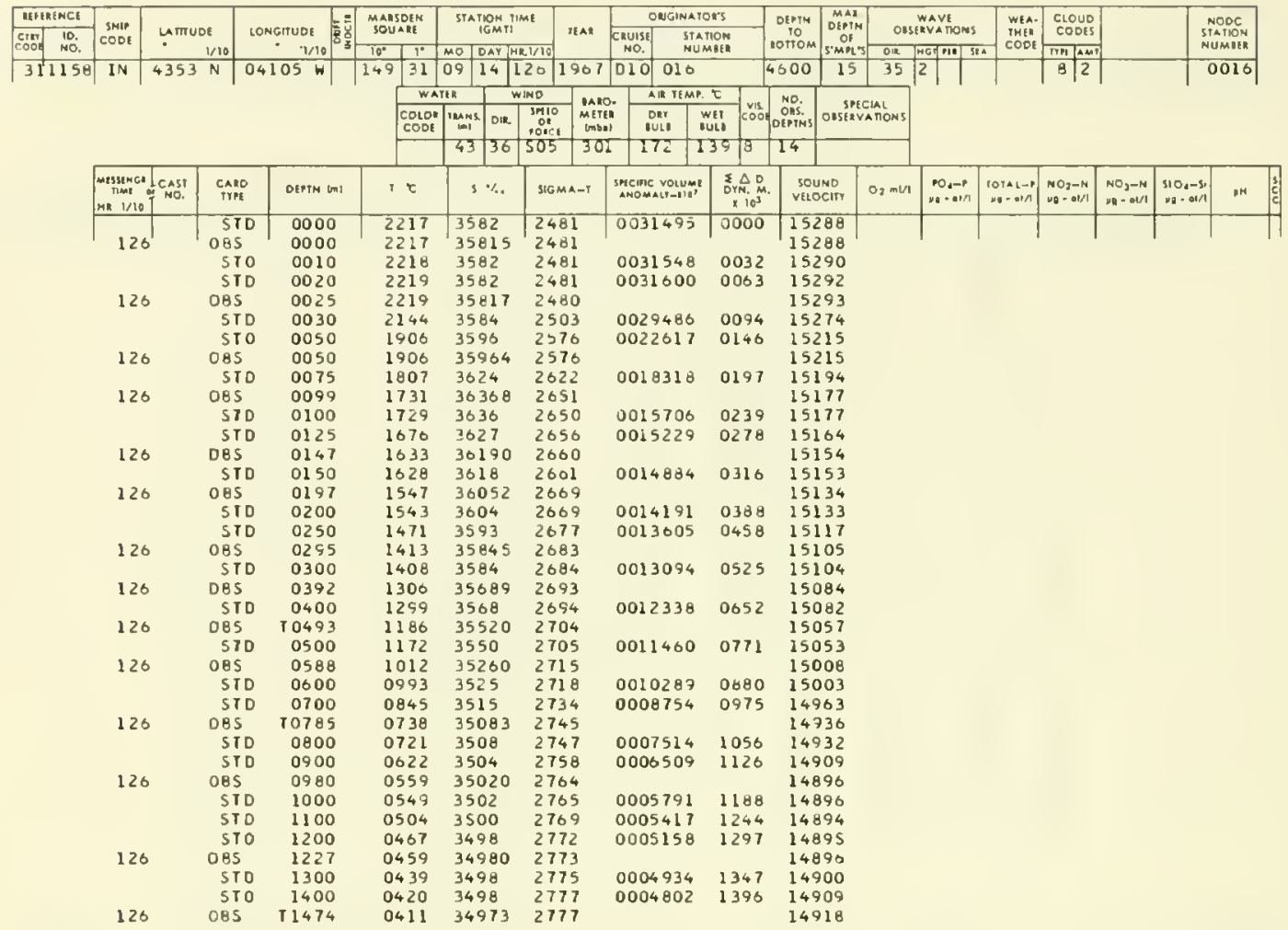

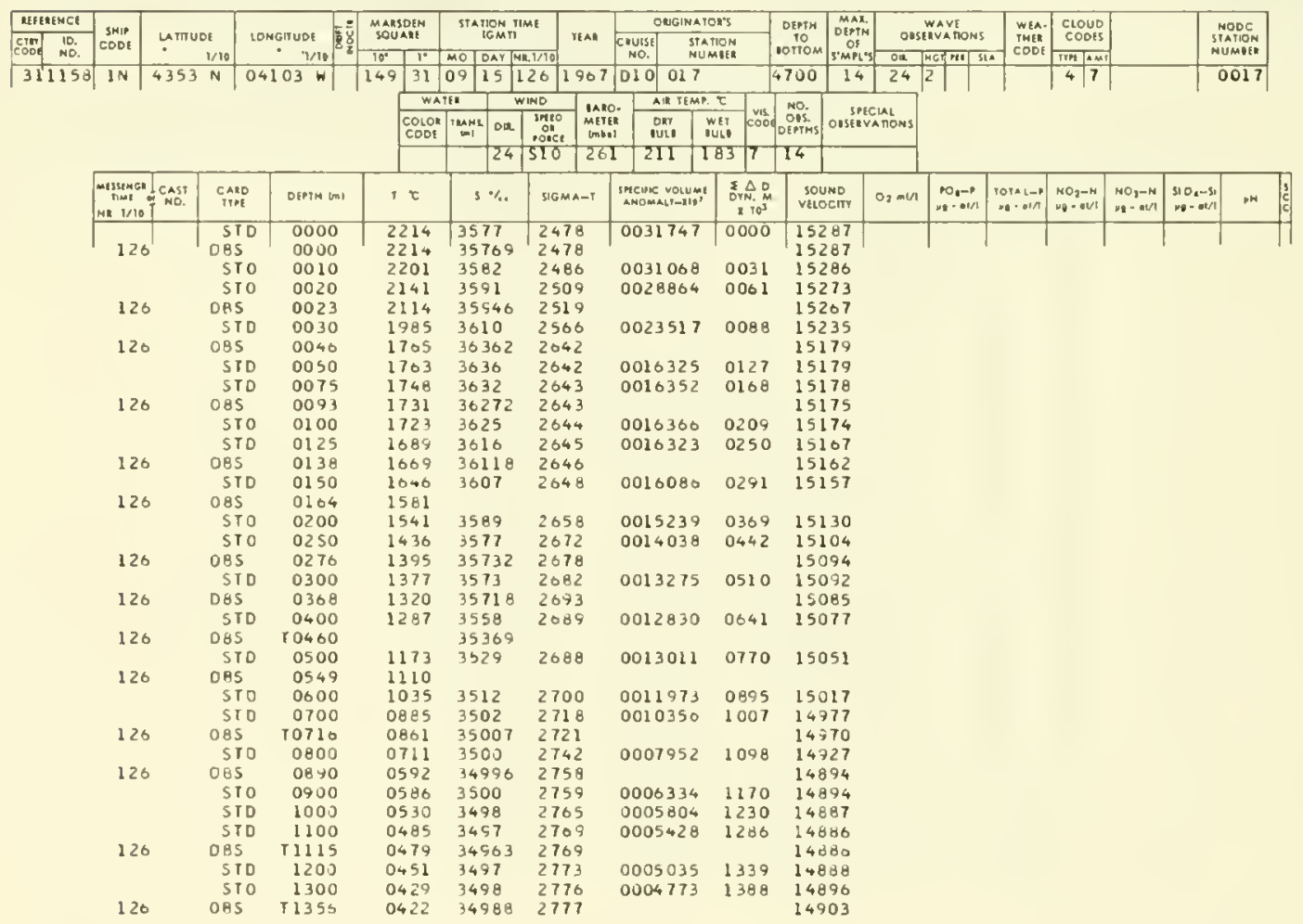


TABLE I.-Continued

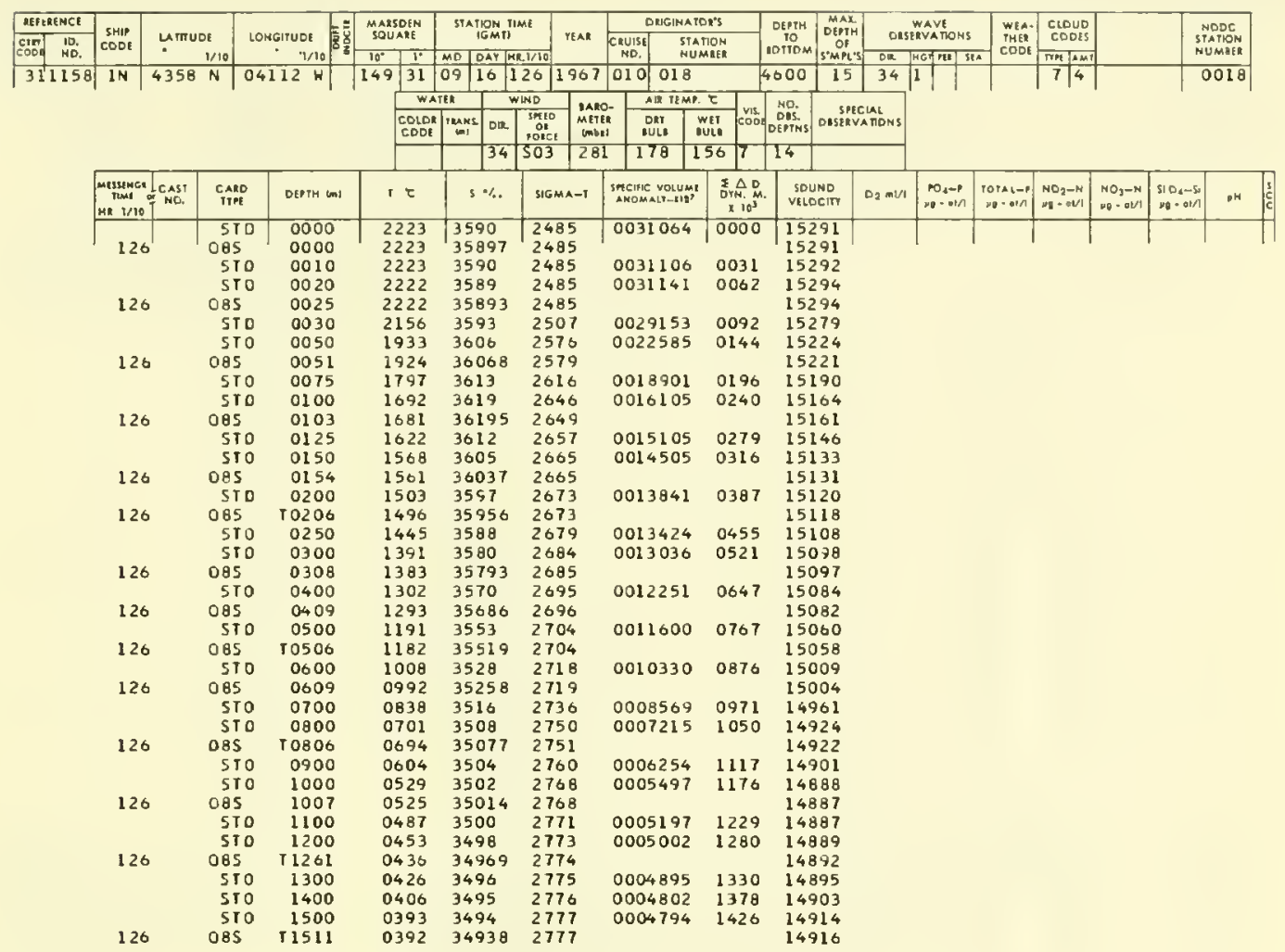


TABLE II.-Observed and interpolated oceanographic data for stations taken by USCGC CHINOCOTEAGUE at Ocean Station DELTA, 22 September-10 October 1967, prepared from NODC listing No. 31-1171 CC.

\begin{tabular}{|c|c|c|c|c|c|c|c|c|c|c|c|c|c|c|c|c|c|}
\hline \multirow{2}{*}{\begin{tabular}{|c|c|} 
REFEALECEE \\
Crey & 10. \\
Cnof & NO. \\
\end{tabular}} & \multirow{2}{*}{$=\begin{array}{l}S N I P \\
C O D E\end{array}$} & \multirow{2}{*}{\multicolumn{2}{|c|}{$\begin{array}{l}\text { LATTUDER } \\
1 / 10\end{array}$}} & \multirow{2}{*}{ IONGIYUER } & $\begin{array}{l}\text { MAASDEN } \\
\text { SOUAHE }\end{array}$ & \multirow{2}{*}{\multicolumn{2}{|c|}{$\underset{\text { STATION FIME }}{\text { TGMTI }}$}} & \multicolumn{2}{|c|}{ OMGINAJOR'S } & \multirow{2}{*}{$\begin{array}{c}\text { Oepri } \\
10 \\
1010 \mathrm{M}\end{array}$} & \multirow{2}{*}{$\mid \begin{array}{c}\text { MAX } \\
\text { OEPYH } \\
\text { Of } \\
\text { S'MPL's }\end{array}$} & \multirow{2}{*}{$\begin{array}{l}\text { WAVE } \\
\text { OASERVATONS }\end{array}$} & \multirow{2}{*}{$\begin{array}{l}\text { WEA- } \\
\text { IHEB } \\
\text { COOE }\end{array}$} & \multirow{2}{*}{$\begin{array}{c}\text { CLOUO } \\
\text { COOES } \\
\text { Tre }\end{array}$} & & \\
\hline & & & & & & & YEAR & $\begin{array}{l}\text { CAUISE: } \\
\text { NO. }\end{array}$ & $\begin{array}{l}\text { STAPION } \\
\text { NU MHER }\end{array}$ & & & & & & & & \\
\hline & $C C$ & 4401 & $\mathrm{~N}$ & $050 \mathrm{~W}$ & \begin{tabular}{|l|l|}
149 & 40
\end{tabular} & \begin{tabular}{l|l|}
09 & 22 \\
\end{tabular} & $\begin{array}{lll}182 & 1967\end{array}$ & 011001 & & 4572 & 14 & 43 & & \begin{tabular}{|l|l|}
8 & 6 \\
\end{tabular} & & & \\
\hline & & & & & WA & TER - & WINO & i. AIR IEAP. & & & & & & & & & \\
\hline & & & & & $\begin{array}{l}\text { COLOR } \\
\text { COOE }\end{array}$ & 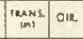 & $\mid \begin{array}{c}3 n t 0 \\
\text { ont } \\
\text { onct }\end{array}$ & \begin{tabular}{|c|c} 
OHY & $W$ \\
out & Bu \\
\end{tabular} & 6006 & ORS. & OSERVATIONS & & & & & & \\
\hline & & & & & & 34 & 4515 & 283 & 61 & 14 & & & & & & & \\
\hline & 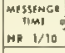 & OP CAST & $\begin{array}{l}\text { CARC } \\
\text { TYPE }\end{array}$ & OEPTH $\mathrm{mI}$ & $1 t$ & $5 \%$ & SIGMA-1 & 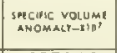 & $\begin{array}{r}5 . \Delta 0 \\
\text { OrN. } \\
\times \quad 0^{1} \\
\end{array}$ & & $\begin{array}{l}\text { UND } \\
\text { OCINY }\end{array}$ & 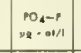 & $\begin{array}{r}\text { rotalat } \\
\text { עo }\end{array}$ & $\begin{array}{l}\mathrm{NO}_{2}-\mathrm{N} \\
\mathrm{N}=0 \mathrm{OV} / 1\end{array}$ & 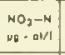 & $\begin{array}{l}510 .-5 i \\
v 8-0,1 / 71\end{array} \mid$ & $\mathrm{pH}$ \\
\hline & & & $5 T 0$ & 0000 & 2158 & 3620 & 2527 & 0027133 & 0000 & 152 & & & & & & & \\
\hline & 182 & & $\begin{array}{l}085 \\
\text { STO }\end{array}$ & $\begin{array}{l}0000 \\
0010\end{array}$ & $\begin{array}{l}2158 \\
2158\end{array}$ & $\begin{array}{l}36201 \\
3620\end{array}$ & $\begin{array}{l}2527 \\
2520\end{array}$ & 0027197 & 0027 & $\begin{array}{l}152 \\
152\end{array}$ & $\begin{array}{l}277 \\
279\end{array}$ & & & & & & \\
\hline & & & STO & 0020 & 2159 & 3620 & 2520 & 0027253 & 0054 & 152 & 281 & & & & & & \\
\hline & 182 & & 085 & 0024 & 2159 & 36197 & 2526 & & & 152 & 282 & & & & & & \\
\hline & & & 510 & 0030 & 2052 & 3626 & 2560 & 0024059 & 0080 & 152 & 255 & & & & & & \\
\hline & 182 & & OBS & 0048 & 1806 & 36386 & 2634 & & & 152 & 191 & & & & & & \\
\hline & & & SIO & 0050 & 1801 & 3639 & 2635 & 0017037 & 0121 & 151 & 190 & & & & & & \\
\hline & & & STO & 0075 & 1735 & 3637 & 2650 & 0015701 & 0162 & 151 & 175 & & & & & & \\
\hline & 182 & & 085 & 0096 & 1685 & 36353 & 2661 & & & 252 & 163 & & & & & & \\
\hline & & & 510 & 0100 & 1677 & 3633 & 2661 & 0014732 & 0200 & 151 & 101 & & & & & & \\
\hline & & & STo & 0125 & 1627 & 3619 & 2662 & 0014708 & 0237 & 151 & 148 & & & & & & \\
\hline & 182 & & 085 & 0145 & & 36111 & & & & & & & & & & & \\
\hline & & & STD & 0150 & 1581 & 3610 & 2665 & 0014426 & 0273 & 151 & 137 & & & & & & \\
\hline & 182 & & 085 & 0196 & 1504 & 35991 & 2674 & & & 151 & 119 & & & & & & \\
\hline & & & STO & 0200 & 1497 & 3598 & 2675 & 0013641 & 0343 & 151 & 118 & & & & & & \\
\hline & & & ST D & 0250 & 1425 & 3584 & 2680 & 0013300 & 0411 & 151 & 101 & & & & & & \\
\hline & 182 & & 085 & 10292 & 1377 & 35759 & 2684 & & & 150 & 092 & & & & & & \\
\hline & & & STO & 0300 & 1375 & 3575 & 2684 & 0013000 & 0477 & 150 & 092 & & & & & & \\
\hline & 202 & & 085 & 0386 & 1315 & 35675 & 2690 & & & 150 & 085 & & & & & & \\
\hline & & & STO & 0400 & 1297 & 3502 & 2690 & 0012735 & 0606 & 150 & 081 & & & & & & \\
\hline & 182 & & OBS & 0483 & 1158 & 35385 & 2699 & & & 150 & 044 & & & & & & \\
\hline & & & STO & 0500 & 1112 & 3537 & 2706 & 0011299 & 0726 & 150 & 031 & & & & & & \\
\hline & 182 & & 085 & T 0576 & 0931 & 35299 & 2732 & & & 149 & 977 & & & & & & \\
\hline & & & $5 T 0$ & 0600 & 0894 & 3528 & 2737 & 0008404 & 0824 & 149 & 967 & & & & & & \\
\hline & & & STO & 0700 & 0755 & 3519 & 2751 & 0007054 & 0902 & 149 & 930 & & & & & & \\
\hline & 182 & & 085 & 0771 & 0671 & 35138 & 2759 & & & 149 & 908 & & & & & & \\
\hline & & & STo & 0800 & 0639 & 3512 & 2762 & 0006020 & 0967 & 149 & 900 & & & & & & \\
\hline & & & 510 & 0900 & 0547 & 3506 & 2769 & 0005322 & 1024 & 148 & 879 & & & & & & \\
\hline & 182 & & OBS & 10768 & 0500 & 35034 & 2772 & & & 148 & 871 & & & & & & \\
\hline & & & $5 T 0$ & 1000 & 0488 & 3503 & 2773 & 0004878 & 2075 & 148 & 871 & & & & & & \\
\hline & & & STO & 1100 & 0457 & 3500 & 2775 & 0004803 & 1123 & 148 & 874 & & & & & & \\
\hline & & & $5 T 0$ & 1200 & 0433 & 3498 & 2776 & 0004742 & 1171 & 148 & 881 & & & & & & \\
\hline & 182 & & 085 & 1209 & 0431 & 34979 & 2776 & & & 148 & 882 & & & & & & \\
\hline & & & STO & 1300 & 0417 & 3497 & 2777 & 0004712 & 1218 & 148 & 891 & & & & & & \\
\hline & & & 5To & 1400 & 0409 & 3496 & 2776 & 0004789 & 1266 & 149 & 904 & & & & & & \\
\hline & 182 & & 085 & $T 1450$ & 0408 & 34951 & 2776 & & & 149 & 912 & & & & & & \\
\hline
\end{tabular}

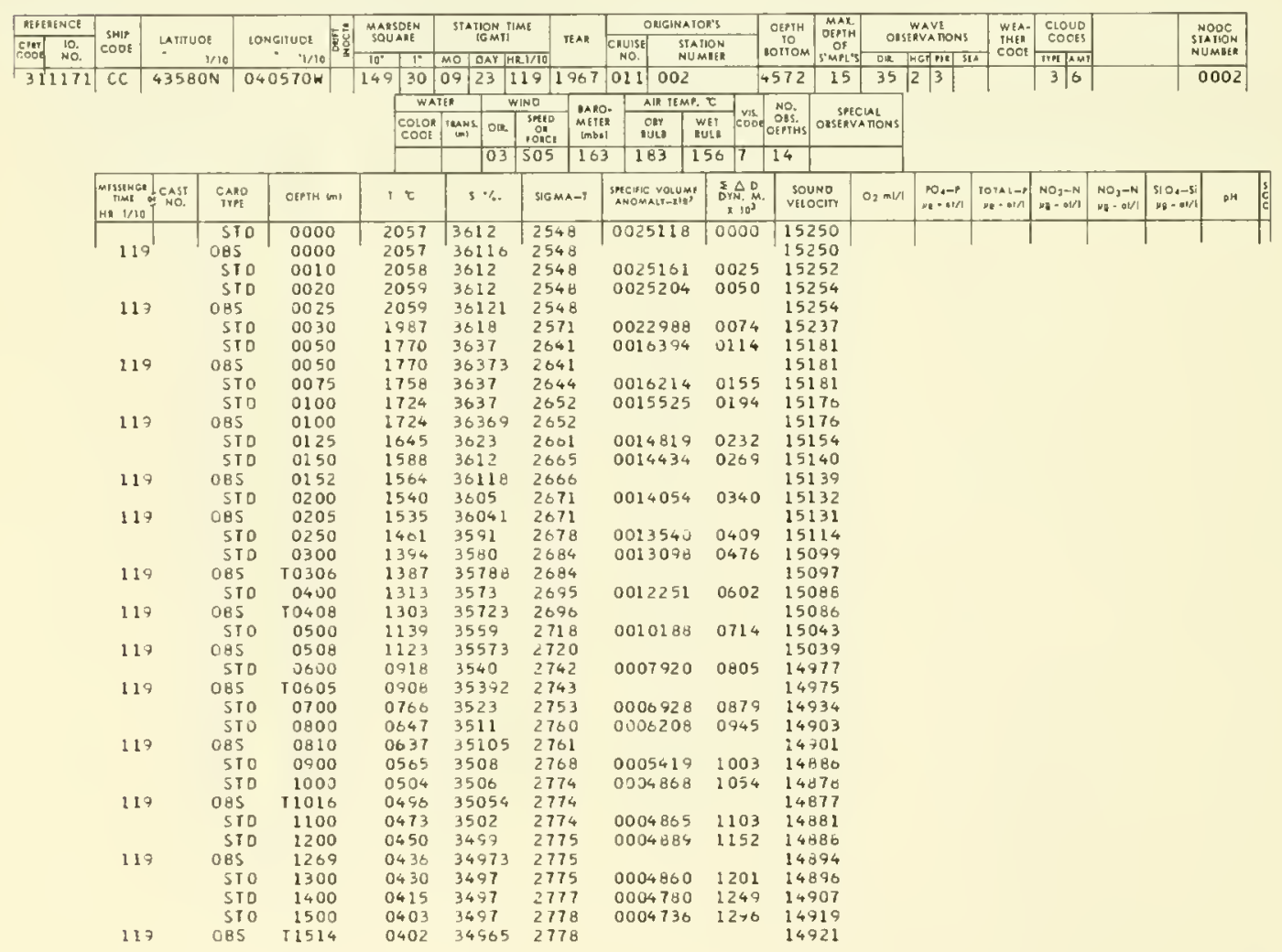


TABle II.-Continued

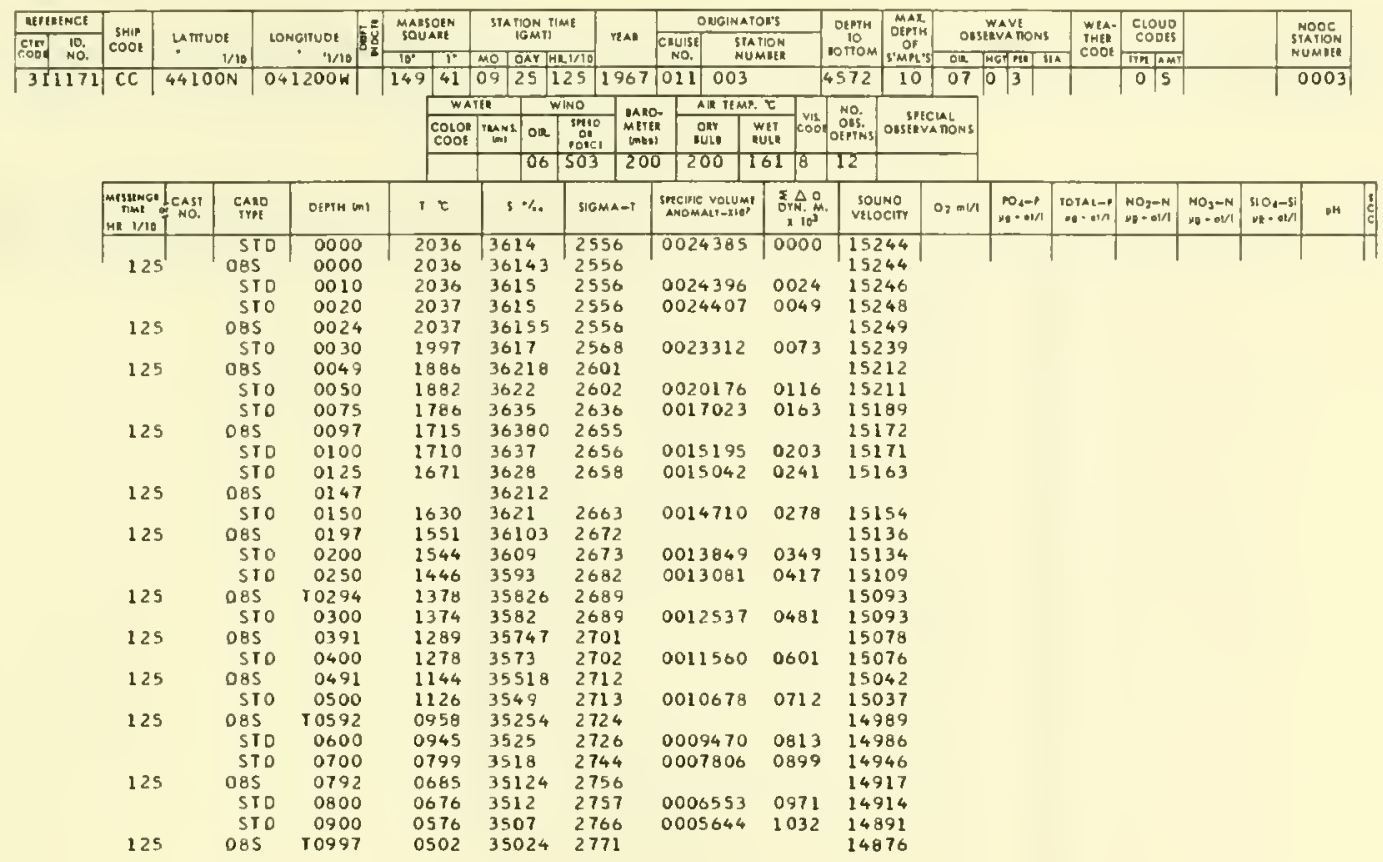

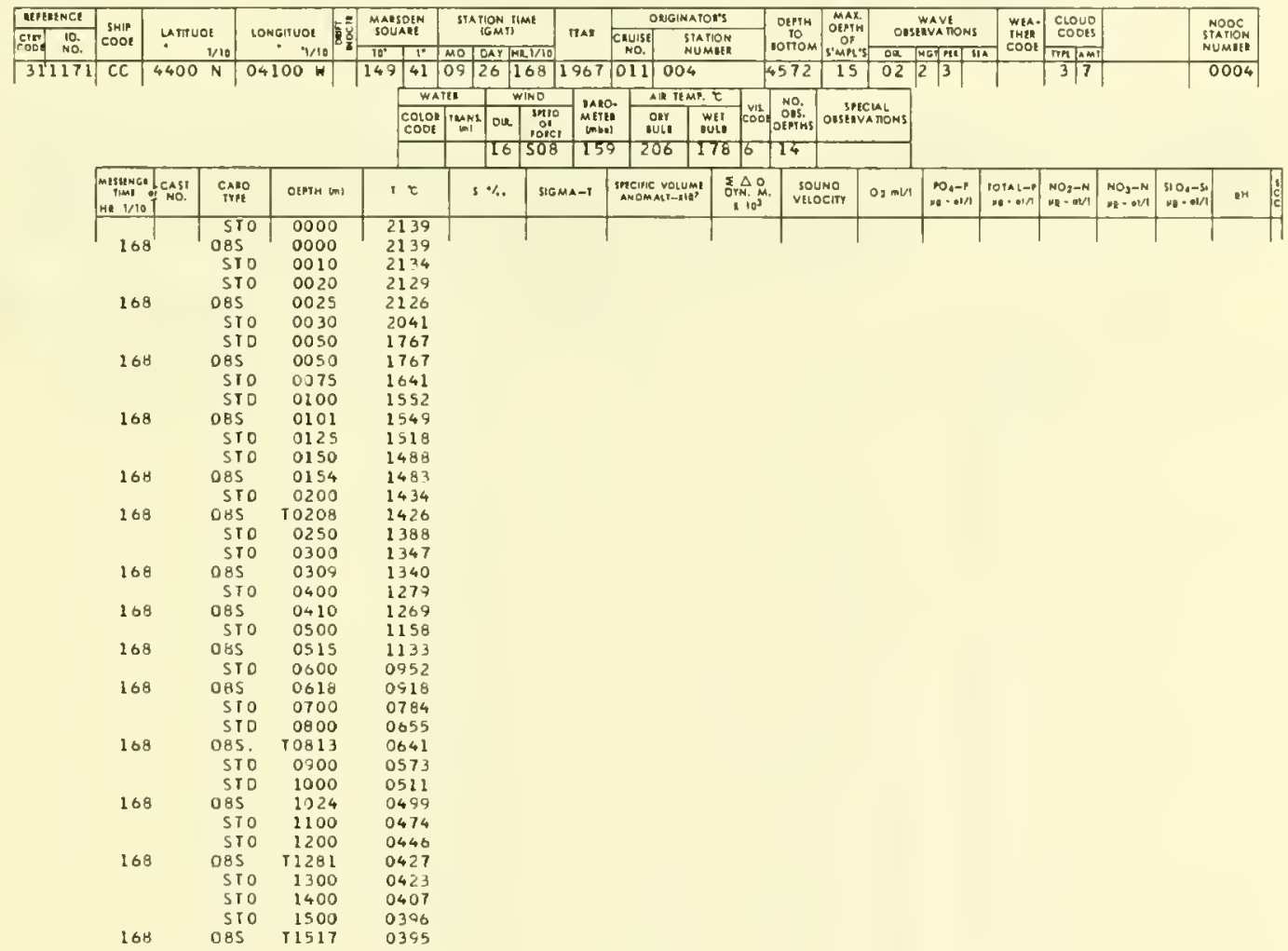


TABLE II.-Continued

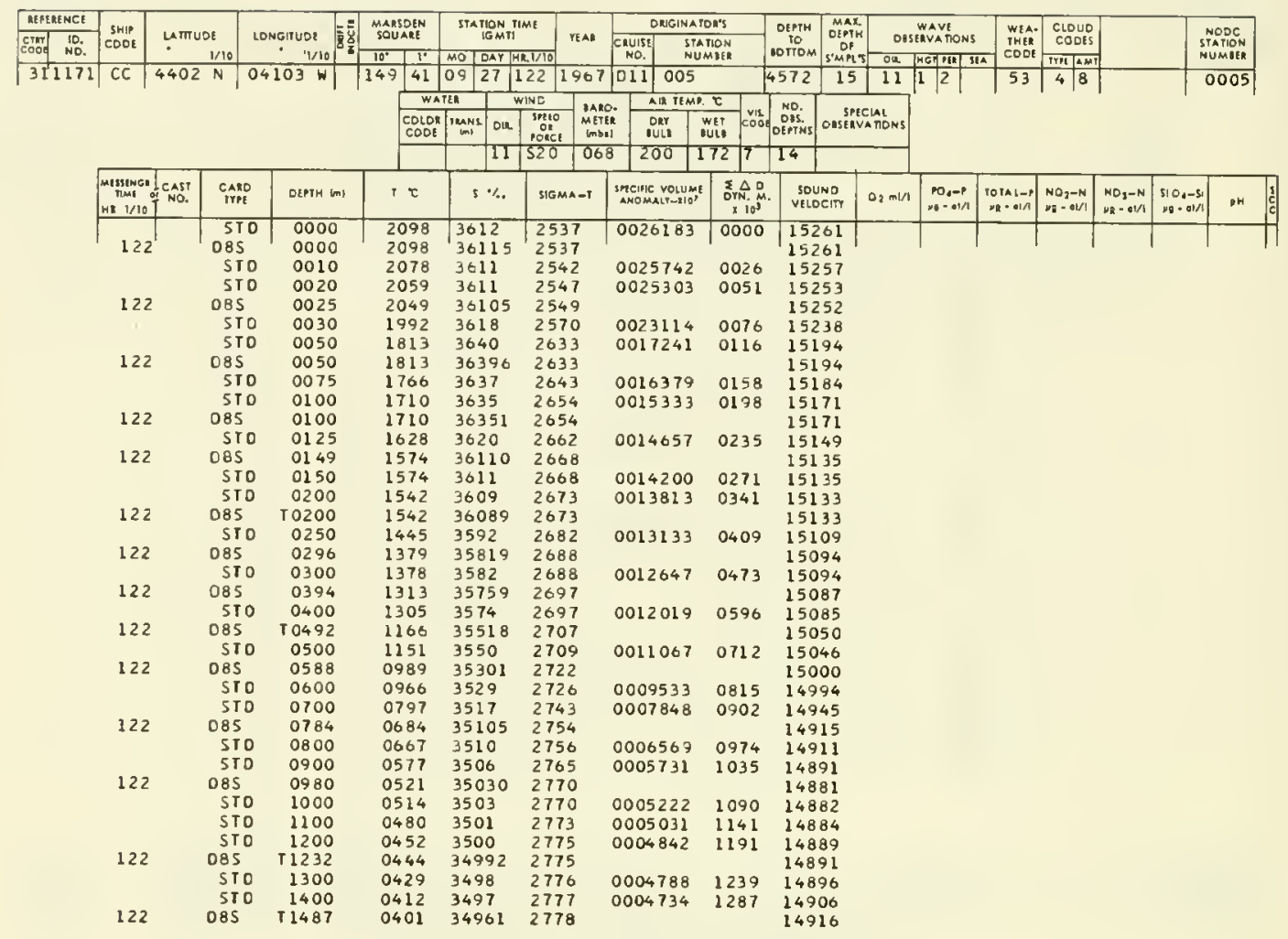

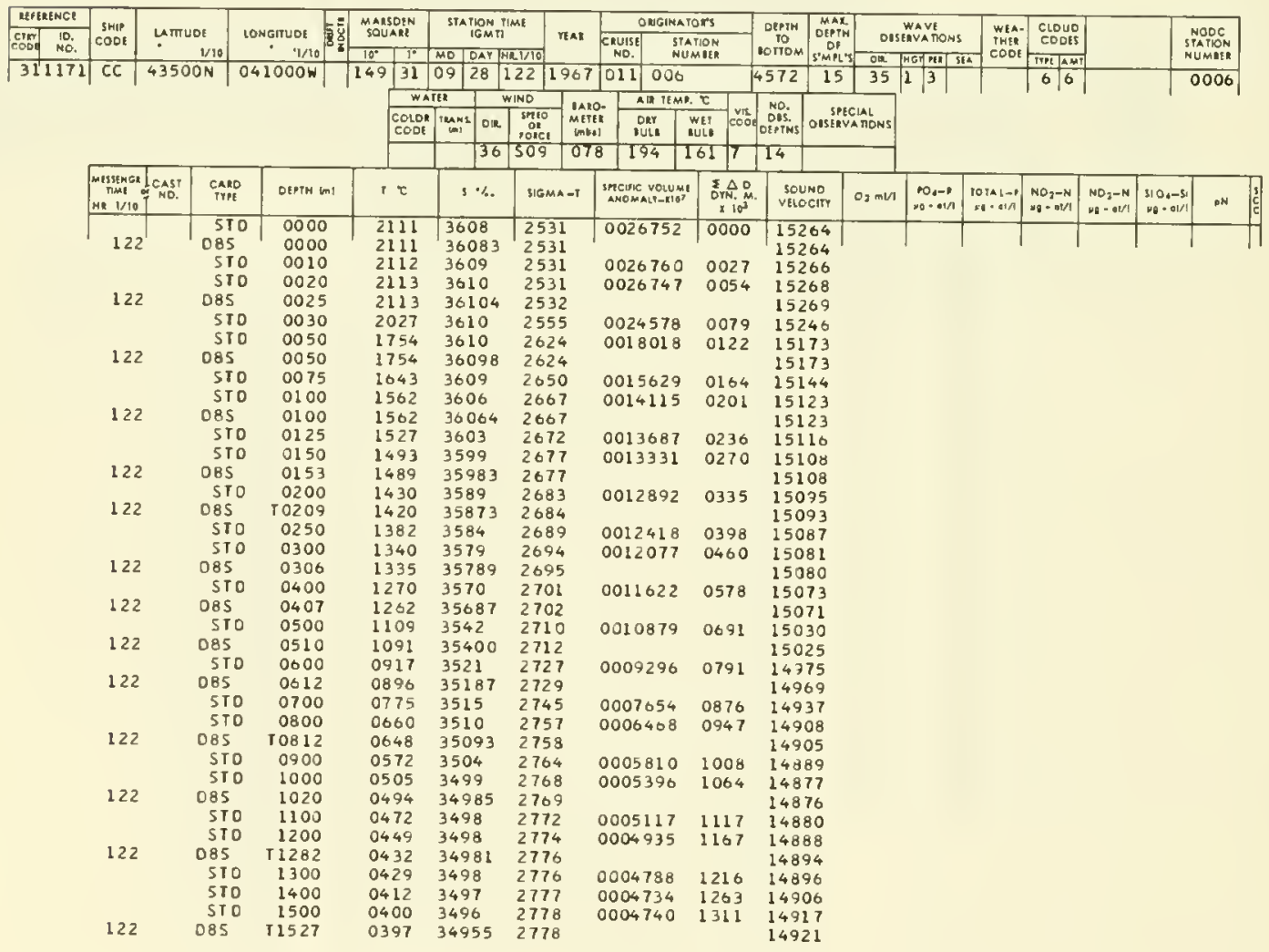




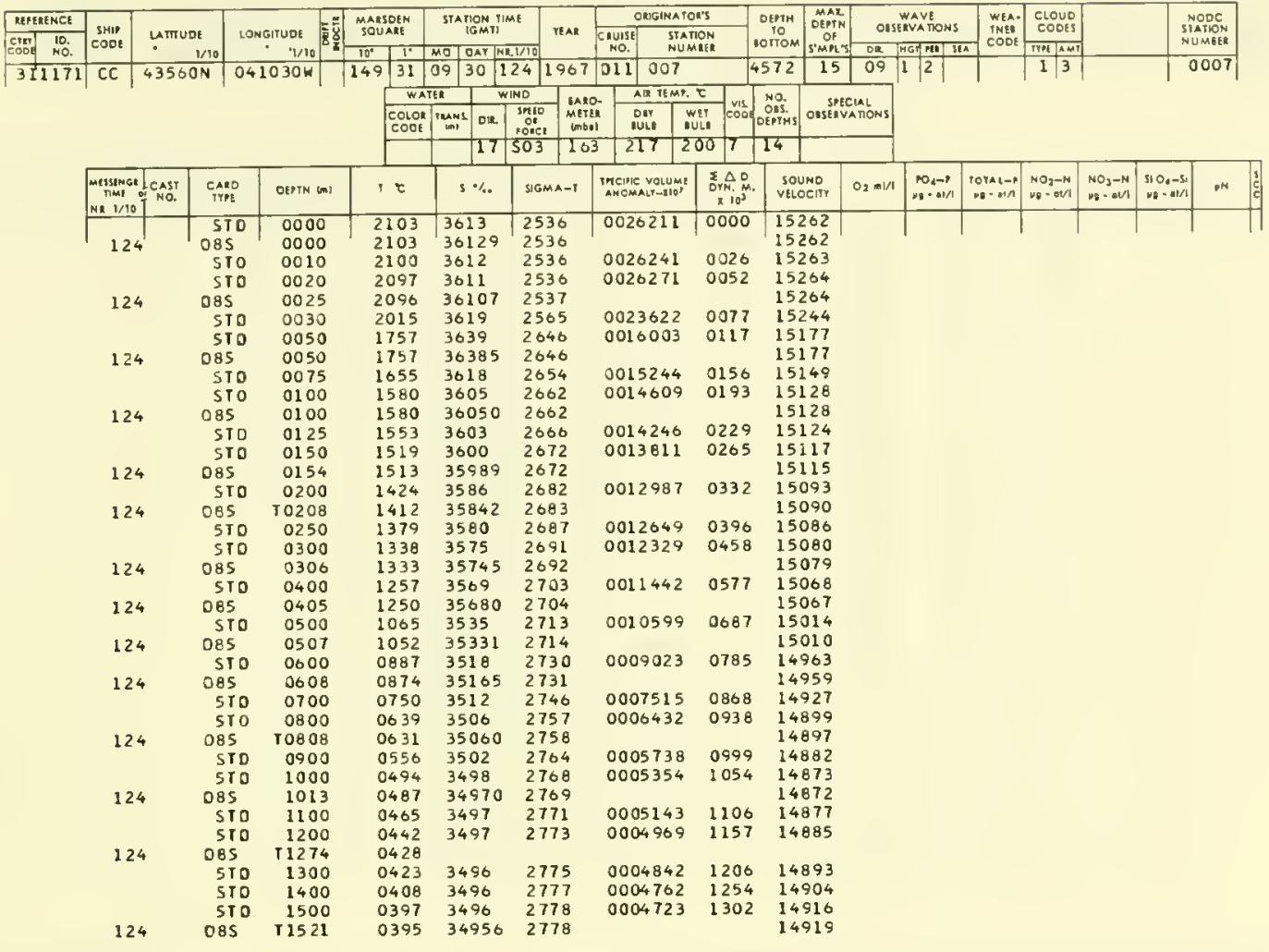

\begin{tabular}{|c|c|c|c|c|c|c|c|c|c|c|c|c|c|c|c|c|c|c|}
\hline \multirow{2}{*}{\begin{tabular}{|c|c|} 
REFEREHCI \\
CINT & ID. \\
COD & NO. \\
\end{tabular}} & \multirow[b]{2}{*}{$\begin{array}{l}\operatorname{SH}_{\mathrm{CH}} \\
\mathrm{COOE}\end{array}$} & \multirow{2}{*}{\multicolumn{2}{|c|}{$\begin{array}{l}\text { umuar } \\
\text { vio }\end{array}$}} & \multirow{2}{*}{ LONGTUDE } & \multirow{2}{*}{ 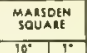 } & \multirow{2}{*}{ 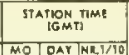 } & \multirow[b]{2}{*}{ reAR } & \multicolumn{2}{|c|}{ OUGINATORS } & \multirow{2}{*}{$\begin{array}{c}\text { DEPTM } \\
\text { TO } \\
10 \text { TOOM }\end{array}$} & \multirow{3}{*}{\begin{tabular}{|c|}
$\begin{array}{c}\text { MAX } \\
\text { OEgTH } \\
\text { Of } \\
\text { SMVL'S }\end{array} \mid$ \\
15 \\
\end{tabular}} & \multicolumn{2}{|c|}{$\begin{array}{l}\text { Wave } \\
\text { Oaseavations }\end{array}$} & \multirow{2}{*}{$\begin{array}{l}\text { WEA } \\
\text { WEA } \\
\text { COOR }\end{array}$} & \multirow{2}{*}{\begin{tabular}{|c|} 
CLOUD \\
CODES \\
rim Thent
\end{tabular}} & & & \multirow{2}{*}{$\begin{array}{l}\text { NOOC } \\
\text { SATION } \\
\text { NUMAER }\end{array}$} \\
\hline & & & & & & & & $\begin{array}{c}\text { CRUISE } \\
\text { No. }\end{array}$ & $\begin{array}{l}\text { STATIOM } \\
\text { NUMAER }\end{array}$ & & & ox & 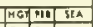 & & & & & \\
\hline 311171 & CC & 4400 & $N$ & $100 *$ & \begin{tabular}{l|l|}
149 & 41
\end{tabular} & \begin{tabular}{|l|l|l}
10 & 01 & 1 \\
\end{tabular} & \begin{tabular}{|l|l|}
124 & 1967 \\
\end{tabular} & \begin{tabular}{|l|l|}
011 & 008 \\
\end{tabular} & & $\frac{10770 \mathrm{M}}{4572}$ & & 06 & \begin{tabular}{|l|l|l|}
2 & 3 & \\
\end{tabular} & & \begin{tabular}{|l|l|}
3 & 2 \\
\end{tabular} & & & 0008 \\
\hline & & & & & WA & \begin{tabular}{|l|l} 
TEA & v \\
\end{tabular} & \begin{tabular}{l|l} 
WIMD & IAro \\
\end{tabular} & O. AR TEMP. & & No. & & & & & & & & \\
\hline & & & & & \begin{tabular}{|l|} 
COLOR \\
Cook
\end{tabular} & $\prod_{m i n}$ on & 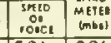 & \begin{tabular}{|l|l|} 
okr & ow \\
ouL & ou \\
\end{tabular} & is coo & ons: & oustave & A MONS & & & & & & \\
\hline & & & & & & 13 & $504 \quad 204$ & 217 & $89 \sqrt{7}$ & 14 & & & & & & & & \\
\hline & 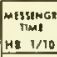 & $\left.\right|_{\text {OAST }} ^{\text {CAST }}$ & $\begin{array}{l}\text { CAED } \\
\text { TWEE }\end{array}$ & DEPTH (m) & 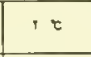 & $s \%$ & SIGAAA-T & 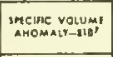 & 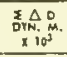 & & $\begin{array}{l}\text { UNo } \\
\text { OCIn }\end{array}$ & $0, \mathrm{~min}$ & $\begin{array}{c}0, p \\
\cdots \\
\cdots\end{array}$ & gotalar & $\begin{array}{l}\mathrm{NO}_{2}-\mathrm{N} \\
\mathrm{NO}=\mathrm{OM} / \mathrm{h}\end{array}$ & $\begin{array}{l}\mathrm{NO}_{3}-\mathrm{N} \\
\mathrm{YO}=\mathrm{av} / 1\end{array}$ & $\begin{array}{l}510,-35 \\
50-a, 1 / 1\end{array} \mid$ & $\mathrm{NM}$ \\
\hline & 124 & & $\begin{array}{l}510 \\
085\end{array}$ & $\begin{array}{l}0000 \\
0000\end{array}$ & $\begin{array}{l}1963 \\
1903\end{array}$ & $\begin{array}{l}3452 \\
34521\end{array}$ & $\begin{array}{l}2451 \\
2451\end{array}$ & 0034305 & 0000 & & $\begin{array}{l}205 \\
205\end{array}$ & & & & & & & \\
\hline & 124 & & $\begin{array}{l}085 \\
510\end{array}$ & 0010 & 2013 & 3490 & 2467 & 0032830 & 0034 & & 225 & & & & & & & \\
\hline & & & STO & 0020 & 2062 & 3524 & 2480 & 0031664 & 0066 & & 244 & & & & & & & \\
\hline & 124 & & OBS & 0024 & 2082 & 35360 & 2484 & & & & 252 & & & & & & & \\
\hline & & & 510 & 0030 & 1990 & 3554 & 2522 & 0027698 & 0095 & & 230 & & & & & & & \\
\hline & 124 & & o8s & 0048 & 1771 & 35954 & 2609 & & & & 176 & & & & & & & \\
\hline & & & SID & 0050 & 1763 & 3596 & 2612 & 0019230 & 0142 & & 174 & & & & & & & \\
\hline & & & STO & 0075 & 1673 & 3604 & 2639 & 0016667 & 0187 & & 152 & & & & & & & \\
\hline & 124 & & 085 & 0097 & 1609 & 36064 & 2656 & & & & 137 & & & & & & & \\
\hline & & & SIO & 0100 & 1602 & 3606 & 2658 & 0015019 & 0227 & 15 & 135 & & & & & & & \\
\hline & & & sTo & 0125 & 1551 & 3600 & 2605 & 0014422 & 0264 & & 123 & & & & & & & \\
\hline & 124 & & 085 & 0145 & 1517 & $35 \$ 58$ & 2669 & & & & 115 & & & & & & & \\
\hline & & & 510 & 0150 & 1513 & 3595 & 2669 & 0014026 & 0299 & 15 & 114 & & & & & & & \\
\hline & 124 & & 085 & 10197 & 1459 & 35907 & 2678 & & & & 104 & & & & & & & \\
\hline & & & STO & 0200 & 1450 & 3589 & 2678 & 0013307 & 0368 & 15 & 102 & & & & & & & \\
\hline & & & STO & 0250 & 1324 & 3562 & 2684 & 0012861 & 0433 & & 065 & & & & & & & \\
\hline & 12 & & 085 & 0291 & 1253 & 35485 & 2688 & & & & 047 & & & & & & & \\
\hline & & & STO & 0300 & 1248 & 3551 & 2691 & 0022323 & 0496 & 15 & 047 & & & & & & & \\
\hline & 12 & & 085 & 0389 & 1201 & 35534 & 2702 & & & & 046 & & & & & & & \\
\hline & & & STO & 0400 & 1187 & 3552 & 2704 & 0011346 & 0614 & 15 & 042 & & & & & & & \\
\hline & 12 & & 085 & 0486 & 1015 & 35287 & 2717 & & & 14 & 993 & & & & & & & \\
\hline & & & STO & 0500 & 0957 & 3519 & 2719 & 0009904 & 0721 & 14 & 973 & & & & & & & \\
\hline & 12 & & 085 & 0583 & 0693 & 34807 & 2730 & & & 14 & 881 & & & & & & & \\
\hline & & & STO & 0600 & 0690 & 3484 & 2733 & 0008510 & 0813 & 14 & 883 & & & & & & & \\
\hline & & & STD & 0700 & 0664 & 3501 & 2750 & 0007038 & 0890 & 14 & 892 & & & & & & & \\
\hline & 12 & & 085 & 10778 & 0628 & 35074 & 2760 & & & 148 & 891 & & & & & & & \\
\hline & & & STO & 0800 & 0606 & 3506 & 2761 & 0005998 & 0956 & & 886 & & & & & & & \\
\hline & & & STO & 0900 & 0525 & 3501 & 2767 & 0005396 & 1013 & & 869 & & & & & & & \\
\hline & 12 & & 085 & 0978 & 0479 & 34980 & 2771 & & & 14 & 863 & & & & & & & \\
\hline & & & STO & 1000 & 0476 & 3498 & 2771 & 0005082 & 1065 & & 865 & & & & & & & \\
\hline & & & SIO & 1100 & 0460 & 3498 & 2773 & 0004967 & 1115 & 14 & 875 & & & & & & & \\
\hline & & & STD & 1200 & 0445 & 3499 & 2775 & 0004853 & 1104 & & 886 & & & & & & & \\
\hline & 12 & & 085 & 11227 & 0441 & 34987 & 2775 & & & & 889 & & & & & & & \\
\hline & & & SIO & 1300 & 0430 & 3498 & 2776 & 0004801 & 2213 & & 896 & & & & & & & \\
\hline & & & 510 & 1400 & 0416 & 3498 & 2777 & 0004713 & 1260 & & 707 & & & & & & & \\
\hline & 12 & & 085 & 11477 & 0405 & 34968 & 2778 & & & 148 & 916 & & & & & & & \\
\hline
\end{tabular}


TABLE II.-Continued

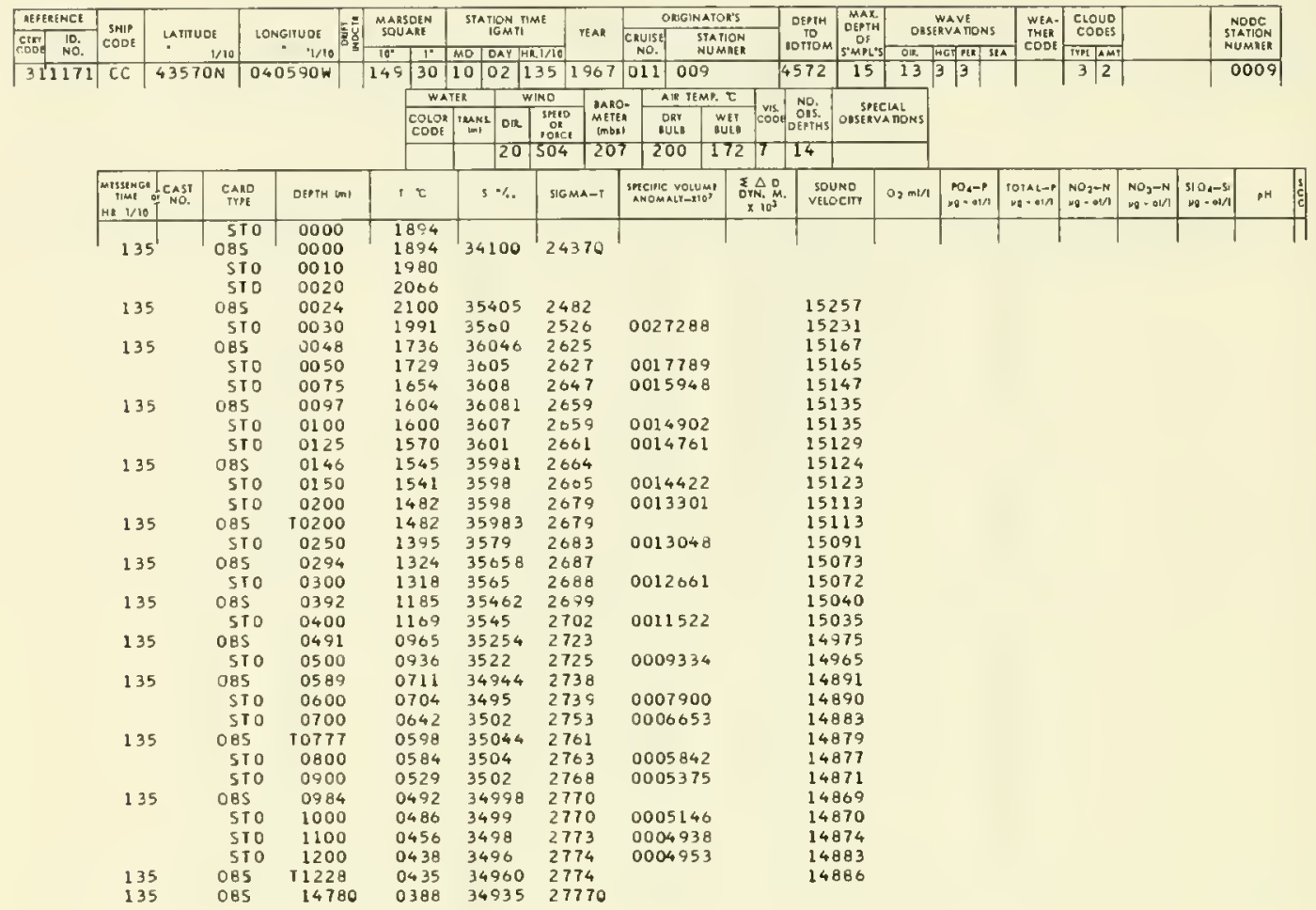

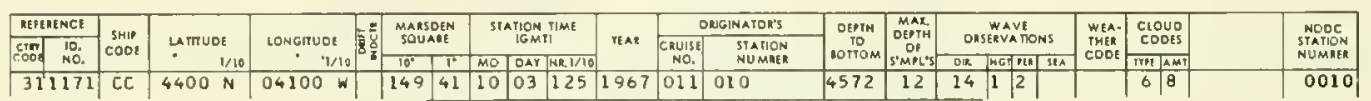

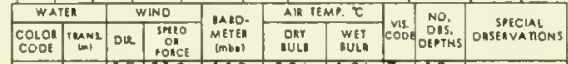

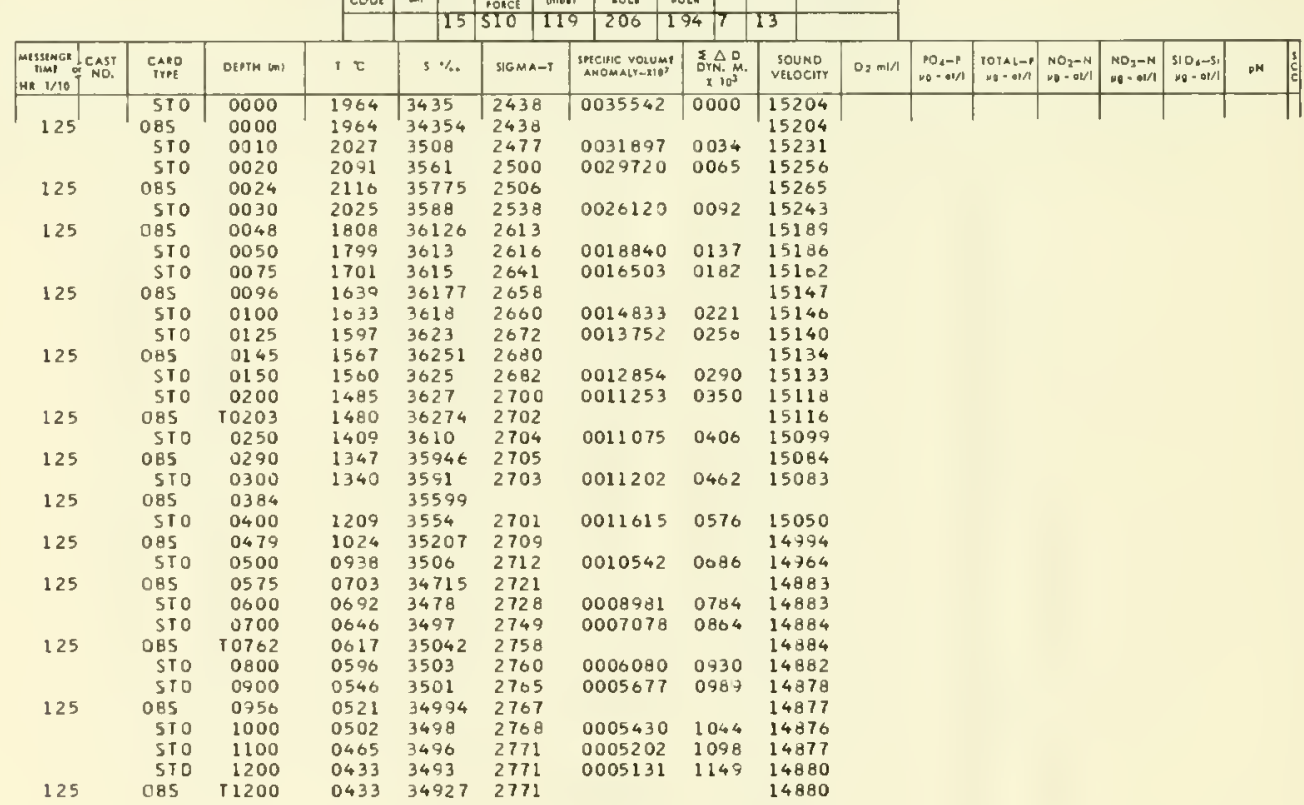




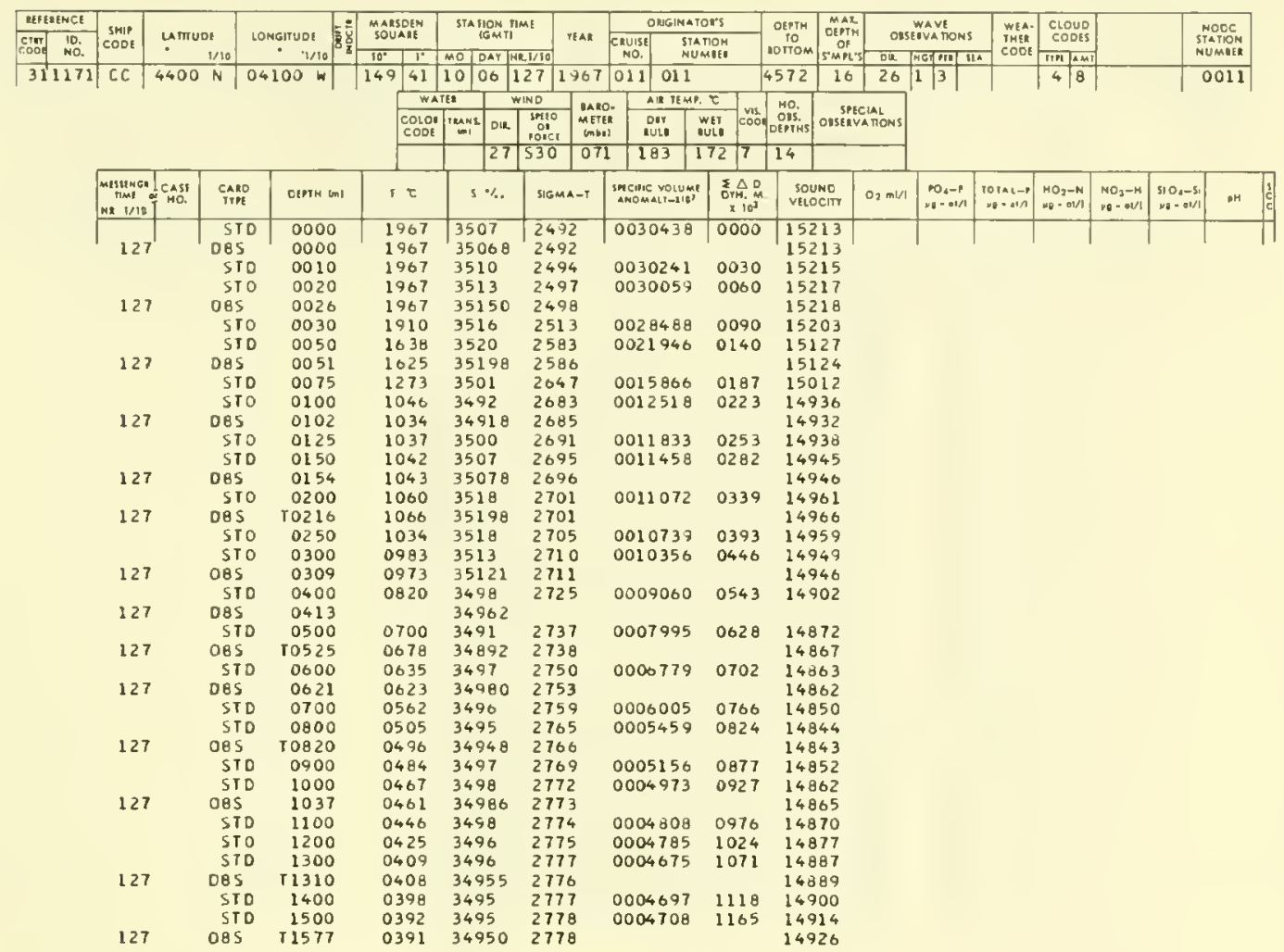

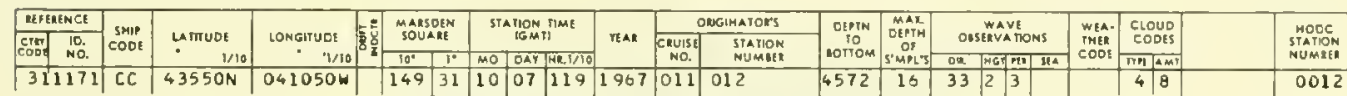

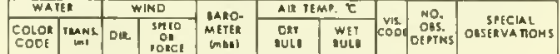

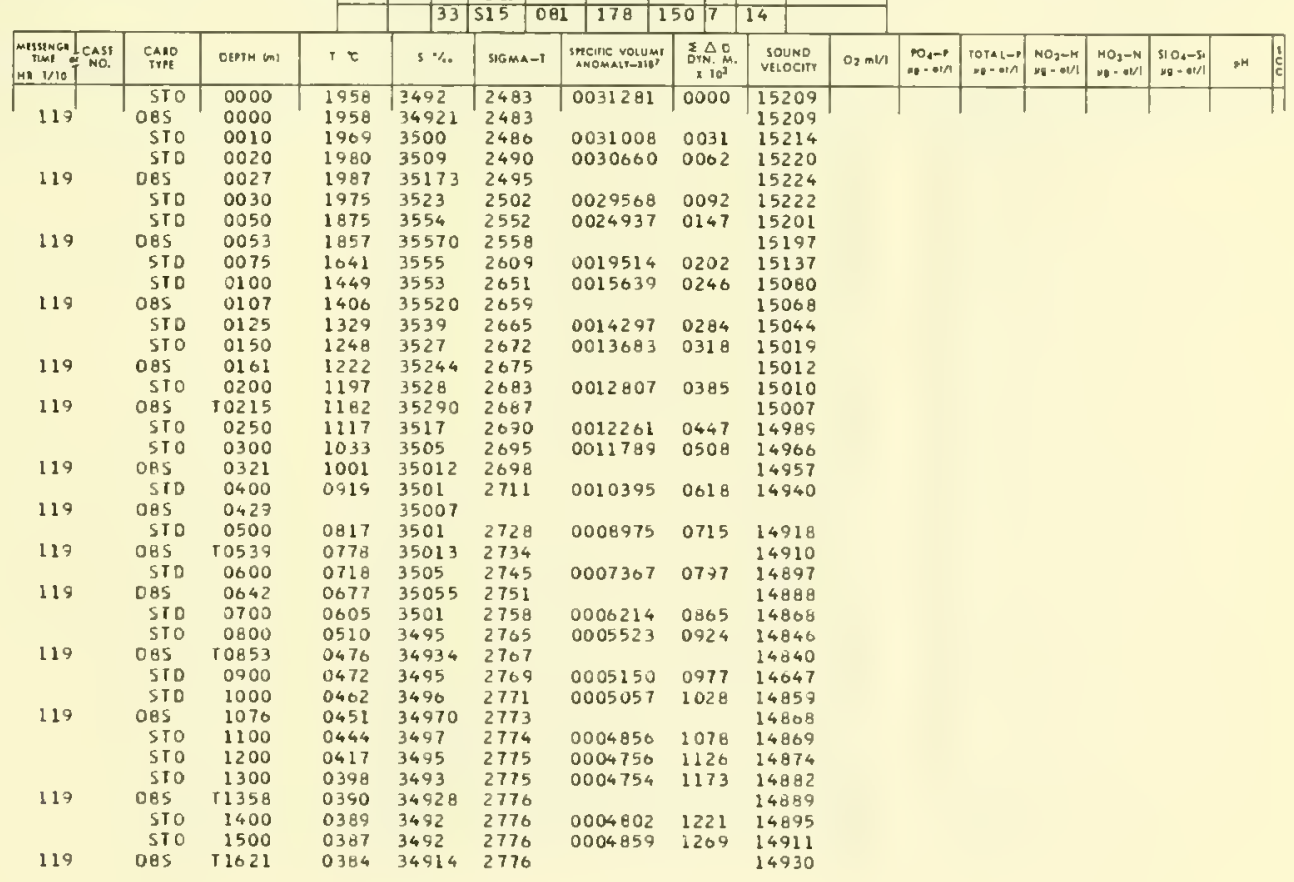


TABle II.-Continued

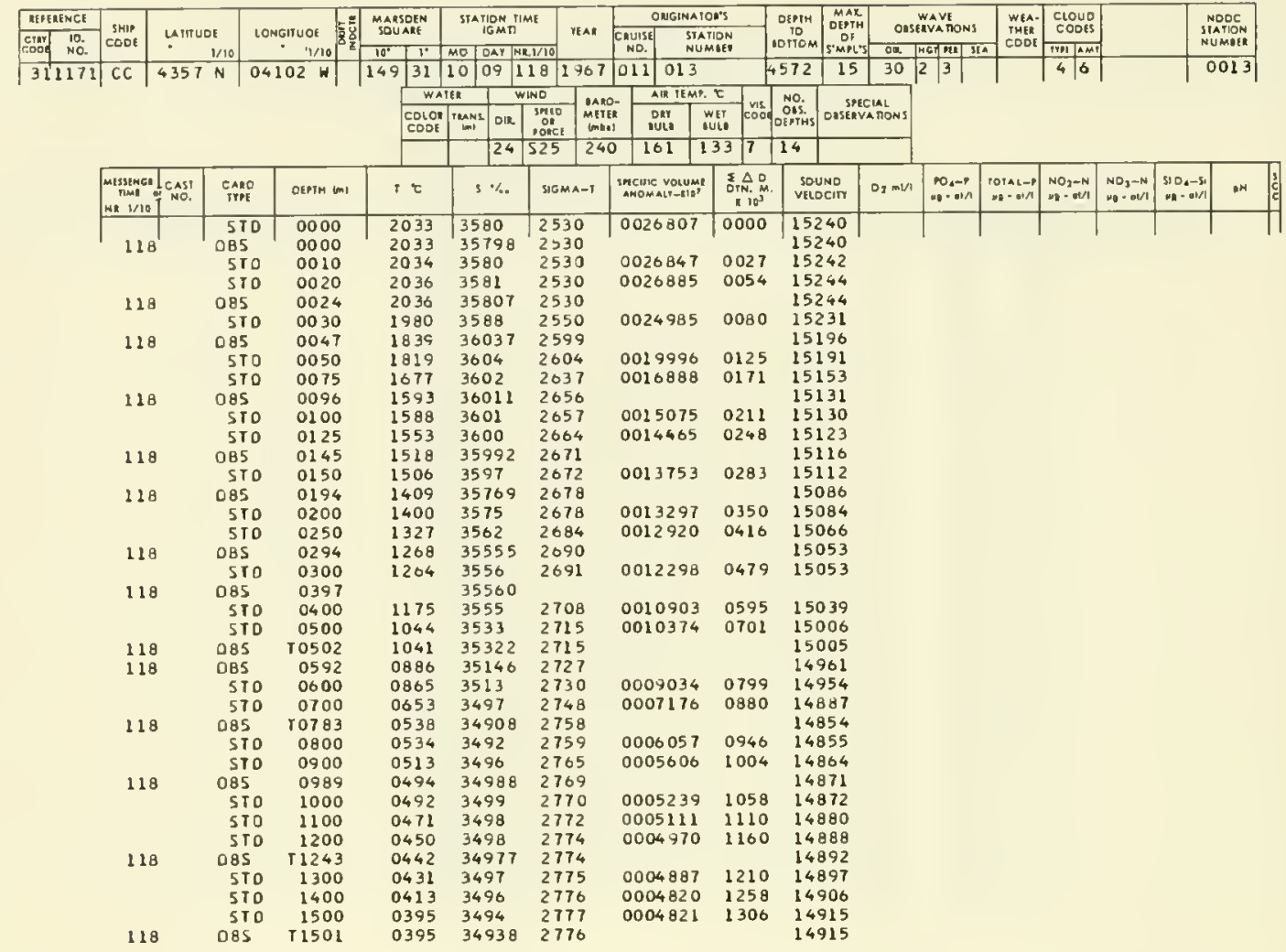

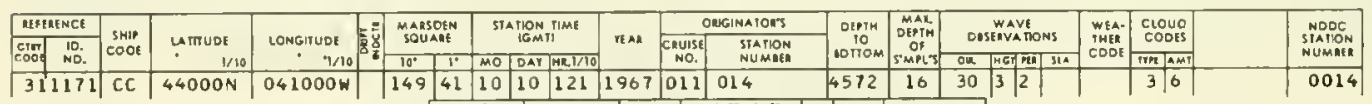

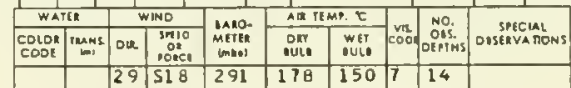

\begin{tabular}{|c|c|c|c|c|c|c|c|c|c|c|c|c|c|c|c|}
\hline 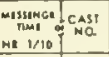 & $\begin{array}{l}\text { CANO } \\
\text { TwhI }\end{array}$ & OENH UI & $: \tau$ & $s \%$ & SIEMA-T & 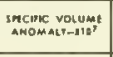 & 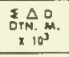 & $\begin{array}{l}\text { souno } \\
\text { vilocitr }\end{array}$ & $O_{2} m / 11$ & $\begin{array}{c}m_{4}-p \\
\cdots \cdots n\end{array}$ & 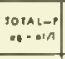 & $\begin{array}{l}\mathrm{NO}_{2}-\mathrm{N} \\
\mathrm{N}=\mathrm{N} / \mathrm{VI}\end{array}$ & $\begin{array}{l}\mathrm{NO}_{1}-\mathrm{N} \\
\mathrm{NO}_{0}-\mathrm{N}\end{array}$ & $\left|\begin{array}{ccc}31 & 0 . & -51 \\
m & -31 / 1\end{array}\right|$ & H \\
\hline \multirow{4}{*}{121} & 510 & 0000 & 1966 & 3545 & 2521 & 0027036 & 0000 & 15217 & & & & & & & \\
\hline & a日s & 0000 & 1966 & 35451 & 2521 & & & 15217 & & & & & & & \\
\hline & STO & 0010 & 1960 & 3548 & 2523 & 0027502 & 0028 & 15220 & & & & & & & \\
\hline & $5 T 0$ & 0020 & 1970 & 3551 & 2525 & 0027375 & 0055 & 15222 & & & & & & & \\
\hline \multirow[t]{3}{*}{121} & o8s & 0026 & 1971 & 35528 & 2526 & & & 15224 & & & & & & & \\
\hline & 510 & 0030 & 1986 & 3559 & 2527 & 0027235 & 0082 & 15229 & & & & & & & \\
\hline & STO & 0050 & 2004 & 3583 & 2540 & 0026008 & 0136 & 15240 & & & & & & & \\
\hline \multirow[t]{3}{*}{121} & DBS & 0050 & 2004 & 35832 & 2540 & & & 15240 & & & & & & & \\
\hline & STO & 0075 & 1781 & 3592 & 2604 & 0020012 & 0193 & 25183 & & & & & & & \\
\hline & STO & 0100 & 1623 & 3601 & 2649 & 0015825 & 0238 & 15141 & & & & & & & \\
\hline \multirow[t]{3}{*}{121} & OAS & 0102 & 1613 & 36020 & 2652 & & & 15138 & & & & & & & \\
\hline & $5 T 0$ & 0125 & 1570 & 3602 & 2602 & 0014688 & 0276 & 15129 & & & & & & & \\
\hline & 510 & 0150 & 1513 & 3597 & 2671 & 0013902 & 0312 & 15114 & & & & & & & \\
\hline \multirow[t]{2}{*}{121} & OBS & 0154 & 1503 & 35953 & 2672 & & & 25112 & & & & & & & \\
\hline & STO & 0200 & 1360 & 3571 & 2684 & 0012781 & 0378 & 15070 & & & & & & & \\
\hline \multirow{3}{*}{121} & $0 B 5$ & 10205 & 1349 & 35689 & 2084 & & & 15067 & & & & & & & \\
\hline & 510 & 0250 & 1328 & 3508 & 2688 & 0012480 & 0442 & 15068 & & & & & & & \\
\hline & $5 T 0$ & 0300 & 1292 & $35 t 8$ & 2695 & 0011952 & 0503 & 15064 & & & & & & & \\
\hline \multirow[t]{2}{*}{121} & OBS & 0307 & 1286 & 35676 & 2696 & & & 15063 & & & & & & & \\
\hline & 570 & 0400 & 1193 & 3554 & 2704 & 0011313 & 0019 & 15045 & & & & & & & \\
\hline \multirow[t]{2}{*}{121} & 085 & 0410 & & 35518 & & & & & & & & & & & \\
\hline & 510 & 0500 & 1020 & 3532 & 2719 & 0010027 & 0726 & 14997 & & & & & & & \\
\hline \multirow[t]{2}{*}{121} & OB5 & 10519 & 0978 & 35279 & 2723 & & & 14985 & & & & & & & \\
\hline & 570 & 0600 & 0757 & 3505 & 2740 & 0007944 & 0816 & 14912 & & & & & & & \\
\hline \multirow[t]{3}{*}{121} & DBS & 0616 & 0722 & 35016 & 2742 & & & 14901 & & & & & & & \\
\hline & 510 & 0700 & 0634 & 3500 & 2753 & 0006688 & 0889 & 14880 & & & & & & & \\
\hline & STD & 0800 & 0550 & 3498 & 2762 & 0005826 & 0951 & 14862 & & & & & & & \\
\hline \multirow[t]{3}{*}{121} & 085 & 10827 & 0532 & 34975 & 2764 & & & 14859 & & & & & & & \\
\hline & STO & 0900 & 0497 & 3497 & 2768 & 0005316 & 1007 & 14857 & & & & & & & \\
\hline & 510 & 1000 & 0459 & 3497 & 2772 & 0004941 & 1059 & 14858 & & & & & & & \\
\hline \multirow[t]{4}{*}{121} & OBS & 1041 & 0447 & 34962 & 2773 & & & 14860 & & & & & & & \\
\hline & Sio & 1100 & 0441 & 3496 & 2773 & 0004877 & 1108 & 14867 & & & & & & & \\
\hline & STO & 1200 & 0431 & 3496 & 2775 & 0004841 & 1150 & 14880 & & & & & & & \\
\hline & STO & 1300 & 0422 & 3496 & 2776 & 0004014 & 1205 & 14893 & & & & & & & \\
\hline \multirow[t]{3}{*}{121} & 085 & $T 1326$ & 0419 & 34964 & 2776 & & & 14896 & & & & & & & \\
\hline & STO & 1400 & 0412 & 3496 & 2776 & 0004807 & 1253 & 14900 & & & & & & & \\
\hline & STO & 1500 & 0402 & 3496 & 2777 & 0004767 & 1301 & 14918 & & & & & & & \\
\hline 121 & OBS & $T 1596$ & 0392 & 34957 & 2778 & & & 14930 & & & & & & & \\
\hline
\end{tabular}


TABLE III.-Observed and interpolated oceanographic data for stations taken by USCGC ESCANABA at Ocean Station DELTA, 12 October-3 November 1967, prepared from NODC listing No. 31-1176 EN.

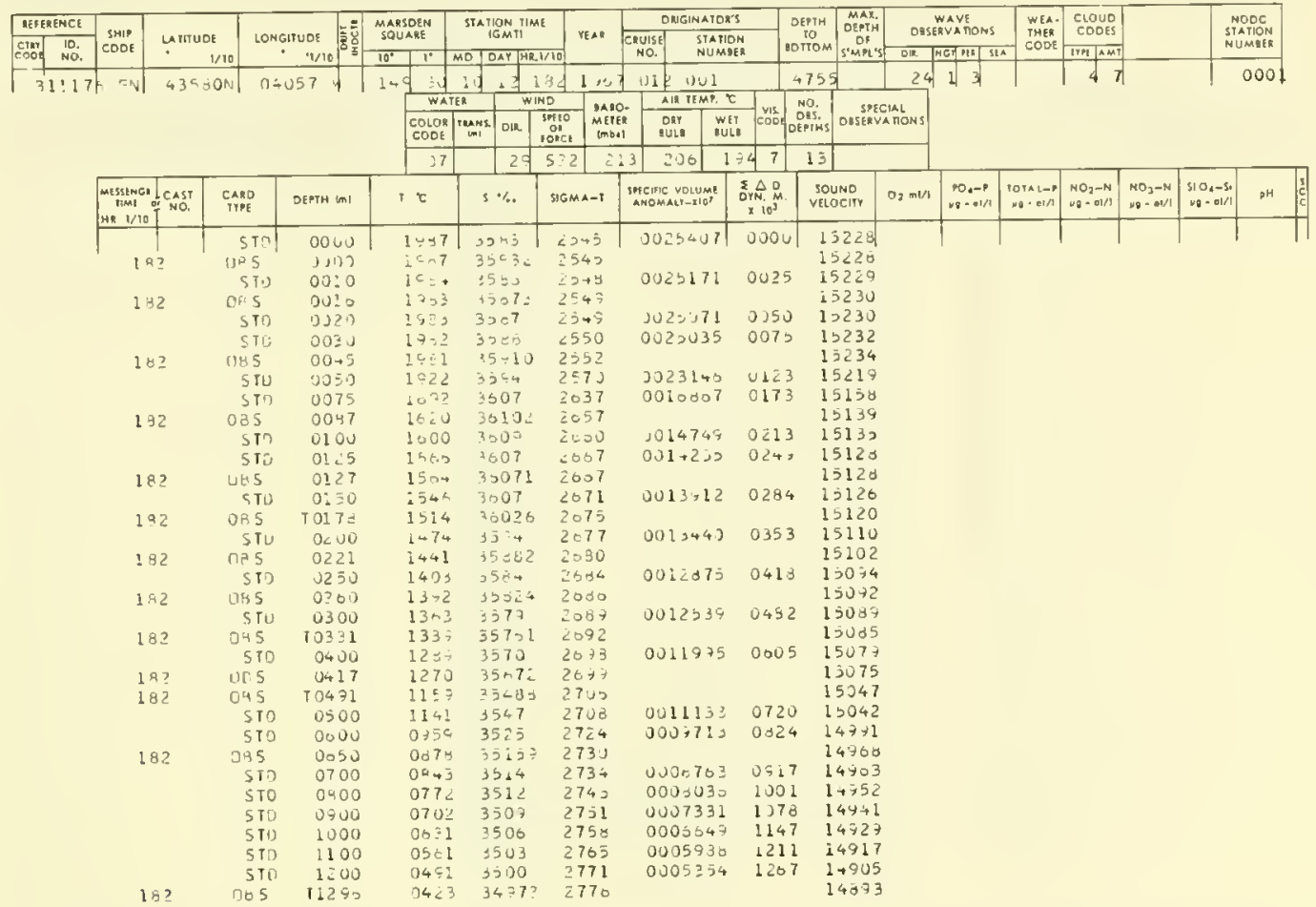

\begin{tabular}{|c|c|c|c|c|c|c|c|c|c|c|c|c|c|c|c|c|}
\hline \multirow{2}{*}{ 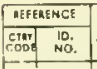 } & \multirow{2}{*}{$\begin{array}{c}5 \mathrm{SHO} \\
\operatorname{coO}\end{array}$} & \multirow{2}{*}{\multicolumn{2}{|c|}{$\begin{array}{l}\text { Ua tITUDE } \\
1 / 10\end{array}$}} & \multirow{2}{*}{ LONGIFUDE } & \multirow{2}{*}{$\begin{array}{c}\text { MAESDEN } \\
\text { SOUARE }\end{array}$} & \multirow{2}{*}{ STATION TIMEE } & \multirow{2}{*}{ IEAs } & \multicolumn{2}{|c|}{ ONGINATOE'S } & \multirow{2}{*}{$\begin{array}{c}\text { O\&gth } \\
10 \\
\text { sotrom }\end{array}$} & \multirow{2}{*}{$\begin{array}{l}\text { WAYE } \\
\text { OSSEVAnONS }\end{array}$} & \multirow{2}{*}{$\begin{array}{l}\text { WEA- } \\
\text { WHER } \\
\text { CODE }\end{array}$} & \multirow{2}{*}{ 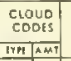 } & & & \multirow{2}{*}{$\begin{array}{l}\text { NODC } \\
\text { SAATION } \\
\text { HUMUER }\end{array}$} \\
\hline & & & & & & & & \begin{tabular}{|c|} 
cauise. \\
No.
\end{tabular} & $\begin{array}{l}\text { STATHON } \\
\text { HUMBER }\end{array}$ & & & & & & & \\
\hline \multirow{39}{*}{311176} & \multirow{4}{*}{ EN } & \multirow{4}{*}{$44 ! 2 \mathrm{~N}$} & N 10 & $050 \sqrt{1}$ & $14=-3$ & $10 i+10$ & 0011707 & $01400 ?$ & & 4.340 & \begin{tabular}{l|l|l|}
27 & 2 & 3
\end{tabular} & & 6. 9 & & & 0002 \\
\hline & & & & & $\frac{W A T}{\text { COLOB }}$ & & \begin{tabular}{l|l} 
IND & IANO \\
SWEED
\end{tabular} & - LAR TEMP. & & & & & & & & \\
\hline & & & & & 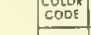 & 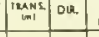 & $\begin{array}{l}0.02 \\
10151 \\
\end{array}$ & $\begin{array}{l}\text { ORy } \\
\text { onta } \\
\end{array}$ & $\begin{array}{ll}67 \\
4\end{array}$ & 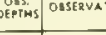 & A MONS & & & & & \\
\hline & & & & & 04 & $2=$ & $\$ 11$ & 200 & \begin{tabular}{|l|l|}
74 & 7 \\
\end{tabular} & 14 & & & & & & \\
\hline & 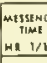 & $\begin{array}{l}\text { CAST } \\
\text { NOS }\end{array}$ & $\begin{array}{l}\text { CARD } \\
\text { Trpe }\end{array}$ & DEPTH GM & 15 & $s \%$ & SIGMA-T & 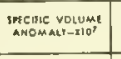 & 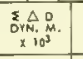 & $\begin{array}{l}\text { SOUNO } \\
\text { VELOEITY }\end{array}$ & 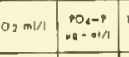 & 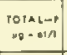 & $\left|\begin{array}{l}\mathrm{NO}^{2}-\mathrm{N} \\
\mathrm{NO}=0 \mathrm{ot/2}\end{array}\right|$ & $\begin{array}{l}\mathrm{NO}_{3}-\mathrm{N} \\
\mathrm{NQ}=\mathrm{OVI} / 1\end{array}$ & $\begin{array}{l}510,-50 \\
00-01 / 1\end{array} \mid$ & 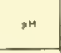 \\
\hline & & & $S T U$ & 0000 & $10: 5$ & 3521 & ¿うらら & $3 J z 52+3$ & गงנol & $1 ; 231$ & & & & & & \\
\hline & & $i$ & (3) 5 & 0000 & $i+i=$ & $\$ 520 \mathrm{~s}$ & 2515 & & & 15201 & & & & & & \\
\hline & & & $\begin{array}{l}5 T 0 \\
570\end{array}$ & $\begin{array}{l}0010 \\
0020\end{array}$ & 152 is & 3,49 & 2510 & 0027700 & $00<0$ & $1520 t$ & & & & & & \\
\hline & & 1 & $\begin{array}{l}510 \\
\text { CSS }\end{array}$ & $\begin{array}{l}0020 \\
0024\end{array}$ & $\begin{array}{l}1730 \\
1 \geqslant 40\end{array}$ & $\begin{array}{l}305 \\
30454\end{array}$ & $\begin{array}{l}2525 \\
524\end{array}$ & 0027340 & 3055 & 15212 & & & & & & \\
\hline & & & STO & 0030 & $1 \div 02$ & $\begin{array}{l}32454 \\
3300\end{array}$ & 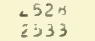 & 0020011 & 0082 & $\begin{array}{l}15214 \\
15223\end{array}$ & & & & & & \\
\hline & & 1 & OES & 0040 & 1074 & $35 \div 34$ & 2535 & $00=0011$ & & 15233 & & & & & & \\
\hline & & & STO & 0050 & 1903 & 3594 & $? 55 y$ & 0024203 & 0133 & $15 \geq 30$ & & & & & & \\
\hline & & & $\begin{array}{l}5 T 13 \\
\text { DPS }\end{array}$ & $\begin{array}{l}0075 \\
0049\end{array}$ & $: 721$ & 3601 & $2 n 20$ & 0017972 & 0180 & 15156 & & & & & & \\
\hline & & $0:$ & $\begin{array}{l}\text { DPS } \\
\text { STD }\end{array}$ & $\begin{array}{l}0049 \\
3100\end{array}$ & 1572 & $3>0+2$ & 2003 & & & $\begin{array}{l}15125 \\
15125\end{array}$ & & & & & & \\
\hline & & & ST & $\begin{array}{l}3120 \\
01 \geq 5\end{array}$ & $\begin{array}{l}1 \leq 71 \\
1540\end{array}$ & 3534 & $2 \sin 3$ & 3014492 & $\begin{array}{l}0220 \\
0 \geq 02\end{array}$ & $\begin{array}{l}15125 \\
15121\end{array}$ & & & & & & \\
\hline & & 31 & DHS & 0125 & $\begin{array}{l}1546 \\
1516\end{array}$ & $300 ?$ & 2 บ० 7 & $001+1 \geqslant 5$ & $0 \geq 02$ & $\begin{array}{l}15121 \\
15112\end{array}$ & & & & & & \\
\hline & & & ST & $11=3$ & $\begin{array}{l}1516 \\
1512\end{array}$ & $\begin{array}{l}35+70 \\
3 ;>0\end{array}$ & $207 ?$ & Jง $1375 J$ & 0257 & 15114 & & & & & & \\
\hline & & $0 !$ & niss & $01 \div 9$ & $1+<5$ & 33321 & 2579 & & & $150 \times 2$ & & & & & & \\
\hline & & & STJ & bran & $1+3$ & jjes & $\therefore, 70$ & 0013200 & $036 \overline{5}$ & $1>0>2$ & & & & & & \\
\hline & & & ST & 0253 & $235 ?$ & 393 & ?c5) & 3012694 & $0+30$ & $: 3087$ & & & & & & \\
\hline & & $a 1$ & 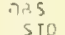 & $\begin{array}{l}02 \div 5 \\
0200\end{array}$ & $134,-1$ & 3,170 & $-\infty=1$ & & & 15003 & & & & & & \\
\hline & & $a 1$ & . & $\begin{array}{l}0200 \\
0397\end{array}$ & 1347 & $\begin{array}{l}2 \div 33 \\
2=7323\end{array}$ & & 0012312 & 0492 & $\begin{array}{l}: 5033 \\
15) 84\end{array}$ & & & & & & \\
\hline & & & $5 \mathrm{Ti}$ & $\begin{array}{l}0397 \\
0<130\end{array}$ & $\begin{array}{l}1 ?: 22 \\
12 \div 7\end{array}$ & $\begin{array}{l}357 ? 3 \\
371\end{array}$ & $\begin{array}{l}20=7 \\
200=7\end{array}$ & 3012353 & $001+$ & $\begin{array}{l}15) 84 \\
15002\end{array}$ & & & & & & \\
\hline & & 01 & 0135 & $10<22$ & $i: 1: 7$ & $15 \div i c$ & 2735 & & & 15032 & & & & & & \\
\hline & & & $5 T$ & voou & $10 \div i$ & 25,44 & 271 & JulJ $5+3$ & 072 d & 1 bue4 & & & & & & \\
\hline & & 21 & 1) 45 & $05 \approx 6$ & 2043 & 3203:- & $=725$ & & & $1-x 44$ & & & & & & \\
\hline & & & ST & $0=00$ & 0,10 & 3504 & $27<0$ & $0 J 0=315$ & $002 y$ & $14 \div 43$ & & & & & & \\
\hline & & & STJ & 070.5 & $07-$ & $3,0)=$ & 2744 & 00070,5 & $0=1 \leftrightarrow$ & $14 \dot{9} 25$ & & & & & & \\
\hline & & JI & าम 5 & $T 37 x ?$ & $0: t 7$ & 25115 & $27: 7$ & & & 14700 & & & & & & \\
\hline & & & ST & $0 \leq 00$ & $a t \sim 0$ & $\approx 510$ & 2757 & 0000400 & $0 \div 05$ & $24 \times 135$ & & & & & & \\
\hline & & 31 & S $(\mathrm{T})$ & 0000 & 057. & 3500 & $27 \leq 5$ & $000=770$ & $104 c$ & $14 y+\angle$ & & & & & & \\
\hline & & & $\begin{array}{l}D+5 \\
5 T 11\end{array}$ & 9,7 & J518 & $3531=$ & $77=8$ & & & $i 400 \overline{2}$ & & & & & & \\
\hline & & & $\begin{array}{l}5 T 11 \\
5 T 0\end{array}$ & 1000 & 0515 & $350 ?$ & $.75=$ & $00053=0$ & 1101 & 14032 & & & & & & \\
\hline & & & $\begin{array}{l}510 \\
510\end{array}$ & $1: 00$ & $0+7:$ & 3427 & 2771 & $000=18-$ & 1154 & 14800 & & & & & & \\
\hline & & 01 & $\begin{array}{c}510 \\
\text { MHS }\end{array}$ & 2200 & $04-7$ & 3424 & $\div 772$ & 0,2500, & $1<0\rangle$ & $\begin{array}{l}14002 \\
14504\end{array}$ & & & & & & \\
\hline & & & $\begin{array}{l}\text { CHS } \\
\text { STJ }\end{array}$ & 12,41 & $\begin{array}{l}04=\mathrm{m} \\
04 \mathrm{i},\end{array}$ & $\begin{array}{l}34 \div 30 \\
3434\end{array}$ & $\begin{array}{l}2773 \\
2774\end{array}$ & 0004725 & 1255 & 14071 & & & & & & \\
\hline & & & $\begin{array}{l}5 \mathrm{TIJ} \\
5 \mathrm{TH}\end{array}$ & $\begin{array}{l}1500 \\
1>00\end{array}$ & $\begin{array}{l}041, \\
040 r\end{array}$ & $\begin{array}{l}3474 \\
3476\end{array}$ & 2775 & $000494-$ & 1334 & 14733 & & & & & & \\
\hline & & & STij & 1500 & $0: 4 i n$ & $3+25$ & 2771 & $000-731$ & 1352 & 14315 & & & & & & \\
\hline & & & yes & 11501 & $03=4$ & $3+=50$ & 2777 & & & $14 \times 15$ & & & & & & \\
\hline
\end{tabular}


TABLE III.-Continued

\begin{tabular}{|c|c|c|c|c|c|c|c|c|c|c|c|c|c|c|c|c|c|}
\hline \multirow{2}{*}{ 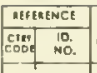 } & \multirow{2}{*}{$\begin{array}{c}\text { snir } \\
\text { CoOt }\end{array}$} & \multirow{2}{*}{\multicolumn{2}{|c|}{ 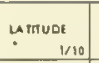 }} & \multirow{2}{*}{ LONGITUOE } & \multirow{2}{*}{$\begin{array}{l}\text { MAESDEKK } \\
\text { SOUARI }\end{array}$} & STATION $_{\text {IGMII }}$ & \multirow[b]{2}{*}{ VEAR } & \multicolumn{2}{|c|}{ ORGIMATOI's } & \multirow{2}{*}{$\begin{array}{c}\text { Depriw } \\
\text { ro } \\
\text { Hotrom } \\
\end{array}$} & \multirow{2}{*}{\multicolumn{2}{|c|}{$\begin{array}{l}\text { Wave } \\
\text { oustivanions }\end{array}$}} & \multirow{2}{*}{$\begin{array}{l}\text { WIA- } \\
\text { TMER } \\
\text { COOE }\end{array}$} & \multirow{2}{*}{ 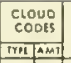 } & & & \multirow{2}{*}{$\begin{array}{l}\text { NOOE } \\
\text { STA IION } \\
\text { NUMBER } \\
\end{array}$} \\
\hline & & & & & & \begin{tabular}{|l|l|} 
& IG MII \\
MO DAY IH
\end{tabular} & & $\begin{array}{l}\text { CRuis: } \\
\text { No. } \\
\end{array}$ & $\begin{array}{l}\text { STARLON } \\
\text { NumBER }\end{array}$ & & & & & & & & \\
\hline \multirow[t]{43}{*}{31117} & $\underline{E N}$ & 4356 & $\mathrm{~N}$ & $04035 W$ & $140 \quad 30$ & $1014_{4}$ & 2331907 & 011003 & & 4801 & 19 & $\left|\begin{array}{ll}\mid & 3\end{array}\right|$ & 63 & 59 & & 1 & 0003 \\
\hline & & & & & WAT & & IND & O. AR TEMP. & .2 & \begin{tabular}{|l|l|} 
No. \\
\end{tabular} & & & & & & & \\
\hline & & & & & $\begin{array}{l}\text { color } \\
\text { cook }\end{array}$ & 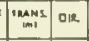 & $\begin{array}{l}\text { shio } \\
\text { or } \\
\text { ox }\end{array}$ & \begin{tabular}{l|l} 
onr & out \\
out & :
\end{tabular} & WEt =oo & fouss oustery & CAATONB & & & & & & \\
\hline & & & & & 01 & 24 & 525 & 200 & $200 \mid 6$ & 14 & & & & & & & \\
\hline & 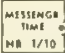 & $\begin{array}{l}\text { ICAst } \\
\text { I NO. }\end{array}$ & $\begin{array}{l}\text { CAlO } \\
\text { Tyef }\end{array}$ & OEPTY UnI & $1 \tau$ & $s=$ & SIGMA-T & $\begin{array}{l}\text { sencific volumy } \\
\text { anamabt-sing }\end{array}$ & 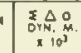 & $\begin{array}{l}\text { SOUND } \\
\text { VELOCIIY }\end{array}$ & $02 m L 1$ & 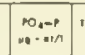 & $\begin{array}{l}\text { rotat-p } \\
\text { ne.ein }\end{array}$ & 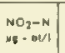 & $\begin{array}{l}\mathrm{m}-\mathrm{OVI} \\
\mathrm{NO}^{3}-\mathrm{N}\end{array}$ & $\left|\begin{array}{ll}31 & 0 \\
38 & -51 / 1\end{array}\right|$ & 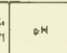 \\
\hline & & & $5 \mathrm{TO}$ & (2) & 1924 & 3,00 & $24>0$ & 0029907 & 0000 & 15230 & & & & & & & \\
\hline & 239 & & DA 5 & 0000 & 1526 & 34750 & 2475 & & & 15200 & & & & & & & \\
\hline & & & $5 \mathrm{TO}$ & 0010 & 1930 & 3507 & 2502 & 0023534 & 0029 & 15205 & & & & & & & \\
\hline & & & $5 \mathrm{TO}$ & 0020 & 1927 & 3520 & 2513 & 302877 , & $005 d$ & 15210 & & & & & & & \\
\hline & 23 & & 085 & 0025 & 1940 & 35229 & 2510 & & & 15212 & & & & & & & \\
\hline & & & $5 T 0$ & 0030 & 1927 & $35+5$ & 2531 & 0026818 & 0026 & 15211 & & & & & & & \\
\hline & & & $5 \mathrm{TJ}$ & 0050 & 2807 & 3594 & $25 \mathrm{~d} 4$ & 0021856 & 0135 & 15203 & & & & & & & \\
\hline & $23 c$ & & 085 & 0052 & 1800 & 35975 & $25 d \mathrm{~d}$ & & & 15202 & & & & & & & \\
\hline & & & 570 & 0075 & 1756 & 3607 & 2021 & 001438. & 0185 & 15177 & & & & & & & \\
\hline & & & $5 \mathrm{TO}$ & 0100 & 1600 & 3617 & 2052 & 3015535 & 0228 & 15154 & & & & & & & \\
\hline & 230 & & OB 5 & 9104 & 1046 & 33183 & 2057 & & & 15251 & & & & & & & \\
\hline & & & $5 \mathrm{TO}$ & 0125 & 1581 & 3007 & 2003 & 0014572 & 0205 & 15133 & & & & & & & \\
\hline & & & $5 \mathrm{TO}$ & 0150 & $151 \%$ & 3590 & 2009 & $00140 d 0$ & 0301 & 15110 & & & & & & & \\
\hline & 236 & & OBS & $0: 54$ & 1511 & 33950 & 2070 & & & 15114 & & & & & & & \\
\hline & & & $5 \mathrm{TO}$ & 0200 & 1440 & $35 y_{4}$ & 2070 & 0013574 & 0370 & 15100 & & & & & & & \\
\hline & 230 & & 085 & 0200 & 1440 & 35843 & 2076 & & & 15100 & & & & & & & \\
\hline & & & STO & 2253 & 1402 & 3579 & 2881 & 0013172 & 0437 & 15093 & & & & & & & \\
\hline & & & $5 \mathrm{TO}$ & 0300 & 1343 & 3571 & 2687 & 0012727 & 0502 & 15001 & & & & & & & \\
\hline & 236 & & DR 5 & 0310 & 1330 & $358 ; 1$ & 2008 & & & 15078 & & & & & & & \\
\hline & & & 570 & 0603 & 1182 & 3544 & 2690 & 0011828 & 0625 & 15039 & & & & & & & \\
\hline & 23 & & Oos & 0412 & 1162 & 35414 & 2700 & & & 15034 & & & & & & & \\
\hline & & & $5 T 0$ & 0500 & 1029 & 3528 & 2714 & 0010451 & 0730 & 15000 & & & & & & & \\
\hline & 236 & & QA 5 & T 3515 & 0997 & 35246 & 2717 & & & 14970 & & & & & & & \\
\hline & & & 510 & 0600 & 0749 & 3472 & 2731 & 0008701 & 0832 & 14907 & & & & & & & \\
\hline & 230 & & 085 & 0516 & 0714 & 34885 & 2733 & & & 14896 & & & & & & & \\
\hline & & & sio & 0700 & 0667 & 3499 & 2748 & 0007222 & 0912 & 14893 & & & & & & & \\
\hline & & & STD & 0800 & 0011 & 3506 & 2701 & 0006077 & 0578 & 14008 & & & & & & & \\
\hline & 230 & & 085 & 70816 & 0502 & 35064 & 2762 & & & 14837 & & & & & & & \\
\hline & & & STO & 0900 & 0543 & 3503 & 2760 & 0005591 & 1037 & 14879 & & & & & & & \\
\hline & & & 570 & 1000 & $04>5$ & $34 \div 9$ & 2707 & 0005258 & 1071 & 14873 & & & & & & & \\
\hline & 230 & & 005 & 1017 & 0487 & 34986 & 2770 & & & 14873 & & & & & & & \\
\hline & & & $5 T 3$ & 1100 & $046 ?$ & 3499 & 2772 & 0005020 & 1142 & 14876 & & & & & & & \\
\hline & & & STO & 1200 & 0434 & 3497 & 2774 & 0004652 & 1192 & 14381 & & & & & & & \\
\hline & 235 & & 085 & $T 1209$ & 0417 & 34901 & 2770 & & & 14850 & & & & & & & \\
\hline & & & STO & 1300 & 0413 & 3496 & 2776 & 0004735 & 1240 & 14839 & & & & & & & \\
\hline & & & 570 & 1400 & 0399 & 3495 & 2777 & 0004672 & 1287 & 14899 & & & & & & & \\
\hline & & & $5 T 0$ & 1500 & 0388 & 3495 & $277 d$ & $000 \div 606$ & 1334 & 14912 & & & & & & & \\
\hline & & & 085 & $T 1537$ & 0356 & 34948 & $277 \mathrm{~d}$ & & & 14918 & & & & & & & \\
\hline
\end{tabular}

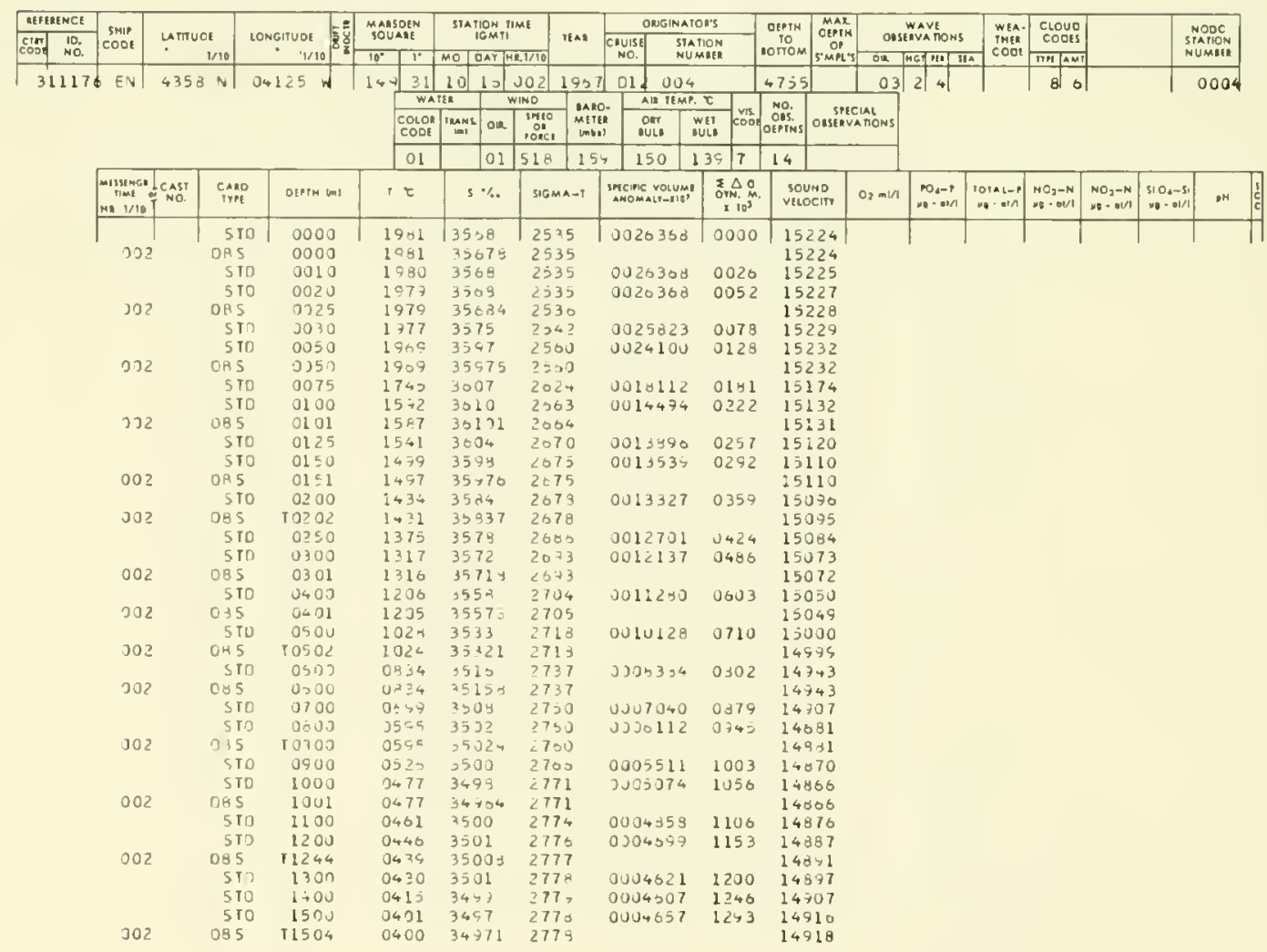




\section{TABle III.-Continued}

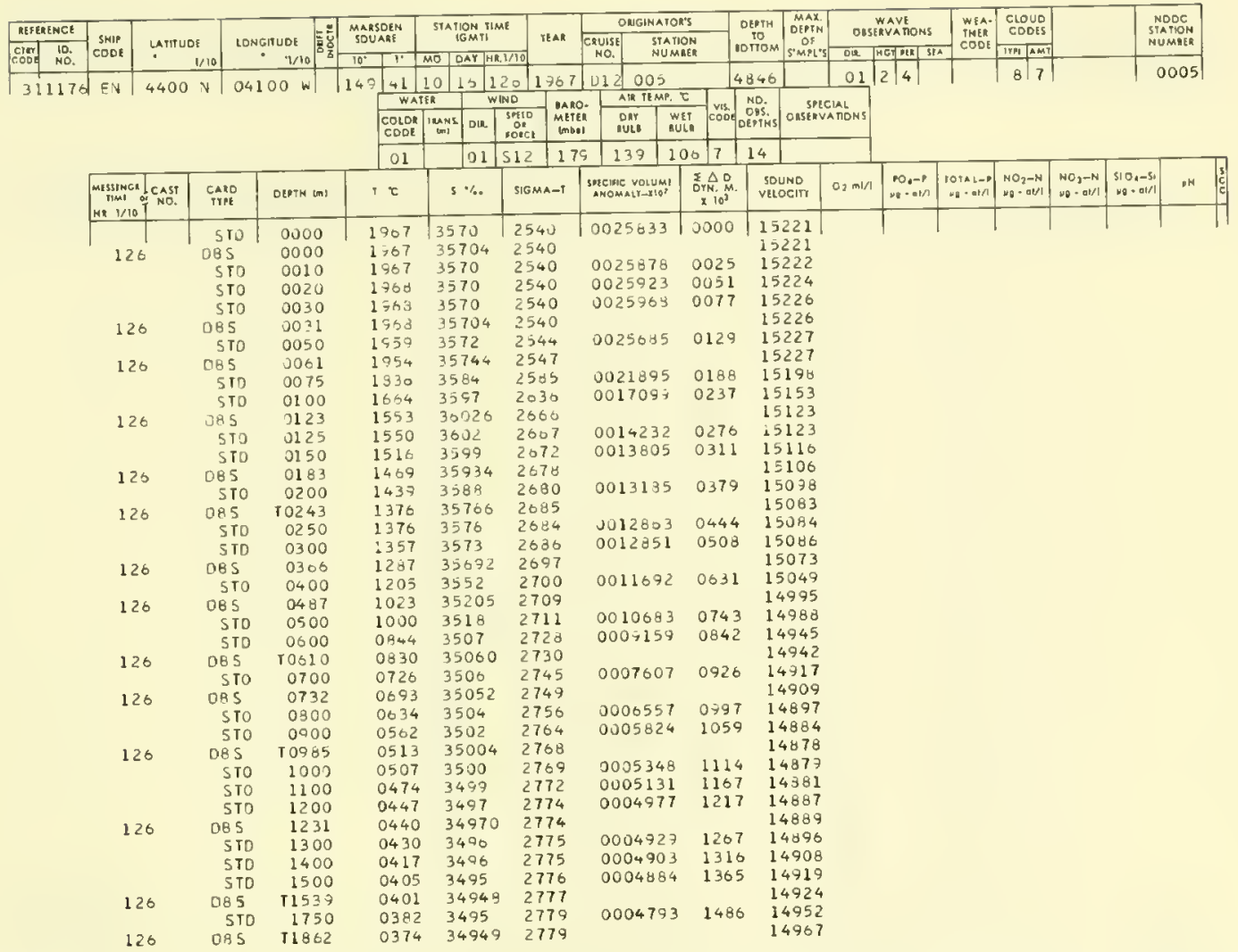

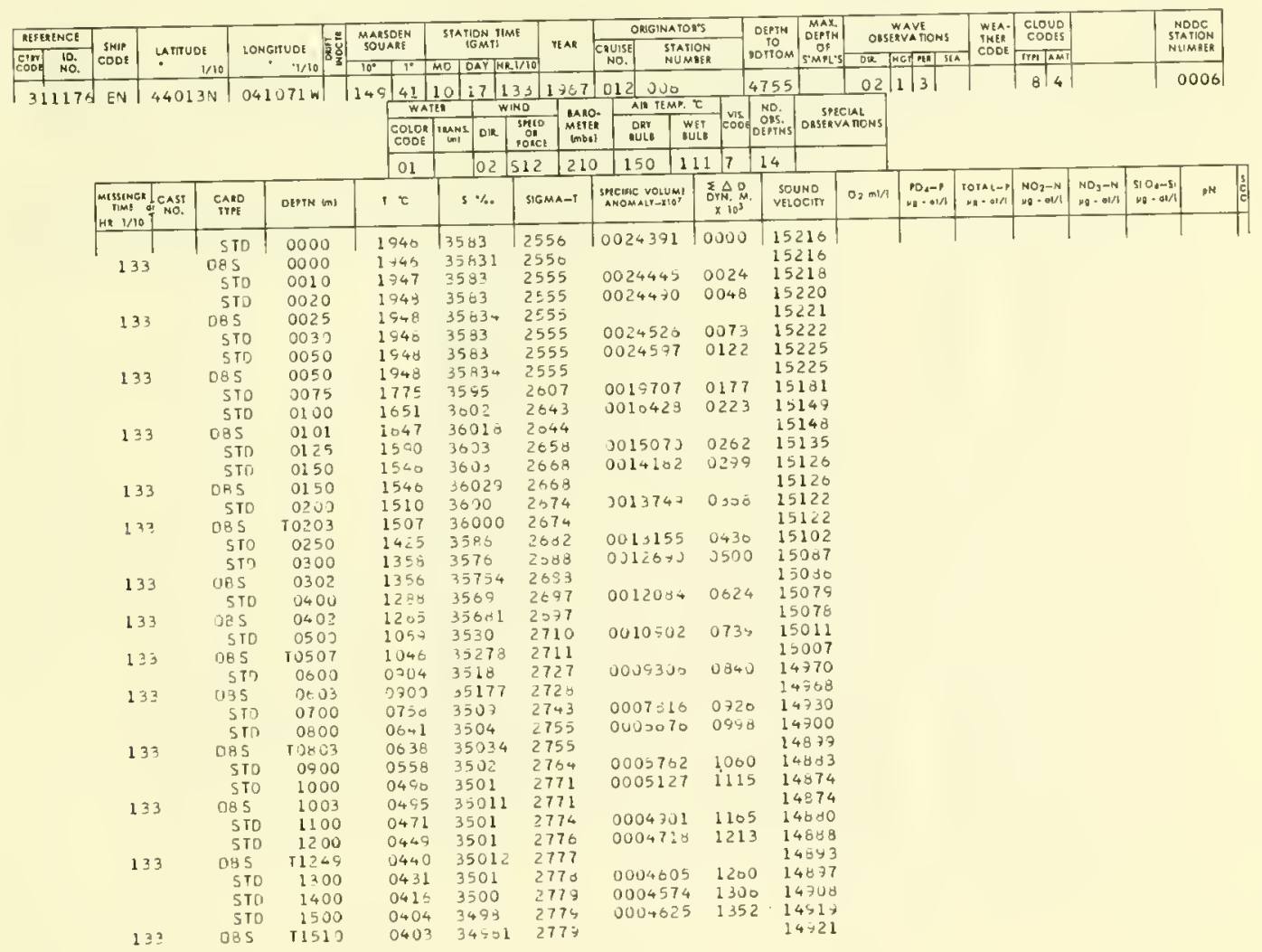


TABle III.-Continued

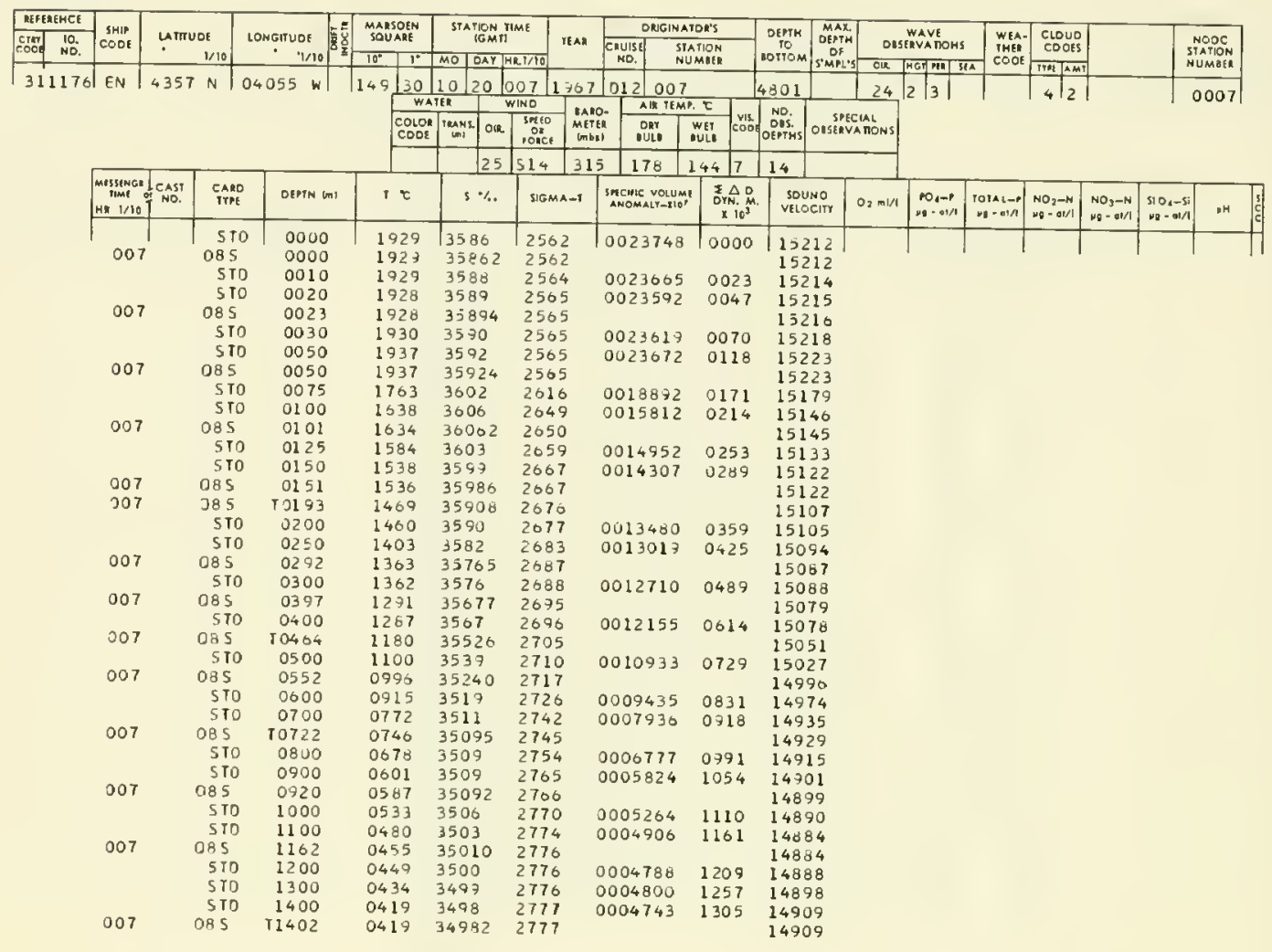

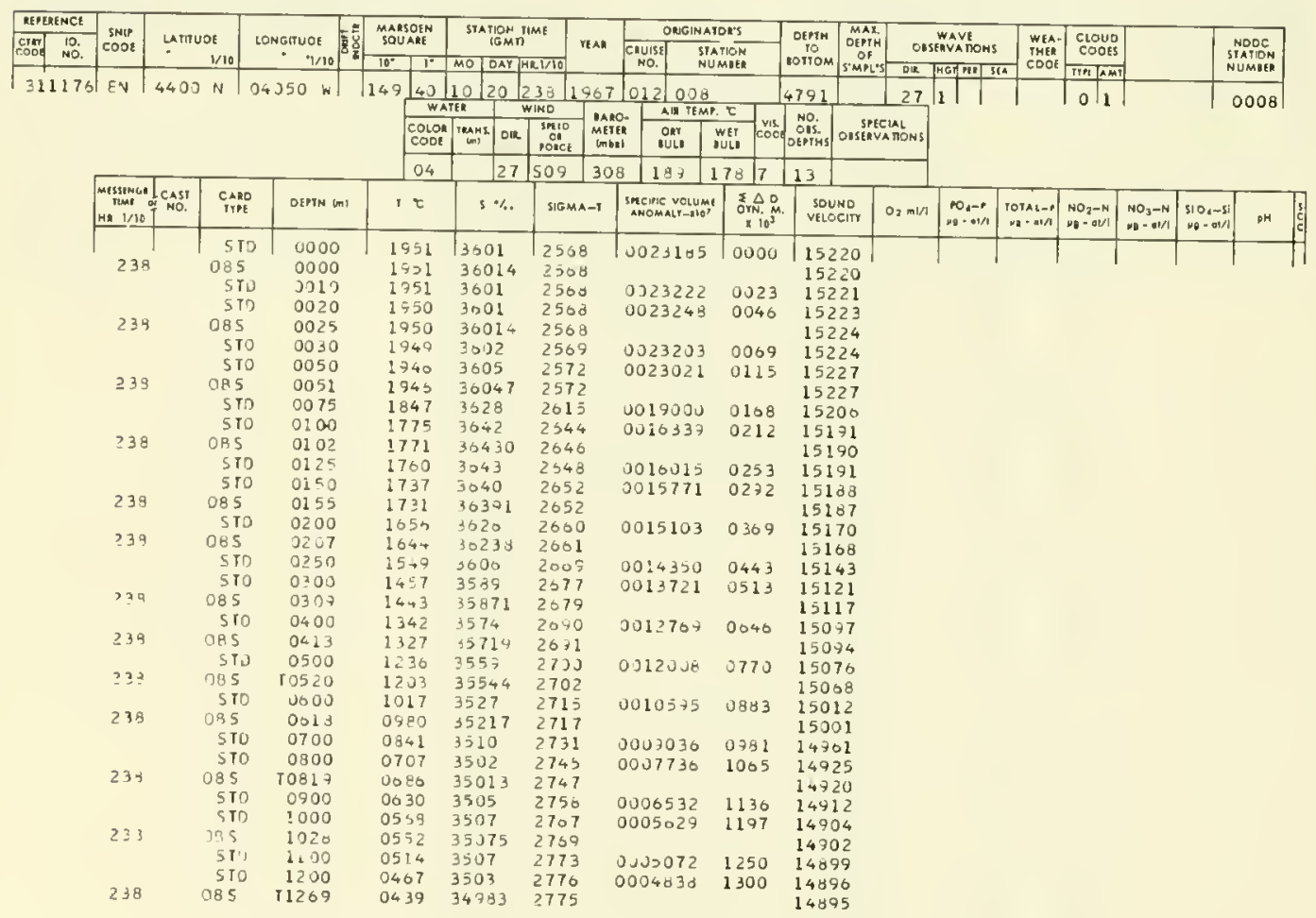


TABLE III.-Continued

\begin{tabular}{|c|c|c|c|c|c|c|c|c|c|c|c|c|c|c|c|c|c|c|c|c|}
\hline \multirow{2}{*}{\begin{tabular}{|l|l|} 
REFERENCE \\
CrIm & 10. \\
NoO & No. \\
\end{tabular}} & \multirow{2}{*}{$\left|\begin{array}{c}s m i p \\
c O D:\end{array}\right|$} & \multirow{2}{*}{\multicolumn{2}{|c|}{\begin{tabular}{|c|} 
Lanrude \\
$1 / 10$
\end{tabular}}} & \multirow{2}{*}{$\begin{array}{c}\text { LONGITUDE } \\
\text { 1/10 }\end{array}$} & \multirow{2}{*}{$\begin{array}{l}\text { MARSDEN } \\
\text { SQUAKE }\end{array}$} & \multirow{2}{*}{\multicolumn{3}{|c|}{$\begin{array}{l}\text { STAYION TIMI } \\
\text { IGMTI }\end{array}$}} & \multirow[b]{2}{*}{ YEAR } & \multicolumn{2}{|c|}{\begin{tabular}{|l} 
ORGINATOR'S \\
\end{tabular}} & \multirow{2}{*}{$\begin{array}{l}\text { DEPTH } \\
\text { TO } \\
\text { OTIOM }\end{array}$} & \multirow{2}{*}{ 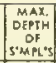 } & \multirow{2}{*}{\multicolumn{2}{|c|}{$\begin{array}{l}\text { WAVE } \\
\text { OUSERVATONS }\end{array}$}} & \multirow{2}{*}{\begin{tabular}{|l|} 
WEA. \\
IHER \\
CODE \\
\end{tabular}} & \multirow{2}{*}{\begin{tabular}{|l|} 
CLOUD \\
CODES \\
PMETAMT \\
\end{tabular}} & & & \multirow{2}{*}{$\begin{array}{l}\text { NODC } \\
\text { SATION } \\
\text { NUMEER } \\
\end{array}$} \\
\hline & & & & & & & & & & $\begin{array}{c}\text { CRuise } \\
\text { No. }\end{array}$ & $\begin{array}{l}\text { STATION } \\
\text { NUMUER }\end{array}$ & & & & & & & & & \\
\hline \multirow[t]{37}{*}{311170} & $E N$ & 4401 & $N \mid O$ & $04102 \mathrm{Wl}$ & 14941 & 10 & 21 & 22 & $1 \geqslant 07$ & 1012009 & & 4840 & & 22 & $|2| 3 \mid$ & & $8 / 5$ & & & 0009 \\
\hline & & & & & WA & & & NiND & & 0. AII IEMP. & 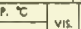 & No. & & & & & & & & \\
\hline & & & & & \begin{tabular}{|l} 
COLOR \\
CODE
\end{tabular} & 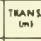 & DIR & \begin{tabular}{|l|} 
Smikg \\
on \\
oonet
\end{tabular} & $\begin{array}{c}\text { Meti } \\
\text { mat }\end{array}$ & \begin{tabular}{l|l} 
Dart \\
vuLi
\end{tabular} & \begin{tabular}{l|l} 
WET & COIS \\
BULI & \\
\end{tabular} & Ce cIS. & OISERY & AMONS & & & & & & \\
\hline & & & & & 04 & & 22 & 517 & 30 & 194 & $103 \sqrt{7}$ & 13 & & & & & & & & \\
\hline & 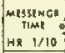 & "LCASI & $\begin{array}{l}\text { CARD } \\
\text { TYPE }\end{array}$ & DEPTM (M) & it & & $\cdot \%$ & & $u a-r$ & $\begin{array}{l}\text { Sme ire volume } \\
\text { ANOMA TF- }\end{array}$ & 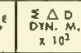 & & $\begin{array}{l}\text { UND } \\
\text { OCITY }\end{array}$ & $02 \mathrm{mb} /$ & 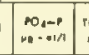 & 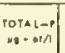 & $\begin{array}{l}\mathrm{NO}_{2}-\mathrm{N} \\
\mathrm{yq}_{2}-\mathrm{al/1}\end{array}$ & $\begin{array}{l}\mathrm{NO}_{3}-\mathrm{N} \\
\mathrm{ni}-\mathrm{OLI} / \mathrm{I}\end{array}$ & $\left|\begin{array}{l}310,-51 \\
40-81 / 1\end{array}\right|$ & PH \\
\hline & & & 510 & 0000 & 1947 & 368 & & 25 & & 0023022 & 0000 & $152-x \cdot \theta$ & 219 & & & & & & & \\
\hline & 125 & & 065 & 0000 & 1947 & & 023 & 25 & & & & & 219 & & & & & & & \\
\hline & & & $5 \mathrm{IO}$ & 0010 & 1946 & 368 & 02 & 25 & & 0023045 & 0023 & & 220 & & & & & & & \\
\hline & 125 & & Des 5 & 0018 & 1940 & & 019 & 25 & & & & & 221 & & & & & & & \\
\hline & & & STO & 0020 & 1946 & 36 & 02 & 25 & & 0023035 & 0046 & & 222 & & & & & & & \\
\hline & & & STO & 0030 & 1947 & 30 & 03 & 25 & & 0023058 & 0069 & 152 & 224 & & & & & & & \\
\hline & 125 & & DBS 5 & 0035 & 1948 & & 041 & 25 & & & & & 225 & & & & & & & \\
\hline & & & STO & 0050 & 1912 & 36 & 22 & 25 & & 0020918 & 0213 & & 219 & & & & & & & \\
\hline & 125 & & OR 5 & 0071 & 1862 & & 381 & 26 & & & & 152 & 211 & & & & & & & \\
\hline & & & STO & 0075 & 1851 & 36 & 39 & 26 & & 0018304 & 0152 & 152 & 208 & & & & & & & \\
\hline & & & sto & 0100 & 1752 & 36. & 42 & 26 & & 0016775 & 0206 & & 196 & & & & & & & \\
\hline & 125 & & 085 & 0111 & 1771 & & 420 & 20 & & & & & 192 & & & & & & & \\
\hline & & & STO & 0125 & 1757 & 30 & 42 & 26 & & 0016033 & 0247 & & 190 & & & & & & & \\
\hline & & & STD & 0150 & 1720 & 36 & 40 & 23 & & 0015597 & 0286 & & 186 & & & & & & & \\
\hline & 125 & & 085 & To1 55 & 1723 & & 393 & 26 & & & & & 185 & & & & & & & \\
\hline & & & 510 & 0200 & 1607 & 36. & 30 & 26 & & 0015061 & 0363 & & 174 & & & & & & & \\
\hline & & & $5 \mathrm{TO}$ & 0250 & 159. & 36 & 17 & 26 & & 0014494 & 0437 & & 159 & & & & & & & \\
\hline & 125 & & 085 & 0272 & 1525 & & 053 & 26 & & & & & 143 & & & & & & & \\
\hline & & & STo & 0300 & 1507 & 36 & 02 & 26 & & 0013881 & 0508 & & 138 & & & & & & & \\
\hline & 125 & & 085 & 10368 & 1380 & & 773 & 26 & & & & & 105 & & & & & & & \\
\hline & & & STo & 0400 & 1347 & 35 & 75 & 26 & & 0012773 & 0641 & & 100 & & & & & & & \\
\hline & 125 & & DRS & 0443 & 1298 & 35 & 630 & 26 & & & & & 089 & & & & & & & \\
\hline & & & 510 & 0500 & 1221 & 35 & 57 & 27 & & 0011877 & 0764 & & 072 & & & & & & & \\
\hline & 125 & & 085 & T0593 & 1073 & & 380 & 27 & & & & & 032 & & & & & & & \\
\hline & & & 510 & 0000 & 1057 & 35 & 36 & 27 & & 0010606 & 0877 & & 028 & & & & & & & \\
\hline & & & 510 & 0700 & 0854 & 35 & & 27 & & 0008886 & 0774 & & 967 & & & & & & & \\
\hline & 125 & & 085 & 0735 & 0794 & & 104 & 27 & & & & & 949 & & & & & & & \\
\hline & & & 5ID & 0800 & 0699 & 35 & 08 & 27 & & 0007215 & 1055 & & 923 & & & & & & & \\
\hline & 125 & & 085 & 10899 & 0591 & 35 & 050 & 27 & & & & 148 & 896 & & & & & & & \\
\hline & & & 5To & 0900 & 0570 & 35 & 05 & 27 & & 0005989 & 1121 & & 896 & & & & & & & \\
\hline & & & STO & 1000 & 0524 & 35 & 04 & 27 & & 0005254 & 1277 & & 886 & & & & & & & \\
\hline & 125 & & 085 & 12086 & 0503 & 35 & 040 & 27 & & & & 14 & 892 & & & & & & & \\
\hline
\end{tabular}

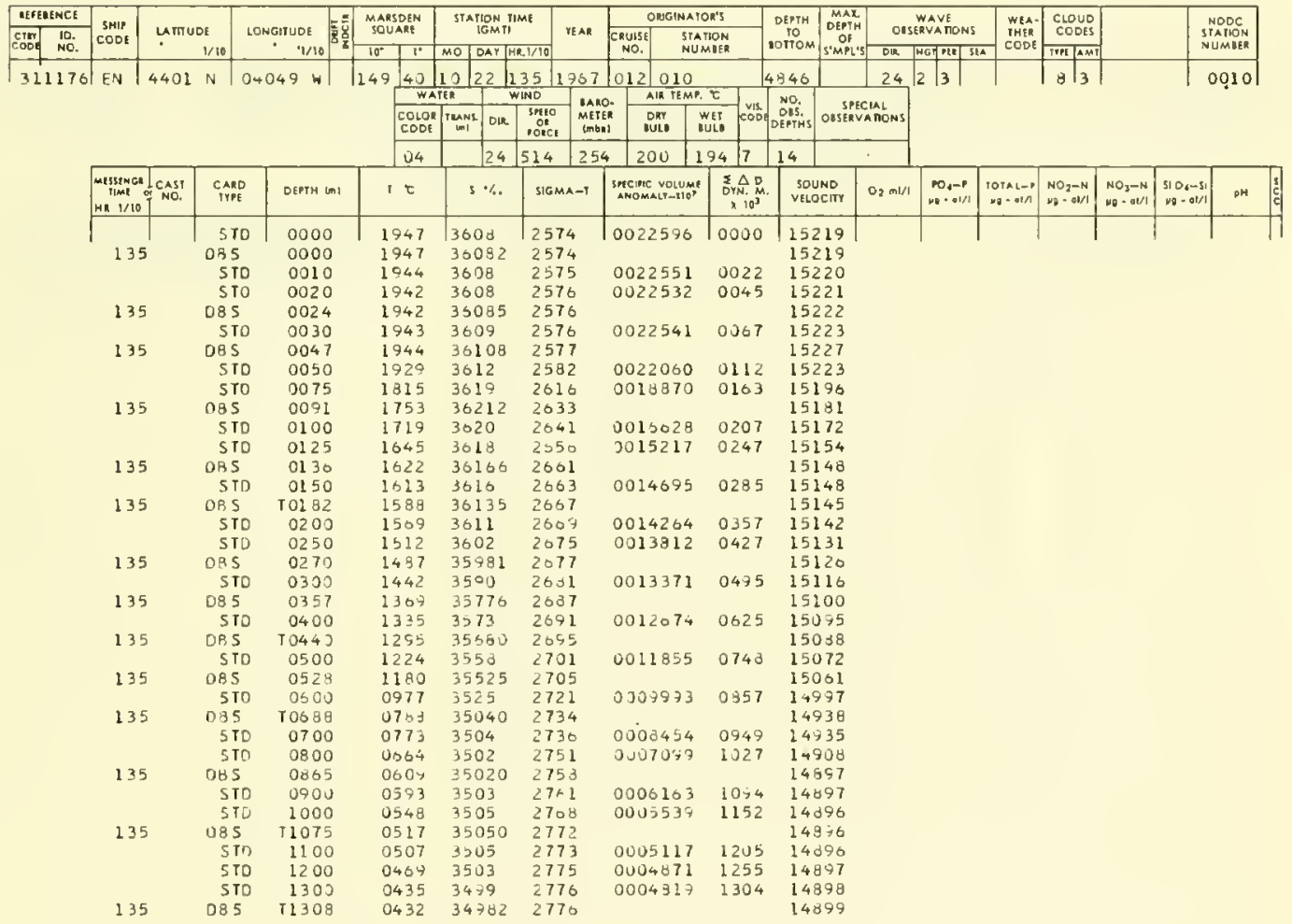


TABLE III.-Continued

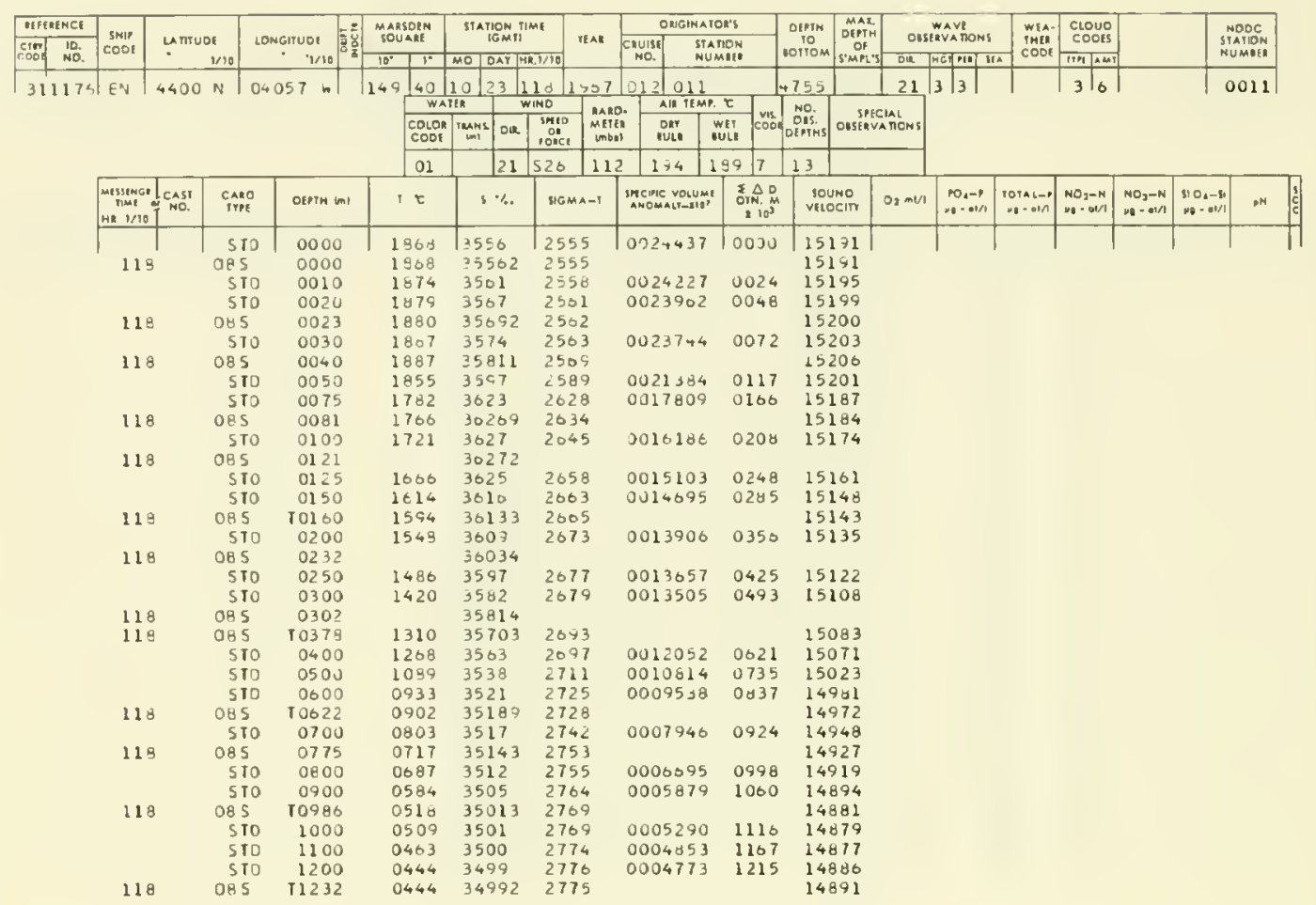

\begin{tabular}{|c|c|c|c|c|c|c|c|c|c|c|c|c|c|c|c|c|c|c|c|c|c|}
\hline AHRAENCE & \multirow[b]{2}{*}{$\begin{array}{l}S H I P \\
C O D\end{array}$} & \multirow{2}{*}{\multicolumn{2}{|c|}{ 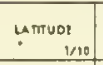 }} & \multirow{2}{*}{$\begin{array}{c}\text { LONGIYUDe } \\
1 / 10\end{array}$} & \multirow{2}{*}{\multicolumn{2}{|c|}{$\begin{array}{c}\text { MAASDLN } \\
\text { SOUARE }\end{array}$}} & \multirow{2}{*}{\multicolumn{3}{|c|}{$\begin{array}{c}\text { SIA MION FIME } \\
\text { IG MTI }\end{array}$}} & \multirow[b]{2}{*}{ reAE } & \multicolumn{2}{|c|}{ DEGINATOH"S } & \multirow{2}{*}{ 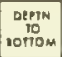 } & \multirow{2}{*}{ 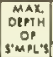 } & \multirow{2}{*}{\multicolumn{2}{|c|}{$\begin{array}{c}\text { Wave } \\
\text { oustrvanons }\end{array}$}} & \multirow{2}{*}{$\begin{array}{l}\text { WAA- } \\
\text { TMEK } \\
\text { COOE }\end{array}$} & \multirow{2}{*}{ 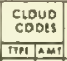 } & & & \multirow{2}{*}{$\begin{array}{l}\text { NOOC } \\
\text { SRTIION } \\
\text { NUMUL" }\end{array}$} \\
\hline 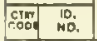 & & & & & & & & & & & \begin{tabular}{|c|c|} 
COUISE & STA \\
NO. & NU \\
\end{tabular} & $\begin{array}{l}\text { ATION } \\
\text { IMUE: }\end{array}$ & & & & & & & & & \\
\hline 311176 & \multirow[t]{4}{*}{ of EN } & \multirow[t]{4}{*}{4357} & \multirow[t]{4}{*}{$N$} & \multirow[t]{4}{*}{$0.057 \mathrm{~W}$} & \multirow[t]{4}{*}{1149} & 30 & 100 & 25 & 011 & 1,007 & $\mid \begin{array}{ll}012 & 012 \\
\end{array}$ & & 4755 & & 01 & $|3| 3 \mid$ & & 145 & & & 0012 \\
\hline & & & & & & WA & ITE & & WINO & & to $A \& \operatorname{TIMP}$ & t. & NO. & & & & & & & & \\
\hline & & & & & & $\begin{array}{l}\text { Coton } \\
\text { COot }\end{array}$ & $\lim _{n \rightarrow 1}$ & 12 Din & $\begin{array}{l}1 n ! \\
0 ! \\
010\end{array}$ & $\begin{array}{c}\text { mere } \\
\text { imbe }\end{array}$ & $\begin{array}{ll}\text { on } \\
\text { iut }\end{array}$ & WEL COO & Dift: & ousinv & VATONS & & & & & & \\
\hline & & & & & & 04 & & 30 & 515 & 234 & 133 & 1067 & 13 & & & & & & & & \\
\hline & $\begin{array}{l}\text { monemen } \\
\text { nat } \\
H \cap 1 / 18\end{array}$ & $\begin{array}{l}\text { CAST } \\
\text { CND. }\end{array}$ & $\begin{array}{l}\text { CARD } \\
\text { Trm }\end{array}$ & DETN WII & & I $c$ & & $* \%$ & & ima-1 & 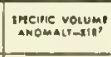 & 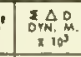 & & & $D_{2} m V_{1}$ & 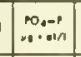 & 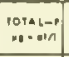 & 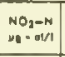 & $\begin{array}{l}\mathrm{MO}, \mathrm{N} \\
\mathrm{N}=\mathrm{OLN}\end{array}$ & $\left|\begin{array}{c|c}310,-8 \\
00=0+1 / 1\end{array}\right|$ & o \\
\hline & & & ST & 0000 & & 1702 & 1360 & 07 & 25 & 85 & 0021533 & 0000 & 152 & 207 & & & & & & & \\
\hline & 011 & & 0.85 & 0000 & & 1902 & & 072 & 25 & 305 & & & & 207 & & & & & & & \\
\hline & & & ST & 0010 & & 1004 & 360 & $O B$ & 25 & 3 & 0021599 & 0021 & 152 & 209 & & & & & & & \\
\hline & 011 & & 025 & $001 \mathrm{R}$ & & 1905 & & 082 & 25 & 85 & & & 152 & 211 & & & & & & & \\
\hline & & & $5 T$ & 0.223 & & 1935 & $36 ?$ & OA & 23 & 85 & 0021634 & 0.043 & 152 & 211 & & & & & & & \\
\hline & & & $5 \mathrm{~T}$ & 0030 & & 1904 & $36 \mathrm{C}$ & 08 & 25 & נ5 & 0021052 & 0004 & 152 & 212 & & & & & & & \\
\hline & 011 & & ons & 0035 & & 1904 & & 002 & 25 & 590 & & & 152 & 213 & & & & & & & \\
\hline & & & ST & 0250 & & $8=3$ & 362 & 25 & 20 & 12 & 0015239 & 0105 & 152 & 203 & & & & & & & \\
\hline & 011 & & OBS & aous & & 1809 & & 376 & 20 & $1<$ & & & 151 & 195 & & & & & & & \\
\hline & & & $5 \mathbf{T}$ & 0075 & & $17 \div 8$ & $36=$ & 33 & 20 & 37 & 0010860 & 0150 & 151 & $1>0$ & & & & & & & \\
\hline & & & ST & 0100 & & 1744 & 363 & 36 & $\angle 0$ & 47 & 3015673 & 0191 & 151 & 332 & & & & & & & \\
\hline & 011 & & DPS & 0112 & & 730 & 36 & 330 & 20 & 52 & & & 151 & 177 & & & & & & & \\
\hline & & & ST & 0125 & & 1727 & 36 & 38 & zo & 53 & 0015507 & 0231 & 151 & 181 & & & & & & & \\
\hline & & & ST & 0150 & & 1712 & $36]$ & 38 & 20 & 56 & 0015332 & 0269 & 151 & 180 & & & & & & & \\
\hline & 11 & & OBS & 13156 & & 1707 & 30 & 381 & 20 & 57 & & & 151 & $1 \forall 0$ & & & & & & & \\
\hline & & & $5 \mathrm{~T}$ & 3200 & & $164 !$ & 362 & 25 & 20 & 03 & $001+337$ & 0345 & 151 & 100 & & & & & & & \\
\hline & 011 & & OPS & 0217 & & $101=$ & $3 y$ & 202 & 20 & 105 & & & 151 & 100 & & & & & & & \\
\hline & & & $5 T$ & 0253 & & 1572 & 351 & 13 & 20 & 70 & $001+350$ & 0419 & 151 & 151 & & & & & & & \\
\hline & 01 & & 085 & $02 \leqslant 2$ & & 1522 & 300 & 042 & 20 & 74 & & & 151 & 140 & & & & & & & \\
\hline & & & $5 T$ & 0300 & & $14=7$ & 35 & 99 & $2 B$ & 77 & 0013719 & 0448 & 151 & 132 & & & & & & & \\
\hline & 311 & & 205 & 10345 & & $\ln 0 t$ & 30 & 540 & 20 & d5 & & & 151 & 111 & & & & & & & \\
\hline & & & $5 T$ & 0400 & & 1906 & $3 s$ & 60 & 25 & 72 & 0012572 & 0013 & 150 & 084 & & & & & & & \\
\hline & 011 & & ORS & 0403 & & 1259 & $35 \mathrm{c}$ & 624 & $<0$ & 92 & & & 150 & 083 & & & & & & & \\
\hline & & & ST & jะ0? & & (13) & 354 & 4) & 27 & כ & 0312410 & 0739 & 150 & 037 & & & & & & & \\
\hline & & & ST & 0600 & & 9947 & 352 & 20 & 27 & ¿? & 0009088 & 0840 & 149 & 780 & & & & & & & \\
\hline & 211 & & oes & $O \rightarrow \geq 5$ & & 503 & 351 & 164 & 27 & 20 & & & & 973 & & & & & & & \\
\hline & & & ST & 0700 & & $37 t 2$ & 350 & 07 & 27 & -1 & ס & 1935 & $1+9$ & 431 & & & & & & & \\
\hline & 311 & & gas & 10756 & & $x=4$ & 350 & $02=$ & 27 & 53 & & & 145 & 997 & & & & & & & \\
\hline & il 11 & & OBS & $0+230$ & & 505 & 356 & 001 & & $0.7 \mathrm{~J}$ & & & & & & & & & & & \\
\hline
\end{tabular}




\section{TABLE III.-Continued}

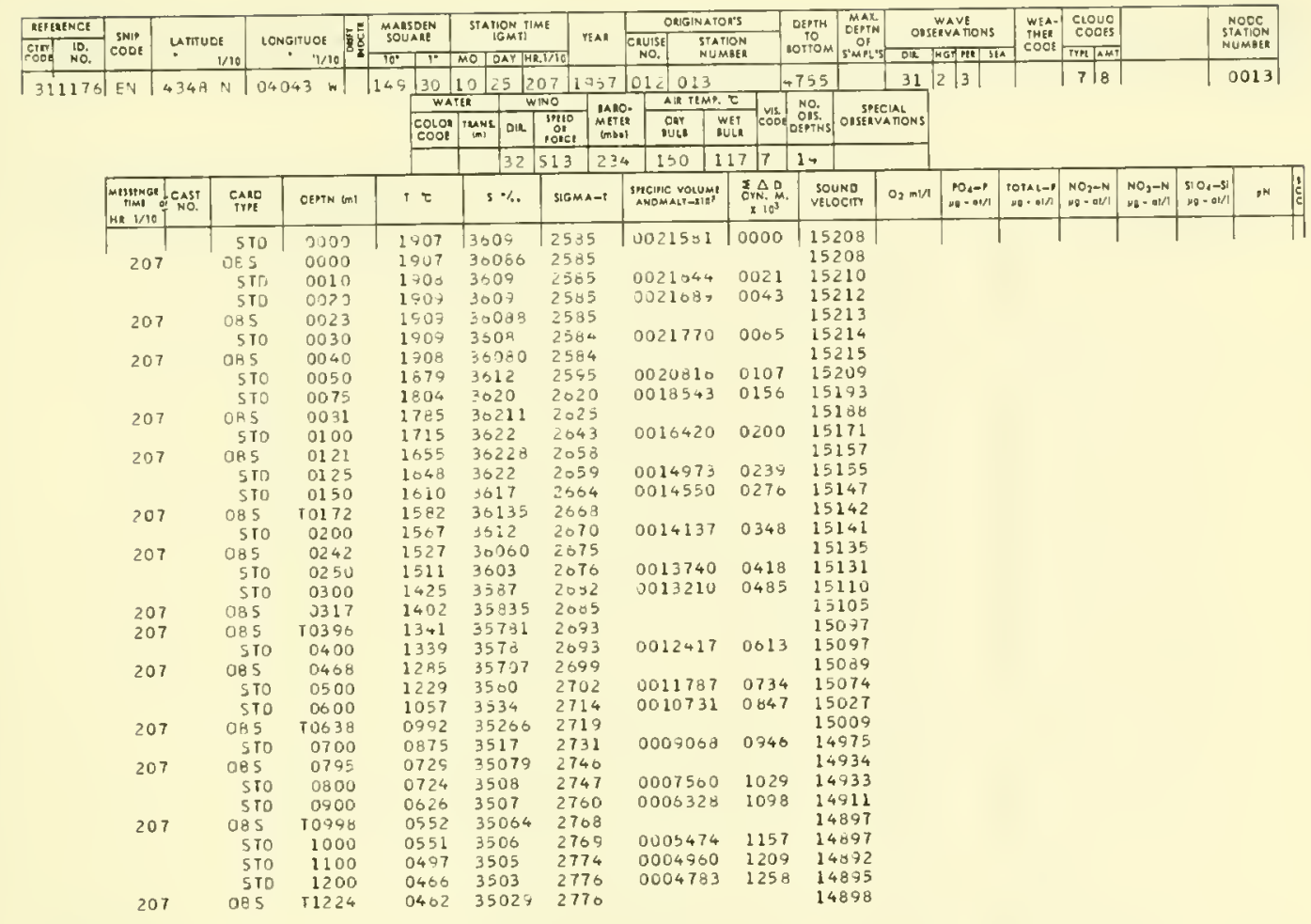


TABle III.-Continued

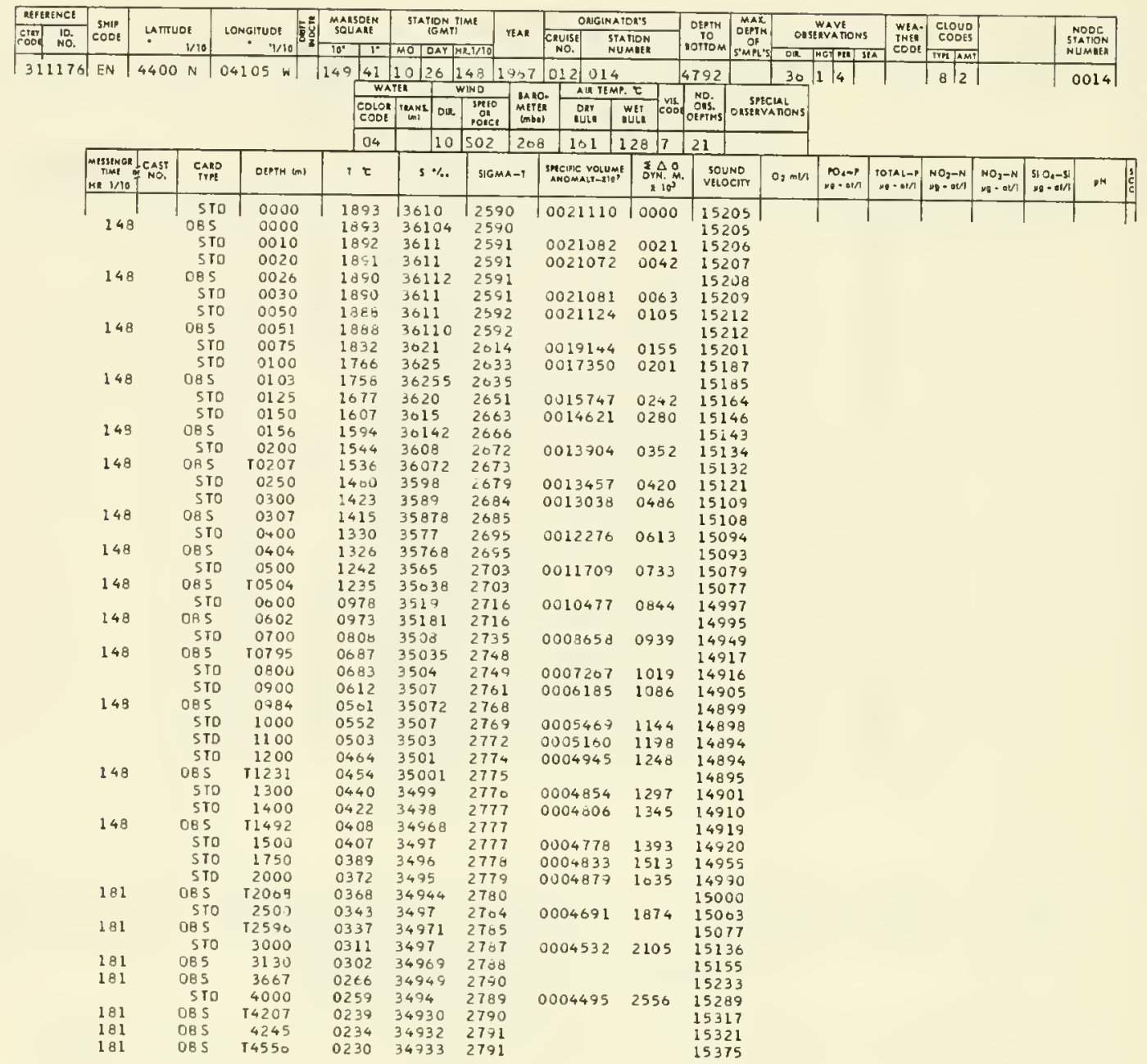


TABLE III_-Continued

\begin{tabular}{|c|c|c|c|c|c|c|c|c|c|c|c|c|c|c|c|c|c|c|}
\hline \multirow{2}{*}{ 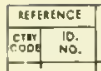 } & \multirow{2}{*}{$\begin{array}{c}S M B \\
C O D B\end{array}$} & \multirow{2}{*}{\multicolumn{2}{|c|}{$\begin{array}{c}0 \text { LITUDE } \\
-\quad 1 / 10 \\
\end{array}$}} & \multirow{2}{*}{ LONGIFUDR } & \multirow{2}{*}{ 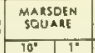 } & \multicolumn{2}{|c|}{$\begin{array}{c}\text { STAPIOM FIME } \\
\text { IGMTI }\end{array}$} & \multirow{2}{*}{ tEAR } & \multicolumn{2}{|c|}{ ORIGINATON'S } & \multirow{2}{*}{\begin{tabular}{|c|} 
DEPTH \\
$T 0$ \\
$10 T 1 O M$ \\
\end{tabular}} & \multirow{2}{*}{\begin{tabular}{|c|} 
MAX. \\
OEPTH \\
OF \\
SMAL'S
\end{tabular}} & \multirow{2}{*}{$\begin{array}{c}\text { WAVE } \\
\text { CHSERYATONS }\end{array}$} & \multirow{2}{*}{$\begin{array}{l}\text { WEA: } \\
\text { TMER } \\
\text { CODE }\end{array}$} & \multirow{2}{*}{ 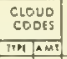 } & & \multirow{2}{*}{$\begin{array}{l}\text { NDDC } \\
\text { STARIDN } \\
\text { NUMAER } \\
\end{array}$} & \multirow{2}{*}{$\begin{array}{l}\text { NDDC } \\
\text { STATION } \\
\text { NUMAER } \\
\end{array}$} \\
\hline & & & & & & \begin{tabular}{l|l} 
MO & DAY \\
\end{tabular} & HE. & & $\begin{array}{c}\text { Cruist } \\
\text { No. }\end{array}$ & $\begin{array}{l}\text { STAAlion } \\
\text { Numatk }\end{array}$ & & & & & & & & \\
\hline \multirow[t]{36}{*}{311176} & \multirow[t]{4}{*}{ EN } & \multirow[t]{4}{*}{4400} & \multirow[t]{4}{*}{ N } & $04054 \mathrm{ml}$ & $14940 \mid$ & $10: 27$ & $\begin{array}{l}133 \\
\end{array}$ & 967 & $101: 015$ & & 4028 & 11 & \begin{tabular}{|l|l|}
2 & 3
\end{tabular} \mid & & $4|4|$ & & & 0015 \\
\hline & & & & & WA & ITER & WINo & & D. All TEMB. & & No. & & & & & & & \\
\hline & & & & & $\begin{array}{l}\text { COLOR } \\
\text { COOE }\end{array}$ & 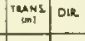 & $\begin{array}{c}\text { sete } \\
0 \\
\text { once } \\
\end{array}$ & $\begin{array}{c}\text { METER } \\
\text { Lmber }\end{array}$ & $\begin{array}{l}\text { DNR } \\
\text { DNUL } \\
\end{array}$ & $\begin{array}{l}\text { WE1 } \\
\text { out }\end{array}$ & DenTHS & OLSLAVATONS & & & & & & \\
\hline & & & & & 04 & 05 & $\$ 12$ & 259 & 133 & 1567 & 14 & & & & & & & \\
\hline & $\begin{array}{l}\text { MISSINGA } \\
\text { TIM] } \\
\text { MR } \quad \forall / 10 \\
\end{array}$ & I CAST & $\begin{array}{l}\text { CARO } \\
\text { IrOH }\end{array}$ & OETH (n) & $i c$ & $s \%$ & SIGMA- & & 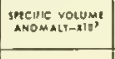 & $\begin{array}{r}E \times N_{0} D \\
\times 10^{3} \\
\end{array}$ & $\begin{array}{l}\text { Sou } \\
\text { YELO }\end{array}$ & $\begin{array}{l}\text { UND } \\
\text { SCIT }\end{array}$ & 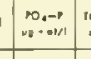 & $\begin{array}{c}\text { rorALto } \\
\text { mo-ain }\end{array}$ & 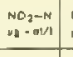 & 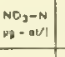 & $\begin{array}{l}510,-5 i \\
58 \div 01 / 1\end{array}$ & PH \\
\hline & & & STD & 0000 & $12 \div 3$ & 3614 & 25132 & & 0020883 & 10000 & 152 & 205 & & & & & & \\
\hline & 133 & & 085 & 0000 & $1 \div: 3$ & 36136 & 2572 & & & & & 205 & & & & & & \\
\hline & 133 & & 085 & $\begin{array}{l}0010 \\
0019\end{array}$ & $\begin{array}{l}1993 \\
1803\end{array}$ & $\begin{array}{l}3014 \\
30134\end{array}$ & $\begin{array}{l}2592 \\
2592\end{array}$ & & 0020919 & 0020 & & $\begin{array}{l}207 \\
206\end{array}$ & & & & & & \\
\hline & 200 & & STO & 0020 & & 3613 & 2552 & & $0020 \div 73$ & 0041 & 152 & 208 & & & & & & \\
\hline & & & STO & 0030 & 1354 & 3013 & $25 \div 2$ & & 0021035 & 0062 & 152 & 210 & & & & & & \\
\hline & 133 & & OBS & 0038 & 1854 & 36130 & 2592 & & & & 152 & 211 & & & & & & \\
\hline & & & STO & 3050 & 1834 & 3025 & 2016 & & 0013830 & 0102 & & 198 & & & & & & \\
\hline & & & STO & 0075 & 1737 & 3638 & 2630 & & 0015673 & 0145 & 151 & 175 & & & & & & \\
\hline & 133 & & oes 5 & 0075 & 1737 & 35378 & 2650 & & & & 151 & 175 & & & & & & \\
\hline & & & $\begin{array}{l}\text { STD } \\
\text { OBS }\end{array}$ & $\begin{array}{l}01013 \\
9110\end{array}$ & $\begin{array}{l}1084 \\
1657\end{array}$ & $\begin{array}{l}3529 \\
30252\end{array}$ & $\begin{array}{l}2656 \\
2057\end{array}$ & & 0015153 & 0134 & 151 & 153 & & & & & & \\
\hline & 133 & & $\begin{array}{l}\text { OBS } \\
\text { STO }\end{array}$ & $\begin{array}{l}0110 \\
0125\end{array}$ & 1647 & $\begin{array}{l}30252 \\
3624\end{array}$ & $\begin{array}{l}2057 \\
2660\end{array}$ & & & & 251 & 157 & & & & & & \\
\hline & & & $\begin{array}{l}\text { STO } \\
\text { STO }\end{array}$ & $\begin{array}{l}0125 \\
0150\end{array}$ & $16<2$ & $\begin{array}{l}3624 \\
3020\end{array}$ & $\begin{array}{l}2660 \\
2663\end{array}$ & & 0014847 & 0221 & 251 & 150 & & & & & & \\
\hline & 133 & & $\begin{array}{l}\text { STO } \\
\text { OBS }\end{array}$ & TO1 55 & 1616 & 36296 & $\begin{array}{l}2663 \\
2064\end{array}$ & & 0014643 & 0256 & & 151 & & & & & & \\
\hline & & & STO & 0200 & 2542 & 3007 & $\begin{array}{l}2064 \\
2673\end{array}$ & & & & 151 & 150 & & & & & & \\
\hline & 133 & & $O B S$ & 0232 & 1474 & 36018 & $\begin{array}{l}2673 \\
2679\end{array}$ & & 0013823 & 0329 & 151 & 133 & & & & & & \\
\hline & & & SID & 0250 & 1466 & 3576 & $\begin{array}{l}2679 \\
2681\end{array}$ & & & & 151 & 123 & & & & & & \\
\hline & & & STo & 0300 & 1402 & 3586 & $\begin{array}{l}2681 \\
2686\end{array}$ & & $\begin{array}{l}0013262 \\
0012839\end{array}$ & 0377 & 151 & 116 & & & & & & \\
\hline & 133 & & OBS & 0300 & 1396 & 35851 & $\begin{array}{l}2686 \\
2687\end{array}$ & & 0012839 & 0462 & 151 & 102 & & & & & & \\
\hline & 133 & & OBS & 10368 & 1340 & 35823 & $\begin{array}{l}2687 \\
2675\end{array}$ & & & & 151 & 101 & & & & & & \\
\hline & & & 510 & 0400 & 1342 & 3582 & $\begin{array}{l}2675 \\
2646\end{array}$ & & & & 150 & 095 & & & & & & \\
\hline & 133 & & OBS & 0442 & 1318 & 35784 & 2698 & & 0012203 & 0538 & 150 & 098 & & & & & & \\
\hline & & & S10 & 0500 & 1253 & 3569 & 2704 & & & & 150 & 097 & & & & & & \\
\hline & 133 & & OBS & 10582 & 2110 & 35497 & 2715 & & 0011629 & 0707 & 150 & 083 & & & & & & \\
\hline & & & STO & 0000 & 1061 & 3541 & 2718 & & & & 150 & 047 & & & & & & \\
\hline & & & STO & 0700 & $0 B 11$ & 3506 & 2733 & & 0010325 & 0817 & 150 & 030 & & & & & & \\
\hline & 133 & & OBS & 0718 & 0776 & 35027 & 2735 & & 0008850 & 0912 & 149 & 949 & & & & & & \\
\hline & & & STO & 0800 & 0670 & 3503 & 2750 & & & & & 939 & & & & & & \\
\hline & 133 & & OBS & 10870 & 0500 & 35034 & 2780 & & 0007128 & 0992 & & $\begin{array}{l}711 \\
895\end{array}$ & & & & & & \\
\hline & & & STD & 0500 & 0576 & 3504 & 27.3 & & $0 \cup 05897$ & 1057 & 148 & 890 & & & & & & \\
\hline & & & STD & 1000 & 0519 & 3504 & 2771 & & 0005207 & 1113 & 148 & $8 B 4$ & & & & & & \\
\hline & 133 & & OBS & $T 1038$ & 0508 & 35044 & 2772 & & & & 148 & 886 & & & & & & \\
\hline
\end{tabular}

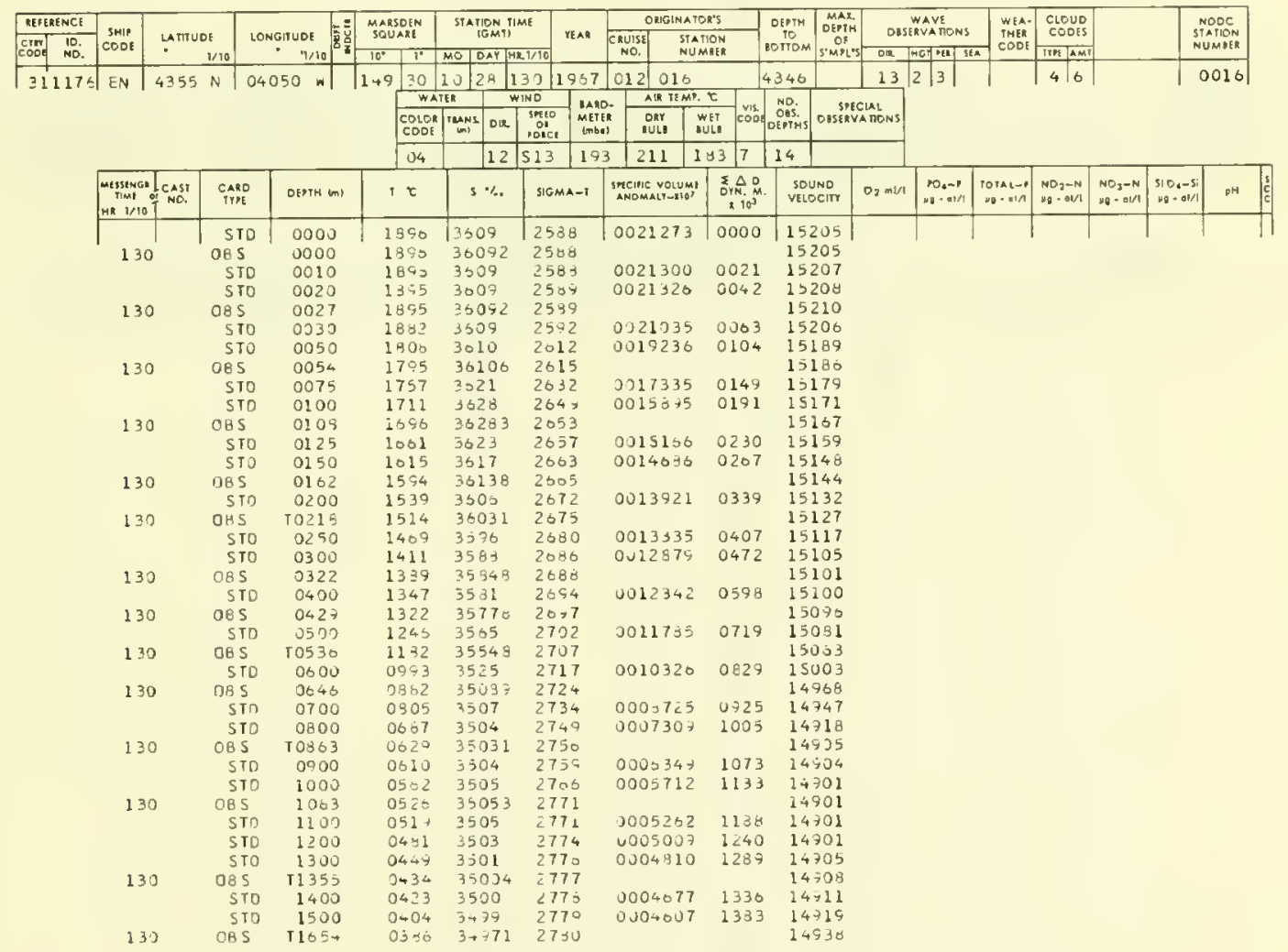


TABLE III.-Continued

\begin{tabular}{|c|c|c|c|c|c|c|c|c|c|c|c|c|c|c|c|c|c|}
\hline RIFERENCE & \multirow{2}{*}{$\begin{array}{c}\text { SNIP } \\
\text { COOE }\end{array}$} & \multirow{2}{*}{\multicolumn{2}{|c|}{$\begin{array}{c}\text { Latrudue } \\
\quad 1 / 10 \\
\end{array}$}} & \multirow{2}{*}{ 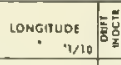 } & \multirow{2}{*}{$\begin{array}{l}\text { MARSOEN } \\
\text { SOUAEE }\end{array}$} & \multirow{2}{*}{\multicolumn{2}{|c|}{ STA nign riME }} & \multicolumn{2}{|c|}{\begin{tabular}{|l} 
ONGINATOR'S \\
\end{tabular}} & \multirow{2}{*}{$\begin{array}{c}\text { OEPTH } \\
\text { TO } \\
\text { LTIOM } \\
\end{array}$} & & \multirow{2}{*}{$\begin{array}{c}\text { WAVE } \\
\text { OBSERVA IONS }\end{array}$} & \multirow{2}{*}{$\begin{array}{c}\text { WEA } \\
\text { IHE: } \\
\text { COOE }\end{array}$} & \multirow{2}{*}{ 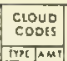 } & & & \multirow{2}{*}{$\begin{array}{l}\text { NODC } \\
\text { SATFíN } \\
\text { NUMAIE }\end{array}$} \\
\hline crer & & & & & & & rean & \begin{tabular}{|l|} 
CRUISE \\
No.
\end{tabular} & $\begin{array}{l}\text { STAFIOA } \\
\text { NUMBER }\end{array}$ & & 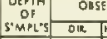 & & & & & & \\
\hline 311276 & EN & 4403 & N & $04 \mathrm{~L} 04 \quad \mathrm{~W}$ & $\begin{array}{lll}145 & 41 \\
\end{array}$ & \begin{tabular}{l|l|l|}
10 & 2 \\
\end{tabular} & $1341>07$ & \begin{tabular}{|l|l|}
512 & 017 \\
\end{tabular} & & 4771 & 16 & \begin{tabular}{|l|l|l}
3 & 3 &
\end{tabular} & & \begin{tabular}{|l|l|}
5 & 8 \\
\end{tabular} & & & 0017 \\
\hline & & & & & WAT & \begin{tabular}{l|l} 
ATER \\
\end{tabular} & \begin{tabular}{l|l} 
WINO & WRO \\
\end{tabular} & - AIR TEMV. & $\tau$ & & & & & & & & \\
\hline & & & & & $\begin{array}{l}\text { Colon } \\
\text { cook } \\
\end{array}$ & 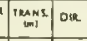 & 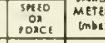 & \begin{tabular}{l|l} 
ORY & 4 \\
IUL & 4 \\
\end{tabular} & 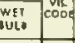 & be ons ons o & oBsizVAADONS & & & & & & \\
\hline & & & & & & 11 & 511 & 211 & \begin{tabular}{l|l}
174 & 7 \\
\end{tabular} & 14 & & & & & & & \\
\hline & 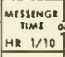 & $\begin{array}{l}\text { Cosst } \\
\text { ons. }\end{array}$ & $\begin{array}{l}\text { CAet } \\
\text { TYret }\end{array}$ & OEPTN $(\mathrm{m})$ & $T \tau$ & $5 \%$ & SIGMA-T & $\begin{array}{l}\text { IHCIFIC VOLUME } \\
\text { ANOMALYTII:" }\end{array}$ & 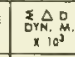 & $\begin{array}{l}\text { seun } \\
\text { veroo }\end{array}$ & $0_{2} \mathrm{~m} / 1$ & 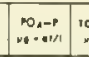 & 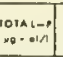 & $\left|\begin{array}{l}\mathrm{NO}_{2}-\mathrm{N} \\
\mathrm{M}=0 \mathrm{Ol} / 1 / 1\end{array}\right|$ & $\begin{array}{l}\mathrm{NO}_{3}-\mathrm{N} \\
\mathrm{NOWU1}\end{array}$ & $\begin{array}{l}510_{4}=-5 i \\
14=0 v 1 / 1\end{array}$ & 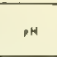 \\
\hline & & & STO & 0000 & 1945 & $35 \mathrm{~s} 2$ & $2>31$ & 002193 & 0000 & 151 & 108 & & & & & 1 & 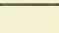 \\
\hline & 139 & & oEs & 0000 & 1845 & 35823 & 2531 & & & 151 & 88 & & & & & & \\
\hline & & & STI & 0010 & 1343 & 3583 & 2582 & $0021 \div 42$ & 0022 & 1510 & & & & & & & \\
\hline & & & STO & 0020 & 1841 & 3583 & 2502 & $0021=95$ & 0043 & 151 & & & & & & & \\
\hline & 139 & & 085 & 0025 & 1840 & 15834 & 2583 & & & 151 & 71 & & & & & & \\
\hline & & & STD & 0033 & 1339 & 3505 & 2594 & 0022753 & 0065 & 151 & 91 & & & & & & \\
\hline & & & STD & 0050 & 1037 & 3593 & 2571 & $002+211$ & 0108 & 151 & 95 & & & & & & \\
\hline & 130 & & DES & 0051 & 1837 & 35933 & 2591 & & & 151 & 95 & & & & & & \\
\hline & & & STD & 3075 & 1799 & 3513 & $2 t 13$ & 0013150 & 0157 & 151 & 90 & & & & & & \\
\hline & & & 510 & 0100 & 1749 & 3620 & $203=$ & 0017338 & 0204 & 151 & 80 & & & & & & \\
\hline & 137 & & DBS & 0102 & 1743 & 30200 & 2035 & & & 1517 & 79 & & & & & & \\
\hline & & & STD & 0125 & $166 \%$ & 3618 & 2651 & 0015790 & 0246 & 1510 & .61 & & & & & & \\
\hline & & & STO & 0150 & 1603 & $36 ! 4$ & 2663 & 0014624 & 0234 & 1514 & 45 & & & & & & \\
\hline & 137 & & DES & 0154 & 1594 & 36132 & 2605 & & & 1514 & & & & & & & \\
\hline & & & STO & 0200 & 1519 & 3500 & 2072 & $0 J 13432$ & 0355 & 1512 & & & & & & & \\
\hline & 139 & & 085 & 0205 & $: 511$ & 35991 & 2673 & & & 1512 & & & & & & & \\
\hline & & & STD & 0250 & 1451 & 3590 & 2679 & 0013407 & 0423 & 1512 & 11 & & & & & & \\
\hline & & & STO & $03 \cup 0$ & 1395 & 3582 & 2015 & 0012901 & 1489 & 1510 & & & & & & & \\
\hline & 139 & & 085 & 0307 & 1388 & 35513 & 2030 & & & 1500 & & & & & & & \\
\hline & & & DT & 0400 & 1324 & 3575 & 2074 & 0012310 & 0616 & 1500 & & & & & & & \\
\hline & 139 & & D85 & 0411 & 1307 & 35723 & 2676 & & & 1500 & & & & & & & \\
\hline & & & 510 & 0500 & 1070 & 3527 & 2700 & 0011254 & 0734 & 1501 & & & & & & & \\
\hline & 137 & & 085 & 10517 & 1042 & 35226 & 2707 & & & 1500 & & & & & & & \\
\hline & & & SIo & 0600 & 1003 & 3520 & 2712 & 0010820 & 0844 & 1500 & & & & & & & \\
\hline & 139 & & 085 & 0621 & 0985 & 35172 & 2715 & & & 1500 & & & & & & & \\
\hline & & & STO & 0700 & 0812 & 3510 & 2735 & 0008592 & 0,41 & 1435 & & & & & & & \\
\hline & & & STO & 0800 & 0649 & 3302 & 2753 & 0005886 & 1018 & 1490 & & & & & & & \\
\hline & 139 & & 085 & 10836 & 0006 & 35034 & 2757 & & & 1489 & 91 & & & & & & \\
\hline & & & STD & 0900 & 0576 & 3501 & 2701 & 0000120 & 1033 & 1489 & 90 & & & & & & \\
\hline & & & SIO & 1000 & 0534 & 3501 & 2766 & 0005637 & 1142 & 1489 & 90 & & & & & & \\
\hline & 139 & & DBS & 1045 & $051 \%$ & 35008 & 2708 & & & 1489 & & & & & & & \\
\hline & & & STO & 1100 & 0502 & 3501 & 2770 & 0005334 & 1197 & 1489 & 93 & & & & & & \\
\hline & & & SID & 1200 & 0475 & 3501 & 2773 & 0005031 & 1249 & 1489 & & & & & & & \\
\hline & & & SIO & 1300 & $0 \multimap 51$ & 3501 & 2770 & 0004868 & 1279 & 1450 & & & & & & & \\
\hline & 139 & & 085 & 1512 & 0443 & 35008 & 2776 & & & 1490 & & & & & & & \\
\hline & & & STO & 1400 & 0430 & 3500 & 2770 & 0004742 & 1347 & 1491 & & & & & & & \\
\hline & & & S10 & 1500 & 4411 & 3497 & 2779 & 0004687 & 1394 & 1472 & 22 & & & & & & \\
\hline & 139 & & 085 & $T 1581$ & 0345 & 34974 & 2779 & & & 1493 & & & & & & & \\
\hline
\end{tabular}

\begin{tabular}{|c|c|c|c|c|c|c|c|c|c|c|c|c|c|c|c|c|c|}
\hline REI ERENCE & \multirow{2}{*}{$\begin{array}{l}\text { SHAP } \\
\text { COOG }\end{array}$} & \multirow{2}{*}{\multicolumn{2}{|c|}{ LamUoe }} & \multirow{2}{*}{ IONGITUDE } & \multirow{2}{*}{$\begin{array}{l}\text { MA2SOEN } \\
\text { SOUART }\end{array}$} & \multicolumn{2}{|c|}{$\begin{array}{l}\text { SIATION FIME } \\
\text { IGMTI }\end{array}$} & \multicolumn{2}{|c|}{ OUGINATOR'S } & \multirow{2}{*}{$\begin{array}{c}\text { OEPTH } \\
\text { PO } \\
101 T 0 \mathrm{M}\end{array}$} & \multirow{2}{*}{$\begin{array}{l}\text { MAXK } \\
\text { OEPIN } \\
\text { OF } \\
\text { SMPL's }\end{array}$} & \multirow{2}{*}{ oustivanions } & \multirow{2}{*}{$\begin{array}{l}\text { WEA. } \\
\text { IMER } \\
\text { COOE }\end{array}$} & \multirow{2}{*}{ 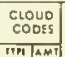 } & & & \multirow{2}{*}{$\begin{array}{l}\text { NOOC } \\
\text { STATION } \\
\text { NUMBER }\end{array}$} \\
\hline \begin{tabular}{l|l}
$\mathrm{CrN}$ & 10. \\
$\mathrm{CoO}$ & $\mathrm{NO}$. \\
\end{tabular} & & & & & & \begin{tabular}{|l|l|}
\multicolumn{1}{|c|}{$16 \mathrm{MT}$} \\
MO. & OAY \\
\end{tabular} & HR.1/10 & $\begin{array}{l}\text { ERUise } \\
\text { No. }\end{array}$ & $\begin{array}{l}\text { A TION } \\
\text { JMUE: }\end{array}$ & & & & & & & & \\
\hline 311176 & \multirow[t]{4}{*}{$S \mid E N$} & \multirow[t]{4}{*}{4359} & \multirow[t]{4}{*}{$N$} & \multirow[t]{4}{*}{$04104 \quad w 1$} & $14 \Rightarrow \equiv 1$ & \begin{tabular}{|l|l|}
10 & 30 \\
\end{tabular} & $125 \quad 1557$ & 0.2010 & & 4528 & 15 & $\begin{array}{lll}0 & 3 & \end{array}$ & 00 & 0 & & & 0018 \\
\hline & & & & & WA & Tiek & WWO & - $\quad$ AII IE $M A$ & $t 5$ & No. & & & & & & & \\
\hline & & & & & $\begin{array}{l}\text { Color } \\
\text { cook }\end{array}$ & $\lim _{\min }$ oth & 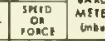 & \begin{tabular}{l|l} 
Ony \\
outh
\end{tabular} & 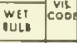 & oirsins & $\begin{array}{l}\text { OISEECALL } \\
\text { OISRYA RONS }\end{array}$ & & & & & & \\
\hline & & & & & & 13 & $5 1 0 \longdiv { 2 2 }$ & 225 & $174 \quad 7$ & 14 & & & & & & & \\
\hline & $\begin{array}{l}\text { Missince } \\
\text { that } \\
\text { HI } 1 / 10\end{array}$ & $\begin{array}{l}\text { cass } \\
\text { ons. } \\
\text { No. }\end{array}$ & $\underset{\text { TYRE }}{\text { CARO }}$ & DEPIH Im & it & $5 \% / 4$ & SIGMA-1 & $\begin{array}{l}\text { sherre voluml } \\
\text { Anomaty-1to }\end{array}$ & 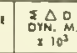 & $\begin{array}{l}\text { SOUN } \\
\text { VELOC }\end{array}$ & $\begin{array}{l}\text { uNo } \\
\text { DeIrY }\end{array}$ & 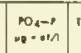 & 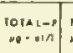 & 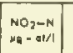 & $\begin{array}{l}\mathrm{Na}-\mathrm{OH} / 1 \\
\mathrm{NO}^{3}-\mathrm{N}\end{array}$ & $\left|\begin{array}{cc}510 & 0,5 n \\
n=1 & -1 / n\end{array}\right|$ & $\cdot N$ \\
\hline & & & STO & 000 & 1544 & $1 \times 563$ & 2585 & 0021572 & 10000 & $: 518$ & 188 & & & & & & \\
\hline & 129 & & aes & 0000 & 1340 & 35378 & 2535 & & & 151 & 136 & & & & & & \\
\hline & & & STJ & 0010 & 1344 & 3590 & 2537 & 0021426 & 0021 & $151=$ & 170 & & & & & & \\
\hline & & & $\begin{array}{l}\text { SIO } \\
\text { IPS }\end{array}$ & $\begin{array}{l}0020 \\
0250\end{array}$ & 1844 & 3552 & $258 \mathrm{~A}$ & $002: 334$ & 0042 & 1510 & 192 & & & & & & \\
\hline & 123 & & $\begin{array}{l}11 P 5 \\
\text { sid }\end{array}$ & 2232 & 1544 & $\begin{array}{l}35927 \\
35=3\end{array}$ & $\begin{array}{l}2509 \\
2508\end{array}$ & $00212=5$ & 0004 & 151 & $\begin{array}{l}193 \\
194\end{array}$ & & & & & & \\
\hline & 129 & & DKS & 0044 & $13+3$ & 15257 & 2540 & & & 51 & 176 & & & & & & \\
\hline & & & sto & 0050 & 2330 & 3575 & 2592 & $002: 004$ & 0106 & 251 & 195 & & & & & & \\
\hline & & & SIO & , & 1774 & $=607$ & $2+16$ & $001=748$ & 0150 & 2510 & & & & & & & \\
\hline & 127 & & DBS & NOG5 & $17 \angle 1$ & 30133 & 2035 & & & 1517 & 171 & & & & & & \\
\hline & & & SID & 0100 & 1704 & 3013 & 2033 & 0010785 & 0200 & 1510 & $\log 7$ & & & & & & \\
\hline & & & sto & $0: 23$ & $16 ? 1$ & 5013 & 2050 & 0313235 & 0240 & 131 & .49 & & & & & & \\
\hline & 129 & & 085 & $0: 43$ & $25 \div 0$ & 36130 & 2000 & & & 151 & 37 & & & & & & \\
\hline & & & STO & 0150 & Isdl & $0: 2$ & 2007 & 0014354 & 0277 & $15 i=$ & 38 & & & & & & \\
\hline & 127 & & $013 \leq$ & $10 ? 0_{2}$ & 1520 & $3604=$ & 2674 & & & $1>12$ & & & & & & & \\
\hline & & & STL & $0=00$ & $1>1<$ & 3602 & 2075 & 0023705 & $03+7$ & 2512 & & & & & & & \\
\hline & & & STO & $0 \geq 50$ & 1457 & $358 \mathrm{~A}$ & 2001 & 0013250 & 0414 & 1510 & 103 & & & & & & \\
\hline & 120 & & Ges & $02 \times 3$ & $13-0$ & $35 \div 13$ & 2084 & & & $150 \mathrm{c}$ & 997 & & & & & & \\
\hline & & & STD & $0 ? 00$ & $13 \leqslant 4$ & $35 ; 0$ & 2030 & 0012313 & 0480 & 1505 & 996 & & & & & & \\
\hline & 129 & & USS & 0377 & $1 \geq 2$ & 35730 & 26,3 & & & 1508 & & & & & & & \\
\hline & & & STO & $u \rightarrow 00$ & 1316 & $3 ; 72$ & 2534 & $001<31 \%$ & 0006 & 1508 & & & & & & & \\
\hline & 127 & & DBS & 10477 & 1223 & $355 \div 1$ & $270 ?$ & & & 1500 & & & & & & & \\
\hline & & & STI & 0รงว & 1150 & $3>05$ & $\angle 700$ & 0011355 & $07<4$ & 1504 & & & & & & & \\
\hline & 127 & & nas & $05=2$ & $0=5$ & $=520 \mathrm{~s}$ & 2710 & & & 1.97 & & & & & & & \\
\hline & & & $S T D$ & 0003 & $0 \leqslant 21$ & 4515 & $\angle 722$ & DJ Jo5\& & 0830 & 1477 & & & & & & & \\
\hline & & & STD & 0T00 & $07>1$ & 3501 & $273 x$ & 1000? 200 & $0>1$ & 1472 & & & & & & & \\
\hline & 127 & & DHS & T07 है & $a+73$ & 34,75 & 2745 & & & $i 4 \rightarrow 0$ & & & & & & & \\
\hline & & & STO & as Jis & $0_{2}:=$ & उ>30 & 2751 & טग07 J 1 & 0997 & $14 ; 0$ & & & & & & & \\
\hline & & & sro & $0=00$ & osuo & 3500 & 2702 & OUNDUS4 & 1003 & 1490 & & & & & & & \\
\hline & 123 & & OHS & $0=5 \%$ & 0573 & 35077 & 2767 & & & 1487 & & & & & & & \\
\hline & & & ST & 1000 & $05: 1$ & 3507 & 2755 & $302=449$ & 1120 & 1489 & & & & & & & \\
\hline & & & STD & 1100 & 0507 & 3504 & 2772 & 0005134 & 1173 & 1487 & & & & & & & \\
\hline & & & STO & 1200 & $0<70$ & 3502 & 2775 & $000 \div 904$ & 1224 & 1409 & & & & & & & \\
\hline & 129 & & DPS & $T \geq 20=$ & $0<09$ & 35062 & 2773 & & & 1409 & 77 & & & & & & \\
\hline & & & STO & 1300 & $0 *+1$ & 3500 & 2777 & 0004777 & $\lfloor 272$ & 1470 & & & & & & & \\
\hline & & & S I0 & 1400 & $04: 0$ & $34 \div a$ & 2777 & 0004756 & 1319 & 1490 & & & & & & & \\
\hline & 129 & & OSS & $T 14 \leq 3$ & 0410 & $34=72$ & 2777 & & & $1+x 1$ & 15 & & & & & & \\
\hline
\end{tabular}




\section{TABLE III.-Continued}

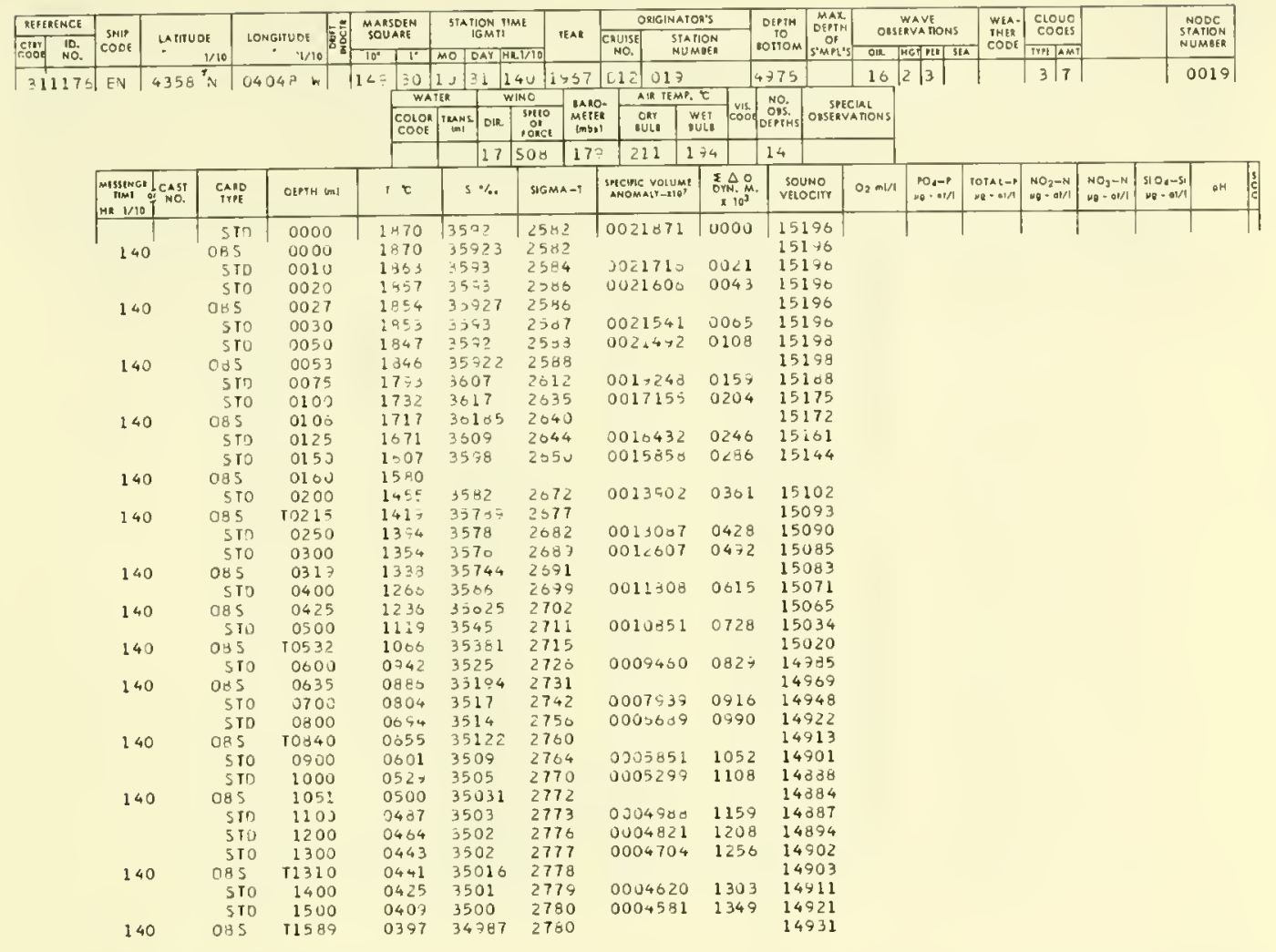

\begin{tabular}{|c|c|c|c|c|c|c|c|c|c|c|c|c|c|c|}
\hline AEffe:NCE & & & & MARSDEN & STATION TIME & & & AGGATAR'S & DEPTH & \begin{tabular}{|c|} 
MAR \\
OEPH
\end{tabular} & WAVE & & $\begin{array}{l}\text { clovo } \\
\text { cooss }\end{array}$ & \\
\hline $\begin{array}{lll}\text { crino } & 10 \\
\text { coog } & \text { No. } \\
\end{array}$ & coot & $\begin{array}{r}\text { Latruoor } \\
-\quad 1 / 10 \\
\end{array}$ & $\begin{array}{c}\text { LONGIUOA } \\
-1 / 10\end{array}$ & SOUAME & $\begin{array}{l}16 \mathrm{MP}+1 \\
\mathrm{MO}\end{array}$ & rean & $\begin{array}{c}\begin{array}{c}\text { Cruise: } \\
\text { No. }\end{array} \\
\end{array}$ & 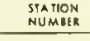 & , & 5 s.men & 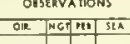 & 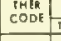 & 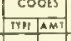 & $\begin{array}{l}\text { SRAtil } \\
\text { MUMA }\end{array}$ \\
\hline 31117 & EN & $4400 \mathrm{~N}$ & $04106 \mathrm{~W}$ & 149 41 & \begin{tabular}{|l|l|l|l|l|l|l|} 
& 01 \\
\end{tabular} & 1907 & 012 & 020 & 4755 & & $04|3| 3 \mid$ & 52 & $5 \longdiv { 5 }$ & 0020 \\
\hline
\end{tabular}

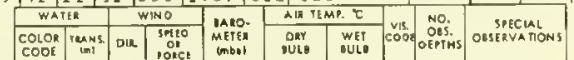

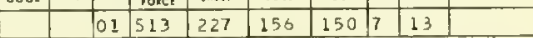

\begin{tabular}{|c|c|c|c|c|c|c|c|c|c|c|c|c|c|c|c|}
\hline 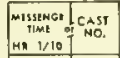 & 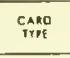 & DEPH (m) & ic & $5 \%$ & SIGMA-T & 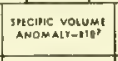 & 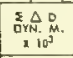 & $\begin{array}{l}\text { SOUNO } \\
\text { VELOCITY }\end{array}$ & $0_{3} \mathrm{~m} / 1$ & 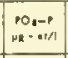 & $\left|\begin{array}{c}\text { rolat, } \\
\text { xacoin }\end{array}\right|$ & $\begin{array}{l}\mathrm{NO}_{2}-\mathrm{N} \\
\mathrm{NO}^{8}-\mathrm{OH} / 1\end{array}$ & 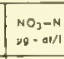 & 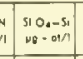 & $\mathrm{PM}$ \\
\hline \multirow{3}{*}{006} & 510 & 0000 & 2834 & |3566 & 2571 & 10022923 & 0000 & 15133 & & & & & & & \\
\hline & 085 & 0000 & 1834 & 35559 & 2571 & & & 15133 & & & & & & & \\
\hline & $\sin$ & an 10 & 1334 & 2506 & 2571 & 0022030 & 0022 & 15194 & & & & & & & \\
\hline \multirow{2}{*}{006} & 510 & 0020 & 1335 & $3>67$ & 2571 & 0022940 & 0045 & 15130 & & & & & & & \\
\hline & OBS & 0021 & 1335 & 35059 & 2571 & & & 25180 & & & & & & & \\
\hline \multirow[t]{3}{*}{006} & $\begin{array}{l}510 \\
045\end{array}$ & $\begin{array}{l}0030 \\
0044\end{array}$ & $\begin{array}{l}1838 \\
1839\end{array}$ & $3568=$ & 2571 & 0022990 & 3008 & 15189 & & & & & & & \\
\hline & $\begin{array}{c}585 \\
510\end{array}$ & 0050 & $\begin{array}{l}1839 \\
1835\end{array}$ & 35085 & $\begin{array}{l}2372 \\
2534\end{array}$ & 0021301 & 0113 & $\begin{array}{l}15291 \\
15193\end{array}$ & & & & & & & \\
\hline & 510 & 0075 & $\begin{array}{l}1835 \\
1215\end{array}$ & $\begin{array}{l}3593 \\
3028\end{array}$ & $\begin{array}{l}25344 \\
2623\end{array}$ & 0013219 & 0103 & $\begin{array}{l}15193 \\
15197\end{array}$ & & & & & & & \\
\hline \multirow[t]{3}{*}{006} & 085 & 0072 & 18530 & $3044 \equiv$ & 26250 & & & & & & & & & & \\
\hline & STO & 0100 & $: 798$ & 3645 & 2643 & $001044 \mathrm{~A}$ & 0207 & 15193 & & & & & & & \\
\hline & $5 T 0$ & 0125 & 1752 & $30+4$ & 2531 & $001>710$ & 0247 & 15189 & & & & & & & \\
\hline \multirow[t]{2}{*}{906} & 085 & 0131 & 1742 & 30444 & 2654 & & & 15187 & & & & & & & \\
\hline & STO & 0150 & 1704 & 3037 & $20>7$ & 0015230 & 0200 & 15178 & & & & & & & \\
\hline \multirow[t]{3}{*}{006} & $n \times 5$ & 10174 & 1057 & 35274 & 2001 & & & 15107 & & & & & & & \\
\hline & STO & $0 ? 00$ & 1506 & 3618 & 2665 & 3014510 & 0300 & 15154 & & & & & & & \\
\hline & $5 T D$ & $02>0$ & $151 \mathrm{C}$ & 3002 & 2675 & 0013021 & 0431 & 15331 & & & & & & & \\
\hline \multirow[t]{2}{*}{006} & OBS & 0260 & i495 & $359+2$ & 2076 & & & 15127 & & & & & & & \\
\hline & sTh & 0302 & $14 \overline{67}$ & 3537 & 2332 & 0013274 & 0475 & 15110 & & & & & & & \\
\hline \multirow[t]{2}{*}{006} & oos & 0346 & 1369 & 35774 & 2637 & & & $150 \div 8$ & & & & & & & \\
\hline & $5 \mathrm{TV}$ & 0400 & 1347 & 3577 & 2091 & 0012074 & 0628 & 15075 & & & & & & & \\
\hline \multirow[t]{2}{*}{006} & OBS & 10431 & $131 \mathrm{~s}$ & 35758 & $2=47$ & & & 15094 & & & & & & & \\
\hline & $5 \mathrm{TO}$ & 0500 & $11 d^{4}$ & 3550 & 2702 & $001170=$ & 0751 & 15059 & & & & & & & \\
\hline \multirow[t]{4}{*}{000} & 085 & 0518 & 1157 & 35445 & 2703 & & & 15050 & & & & & & & \\
\hline & STO & on ou & 0930 & 3531 & 2724 & 3009743 & 0858 & $1503 t$ & & & & & & & \\
\hline & STO & 0700 & 0812 & 3517 & 2741 & $000503=$ & $0 \times 47$ & 14951 & & & & & & & \\
\hline & STO & 0900 & 0673 & $350 \mathrm{~B}$ & 2754 & 0006830 & 1021 & 14913 & & & & & & & \\
\hline \multirow[t]{3}{*}{006} & OES & 0265 & 0002 & 35031 & 2760 & & & 14375 & & & & & & & \\
\hline & STo & 0500 & 0578 & 3532 & 2762 & 0005013 & 1056 & 14371 & & & & & & & \\
\hline & $5 \mathrm{Tn}$ & 1000 & 0519 & 3501 & 2769 & 0005473 & 1143 & 14383 & & & & & & & \\
\hline \multirow[t]{4}{*}{006} & & T1 099 & 0474 & 24901 & 2772 & & & 14581 & & & & & & & \\
\hline & $\sin$ & 1100 & 0674 & 3493 & 2772 & 3005095 & 1196 & 24831 & & & & & & & \\
\hline & SID & 1200 & 0441 & 2498 & 2775 & 0004345 & 1246 & 14334 & & & & & & & \\
\hline & $5 \mathbf{T} 0$ & 1300 & 0422 & 3457 & 2770 & 0004754 & 1294 & 14893 & & & & & & & \\
\hline 005 & $O B S$ & 11357 & 0417 & 3475 ? & 2770 & & & 14970 & & & & & & & \\
\hline
\end{tabular}


TABLE III.-Continued

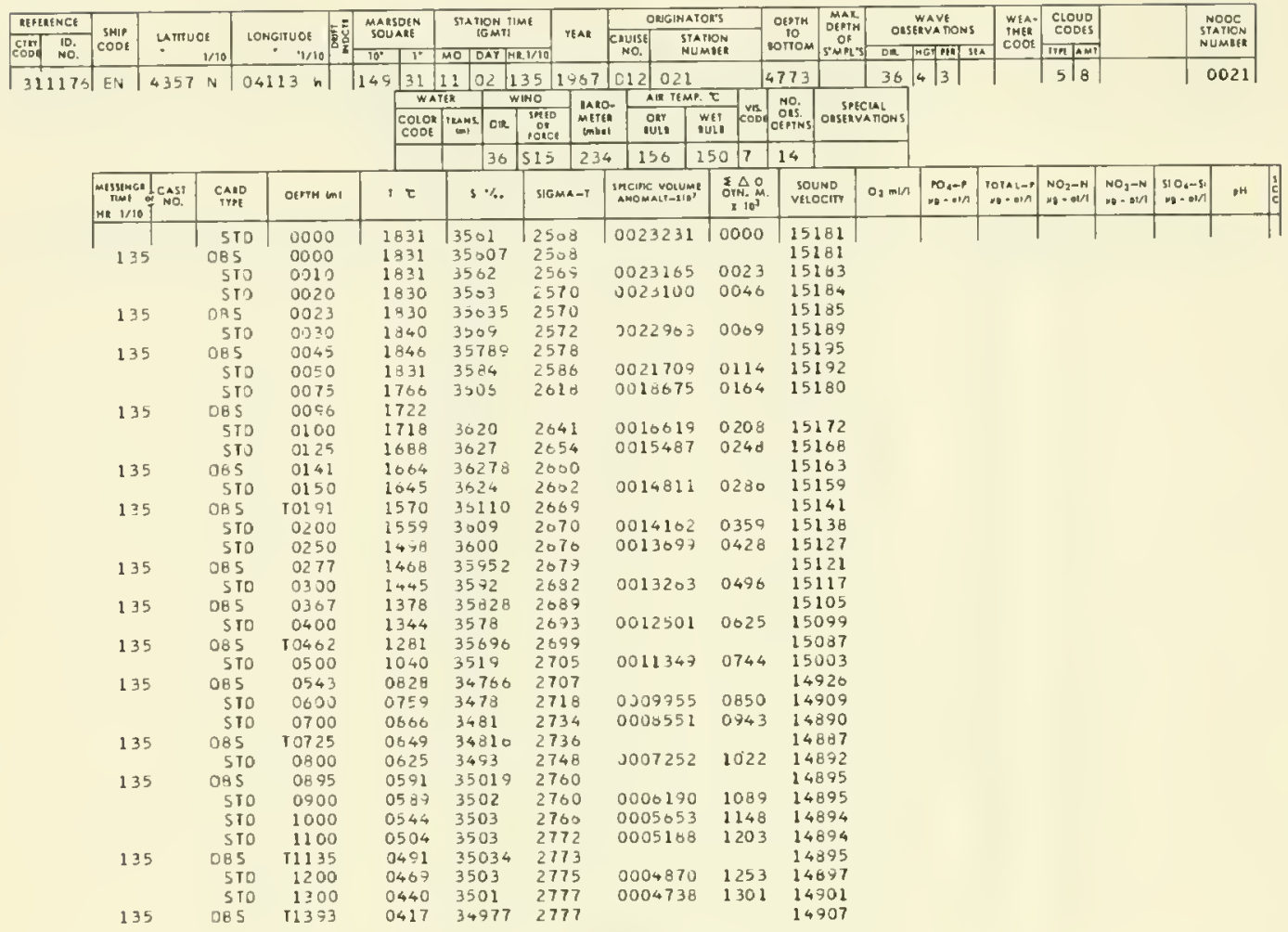

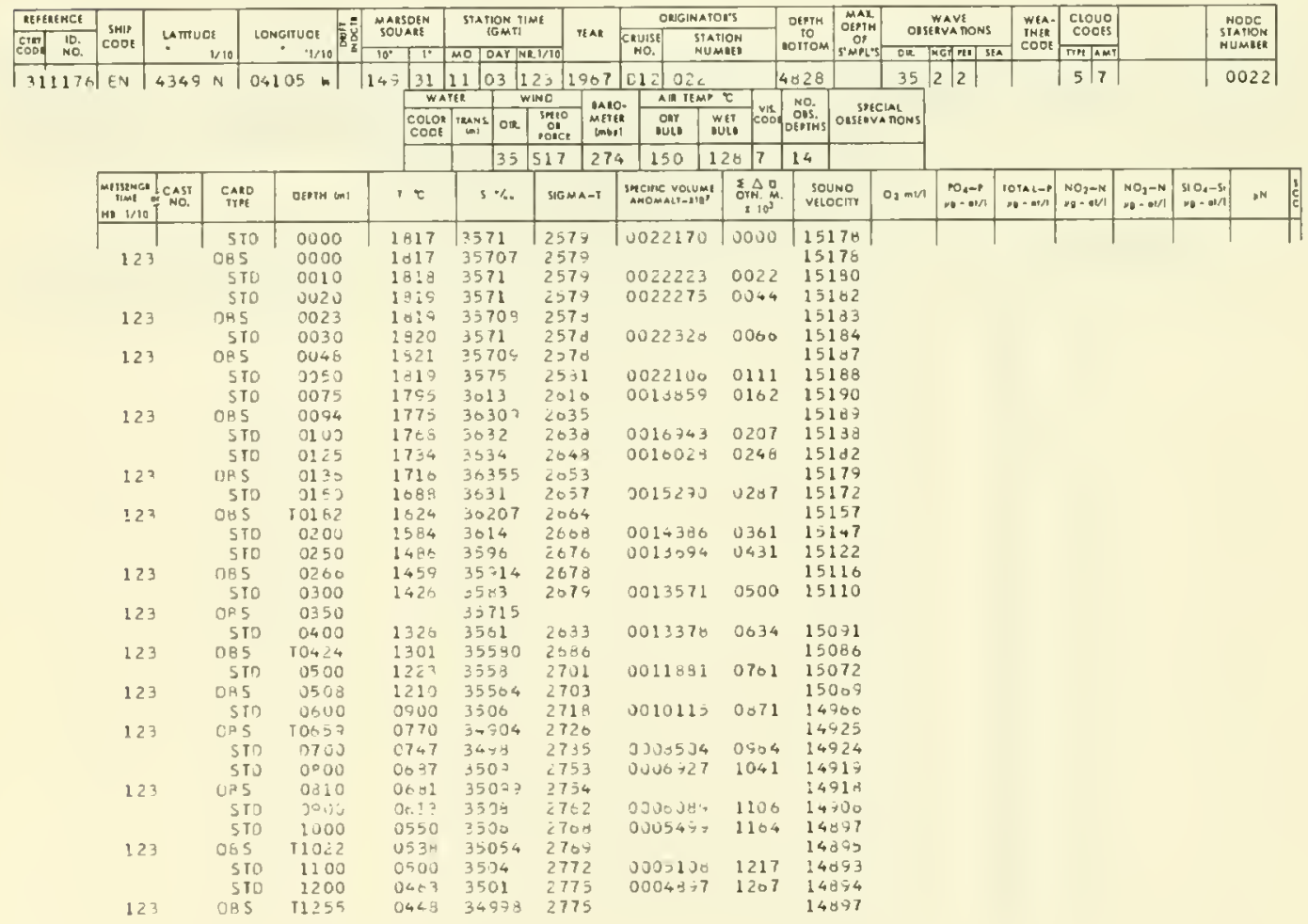


TABLE IV.-Observed and interpolated oceanographic data for stations taken by USCGC OWASCO at Ocean Station DELTA, 30 November-15 December 1967, prepared from NODC listing No. 31-1184 OW.

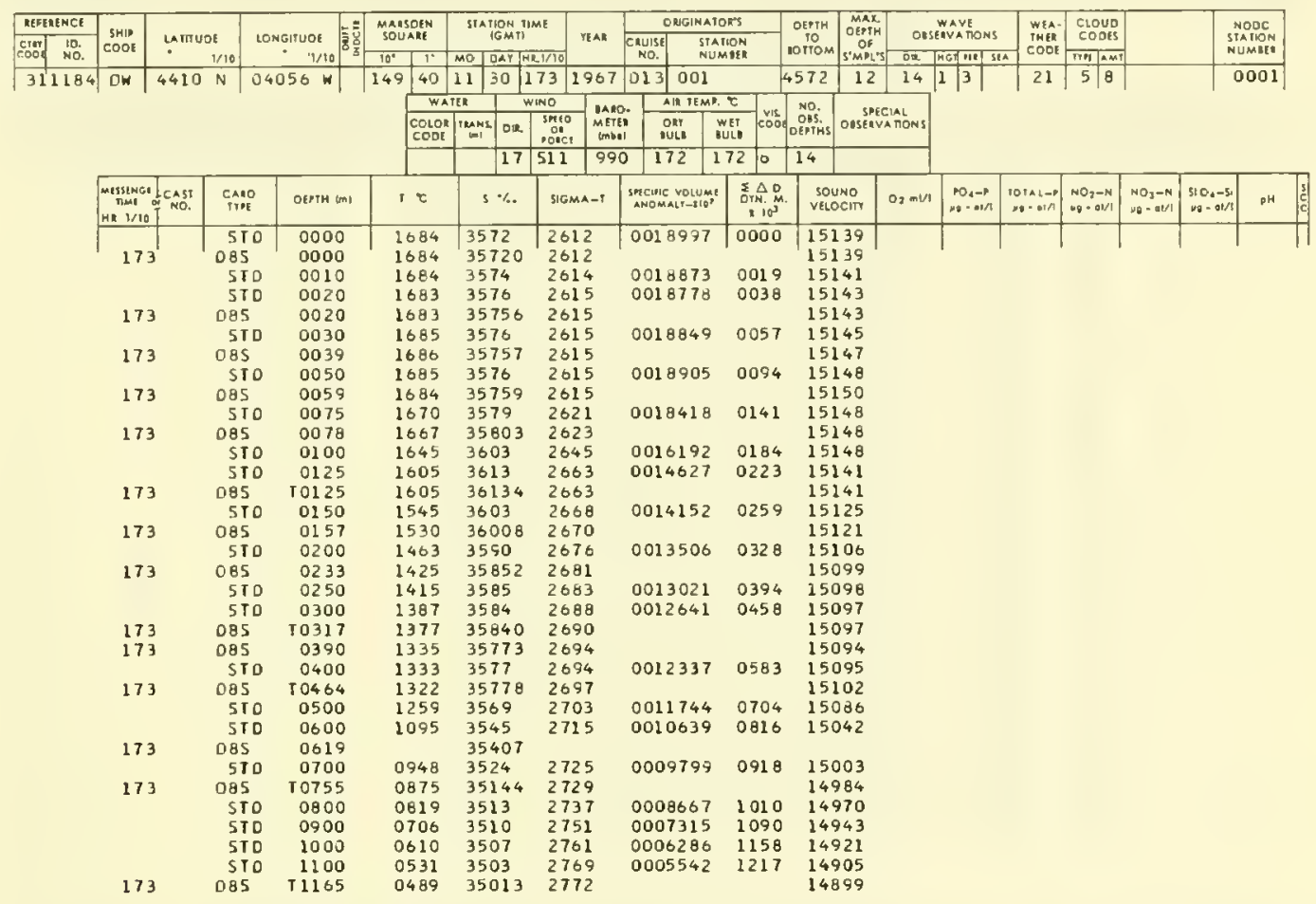

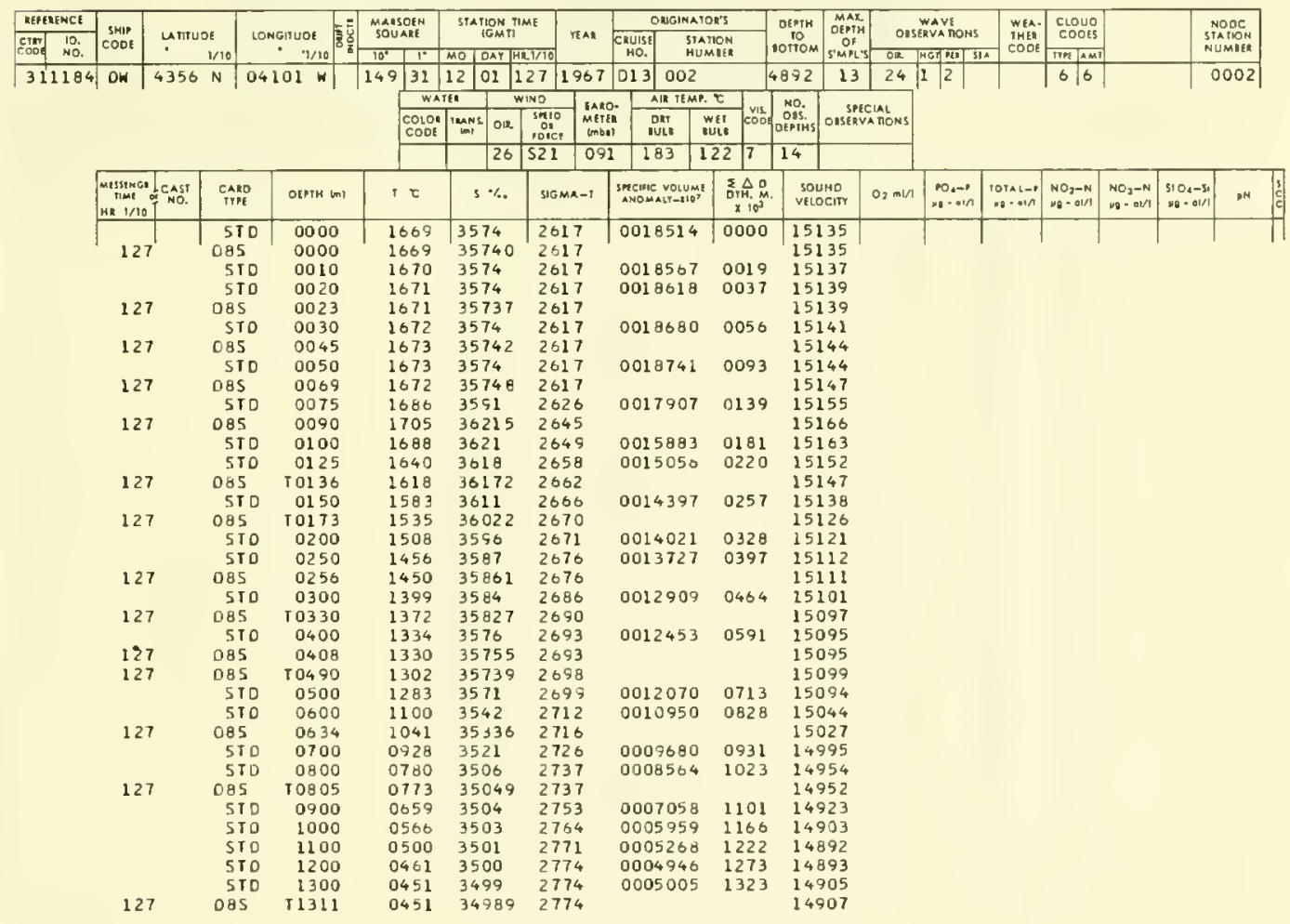


TABLE IV.—Continued

\begin{tabular}{|c|c|c|c|c|c|c|c|c|c|c|c|c|c|c|c|c|c|c|c|}
\hline \multirow{2}{*}{ 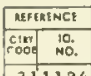 } & \multirow{2}{*}{$\begin{array}{l}\text { sulp } \\
\text { coot }\end{array}$} & \multirow{2}{*}{\multicolumn{2}{|c|}{ untruos }} & \multirow{2}{*}{ LONGIIUat } & \multirow{2}{*}{$\begin{array}{c}\text { MAlsokN } \\
\text { SOUAGE }\end{array}$} & \multirow{2}{*}{\multicolumn{2}{|c|}{\begin{tabular}{|c|} 
STAYION \\
$16 \mathrm{MriM}$ \\
\end{tabular}}} & \multirow[b]{2}{*}{ TEAR } & \multicolumn{2}{|c|}{ ONGINA POA's } & \multirow{2}{*}{\begin{tabular}{|l|} 
oefph \\
10 \\
contom
\end{tabular}} & \multirow{2}{*}{ 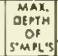 } & & \multicolumn{2}{|c|}{ WEA- Croug } & & \multirow{2}{*}{\multicolumn{2}{|c|}{ 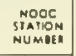 }} \\
\hline & & & & & & & & & $\begin{array}{c}\text { couss } \\
\text { no. }\end{array}$ & $\begin{array}{l}\text { STAMON } \\
\text { NUMUEA }\end{array}$ & & & \multicolumn{2}{|c|}{ 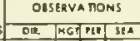 } & \multicolumn{2}{|c|}{ 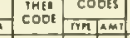 } & & & \\
\hline & OW & 4403 & $\mathrm{~N}$ & $04105 \mathrm{~W}$ & 14961 & \begin{tabular}{|l|l|l|}
12 & 03 \\
\end{tabular} & \begin{tabular}{|l|l}
123 & 1 \\
\end{tabular} & 967 & \begin{tabular}{l|l|l|l|l|l}
013 & 003 \\
\end{tabular} & & $\begin{array}{l}\text { 10riom } \\
4572\end{array}$ & 14 & 154 & \begin{tabular}{|l|l|}
4 & 2 \\
\end{tabular} & & 616 & & & 0003 \\
\hline & & & & & $w$ & 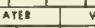 & Wimo & IA & o. & $\tau$ & No. & & & & & & & & \\
\hline & & & & & 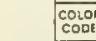 & 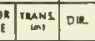 & 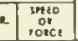 & Metreg & \begin{tabular}{l|l} 
ogr & W \\
auti & ut
\end{tabular} & $\begin{array}{l}\text { WEri } \\
\text { ouit }\end{array}$ & ouss & arserve & Nanows & & & & & & \\
\hline & & & & & & \begin{tabular}{l|l}
14 \\
\end{tabular} & 4510 & 130 & 177 & \begin{tabular}{l|l}
172 & 7
\end{tabular} & 14 & & & & & & & & \\
\hline & 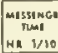 & tcast & CAnO & OERTH $(m)$ & $i c$ & $3 \%$ & झEMA & an-1 & 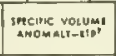 & 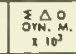 & & $\begin{array}{l}\text { uno } \\
\text { oeiry }\end{array}$ & $0, \mathrm{~min}$ & $\left|\begin{array}{ll}0 .,-1 \\
\cdots, \cdots, n\end{array}\right|$ & 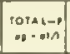 & $\mid$\begin{tabular}{|c|}
$\mathrm{NO}_{2}-\mathrm{N}$ \\
$\mathrm{NO}_{2}$
\end{tabular} & 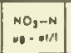 & 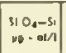 & on \\
\hline & & & 510 & 0000 & 1703 & 3578 & 2612 & & 0018991 & 0000 & 151 & 146 & & & & & & & \\
\hline & 123 & & 085 & 0000 & 1703 & 35780 & 2612 & & & & 151 & 146 & & & & & & & \\
\hline & & & SID & 0010 & 1701 & 3578 & 2612 & & 0019007 & 0019 & 151 & 147 & & & & & & & \\
\hline & & & STO & 0020 & 1696 & 3577 & 261 & & 0018956 & 0038 & 151 & 147 & & & & & & & \\
\hline & 12 & & 085 & 0023 & 1695 & 35771 & 2616 & & & & & 247 & & & & & & & \\
\hline & & & sto & 0030 & 1690 & 3577 & 2615 & & 0018869 & 0057 & $15 !$ & 147 & & & & & & & \\
\hline & 12 & & 085 & 0040 & 1679 & 35758 & 2616 & & & & 151 & 146 & & & & & & & \\
\hline & & & 510 & 0050 & 1676 & 3576 & 201 & & 0018689 & 0094 & 151 & 146 & & & & & & & \\
\hline & 12 & & 085 & 0068 & 1669 & 35763 & 262 & & & & 252 & 247 & & & & & & & \\
\hline & & & 570 & 0075 & 1669 & 3577 & 261 & & 0018555 & 0141 & 151 & 148 & & & & & & & \\
\hline & 12 & & 085 & 0091 & 1069 & 35780 & 262 & & & & & 151 & & & & & & & \\
\hline & & & sio & 0100 & 1668 & 3593 & $263 i$ & & 0017431 & 0186 & & 153 & & & & & & & \\
\hline & & & STO & 0125 & 1665 & 3621 & 265 & & 0015406 & 0227 & & 160 & & & & & & & \\
\hline & 123 & & 085 & 10237 & 1663 & 36262 & 265 & & & & & 162 & & & & & & & \\
\hline & & & 570 & 0150 & 1628 & 3619 & 2661 & & 0014811 & 0265 & 151 & 153 & & & & & & & \\
\hline & 123 & & 085 & 0181 & 1554 & 36055 & 266 & & & & 151 & 133 & & & & & & & \\
\hline & & & STO & 0200 & 2514 & 3599 & 267 & & 0013931 & 0337 & 151 & 123 & & & & & & & \\
\hline & & & STD & 0250 & 1430 & 3586 & 2680 & & 0013258 & 0405 & 151 & 103 & & & & & & & \\
\hline & 123 & & OBS & 0271 & 1405 & 35826 & 2683 & & & & & 098 & & & & & & & \\
\hline & 121 & & STO & 0300 & 1390 & 3582 & 2680 & & 0012870 & 0470 & & 098 & & & & & & & \\
\hline & 123 & & 085 & $T 0363$ & 1360 & 35806 & 2691 & & & & & 098 & & & & & & & \\
\hline & & & STO & 0400 & 1352 & 3580 & 2692 & & 0012525 & 0597 & 151 & 102 & & & & & & & \\
\hline & 127 & & OBS & 0451 & 1327 & 35770 & 2096 & & & & 151 & 101 & & & & & & & \\
\hline & & & STO & 0500 & 1288 & 3572 & 269 & & 0012097 & 0720 & 150 & 096 & & & & & & & \\
\hline & 123 & & 085 & 10537 & 1248 & 35657 & 2702 & & & & 150 & 088 & & & & & & & \\
\hline & & & STO & 0600 & 1128 & 3546 & 2710 & & 0011173 & 0836 & 150 & 054 & & & & & & & \\
\hline & & & 510 & 0700 & 0955 & 3522 & 2722 & & 0010065 & 0943 & 150 & 005 & & & & & & & \\
\hline & 123 & & 085 & 0721 & 0921 & 35181 & 272 & & & & 149 & 996 & & & & & & & \\
\hline & & & STO & 0800 & 0801 & 3512 & 273 & & 0008447 & 1035 & 149 & 963 & & & & & & & \\
\hline & & & STO & 0900 & 0673 & 3504 & 2751 & & 0007219 & 1113 & 149 & 929 & & & & & & & \\
\hline & 12. & & Dos & 0901 & 0672 & 35043 & 2751 & & & & 249 & 929 & & & & & & & \\
\hline & & & STO & 1000 & 0622 & 3503 & 275 & & 0000730 & 1183 & 149 & 925 & & & & & & & \\
\hline & & & STO & 1100 & 0572 & 3501 & 2762 & & 0006247 & 1248 & 149 & 921 & & & & & & & \\
\hline & & & STO & 1200 & 0520 & 3500 & 2767 & & 0005768 & 1308 & & 917 & & & & & & & \\
\hline & & & STO & 1300 & 0469 & 3498 & 2772 & & 0005293 & 1363 & & 912 & & & & & & & \\
\hline & 12 & & 085 & $T 1358$ & 0439 & 34974 & 2775 & & & & 149 & 910 & - & & & & & & \\
\hline
\end{tabular}

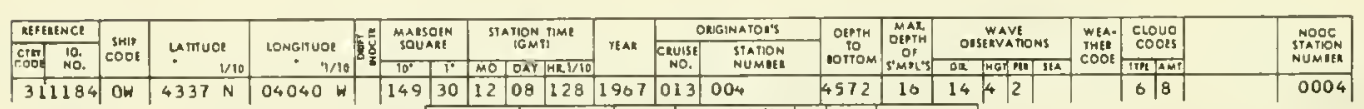

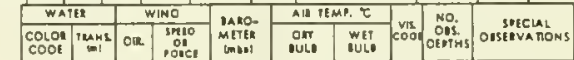

\begin{tabular}{|c|c|c|c|c|c|c|c|c|c|c|c|c|c|c|c|}
\hline & & & WAA & & & Al Rem? & $\tau$ vis & No: s & & & & & & & \\
\hline & & & \begin{tabular}{|l|l|} 
colo: \\
coot
\end{tabular} & 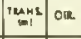 & 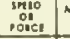 & \begin{tabular}{l|l} 
our \\
out
\end{tabular} & 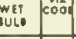 & $\begin{array}{l}\text { Oints } \\
\text { cirts }\end{array}$ & V. & & & & & & \\
\hline & & & & 14 & 518 & 180 & 170 b & 14 & & & & & & & \\
\hline 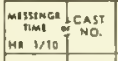 & $\underset{T T M B}{\text { CANO }}$ & OEtrH (m) & 12 & $8 \%$ & $S G \mathrm{Ma}-$ & $\begin{array}{l}\text { smcinc votumt } \\
\text { anomat? } \rightarrow \text { if }\end{array}$ & 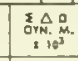 & $\begin{array}{c}1 \\
\text { souno } \\
\text { velociry }\end{array}$ & $0, \min$ & 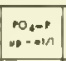 & rozatis & $\left|\begin{array}{c}\mathrm{NO}_{3}-\mathrm{N} \\
\mathrm{No}-\mathrm{WN}\end{array}\right|$ & 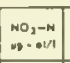 & 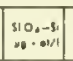 & M \\
\hline & STD & 0000 & 1645 & 3579 & 2627 & 0017600 & 0000 & 15129 & & & & & & & \\
\hline 128 & $\begin{array}{l}\text { OBS } \\
\text { SIO }\end{array}$ & $\begin{array}{l}0000 \\
0010\end{array}$ & $\begin{array}{l}1645 \\
1645\end{array}$ & $\begin{array}{l}35792 \\
3570\end{array}$ & 2627 & 0017623 & 0018 & 15129 & & & & & & & \\
\hline & $\begin{array}{l}510 \\
510\end{array}$ & 0020 & 1644 & $\begin{array}{l}3579 \\
3579\end{array}$ & $\begin{array}{l}2627 \\
2627\end{array}$ & 0017637 & 0035 & & & & & & & & \\
\hline 128 & 085 & 0023 & 1044 & 35793 & 2627 & & & 15132 & & & & & & & \\
\hline & $5 T 0$ & 0030 & 1644 & 3579 & 2027 & 0017607 & 0053 & 15133 & & & & & & & \\
\hline 128 & OBS & 0044 & 1644 & 35793 & 2627 & & & 15135 & & & & & & & \\
\hline & STO & 0050 & 1644 & 3579 & 2027 & 0017732 & 0088 & 15136 & & & & & & & \\
\hline 128 & Des & 0070 & 1644 & 35793 & 2627 & & & 15140 & & & & & & & \\
\hline & STD & 0075 & 1646 & 3582 & 2328 & 0017734 & 0133 & 15141 & & & & & & & \\
\hline 128 & OBS & 0093 & 1648 & 35868 & 2632 & & & 15146 & & & & & & & \\
\hline & STO & 0100 & 1644 & 3593 & 2638 & 0016897 & 0176 & 15146 & & & & & & & \\
\hline & STO & 0125 & 1020 & 3609 & 2656 & 0015279 & 0216 & 15145 & & & & & & & \\
\hline 128 & D8S & $T 0145$ & 1589 & 36127 & 2600 & & & 15139 & & & & & & & \\
\hline & 510 & 0150 & 1577 & 3611 & 2607 & 0014265 & 0253 & 15136 & & & & & & & \\
\hline 128 & DBS & 0177 & 1510 & 36017 & 2674 & & & 15120 & & & & & & & \\
\hline & STO & 0200 & 1479 & 3596 & 2678 & 0023405 & 0322 & 15112 & & & & & & & \\
\hline & STO & 0250 & 1415 & 3587 & 2684 & 0012875 & 0398 & 15098 & & & & & & & \\
\hline 128 & DAS & 0272 & 1393 & 35843 & 2087 & & & 15095 & & & & & & & \\
\hline & $5 T 0$ & 0300 & 1379 & 3583 & 2689 & 0012573 & 0452 & 15094 & & & & & & & \\
\hline 128 & OBS & T0370 & 1348 & 35799 & 2693 & & & 15095 & & & & & & & \\
\hline & STO & 0400 & 1344 & 3579 & 2693 & 0012428 & 0577 & 15099 & & & & & & & \\
\hline 128 & DAS & 0464 & 1319 & 35774 & 2697 & & & 15101 & & & & & & & \\
\hline & STO & 0500 & 1299 & 3573 & 2098 & 0012243 & 0700 & 15100 & & & & & & & \\
\hline 128 & 085 & 0562 & 1242 & 35642 & 2702 & & & 15087 & & & & & & & \\
\hline & STO & 0600 & 1159 & 3552 & 2709 & 0011315 & 0818 & 15066 & & & & & & & \\
\hline & STO & 0700 & 0764 & 3527 & 2724 & 0009854 & 0924 & 15009 & & & & & & & \\
\hline 128 & 085 & 0777 & 0835 & 35128 & 2734 & & & 14972 & & & & & & & \\
\hline & 510 & 0800 & 0803 & 3511 & 2738 & 0008559 & 1016 & 14964 & & & & & & & \\
\hline & STO & 0900 & 0676 & 3505 & 2751 & 0007219 & $\begin{array}{l}1095 \\
1095\end{array}$ & 14730 & & & & & & & \\
\hline 128 & 085 & 10998 & 0573 & 35007 & 2761 & & & 14905 & & & & & & & \\
\hline & $5 T 0$ & 1000 & 0572 & 3501 & 2701 & 0000188 & 1162 & 14905 & & & & & & & \\
\hline & STO & 1100 & 0542 & 3500 & 2765 & 0005950 & 1222 & 14909 & & & & & & & \\
\hline & STO & 1200 & 0512 & 3499 & 2768 & 0005710 & 1281 & 14914 & & & & & & & \\
\hline & STO & 1300 & 0481 & 3498 & 2770 & 0005465 & 1336 & 14918 & & & & & & & \\
\hline & STO & 1400 & 0451 & 3498 & 2773 & 0005208 & 1390 & 14922 & & & & & & & \\
\hline & 510 & 1500 & 0421 & 3497 & 2776 & 0004955 & 1441 & 14926 & & & & & & & \\
\hline 128 & 085 & 11501 & 0402 & 34963 & 2778 & & & 14928 & & & & & & & \\
\hline
\end{tabular}


TABLE IV.-Continued

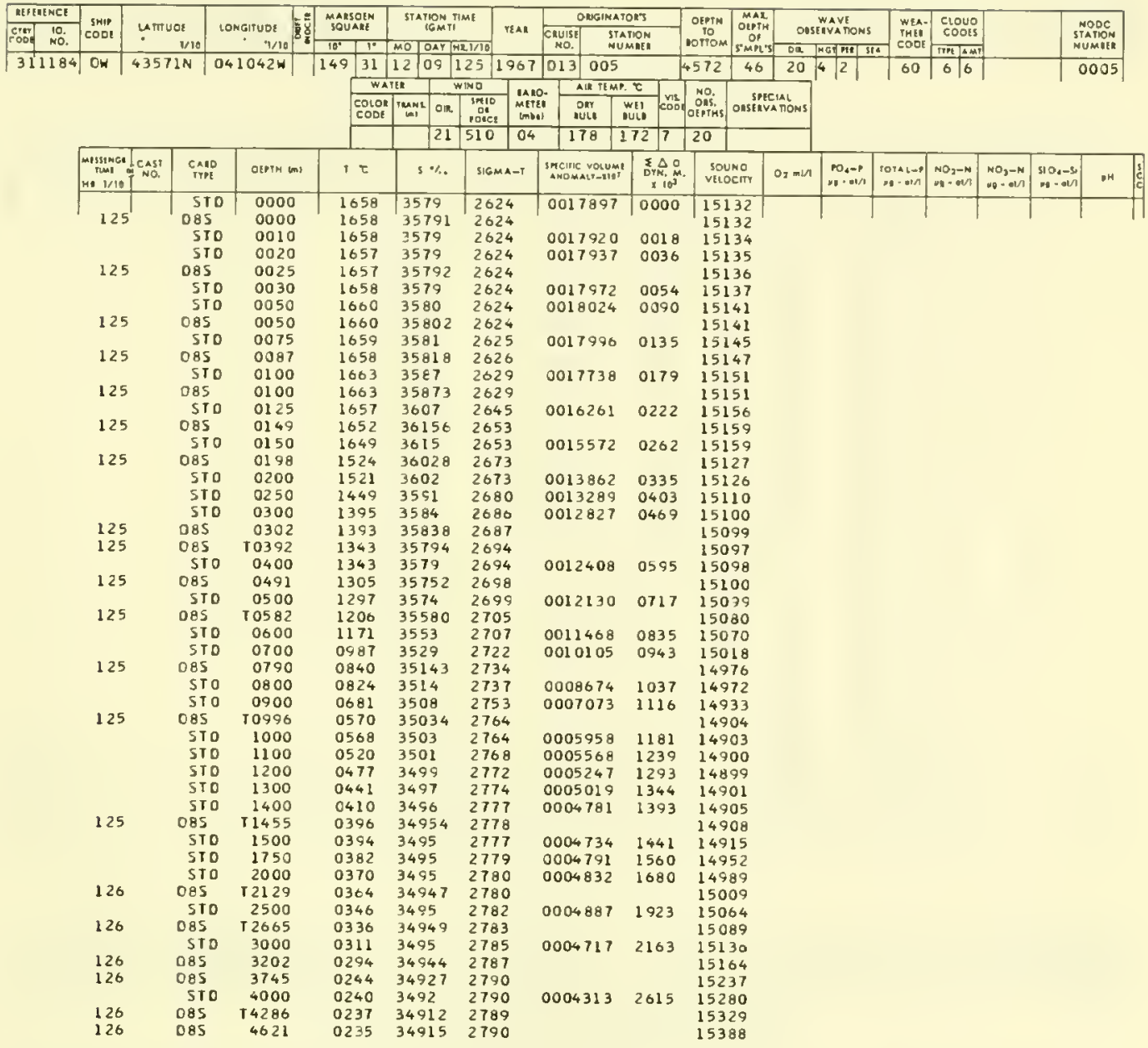


TABLE IV.—Continued

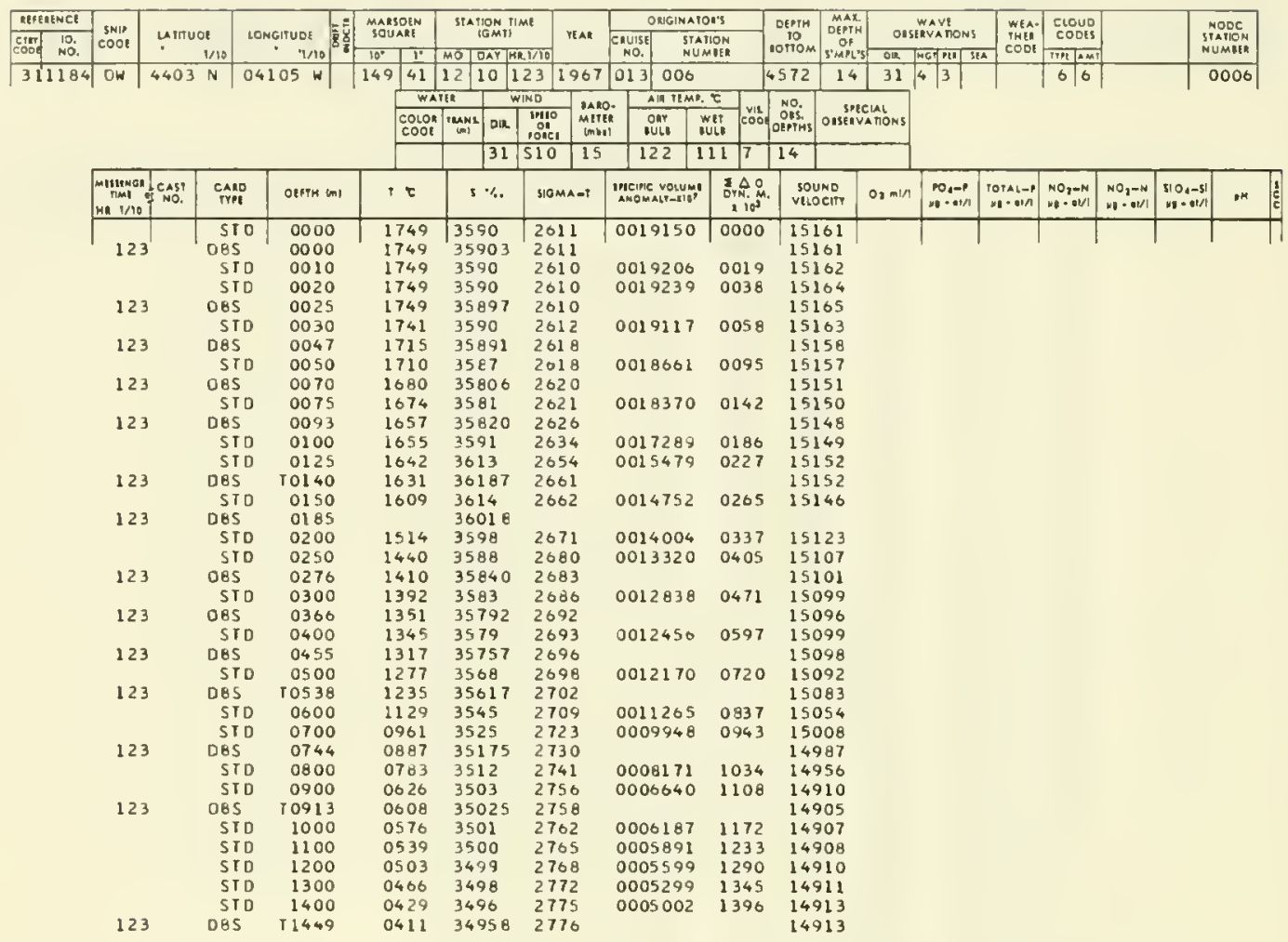

\begin{tabular}{|c|c|c|c|c|c|c|c|c|c|c|c|c|c|c|c|c|c|c|}
\hline \multirow{3}{*}{\begin{tabular}{|l|l|} 
AEFEEACE \\
CEI & 10. \\
COOE & No. \\
\end{tabular}} & \multirow{2}{*}{$\begin{array}{l}\text { SNIR } \\
\text { CODi }\end{array}$} & \multirow{2}{*}{\multicolumn{2}{|c|}{$\begin{array}{l}\text { umTUO: } \\
\quad 1 / 10 \\
\end{array}$}} & \multirow{2}{*}{$\begin{array}{c}\text { LONGITUDE } \\
1 / 10\end{array}$} & \multirow{2}{*}{$\begin{array}{l}\text { MARSDEN } \\
\text { SOUAME }\end{array}$} & \multirow{2}{*}{\multicolumn{2}{|c|}{$\begin{array}{l}\text { STATLON FiMt } \\
\text { (GMI) }\end{array}$}} & \multicolumn{2}{|c|}{ ORGINATOAS } & \multirow{2}{*}{$\begin{array}{c}\text { OLe? } \\
\text { To } \\
\text { soriom }\end{array}$} & \multirow{2}{*}{ 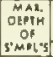 } & \multirow{2}{*}{\multicolumn{2}{|c|}{$\begin{array}{l}\text { WAVE } \\
\text { OHSEVATONS }\end{array}$}} & \multirow{3}{*}{$-\begin{array}{c}\text { WEA } \\
\text { INE } \\
\operatorname{coD} \\
63\end{array}$} & \multirow{2}{*}{ 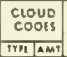 } & & & \multirow{2}{*}{$\begin{array}{l}\text { NOOC } \\
\text { SYAION } \\
\text { NUMUER }\end{array}$} \\
\hline & & & & & & & reAR & $\begin{array}{c}\text { CRuist } \\
\text { No: }\end{array}$ & $\begin{array}{l}\text { SNATION } \\
\text { NUMU:A }\end{array}$ & & & & & & & & & \\
\hline & ON & $43 \overline{55}$ & $N$ & $04053 \mathrm{~W}$ & 14930 & 1211 & $1 2 6 \longdiv { 1 9 6 7 }$ & \begin{tabular}{|l|l|}
013 & 007 \\
\end{tabular} & & 4572 & 10 & 30 & \multirow{2}{*}{\multicolumn{2}{|c|}{\begin{tabular}{|l|l|l|l|} 
Mat & 11: \\
3 & & & 63 \\
\end{tabular}}} & 58 & & & $\overline{0007}$ \\
\hline & & & & & WAI & & \begin{tabular}{l|l} 
WIND & IAAC \\
W
\end{tabular} & 0. AIR TEM" & & & & & & & & & & \\
\hline & & & & & $\begin{array}{l}\text { color } \\
\text { cook }\end{array}$ & remint oir & $\begin{array}{l}1710 \\
010 \\
1061\end{array}$ & 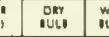 & Niti cood & ONS. & ousing & A MONS & & & & & & \\
\hline & & & & & & 30 & 518 & 111 & \begin{tabular}{|l|l|}
11 & 6 \\
\end{tabular} & 13 & & & & & & & & \\
\hline & $\begin{array}{l}\text { MBIMNG" } \\
\text { nMI } \\
\text { HR } 1 / 10\end{array}$ & $\begin{array}{l}\text { CAst } \\
\text { CNO? }\end{array}$ & $\underset{\text { GARD }}{\text { gAl }}$ & OENTH ImI & $T E$ & $1 \%$ & SIGMA-t & "me, Are volumy & ${ }_{10} \Delta 0^{3}$ & & & $0, \mathrm{~m} / \mathrm{n}$ & n...p & $\begin{array}{l}\text { rotabap } \\
\text { maten }\end{array}$ & 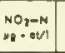 & $\begin{array}{l}N_{1} O_{2}-N \\
M=M / 1\end{array}$ & 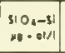 & on \\
\hline & 126 & & $\begin{array}{l}\text { STD } \\
\text { DBS }\end{array}$ & $\begin{array}{l}0000 \\
0000\end{array}$ & $\begin{array}{l}1696 \\
1656\end{array}$ & $\begin{array}{l}3583 \\
35833\end{array}$ & $\begin{array}{l}2618 \\
2018\end{array}$ & 0018446 & 0000 & $\begin{array}{l}151 \\
251\end{array}$ & & & & & & & & \\
\hline & & & STD & 0010 & 1698 & 3584 & 2618 & 0018488 & 0018 & 151 & 47 & & & & & & & \\
\hline & & & STO & 0020 & 1699 & 3584 & 2618 & 0018507 & 0037 & 151 & .49 & & & & & & & \\
\hline & 126 & & D85 & 0029 & 1700 & 35848 & 2618 & & & 151 & so & & & & & & & \\
\hline & & & 510 & 0030 & 1700 & 3585 & 2618 & 0018527 & 0055 & 151 & $\$ 1$ & & & & & & & \\
\hline & & & STD & 0050 & 1701 & 3585 & 2618 & 0018624 & 0093 & 151 & .54 & & & & & & & \\
\hline & 126 & & DBS & $00 \$ 7$ & 1701 & 35845 & 2618 & & & 151 & 55 & & & & & & & \\
\hline & & & \$ID & 0075 & 1702 & 3586 & 2618 & 0018661 & 0139 & 151 & .59 & & & & & & & \\
\hline & 126 & & DBS & 0086 & 2702 & 35061 & 2615 & & & 151 & 61 & & & & & & & \\
\hline & & & SID & 0100 & 1705 & 3014 & 2040 & 0016742 & $0: 83$ & 151 & .67 & & & & & & & \\
\hline & 126 & & 085 & 0113 & 1707 & 36315 & 2652 & & & 151 & 172 & & & & & & & \\
\hline & & & SID & 0125 & 1665 & $362 B$ & 2655 & 0015361 & 0224 & 151 & 167 & & & & & & & \\
\hline & & & STO & 0150 & 1643 & 3622 & 2000 & 0014929 & 0261 & 151 & so & & & & & & & \\
\hline & 126 & & DES & 10172 & 1608 & 36175 & 2605 & & & 151 & so & & & & & & & \\
\hline & & & SID & 0200 & 1576 & 3613 & 2669 & 0014256 & 0334 & 251 & .44 & & & & & & & \\
\hline & 126 & & DBS & 0227 & 1534 & 36064 & 2673 & & & 151 & 35 & & & & & & & \\
\hline & & & SID & 0250 & 1472 & 3597 & 2680 & 0013335 & 0403 & 151 & 18 & & & & & & & \\
\hline & 126 & & 085 & 0294 & 1380 & 35825 & 2088 & & & 150 & 994 & & & & & & & \\
\hline & & & SID & 0300 & 1379 & 3582 & 2688 & 0012624 & 0408 & 150 & 994 & & & & & & & \\
\hline & & & STO & 0400 & 1346 & 3579 & 2693 & 0012462 & 0594 & 150 & 999 & & & & & & & \\
\hline & 126 & & Des & 10436 & 1324 & 35780 & 2697 & & & 150 & 998 & & & & & & & \\
\hline & & & STO & 0500 & 1281 & 3570 & 2699 & 0012104 & 0717 & 150 & 93 & & & & & & & \\
\hline & 120 & & Des & 0533 & 1230 & 35624 & 2702 & & & 150 & 82 & & & & & & & \\
\hline & & & 510 & 0600 & 1077 & 3538 & 2713 & 0010823 & 0.831 & 150 & 35 & & & & & & & \\
\hline & 126 & & DBS & 0628 & 1016 & 35292 & 2717 & & & 150 & 17 & & & & & & & \\
\hline & & & SID & 0700 & 0869 & 3517 & 2732 & 0008996 & 0930 & 149 & 973 & & & & & & & \\
\hline & & & STD & 0800 & 0708 & 3505 & 2747 & 0007540 & 1013 & 149 & 226 & & & & & & & \\
\hline & 126 & & DBS & 0824 & 0677 & 35028 & 2749 & & & 149 & 18 & & & & & & & \\
\hline & & & SID & 0900 & 0599 & 3502 & 2759 & 0000301 & 1082 & 148 & 99 & & & & & & & \\
\hline & & & 510 & 1000 & 0541 & 3500 & 2765 & 0005800 & 1143 & 148 & 92 & & & & & & & \\
\hline & 126 & & DBS & 11017 & 0536 & 34997 & 2705 & & & 148 & 93 & & & & & & & \\
\hline
\end{tabular}




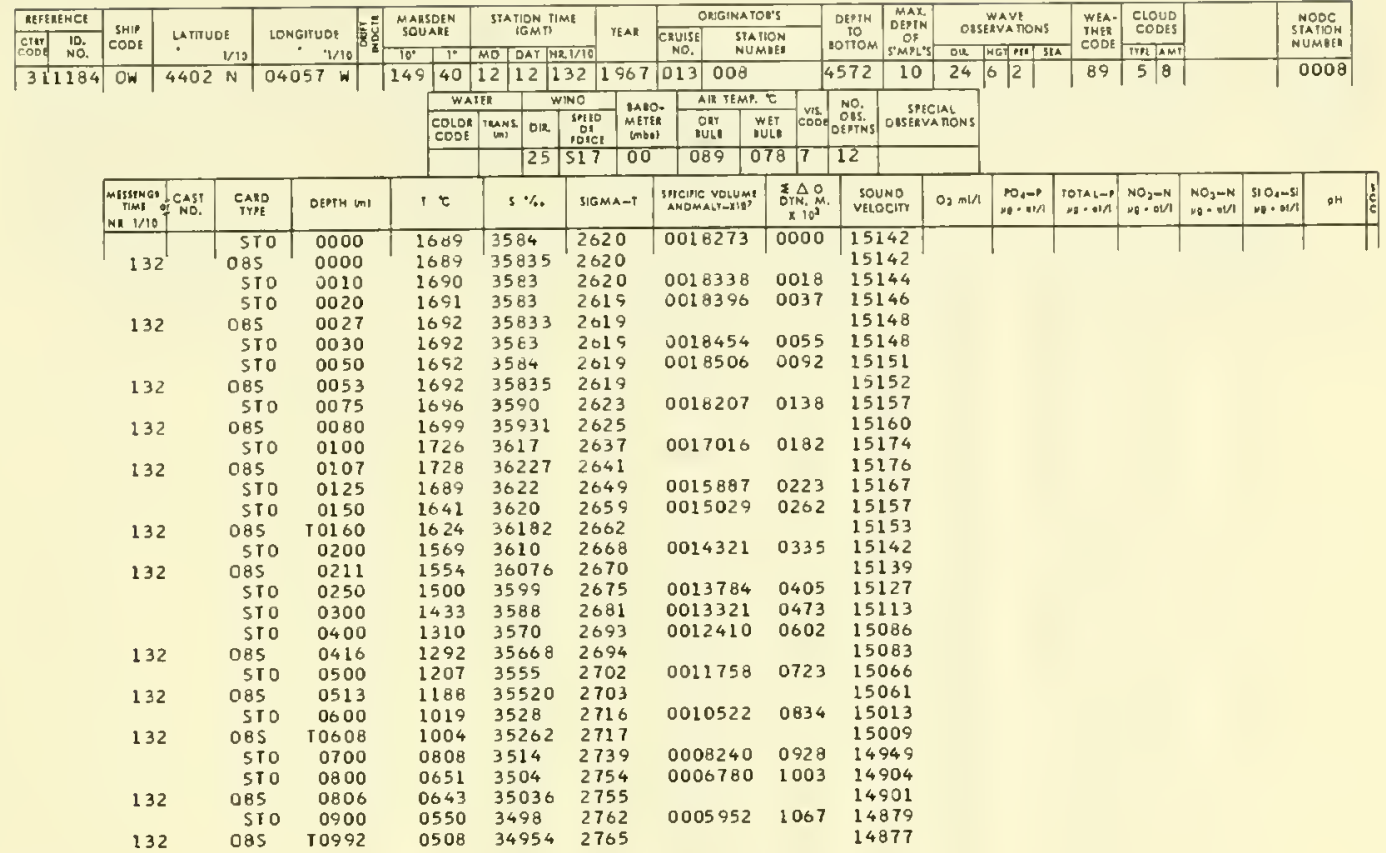

\begin{tabular}{|c|c|c|c|c|c|c|c|c|c|c|c|c|c|c|c|c|c|c|c|}
\hline \multicolumn{2}{|c|}{ MifEREWE: } & \multirow[b]{2}{*}{$\begin{array}{c}S H E \\
C O D E\end{array}$} & \multirow[b]{2}{*}{ UTITUd } & \multirow{2}{*}{\multicolumn{2}{|c|}{ LONGITUDR }} & \multirow{2}{*}{\multicolumn{2}{|c|}{$\begin{array}{l}\text { MAESDEN } \\
\text { SOUARE }\end{array}$}} & \multirow{2}{*}{\multicolumn{3}{|c|}{ 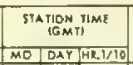 }} & \multirow[b]{2}{*}{ rak } & \multicolumn{2}{|c|}{ ORGINATOR"S } & \multirow{2}{*}{$\begin{array}{c}\text { DEPIM } \\
\text { POTOM }\end{array}$} & \multirow{2}{*}{\begin{tabular}{|l|}
$M A X$ \\
$D E P T M$ \\
$O F$ \\
$5 M P L S$
\end{tabular}} & \multirow{2}{*}{$\begin{array}{c}\text { WAVE } \\
\text { DISERVANONS }\end{array}$} & \multirow{2}{*}{$\begin{array}{l}\text { WEA- } \\
\text { THER } \\
\text { COOE }\end{array}$} & \multirow{2}{*}{ 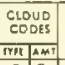 } & \multirow{2}{*}{$\begin{array}{l}\text { NoDC } \\
\text { SiAnOH } \\
\text { NUMBSt }\end{array}$} \\
\hline $\operatorname{crm}$ & $\begin{array}{l}\text { ID. } \\
\text { NO. }\end{array}$ & & & & & & & & & & & $\begin{array}{l}\text { equise: } \\
\text { No. }\end{array}$ & $\begin{array}{l}\text { STAFION } \\
\text { NUMEE }\end{array}$ & & & & & & \\
\hline $3 \hat{1}$ & 2184 & OW & $4403 \mathrm{~N}$ & $04058 \mathrm{w}$ & & 149 & 40 & 121 & \begin{tabular}{l|l}
14 & 1
\end{tabular} & 124 & 1907 & 013 & 009 & 4663 & 16 & \begin{tabular}{l|l|l|l}
03 & 1 & 3 & \\
\end{tabular} & & \begin{tabular}{|l|l|}
5 & 5 \\
\end{tabular} & 0009 \\
\hline
\end{tabular}

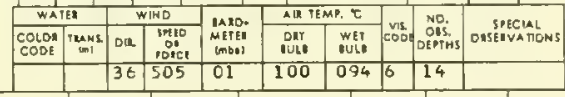

\begin{tabular}{|c|c|c|c|c|c|c|c|c|c|c|c|c|c|c|c|}
\hline 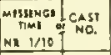 & CAND & D(PTN in) & $+\tau$ & $s \%$ & SIGMA-S & $\begin{array}{l}\text { SPGIFC VDLUM, } \\
\text { ANOMALT }\end{array}$ & 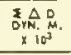 & $\begin{array}{l}\text { 3DUND } \\
\text { VELDCIN }\end{array}$ & $0.2 \mathrm{mLA}$ & 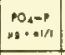 & 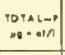 & 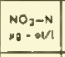 & $\begin{array}{l}\mathrm{HO}_{3}-\mathrm{H} \\
\mathrm{H}-\mathrm{N} / \mathrm{VI}\end{array}$ & $\begin{array}{l}510,05 i \\
w 1-s i n\end{array}$ & $\mathrm{OH}$ \\
\hline \multirow{4}{*}{124} & 510 & 0000 & 1670 & 3576 & 2618 & 0018420 & 0000 & 15136 & & & & & & & \\
\hline & 085 & 0000 & 1670 & 35756 & 2618 & & & 15136 & & & & & & & \\
\hline & STO & 0010 & 1670 & 3576 & 2619 & 0018429 & 0018 & 15137 & & & & & & & \\
\hline & STO & 0020 & 1669 & 3576 & 2619 & 0018446 & 0037 & 15139 & & & & & & & \\
\hline \multirow{3}{*}{124} & 085 & 0025 & 1669 & 35760 & 2619 & & & 15139 & & & & & & & \\
\hline & STO & 0030 & 1670 & 3576 & 2619 & 0018482 & 0055 & 15141 & & & & & & & \\
\hline & STO & 0050 & 1672 & 3576 & 2618 & 0018570 & 0092 & 15145 & & & & & & & \\
\hline \multirow[t]{2}{*}{124} & 085 & 0050 & 1672 & 35764 & 2618 & & & 15145 & & & & & & & \\
\hline & STO & 0075 & 2671 & 3576 & 2619 & 0018636 & 0139 & 15148 & & & & & & & \\
\hline \multirow[t]{2}{*}{124} & 085 & 0076 & 1671 & 35763 & 2619 & & & 15148 & & & & & & & \\
\hline & $5 T 0$ & 0100 & 1674 & 3577 & 2618 & 0018742 & 0186 & 15153 & & & & & & & \\
\hline \multirow[t]{3}{*}{124} & 085 & 0100 & 1674 & 35769 & 2618 & & & 15153 & & & & & & & \\
\hline & STO & 0125 & 1608 & 3605 & 2641 & 0016652 & 0230 & 15159 & & & & & & & \\
\hline & STO & 0150 & 1663 & 3619 & 2653 & 0015591 & 0270 & 15163 & & & & & & & \\
\hline \multirow[t]{2}{*}{124} & 085 & 10153 & 1662 & 36202 & 2654 & & & 15164 & & & & & & & \\
\hline & STO & 0200 & 1580 & 3620 & 2666 & 0014555 & 0346 & 15145 & & & & & & & \\
\hline \multirow[t]{2}{*}{124} & 085 & 0200 & 1580 & 36101 & 2666 & & & 15145 & & & & & & & \\
\hline & 510 & 0250 & 1486 & 3555 & 2075 & 0013777 & 0416 & 15122 & & & & & & & \\
\hline \multirow[t]{2}{*}{124} & 085 & 0298 & 1417 & 35844 & 2062 & & & 15107 & & & & & & & \\
\hline & STO & 0300 & 1416 & 3584 & 2682 & 0013259 & 0484 & 15107 & & & & & & & \\
\hline \multirow[t]{2}{*}{124} & 085 & 10391 & 1342 & 35763 & 2092 & & & 15096 & & & & & & & \\
\hline & SIO & 0400 & 1328 & 3574 & 2693 & 0012478 & 0613 & 15093 & & & & & & & \\
\hline \multirow[t]{2}{*}{124} & 085 & 0489 & 1183 & 35497 & 2703 & & & 15055 & & & & & & & \\
\hline & 510 & 0500 & 1162 & 3547 & 2704 & 0011491 & 0732 & 15050 & & & & & & & \\
\hline \multirow[t]{3}{*}{124} & 085 & 10583 & 1008 & 35278 & 2717 & & & 15006 & & & & & & & \\
\hline & 510 & 0600 & 0970 & 3524 & 2721 & 0009967 & 0840 & 14995 & & & & & & & \\
\hline & STO & 0700 & 0776 & 3508 & 2739 & 0008183 & 0931 & 14936 & & & & & & & \\
\hline \multirow[t]{3}{*}{124} & OBS & 0767 & 0675 & 35005 & 2748 & & & 14907 & & & & & & & \\
\hline & 510 & 0800 & 0041 & 3499 & 2751 & 0007005 & 1006 & 14899 & & & & & & & \\
\hline & 510 & 0900 & 0552 & 3496 & 2760 & 0006126 & 1072 & 14879 & & & & & & & \\
\hline \multirow[t]{6}{*}{124} & 085 & T0954 & 0512 & 34949 & 2764 & & & 24872 & & & & & & & \\
\hline & SIO & 1000 & 0502 & 3495 & 2765 & 0005662 & 1131 & 14876 & & & & & & & \\
\hline & STO & 1200 & 0481 & 3495 & 2768 & 0005497 & 1187 & 14884 & & & & & & & \\
\hline & STO & 1200 & 0460 & 3495 & 2770 & 0005326 & 1241 & 14892 & & & & & & & \\
\hline & STO & 1300 & 0439 & 3495 & 2772 & 0005151 & 1293 & 14700 & & & & & & & \\
\hline & STO & 1400 & 0418 & 3495 & 2775 & 0004970 & 1344 & 14908 & & & & & & & \\
\hline 124 & 085 & $T 1449$ & 0408 & 34949 & 2776 & & & 14912 & & & & & & & \\
\hline
\end{tabular}


Table IV.-Continued

\begin{tabular}{|c|c|c|c|c|c|c|c|c|c|c|c|c|c|c|c|c|c|c|}
\hline \multirow{2}{*}{ 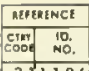 } & \multirow{2}{*}{$\begin{array}{c}\text { SNIP } \\
\text { COOE }\end{array}$} & \multirow{2}{*}{\multicolumn{2}{|c|}{ umuat }} & \multirow{2}{*}{ LONGITUOE } & $\begin{array}{l}\text { MARSDEN } \\
\text { SOUARE }\end{array}$ & \multicolumn{2}{|c|}{$\begin{array}{l}\text { STATION TIME } \\
\text { IGMII }\end{array}$} & \multicolumn{2}{|c|}{ OMGIMATOA'S } & \multirow{2}{*}{$\begin{array}{c}\text { OEPTH } \\
\text { To } \\
\text { ortiom }\end{array}$} & \multirow{2}{*}{$\begin{array}{c}\text { MAX } \\
\text { otrin } \\
\text { OF } \\
\text { SML'S }\end{array}$} & \multicolumn{2}{|c|}{$\begin{array}{l}\text { WAVI } \\
\text { OISERAATONS }\end{array}$} & \multirow{2}{*}{$\begin{array}{l}\text { WEA. } \\
\text { INER } \\
\text { CODE }\end{array}$} & \multirow{2}{*}{ 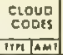 } & & & \multirow{2}{*}{$\begin{array}{l}\text { NOOC } \\
\text { STATKN } \\
\text { NUMIEE }\end{array}$} \\
\hline & & & & & $10^{\circ} \quad 15$ & \begin{tabular}{|l|l|} 
MOAY \\
\end{tabular} & teAs & \begin{tabular}{c|} 
CRUISE: \\
NO.
\end{tabular} & $\begin{array}{l}\text { STATION } \\
\text { NUMIEA }\end{array}$ & & & Dig & HG Pा IIL & & & & & \\
\hline & OH & 4358 & & 340104 & 14934 & 12.15 & \begin{tabular}{|l|l|}
133 & 1967 \\
\end{tabular} & \begin{tabular}{|l|l|}
013 & 010 \\
\end{tabular} & & 4692 & 17 & 20 & 32 & & \begin{tabular}{|l|l|}
6 & 5 \\
\end{tabular} & & & 0010 \\
\hline & & & & & WAT & \begin{tabular}{l|l} 
itek & \\
\end{tabular} & WINO & \begin{tabular}{l|l}
0. & $A R$ TEMP \\
\end{tabular} & & พо. & & & & & & & & \\
\hline & & & & & \begin{tabular}{|l|} 
color \\
coot
\end{tabular} & Trans do & $\mid \begin{array}{l}\sin 10 \\
\text { of } \\
\text { ofces }\end{array}$ & \begin{tabular}{c|c} 
DRY & w \\
xuL & ou \\
\end{tabular} & it coog & ois: & onseres. & AnONS: & & & & & & \\
\hline & & & & & & 23 & 523 & 122 & 977 & 14 & & & & & & & & \\
\hline & 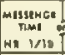 & $\begin{array}{l}\text { cast } \\
\text { cost }\end{array}$ & $\begin{array}{l}\text { CARD } \\
\text { IIPE }\end{array}$ & OEPTH (m) & $1 c$ & $5 \%$ & SIGMA-P & 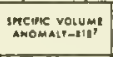 & 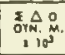 & $\begin{array}{l}\text { sour } \\
\text { velo }\end{array}$ & $\begin{array}{l}\text { UNa } \\
\text { ociry }\end{array}$ & Oy $\rightarrow L / 4$ & $\infty_{4,1}^{\infty}$ & 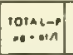 & $\mid \begin{array}{l}\mathrm{NO}_{2}-\mathrm{N} \\
\mathrm{H}-\mathrm{OVI}\end{array}$ & $\begin{array}{l}N_{2}-\mathrm{N} \\
\mathrm{n}=\mathrm{On}\end{array}$ & 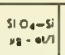 & PH \\
\hline & & & STO & 0000 & 1677 & 3580 & 2620 & 0018228 & 0000 & 151 & 138 & & & & & & & \\
\hline & 133 & & 085 & 0000 & 1677 & 35804 & 2620 & & & & 138 & & & & & & & \\
\hline & & & STO & 0010 & 1678 & 3580 & 2620 & 0018283 & 0018 & 151 & 140 & & & & & & & \\
\hline & & & $5 T 0$ & 0020 & 1678 & 3580 & 2020 & 0018323 & 0037 & & & & & & & & & \\
\hline & 133 & & 085 & 0029 & 1679 & 35803 & 2620 & & & 151 & 144 & & & & & & & \\
\hline & & & STO & 0030 & 1679 & 3580 & 2620 & 0018381 & 0055 & 151 & 144 & & & & & & & \\
\hline & & & STO & 0050 & 1681 & 3580 & 2620 & 0018471 & 0092 & 151 & 148 & & & & & & & \\
\hline & 133 & & 085 & 0057 & 1681 & 35604 & 2619 & & & 151 & 149 & & & & & & & \\
\hline & & & STO & 0075 & 1680 & 3581 & 2620 & 0018536 & 0138 & 151 & 152 & & & & & & & \\
\hline & 133 & & OBS & 0085 & 1680 & 35807 & 2620 & & & & 153 & & & & & & & \\
\hline & & & STO & 0100 & 2694 & 3590 & 2624 & 0018244 & 0184 & 151 & 161 & & & & & & & \\
\hline & 133 & & OBS & 0113 & 1700 & 35979 & 2628 & & & 151 & 166 & & & & & & & \\
\hline & & & STO & 0125 & 1700 & 3608 & 2636 & 0017156 & 0228 & 151 & 169 & & & & & & & \\
\hline & & & $5 T 0$ & 0150 & 1685 & 3621 & 2650 & 0015952 & 0270 & 151 & 170 & & & & & & & \\
\hline & 133 & & OBS & T 0171 & 1657 & 36236 & 2658 & & & 152 & 266 & & & & & & & \\
\hline & & & 570 & 0200 & 1579 & 3610 & 2666 & 0014540 & 0346 & 151 & 145 & & & & & & & \\
\hline & 133 & & OBS & 0223 & 1524 & 36009 & 2671 & & & 151 & 130 & & & & & & & \\
\hline & & & STO & 0250 & 1472 & 3592 & 2676 & 0013699 & 0426 & 151 & 117 & & & & & & & \\
\hline & & & $5 T 0$ & 0300 & 1389 & 3579 & 2684 & 0013068 & 0483 & 150 & 097 & & & & & & & \\
\hline & 133 & & 085 & 0332 & 1346 & 35731 & 2688 & & & 150 & 088 & & & & & & & \\
\hline & & & STO & 0400 & 1302 & 3570 & 2695 & 0012251 & 0010 & 150 & $O B_{4}$ & & & & & & & \\
\hline & 133 & & 085 & Т0 0443 & 1256 & 35637 & 2699 & & & 150 & 075 & & & & & & & \\
\hline & & & STO & 0500 & 1169 & 3550 & 2705 & 0011404 & $072 B$ & 150 & 052 & & & & & & & \\
\hline & 133 & & oes & 0547 & 1083 & 35376 & 2712 & & & 150 & 029 & & & & & & & \\
\hline & & & STO & 0600 & $096 \mathrm{~B}$ & 3524 & 2721 & 0009933 & 0835 & 149 & 994 & & & & & & & \\
\hline & 133 & & 085 & $T 0658$ & D853 & 35119 & 2731 & & & $2 \div 9$ & 959 & & & & & & & \\
\hline & & & STO & 0700 & 0781 & 3507 & 2738 & 0008334 & 0926 & 149 & 938 & & & & & & & \\
\hline & & & STO & $0 B 00$ & 0639 & 3499 & 2751 & 0006976 & 1003 & 148 & 898 & & & & & & & \\
\hline & 133 & & OBS & $O B B 2$ & 0554 & 34948 & 2759 & & & 148 & 877 & & & & & & & \\
\hline & & & STO & 0900 & 0545 & 3495 & 2760 & 0006105 & 2068 & 148 & 876 & & & & & & & \\
\hline & & & STO & 1000 & 0502 & 3496 & 2766 & 0005577 & 1127 & 148 & 876 & & & & & & & \\
\hline & 133 & & OBS & Т 1090 & 0468 & 34971 & 2771 & & & 148 & 877 & & & & & & & \\
\hline & & & STO & 2100 & 0465 & 3497 & 2771 & 0005121 & 1180 & 148 & 877 & & & & & & & \\
\hline & & & STo & 1200 & 0434 & 3497 & 2774 & 0004865 & 2230 & $14 \mathrm{~B}$ & 881 & & & & & & & \\
\hline & & & STO & 1300 & 0410 & 3496 & 2777 & 0004688 & 1278 & 248 & $88 \mathrm{~B}$ & & & & & & & \\
\hline & & & 570 & 1400 & 0393 & 3495 & $277 B$ & 0004603 & 1324 & 148 & 897 & & & & & & & \\
\hline & & & STO & 1500 & 0382 & 3495 & 2779 & 0004585 & 1370 & 149 & 910 & & & & & & & \\
\hline & 133 & & 085 & 11671 & 0379 & 34939 & 2778 & & & 149 & 937 & & & & & & & \\
\hline
\end{tabular}


TABLE V.-Observed and interpolated oceanographic data for stations taken by USCGC ABSECON at Ocean Station DELTA, 13 January-30 January 1968, prepared from NODC listing No. 31-1202 AZ.

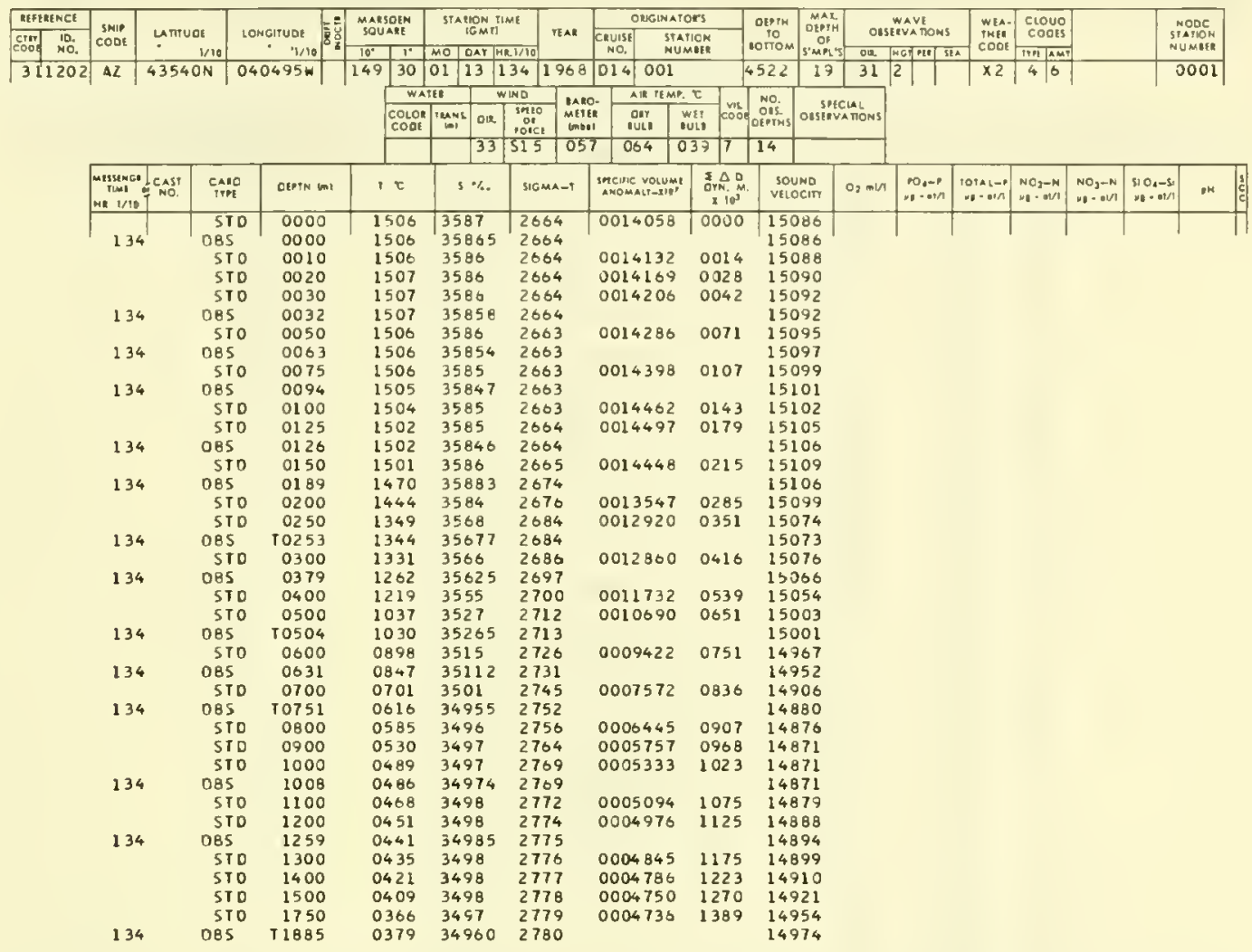

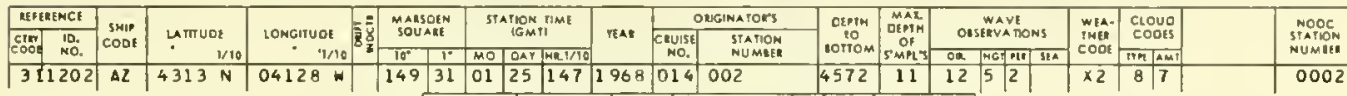

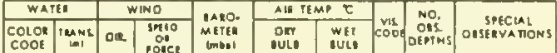
\begin{tabular}{|l|l|l|l|l|l|l|l}
\hline & 13 & 519 & 234 & 157 & 138 & 7 & 14 \\
\hline
\end{tabular}

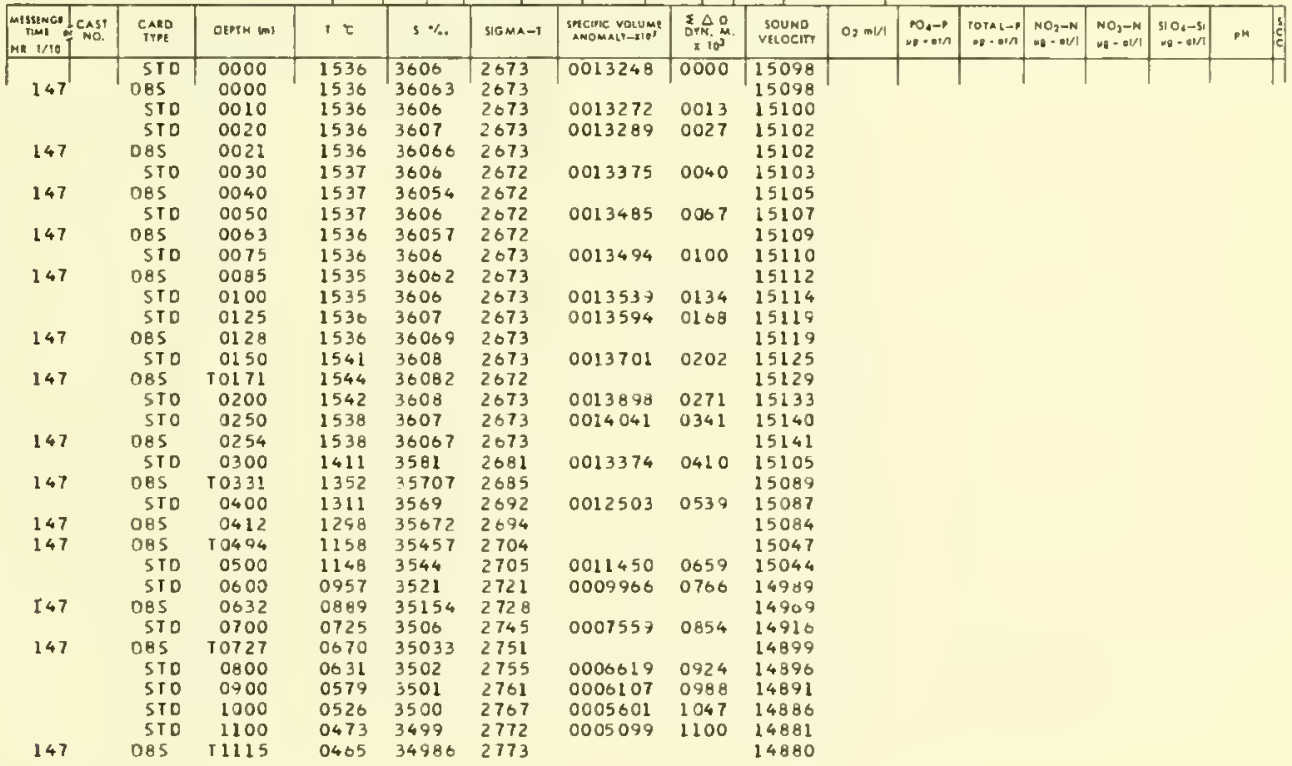


TABle V.-Continued

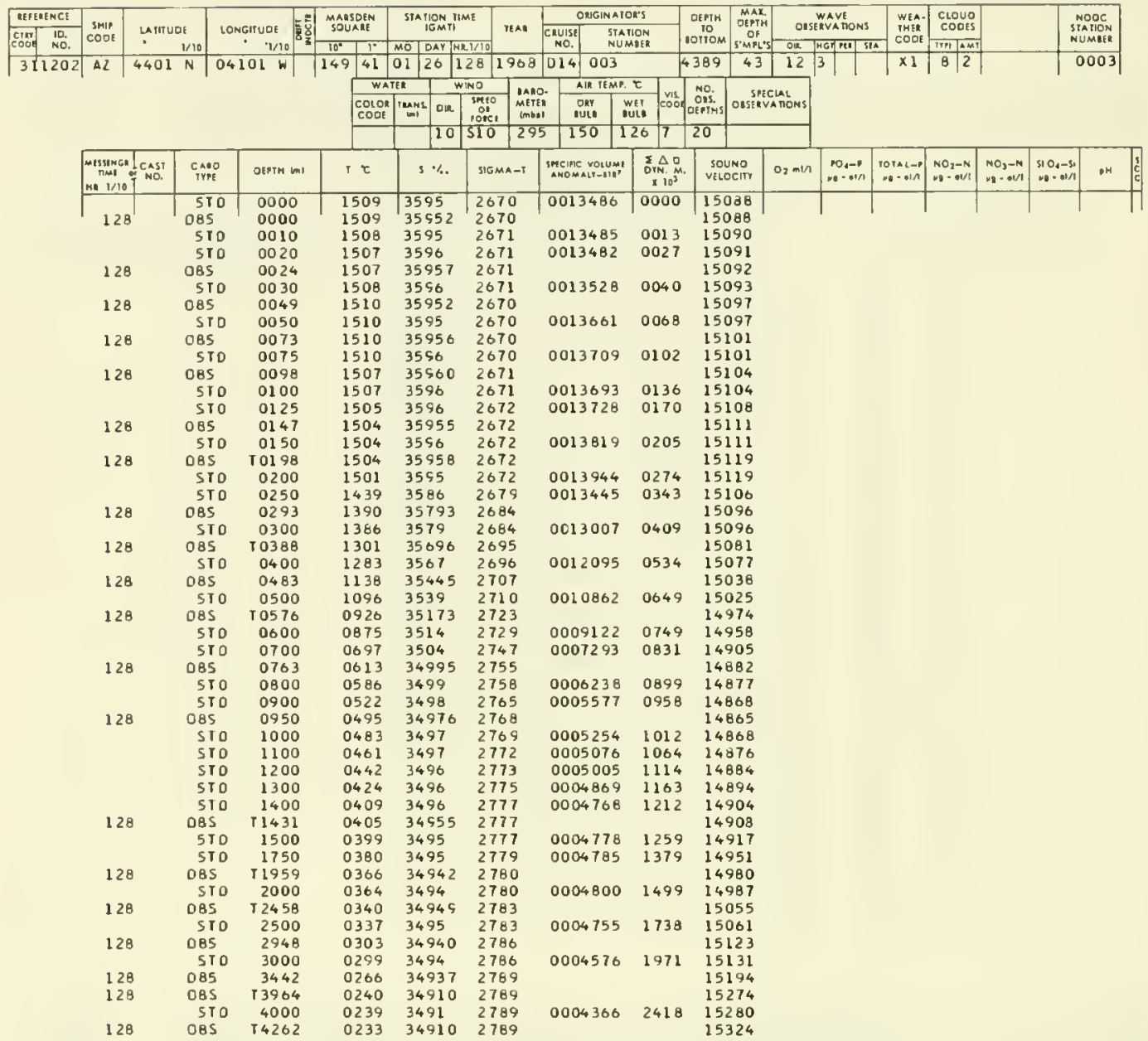


TABLE V.-Continued

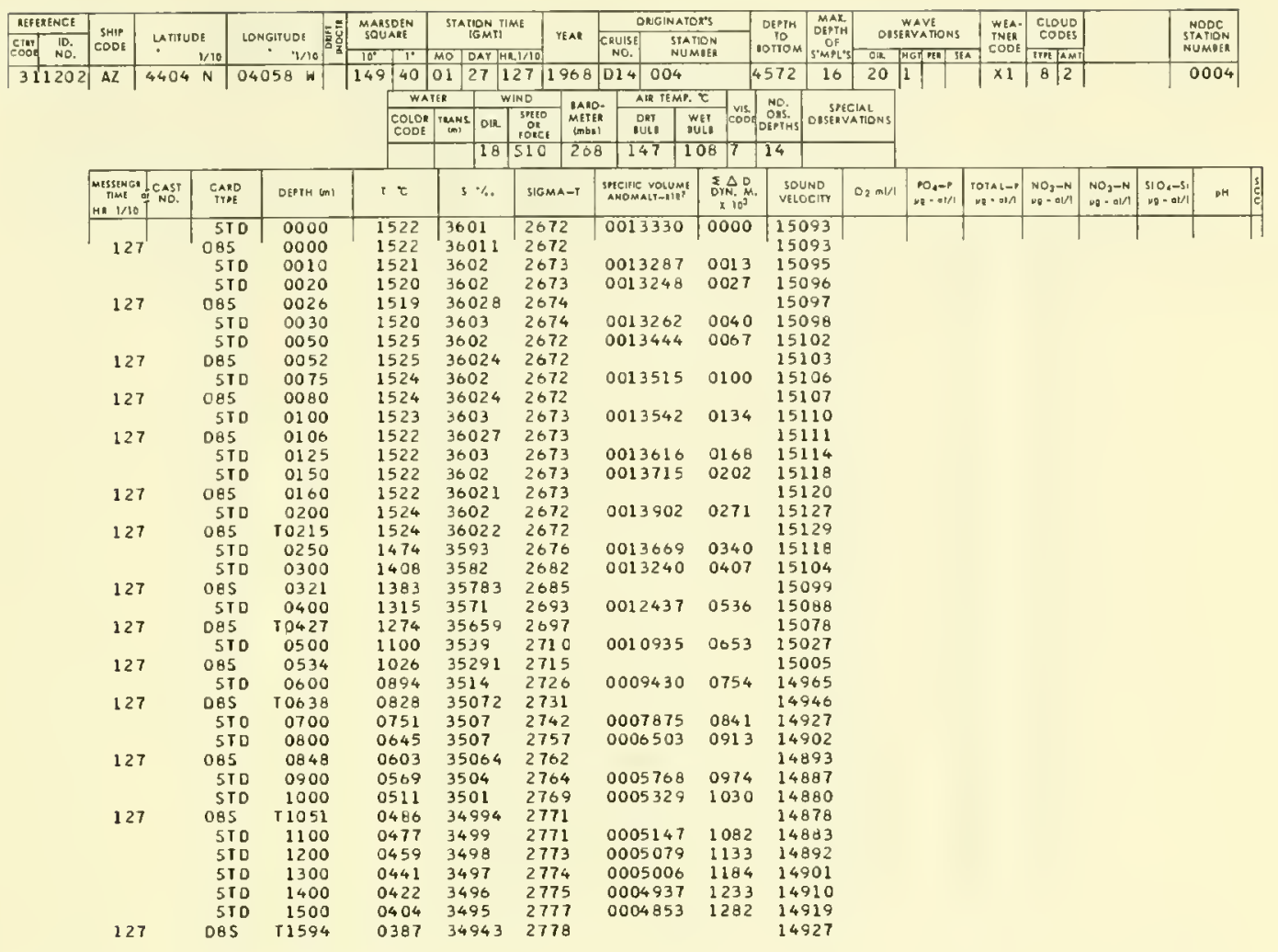

\begin{tabular}{|c|c|c|c|c|c|c|c|c|c|c|c|c|c|c|c|c|c|c|}
\hline \multirow{2}{*}{\begin{tabular}{|c|c|}
\multicolumn{2}{|c|}{ |eferenCE } \\
CrIm & 10. \\
coog & NO. \\
\end{tabular}} & \multirow{2}{*}{$\begin{array}{c}\text { SHEP } \\
C O O E\end{array}$} & \multirow{2}{*}{\multicolumn{2}{|c|}{ UATUDE }} & \multirow{2}{*}{ LONGITUDE } & \multirow{2}{*}{$\begin{array}{l}\text { MAASOEN } \\
\text { SQUARE }\end{array}$} & \multirow{2}{*}{\multicolumn{2}{|c|}{$\begin{array}{c}\text { STATION TIME } \\
\text { IOMTI }\end{array}$}} & \multicolumn{2}{|c|}{ ONGINATOR'S } & \multirow{2}{*}{\begin{tabular}{|c|} 
DEPTH \\
ID \\
IOTOM
\end{tabular}} & \multirow{2}{*}{$\begin{array}{l}\text { MAK } \\
\text { OEPTN } \\
\text { OF } \\
\text { SMPL'S }\end{array}$} & \multirow{2}{*}{\multicolumn{2}{|c|}{$\begin{array}{c}\text { WAYE } \\
\text { OUSERYAONS }\end{array}$}} & \multirow{2}{*}{$\begin{array}{l}\text { WEA- } \\
\text { THER } \\
\text { CODE }\end{array}$} & \multirow{2}{*}{$\begin{array}{l}\text { ClOUD } \\
\text { CODES } \\
\text { TST AMT }\end{array}$} & & & \multirow{2}{*}{$\begin{array}{l}\text { NOOE } \\
\text { STATON } \\
\text { NUMHER }\end{array}$} \\
\hline & & & & & & & VEAR & $\begin{array}{l}\text { Covise } \\
\text { No. }\end{array}$ & $\begin{array}{l}\text { STATION } \\
\text { NUMUEE }\end{array}$ & & & & & & & & & \\
\hline 311202 & $\Delta Z$ & 4407 & $\mathrm{~N}$ & $04051 \mathrm{~W}$ & \begin{tabular}{|l|l|}
149 & 40 \\
\end{tabular} & \begin{tabular}{l|l|}
01 & 28 \\
\end{tabular} & \begin{tabular}{|l|l|}
132 & 1968
\end{tabular} & \begin{tabular}{|l|l|}
014 & 005 \\
\end{tabular} & & :otrom & 15 & 20 & 3 & $\times 1$ & \begin{tabular}{|l|l|}
35 \\
3
\end{tabular} & & & 0005 \\
\hline & & & & & WA & TET & WIND I A & A. TEMP. & & No. & & & & & & & & \\
\hline & & & & & $\begin{array}{l}\text { colon } \\
\text { CODt }\end{array}$ & Pmust OIR. & $\begin{array}{l}\operatorname{smid} \\
\text { or } \\
\text { sonef }\end{array}$ & \begin{tabular}{l|l} 
our & out \\
put & nu
\end{tabular} & $\begin{array}{l}\text { VET coos } \\
\text { UL: coos }\end{array}$ & OAS: & $\begin{array}{l}\text { SPEC } \\
\text { OASERVA }\end{array}$ & At Al & & & & & & \\
\hline & & & & & & 20 & 513 & 150 & \begin{tabular}{l|l}
18 & 7
\end{tabular} & 14 & & & & & & & & \\
\hline & 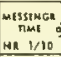 & DAST & $\begin{array}{l}\text { CAMD } \\
\text { TYPF }\end{array}$ & DEITM (n) & 12 & $s \%$ & SLGMA-I & $\begin{array}{l}\text { spenle volumi } \\
\text { ANOMALY-X107? }\end{array}$ & 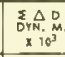 & $\begin{array}{l}\text { sou } \\
\text { velo }\end{array}$ & $\begin{array}{l}\text { JND } \\
\text { Ciry }\end{array}$ & $0, \mathrm{~m} / 1 / 1$ & 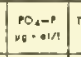 & 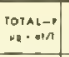 & $\begin{array}{l}\mathrm{NO}_{2}-\mathrm{N} \\
\mathrm{NO}=\mathrm{a} / 1 / 1\end{array}$ & $\begin{array}{l}\text { NO },-N \\
\text { Wu }=\text { ovil }\end{array}$ & $\left|\begin{array}{ll}310<-51 \\
m_{0}-0+1 / 1\end{array}\right|$ & $\mathrm{OH}$ \\
\hline & 132 & & $\begin{array}{l}510 \\
085\end{array}$ & $\begin{array}{l}0000 \\
0000\end{array}$ & $\begin{array}{l}1518 \\
1518\end{array}$ & $\begin{array}{l}3601 \\
36006\end{array}$ & $\begin{array}{l}2672 \\
2672\end{array}$ & 0013282 & 0000 & & $\begin{array}{l}092 \\
092\end{array}$ & & & & & & & \\
\hline & 132 & & $\begin{array}{l}085 \\
510\end{array}$ & $\begin{array}{l}0000 \\
0010\end{array}$ & $\begin{array}{l}1518 \\
1516\end{array}$ & $\begin{array}{l}36006 \\
3601\end{array}$ & $\begin{array}{l}2672 \\
2673\end{array}$ & 0013284 & 0013 & $\begin{array}{l}150 \\
150\end{array}$ & $\begin{array}{l}092 \\
093\end{array}$ & & & & & & & \\
\hline & & & 570 & 0020 & 1515 & 3600 & 2673 & 0013288 & 0027 & 150 & 094 & & & & & & & \\
\hline & 132 & & 085 & 0024 & 1514 & 36004 & 2673 & & & 150 & 095 & & & & & & & \\
\hline & & & $5 \mathbf{I} 0$ & 0030 & 1515 & 3600 & 2673 & 0013333 & 0040 & 150 & 076 & & & & & & & \\
\hline & 132 & & 085 & 0048 & 1516 & 36001 & 2673 & & & 150 & 099 & & & & & & & \\
\hline & & & STD & 0050 & 1516 & 3600 & 2672 & 0013435 & 0067 & 151 & 100 & & & & & & & \\
\hline & 132 & & 085 & 0074 & 1518 & 36004 & 2672 & & & 151 & 104 & & & & & & & \\
\hline & & & STD & 0075 & 1518 & 3600 & 26.72 & 0013529 & 0100 & 151 & 104 & & & & & & & \\
\hline & 132 & & 085 & 0098 & 1516 & $3600 t$ & 2673 & & & 151 & 107 & & & & & & & \\
\hline & & & STD & 0100 & 1516 & 3601 & 2673 & 0013549 & 0134 & 151 & 108 & & & & & & & \\
\hline & & & 5T D & 0125 & 1516 & 3600 & 2673 & 0013638 & 0168 & 151 & 112 & & & & & & & \\
\hline & 132 & & 085 & 0147 & 1515 & 36001 & 2673 & & & 151 & 115 & & & & & & & \\
\hline & & & 510 & 0150 & 1516 & 3600 & 2672 & 0013747 & 0202 & 151 & 116 & & & & & & & \\
\hline & 132 & & 085 & T 0194 & 1518 & 35995 & 2672 & & & 151 & 124 & & & & & & & \\
\hline & & & STD & 0200 & 1514 & 3599 & 2672 & 0013931 & 0272 & 151 & 123 & & & & & & & \\
\hline & & & 5 ro & 0250 & 1473 & 3593 & 2677 & 0013647 & 0341 & 151 & 118 & & & & & & & \\
\hline & 132 & & D85 & 0283 & 1442 & 35878 & 2679 & & & $13 i$ & 113 & & & & & & & \\
\hline & & & 5T0 & 0300 & 1426 & 3585 & 2681 & 0013394 & 0408 & 151 & 110 & & & & & & & \\
\hline & 132 & & 085 & Т 0387 & 1320 & 35698 & 2691 & & & 150 & 088 & & & & & & & \\
\hline & & & $5 T 0$ & 0400 & 1300 & 3567 & 2693 & 0012430 & 0537 & 150 & 083 & & & & & & & \\
\hline & 132 & & DES & 0487 & 1142 & 35446 & 2706 & & & 150 & 040 & & & & & & & \\
\hline & & & STO & 0500 & 1109 & 3540 & 2709 & 0011025 & 0655 & 150 & 030 & & & & & & & \\
\hline & 132 & & 085 & T0586 & 0912 & 35159 & 2724 & & & 149 & 970 & & & & & & & \\
\hline & & & 5TO & 0600 & 0882 & 3514 & 2728 & 0009235 & 0756 & & 901 & & & & & & & \\
\hline & & & $5 T 0$ & 0700 & 0702 & 3502 & 2745 & 0007514 & 0840 & & 907 & & & & & & & \\
\hline & 132 & & 085 & 0778 & 0603 & 34963 & 2754 & & & 148 & 880 & & & & & & & \\
\hline & & & ST & 0800 & 0552 & 3497 & 2756 & 0006467 & 0910 & 148 & 879 & & & & & & & \\
\hline & & & STD & 0900 & 0546 & 3499 & 2763 & 0005824 & 0971 & 148 & 877 & & & & & & & \\
\hline & 132 & & 085 & 10978 & 0514 & 35002 & 2768 & & & 148 & 978 & & & & & & & \\
\hline & & & STD & 1000 & 0506 & 3500 & 2769 & 0005351 & 1027 & 148 & $\$ 78$ & & & & & & & \\
\hline & & & $5 T D$ & 1100 & 0471 & 3499 & 2772 & 0005096 & 1079 & 148 & 880 & & & & & & & \\
\hline & & & $5 T 0$ & 1200 & 0443 & 3497 & 2774 & 0004930 & 1129 & 148 & 885 & & & & & & & \\
\hline & & & $5 T D$ & 1300 & 0420 & 3496 & 2775 & 0004817 & 1178 & 148 & 892 & & & & & & & \\
\hline & & & STD & 1400 & 0403 & 3495 & 2776 & 0004784 & 1220 & 149 & 902 & & & & & & & \\
\hline & 132 & & 085 & 11482 & 0394 & 34936 & 2776 & & & 149 & 911 & & & & & & & \\
\hline
\end{tabular}


Table V.-Continued

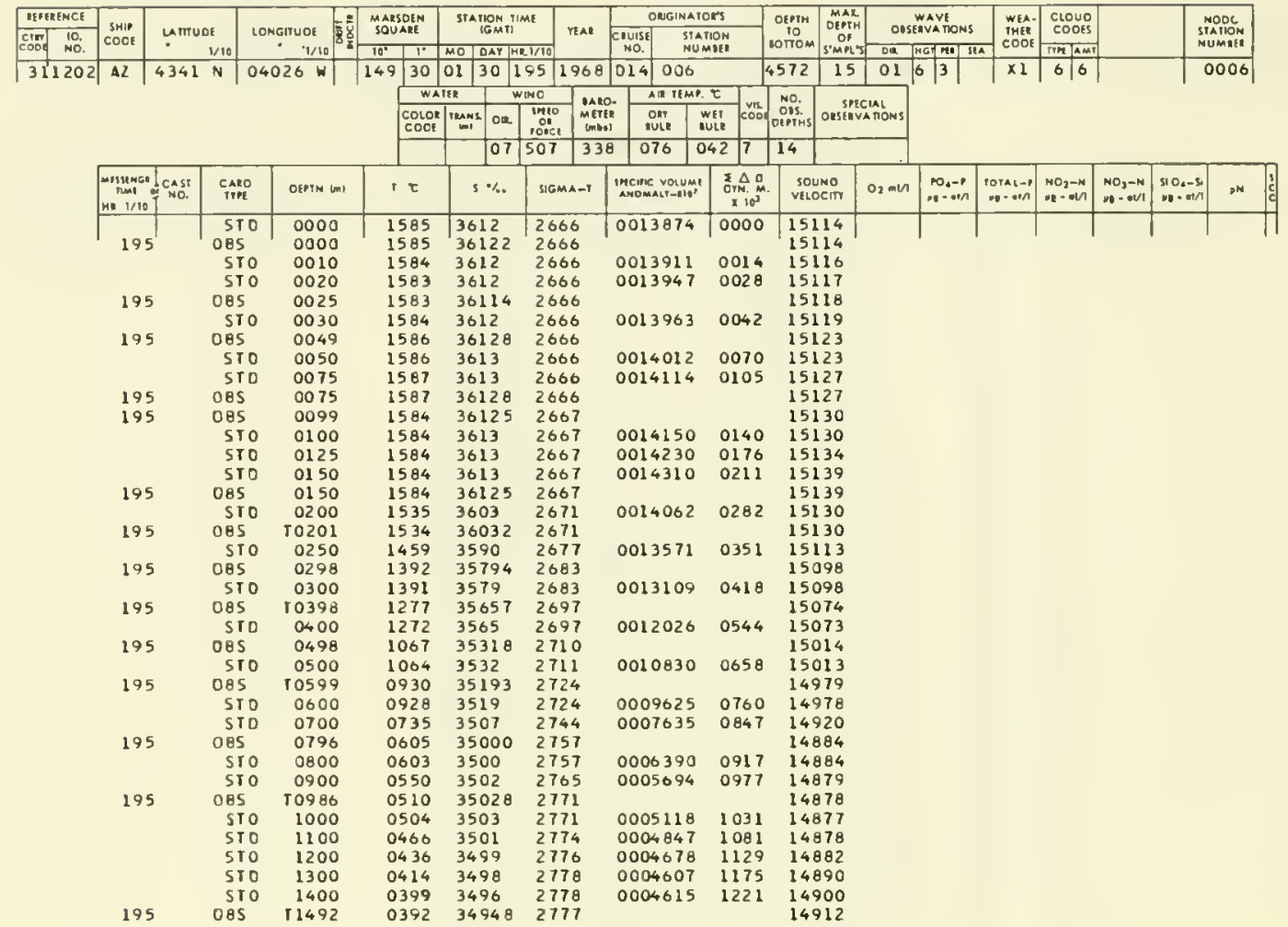


TABLE VI.-Observed and interpolated oceanographic data for stations taken by USCGC CASCO at Ocean Station DELTA, 28 February-19 March 1968, prepared from NODC listing No. 31-1210 CS.

\begin{tabular}{|c|c|c|c|c|c|c|c|c|c|c|c|c|c|c|c|c|c|c|}
\hline IEFERENCE & & & & & $\begin{array}{l}\text { MALSDEN } \\
\text { SOUARE }\end{array}$ & $\begin{array}{l}\text { STAPION } \\
\text { IGMTI }\end{array}$ & TIME & ORGIMATO & & DEPTH & $\begin{array}{l}\text { MRX } \\
\text { OEPTH }\end{array}$ & & $\begin{array}{l}\text { WAVE } \\
\text { SEVATIONS }\end{array}$ & wus- & $\begin{array}{l}\text { CLOUD } \\
\text { COOES }\end{array}$ & & & \\
\hline $\begin{array}{lll}\operatorname{lin} & 10 . \\
\cos & \text { No. }\end{array}$ & coDe & uttu & VIO & $\begin{array}{c}1 / 10 \\
0\end{array}$ & 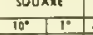 & 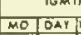 & HAR 1/10 & $\begin{array}{c}\text { CAuIs: } \\
\text { NOS. }\end{array}$ & & Loriom & $\begin{array}{l}\text { of } \\
\text { sinses }\end{array}$ & $D$ D & सG FI IEA & & \begin{tabular}{|l|} 
Tथम 7 ISMT \\
\end{tabular} & & & matt \\
\hline 311210 & CS & 4401 & $\mathbf{N}$ & $04 \overline{103}$ & \begin{tabular}{l|l|}
149 & 41 \\
\end{tabular} & \begin{tabular}{|l|l|}
02 & 28 \\
\end{tabular} & \begin{tabular}{|l|l|}
130 & 1968 \\
\end{tabular} & 015001 & & 4527 & 15 & 322 & \begin{tabular}{|l|l|}
2 & 3 \\
\end{tabular} & $x 1$ & \begin{tabular}{l|l|}
8 & 4
\end{tabular} & & & 001 \\
\hline & & & & & WaT & aten & Wimo & All TEM?. & & No. & & & & & & & & \\
\hline & & & & & $\begin{array}{l}\text { Cotor } \\
\text { CODE }\end{array}$ & Thans or & $\begin{array}{c}\sin 10 \\
\text { of } \\
10 \mathrm{cct}\end{array}$ & \begin{tabular}{l|l} 
Der & $W$ \\
IUL & W \\
\end{tabular} & Ui. coos & Ools & onsenv & A IONS & & & & & & \\
\hline & & & & & & 33 & 311 & 092 & 577 & 14 & & & & & & & & \\
\hline & $\begin{array}{l}\text { MISEREt } \\
\text { nmL } \\
\text { MR VIO }\end{array}$ & $\begin{array}{l}\text { Cast cast } \\
\text { No. }\end{array}$ & $\begin{array}{l}\text { CAED } \\
\text { TWPE }\end{array}$ & OEPTH GI & is & $s \%$ & SIGMA-1 & 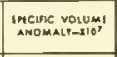 & 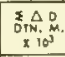 & & $\begin{array}{l}\text { UND } \\
\text { octirs }\end{array}$ & $\mathrm{O}_{2} \mathrm{~m} / \mathrm{ll}$ & 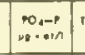 & 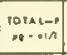 & 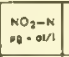 & $\begin{array}{l}\mathrm{NO}_{3}-\mathrm{N} \\
\mathrm{NO}^{-}=\mathrm{BV1}\end{array}$ & $\begin{array}{l}510,-5 i \\
10 \div 0 \mathrm{at} / 1\end{array}$ & on \\
\hline & & & STD & $\begin{array}{l}0000 \\
0000\end{array}$ & 1501 & 3579 & 2600 & 0014486 & 0000 & & 084 & & & & & & & \\
\hline & 130 & & $\begin{array}{l}085 \\
\text { STD }\end{array}$ & $\begin{array}{l}0000 \\
0010\end{array}$ & $\begin{array}{l}1501 \\
1508\end{array}$ & & $\begin{array}{l}2660 \\
2669\end{array}$ & 0013656 & 0014 & $\begin{array}{l}15 \\
15\end{array}$ & $\begin{array}{l}084 \\
090\end{array}$ & & & & & & & \\
\hline & & & STD & 0020 & $\begin{array}{l}1508 \\
1513\end{array}$ & $\begin{array}{l}3593 \\
3602\end{array}$ & 2675 & 0013136 & 0027 & & 094 & & & & & & & \\
\hline & 130 & & Des & 0022 & 1514 & 36030 & 2675 & & & & 095 & & & & & & & \\
\hline & & & $5 T 0$ & 0030 & 1514 & 3603 & 2675 & 0013121 & 0041 & 15 & 096 & & & & & & & \\
\hline & 130 & & Des & 0045 & 1515 & 36026 & 2675 & & & & 099 & & & & & & & \\
\hline & & & STD & 0050 & 1515 & 3603 & 2675 & 0013220 & 0067 & & 099 & & & & & & & \\
\hline & 130 & & 085 & 0070 & 1515 & 36029 & 2675 & & & & 103 & & & & & & & \\
\hline & & & $5 T 0$ & 0075 & & $\begin{array}{l}3603 \\
36027\end{array}$ & $\begin{array}{l}2675 \\
2675\end{array}$ & 0013283 & 0100 & $\begin{array}{l}15 \\
15\end{array}$ & $\begin{array}{l}104 \\
106\end{array}$ & & & & & & & \\
\hline & 130 & & Des & $\begin{array}{l}0092 \\
0100\end{array}$ & $\begin{array}{l}1515 \\
1515\end{array}$ & $\begin{array}{l}36028 \\
3603\end{array}$ & 2675 & 0013379 & 0133 & 15 & 108 & & & & & & & \\
\hline & & & 570 & 0125 & 1516 & 3603 & 2674 & 0013474 & 0167 & 15 & 112 & & & & & & & \\
\hline & 130 & & OBS & 0140 & 1516 & 36025 & 2674 & & & 15 & 114 & & & & & & & \\
\hline & & & STO & 0150 & 1516 & 3603 & 2674 & 0013569 & 0201 & 15 & 116 & & & & & & & \\
\hline & 130 & & 085 & 10188 & 1517 & 36026 & 2674 & & & 15 & 123 & & & & & & & \\
\hline & & & STD & 0200 & 1517 & 3603 & 2674 & 0013738 & 0269 & 15 & 125 & & & & & & & \\
\hline & & & STD & 0250 & 1516 & 3602 & 2674 & 0013893 & 0338 & & 133 & & & & & & & \\
\hline & 130 & & DBS & 0280 & 1516 & 36021 & 2674 & & & 15 & 137 & & & & & & & \\
\hline & & & $5 T 0$ & 0300 & 1509 & 3601 & 2675 & 0013986 & 0408 & 15 & 138 & & & & & & & \\
\hline & 130 & & OBS & 0385 & 1478 & 35958 & 2678 & & & 15 & 142 & & & & & & & \\
\hline & & & SID & 0400 & 1435 & 3589 & 2682 & 0013581 & 0546 & 15 & 130 & & & & & & & \\
\hline & 130 & & 085 & 0474 & 1249 & 35599 & 2690 & & & 15 & 077 & & & & & & & \\
\hline & & & SIO & 0500 & 1202 & 3553 & 2701 & 0011809 & 0673 & & 064 & & & & & & & \\
\hline & 130 & & DBS & 10575 & 1064 & 35344 & 2713 & & & 15 & 026 & & & & & & & \\
\hline & & & STO & 0600 & 1004 & 3529 & 2719 & 0010187 & 0783 & 15 & 008 & & & & & & & \\
\hline & & & STD & 0700 & 0800 & 3512 & 2739 & 0008262 & 0875 & 14 & 946 & & & & & & & \\
\hline & 130 & & 085 & 0771 & 0687 & 35042 & 2749 & & & 14 & 913 & & & & & & & \\
\hline & & & SID & 0800 & 0658 & 3504 & 2753 & 0006880 & 0951 & 14 & 906 & & & & & & & \\
\hline & & & STD & 0900 & 0568 & 3503 & 2764 & $000582 \theta$ & 1014 & & 887 & & & & & & & \\
\hline & 130 & & $08 \mathrm{~S}$ & T0975 & 0513 & 35019 & 2770 & & & 14 & 877 & & & & & & & \\
\hline & & & STO & 1000 & 0508 & 3502 & 2770 & 0005238 & 1069 & 14 & 879 & & & & & & & \\
\hline & & & STO & 1200 & 0486 & 3500 & 2772 & 0005151 & 1121 & 14 & 886 & & & & & & & \\
\hline & & & S10 & 1200 & 0464 & 3499 & 2773 & 0005049 & 1172 & 14 & 699 & & & & & & & \\
\hline & & & STD & 1300 & 0442 & 3498 & 2775 & 0004952 & 1222 & 14 & 901 & & & & & & & \\
\hline & & & ס & 1400 & 0420 & 3497 & 2776 & 0004848 & 1271 & & 909 & & & & & & & \\
\hline & 130 & & DBS & T 1492 & 0400 & 34958 & 2777 & & & 14 & 916 & & & & & & & \\
\hline
\end{tabular}

\begin{tabular}{|c|c|c|c|c|c|c|c|c|c|c|c|c|c|c|}
\hline GEFERENCE & \multirow{2}{*}{$\begin{array}{l}3 \mathrm{SNIF} \\
\text { COOL }\end{array}$} & \multirow[b]{2}{*}{$\begin{array}{l}\text { utTUde } \\
. \quad 1 / 10\end{array}$} & \multirow{2}{*}{ LONGTIUDE } & \multirow{2}{*}{\multicolumn{2}{|c|}{$\begin{array}{c}\text { Marsoin } \\
\text { SOUAME }\end{array}$}} & \multirow{2}{*}{$\begin{array}{l}\text { STATION TIME } \\
\text { IGMT }\end{array}$} & \multirow[b]{2}{*}{ reat } & \multicolumn{2}{|r|}{ ONGINATERS } & \multirow{2}{*}{$\begin{array}{c}\text { DEPTH } \\
\text { PO } \\
\text { LITOM }\end{array}$} & \multirow{2}{*}{ 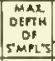 } & \multirow{2}{*}{ 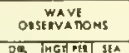 } & \multirow{2}{*}{ 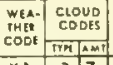 } & \multirow{2}{*}{$\begin{array}{l}\text { NOOC } \\
\text { STANION } \\
\text { NUMAEK }\end{array}$} \\
\hline 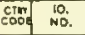 & & & & & & & & $\begin{array}{c}\text { EUU1SE } \\
\text { No. }\end{array}$ & $\begin{array}{l}\text { STAFION } \\
\text { NUMIER }\end{array}$ & & & & & \\
\hline 321210 & cs & $4358 \mathrm{~N}$ & $04103 W$ & 149 & 31 & \begin{tabular}{|l|l|l|}
02 & 29 & 127
\end{tabular} & 1968 & 015 & 002 & 4170 & 15 & \begin{tabular}{l|l|l|l|}
32 & 1 & 3 &
\end{tabular} & \begin{tabular}{|l|l|l|}
$x_{1}$ & 3 & 7
\end{tabular} & 0002 \\
\hline
\end{tabular}

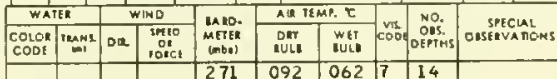

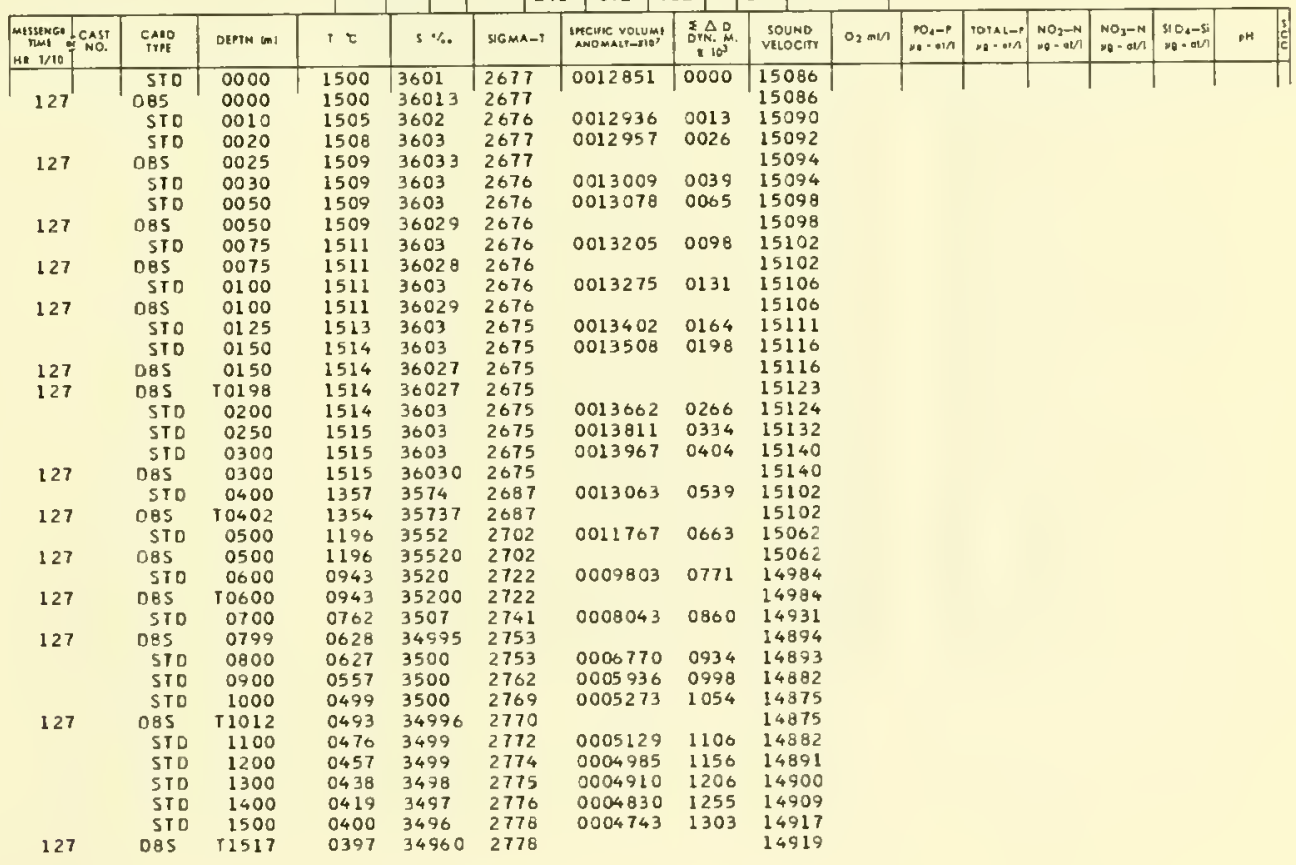


TABle VI.-Continued

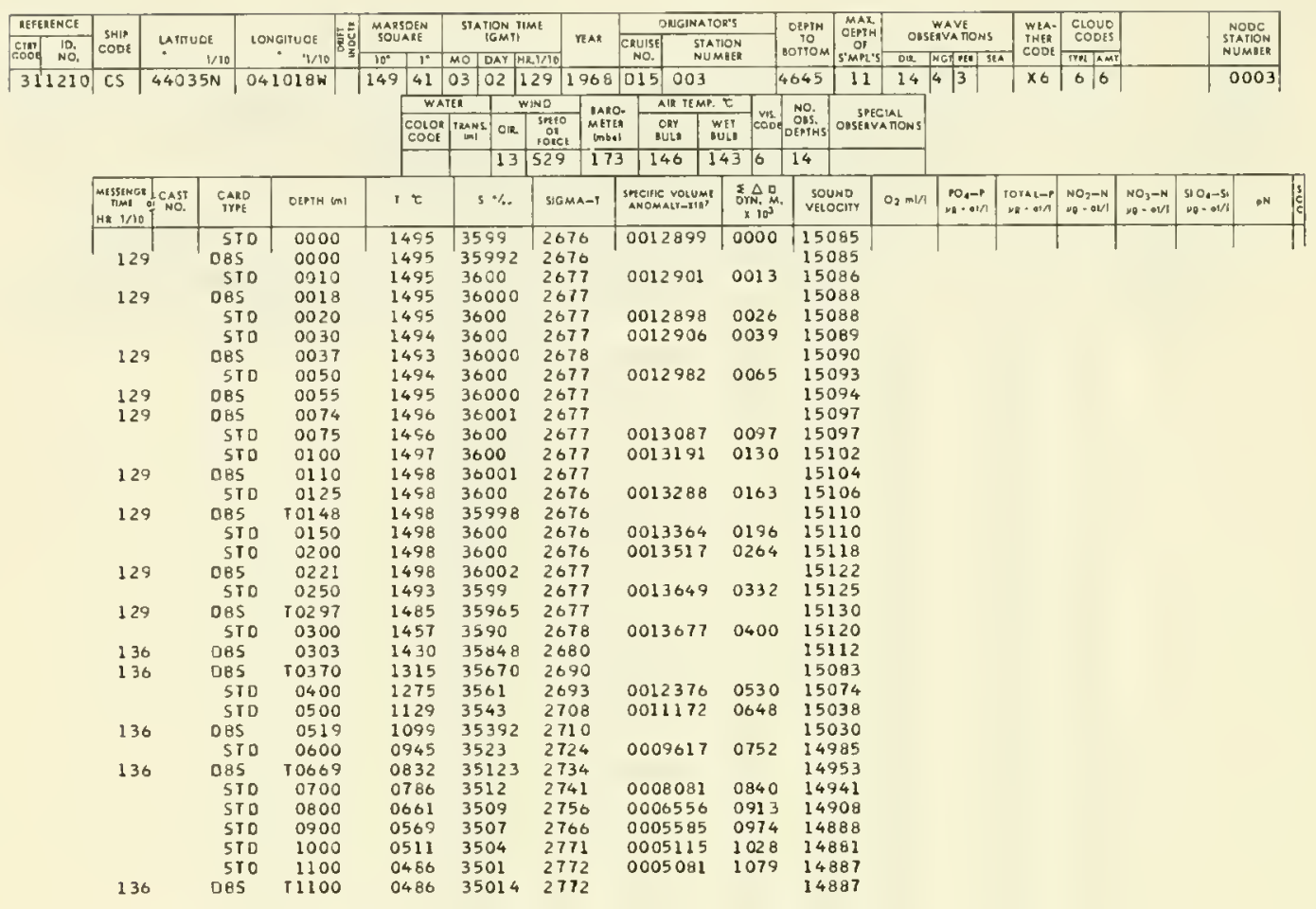

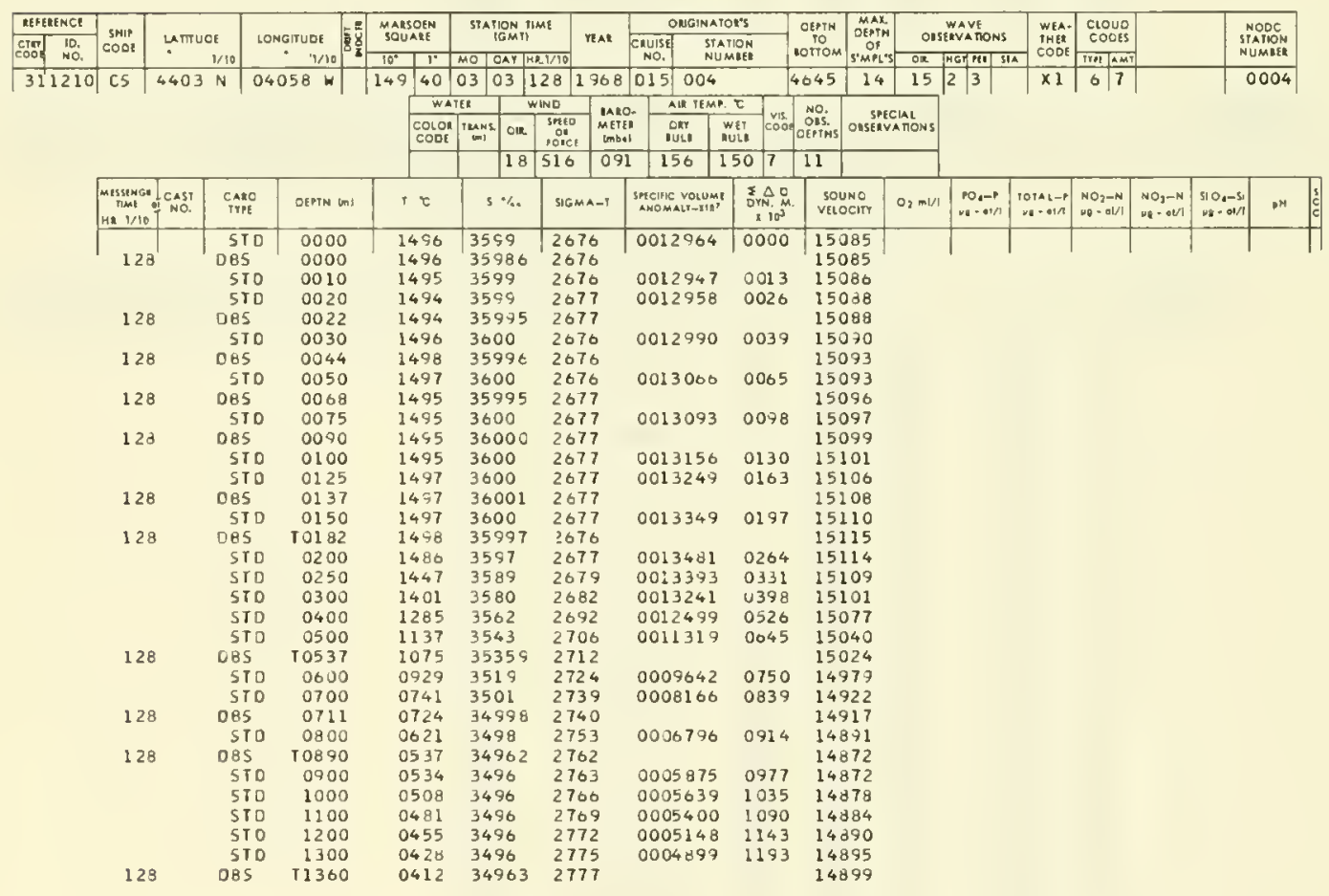




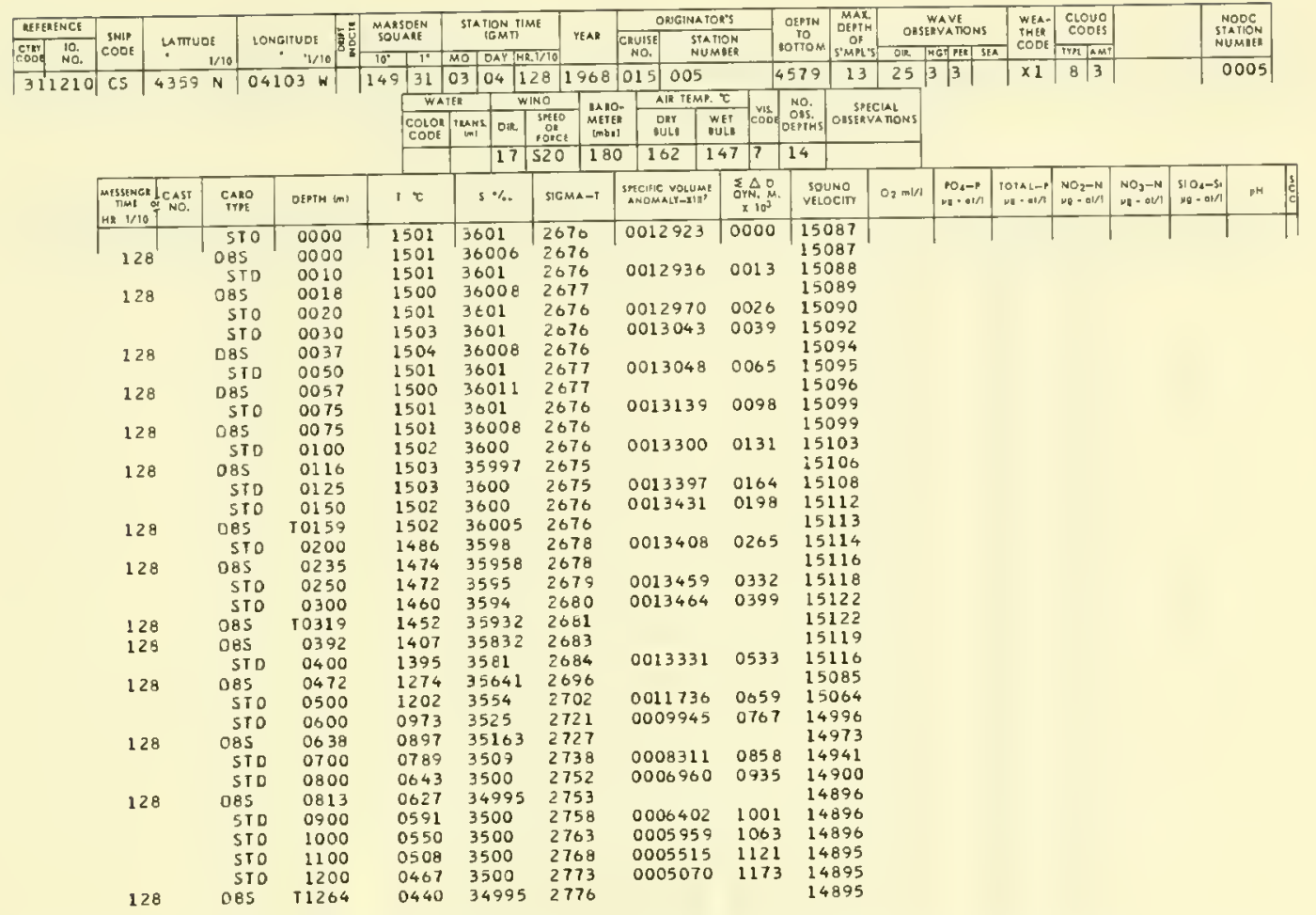

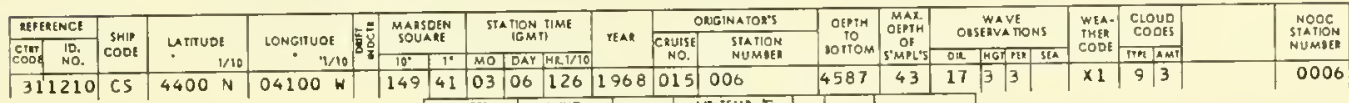

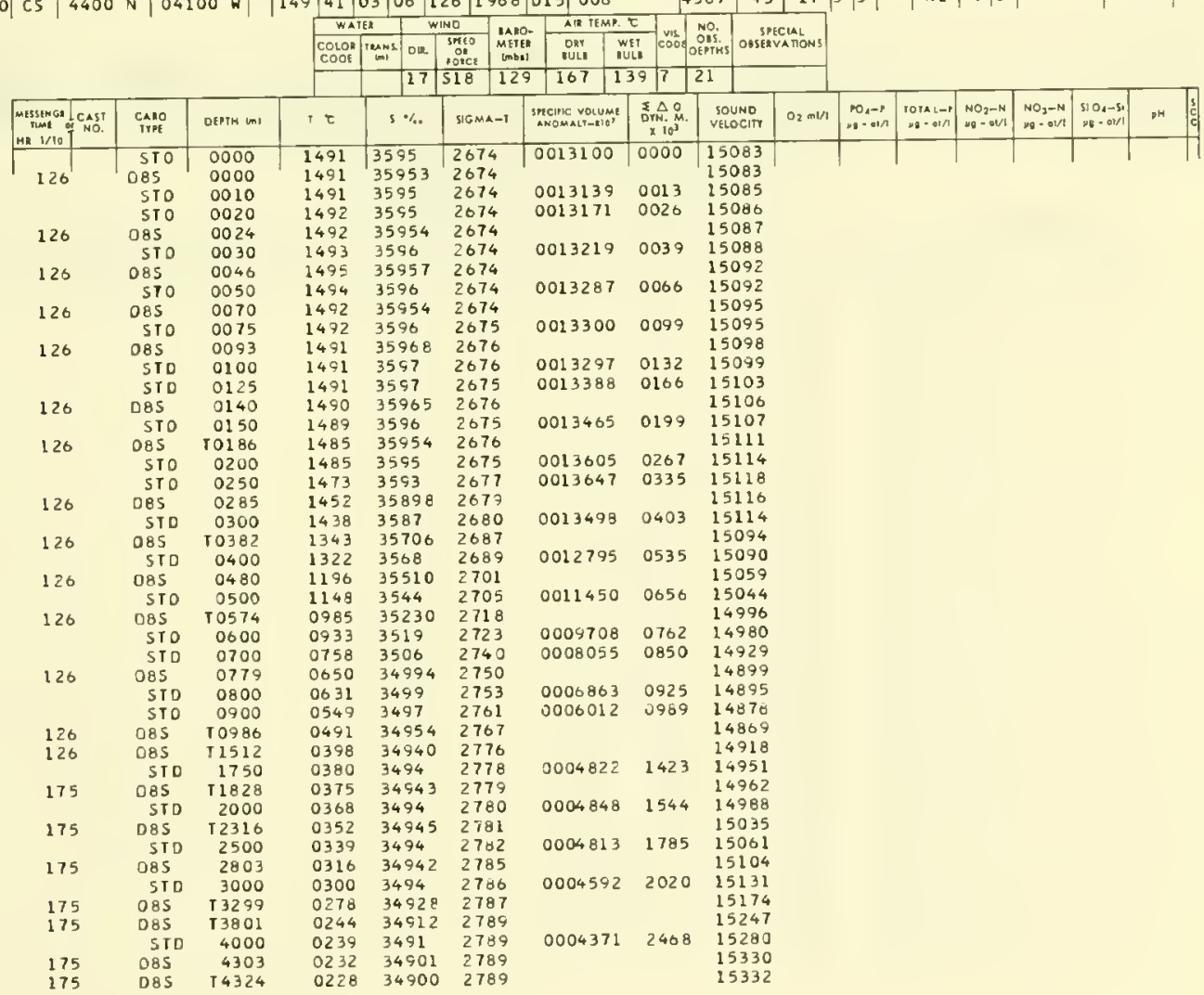


TABLE VI.-Continued

\begin{tabular}{|c|c|c|c|c|c|c|c|c|c|c|c|c|c|c|c|c|c|c|}
\hline MrFEREGL & sнің & & & $=E$ & MAISBEA & MTAPION? & fime & ONGINAT & Tors & OtPTH & MAX. & & WAVE & & Etouo & & & \\
\hline \begin{tabular}{|l|l|} 
criom & to. \\
noo.
\end{tabular} & $\cos$ & yntuc & the & IONGITUOT & $\frac{\text { SOUAnt }}{10^{\circ}}$ & $\begin{array}{l}\text { "GMTI } \\
\text { MO TOAYT }\end{array}$ & |WTivio & $\begin{array}{l}\text { Cauist } \\
\text { No. }\end{array}$ & AnIOH & 10 & 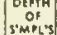 & & StRVATIONS & $\begin{array}{l}\text { TME } \\
\text { COOL }\end{array}$ & \begin{tabular}{|c|} 
CoOEs \\
Thest
\end{tabular} & & & $\begin{array}{l}\text { STATHON } \\
\text { Numith }\end{array}$ \\
\hline 311210 & CS & 4400 & $N$ & $04102 \mathrm{H}$ & 14941 & \begin{tabular}{|l|l|}
03 & 07 \\
\end{tabular} & \begin{tabular}{|l|l|}
129 & 1988 \\
\end{tabular} & \begin{tabular}{|l|l|}
015 & 007 \\
\end{tabular} & & 4827 & 15 & 12 & \begin{tabular}{ll|}
1 & 3
\end{tabular} & $x 1$ & $4 \mid 5$ & & & 0007 \\
\hline & & & & & WAT & TER & WINO & AII IEMP. & p. $\mathrm{c}$ & NO. & & & & & & & & \\
\hline & & & & & \begin{tabular}{l|} 
COLOE: \\
COOE
\end{tabular} & $\operatorname{Timams}_{\text {tmi }}$ DiR & $\begin{array}{l}\sin _{0} 10 \\
\text { on } \\
\text { ontel }\end{array}$ & \begin{tabular}{l|l} 
ont \\
out
\end{tabular} & $\begin{array}{lll}\text { wet } \\
\text { out }\end{array}$ & of ons. & oustave, & CAMONS & & & & & & \\
\hline & & & & & & 12 & 2510 & 144 & \begin{tabular}{l|l}
133 & 7
\end{tabular} & 14 & & & & & & & & \\
\hline & MIssimes & leAst & $\begin{array}{l}\text { Calo } \\
\text { TYPE }\end{array}$ & ot $n$ in $\mathrm{Cm}$ & $1 x$ & $\$ \%$ & MIGMA-T & smene volumt & 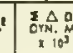 & $\begin{array}{c}30 u \\
V E 10\end{array}$ & $\begin{array}{l}\text { UND } \\
\text { Dein }\end{array}$ & $0_{2} \mathrm{mul}$ & 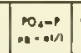 & 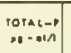 & $\begin{array}{l}\mathrm{NO}_{2}-\mathrm{N} \\
\mathrm{H}: \mathrm{OUVI}\end{array}$ & $\begin{array}{l}\mathrm{HO}_{2}, \mathrm{~N} \\
{ }_{\mathrm{O}}=\mathrm{N}\end{array}$ & $\begin{array}{l}310,-8 i \\
n a+\infty\end{array}$ & $8 i$ \\
\hline & & & 510 & 0000 & 1481 & 3594 & 2675 & 0012993 & 0000 & 150 & 079 & & & & & & & \\
\hline & 129 & & 085 & 0000 & 1481 & 35939 & 2675 & & & 150 & 079 & & & & & & & \\
\hline & & & Sio & 0010 & 1480 & 3594 & 2676 & 0012990 & 0013 & 150 & 081 & & & & & & & \\
\hline & & & 510 & 0020 & 1478 & 3594 & 2676 & 0012993 & 0026 & 150 & 082 & & & & & & & \\
\hline & 129 & & 085 & 0023 & 1478 & 35942 & 2676 & & & & 082 & & & & & & & \\
\hline & & & STO & 0030 & 1479 & 3554 & 2676 & 0013028 & 0039 & 150 & 084 & & & & & & & \\
\hline & 129 & & 085 & 0046 & 1480 & 35936 & 2675 & & & 150 & 087 & & & & & & & \\
\hline & & & 510 & 0050 & 1479 & 3594 & 2676 & 0013097 & 0065 & 150 & 087 & & & & & & & \\
\hline & 129 & & 085 & 0068 & 1474 & $35 \$ 45$ & 2677 & & & 150 & 088 & & & & & & & \\
\hline & & & 5.TO & 0075 & 1469 & 3594 & 2678 & 0012904 & 0098 & 150 & 088 & & & & & & & \\
\hline & 129 & & 085 & 0092 & 1460 & 35928 & 2679 & & & 150 & 088 & & & & & & & \\
\hline & & & $5 T 0$ & 0100 & 1459 & 3592 & 2679 & 0012948 & 0130 & 150 & 089 & & & & & & & \\
\hline & & & 510 & 0125 & 1454 & 3591 & 2679 & 0013007 & 0163 & 150 & 091 & & & & & & & \\
\hline & 129 & & 085 & 0136 & 1450 & 35906 & 2680 & & & 150 & 091 & & & & & & & \\
\hline & & & STO & 0150 & 1442 & 3589 & 2680 & 0012993 & 0195 & 150 & 091 & & & & & & & \\
\hline & 129 & & 085 & 10181 & 1428 & 35866 & 2681 & & & 15.0 & 091 & & & & & & & \\
\hline & & & 570 & 0200 & 1424 & 3586 & 2682 & 0012987 & 0260 & 150 & 093 & & & & & & & \\
\hline & & & $5 T 0$ & 0250 & 1412 & 3583 & 2682 & 0013105 & 0325 & 150 & 097 & & & & & & & \\
\hline & 129 & & 085 & 0271 & 1406 & 35820 & 2683 & & & 150 & 098 & & & & & & & \\
\hline & & & 5 TO & 0300 & 1401 & 3579 & 2681 & 0013321 & 0391 & 151 & 101 & & & & & & & \\
\hline & 129 & & 085 & 10350 & 1375 & & & & & & & & & & & & & \\
\hline & & & STO & 0400 & 1350 & 3568 & 2683 & 0013358 & 0525 & 150 & & & & & & & & \\
\hline & 129 & & Des & 0448 & 1285 & 35628 & 2693 & & & 150 & 085 & & & & & & & \\
\hline & & & $5 T 0$ & 0500 & 1148 & 3541 & 2702 & 0011609 & 0650 & 150 & 044 & & & & & & & \\
\hline & 129 & & DES & 10538 & 1058 & 35287 & 2709 & & & & 017 & & & & & & & \\
\hline & & & $5 T 0$ & 0000 & 0934 & 3518 & 2722 & 0009798 & 0757 & 149 & 981 & & & & & & & \\
\hline & & & 510 & 0700 & 0760 & 3506 & 2739 & 0008177 & 0847 & 149 & 932 & & & & & & & \\
\hline & 129 & & 085 & 0720 & 0737 & 35047 & 2742 & & & 149 & 924 & & & & & & & \\
\hline & & & STO & 0800 & 0645 & 3502 & 2753 & 0006812 & 0922 & 149 & 901 & & & & & & & \\
\hline & & & 510 & 0900 & 0549 & 3500 & 2763 & 0005828 & 0985 & 148 & 379 & & & & & & & \\
\hline & 129 & & Dos & 10903 & 0546 & 34994 & 2764 & & & & 78 & & & & & & & \\
\hline & & & $5 T 0$ & 1000 & 0522 & 3499 & 2760 & 0005630 & 1042 & 148 & 384 & & & & & & & \\
\hline & & & STO & 1100 & 0497 & 3498 & 2769 & 0005452 & 1098 & 148 & 991 & & & & & & & \\
\hline & & & STO & 1200 & 0473 & 3498 & 2771 & 0005276 & 1151 & 148 & 897 & & & & & & & \\
\hline & & & $5 T 0$ & 1300 & 0448 & 3497 & 2773 & 0005087 & 1203 & 149 & 304 & & & & & & & \\
\hline & & & STO & 1400 & 0423 & 3497 & 2776 & 0004895 & 1253 & 149 & 910 & & & & & & & \\
\hline & 129 & & 085 & 11453 & 0410 & 34965 & 2777 & & & 149 & 914 & & & & & & & \\
\hline
\end{tabular}

\begin{tabular}{|c|c|c|c|c|c|c|c|c|c|c|c|c|c|c|c|c|c|c|}
\hline \multirow{3}{*}{ 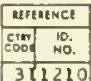 } & \multirow{3}{*}{ 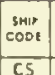 } & \multirow{2}{*}{\multicolumn{2}{|c|}{$\begin{array}{c}\text { umrmud } \\
1 / 10 \\
\end{array}$}} & \multirow{2}{*}{$\begin{array}{c}\text { Longruo: } \\
\text { MII }\end{array}$} & \multirow{2}{*}{$\begin{array}{l}\text { MARSOEN } \\
\text { SOUARE }\end{array}$} & \multicolumn{2}{|c|}{ 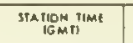 } & \multicolumn{2}{|c|}{ ORGINATERS } & & \multirow{2}{*}{ 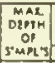 } & \multirow{2}{*}{\multicolumn{2}{|c|}{ OUSEAvA nons }} & \multirow{2}{*}{$\left|\begin{array}{c}\text { WEA- } \\
\text { TNER } \\
\text { COOt }\end{array}\right|$} & \multirow{2}{*}{ 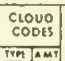 } & & & \multirow{2}{*}{$\begin{array}{l}\text { NOOC } \\
\text { STAYHO } \\
\text { NUMUIR }\end{array}$} \\
\hline & & & & & & \begin{tabular}{|l|l|}
\multicolumn{1}{|c|}{ IGM T } \\
$M O$
\end{tabular} & rest & $\begin{array}{c}\text { CEUIst } \\
\text { NO. } \\
\end{array}$ & $\begin{array}{l}\text { SIATION } \\
\text { Numat }\end{array}$ & & & & & & & & & \\
\hline & & 4408 & \begin{tabular}{l|l} 
& 0
\end{tabular} & $04059 \times$ & \begin{tabular}{|l|l|}
49 & 40 \\
\end{tabular} & \begin{tabular}{|l|l|}
03 & 08 \\
\end{tabular} & \begin{tabular}{|l|l|}
30 & 1968 \\
\end{tabular} & 015,008 & & $\begin{array}{c}10 \\
10170 \mathrm{M} \\
4827\end{array}$ & 16 & 182 & \begin{tabular}{|l|l|l}
2 & 3 & \\
\end{tabular} & $|x|$ & $8 / 4$ & & & 0008 \\
\hline & & & & & WAT & riti & WIND & 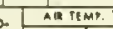 & & & & & & & & & & \\
\hline & & & & & $\begin{array}{l}\mathrm{COLO} \\
\mathrm{COOt}\end{array}$ & inams on & 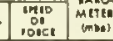 & \begin{tabular}{l|l} 
ony & ow \\
outu & ou \\
\end{tabular} & ait $=000$ & | oist. & onstove & CAALONS & & & & & & \\
\hline & & & & & & 16 & \begin{tabular}{l|l}
525 & 149
\end{tabular} & 158 & 5477 & 13 & & & & & & & & \\
\hline & $\begin{array}{l}\text { messemGn } \\
\text { sumt } \\
\text { Hit } 1 / 10\end{array}$ & $\begin{array}{l}\text { DCAST } \\
\text { DeAD. }\end{array}$ & $\underset{\substack{\text { CARC } \\
\text { IYpi }}}{ }$ & DUTIM (m) & t $t$ & $3 \%$ & SIGMA-T & $\begin{array}{l}\text { 'Meinc volum! } \\
\text { ANOHA }\end{array}$ & 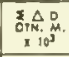 & $\begin{array}{l}\text { sou } \\
\text { vilo }\end{array}$ & $\begin{array}{l}\text { JNO } \\
\text { SCIT }\end{array}$ & $O_{2} m V 1$ & $\left|\begin{array}{l}m_{4}-p \\
m=\cdots / 1 / 1\end{array}\right|+$ & 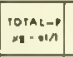 & $\begin{array}{l}\mathrm{NO}_{2}-\mathrm{N} \\
\mathrm{nO}=\mathrm{OVI}\end{array}$ & $\begin{array}{l}\mathrm{NO}_{2}-\mathrm{N} \\
\mathrm{NOM}=\mathrm{NU}\end{array}$ & $\left|\begin{array}{l}310+-5 \\
40=01 / 1\end{array}\right|$ & H H \\
\hline & & & SIO & 0000 & 1501 & 3598 & 2674 & 0013098 & 0000 & & & & & & & & & \\
\hline & 130 & & $\begin{array}{l}085 \\
\text { S10 }\end{array}$ & $\begin{array}{l}0000 \\
0010\end{array}$ & $\begin{array}{l}1501 \\
1501\end{array}$ & $\begin{array}{l}35982 \\
3598\end{array}$ & $\begin{array}{l}2674 \\
2674\end{array}$ & 0013123 & 0013 & $\begin{array}{l}150 \\
150\end{array}$ & 586 & & & & & & & \\
\hline & & & STO & 0020 & 1502 & 3549 & 2075 & 0013140 & 0026 & 150 & 990 & & & & & & & \\
\hline & 130 & & 085 & 0025 & 1502 & 35988 & 2075 & & & 150 & 91 & & & & & & & \\
\hline & & & STO & 0030 & 1501 & 3599 & 2675 & 0013147 & 0.039 & 150 & 991 & & & & & & & \\
\hline & & & 510 & 0050 & 1496 & 3599 & 2676 & 0013096 & 0006 & 150 & 993 & & & & & & & \\
\hline & 130 & & 085 & 0050 & 1490 & 35989 & 2676 & & & 150 & 993 & & & & & & & \\
\hline & & & SID & 0075 & 1494 & 3599 & 2676 & 0013168 & 0098 & 150 & 997 & & & & & & & \\
\hline & 130 & & 085 & 0096 & & 35982 & & & & & & & & & & & & \\
\hline & & & STO & 0100 & 1493 & 3598 & 2676 & 0013235 & 0131 & 251 & 100 & & & & & & & \\
\hline & & & 510 & 0125 & 1491 & 3598 & 2677 & 0013277 & 0265 & 151 & 104 & & & & & & & \\
\hline & 130 & & 085 & 0146 & 1490 & 35482 & 2677 & & & $15 i$ & 107 & & & & & & & \\
\hline & & & STO & 0150 & 1488 & 3558 & 2077 & 0013298 & 0198 & 151 & 107 & & & & & & & \\
\hline & 130 & & 085 & T0190 & 1464 & 35937 & 2679 & & & 151 & 105 & & & & & & & \\
\hline & & & STD & 0200 & 1460 & 3593 & $267 x$ & 0013225 & 0264 & 151 & 05 & & & & & & & \\
\hline & & & 510 & 0250 & 1436 & 3588 & 2681 & 0013236 & 0330 & 151 & los & & & & & & & \\
\hline & 130 & & 085 & 0287 & 1413 & 35836 & 2682 & & & 151 & 103 & & & & & & & \\
\hline & & & SIO & 0300 & 1400 & 3582 & 2683 & 0013198 & 0390 & 151 & 103 & & & & & & & \\
\hline & 130 & & 085 & T0387 & 1329 & $3570 \mathrm{C}$ & 2689 & & & 150 & 91 & & & & & & & \\
\hline & & & $5 T 0$ & 0400 & 1313 & 3567 & 2690 & 0012688 & 0526 & 150 & 687 & & & & & & & \\
\hline & 130 & & 085 & 0489 & 1178 & 35476 & 2702 & & & 150 & 053 & & & & & & & \\
\hline & & & $5 T 0$ & 0500 & 1154 & 3545 & 2704 & $001 \div 488$ & 0647 & 150 & 946 & & & & & & & \\
\hline & 130 & & OBS & $T 0587$ & 0978 & 35227 & 2719 & & & 149 & 995 & & & & & & & \\
\hline & & & SID & 0600 & 0952 & 3521 & 2722 & 0009881 & 0754 & 149 & & & & & & & & \\
\hline & & & 510 & 0700 & 0775 & 3508 & 2739 & 0008108 & 0844 & 149 & 36 & & & & & & & \\
\hline & & & 510 & 0800 & 0638 & 3500 & 2752 & 0006896 & 0919 & 148 & 398 & & & & & & & \\
\hline & 130 & & 085 & 0800 & $\operatorname{los} 38$ & 34995 & 2752 & & & 148 & & & & & & & & \\
\hline & & & SID & 0900 & 0556 & 3458 & 2761 & 0006055 & 0984 & 148 & & & & & & & & \\
\hline & & & 510 & 1000 & 0488 & 3496 & 2707 & 0005430 & 1041 & 140 & & & & & & & & \\
\hline & 130 & & 085 & T1012 & 0481 & 34952 & 2768 & & & 148 & 109 & & & & & & & \\
\hline & & & 510 & 1100 & 0466 & 3495 & 2709 & 0005314 & 1095 & 148 & 77 & & & & & & & \\
\hline & & & 5To & 1200 & 0448 & 3494 & 2771 & 0005243 & 1148 & 148 & & & & & & & & \\
\hline & & & SID & 1300 & 0431 & 3493 & 2772 & 0005166 & 1200 & 148 & 96 & & & & & & & \\
\hline & & & 510 & 1400 & 0414 & 3493 & 2773 & 0005084 & 1251 & 147 & 06 & & & & & & & \\
\hline & & & SID & 1500 & 0396 & 3492 & 2775 & 0004999 & 1301 & 149 & & & & & & & & \\
\hline & 130 & & 085 & T1588 & 0381 & 34912 & 2776 & & & 149 & 24 & & & & & & & \\
\hline
\end{tabular}


TABLE VI.-Continued

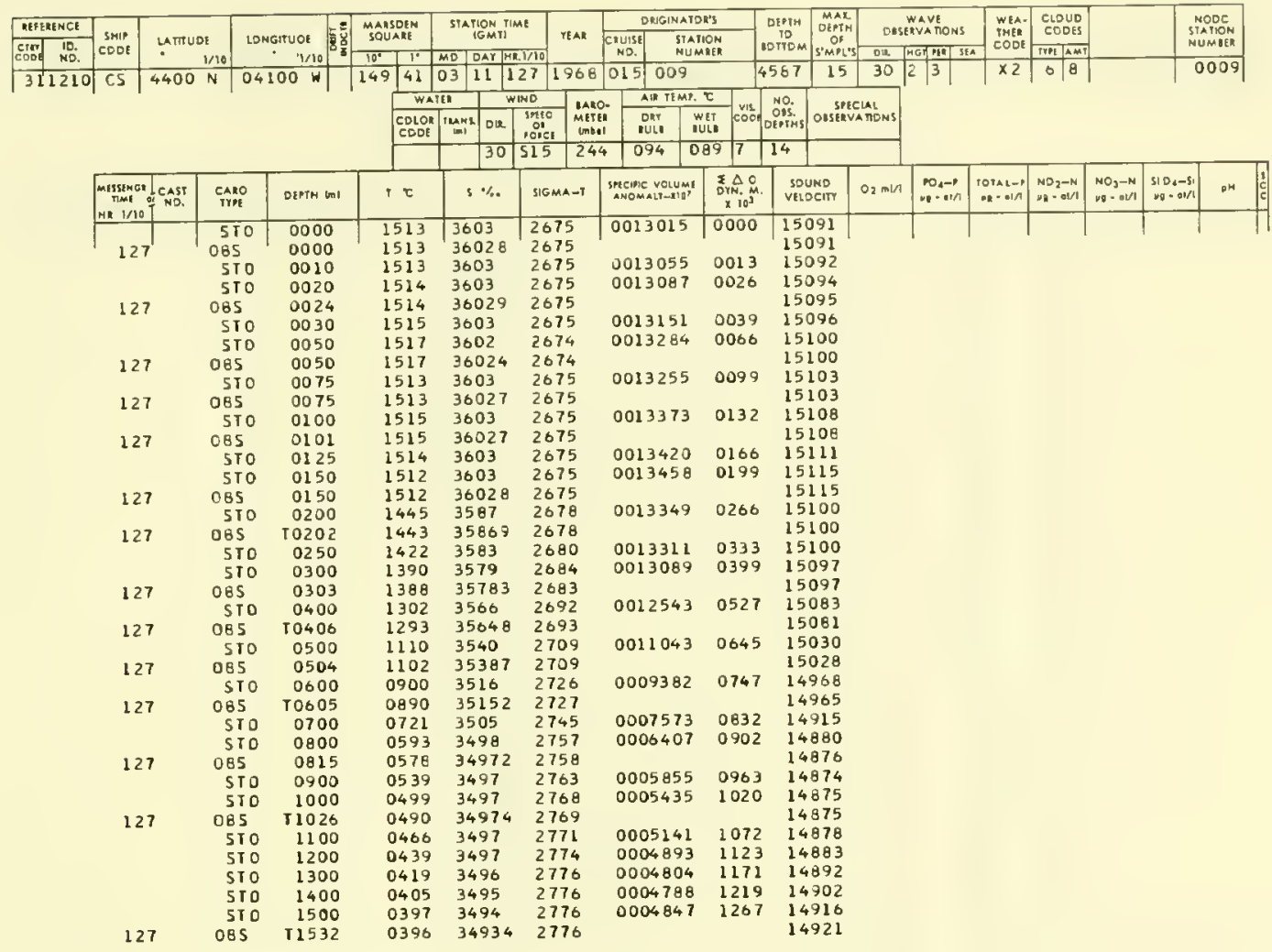




\section{TABLE VI.-Continued}

\begin{tabular}{|c|c|c|c|c|c|c|c|c|c|c|c|c|c|c|c|c|c|c|}
\hline \multirow{2}{*}{\begin{tabular}{|c|c|} 
KIT ERENCE \\
CIRr \\
Cox & NO. \\
\end{tabular}} & \multirow{2}{*}{ CoOR } & \multirow{2}{*}{\multicolumn{2}{|c|}{$\begin{array}{c}\text { ZATTUDe } \\
\quad 1 / 10\end{array}$}} & \multirow{2}{*}{ LONGITUDE } & \multirow{2}{*}{$\begin{array}{l}\text { MARsoen } \\
\text { SOUAKt }\end{array}$} & \multirow{2}{*}{\multicolumn{2}{|c|}{$\begin{array}{c}\text { STATION TIME } \\
\text { TGMTI }\end{array}$}} & \multicolumn{2}{|c|}{ ORGGINAIOR'S } & \multirow{2}{*}{$\begin{array}{c}\text { DEFYH } \\
\text { PO } \\
\text { OOTTOM }\end{array}$} & \multirow{2}{*}{$\mid \begin{array}{c}\text { MAX } \\
\text { OtFrM } \\
\text { Of } \\
\text { S'MPL's }\end{array}$} & \multirow{2}{*}{\multicolumn{2}{|c|}{$\begin{array}{l}\text { WAVE } \\
\text { DOSEAVATONS }\end{array}$}} & \multirow{3}{*}{\begin{tabular}{|c|} 
WEA- \\
WNE \\
COO \\
$\times 1$ \\
\end{tabular}} & \multirow{2}{*}{ 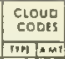 } & & & \\
\hline & & & & & & & veAk & \begin{tabular}{|c|c|} 
CRUISEE & STA \\
NOD. & NU \\
\end{tabular} & MOEN & & & & & & & & & $\begin{array}{l}\text { SATHON } \\
\text { NüLER }\end{array}$ \\
\hline & CS & 4400 & $N$ & $24100 \mathrm{~W}$ & \begin{tabular}{|l|l|}
49 & 41 \\
\end{tabular} & 03,12 & $127 \mid 1908$ & \begin{tabular}{|l|l|}
015 & 010 \\
\end{tabular} & & 4587 & 39 & 13 & \begin{tabular}{l|l|l|}
1 & 2 \\
\end{tabular} & & \begin{tabular}{|l|l|}
8 & 2 \\
\end{tabular} & & & 0010 \\
\hline & & & & & $W_{A}$ & atin & WIND & D. Ail $T E M F$. & & No. & & & & & & & & \\
\hline & & & & & $\begin{array}{l}\text { coton } \\
\text { cose }\end{array}$ & PMans ove & $\begin{array}{l}3410 \\
010 \\
10101\end{array}$ & \begin{tabular}{c|c} 
onr \\
out
\end{tabular} & 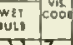 & gous rins & onsenv & TONS & & & & & & \\
\hline & & & & & & 14 & $4 \mid 510$ & 117 & 117 & 20 & & & & & & & & \\
\hline & 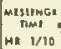 & I & $\begin{array}{l}\text { CAFD } \\
\text { IFPE }\end{array}$ & DEPTH ims & 15 & $5 \%$ & SlGma-r & 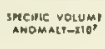 & 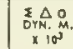 & & & $\mathrm{O}_{2} \mathrm{mLI}$ & || $\begin{array}{ll}00,-1 \\
0.11 / 1\end{array} \mid$ & 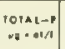 & $\left|\begin{array}{l}\mathrm{NO}_{2}-\mathrm{N} \\
\mathrm{NO}-\mathrm{OH/1}\end{array}\right|$ & $\begin{array}{l}\mathrm{NO}_{3}-\mathrm{N} \\
\mathrm{yg} \cdot \mathrm{at} / 1\end{array}$ & $\begin{array}{l}350,-51 \\
21=01 / 1\end{array}$ & DH \\
\hline & 127 & & $\begin{array}{l}5 \mathrm{TO} \\
085\end{array}$ & $\begin{array}{l}000 \overline{0} \\
0000\end{array}$ & $\begin{array}{l}1503 \\
1503\end{array}$ & $\begin{array}{l}3600 \\
36004\end{array}$ & $\begin{array}{l}2676 \\
2676\end{array}$ & 0012979 & 0000 & $\begin{array}{l}150 \\
150\end{array}$ & $\begin{array}{l}087 \\
087\end{array}$ & & & & & & & \\
\hline & $12 \pi$ & & STO & 0010 & $\begin{array}{l}1503 \\
1502\end{array}$ & 36004 & $\begin{array}{l}2676 \\
2676\end{array}$ & 0012988 & 0013 & $\begin{array}{l}150 \\
150\end{array}$ & 088 & & & & & & & \\
\hline & & & STD & 0020 & 1500 & 3600 & 2676 & 0012999 & 0026 & 150 & 090 & & & & & & & \\
\hline & 127 & & DBS & 0022 & 1500 & 36002 & $267 b$ & & & 150 & 090 & & & & & & & \\
\hline & & & 510 & 0030 & 1498 & 3600 & 2676 & 0012996 & 0039 & 150 & 091 & & & & & & & \\
\hline & 127 & & 085 & 0045 & 1497 & 35996 & 2676 & & & 150 & 093 & & & & & & & \\
\hline & & & 5 IO & 0050 & 1497 & 3600 & 2677 & $001304\}$ & 0065 & 150 & 094 & & & & & & & \\
\hline & 127 & & 985 & 0067 & 1498 & 36003 & 2677 & & & 150 & 097 & & & & & & & \\
\hline & & & $5 T 0$ & 0075 & 1498 & 3600 & 2676 & 0013143 & 0098 & 150 & 098 & & & & & & & \\
\hline & 127 & & 085 & 0089 & 1499 & 35598 & 2676 & & & 151 & 100 & & & & & & & \\
\hline & & & $5 \mathrm{TO}$ & 0100 & 1486 & $35 \subseteq 8$ & 2678 & 0013104 & 0131 & 150 & 098 & & & & & & & \\
\hline & & & STO & 0125 & 1463 & $35 \$ 5$ & 2680 & 0012917 & 0163 & 150 & 094 & & & & & & & \\
\hline & 127 & & 085 & 0133 & 1457 & 35937 & 2681 & & & 150 & 094 & & & & & & & \\
\hline & & & $5 T 0$ & 0150 & 1451 & 3593 & 2681 & 0012888 & 0195 & 150 & 094 & & & & & & & \\
\hline & 127 & & 085 & T0176 & 1442 & 35911 & 2682 & & & 150 & & & & & & & & \\
\hline & & & STO & 0200 & 1437 & 3590 & 2682 & 0012964 & 0260 & 150 & 098 & & & & & & & \\
\hline & & & 510 & 0250 & 1418 & 3585 & 2682 & 0013082 & 0325 & 150 & 099 & & & & & & & \\
\hline & 127 & & 085 & 0266 & 1409 & 35838 & 2683 & & & 150 & 099 & & & & & & & \\
\hline & & & STO & 0300 & 1390 & 3580 & 2684 & 0013016 & 0390 & 150 & 098 & & & & & & & \\
\hline & 127 & & Dos & T0359 & 1337 & 35718 & 2689 & & & 150 & 089 & & & & & & & \\
\hline & & & SID & 0400 & 1287 & 3565 & 2694 & 0012320 & 0517 & 150 & 078 & & & & & & & \\
\hline & 127 & & 085 & 0443 & 1218 & 3555 与 & 2701 & & & 150 & 061 & & & & & & & \\
\hline & & & SIO & 0500 & 1094 & 3538 & 2710 & 0010899 & $0+33$ & 150 & & & & & & & & \\
\hline & 127 & & 085 & T0527 & 1038 & 35304 & 2714 & & & 150 & 008 & & & & & & & \\
\hline & & & SIO & 0600 & 0886 & 3515 & 2728 & 0009227 & 0734 & 149 & 962 & & & & & & & \\
\hline & & & STO & 0700 & 0721 & 3501 & 2742 & 0007867 & 0819 & 149 & 914 & & & & & & & \\
\hline & 127 & & 085 & 0703 & 0717 & 35003 & 2742 & & & 149 & 913 & & & & & & & \\
\hline & & & SID & 0800 & 0617 & 3498 & 2754 & 0006740 & 0892 & 148 & & & & & & & & \\
\hline & 127 & & 085 & T0877 & 0552 & 34972 & 2761 & & & 148 & 876 & & & & & & & \\
\hline & & & 510 & 0900 & 0542 & 3497 & 2702 & 0005917 & 0956 & $14 \theta$ & 876 & & & & & & & \\
\hline & & & 510 & 1000 & 0503 & 3496 & 2766 & 0005590 & 1013 & 148 & & & & & & & & \\
\hline & & & 5 TO & 1100 & 0469 & 3455 & 2769 & 0005327 & 1008 & 148 & 879 & & & & & & & \\
\hline & & & $5 \mathrm{JO}$ & 1200 & 0440 & 3494 & 2772 & 0005126 & 1120 & 148 & 883 & & & & & & & \\
\hline & & & $5 \mathrm{TO}$ & 1300 & 0417 & 3494 & 2774 & 0004925 & 1170 & 148 & 891 & & & & & & & \\
\hline & 127 & & 085 & r1335 & 0410 & 34937 & 2775 & & & 148 & 893 & & & & & & & \\
\hline & & & 510 & 1400 & 0404 & 3494 & 2775 & 0004871 & 1219 & 149 & 902 & & & & & & & \\
\hline & & & 510 & 1500 & 0396 & 3494 & 2776 & 0004850 & 1268 & 149 & 915 & & & & & & & \\
\hline & 183 & & 085 & 11736 & 0380 & 34938 & 2778 & & & 149 & 948 & & & & & & & \\
\hline & & & 510 & 1750 & 0380 & 3494 & 2778 & 0004851 & 1389 & 149 & 951 & & & & & & & \\
\hline & & & 510 & 2000 & 0370 & 3493 & 2779 & 0004956 & 1512 & 149 & 969 & & & & & & & \\
\hline & 183 & & 085 & 2177 & 0361 & 34930 & 2779 & & & 150 & 015 & & & & & & & \\
\hline & & & 510 & 2500 & 0338 & 3494 & 2782 & 0004863 & 1757 & 150 & 061 & & & & & & & \\
\hline & 163 & & 085 & 2748 & 0321 & 34938 & 2784 & & & 150 & 096 & & & & & & & \\
\hline & & & SID & 3000 & 0307 & 3493 & 2784 & 0004770 & 1998 & 151 & 134 & & & & & & & \\
\hline & 183 & & 085 & T 3264 & 0290 & 34925 & 2786 & & & 151 & 173 & & & & & & & \\
\hline & 283 & & o8s & I 3797 & 0247 & 34917 & 2789 & & & 152 & 247 & & & & & & & \\
\hline & 183 & & 085 & 39020 & 0236 & 34912 & 27890 & & & & & & & & & & & \\
\hline
\end{tabular}


TABLE VI.-Continued

\begin{tabular}{|c|c|c|c|c|c|c|c|c|c|c|c|c|c|c|c|c|c|c|}
\hline \multirow{3}{*}{ 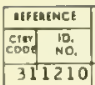 } & \multirow{2}{*}{ SHir } & \multirow{2}{*}{\multicolumn{2}{|c|}{ 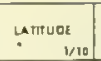 }} & \multirow{2}{*}{\begin{tabular}{|r|r|} 
LONGITUOE & $=0$ \\
V/MO & $=$ \\
\end{tabular}} & \multirow{2}{*}{$\begin{array}{c}\text { MABSDEN } \\
\text { SOUARE } \\
\end{array}$} & $\begin{array}{l}\text { STATION FIME } \\
\text { TGM HI }\end{array}$ & TEA & \multicolumn{2}{|c|}{ ONGINATO'S } & \multirow{2}{*}{\begin{tabular}{|c|} 
aEpm \\
10 \\
LTTOM
\end{tabular}} & \multirow{2}{*}{ 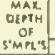 } & \multirow{2}{*}{\multicolumn{2}{|c|}{$\begin{array}{l}\text { wave } \\
\text { onseavations }\end{array}$}} & \multirow{2}{*}{$\begin{array}{c}\text { NEA } \\
\text { IME } \\
\text { COOE }\end{array}$} & \multirow{2}{*}{ 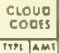 } & \multirow{2}{*}{\multicolumn{3}{|c|}{$\begin{array}{l}\text { NODC } \\
\text { STA1DON } \\
\text { NUMAE: }\end{array}$}} \\
\hline & & & & & & & Nento & \begin{tabular}{|c|} 
Cnuist \\
no. \\
015
\end{tabular} & $\begin{array}{l}\text { STATION } \\
\text { NuMAQG }\end{array}$ & & & & & & & & & \\
\hline & CS & 4400 & N 104 & $100 \mathrm{~W}$ & \begin{tabular}{l|l|}
149 & 41
\end{tabular} & \begin{tabular}{l|l|l}
03 & 13
\end{tabular} & $128 \mid 1766$ & \begin{tabular}{|l|l|}
015 & 011 \\
\end{tabular} & 4 & $\frac{10 \pi \mathrm{m}}{4554}$ & 17 & 241 & \begin{tabular}{l|l|l}
1 & 3
\end{tabular} & $\times 2$ & \begin{tabular}{|l|l|}
6 & 8 \\
\end{tabular} & & & 011 \\
\hline & & & & & WAT & & WIND & 10- AII TEMAP. & & \begin{tabular}{|l|} 
No. \\
\end{tabular} & & & & & & & & \\
\hline & & & & & $\begin{array}{ll}\text { Colo: } \\
\text { COOE }\end{array}$ & 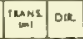 & $\begin{array}{l}\text { sinto } \\
\text { oo } \\
\text { oose }\end{array}$ & \begin{tabular}{l|l} 
\\
on \\
ont
\end{tabular} & $\begin{array}{ll}\text { WE1 } \\
\text { uiv coof }\end{array}$ & oins. & oustive & Anows & & & & & & \\
\hline & & & & & & 35 & 518 & 133 & & 14 & & & & & & & & \\
\hline & 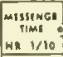 & CeAst & $\begin{array}{l}\text { CABO } \\
\text { TYME }\end{array}$ & OLPTN $(m)$ & $1 \tau$ & $s \%$ & SIGMA-T & 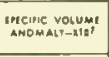 & 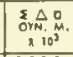 & $\begin{aligned} 50 u \\
\text { veto } \\
\end{aligned}$ & & $0, \mathrm{~mL} / 1$ & 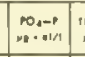 & $\begin{array}{l}\text { roraten } \\
\text { packin }\end{array}$ & 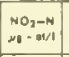 & $\begin{array}{l}\mathrm{MO}_{\mathrm{y}}-\mathrm{H} \\
\mathrm{m}=\mathrm{M}_{1 / 1}\end{array}$ & 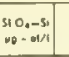 & ,H \\
\hline & & & STO & 0000 & 1465 & 3594 & 2679 & 0012639 & 0000 & 150 & 674 & & & & & & & \\
\hline & 128 & & OBS & 0000 & 1465 & 35942 & 2679 & & & 150 & 074 & & & & & & & \\
\hline & & & STO & 0010 & 1464 & 3594 & 2680 & 0012639 & 0013 & & 976 & & & & & & & \\
\hline & & & STO & 0020 & 1463 & 3595 & 2680 & 0012632 & 0025 & & 977 & & & & & & & \\
\hline & 128 & & 085 & 0027 & 1402 & 35946 & 2680 & & & 150 & 378 & & & & & & & \\
\hline & & & SIO & 0030 & 1462 & 3595 & 2680 & 0012652 & 0038 & 150 & 78 & & & & & & & \\
\hline & & & STO & 0050 & 1465 & 3594 & 2679 & 0012792 & 0063 & 150 & 82 & & & & & & & \\
\hline & 128 & & OBS & 0053 & 1465 & 35940 & 2679 & & & 150 & 83 & & & & & & & \\
\hline & & & SIO & 0075 & 1461 & 3594 & 2080 & 0012783 & 0095 & 150 & 85 & & & & & & & \\
\hline & 128 & & 085 & 0081 & 1460 & 35942 & 2680 & & & 150 & 386 & & & & & & & \\
\hline & & & STO & 0100 & 1459 & 3553 & 2680 & 0012904 & 0227 & 15 & 089 & & & & & & & \\
\hline & 128 & & 085 & 0107 & 1458 & 35926 & 2680 & & & 15 & 689 & & & & & & & \\
\hline & & & SIO & 0125 & 1444 & 3590 & 2681 & 0012887 & 0160 & 15 & 88 & & & & & & & \\
\hline & & & SIO & 0150 & 1429 & 3587 & 2681 & 0012670 & 0192 & 150 & 87 & & & & & & & \\
\hline & 128 & & OBS & 0163 & 1422 & 35863 & 2682 & & & 15 & 086 & & & & & & & \\
\hline & & & SIO & 0200 & 1412 & 3585 & 2684 & 0012813 & 0256 & 15 & 389 & & & & & & & \\
\hline & 128 & & 085 & 10217 & 1400 & 35839 & 2684 & & & 150 & 990 & & & & & & & \\
\hline & & & STO & 0250 & 1393 & 3581 & 2685 & 0012861 & 0320 & 150 & 990 & & & & & & & \\
\hline & & & STO & 0300 & 1361 & 3576 & 2087 & 0012713 & 0384 & 250 & 288 & & & & & & & \\
\hline & 128 & & OBS & 0326 & 1339 & 35720 & 2689 & & & 150 & 984 & & & & & & & \\
\hline & & & STO & 0400 & 1251 & 3558 & 2696 & 0012128 & 0508 & 150 & 965 & & & & & & & \\
\hline & 128 & & 085 & T0437 & 1198 & 35508 & 2701 & & & & 052 & & & & & & & \\
\hline & & & SIO & 0500 & 1053 & 3535 & 2708 & 0011101 & 0625 & 150 & 024 & & & & & & & \\
\hline & 128 & & 085 & 0550 & 1001 & 35234 & 2715 & & & 14 & 998 & & & & & & & \\
\hline & & & STO & 0600 & 0885 & 3512 & 2726 & 0009430 & 0727 & 148 & 962 & & & & & & & \\
\hline & 128 & & OBS & 0602 & 0766 & 35008 & 2735 & & & 148 & 925 & & & & & & & \\
\hline & & & STO & 0700 & 0721 & 3500 & 2741 & $000794:$ & 0814 & 140 & 914 & & & & & & & \\
\hline & & & STO & 0800 & 0021 & 3497 & 2752 & 0006870 & 0888 & 148 & 391 & & & & & & & \\
\hline & 128 & & OAS & 0886 & 0554 & 34962 & 2760 & & & 148 & 378 & & & & & & & \\
\hline & & & STO & 0900 & 0547 & 3496 & 2761 & 0006044 & 0953 & 148 & 377 & & & & & & & \\
\hline & & & Sto & 1000 & 0504 & 3497 & 2766 & 0005567 & 1011 & 148 & 377 & & & & & & & \\
\hline & & & STo & 1100 & 0468 & 3497 & 2771 & 0005182 & 1065 & 148 & 979 & & & & & & & \\
\hline & 128 & & OBS & 51109 & 0465 & 34968 & 2771 & & & & 379 & & & & & & & \\
\hline & & & STO & 1200 & 0438 & 3496 & 2774 & 0004939 & 1115 & 148 & 883 & & & & & & & \\
\hline & & & STO & 1300 & 0415 & 3496 & 2776 & 0004782 & 2164 & 148 & 390 & & & & & & & \\
\hline & & & STO & 1400 & 0398 & 3495 & 2777 & 0004697 & 1211 & 148 & 900 & & & & & & & \\
\hline & & & STO & 1500 & 0388 & 3494 & 2778 & 0004699 & 1258 & 140 & 912 & & & & & & & \\
\hline & 128 & & OBS & $T 1060$ & 0386 & 34934 & 2777 & & & 149 & 938 & & & & & & & \\
\hline
\end{tabular}

\begin{tabular}{|c|c|c|c|c|c|c|c|c|c|c|c|c|c|c|}
\hline \multirow{3}{*}{ 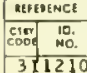 } & \multirow{2}{*}{$\begin{array}{l}\text { SMIP } \\
\text { CODE }\end{array}$} & \multirow[b]{2}{*}{$\begin{array}{l}\text { YATTUDE } \\
\text { y/10 }\end{array}$} & \multirow{2}{*}{ LONGIIUAE } & \multirow{2}{*}{$\begin{array}{l}\text { MAYSOEM } \\
\text { SOUAK: }\end{array}$} & \multirow{2}{*}{$\begin{array}{c}\text { SPA THON TIMMI } \\
\text { IGMTI }\end{array}$} & \multirow[b]{2}{*}{ YEAE } & \multicolumn{2}{|r|}{ OMIEINATOR'S } & \multirow{2}{*}{$\begin{array}{c}\text { OCHM } \\
10 \\
\text { :OTTOM }\end{array}$} & \multirow{2}{*}{\begin{tabular}{|c|} 
MAR \\
DEPTH \\
OI \\
$5^{2} M P L^{\prime} S$
\end{tabular}} & \multirow{2}{*}{$\begin{array}{c}\text { WAVEE } \\
\text { OASEVATONS }\end{array}$} & \multirow{2}{*}{$\begin{array}{c}\text { WEA- } \\
\text { PHE: } \\
\text { CODE }\end{array}$} & \multirow{2}{*}{ 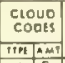 } & \multirow{2}{*}{$\begin{array}{l}\text { NODC } \\
\text { STANION } \\
\text { NUMIER } \\
\end{array}$} \\
\hline & & & & & & & $\begin{array}{c}\text { EuUis: } \\
\text { No. }\end{array}$ & $\begin{array}{l}\text { SPATION } \\
\text { NUMUIV }\end{array}$ & & & & & & \\
\hline & CS & $4401 \mathrm{~N}$ & 04059 W & 14940 & \begin{tabular}{|l|l|l|}
03 & 14 & 129
\end{tabular} & 1968 & 015 & 012 & 4572 & 15 & 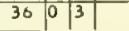 & $x 1$ & \begin{tabular}{|l|l|}
6 & 5 \\
\end{tabular} & 0012 \\
\hline
\end{tabular}

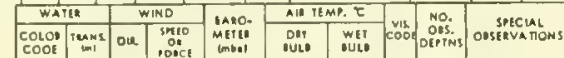

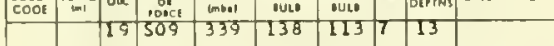

\begin{tabular}{|c|c|c|c|c|c|c|c|c|c|c|c|c|c|c|c|}
\hline 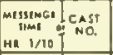 & $\begin{array}{l}\text { CARO } \\
\text { TYP! }\end{array}$ & OEPTN (m) & $7 t$ & $s \%$ & SHGMA-T & 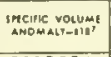 & 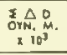 & $\begin{array}{l}\text { SOUNO } \\
\text { VELOCIIS }\end{array}$ & $0, \mathrm{ml} / 1$ & 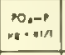 & 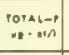 & 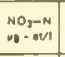 & 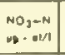 & $\begin{array}{l}\$ 10,-51 \\
n_{8}-\infty .1 / 1\end{array} \mid$ & AN \\
\hline \multirow{4}{*}{$129^{\circ}$} & STo & 0000 & 1492 & 3599 & 2677 & 0012836 & 0000 & 15084 & & & & & & & \\
\hline & OBS & 0000 & 1492 & 35992 & 2077 & & & 15084 & & & & & & & \\
\hline & SIO & 0010 & 1491 & 3599 & 2677 & 0012857 & 0013 & 15085 & & & & & & & \\
\hline & STO & 0020 & 1490 & 3599 & 2677 & 0012879 & 0026 & 15086 & & & & & & & \\
\hline \multirow[t]{3}{*}{129} & OBS & 0025 & 1490 & 35990 & 2677 & & & 15087 & & & & & & & \\
\hline & SIO & 0030 & 1490 & 3599 & 2677 & 0012905 & 0039 & 15088 & & & & & & & \\
\hline & STO & 0050 & 1491 & 3559 & 2677 & 0012983 & 0064 & 15091 & & & & & & & \\
\hline \multirow[t]{2}{*}{129} & OBS & 0051 & 1491 & 35990 & 2077 & & & 15092 & & & & & & & \\
\hline & STO & 0075 & 1487 & 3599 & 2878 & 0012973 & 0097 & 15094 & & & & & & & \\
\hline \multirow[t]{2}{*}{129} & 085 & 0076 & 1487 & 35991 & 2678 & & & 15094 & & & & & & & \\
\hline & STo & 0100 & 1488 & 3599 & 2678 & 0013071 & 0129 & 15099 & & & & & & & \\
\hline \multirow[t]{3}{*}{129} & aBs & 0202 & 1488 & 35986 & 2678 & & & 15099 & & & & & & & \\
\hline & SID & 0125 & 1490 & 3599 & 2677 & 0013219 & 0162 & 15103 & & & & & & & \\
\hline & ST 0 & 0150 & 1492 & 3599 & 2677 & 0013337 & 0196 & 15108 & & & & & & & \\
\hline \multirow[t]{2}{*}{129} & OBS & 0151 & 1492 & 35986 & 2677 & & & 15108 & & & & & & & \\
\hline & STO & 0200 & 1455 & 3594 & 2681 & 0013047 & 0262 & 15104 & & & & & & & \\
\hline \multirow[t]{3}{*}{127} & 085 & T 0202 & 1454 & 35936 & 2681 & & & 15104 & & & & & & & \\
\hline & SIO & 0250 & 1440 & 3589 & 2681 & 0013247 & 0327 & 15107 & & & & & & & \\
\hline & STO & 0300 & 1412 & 3583 & 2682 & 0013249 & 0393 & 15105 & & & & & & & \\
\hline \multirow[t]{2}{*}{129} & O BS & 0303 & 1410 & 35829 & 2682 & & & 15105 & & & & & & & \\
\hline & STO & 0400 & 1317 & 3568 & 2690 & 0012695 & 0523 & 15088 & & & & & & & \\
\hline \multirow[t]{2}{*}{129} & 085 & $T 0402$ & 1314 & 35075 & 2690 & & & 15088 & & & & & & & \\
\hline & STO & 0500 & 1154 & 3544 & 2704 & 0011561 & 0644 & 15046 & & & & & & & \\
\hline \multirow[t]{2}{*}{129} & 085 & 0505 & 1144 & 35433 & 2705 & & & 15044 & & & & & & & \\
\hline & STO & 0600 & 0929 & 3527 & 2730 & 0009055 & 0748 & 14980 & & & & & & & \\
\hline \multirow[t]{3}{*}{129} & OBS & T0607 & 0915 & $3412 P$ & 26430 & & & & & & & & & & \\
\hline & STO & 0700 & 0700 & 3512 & 2745 & 0007645 & 0831 & 14931 & & & & & & & \\
\hline & STO & 0800 & 0619 & 3500 & 2755 & 0006621 & 0902 & 14890 & & & & & & & \\
\hline 129 & OBS & 0813 & 0603 & 34988 & 2756 & & & 14886 & & & & & & & \\
\hline 129 & $O B S$ & T 1534 & 0397 & 34933 & 2776 & & & 14921 & & & & & & & \\
\hline
\end{tabular}


TABLE VI.-Continued

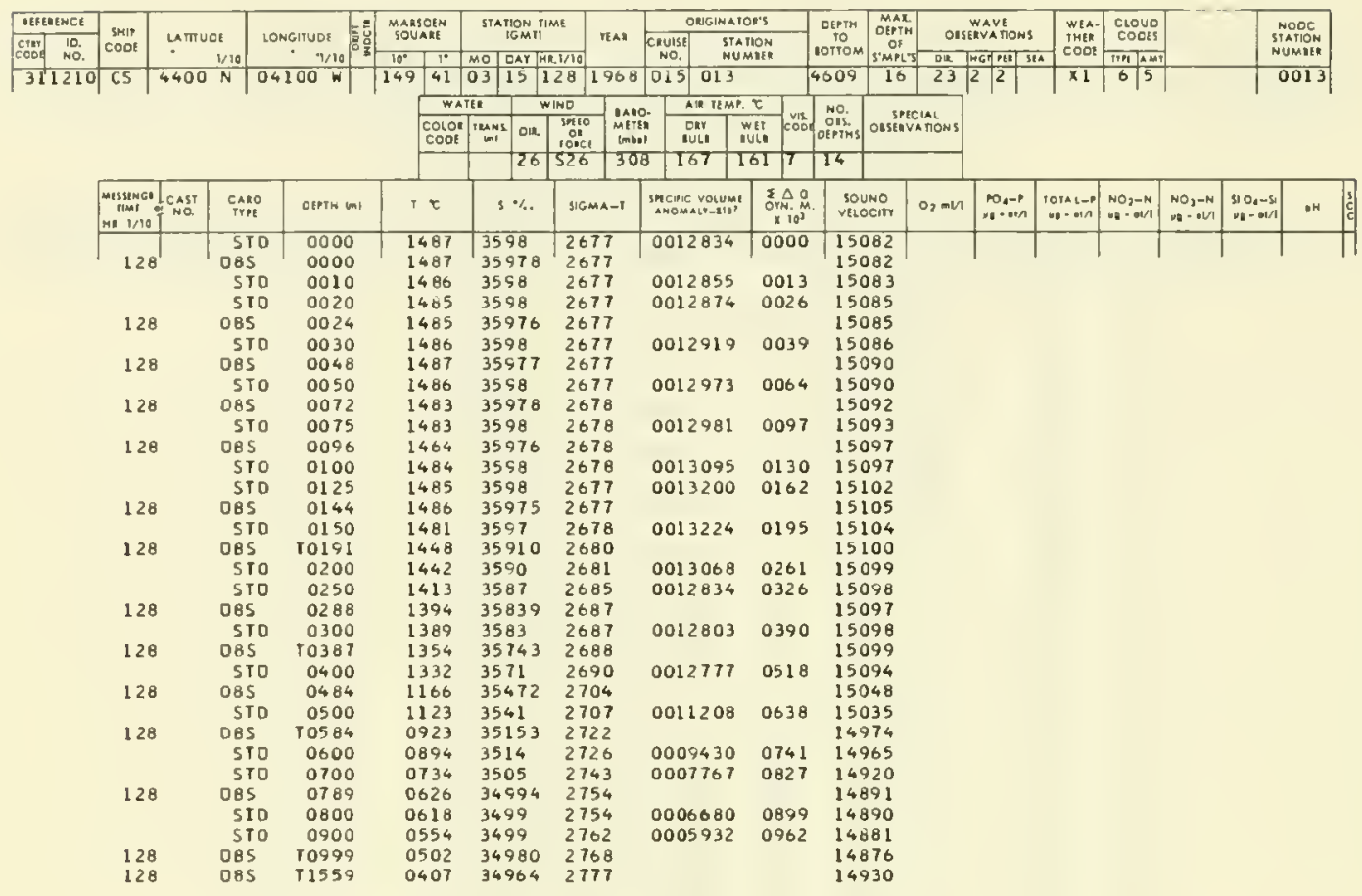

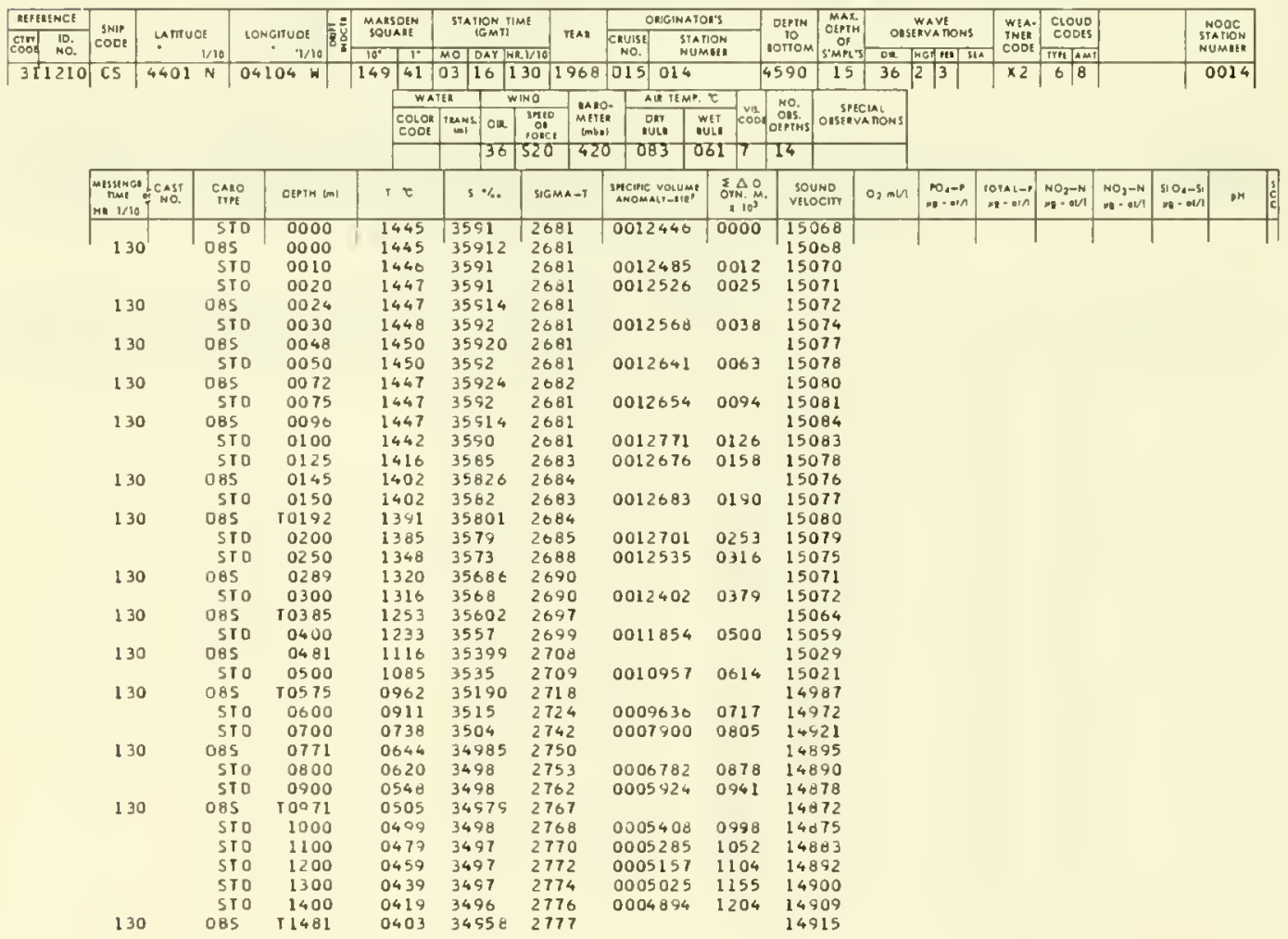


TABLE VI.-Continued

\begin{tabular}{|c|c|c|c|c|c|c|c|c|c|c|c|c|c|c|c|c|c|c|}
\hline \begin{tabular}{|l} 
Reget: NCI \\
\end{tabular} & \multirow{2}{*}{$\begin{array}{l}S N \mathrm{NIP} \\
C O D E\end{array}$} & \multirow{2}{*}{\multicolumn{2}{|c|}{$\begin{array}{l}\text { LATITLDE } \\
1 / 10\end{array}$}} & \multirow{2}{*}{ LONGITUDE } & $\begin{array}{l}\text { MARSDEN } \\
\text { SOUARE }\end{array}$ & STATION TIME & \multirow[b]{2}{*}{ veat } & \multicolumn{2}{|c|}{ ONGINATOR'S } & \multirow{2}{*}{\begin{tabular}{|l|} 
DEPH \\
TO \\
DOTOM M
\end{tabular}} & \multirow{2}{*}{ 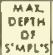 } & \multicolumn{2}{|c|}{$\begin{array}{l}\text { WAVE } \\
\text { desenvations }\end{array}$} & \multirow{2}{*}{$\begin{array}{c}\text { WEA- } \\
\text { PHEQ } \\
\text { CODE }\end{array}$} & \multirow{2}{*}{\begin{tabular}{|l|} 
CLOUD \\
CODHS \\
\end{tabular}} & & & \multirow{2}{*}{$\begin{array}{l}\text { MODC } \\
\text { STATION } \\
\text { NUMALER }\end{array}$} \\
\hline \begin{tabular}{|l|l|} 
CInT & it. \\
Coge & ND. \\
\end{tabular} & & & & & \begin{tabular}{l|l}
\multicolumn{2}{c}{ SouAnt } \\
$10^{6}$ & $1^{\circ}$
\end{tabular} & \begin{tabular}{|l|l|} 
& EGMV11 \\
WO DAYY
\end{tabular} & & \begin{tabular}{|c|c|} 
CNUISE & STAP \\
\end{tabular} & & & & Du & 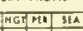 & & & & & \\
\hline & \multirow[t]{4}{*}{$\longdiv { C 5 }$} & \multirow[t]{4}{*}{4400} & \multirow[t]{4}{*}{$N$} & $04100 \mathrm{~W}$ & \begin{tabular}{|l|l|}
149 & 41 \\
\end{tabular} & \begin{tabular}{|l|l|}
03 & 17 \\
\end{tabular} & \begin{tabular}{|l|l|}
130 & 1968 \\
\end{tabular} & \begin{tabular}{l|l|}
015 & 015
\end{tabular} & & 4590 & 15 & 32 & \begin{tabular}{|l|l|}
1 & 4
\end{tabular} & $\times 2$ & \begin{tabular}{l|l}
0 & 8 \\
\end{tabular} & & & 0015 \\
\hline & & & & & WA & Iter & WINO IAxC & AL TEAP. & & No. & & & & & & & & \\
\hline & & & & & $\begin{array}{l}\text { Colot } \\
\text { CODt }\end{array}$ & 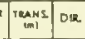 & 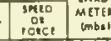 & \begin{tabular}{c|c} 
Ont & W \\
Iuts & in
\end{tabular} & vis coos & DE OES. & orseren & А понь & & & & & & \\
\hline & & & & & & 34 & 503 & 106 & \begin{tabular}{l|l}
72 & 1
\end{tabular} & 14 & & & & & & & & \\
\hline & $\begin{array}{l}\text { Messimas } \\
\text { THWL } \\
\text { He } 1 / 10\end{array}$ & TOSO & $\underset{T \text { TARE }}{\text { CARD }}$ & DEPTH \&mI & $T \tau$ & $s \cdot \%$ & SIGMA-1 & $\begin{array}{l}\text { SHene volum } \\
\text { ANGMAIT-210' }\end{array}$ & 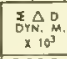 & $\underset{\text { Seu }}{\text { SeL }}$ & $\begin{array}{l}\text { UND } \\
\text { OCIIT }\end{array}$ & $0_{2} \mathrm{mb} / 1$ & 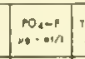 & 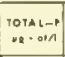 & $\left|\begin{array}{l}\mathrm{NO}^{3}-\mathrm{N} \\
\mathrm{No}-\mathrm{OQt} / \mathrm{t}\end{array}\right|$ & 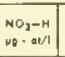 & $\begin{array}{l}510_{4}-s_{1} \\
\mathrm{~W}=\mathrm{sil}\end{array}$ & $\mathrm{NH}$ \\
\hline & & & STO & 0000 & 1479 & 3559 & 2680 & 0012557 & 0000 & 150 & 079 & & & & & & & \\
\hline & 130 & & $\begin{array}{l}085 \\
\text { STO }\end{array}$ & $\begin{array}{l}0000 \\
0010\end{array}$ & $\begin{array}{l}1479 \\
1479\end{array}$ & $\begin{array}{l}35993 \\
3559\end{array}$ & $\begin{array}{l}2680 \\
2680\end{array}$ & 0012596 & 0013 & $\begin{array}{l}150 \\
150\end{array}$ & $\begin{array}{l}079 \\
081\end{array}$ & & & & & & & \\
\hline & & & 5TO & $\begin{array}{l}0010 \\
0020\end{array}$ & $\begin{array}{l}1479 \\
1480\end{array}$ & 3559 & 2680 & 0012635 & 0025 & 150 & 083 & & & & & & & \\
\hline & 130 & & DBS & 0024 & 1480 & 35993 & 2680 & & & 150 & 084 & & & & & & & \\
\hline & & & STO & 0030 & 1482 & 3599 & 2679 & 0012733 & 0038 & 150 & 085 & & & & & & & \\
\hline & 130 & & 085 & 0049 & 1484 & 35989 & 2679 & & & 150 & 089 & & & & & & & \\
\hline & & & STO & 0050 & 1484 & 3599 & 2679 & 0012836 & 0063 & 150 & 089 & & & & & & & \\
\hline & 130 & & 085 & 0073 & 1480 & 35991 & 2680 & & & 150 & 092 & & & & & & & \\
\hline & & & STO & 0075 & 1480 & 3559 & 2680 & 0012826 & 0096 & 150 & 092 & & & & & & & \\
\hline & 130 & & 085 & 0098 & 1482 & 35990 & 2679 & & & 150 & 096 & & & & & & & \\
\hline & & & STO & 0100 & 1462 & 3599 & 2679 & 0012949 & 0128 & 150 & 097 & & & & & & & \\
\hline & & & STO & 0125 & 1484 & 3599 & 2679 & 0013044 & 0160 & 151 & 101 & & & & & & & \\
\hline & 130 & & 085 & 0147 & 1485 & 35994 & 2679 & & & 151 & 105 & & & & & & & \\
\hline & & & STo & 0150 & 1485 & 3559 & 2679 & 0013133 & 0193 & 151 & 106 & & & & & & & \\
\hline & 130 & & 085 & T0195 & 1484 & 35993 & 2679 & & & 151 & 113 & & & & & & & \\
\hline & & & ST0 & 0200 & 1483 & 3559 & 2679 & 0013271 & 0259 & 151 & 113 & & & & & & & \\
\hline & & & 570 & 0250 & 1459 & 3593 & 2680 & 0013353 & 0326 & 152 & 113 & & & & & & & \\
\hline & 130 & & 085 & 0294 & 1425 & 35868 & 2682 & & & 151 & 109 & & & & & & & \\
\hline & & & STO & 0300 & 1418 & 3586 & 2693 & 0013155 & 0392 & 151 & 107 & & & & & & & \\
\hline & 130 & & 085 & $T 0395$ & 1296 & 35657 & 2693 & & & 150 & 080 & & & & & & & \\
\hline & & & STO & 0400 & 1289 & 3565 & 2694 & 0012359 & 0519 & 150 & 079 & & & & & & & \\
\hline & 130 & & 085 & 0492 & 1141 & 35420 & 2705 & & & 150 & 040 & & & & & & & \\
\hline & & & ST & 0500 & 1122 & 3540 & 2707 & 0011262 & 0637 & 150 & 035 & & & & & & & \\
\hline & 130 & & 085 & T0586 & 0933 & 35172 & 2721 & & & 149 & 980 & & & & & & & \\
\hline & & & STo & 0600 & 0909 & 3515 & 2724 & 0009603 & 0742 & 149 & 971 & & & & & & & \\
\hline & & & $5 T 0$ & 0700 & 0730 & 3504 & 2743 & 0007780 & 0829 & 149 & 710 & & & & & & & \\
\hline & 130 & & 085 & 0789 & 0610 & 34970 & 2754 & & & 148 & 385 & & & & & & & \\
\hline & & & STO & 0800 & 0601 & 3497 & 2755 & 0006591 & 0901 & 148 & 883 & & & & & & & \\
\hline & & & STO & 0900 & 0531 & 3496 & 2763 & 0005844 & 0963 & 148 & 971 & & & & & & & \\
\hline & 130 & & 085 & 10993 & 0478 & 34950 & 2768 & & & 148 & 865 & & & & & & & \\
\hline & & & STO & 1000 & 0477 & 3495 & 2768 & 0005322 & 1019 & 148 & 865 & & & & & & & \\
\hline & & & 510 & 1100 & 0461 & 3454 & 2769 & 0005290 & 1072 & 148 & 975 & & & & & & & \\
\hline & & & 5TO & 1200 & 0444 & 3494 & 2771 & 0005180 & 1124 & 148 & 85 & & & & & & & \\
\hline & & & 5To & 1300 & 0428 & 3494 & 2773 & 0005084 & 1175 & 148 & 395 & & & & & & & \\
\hline & & & $5 T 0$ & 1400 & 0411 & 3494 & 2775 & 0004944 & 1225 & 149 & 305 & & & & & & & \\
\hline & 130 & & DBS & T 1481 & 0399 & 34940 & 2776 & & & 149 & 913 & & & & & & & \\
\hline
\end{tabular}

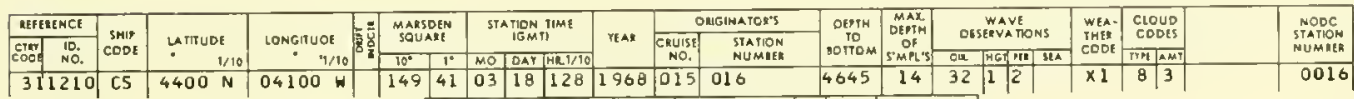

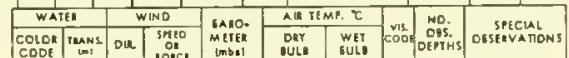

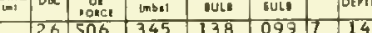

\begin{tabular}{|c|c|c|c|c|c|c|c|c|c|c|c|c|c|c|c|}
\hline 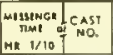 & $\underset{\text { THYI }}{\text { CAlD }}$ & DEPTN UnI & $i c$ & $5 \%$ & SIGMA-T & 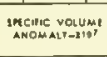 & 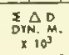 & $\begin{array}{l}\text { SOUND } \\
\text { veloeiry }\end{array}$ & $0, \pi / 4$ & 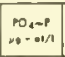 & 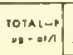 & 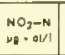 & 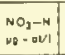 & $\begin{array}{l}510,0<-58 \\
080-01 / 10\end{array}$ & 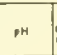 \\
\hline \multirow{4}{*}{$128^{\circ}$} & $5 T 0$ & 0000 & 1419 & 3587 & 2684 & 0012208 & 0000 & 15059 & & & & & & & \\
\hline & 085 & 0000 & 1419 & 35872 & 2684 & & & 15059 & & & & & & & \\
\hline & $5 T 0$ & 0010 & 1421 & 3588 & 2684 & 0012262 & 0012 & 15061 & & & & & & & \\
\hline & STo & 0020 & 1424 & 3588 & 2683 & 0012322 & 0025 & 15064 & & & & & & & \\
\hline \multirow[t]{2}{*}{128} & 085 & 0022 & 1424 & 35878 & 2683 & & & 15064 & & & & & & & \\
\hline & STO & 0030 & 1426 & $358 \mathrm{\theta}$ & 2683 & 0012403 & 0037 & 15066 & & & & & & & \\
\hline \multirow[t]{2}{*}{128} & 085 & 0045 & 1427 & 35875 & 2682 & & & 15069 & & & & & & & \\
\hline & STO & 0050 & 1426 & 3588 & 2682 & 0012477 & 0062 & 15069 & & & & & & & \\
\hline \multirow[t]{2}{*}{128} & 085 & 0065 & 1423 & 35876 & 2683 & & & 15071 & & & & & & & \\
\hline & $5 T 0$ & 0075 & 1424 & 3588 & 2683 & 0012485 & 0093 & 15073 & & & & & & & \\
\hline \multirow[t]{3}{*}{128} & 085 & 0086 & 1424 & 35878 & 2683 & & & 15075 & & & & & & & \\
\hline & STO & 0100 & 1422 & 3588 & 2684 & 0012506 & 0124 & 15076 & & & & & & & \\
\hline & STD & 0125 & 1420 & 3588 & 2684 & 0012539 & 0156 & 15080 & & & & & & & \\
\hline \multirow[t]{2}{*}{128} & DB5 & 0130 & 1419 & 35881 & 2084 & & & 15080 & & & & & & & \\
\hline & 510 & 0150 & 1417 & 3588 & 2685 & 0012551 & 0187 & 15083 & & & & & & & \\
\hline \multirow[t]{3}{*}{128} & 085 & 10170 & 1415 & 35877 & 2685 & & & 15085 & & & & & & & \\
\hline & STO & 0200 & 1412 & 3587 & 2685 & 0012667 & 0250 & 15089 & & & & & & & \\
\hline & 510 & 0250 & 2401 & 3584 & 2685 & 0012806 & 0314 & 15093 & & & & & & & \\
\hline \multirow[t]{2}{*}{128} & 085 & 0258 & 1399 & 35834 & 2685 & & & 15094 & & & & & & & \\
\hline & $5 T 0$ & 0300 & 1391 & 3581 & 2685 & 0012903 & 0378 & $\$ 5098$ & & & & & & & \\
\hline \multirow[t]{2}{*}{128} & 085 & 10345 & $13+3$ & 35757 & 2687 & & & 15096 & & & & & & & \\
\hline & STO & 0400 & 1304 & 3560 & 2691 & 0012582 & 0506 & 15084 & & & & & & & \\
\hline \multirow[t]{2}{*}{128} & 085 & 0432 & 1253 & 35589 & 2696 & & & 15071 & & & & & & & \\
\hline & STO & 0500 & 1058 & 3537 & 2709 & 0011045 & 0624 & 15026 & & & & & & & \\
\hline \multirow[t]{3}{*}{128} & 085 & $r 0518$ & 1059 & 35321 & 2712 & & & 15014 & & & & & & & \\
\hline & STO & 0600 & 0880 & 3515 & 2729 & 0009129 & 0725 & 14960 & & & & & & & \\
\hline & STO & 0700 & 0711 & 3501 & 2743 & 0007719 & 0807 & 14910 & & & & & & & \\
\hline \multirow[t]{2}{*}{128} & 085 & 0702 & 0708 & 35006 & 2743 & & & 14709 & & & & & & & \\
\hline & STO & 0800 & 0611 & 3498 & 2754 & 0006656 & 0881 & 14887 & & & & & & & \\
\hline \multirow[t]{6}{*}{128} & OBS & T0894 & 0535 & 34906 & 2763 & & & 14872 & & & & & & & \\
\hline & 510 & 0900 & 0533 & 3497 & 2703 & 0005817 & 0943 & 14872 & & & & & & & \\
\hline & STO & 1000 & 0507 & 3456 & 2766 & 0005021 & 1000 & 14878 & & & & & & & \\
\hline & STD & 1100 & 0481 & 3496 & 2769 & 0005421 & 1056 & 14884 & & & & & & & \\
\hline & STO & 1200 & 0454 & 3495 & 2771 & 0005216 & 1109 & 14889 & & & & & & & \\
\hline & STO & 1300 & 0428 & 3455 & 2774 & 0005006 & 1160 & 14895 & & & & & & & \\
\hline 128 & $0 E 5$ & T1386 & 0405 & 34944 & 2776 & & & 14900 & & & & & & & \\
\hline
\end{tabular}


Table VI.-Continued

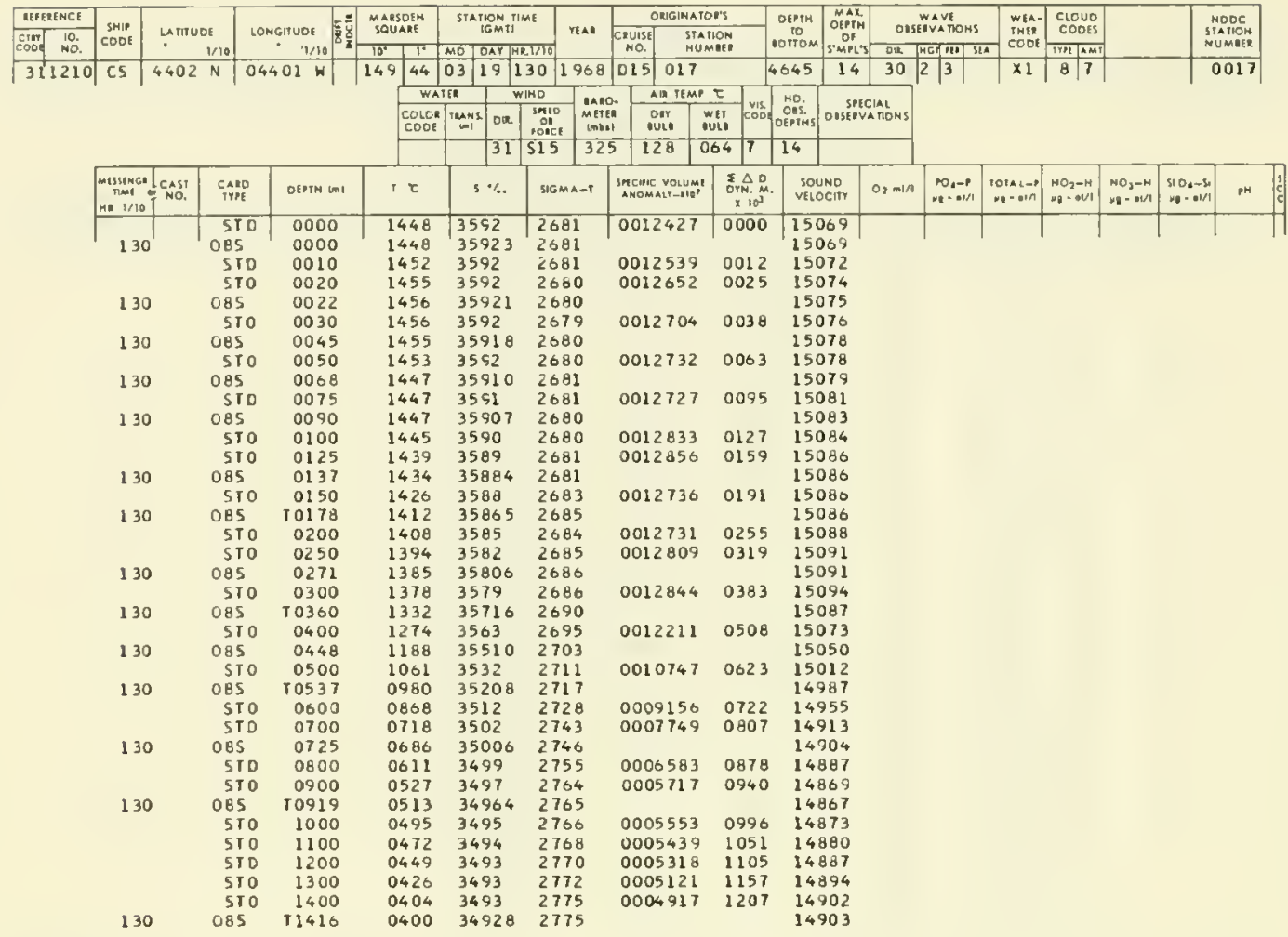


TABLE VII.-Observed and interpolated oceanographic data for stations taken by USCGC ABSECON at Ocean Station DELTA, 20 March-8 April 1968, prepared from NODC listing No. 31-1238 $\mathrm{AZ}$.

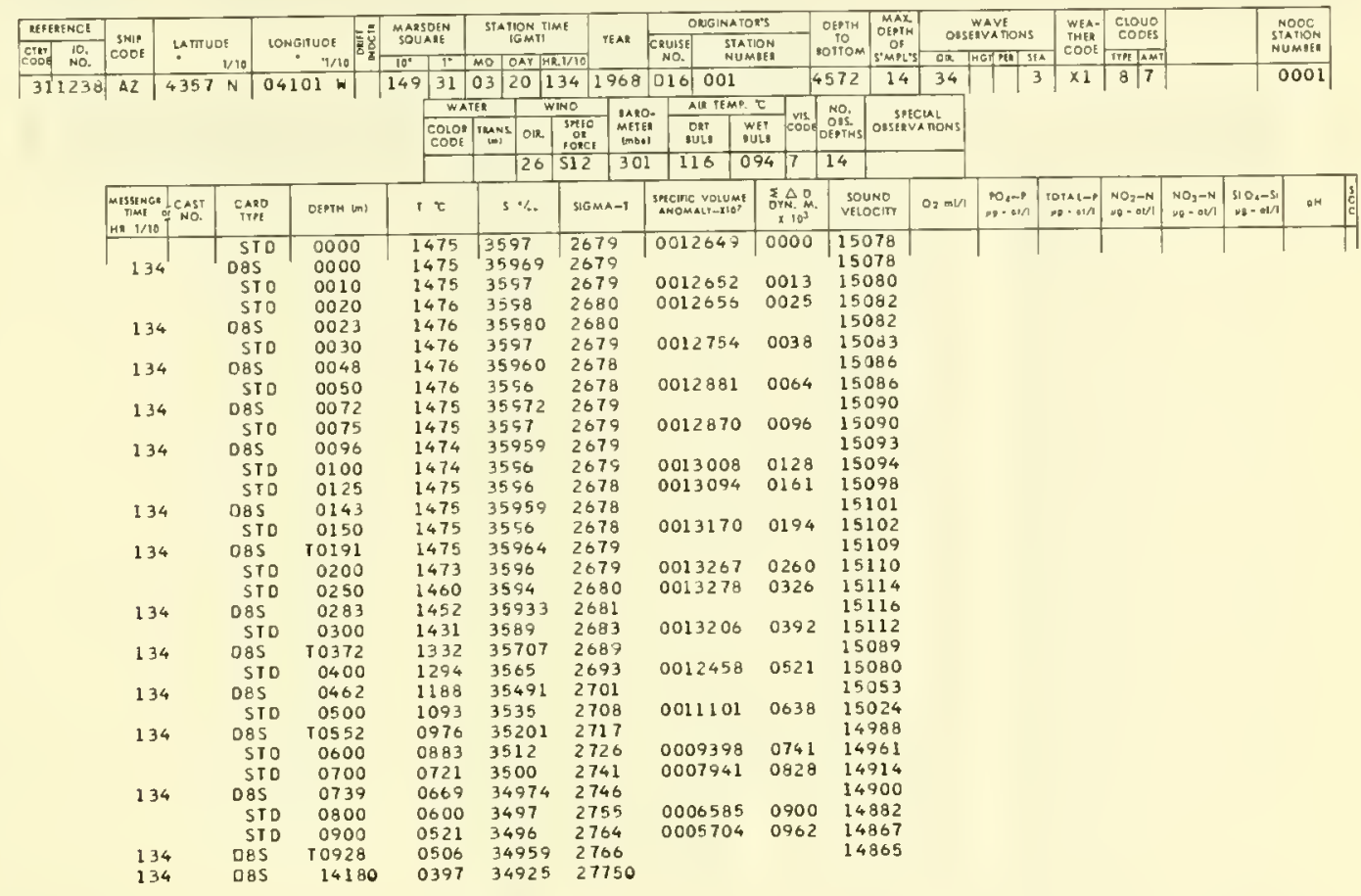

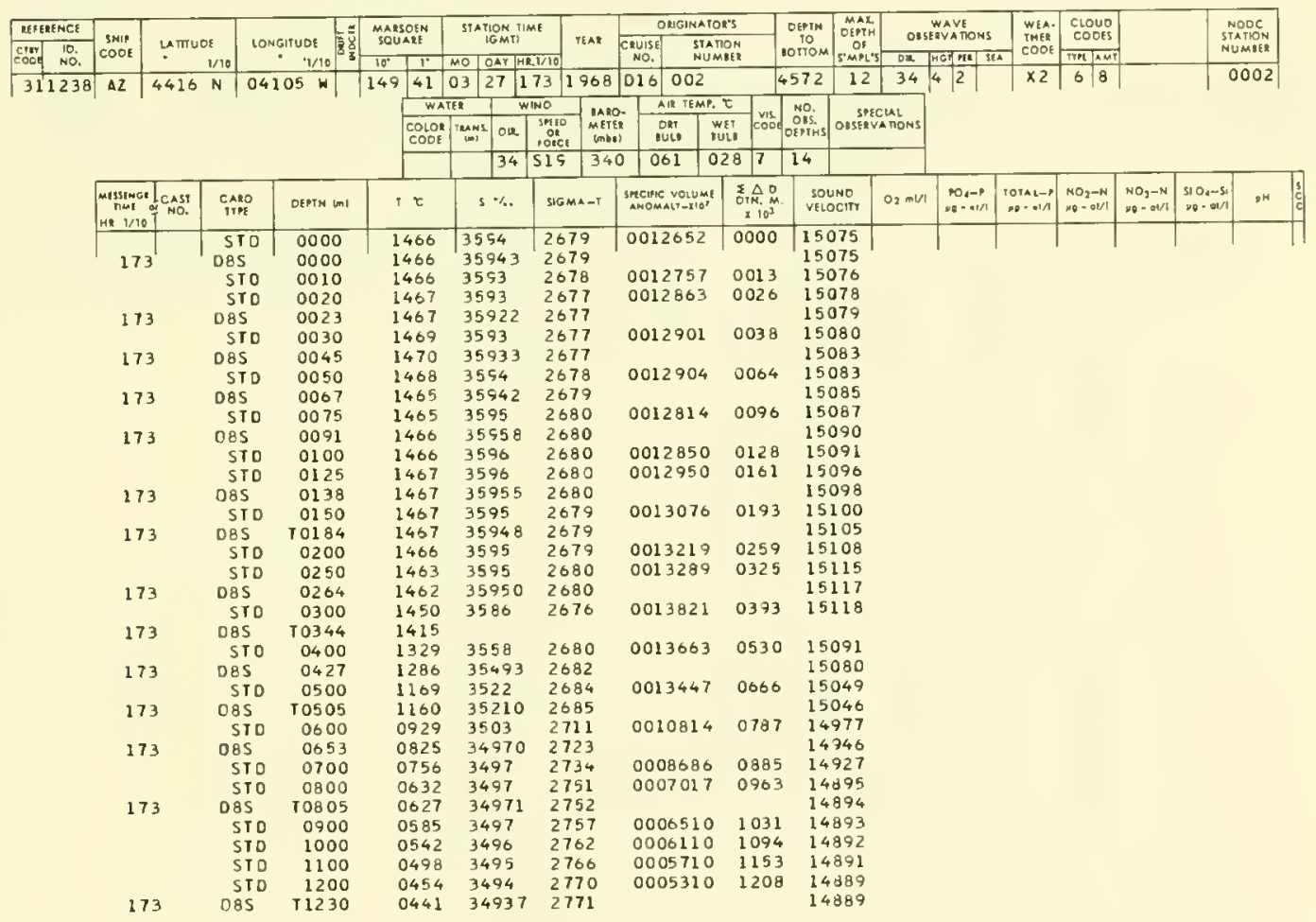


TABLE VII.-Continued

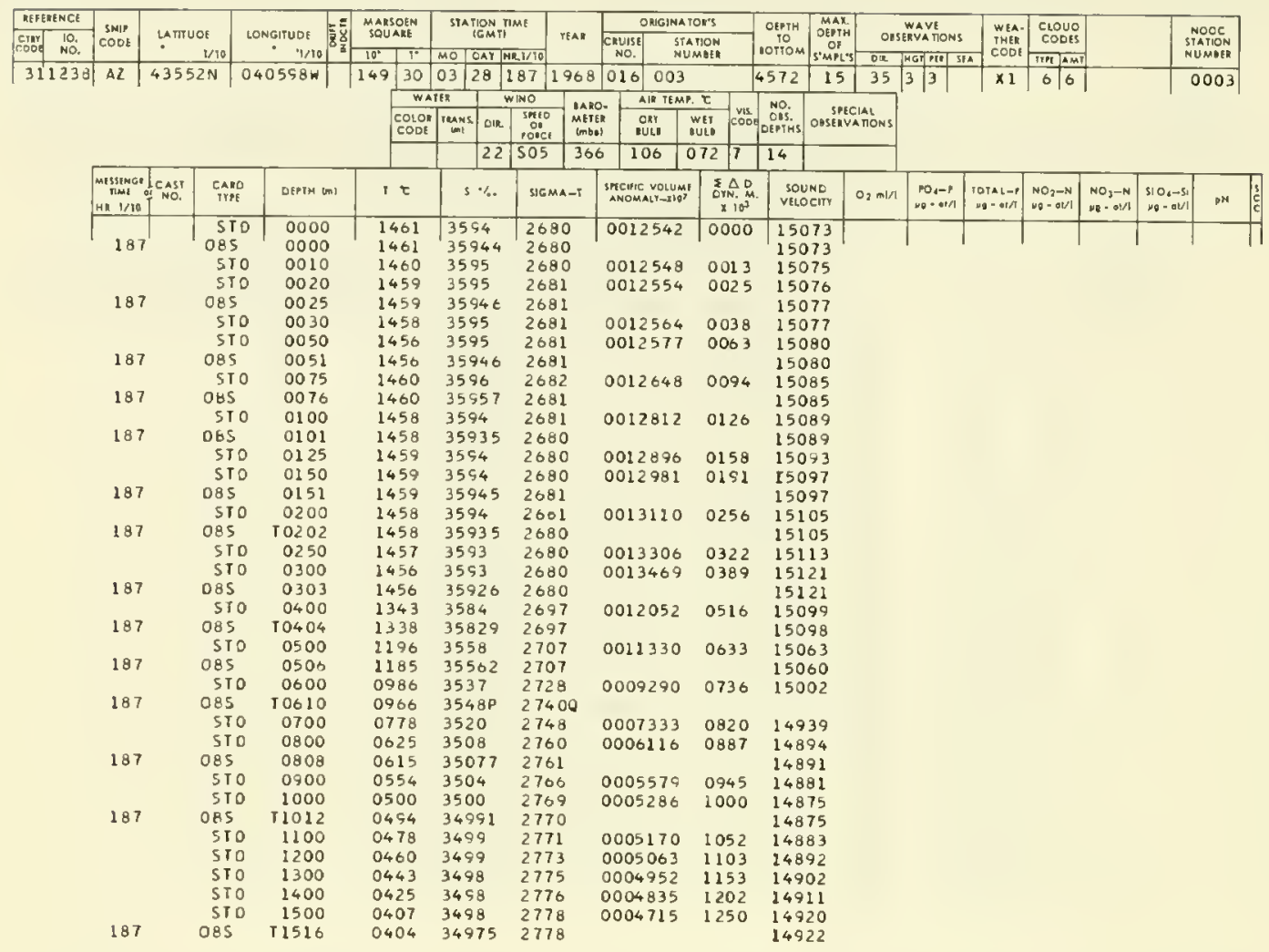

\begin{tabular}{|c|c|c|c|c|c|c|c|c|c|c|c|c|c|c|c|c|c|}
\hline AtFErENeE & \multirow{2}{*}{$\begin{array}{l}S A 1 * \\
\operatorname{SODE}\end{array}$} & \multirow{2}{*}{\multicolumn{2}{|c|}{$\begin{array}{c}\text { ymuot } \\
\text { 1/10 }\end{array}$}} & \multirow{2}{*}{ LONGITUOE } & \multirow{2}{*}{$\begin{array}{l}\text { MANSOEN } \\
\text { SOUAHE }\end{array}$} & \multirow{2}{*}{\multicolumn{2}{|c|}{\begin{tabular}{|c|} 
STATION TMME \\
IGMHI \\
\end{tabular}}} & \multicolumn{2}{|c|}{\begin{tabular}{|l|} 
ONginafor's \\
\end{tabular}} & \multirow{2}{*}{$\begin{array}{c}\text { orrin } \\
\text { so } \\
\text { otrom }\end{array}$} & \multirow{2}{*}{\begin{tabular}{|l|} 
MAXX \\
OEPTH \\
OI \\
SMAPL'S
\end{tabular}} & \multirow{2}{*}{$\begin{array}{c}\text { WAVE } \\
\text { OUSERYA NONS }\end{array}$} & \multirow{2}{*}{$\begin{array}{l}\text { WiA- } \\
\text { THER } \\
\text { CODE }\end{array}$} & \multirow{2}{*}{ 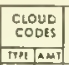 } & & & \multirow{2}{*}{$\begin{array}{l}\text { NOOC } \\
\text { STARHON } \\
\text { NUMASk }\end{array}$} \\
\hline Crog. & & & & & & & reat & $\begin{array}{l}\text { CRUISE } \\
\text { no. }\end{array}$ & $\begin{array}{l}\text { STANION } \\
\text { number }\end{array}$ & & & & & & & & \\
\hline \multirow[t]{39}{*}{$3 ! 1238$} & \begin{tabular}{|l|l}
$A 2$ &
\end{tabular} & 4345 & N & 04103 & \begin{tabular}{|l|l|}
149 & 31 \\
\end{tabular} & 0401 & \begin{tabular}{l|l}
185 & 1908
\end{tabular} & \begin{tabular}{|l|l|}
016 & 004 \\
\end{tabular} & & 4572 & 13 & 8 & $\times 1$ & \begin{tabular}{|l|l|}
8 & 6 \\
\end{tabular} & & & 0004 \\
\hline & & & & & WAI & 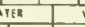 & \begin{tabular}{l|l} 
Wino & aro \\
\end{tabular} & 0. $A$ AR TEMP & $x$ & & & & & & & & \\
\hline & & & & & $\begin{array}{l}\text { cotor } \\
\text { coote }\end{array}$ & $\operatorname{limin}_{\ln 1}$ ore & 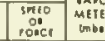 & \begin{tabular}{c|c} 
CRY \\
AULA
\end{tabular} & $\begin{array}{c}\text { WEI } \\
\text { BUt. }\end{array}$ & of 1 of & $\begin{array}{c}\text { SPECIAL } \\
\text { ORSEHVATIOMS }\end{array}$ & & & & & & \\
\hline & & & & & & 22 & 520 & 124 & \begin{tabular}{l|l}
090 & 7
\end{tabular} & 14 & & & & & & & \\
\hline & 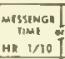 & $\begin{array}{l}\text { CAST } \\
\text { CNO. }\end{array}$ & $\underset{\text { TYMO }}{\text { CAMO }}$ & $D E P T M(m)$ & $r x$ & $s \%$ & SIGMA-T & $\begin{array}{l}\text { Smecinc volumi } \\
\text { ANOMALTSB:D" }\end{array}$ & $\begin{array}{l}\text { I } \\
\text { OrN. } 0 \\
x 10^{3}\end{array}$ & $\begin{array}{l}\text { soun } \\
\text { veLo }\end{array}$ & $\begin{array}{l}\text { JND } \\
\text { JEIT }\end{array}$ & 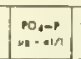 & 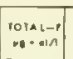 & 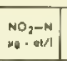 & $\mid \begin{array}{c}\mathrm{NO}_{3}-\mathrm{N} \\
\mathrm{H}-\mathrm{NON}\end{array}$ & 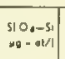 & $\mathbf{P M}$ \\
\hline & $185^{\prime}$ & & $\begin{array}{l}\text { STO } \\
085\end{array}$ & $\begin{array}{l}0000 \\
0000\end{array}$ & $\begin{array}{l}1459 \\
1459\end{array}$ & $\begin{array}{l}3600 \\
35999\end{array}$ & $\begin{array}{l}2676 \\
2676\end{array}$ & 0012932 & 0000 & $\begin{array}{l}150 \\
150\end{array}$ & & & & & & & \\
\hline & & & STO & 0010 & 1499 & $\begin{array}{l}35999 \\
3599\end{array}$ & $\begin{array}{l}2616 \\
2675\end{array}$ & 0013028 & 0013 & 150 & $\begin{array}{l}86 \\
087\end{array}$ & & & & & & \\
\hline & & & STO & 0020 & 1499 & 3559 & 2675 & $001308 d$ & 0026 & 150 & 89 & & & & & & \\
\hline & 185 & & DBS & 0020 & 1499 & 35586 & 2075 & & & 150 & 89 & & & & & & \\
\hline & & & SIO & 0030 & 1501 & 3559 & 2675 & 0013161 & 0039 & 150 & 991 & & & & & & \\
\hline & 185 & & 085 & 0043 & 1502 & 35985 & 2076 & & & 150 & 994 & & & & & & \\
\hline & & & STO & 0050 & 1501 & 3600 & 2676 & 0013123 & 0065 & 150 & 35 & & & & & & \\
\hline & 185 & & 085 & 0065 & 1699 & 36006 & 2677 & & & 150 & 997 & & & & & & \\
\hline & & & SIO & 0075 & 1489 & $35>8$ & 2677 & 0013091 & 0098 & 150 & 75 & & & & & & \\
\hline & 185 & & 085 & 0080 & 1478 & 35960 & 2678 & & & 150 & 93 & & & & & & \\
\hline & & & STO & 0100 & 2471 & 3593 & 2677 & 0013154 & 0131 & 150 & 93 & & & & & & \\
\hline & & & STO & 0125 & 1451 & 3587 & 2677 & 0013251 & 0164 & 150 & ac & & & & & & \\
\hline & 185 & & 085 & $0 \div 31$ & 1445 & 35855 & 2077 & & & 1508 & & & & & & & \\
\hline & & & STO & 0150 & 1410 & 3579 & 2679 & 0013065 & 0197 & 1507 & & & & & & & \\
\hline & 185 & & D\&S & T0174 & 1321 & 35730 & 2081 & & & 1507 & & & & & & & \\
\hline & & & SIO & 0200 & 1369 & 3576 & 2602 & 0012971 & 0202 & 1508 & & & & & & & \\
\hline & & & SIO & 0250 & 1403 & 3583 & 2004 & 0012958 & 0327 & 150 & & & & & & & \\
\hline & 185 & & 085 & 0260 & 1406 & 35838 & 2664 & & & 150 & & & & & & & \\
\hline & & & STO & 0300 & 1378 & 3577 & 2085 & 0012990 & 0392 & 150 & & & & & & & \\
\hline & 185 & & 085 & 10342 & i 354 & 35720 & 2686 & & & 150 & & & & & & & \\
\hline & & & 510 & 0400 & 1349 & 3570 & 2685 & 0013170 & 0523 & 1509 & & & & & & & \\
\hline & 185 & & OBS & 0422 & 1321 & 35696 & 2091 & & & $150^{\circ}$ & & & & & & & \\
\hline & 185 & & GRS & 10497 & 1109 & 35324 & 2703 & & & 1502 & & & & & & & \\
\hline & & & STO & 0500 & 1107 & 3532 & 2703 & 0011573 & 0646 & 1502 & & & & & & & \\
\hline & & & STO & 0600 & 0945 & 3521 & 2723 & $000 \$ 763$ & 0753 & 1498 & & & & & & & \\
\hline & 185 & & 085 & 0644 & 0877 & 35165 & 2730 & & & 1496 & & & & & & & \\
\hline & & & 510 & 0700 & 0767 & 3511 & 2740 & 0008133 & 0842 & 1494 & & & & & & & \\
\hline & & & STO & 0800 & 0652 & 3503 & 2753 & 0000868 & 0917 & 1490 & & & & & & & \\
\hline & 185 & & 085 & TOB 24 & 0624 & 35005 & 2755 & & & 1489 & & & & & & & \\
\hline & & & STO & 0900 & 0593 & 3500 & 2759 & 0006367 & 0984 & 1489 & & & & & & & \\
\hline & & & STO & 1000 & 0551 & 3500 & 2763 & 0005977 & 1045 & $148 y$ & & & & & & & \\
\hline & & & STO & 1100 & 0510 & 3499 & 2708 & 0005593 & 1103 & 1489 & & & & & & & \\
\hline & & & STO & 1200 & 0469 & 3498 & 2772 & 0005210 & 1157 & 1489 & & & & & & & \\
\hline & 185 & & OHS & T $127 \%$ & 0430 & 34574 & 2775 & & & 1489 & & & & & & & \\
\hline
\end{tabular}


Table VII.-Continued

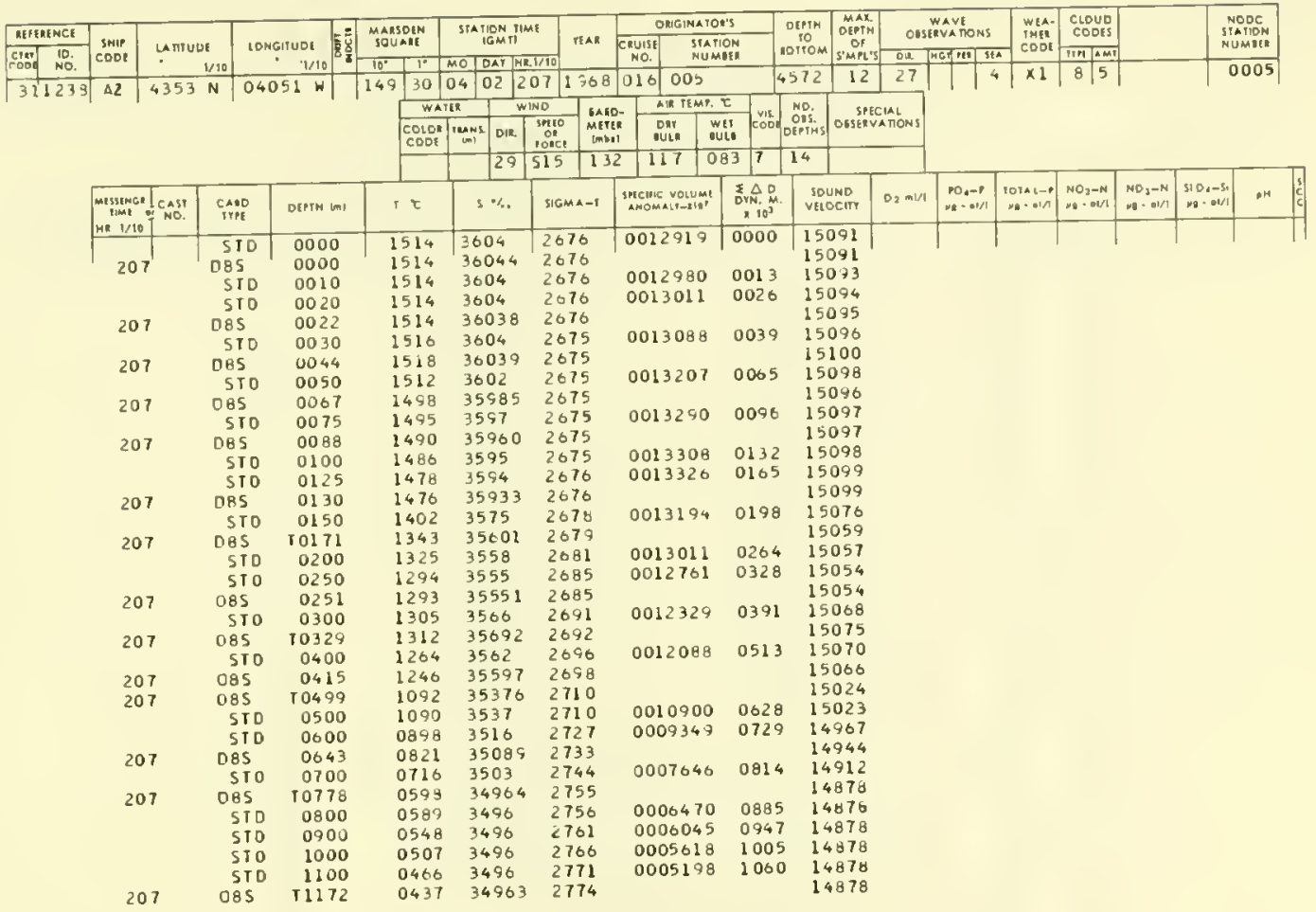

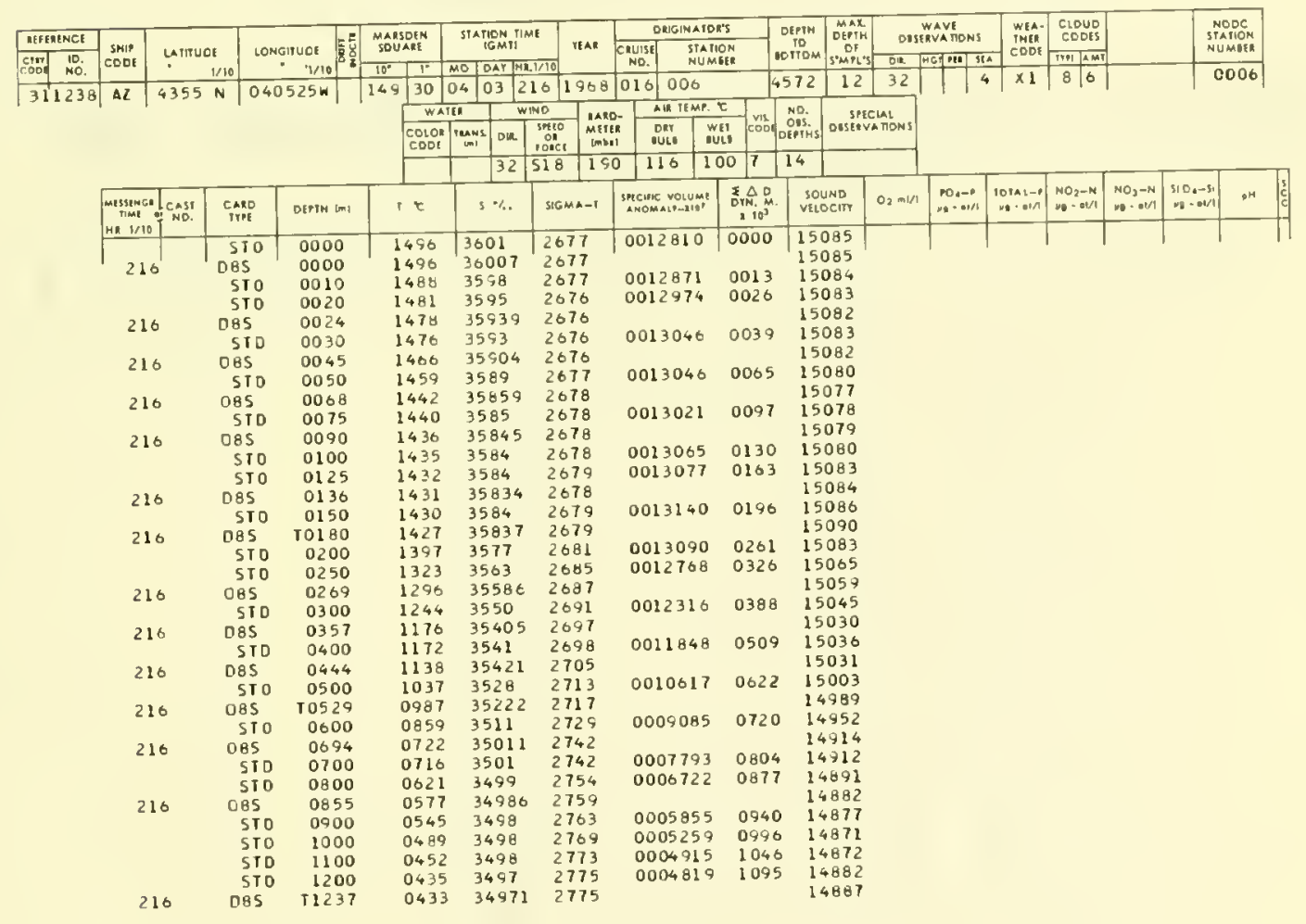


TABLE VII.-Continued

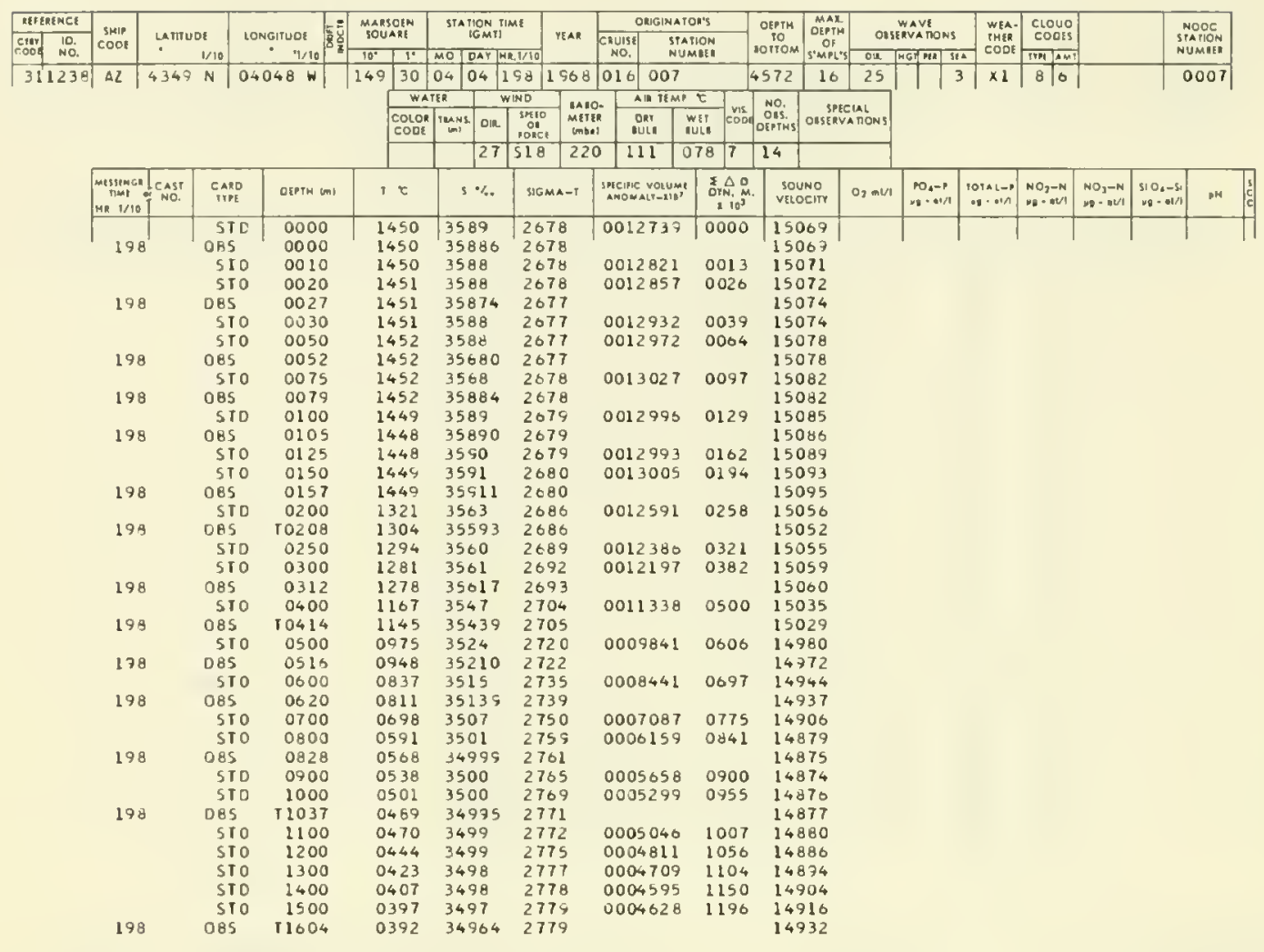

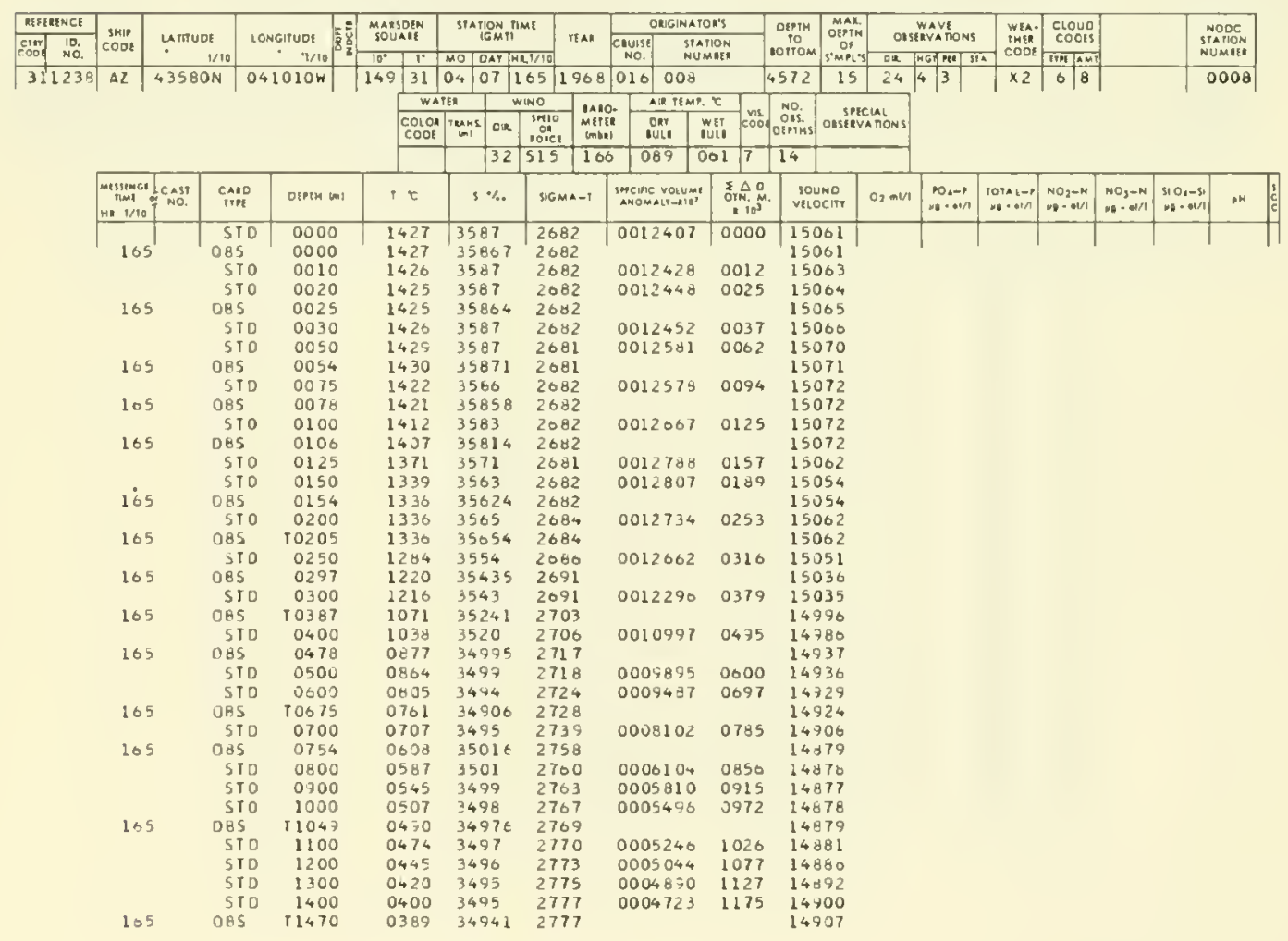


TABlE VII.-Continued

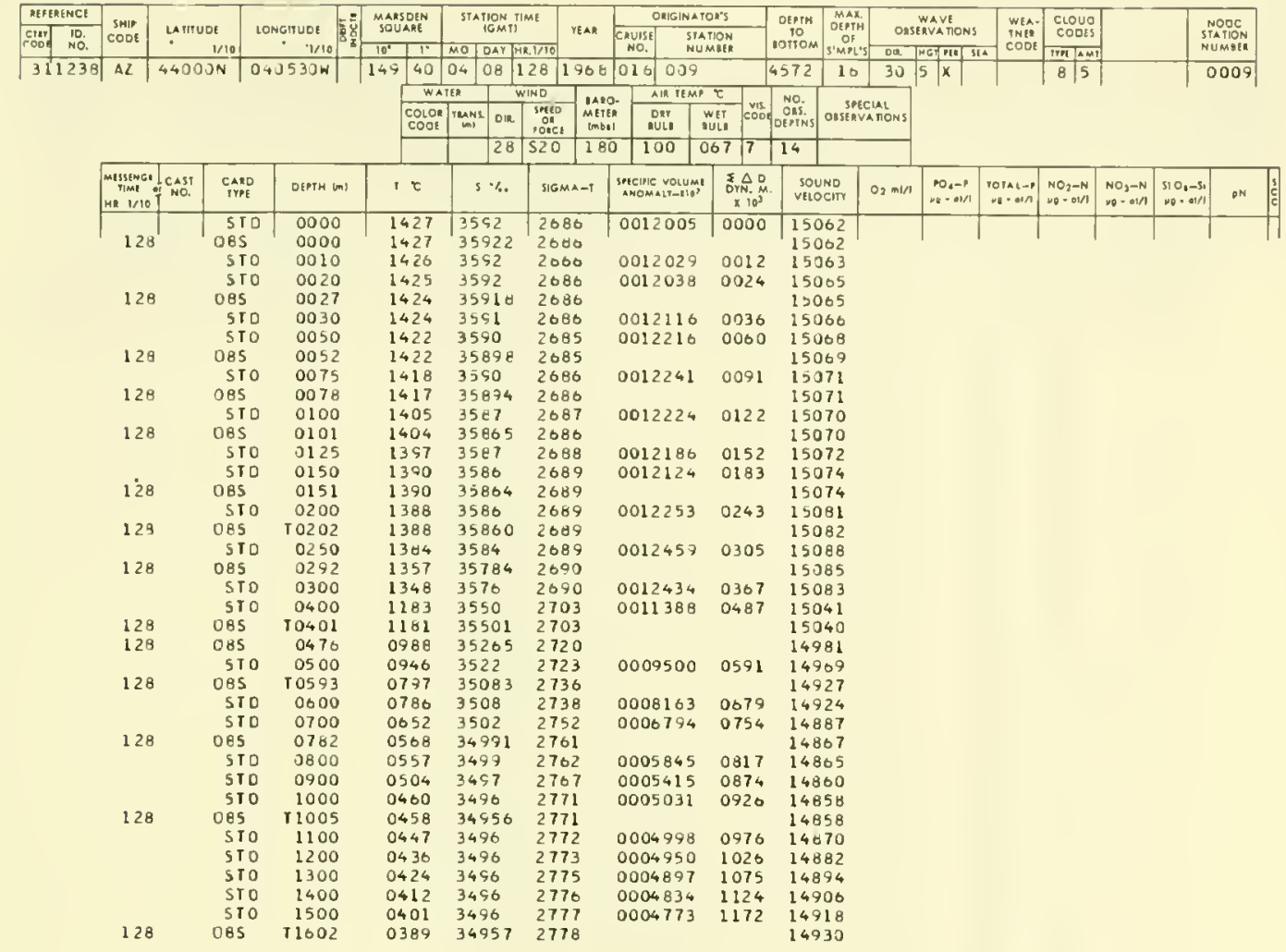


TABLE VIII.-Observed and interpolated oceanographic data for stations taken by USCGC COOK INLET at Ocean Station DELTA, 14 April-5 May 1968, prepared from NODC listing No. 31-1241 $\mathrm{CO}$.

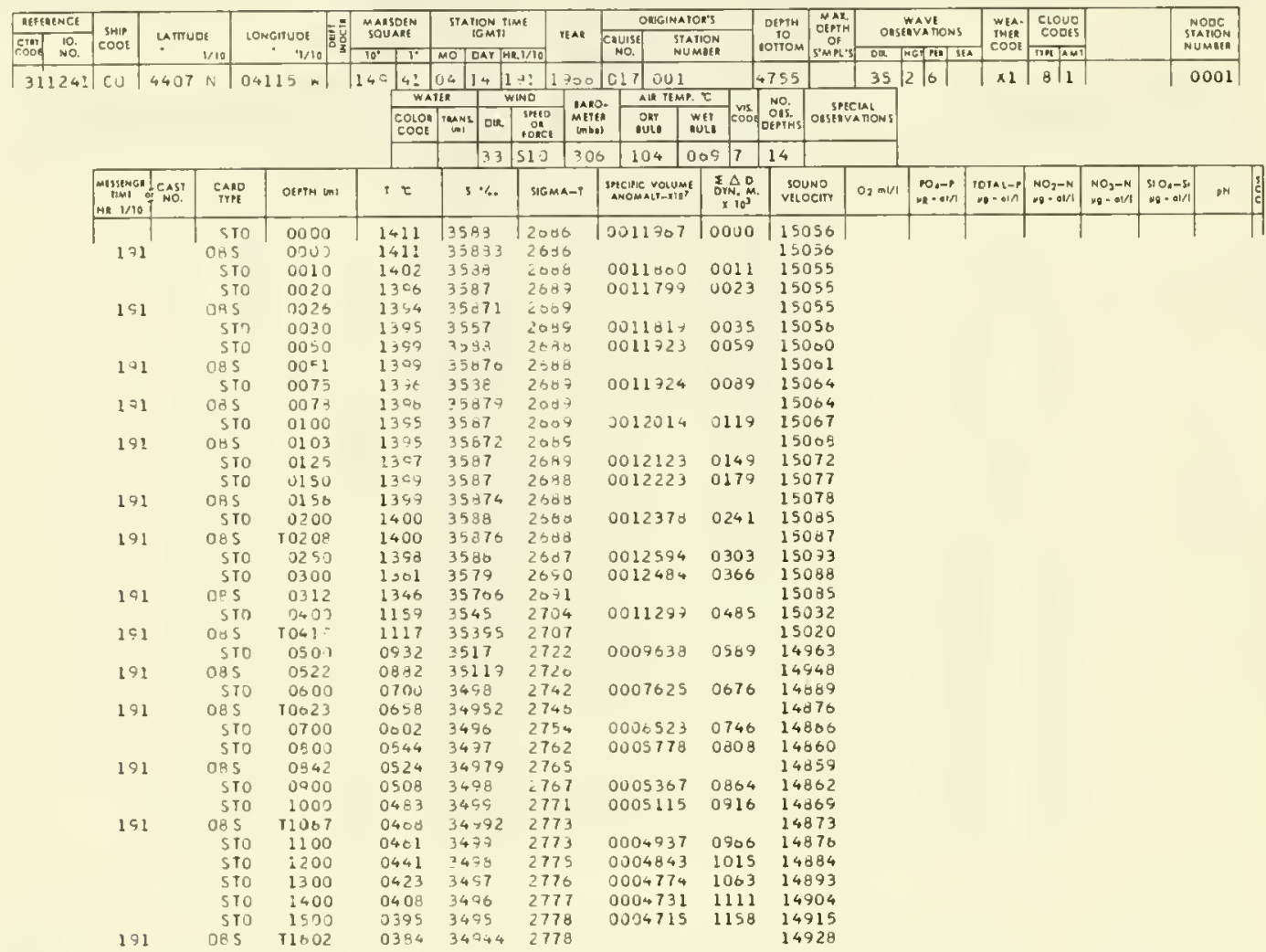

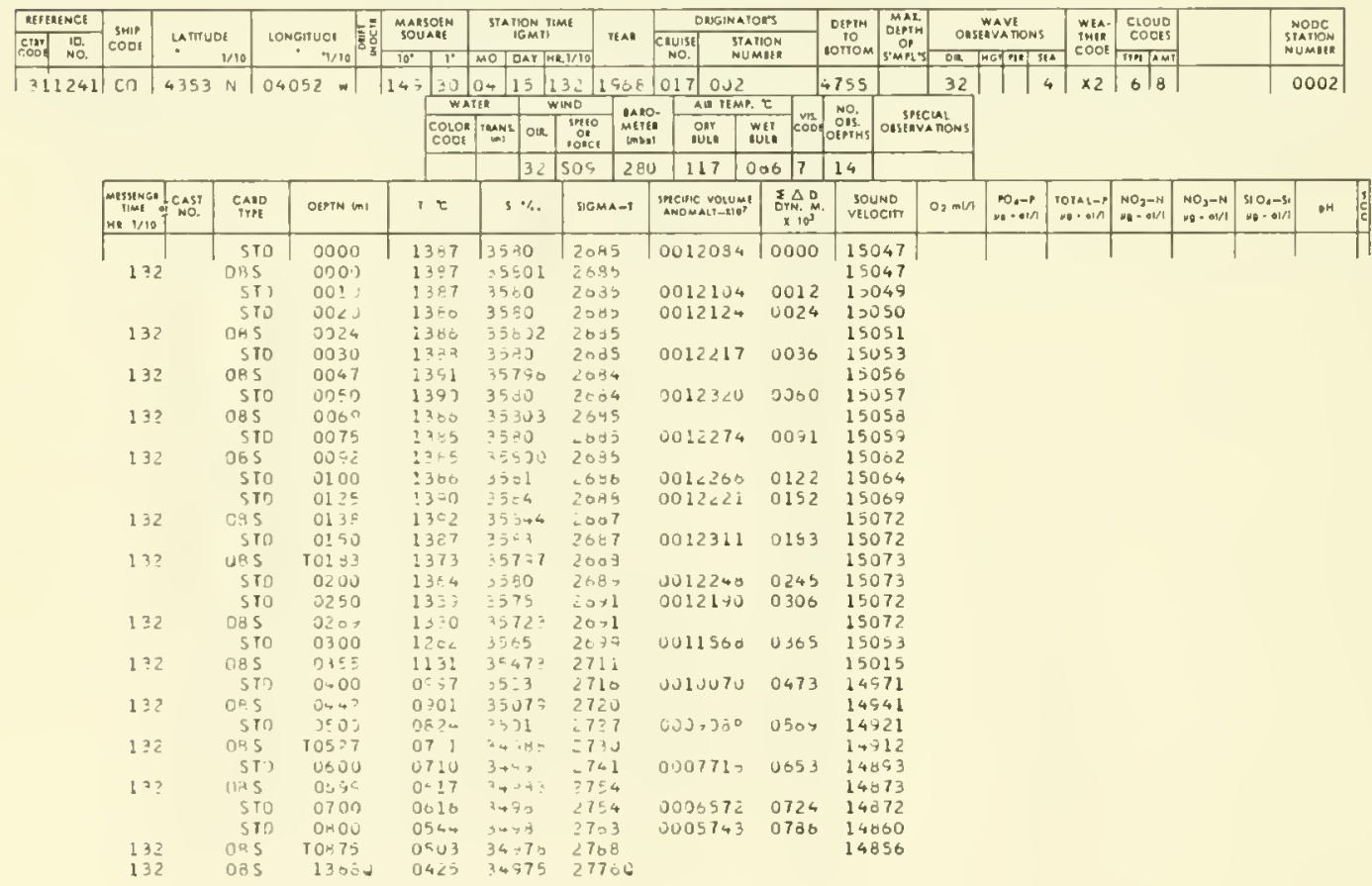




\section{TABLE VIII.-Continued}

\begin{tabular}{|c|c|c|c|c|c|c|c|c|c|c|c|c|c|c|c|c|c|}
\hline \multirow{2}{*}{ 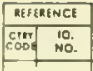 } & \multirow[b]{2}{*}{$\begin{array}{c}\text { SHIP } \\
\text { coot }\end{array}$} & \multirow{2}{*}{\multicolumn{2}{|c|}{$\begin{array}{c}\text { unmuot } \\
\quad 1 / 10\end{array}$}} & \multirow{2}{*}{ LONGruot } & \multirow{2}{*}{$\begin{array}{c}\text { MARSOEN } \\
\text { SQUARE }\end{array}$} & \multirow{2}{*}{$\begin{array}{c}\text { STAfION fimt } \\
\text { tGM川1 }\end{array}$} & \multirow[b]{2}{*}{ YEAI } & \multicolumn{2}{|c|}{ ONGINATORS } & \multirow{2}{*}{\begin{tabular}{|c|} 
OEPTM \\
10 \\
$10 T 10 \mathrm{M}$ \\
\end{tabular}} & \multirow{2}{*}{$\mid$\begin{tabular}{c|}
$\max$ \\
DEPIN \\
OB \\
$\operatorname{SimpL}$
\end{tabular}} & \multirow{2}{*}{$\begin{array}{c}\text { WAVEE } \\
\text { OASERYATONS }\end{array}$} & \multirow{2}{*}{$\mid \begin{array}{c}\text { WIA- } \\
\text { BNER } \\
\text { COOE }\end{array}$} & & & & \\
\hline & & & & & & & & \begin{tabular}{c|c} 
CRUISER \\
NO. STA \\
NUM
\end{tabular} & $\begin{array}{l}\text { THON } \\
\text { MIIR }\end{array}$ & & & & & \begin{tabular}{|c|} 
Coots \\
PHi A AMP \\
\end{tabular} & & & 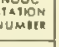 \\
\hline & co & 4252 & $N$ & $04106 \mathrm{k}$ & 14931 & $04: 31$ & $125 \quad 1705$ & \begin{tabular}{|l|l|}
017 & 003 \\
\end{tabular} & & 4064 & 35 & & $x_{1}$ & 416 & & & 0003 \\
\hline & & & & & WAT & \begin{tabular}{l|l}
11 & $w$ \\
\end{tabular} & Nino & 0.4 Aाल & $E$ & No. & & & & & & & \\
\hline & & & & & $\begin{array}{l}\text { Colon } \\
\text { coot } \\
\end{array}$ & \begin{tabular}{|l|l|} 
Teuns \\
ind
\end{tabular} & \begin{tabular}{|l|} 
shit \\
of \\
of
\end{tabular} & \begin{tabular}{l|l} 
ORr & $y$ \\
IuLI & $n$ \\
\end{tabular} & $\begin{array}{ll}\text { WEF COD } \\
\text { DULI COD }\end{array}$ & Ooist & OISERYATONS & & & & & & \\
\hline & & & & & & 36 & $50 \leqslant$ & 13, & 1337 & 21 & & & & & & & \\
\hline & 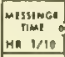 & 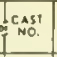 & $\underset{\text { TrNE }}{\text { CANO }}$ & OEPIN Um! & T $t$ & $\$ \%$ & SIGMA-I & 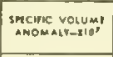 & 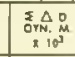 & $\begin{array}{l}\text { Soun } \\
\text { VELoc }\end{array}$ & $\begin{array}{l}\text { UNO } \\
\text { OCINY }\end{array}$ & $\mathrm{PO}_{4}=\mathrm{P}$ & 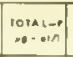 & 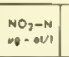 & 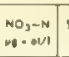 & $\begin{array}{l}310,-50 \\
1600+11\end{array} \mid$ & $D H$ \\
\hline & & & SIO & 0400 & $13 \div 5$ & $35 E ?$ & $258 \Rightarrow$ & Ju11.732 & 0000 & 150 & 051 & & & & & & \\
\hline & 129 & & $\begin{array}{l}\text { Das } \\
\text { SID }\end{array}$ & $\begin{array}{l}0000 \\
0010\end{array}$ & $\begin{array}{l}13 \div 5 \\
1354\end{array}$ & $\begin{array}{l}15472 \\
3557\end{array}$ & $\begin{array}{l}2509 \\
268\end{array}$ & & & & $\begin{array}{l}051 \\
052\end{array}$ & & & & & & \\
\hline & & & 510 & 0020 & $13>3$ & 157R & $\begin{array}{l}268= \\
2070\end{array}$ & $\begin{array}{l}0011716 \\
0011707\end{array}$ & $\begin{array}{l}0011 \\
0023\end{array}$ & $\begin{array}{l}150 \\
150\end{array}$ & 054 & & & & & & \\
\hline & 120 & & DHS & 0026 & $13 \fallingdotseq ?$ & 35575 & $25 ; 3$ & & & 150 & 054 & & & & & & \\
\hline & & & SIT & 0030 & 1303 & 3507 & 20,0 & 0011701 & 0035 & 150 & 054 & & & & & & \\
\hline & & & STO & 0050 & 1370 & 3535 & 2071 & 0011714 & 0058 & 150 & 053 & & & & & & \\
\hline & $12^{\circ}$ & & 085 & 0052 & 1377 & $3>046$ & 2671 & & & 150 & 953 & & & & & & \\
\hline & & & STO & 0075 & 1370 & 3584 & $2 t>2$ & 0011040 & 0087 & 150 & 055 & & & & & & \\
\hline & 129 & & OBS & 0079 & 1369 & $35844^{\circ}$ & 2092 & & & 150 & 055 & & & & & & \\
\hline & & & STO & 0100 & 13.07 & 3554 & 2572 & 3311711 & 3116 & $150=$ & 958 & & & & & & \\
\hline & 129 & & 085 & 0105 & 1306 & 35234 & 2672 & & & 150 & 058 & & & & & & \\
\hline & & & STO & 0125 & 1304 & 2583 & 2092 & 0011807 & $01+6$ & $150 t$ & 961 & & & & & & \\
\hline & & & SIO & 0150 & 1362 & $35 t 2$ & 2692 & 0011870 & 0176 & 1506 & 364 & & & & & & \\
\hline & 127 & & DBS & 0158 & 1302 & 35816 & 2672 & & & 1506 & 065 & & & & & & \\
\hline & & & STO & 0200 & 1360 & $350 ?$ & 2672 & 0011995 & 0235 & 1507 & 972 & & & & & & \\
\hline & 129 & & 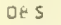 & 10210 & 1300 & $35 \$ 20$ & $2=92$ & & & 1507 & 973 & & & & & & \\
\hline & & & STO & 0250 & 1320 & 3575 & $26+3$ & 0011907 & 0295 & $150 \mathrm{c}$ & 368 & & & & & & \\
\hline & & & $5 \mathrm{TO}$ & 0300 & 1267 & 3504 & $2 \dot{097}$ & 0011751 & 0355 & 1505 & 955 & & & & & & \\
\hline & 129 & & 085 & 0313 & 1248 & 35006 & 2678 & & & 150 & & & & & & & \\
\hline & & & STD & 0400 & 1073 & 3533 & 2710 & 00110695 & 0467 & 1500 & 30 & & & & & & \\
\hline & 129 & & Des & 10422 & 1028 & 35264 & 2713 & & & 1498 & 87 & & & & & & \\
\hline & & & $5 \mathrm{TO}$ & 0500 & 0857 & 3507 & 2726 & $00091 \times 4$ & 0566 & 149 & 34 & & & & & & \\
\hline & 129 & & 085 & 0520 & 0820 & 35032 & 2729 & & & 1472 & 23 & & & & & & \\
\hline & & & STO & 0600 & 0702 & 3659 & 2742 & 0007672 & 0651 & 1489 & 90 & & & & & & \\
\hline & 125 & & DB 5 & T0821 & 0676 & 34557 & 2745 & & & 1408 & 183 & & & & & & \\
\hline & & & $5 T 2$ & 0700 & 0617 & 3493 & 2754 & 0000591 & 0722 & 1487 & 173 & & & & & & \\
\hline & & & STO & 0900 & 0554 & 3407 & 2762 & 0005757 & 0704 & 1406 & 64 & & & & & & \\
\hline & 129 & & DBS & 0833 & 0536 & 34992 & 2705 & & & 1480 & 102 & & & & & & \\
\hline & & & STD & 0900 & 0507 & 3498 & 2758 & 0005347 & 0940 & 1480 & 102 & & & & & & \\
\hline & & & STO & 1000 & 0470 & 3497 & 2771 & 0005047 & 0891 & 1486 & 63 & & & & & & \\
\hline & 127 & & 085 & 11045 & 0455 & 34571 & 2773 & & & 1486 & 164 & & & & & & \\
\hline & & & S To & 1100 & 0444 & 3497 & 2773 & 0004984 & 0941 & 1486 & & & & & & & \\
\hline & & & STO & 1200 & 0426 & 3496 & 2775 & 0004811 & 0990 & 1487 & 78 & & & & & & \\
\hline & & & STO & 1300 & 0411 & 3495 & 2776 & 0004754 & 1037 & 1485 & 38 & & & & & & \\
\hline & & & STO & 1400 & 0393 & 3495 & 2777 & 0004716 & 1085 & $14 d 3$ & 99 & & & & & & \\
\hline & & & STO & 1500 & 0387 & 3404 & 2778 & 9004680 & 1132 & 1471 & 12 & & & & & & \\
\hline & 129 & & 085 & $T 1574$ & $03 E 1$ & 34942 & $277 d$ & & & 1492 & 22 & & & & & & \\
\hline & & & STO & 1750 & 0370 & 3434 & 2779 & $000+750$ & 1250 & 1494 & 49 & & & & & & \\
\hline & 152 & & 085 & T1907 & 0370 & 34945 & 2779 & & & 1477 & 74 & & & & & & \\
\hline & & & STO & 2000 & 0358 & 3455 & 2750 & 0004822 & 1369 & 1498 & & & & & & & \\
\hline & 152 & & DBS & 2409 & 0349 & 34547 & $27 d 2$ & & & 1505 & & & & & & & \\
\hline & & & \$IO & 2500 & 0341 & $3+55$ & 2783 & 0004811 & 1010 & 1506 & & & & & & & \\
\hline & 152 & & 085 & 2910 & 0310 & 34546 & 2785 & & & 1512 & & & & & & & \\
\hline & & & STO & 3000 & 0304 & 3494 & 2786 & 0004625 & 1840 & 1513 & & & & & & & \\
\hline & 152 & & D85 & 3414 & 0278 & 34933 & 2797 & & & 1519 & & & & & & & \\
\hline & 152 & & OAS & 13929 & 0245 & 34921 & 2767 & & & 1527 & & & & & & & \\
\hline & & & SIO & 4000 & 0244 & 3492 & 2709 & 0004368 & 2297 & 1528 & & & & & & & \\
\hline & 152 & & OPS & 4368 & 0237 & 34507 & 2789 & & & 1534 & & & & & & & \\
\hline & 152 & & 085 & 14454 & 0227 & 34912 & 2790 & & & 1535 & 56 & & & & & & \\
\hline
\end{tabular}


Table VIII.-Continued

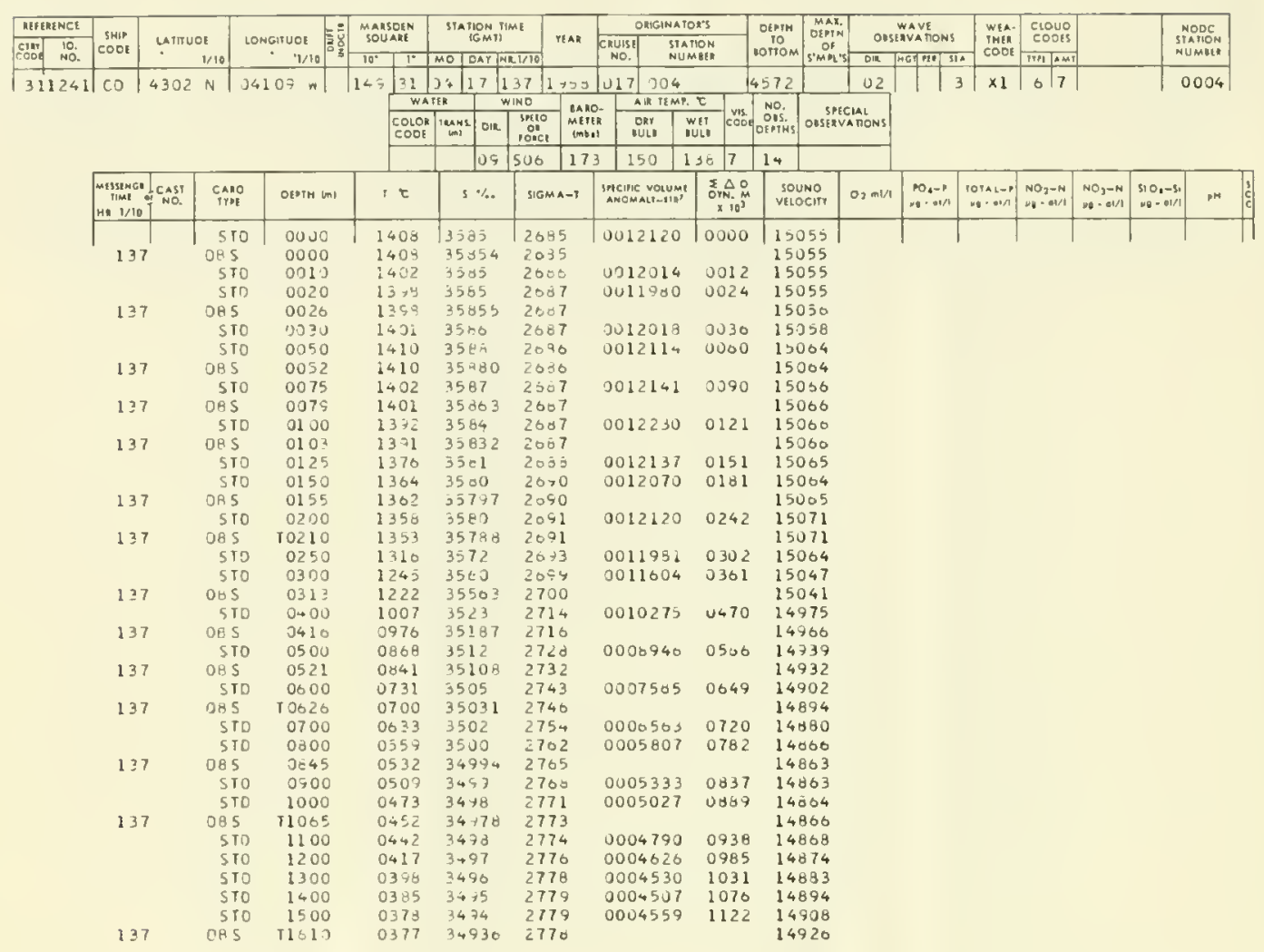

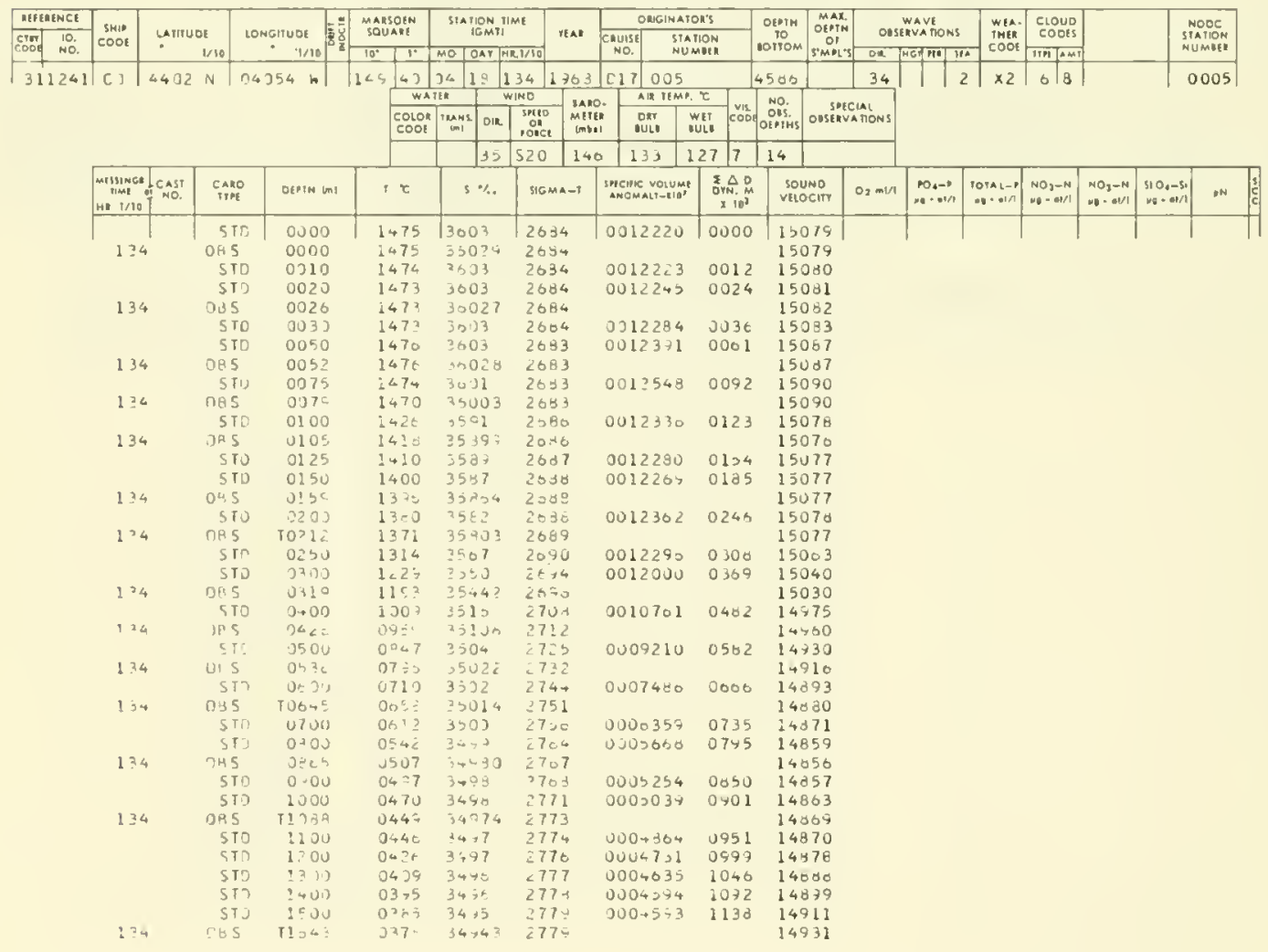


TABLE VIII.-Continued

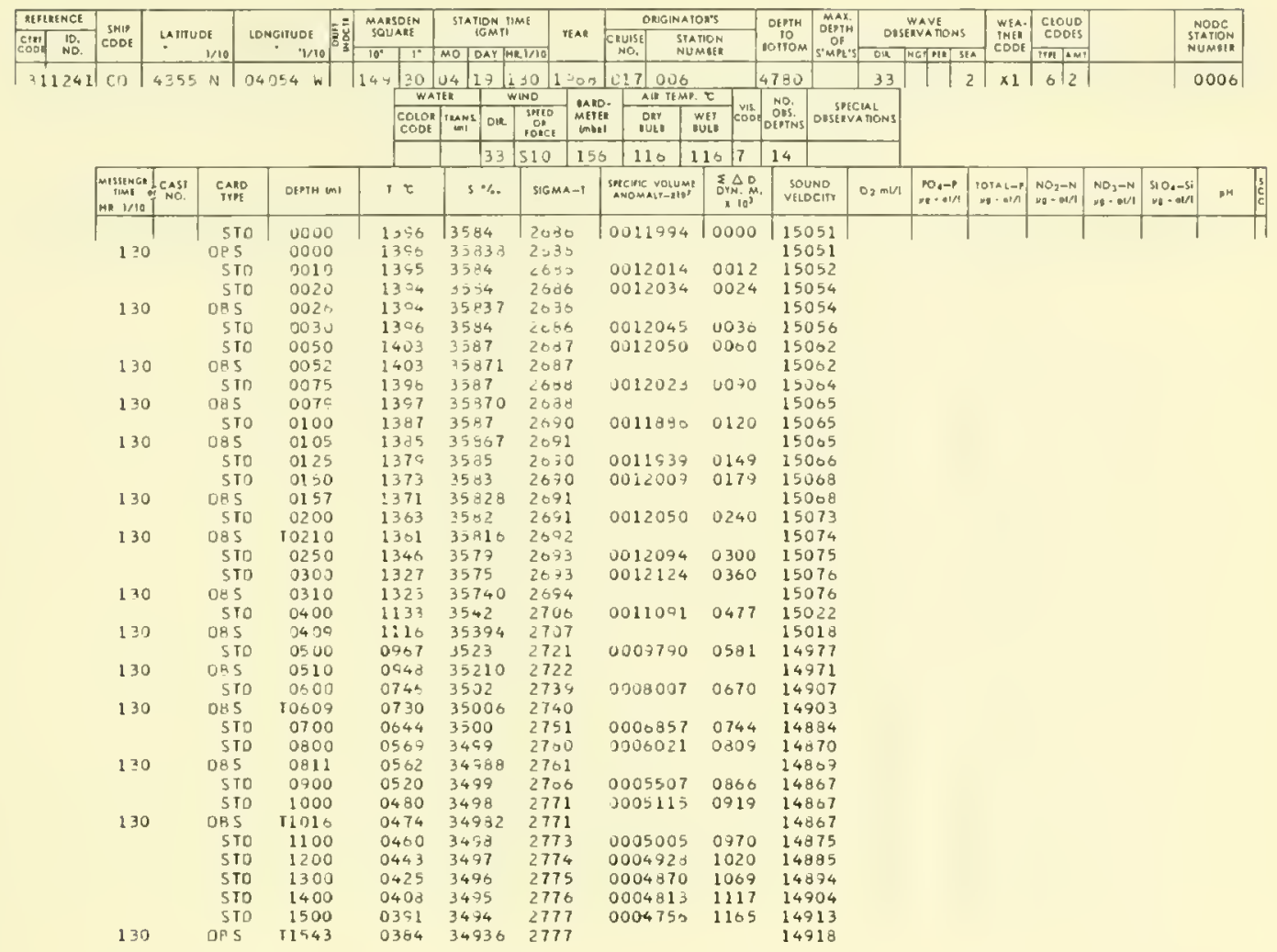

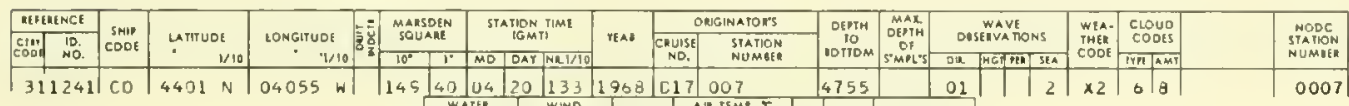

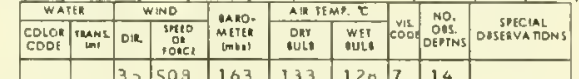

\begin{tabular}{|c|c|c|c|c|c|c|c|c|c|c|c|c|c|c|c|}
\hline 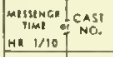 & $\underset{\text { TIMP }}{\text { CAnd }}$ & OEVTH $(\mathrm{m})$ & $r \tau$ & $5 \%$ & SIGMA-T & 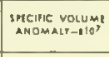 & 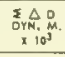 & $\begin{array}{l}\text { SOUND } \\
\text { VEtDCITY }\end{array}$ & $\mathrm{O}_{2} \mathrm{~m} / \mathrm{m} / \mathrm{s}$ & 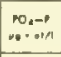 & TORAL" & $\begin{array}{c}M O^{\prime}-N \\
n\end{array}$ & $\begin{array}{l}\mathrm{NO}_{3}-\mathrm{N} \\
\mathrm{N}=\mathrm{OI} / \mathrm{I}\end{array}$ & $\left|\begin{array}{cc}510 & 0<-5 t \\
0 & -0+1 / 4\end{array}\right|$ & $\because H$ \\
\hline & STO & 0000 & $1+70$ & 3603 & 2635 & 0012075 & 0000 & 15077 & & & & & & & \\
\hline \multirow[t]{3}{*}{133} & 0.5 & 0000 & 1470 & 36034 & 2045 & & & 15077 & & & & & & & \\
\hline & $5 T 0$ & 0010 & 1470 & 3603 & Loes 5 & 0012097 & 0012 & 15079 & & & & & & & \\
\hline & STO & 0020 & 1470 & 3004 & 2082 & 0012127 & 0024 & 15080 & & & & & & & \\
\hline \multirow[t]{3}{*}{133} & oas & 0025 & 1470 & 30035 & 2625 & & & 15081 & & & & & & & \\
\hline & STO & 0030 & 1458 & 3001 & 2000 & 0012094 & 0036 & 15078 & & & & & & & \\
\hline & STD & 0050 & 1413 & 3591 & 2688 & 0011977 & 0060 & 25065 & & & & & & & \\
\hline \multirow[t]{2}{*}{133} & 085 & 0051 & $1+11$ & 35903 & 2638 & & & 25065 & & & & & & & \\
\hline & STO & 0075 & 1398 & 3580 & 2030 & 0012008 & 0090 & 15004 & & & & & & & \\
\hline \multirow[t]{2}{*}{133} & ORs & 0077 & 1357 & 35902 & 2008 & & & 15064 & & & & & & & \\
\hline & STD & 0100 & 1394 & 3538 & 2049 & 0011909 & 0120 & 15067 & & & & & & & \\
\hline \multirow[t]{3}{*}{133} & Do 5 & 3102 & 1394 & 35877 & 2669 & & & 15007 & & & & & & & \\
\hline & $5 \mathrm{TU}$ & 0125 & 1384 & 3587 & 2609 & 0012073 & 0150 & 15071 & & & & & & & \\
\hline & STO & 0150 & 1393 & 3586 & 2089 & 0012195 & 0180 & 15075 & & & & & & & \\
\hline \multirow[t]{2}{*}{133} & 085 & 0155 & $13<3$ & 3500.1 & 2088 & & & 15076 & & & & & & & \\
\hline & STO & 0200 & 1379 & 3584 & $26>0$ & 0012130 & 0241 & 15078 & & & & & & & \\
\hline \multirow[t]{3}{*}{133} & 065 & T0207 & 1377 & 35.841 & 2670 & & & 15079 & & & & & & & \\
\hline & STO & 0250 & 1371 & 3583 & 2691 & 0012285 & 0303 & 15084 & & & & & & & \\
\hline & STO & 0300 & 1305 & $35 y 2$ & 2631 & $00123 \times 6$ & 0364 & 15090 & & & & & & & \\
\hline \multirow[t]{2}{*}{133} & 085 & 0308 & 1304 & 35313 & $\therefore x y 1$ & & & 15091 & & & & & & & \\
\hline & STO & 0400 & 1275 & 3564 & 2696 & 0012105 & 0487 & 15074 & & & & & & & \\
\hline \multirow[t]{2}{*}{133} & o8s & 0409 & 1255 & 35014 & 2057 & & & $15070^{\circ}$ & & & & & & & \\
\hline & $5 T n$ & 0500 & 1012 & 35.20 & 2716 & 0010291 & 0549 & 14994 & & & & & & & \\
\hline \multirow[t]{2}{*}{133} & DF 5 & 0515 & 0977 & 35222 & 2718 & & & 14983 & & & & & & & \\
\hline & $5 \mathrm{TO}$ & $060)$ & 2816 & 3509 & 2733 & 0006650 & 0674 & 14935 & & & & & & & \\
\hline \multirow[t]{3}{*}{$1 \geq 3$} & QRS & T0015 & 0701 & 35057 & 2735 & & & 14928 & & & & & & & \\
\hline & $5 \mathrm{TD}$ & 0700 & 0003 & 3502 & 2748 & 0007212 & 0773 & 14899 & & & & & & & \\
\hline & sin & 3600 & 0594 & 3500 & 2759 & 0306140 & 0840 & 14870 & & & & & & & \\
\hline \multirow[t]{3}{*}{133} & DBS & 0630 & 0500 & 34723 & 2702 & & & 14872 & & & & & & & \\
\hline & STO & 0900 & 0520 & $3+99$ & 2765 & 0005547 & 0899 & 14870 & & & & & & & \\
\hline & STO & 1000 & 0485 & 3499 & 2771 & 0005117 & 0952 & 14869 & & & & & & & \\
\hline \multirow[t]{6}{*}{133} & OAS & $T 1045$ & 0469 & 34591 & 2773 & & & 14870 & & & & & & & \\
\hline & 5 TO & 1100 & 0459 & 3497 & 2773 & 0004915 & 1002 & 14875 & & & & & & & \\
\hline & $5 T 0$ & 1200 & 0443 & 3498 & 2775 & 0004842 & 1051 & 14085 & & & & & & & \\
\hline & 5 TO & 1300 & 0427 & 3494 & 2776 & 0004778 & $10 \% 9$ & 14895 & & & & & & & \\
\hline & $5 \mathrm{TO}$ & 1400 & 0411 & 3457 & 2777 & 0004734 & 1147 & 14905 & & & & & & & \\
\hline & STO & 1500 & 0395 & 3436 & 2778 & 0004691 & 1194 & 14915 & & & & & & & \\
\hline 133 & 085 & TI 582 & 0382 & 34949 & 2779 & & & 14923 & & & & & & & \\
\hline
\end{tabular}




\section{TABLE VIII.-Continued}

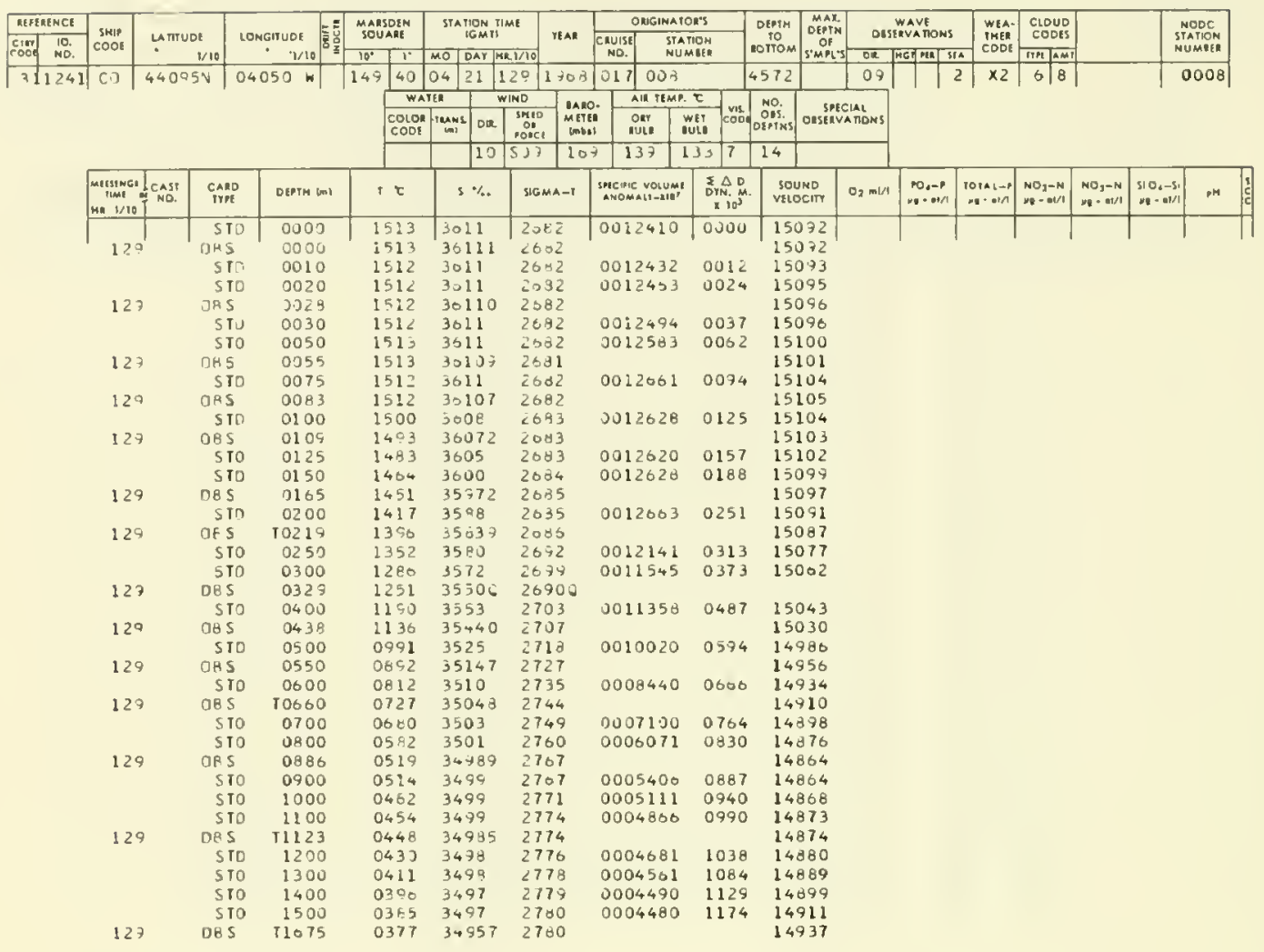

\begin{tabular}{|c|c|c|c|c|c|c|c|c|c|c|c|c|c|c|c|c|c|c|}
\hline & \multirow{2}{*}{$\begin{array}{c}3 \mathrm{NAP} \\
\text { cook }\end{array}$} & \multirow{2}{*}{\multicolumn{2}{|c|}{$\begin{array}{l}\text { untruor } \\
1 / 10\end{array}$}} & \multirow{2}{*}{ IONGISUD } & \multirow{2}{*}{$\begin{array}{l}\text { MALSDEN } \\
\text { SOUALE }\end{array}$} & \multicolumn{2}{|c|}{ STA MOON FIME } & \multicolumn{2}{|c|}{ ONGIMATOR'S } & \multirow{2}{*}{$\begin{array}{c}0 \mathrm{OBTH} \\
\text { To } \\
\mathrm{corTOM}\end{array}$} & \multirow{2}{*}{\begin{tabular}{|l|} 
MA1 \\
DEFIN \\
Of \\
SWPIS \\
\end{tabular}} & \multirow{2}{*}{\multicolumn{2}{|c|}{$\begin{array}{l}\text { WAVE } \\
\text { OSSERVA TONS }\end{array}$}} & \multirow{2}{*}{$\begin{array}{l}\text { WEA- } \\
\text { WHEI } \\
\text { CODE }\end{array}$} & \multirow{2}{*}{\begin{tabular}{|l|} 
Cloud \\
coofs \\
In $\mid$ |am \\
\end{tabular}} & & & \multirow{2}{*}{$\begin{array}{l}\text { NODC } \\
\text { STAYON } \\
\text { NUMEER }\end{array}$} \\
\hline \begin{tabular}{l|l} 
cering & 10. \\
rod & NO.
\end{tabular} & & & & & & \begin{tabular}{l|l} 
& $16 M T I$ \\
MO & DAYY
\end{tabular} & YEAR & $\begin{array}{r}\text { Cnuisi } \\
\text { no. }\end{array}$ & STATION & & & Olsevanans & & & & & & \\
\hline 311241 & $\mathrm{CO}$ & 4355 & $N$ & 04057 w & 145 & \begin{tabular}{l|l|}
04 & 26 \\
\end{tabular} & \begin{tabular}{l|l}
173 & 1938
\end{tabular} & 017009 & & 4581 & & 35 & 7 & $x_{0}$ & 10 & & & 0009 \\
\hline & & & & & WA & TER & WIND & $\begin{array}{l}1 / R T I M P \\
\end{array}$ & 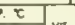 & No. & & & & & & & & \\
\hline & & & & & $\begin{array}{l}\text { Colok } \\
\text { coot }\end{array}$ & $\lim _{i=1}$ Dat & $\begin{array}{l}\text { sento } \\
\text { oncs } \\
\text { onct }\end{array}$ & \begin{tabular}{l|l} 
PRT & Rut \\
\end{tabular} & $\begin{array}{lll}\text { WEt } & \text { Whs } \\
\text { Eute }\end{array}$ & Oos. & ousenve & ATIONS & & & & & & \\
\hline & & & & & & 54 & $\$ 20$ & 139 & \begin{tabular}{l|l}
59 & 7 \\
\end{tabular} & 14 & & & & & & & & \\
\hline & 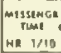 & $\begin{array}{l}\text { CAST } \\
\text { INO. }\end{array}$ & $\begin{array}{c}\text { CAnO } \\
\text { iYME }\end{array}$ & DSPTH $\mathrm{um}$ & I $\tau$ & $s \%$ & SIGMA-F & 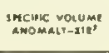 & 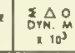 & & & $0, \mathrm{~m} / \mathrm{n}$ & 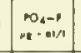 & 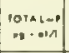 & 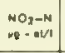 & $\begin{array}{l}\mathrm{NO}_{1}-\mathrm{N} \\
\mathrm{n} \cdot \mathrm{nth}\end{array}$ & $\left|\begin{array}{ll}510,-51 \\
x=0.1 / 1\end{array}\right|$ & אי \\
\hline & & & STO & 0000 & 1521 & 3010 & 2079 & 0012045 & 0000 & 150 & 094 & & & & & & & \\
\hline & 173 & & Ons 5 & $\begin{array}{l}2002 \\
0010\end{array}$ & i5:1 & 35103 & $<679$ & & 0012 & & 094 & & & & & & & \\
\hline & & & S TI) & 0020 & $\begin{array}{l}1519 \\
1516\end{array}$ & 3010 & $\begin{array}{l}\angle 080 \\
2080\end{array}$ & 0012622 & $\begin{array}{l}0012 \\
0025\end{array}$ & 150 & 076 & & & & & & & \\
\hline & 173 & & a\&s & 3024 & is 15 & 35101 & 2660 & & & 150 & 097 & & & & & & & \\
\hline & & & $S I^{\prime}$ & 0010 & $15: 5$ & 3010 & 2080 & 0012021 & 0037 & & 097 & & & & & & & \\
\hline & & & S10 & 0050 & 2512 & 3010 & 2031 & 0012056 & 0003 & 25 & 100 & & & & & & & \\
\hline & 173 & & Des & 0055 & $15: 2$ & $3639 \%$ & 2681 & & & 15 & 100 & & & & & & & \\
\hline & & & SIO & 0075 & $25: 0$ & 3620 & 2661 & $001207+$ & 0094 & 15 & 103 & & & & & & & \\
\hline & 173 & & Je 5 & 0003 & 1504 & 36099 & 2083 & & & 15 & 102 & & & & & & & \\
\hline & & & $\$ 10$ & 0100 & 1475 & 3 कins & 2080 & 0012290 & 0126 & 150 & 090 & & & & & & & \\
\hline & 173 & & Oos & 0109 & 2404 & 36040 & 2687 & & & 15 & 073 & & & & & & & \\
\hline & & & STo & 0125 & 2440 & 2599 & 2688 & 3012109 & 0150 & 150 & 087 & & & & & & & \\
\hline & & & SID & 0150 & $1<12$ & 3542 & 2089 & 0012164 & 0107 & 15 & 032 & & & & & & & \\
\hline & 173 & & $7+5$ & 3164 & 1403 & 35979 & 2005 & & & & 001 & & & & & & & \\
\hline & & & 510 & 0200 & 1400 & 3580 & 2685 & 3012252 & $0<4 B$ & 150 & 086 & & & & & & & \\
\hline & 173 & & Des 5 & $021 \mathrm{c}$ & $13<7$ & Зรत3 & 2050 & & & 150 & $0 \otimes \theta$ & & & & & & & \\
\hline & & & $5 r 0$ & 0250 & $13 \div 5$ & $35 y^{2}$ & 2070 & 3012347 & 0309 & 150 & $0 \$ 2$ & & & & & & & \\
\hline & & & 513 & 0300 & 1373 & 3525 & 2692 & 0012327 & 0371 & 150 & 093 & & & & & & & \\
\hline & 173 & & ne 5 & 0327 & $13 ; 1$ & $2 j \neq 07$ & 2093 & & & $15 c$ & 089 & & & & & & & \\
\hline & & & STD & 3400 & $1=5 ?$ & $35+1$ & $26 y 0$ & 0011914 & 0492 & 150 & 065 & & & & & & & \\
\hline & 17 & & nos & 10440 & $i 1 \circ 1$ & $33+74$ & 2703 & & & 150 & 047 & & & & & & & \\
\hline & & & SIO & 0500 & $103 \%$ & $; 529$ & 2712 & 0010658 & 0005 & 150 & 003 & & & & & & & \\
\hline & 173 & & 085 & 0542 & 0945 & $3 \div 102$ & 2713 & & & 148 & 970 & & & & & & & \\
\hline & & & STu & 0000 & 0357 & $=520$ & 2728 & U & 0704 & 14 & 951 & & & & & & & \\
\hline & 173 & & nEs & TUמ子? & $07 \doteqdot 1$ & 35052 & 2736 & & & 14 & 930 & & & & & & & \\
\hline & & & STD & 0700 & 0721 & 3304 & 2746 & 0007071 & 0780 & 14 & 714 & & & & & & & \\
\hline & & & STO & 0900 & Dels & 3501 & 2756 & $00364 \mp 0$ & 0859 & $14 \mathrm{c}$ & d49 & & & & & & & \\
\hline & 173 & & aes & $0 \times 74$ & $055_{4}$ & $34 \div \div 0$ & $270 ?$ & & & 14 & 876 & & & & & & & \\
\hline & & & STO & 0700 & $054 !$ & $3+77$ & 2704 & 3005128 & 0920 & 14 & 876 & & & & & & & \\
\hline & & & sio & 1000 & 0.70 & 3499 & $27: 9$ & 3035255 & 0975 & 148 & 874 & & & & & & & \\
\hline & & & srit & 1100 & 0455 & $3+93$ & 2773 & $00049 \mathrm{hb}$ & 1020 & 145 & 375 & & & & & & & \\
\hline & $17=$ & & DP 5 & 11104 & 0457 & $34 \times 30$ & 2773 & & & 148 & 875 & & & & & & & \\
\hline & & & sin & 1200 & 0.43 & $5 \infty \div 7$ & 2774 & 0004922 & 1376 & $14:$ & 485 & & & & & & & \\
\hline & & & ST & 1300 & $04 a$ & 3457 & 2775 & $000 \times 5 \times 1$ & 1125 & 14 & $d>6$ & & & & & & & \\
\hline & & & $51)$ & 1400 & $C+15$ & 3420 & 2770 & $000485 ?$ & 1173 & 146 & 907 & & & & & & & \\
\hline & & & ST) & 1500 & 0401 & 3495 & 2777 & 0004007 & 1222 & 14 & 916 & & & & & & & \\
\hline & 173 & & nes & $I i=47$ & 0ख्र० & 24542 & 2779 & & & 149 & 933 & & & & & & & \\
\hline
\end{tabular}


TABLE VIII.-Continued

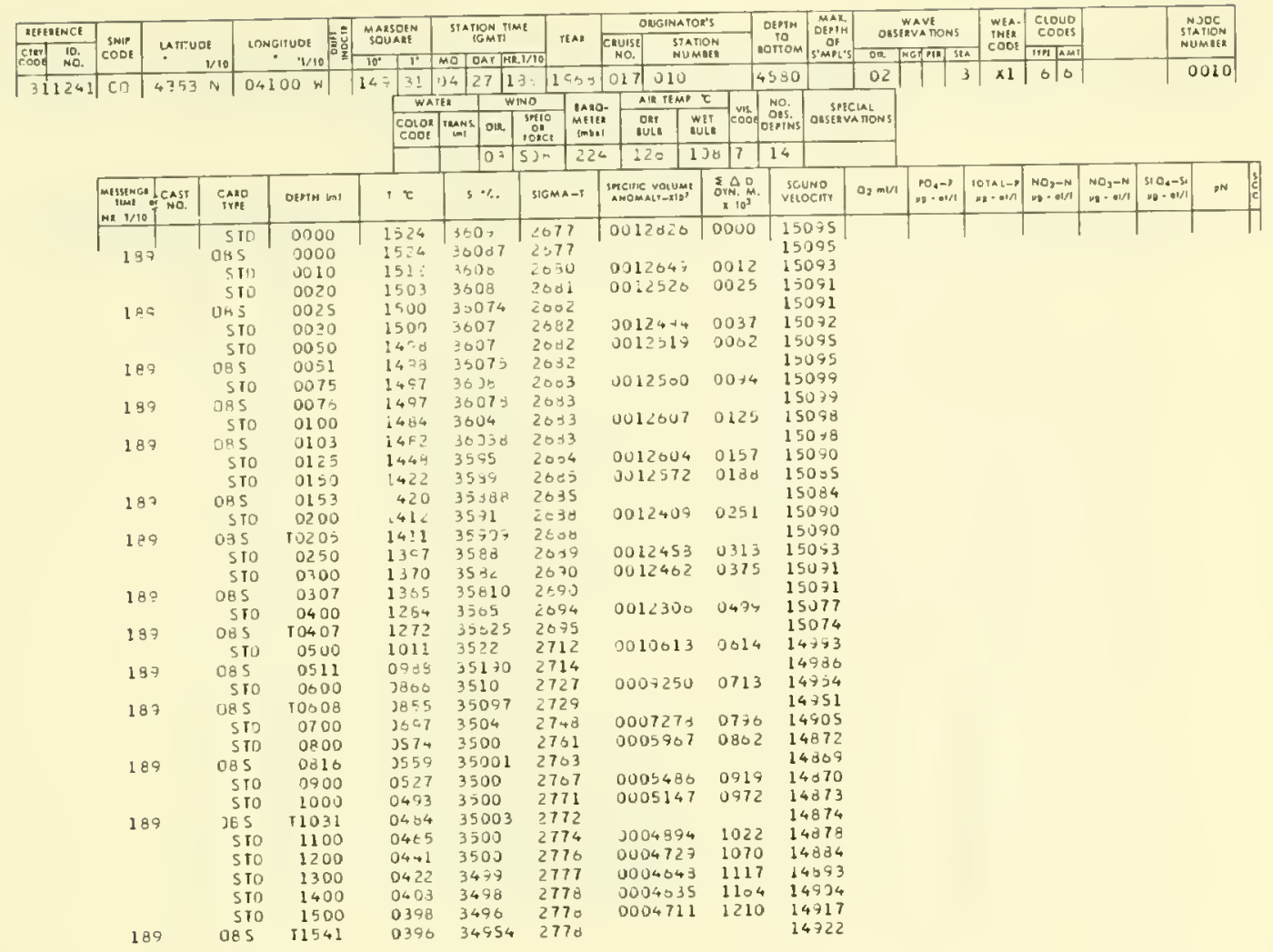

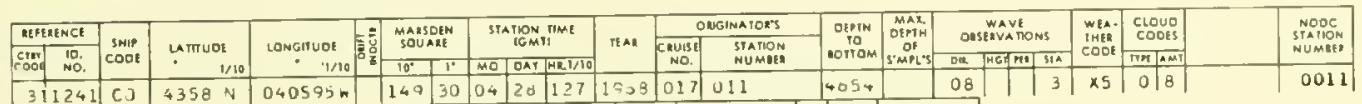

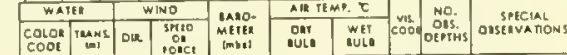

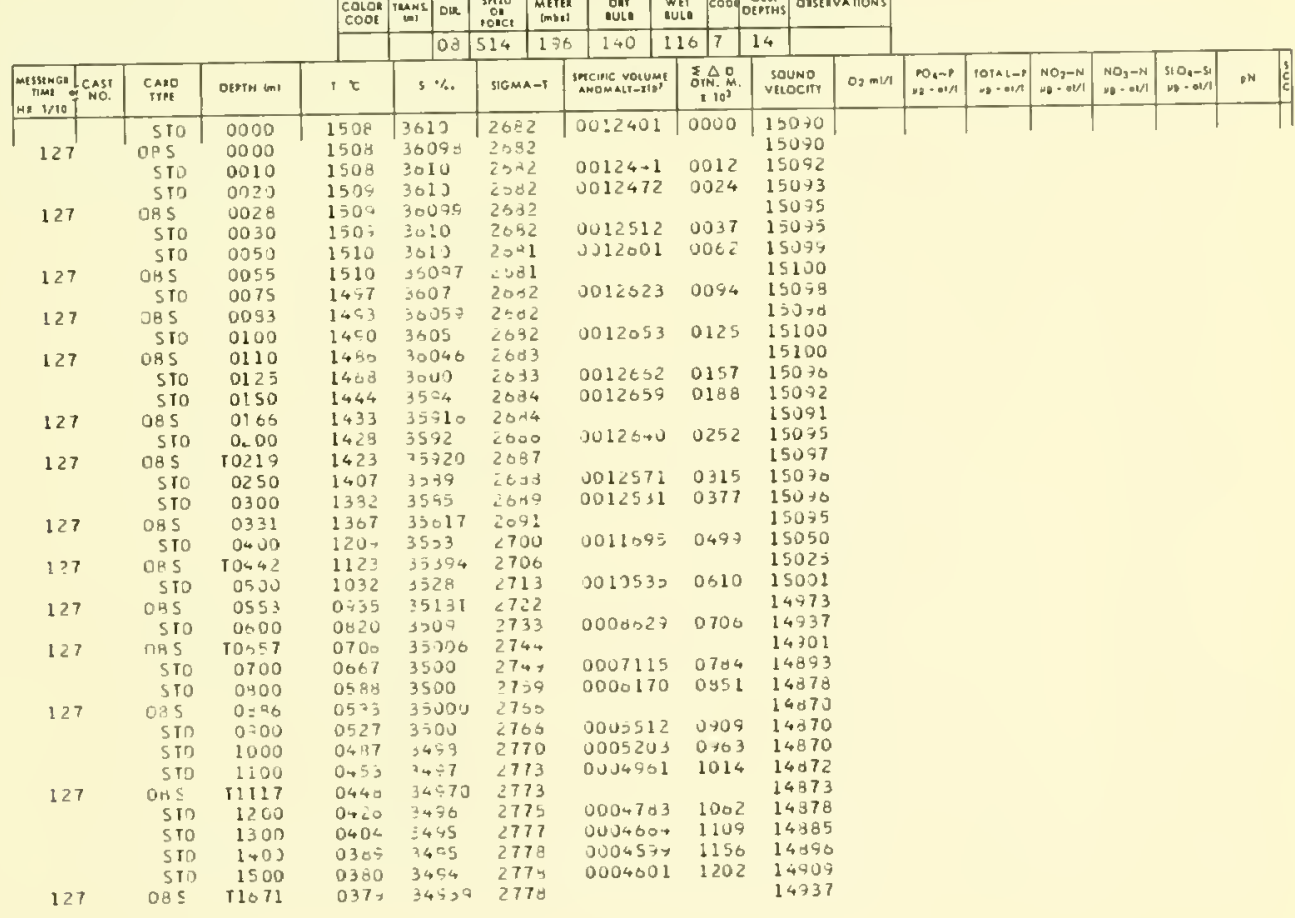


Table VIII.-Continued

\begin{tabular}{|c|c|c|c|c|c|c|c|c|c|c|c|c|c|c|c|c|c|c|}
\hline \multirow{2}{*}{\begin{tabular}{|c|c|} 
AEFERENCE \\
CTNy & 10. \\
coo & NO. \\
\end{tabular}} & \multirow{2}{*}{$\begin{array}{l}\text { SHIP } \\
\text { COOE }\end{array}$} & \multirow{2}{*}{\multicolumn{2}{|c|}{$\begin{array}{c}\text { UATUVE } \\
1 / 10 \\
\end{array}$}} & \multirow{2}{*}{ LONGIYUOE } & \multirow{2}{*}{$\begin{array}{l}\text { MARSDEN } \\
\text { SOUARE }\end{array}$} & \multirow{2}{*}{\multicolumn{2}{|c|}{$\begin{array}{l}\text { STARION FIME } \\
\text { IGM TIME }\end{array}$}} & \multicolumn{2}{|c|}{ ONGINATOR'S } & & \multirow{2}{*}{$\begin{array}{c}\text { MAX. } \\
\text { DEPTH } \\
\text { OF } \\
\text { S'MPLS }\end{array}$} & \multirow{2}{*}{\multicolumn{2}{|c|}{$\begin{array}{c}\text { WaVE } \\
\text { OUSEAVATONS }\end{array}$}} & \multirow{2}{*}{$\begin{array}{l}\text { WEA- } \\
\text { TNER } \\
\text { CODE }\end{array}$} & \multirow{2}{*}{\begin{tabular}{|l|} 
CLOUD \\
CODKS \\
MPI $\mid A W H$ \\
\end{tabular}} & & & \multirow{2}{*}{$\begin{array}{l}\text { NOOC } \\
\text { SPAIION } \\
\text { NUMEER }\end{array}$} \\
\hline & & & & & & & reAR & \begin{tabular}{|c|} 
CRuist \\
No.
\end{tabular} & $\begin{array}{l}\text { SIA TION } \\
\text { NUMBER }\end{array}$ & & & & & & & & & \\
\hline & co & 4400 & $N$ & $04107 \mathrm{w}$ & 14741 & $0 \rightarrow 30$ & \begin{tabular}{|l|l|}
150 & 1700
\end{tabular} & 017012 & & \begin{tabular}{|l|}
$10110 \mathrm{M}$ \\
4572
\end{tabular} & & 10 & \begin{tabular}{l|l|l} 
& & 3
\end{tabular} & \begin{tabular}{|l|l|}
3 & 15 \\
\end{tabular} & \begin{tabular}{|l|l|}
45 \\
\end{tabular} & & & 00121 \\
\hline & & & & & WA & & WIND & O- AIR TEMP. & $\tau$ & No. & & & & & & & & \\
\hline & & & & & $\begin{array}{l}\text { Colon } \\
\text { CODE }\end{array}$ & $\begin{array}{c}\mid r \text { renss } \\
\text { lams } \\
\text { oir }\end{array}$ & 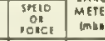 & \begin{tabular}{l|l} 
DAT \\
ivi
\end{tabular} & $\begin{array}{lll}\text { Wet } & \text { no } \\
\text { out } & \text { coo } \\
\end{array}$ & oons. & OASERV & A NONS & & & & & & \\
\hline & & & & & & 15 & 507 & 151 & \begin{tabular}{l|l}
132 & 7 \\
\end{tabular} & 14 & & & & & & & & \\
\hline & $\begin{array}{l}\text { MessismGe } \\
\text { MiN1 } \\
\text { MR } 1 / 10\end{array}$ & $\begin{array}{l}\text { CAST } \\
\text { i NO. }\end{array}$ & cAnD & OEPTM (n) & $1 \tau$ & $s \cdot \%$ & SIGMa-T & 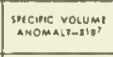 & 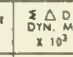 & & & $02 \mathrm{~m} / \mathrm{t}$ & 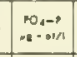 & 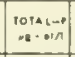 & 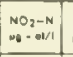 & $\begin{array}{l}N O,-N \\
N=-N / 1\end{array}$ & 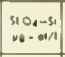 & 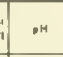 \\
\hline & & & STID & 0300 & 1605 & 3617 & 2607 & 0013830 & 0000 & is & 121 & & & & & & & \\
\hline & 156 & & ORS & 0000 & 2605 & 30297 & 2667 & & & is & 121 & & & & & & & \\
\hline & & & sin & 0010 & Is 92 & 3018 & 2604 & 0013027 & 0013 & 15 & 119 & & & & & & & \\
\hline & & & SID & 0320 & 1St 3 & 3010 & $2 \in 71$ & 0013476 & 0027 & 15 & 118 & & & & & & & \\
\hline & 150 & & $0 \in 5$ & 0023 & 1570 & 30175 & 2672 & & & is & 117 & & & & & & & \\
\hline & & & ST? & 0030 & 1578 & 3513 & 2072 & 0013438 & 0040 & 15 & 128 & & & & & & & \\
\hline & & & STJ & a)so & 2579 & $36 ! 3$ & 2572 & 0013484 & 0067 & is & 121 & & & & & & & \\
\hline & $15 t$ & & DES & 0055 & 1575 & 36170 & 1072 & & & 15 & 122 & & & & & & & \\
\hline & & & STO & 0075 & 1571 & 3010 & 2672 & 0013520 & 0101 & 15 & 123 & & & & & & & \\
\hline & 156 & & OBS & OJHs & 2565 & 35145 & 2073 & & & 15 & .22 & & & & & & & \\
\hline & & & STO & 0100 & 1544 & $36: 0$ & 2074 & 0013430 & 0135 & 15 & 118 & & & & & & & \\
\hline & 156 & & OBS & 0110 & 15,2 & 36031 & 2675 & & & 25 & 115 & & & & & & & \\
\hline & & & sio & $01<5$ & 1510 & 3605 & $\dot{2}>76$ & 00133.4 & 0168 & is & 113 & & & & & & & \\
\hline & & & STO & 0150 & $145 \overline{2}$ & 3601 & 2678 & 0013213 & 0201 & 15 & 100 & & & & & & & \\
\hline & 156 & & OP.S & 0166 & 1470 & 35977 & 2079 & & & 15 & 106 & & & & & & & \\
\hline & & & sio & 0200 & $14 \leq 3$ & 3592 & $2 \in 80$ & 0013144 & 0267 & 15 & 103 & & & & & & & \\
\hline & 156 & & 085 & 10219 & 1437 & 35890 & $\angle 0 B I$ & & & 15 & 101 & & & & & & & \\
\hline & & & STD & 0250 & 1412 & 3556 & 2684 & 0012306 & 0332 & 15 & 097 & & & & & & & \\
\hline & & & STD & 0300 & 1357 & 3578 & 2090 & 0012500 & 0396 & 15 & 037 & & & & & & & \\
\hline & $15 t$ & & OBS & 0331 & 1315 & 35716 & 2693 & & & 15 & 077 & & & & & & & \\
\hline & & & SII & 0400 & 1153 & 3551 & 2702 & 0011540 & 0510 & 15 & 044 & & & & & & & \\
\hline & 156 & & Des & 0446 & 1103 & 35300 & 2708 & & & 15 & 019 & & & & & & & \\
\hline & & & sio & 0500 & 0973 & 3521 & 2718 & 0010020 & 0624 & 14 & 979 & & & & & & & \\
\hline & 156 & & $08 \mathrm{~s}$ & OS 52 & 0863 & 35104 & 2727 & & & 14 & 947 & & & & & & & \\
\hline & & & STO & $0 \subset 00$ & $070 \bar{z}$ & 3507 & $273 t$ & 0008299 & 0715 & 14 & 926 & & & & & & & \\
\hline & 156 & & ORS & 10656 & 0715 & 35346 & 2745 & & & 14 & 905 & & & & & & & \\
\hline & & & STD & 0700 & OQOS & 1503 & 2751 & 0000937 & 0792 & 14 & 894 & & & & & & & \\
\hline & & & Sro & 0400 & 0580 & 3501 & $<761$ & 0000023 & 0850 & 14 & 875 & & & & & & & \\
\hline & 156 & & 0.5 & 0083 & 0525 & 34974 & 2700 & & & 14 & 806 & & & & & & & \\
\hline & & & STO & 0500 & 0519 & $34 \div 5$ & 2707 & 0005443 & 0914 & 14 & 867 & & & & & & & \\
\hline & & & STO & 1000 & 0499 & 3459 & 2770 & 0003171 & 0367 & 14 & 871 & & & & & & & \\
\hline & & & sio & 1100 & 0402 & 3699 & 2773 & 0004905 & 1017 & 14 & 870 & & & & & & & \\
\hline & 156 & & 085 & 11110 & $045 ?$ & $34 \div 56$ & 2773 & & & & 877 & & & & & & & \\
\hline & & & Sin & 1200 & 0436 & $34 \times 9$ & 2775 & $000+801$ & 1000 & 14 & 883 & & & & & & & \\
\hline & & & sio & 1300 & 0.19 & 3457 & 2777 & 0004650 & 1114 & 14 & 892 & & & & & & & \\
\hline & & & STD & 1400 & 0403 & 3497 & 2778 & 0004635 & 1260 & & 902 & & & & & & & \\
\hline & & & STO & 1500 & 0391 & $34=0$ & 2778 & 0004039 & 1207 & 14 & 913 & & & & & & & \\
\hline & 156 & & OAS & 11656 & 0379 & 34940 & $277 \mathrm{~B}$ & & & 14 & 935 & & & & & & & \\
\hline
\end{tabular}


TABLE VIII.-Continued

\begin{tabular}{|c|c|c|c|c|c|c|c|c|c|c|c|c|c|c|c|c|c|}
\hline \multirow{2}{*}{ 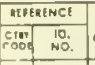 } & \multirow{2}{*}{ SMIP } & \multirow{2}{*}{\multicolumn{2}{|c|}{$\begin{array}{c}\text { LATTUUOE } \\
1 / 10 \\
\end{array}$}} & \multirow{2}{*}{$\begin{array}{c}\text { IONGIIUOE } \\
-1 / 10\end{array}$} & \multirow{2}{*}{$\begin{array}{l}\text { MAESOEN } \\
\text { SOUAAEE }\end{array}$} & \multirow{2}{*}{$\begin{array}{l}\text { STATION TIME } \\
\text { lGMAT1 }\end{array}$} & \multirow[b]{2}{*}{ YEAR } & \multicolumn{2}{|c|}{ ONGINATOR'S } & \multirow{2}{*}{$\begin{array}{c}\text { Derth } \\
\text { ro } \\
\text { noto }\end{array}$} & \multicolumn{2}{|c|}{$\begin{array}{l}\text { WAVE } \\
\text { OBSERYANONS }\end{array}$} & \multirow{2}{*}{$\begin{array}{l}\text { WEA- } \\
\text { IMER } \\
\text { COOE }\end{array}$} & \multirow{2}{*}{ 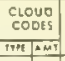 } & \multirow{2}{*}{\multicolumn{3}{|c|}{$\begin{array}{l}\text { NOOC } \\
\text { SIAIION } \\
\text { NUMAER }\end{array}$}} \\
\hline & & & & & & & & $\begin{array}{c}\text { Evilse } \\
\text { NO. }\end{array}$ & $\begin{array}{l}\text { Arion } \\
\text { AMBEX }\end{array}$ & & ou & सद्नणा का & & & & & \\
\hline $3: 12-1$ & 15 & 4254 & v & $0 \rightarrow 124 N 1$ & $16- \pm 1$ & \begin{tabular}{|l|l|l}
05 & 01 & 1 \\
\end{tabular} & $1+4.303$ & $017) 011$ & & 4018 & 33 & & 15 & \begin{tabular}{|l|l|}
2 & 7
\end{tabular} & & & 0013 \\
\hline & & & & & WAT & \begin{tabular}{l|l} 
TER & \\
\end{tabular} & WINO & o. Ait Tem. & .5 & \begin{tabular}{|l|l} 
No. & stee \\
\end{tabular} & & & & & & & \\
\hline & & & & & $\begin{array}{l}\text { COLoa } \\
\text { cook }\end{array}$ & $\begin{array}{c}\begin{array}{c}\text { Funs } \\
\text { int }\end{array} \\
\end{array}$ & \begin{tabular}{c|c} 
shet \\
or \\
roncis
\end{tabular} & \begin{tabular}{c|c} 
onv & w \\
out & uu \\
\end{tabular} & $\begin{array}{l}\text { WET } \\
\text { UuLL COOS }\end{array}$ & oiniws Ousetva & nows & & & & & & \\
\hline & & & & & & 21 & $50+1:=$ & 114 & \begin{tabular}{l|l}
0.7 & 7 \\
\end{tabular} & 21 & & & & & & & \\
\hline & 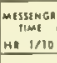 & ${ }^{2} \mathrm{CAST}$ & CARO & OETH Mn & $T \tau$ & $s \%$ & SIGMA-T & 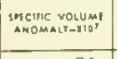 & 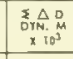 & $\begin{array}{l}\text { SOUND } \\
\text { VELOCITY }\end{array}$ & $02 \mathrm{ml} / \mathrm{s}$ & $\left|\begin{array}{c|}m_{0}, \ldots \\
\ldots, \ldots 11\end{array}\right|$ & 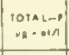 & $\left|\begin{array}{l}\mathrm{NO}_{2}-\mathrm{N} \\
\mathrm{NO}-\mathrm{OV} / 1\end{array}\right|$ & 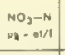 & \begin{tabular}{|l}
$510,-50$ \\
$60-01 / 1$
\end{tabular} & 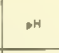 \\
\hline & & & 517 & 0003 & $15 ?$ & 2sus & 20.77 & 0312371 & 3030 & $150=5$ & & & & & & & \\
\hline & 173 & & $\begin{array}{l}\text { OAS } \\
\text { OAS }\end{array}$ & $\begin{array}{l}0000 \\
0003\end{array}$ & $15<4$ & $=30+0$ & 2977 & & & 25035 & & & & & & & \\
\hline & 17 & & $\begin{array}{l}035 \\
5 T 0\end{array}$ & $\begin{array}{l}0003 \\
2010\end{array}$ & $\begin{array}{l}1510 \\
15.7\end{array}$ & $\begin{array}{l}35003 \\
\text { E004 }\end{array}$ & 2577 & & & 15093 & & & & & & & \\
\hline & & & 510 & $\begin{array}{l}2010 \\
0020\end{array}$ & $\begin{array}{l}j 547 \\
14 \div 7\end{array}$ & $\begin{array}{l}300 k \\
3003\end{array}$ & $\begin{array}{l}\cos 7 y \\
2679\end{array}$ & $\begin{array}{l}0012537 \\
0012701\end{array}$ & $\begin{array}{l}0012 \\
0025\end{array}$ & $\begin{array}{l}150>3 \\
15089\end{array}$ & & & & & & & \\
\hline & & & STI & 0030 & 14,4 & 3000 & $\angle x B O$ & 0012072 & 0030 & 15000 & & & & & & & \\
\hline & & & STO & 3053 & 1400 & $3 ; 47$ & 3532 & 3312540 & 0063 & 15003 & & & & & & & \\
\hline & $1=$ & & NAS & 0055 & 1403 & 35572 & 2642 & & & 15083 & & & & & & & \\
\hline & 107 & & $\begin{array}{l}\text { DA } 5 \\
\text { STI }\end{array}$ & $\begin{array}{l}2753 \\
0100\end{array}$ & $\begin{array}{l}i<50 \\
14 \rightarrow r\end{array}$ & $\begin{array}{l}3,3,78 \\
3,77\end{array}$ & $\begin{array}{l}2084 \\
2000\end{array}$ & & & $\begin{array}{l}15030 \\
15035\end{array}$ & & & & & & & \\
\hline & 19 & & ad 5 & 0111 & 1439 & 15507 & $\begin{array}{l}2000 \\
2637\end{array}$ & 0012303 & 0125 & $\begin{array}{l}15035 \\
15035\end{array}$ & & & & & & & \\
\hline & & & STO & $01 \measuredangle \vdots$ & 1435 & $35: 5$ & 2007 & $301 \geq 254$ & 0156 & 15000 & & & & & & & \\
\hline & & & $5 T 0$ & 0150 & $142 ?$ & 3574 & 2667 & $001<34$, & 0197 & 15048 & & & & & & & \\
\hline & 17 & & OHS & 0167 & 1425 & $35 \mp 32$ & 2687 & & & 15039 & & & & & & & \\
\hline & 19 & & $\begin{array}{l}\text { STD } \\
\text { OBS }\end{array}$ & $\begin{array}{r}0200 \\
10222\end{array}$ & $\begin{array}{l}1332 \\
1360\end{array}$ & $\begin{array}{l}35 e^{4} \\
35901\end{array}$ & $\begin{array}{l}26>0 \\
2091\end{array}$ & 0012245 & 0249 & 15079 & & & & & & & \\
\hline & & & sin & $\begin{array}{r}10222 \\
0250\end{array}$ & $\begin{array}{l}1360 \\
1354\end{array}$ & 3540 & $\begin{array}{l}2091 \\
2071\end{array}$ & & & 15075 & & & & & & & \\
\hline & & & STO & & 1345 & 3577 & $\begin{array}{l}2071 \\
2073\end{array}$ & $\begin{array}{l}0012137 \\
0012206\end{array}$ & $\begin{array}{l}0310 \\
0371\end{array}$ & $\begin{array}{l}15078 \\
15083\end{array}$ & & & & & & & \\
\hline & 19 & & DAS & 0333 & $1: 3 y$ & 35779 & $\begin{array}{l}<0+3 \\
2074\end{array}$ & $0012<00$ & 0371 & 15086 & & & & & & & \\
\hline & & & 510 & 0400 & 1175 & 3550 & 2704 & 3013284 & $04 B B$ & 15038 & & & & & & & \\
\hline & 19 & & 035 & 1044. & 1079 & 3,355 & 2711 & & & 15010 & & & & & & & \\
\hline & 19 & & $\begin{array}{l}\text { Sto } \\
085\end{array}$ & $\begin{array}{l}0500 \\
0550\end{array}$ & 0575 & 3524 & 2722 & ग) 04001 & 0574 & 14930 & & & & & & & \\
\hline & 19 & & 085 & $1066 ?$ & $\begin{array}{l}0756 \\
0082\end{array}$ & 3503 , & 2747 & $000+174$ & 0084 & $\begin{array}{l}14523 \\
14873\end{array}$ & & & & & & & \\
\hline & & & \$ID & 0700 & 0643 & 3500 & 2752 & 00Jbel t & 3759 & 14383 & & & & & & & \\
\hline & & & 510 & 0800 & 0557 & $3+\overline{9}$ & 2751 & jo0s\$07 & 0823 & 14805 & & & & & & & \\
\hline & 19 & & 085 & 0351 & 0428 & 34958 & 2707 & & & $1+850$ & & & & & & & \\
\hline & & & STO & 0900 & $04 \leqslant 5$ & 3497 & 2708 & 0005321 & 0079 & 14056 & & & & & & & \\
\hline & & & \$10 & 2000 & 0466 & 3496 & 2771 & 0005032 & 0931 & 14861 & & & & & & & \\
\hline & & & STO & 1100 & $04<0$ & 3493 & 2773 & 0004692 & 0931 & 14857 & & & & & & & \\
\hline & 17 & & 085 & T1119 & 0426 & 34550 & 2714 & & & 14008 & & & & & & & \\
\hline & & & 510 & 1200 & 0423 & 3475 & 2774 & $000-319$ & 1027 & 14876 & & & & & & & \\
\hline & & & STD & 1300 & 0407 & $3+44$ & 2775 & 0004793 & 1077 & 14.537 & & & & & & & \\
\hline & & & STO & 1400 & 0356 & 3454 & 2776 & 000.755 & 1125 & 14837 & & & & & & & \\
\hline & & & STO & 1500 & $03+6$ & 3474 & 2777 & 0004737 & 1172 & 14311 & & & & & & & \\
\hline & 19 & & ORS & $T 1672$ & 0373 & 3.533 & 2778 & & & 14735 & & & & & & & \\
\hline & & & 5ाn & $175 \%$ & 0372 & 3454 & 2779 & $030+754$ & 1291 & 14948 & & & & & & & \\
\hline & & & ST & 2000 & 0307 & $34=4$ & 2700 & 0004835 & 1411 & $1433 d$ & & & & & & & \\
\hline & 21 & & 085 & 12051 & 0365 & $3+544$ & 2700 & & & 14936 & & & & & & & \\
\hline & & & $\$ 10$ & 2500 & $33<2$ & 3475 & 2732 & $0004331)$ & 1652 & $150 \leq 3$ & & & & & & & \\
\hline & 21 & & 035 & T25E? & $0=34$ & $3 .+46$ & 2753 & & & 15071 & & & & & & & \\
\hline & & & SID & 3000 & 0300 & 3494 & 2785 & $000465 ;$ & 1870 & 15134 & & & & & & & \\
\hline & 21 & & 785 & 3077 & 0300 & $\$ 4943$ & 2780 & & & 15144 & & & & & & & \\
\hline & 21 & & 085 & $\mathbf{T} 3591$ & 0204 & 34926 & 2730 & & & 15219 & & & & & & & \\
\hline & & & SIO & 4000 & 0239 & $3+91$ & 2739 & 0004329 & 2339 & 15280 & & & & & & & \\
\hline & 21 & & 085 & 14113 & 0235 & $3+411$ & 2789 & & & 15298 & & & & & & & \\
\hline & 21 & & 085 & 4521 & 0230 & $34=07$ & 2785 & & & 15368 & & & & & & & \\
\hline & 21 & & DRS & $T 4622$ & 0228 & $36>07$ & 2770 & & & 15385 & & & & & & & \\
\hline
\end{tabular}


TABLE VIII.-Continued

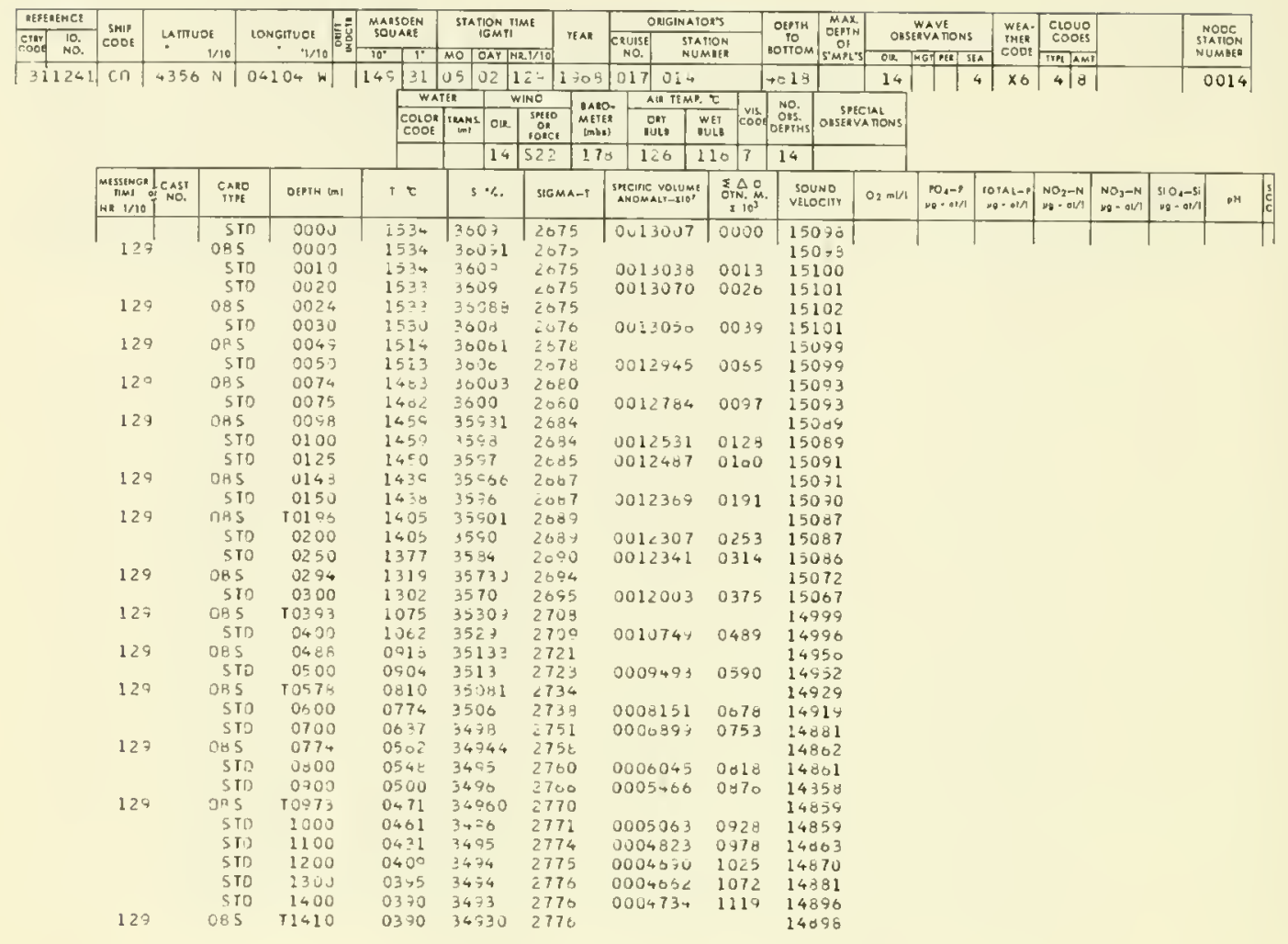

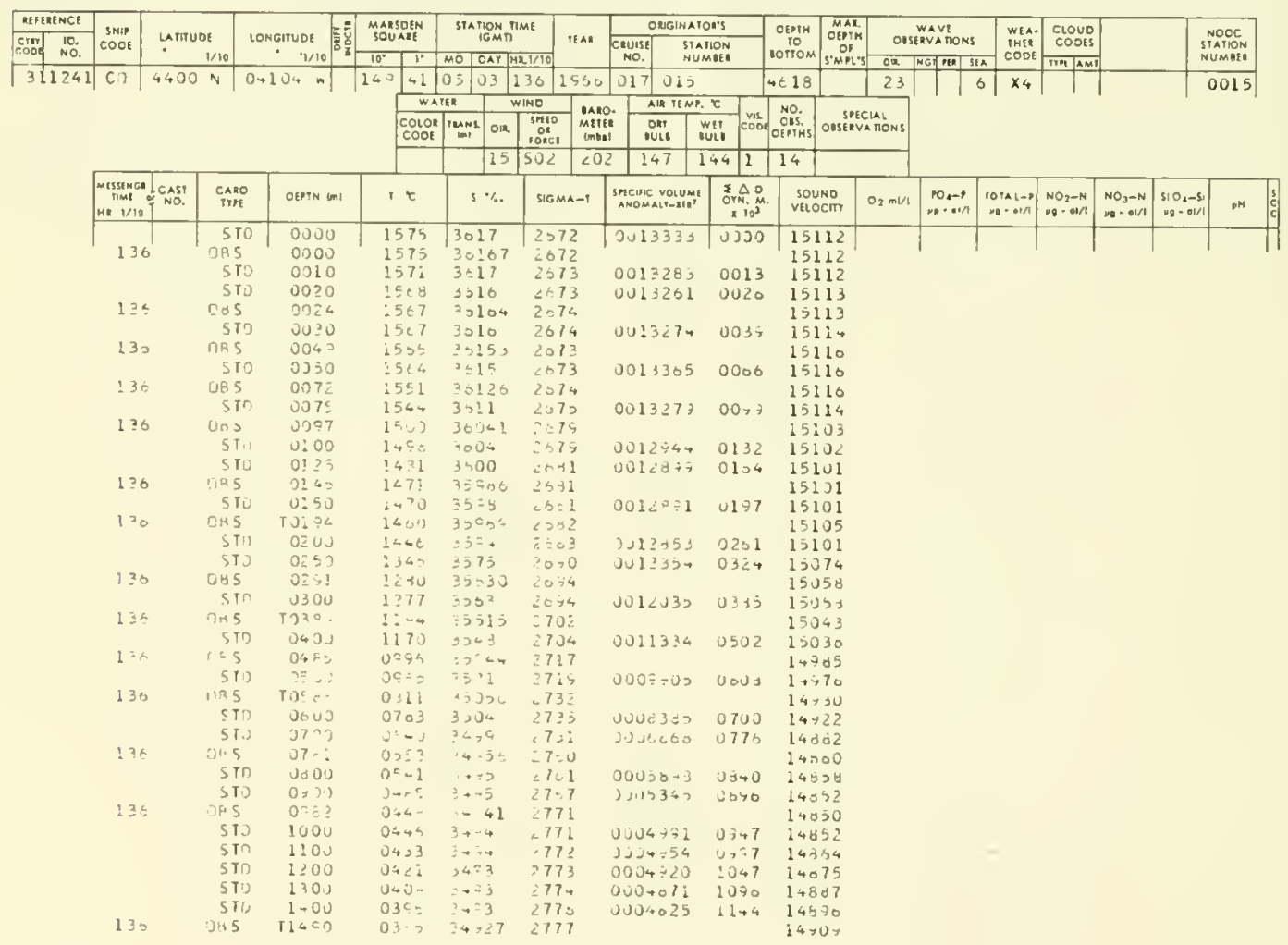


TABLE VIII.-Continued

\begin{tabular}{|c|c|c|c|c|c|c|c|c|c|c|c|c|c|c|c|c|c|c|}
\hline \multirow{2}{*}{\begin{tabular}{|c|c|} 
RISERENCE \\
Cray & 10. \\
coof & NO. \\
\end{tabular}} & \multirow{2}{*}{$\begin{array}{c}\text { SHIP } \\
\text { COOE }\end{array}$} & \multirow{2}{*}{\multicolumn{2}{|c|}{ untruor }} & \multirow{2}{*}{ LONGIIUCe } & \multirow{2}{*}{$\begin{array}{c}\text { MAESDER } \\
\text { SOUARE }\end{array}$} & \multirow{2}{*}{$\begin{array}{c}\text { STA PION IIME } \\
\text { "GM? }\end{array}$} & \multirow[b]{2}{*}{ TEAR } & \multicolumn{2}{|c|}{ ORIGINATOE'S } & \multirow{2}{*}{$\mid \begin{array}{c}\text { DEpmo } \\
10 \\
\text { sottom }\end{array}$} & \multirow{2}{*}{\begin{tabular}{|l|} 
MAR \\
DEPTH \\
OF \\
SMMLSS
\end{tabular}} & \multirow{2}{*}{\multicolumn{2}{|c|}{$\begin{array}{c}\text { WAVE } \\
\text { OUSERVATONS }\end{array}$}} & \multirow{2}{*}{$\begin{array}{l}\text { Wia: } \\
\text { Twak } \\
\text { coof }\end{array}$} & \multirow{2}{*}{$\begin{array}{l}\text { Eloua } \\
\text { cocis }\end{array}$} & \multirow{2}{*}{\multicolumn{3}{|c|}{$\begin{array}{l}\text { NOOC } \\
\text { SATFion } \\
\text { NUMUER }\end{array}$}} \\
\hline & & & & & & & & \begin{tabular}{|l|} 
Cnuis: \\
No.
\end{tabular} & $\begin{array}{l}\text { STAFION } \\
\text { NUMBER }\end{array}$ & & & Da & & & & & & \\
\hline & $\mathrm{Co}$ & 4405 & $N$ & $04106 \times 1$ & \begin{tabular}{|l|l|}
$1+7+1$ \\
\end{tabular} & 05104 & $12,150=$ & \begin{tabular}{|l|l|}
17 & $0: 0$ \\
\end{tabular} & & $\frac{80110 \mathrm{~m}}{-5,1 y}$ & & 03 & \multirow{4}{*}{$1 / 3$} & $3 \times 1$ & $2 / 4$ & & & 0016 \\
\hline & & & & & WAI & \begin{tabular}{l|l} 
TER & \\
\end{tabular} & WINO & Ale TEMP. & & NO. & & & & & & & & \\
\hline & & & & & \begin{tabular}{|l|} 
COLOR \\
COOE \\
\end{tabular} & \begin{tabular}{|c|c} 
reans \\
ind
\end{tabular} & 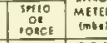 & \begin{tabular}{l|l} 
ong & $w$ \\
sutu & iu \\
\end{tabular} & Et & 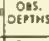 & 5 OASERVA & DONS & & & & & & \\
\hline & & & & & & $9 ?$ & 513 & 149 & \begin{tabular}{l|l}
30 & 7
\end{tabular} & 14 & & & & & & & & \\
\hline & $\begin{array}{l}\text { WISSINGC } \\
\operatorname{liml} \\
\text { HR } 1 / 10 \\
\end{array}$ & I Lass. & $\begin{array}{l}\text { CARD } \\
\text { Trof }\end{array}$ & DEPTN UII & 12 & $s \%$ & SIGMA-1 & 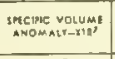 & 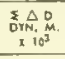 & $\begin{array}{l}\text { se } \\
\text { vet }\end{array}$ & $\begin{array}{l}\text { SUNO } \\
\text { LoentT }\end{array}$ & $0, \mathrm{~m} / 1$ & 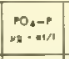 & 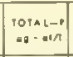 & $\begin{array}{l}\mathrm{NO}_{2}-\mathrm{N} \\
\mathrm{Ne}-\mathrm{OH/2}\end{array}$ & 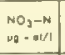 & $\left|\begin{array}{c|c}510.451 \\
28-81 / 4\end{array}\right|$ & DH \\
\hline & 12 & & $\begin{array}{l}\text { OBS } \\
\text { Sin }\end{array}$ & $\begin{array}{l}0000 \\
3012\end{array}$ & $\begin{array}{l}1574 \\
1555\end{array}$ & $\begin{array}{l}30129 \\
3011\end{array}$ & $\begin{array}{l}<=5 \\
2 \leqslant 72\end{array}$ & จग 13364 & 01213 & 15 & 111 & & & & & & & \\
\hline & & & $<\mathrm{I}$, & บบㄴ & $15=7$ & $330-$ & 2074 & 5013169 & 6020 & & 132 & & & & & & & \\
\hline & $: 21$ & & $y=5$ & $\mathrm{Oug}_{2} 4$ & 1531 & $\therefore 20 y 0$ & 2075 & & & & $\mathrm{LJi}$ & & & & & & & \\
\hline & & & 310 & $3: 7$ & 152. & 3003 & $\angle D 7 D$ & 3013001 & 0037 & & 100 & & & & & & & \\
\hline & 12 & & $\begin{array}{l}\text { UHS } \\
\text { SIO }\end{array}$ & $\begin{array}{l}0050 \\
0075\end{array}$ & 1499 & $300+4$ & 2630 & & & & 5095 & & & & & & & \\
\hline & 12 & & oas & $\begin{array}{l}0073 \\
0075\end{array}$ & $\begin{array}{l}1400 \\
1450\end{array}$ & $\begin{array}{l}j 596 \\
55 \geqslant 60\end{array}$ & $200 ?$ & 9312036 & 0957 & & 5035 & & & & & & & \\
\hline & & & 510 & 0100 & 1427 & $\begin{array}{l}35 \div 60 \\
3587\end{array}$ & 2002 & 0012527 & 0126 & $\begin{array}{l}1 \\
1\end{array}$ & $\begin{array}{l}5085 \\
5073\end{array}$ & & & & & & & \\
\hline & 12 & & 085 & 0100 & 1427 & $\begin{array}{l}3547 \\
35=92\end{array}$ & $\begin{array}{l}2084 \\
2884\end{array}$ & 0012325 & $1<0$ & & 5078 & & & & & & & \\
\hline & & & sto & $01<5$ & 1423 & $35 \subseteq 1$ & 2085 & $00: 2473$ & 0100 & & 5083 & & & & & & & \\
\hline & & & 510 & 0150 & 1427 & 3593 & 2586 & $00+2+21$ & 0191 & & 5087 & & & & & & & \\
\hline & 120 & & OB 5 & 0150 & 1427 & $\equiv j=3 i$ & 2640 & & & & 5087 & & & & & & & \\
\hline & & & STO & 0203 & 1354 & $3>75$ & 2000 & 0012387 & 0253 & & 5067 & & & & & & & \\
\hline & 12 & & OBS & TOZOU & 1354 & $3574 \theta$ & 2085 & & & & 5009 & & & & & & & \\
\hline & & & STD & 0253 & 1327 & 3571 & 2590 & 0012235 & 0314 & & 5366 & & & & & & & \\
\hline & & & STD & 0300 & 1285 & 3555 & 2694 & 0012021 & 0375 & & 5001 & & & & & & & \\
\hline & 12 & & OAS & 0301 & 1254 & 32040 & 2596 & & & & 5001 & & & & & & & \\
\hline & & & STD & 0400 & $11 \equiv 7$ & 3542 & 2706 & 0011130 & 0451 & & 5024 & & & & & & & \\
\hline & 12 & & TRS & 0402 & 1134 & $35+20$ & 2706 & & & & 5023 & & & & & & & \\
\hline & & & STO & 0500 & $05 B 1$ & $35 \geq 7$ & 2721 & 0009750 & 0595 & & 4503 & & & & & & & \\
\hline & 12 & & 085 & 0504 & $0 \div 74$ & उड்क0 & 2722 & & & & 4981 & & & & & & & \\
\hline & & & sto & 0000 & $07 \bullet 3$ & 3503 & 2737 & 0005191 & 0085 & & $4+14$ & & & & & & & \\
\hline & 12 & & OH $S$ & 10504 & 0750 & 35024 & 2730 & & & & 4912 & & & & & & & \\
\hline & & & 5 TD & 0700 & 0056 & 3501 & 2.751 & 0006903 & 0760 & & 4589 & & & & & & & \\
\hline & & & $5 T 0$ & 0800 & 0575 & 3501 & 2701 & 0005968 & 0825 & & 4873 & & & & & & & \\
\hline & 12 & & OP 5 & $081 \div$ & 05 bi & 35004 & $27>2$ & & & & 4071 & & & & & & & \\
\hline & & & $5 T 0$ & 3930 & 0526 & 3509 & 2760 & 3005493 & 0,82 & & 4870 & & & & & & & \\
\hline & & & SID & 1000 & 0407 & $34 ; 9$ & 2771 & 0005131 & 0335 & & 4070 & & & & & & & \\
\hline & 12 & & JA5 & 11024 & 047 & 14772 & 2772 & & & & 4670 & & & & & & & \\
\hline & & & SIO & 1100 & 0454 & 3497 & 2774 & 0004050 & 3905 & & 4873 & & & & & & & \\
\hline & & & 510 & 1200 & 0427 & $34 c_{3}$ & 2776 & 0004082 & 1033 & & 4879 & & & & & & & \\
\hline & & & 510 & 1300 & $040 d$ & 3477 & 2777 & 0004570 & 1079 & & 4987 & & & & & & & \\
\hline & & & STO & 1400 & 0395 & 3676 & $277 d$ & $300+593$ & 1125 & & 4895 & & & & & & & \\
\hline & & & 510 & 1500 & $036 ?$ & 3495 & 2778 & v004063 & 1172 & & 4913 & & & & & & & \\
\hline & 12 & & oHs & $I 1 \leq 30$ & 0380 & 34244 & 2777 & & & & 4918 & & & & & & & \\
\hline
\end{tabular}

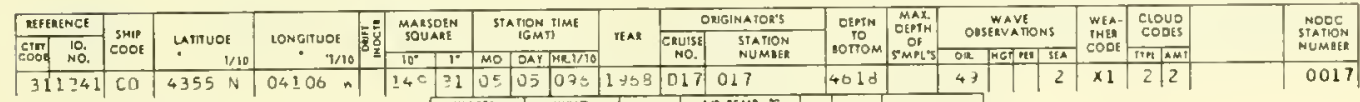

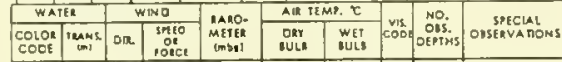

\begin{tabular}{|c|c|c|c|c|c|c|c|c|c|c|c|c|c|c|c|}
\hline 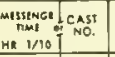 & $\underset{\substack{\text { CARD } \\
\text { TWFE }}}{ }$ & OEPYM Un! & $\tau \tau$ & $5 * /$ & SIGMA-T & $\begin{array}{l}\text { smeine volumt } \\
\text { ANOMALIIIIt? }\end{array}$ & $\begin{array}{l}\text { IrA. } \\
\text { orn. } \\
\times 10^{3} \\
\end{array}$ & $\begin{array}{c}\text { SOUNO } \\
\text { YELOCITT }\end{array}$ & $O_{2} \mathrm{~m} / \mathrm{A}$ & 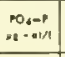 & 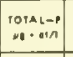 & 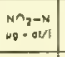 & $\begin{array}{l}\mathrm{NO}_{3}-\mathrm{N} \\
\mathrm{NO}-\mathrm{MN}\end{array}$ & $\begin{array}{l}510_{4}-5.5 \\
00-0111\end{array}$ & $\mathrm{OH}$ \\
\hline \multirow{4}{*}{$0: 6$} & 510 & 3000 & 140 & 2590 & $? 664$ & 5013004 & Јふ৩ง & 12034 & & & & & & & \\
\hline & OHS & 0000 & $14=c$ & $35+39$ & $200 x$ & & & 15084 & & & & & & & \\
\hline & STO & 0010 & $14 c_{3}$ & 3543 & 2675 & 0013117 & 0013 & 15007 & & & & & & & \\
\hline & 5Tว & 0320 & 1501 & 300, & $267 t$ & 0312842 & $0\lrcorner 26$ & $150 \div 0$ & & & & & & & \\
\hline \multirow[t]{2}{*}{$0>0$} & DP 5 & 0024 & 2502 & 30030 & 2670 & & & 15091 & & & & & & & \\
\hline & STO & 0030 & $1+51$ & 3603 & $\therefore 077$ & 3012743 & 0035 & 15091 & & & & & & & \\
\hline \multirow[t]{2}{*}{095} & กณ 5 & 0042 & $1+84$ & $\$ 0000$ & 2000 & & & 10089 & & & & & & & \\
\hline & sin & 0050 & $14=?$ & $300 !$ & 2530 & 0012700 & 0064 & $1500 \bar{y}$ & & & & & & & \\
\hline \multirow[t]{2}{*}{096} & กค. 5 & 0073 & $1 \rightarrow 00$ & 35575 & 2643 & & & 15085 & & & & & & & \\
\hline & STO & $007=$ & 1456 & 3597 & 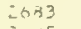 & $001<507$ & 0076 & 15004 & & & & & & & \\
\hline \multirow[t]{3}{*}{376} & DAS & $0 \supset S_{3}$ & $14: 7$ & 35378 & 2005 & & & 15074 & & & & & & & \\
\hline & $5 \pi 0$ & ul oo & 1415 & 3335 & 2085 & J012:27 & 0127 & 15074 & & & & & & & \\
\hline & STn & $0 ! 25$ & 1205 & 3586 & 20007 & 001222 , & 3158 & 15073 & & & & & & & \\
\hline \multirow[t]{2}{*}{$1 \subseteq 5$} & Dos 5 & $0 i \leftarrow 7$ & 1363 & $35=38$ & $\sum 609$ & & & 15071 & & & & & & & \\
\hline & STO & 0150 & 13.31 & 3573 & $200 \div$ & $00121>5$ & 0138 & 15070 & & & & & & & \\
\hline \multirow[t]{3}{*}{$0 \subseteq 5$} & Uิes & $T 0190$ & 1364 & 35760 & $\angle 671$ & & & 15005 & & & & & & & \\
\hline & STO & $0: 03$ & 1341 & 1576 & $i t=1$ & 3012351 & 3247 & 13005 & & & & & & & \\
\hline & sin & 0250 & $1: 01$ & $\$ 507$ & $2 t-3$ & Ju12011 & 0309 & $1>050$ & & & & & & & \\
\hline \multirow[t]{2}{*}{$0=6$} & 065 & $0 \geq 05$ & $1=51$ & 35590 & 2597 & & & $15040^{\circ}$ & & & & & & & \\
\hline & $5 \pi$ & 9300 & 1243 & रइ5त & $20 ; 7$ & $321: 743$ & U 100 & $150+7$ & & & & & & & \\
\hline \multirow[t]{2}{*}{$0 \div 6$} & 095 & $10 ; 95$ & $1: 01$ & 253,4 & 2700 & & & 15010 & & & & & & & \\
\hline & SI & $0+00$ & $10=\angle$ & 3537 & 2710 & $J J: 0 b>1$ & 0400 & 15007 & & & & & & & \\
\hline \multirow[t]{2}{*}{$2 \subseteq 4$} & ges & 0,52 & $0=53$ & $=5172$ & 2723 & & & 14960 & & & & & & & \\
\hline & STR & 0500 & 02314 & $35: 0$ & 2764 & $000=422$ & $\cup 501$ & $1+750$ & & & & & & & \\
\hline \multirow[t]{3}{*}{$0=0$} & nis & TO5 40 & 0771 & 35020 & 2735 & & & 14915 & & & & & & & \\
\hline & 510 & 0000 & $07=5$ & 2502 & $\therefore 730$ & $000 \times 125$ & $0=69$ & 14511 & & & & & & & \\
\hline & 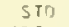 & 373 & $06 \times 5$ & $2+77$ & $275 !$ & $3002>0$, & 0744 & $1 \leftrightarrow 2 \$ 0$ & & & & & & & \\
\hline \multirow[t]{4}{*}{$0=5$} & .105 & $076=$ & $05 E 0$ & $34 \times 45$ & $75 \div$ & & & 14801 & & & & & & & \\
\hline & เก & ט:09 & $0=-7$ & $3 \ldots .5$ & 2700 & (0)נ & usjo & $1+351$ & & & & & & & \\
\hline & 510 & 0.00 & 36,3 & 3455 & 2765 & 0035507 & 0506 & 14855 & & & & & & & \\
\hline & SI) & 1000 & $045=$ & $3+\div 5$ & 2770 & jou>102 & $0=19$ & 14550 & & & & & & & \\
\hline \multirow[t]{6}{*}{$09 n$} & 025 & 11000 & $\mathrm{O}_{4}=5$ & $3+745$ & 2770 & & & $1 \rightarrow 050$ & & & & & & & \\
\hline & 510 & 1103 & $0 \leftrightarrow 3$ & 3495 & -772 & susouls & 3970 & 1400 & & & & & & & \\
\hline & STI & 1200 & $0-3$ & 34.75 & 2773 & $00345+0$ & 1020 & 14879 & & & & & & & \\
\hline & $5 T:$ & 1700 & $04: 3$ & 3434 & 2775 & 00uses & $105 y$ & 14870 & & & & & & & \\
\hline & STR & {$[40\}$} & $04: 1$ & 3434 & $\angle 770$ & $000+7 ; 3$ & $\$ 117$ & 14701 & & & & & & & \\
\hline & STD & $: 500$ & $03=?$ & $34: 4$ & 6777 & 10004740 & 1105 & $1+911$ & & & & & & & \\
\hline 075 & QRS & $T: 50=$ & $03-6$ & 34930 & 2777 & & & $1 \rightarrow 912$ & & & & & & & \\
\hline
\end{tabular}


TABLE IX.-Observed and interpolated oceanographic data for stations taken by USCGC DALLAS at Ocean Station DELTA, 6 May-28 May 1968, prepared from NODC listing No. 31-1265 DG.

\begin{tabular}{|c|c|c|c|c|c|c|c|c|c|c|c|c|c|c|c|c|c|c|}
\hline \multirow{3}{*}{\begin{tabular}{|c|c|}
\multicolumn{2}{|c|}{ REF ERENCE } \\
Crisy & $\begin{array}{c}10 . \\
\text { eoo }\end{array}$ \\
31126. \\
31265
\end{tabular}} & \multirow{3}{*}{\begin{tabular}{|c|} 
SHIP \\
COOE \\
$O G$ \\
\end{tabular}} & \multirow{2}{*}{\multicolumn{2}{|c|}{$\begin{array}{c}\text { Latituoe } \\
1 / 10 \\
\end{array}$}} & \multirow{2}{*}{$\begin{array}{c}\text { LONGITUOE } \\
11 / 10\end{array}$} & \multirow{2}{*}{$\begin{array}{l}\text { MARSOEN } \\
\text { SOUARE }\end{array}$} & \multirow{2}{*}{\multicolumn{2}{|c|}{$\begin{array}{c}\text { STATION TIME } \\
\text { IGMVI }\end{array}$}} & \multicolumn{2}{|c|}{ ONGINATOR'S } & \multirow{2}{*}{$\begin{array}{c}\text { DEPH } \\
\text { ro } \\
\text { DOTOM }\end{array}$} & \multirow{2}{*}{\begin{tabular}{|l|} 
MAN: \\
DEPTH \\
OF \\
S'MPL's
\end{tabular}} & \multirow{2}{*}{\multicolumn{2}{|c|}{$\begin{array}{l}\text { WAVF } \\
\text { OISREATONS }\end{array}$}} & \multirow{2}{*}{$\begin{array}{l}\text { WEA- } \\
\text { YHER } \\
\text { CODE }\end{array}$} & \multirow{2}{*}{$\begin{array}{l}\text { clovo } \\
\text { cooks }\end{array}$} & & & \multirow{2}{*}{$\begin{array}{l}\text { NOOC } \\
\text { SHATHON } \\
\text { NUMAER }\end{array}$} \\
\hline & & & & & & & YEAR & $\begin{array}{c}\text { Cauts: } \\
\text { no. }\end{array}$ & $\begin{array}{l}\text { STATION } \\
\text { NUMBEE }\end{array}$ & & & & & & & & & \\
\hline & & 4401 & N & $04056 \mathrm{~W}$ & \begin{tabular}{|c|c|}
$10^{\circ}$ & $1^{\circ}$ \\
\end{tabular} & \begin{tabular}{|l|l|}
05 & 00 \\
\end{tabular} & \begin{tabular}{|l|l|}
75 & 1968 \\
\end{tabular} & \begin{tabular}{|l|l|}
018 & 001 \\
\end{tabular} & & & 13 & 36 & \begin{tabular}{|l|l|l|}
1 & 3 &
\end{tabular} & $\times 4$ & \begin{tabular}{|l|l|}
3 & 4
\end{tabular} & & & 0001 \\
\hline & & & & & $\frac{1}{W / A}$ & TER & \begin{tabular}{l|l} 
WIND & \\
\end{tabular} & A. AlR Timp. & & & & & & & & & & \\
\hline & & & & & $\begin{array}{l}\text { colok } \\
\text { codit }\end{array}$ & 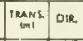 & 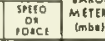 & \begin{tabular}{l|l} 
oky & Iut \\
Iut
\end{tabular} & $\begin{array}{lll}4 E 1 \\
\text { Vit }\end{array}$ & ons, & $\begin{array}{c}\text { SERG } \\
\text { OAsEA }\end{array}$ & IONS & & & & & & \\
\hline & & & & & & 23 & 505 & 200 & $72 \sqrt{7}$ & 14 & & & & & & & & \\
\hline & $\begin{array}{l}\text { Mutsanen } \\
\text { Timat } \\
\text { He } 1 / 10\end{array}$ & $\begin{array}{l}\text { Scst } \\
\text { Tos. }\end{array}$ & $\begin{array}{l}\text { CARO } \\
\text { TYPE }\end{array}$ & OEPTH in! & it & $3 \%$ & SIGHA-1 & 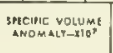 & 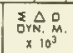 & $\begin{array}{l}\text { SOUN } \\
\text { VELOC }\end{array}$ & 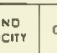 & $02 \mathrm{~m} / 1$ & 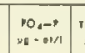 & 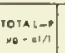 & $\begin{array}{l}\mathrm{NO}_{3}-\mathrm{N} \\
\mathrm{HO}^{2}=\mathrm{OWN1}\end{array}$ & $\begin{array}{l}\mathrm{NO}_{3}-\mathrm{N} \\
\mathrm{We}_{\mathrm{O}} \mathrm{OH/1}\end{array}$ & $\begin{array}{l}510 .-5 i \\
y=001 / 4\end{array}$ & PH \\
\hline & & & 510 & 0000 & 1642 & 3611 & 2652 & 0015194 & 0000 & 1513 & 32 & & & & & & & \\
\hline & 175 & & 085 & 0000 & 1642 & 36113 & 2652 & & & 1513 & 32 & & & & & & & \\
\hline & & & $5 \mathrm{IO}$ & 0010 & 1584 & 3611 & 2666 & 0013950 & 0015 & 1511 & & & & & & & & \\
\hline & & & $5 \mathrm{TO}$ & 0020 & 1545 & 3611 & 2675 & 0013140 & 0028 & 1510 & 05 & & & & & & & \\
\hline & 175 & & OAS & 0022 & 1540 & 36112 & 2670 & & & 1510 & .04 & & & & & & & \\
\hline & & & sta & 0030 & 1540 & 3611 & 2676 & 0013056 & 0041 & 1510 & 05 & & & & & & & \\
\hline & 175 & & D85 & 0047 & 1536 & 36117 & 2677 & & & 1510 & 07 & & & & & & & \\
\hline & & & STO & 0050 & 1534 & 3611 & 2677 & 0013019 & 0067 & 1510 & .06 & & & & & & & \\
\hline & 175 & & DBS & 0070 & 1520 & 36079 & 2678 & & & 1510 & 05 & & & & & & & \\
\hline & & & $5 T 0$ & 0075 & 1516 & 3608 & 2678 & 0012961 & 0100 & 1510 & 04 & & & & & & & \\
\hline & 175 & & D85 & 0094 & 1498 & 36064 & 2681 & & & 1510 & 02 & & & & & & & \\
\hline & & & STD & 0100 & 1488 & 3603 & 2681 & 0012781 & 0132 & 150 & 199 & & & & & & & \\
\hline & & & $5 \pi 0$ & 0125 & 1453 & 3554 & 2682 & 0012782 & 0164 & 150 & 91 & & & & & & & \\
\hline & 175 & & D85 & 0141 & 1437 & 35896 & 2682 & & & 1508 & 188 & & & & & & & \\
\hline & & & STo & 0150 & 1435 & 3589 & 2662 & 0012826 & 0196 & 1508 & 89 & & & & & & & \\
\hline & 175 & & DBS & 10187 & 1417 & 35881 & 2685 & & (2) & 1508 & 89 & & & & & & & \\
\hline & & & $5 T 0$ & 0200 & 1402 & 3586 & 2686 & 0012530 & 0259 & 1508 & 86 & & & & & & & \\
\hline & & & STO & 0250 & 1344 & 3575 & 2090 & 0012309 & 0321 & $150 ?$ & 74 & & & & & & & \\
\hline & 175 & & DBS & 0277 & 1313 & 35693 & 2692 & & & 1506 & 67 & & & & & & & \\
\hline & & & STO & 0300 & 1291 & 3564 & 2692 & 0012202 & 0383 & 1506 & 163 & & & & & & & \\
\hline & 175 & & DBS & 0362 & 1213 & 35498 & 2697 & & & 1504 & 145 & & & & & & & \\
\hline & & & 510 & 0400 & 1148 & 3543 & 2704 & 0011280 & 0500 & 1502 & 28 & & & & & & & \\
\hline & 175 & & DBS & 0444 & 1071 & 35342 & 2711 & & & 1500 & 107 & & & & & & & \\
\hline & & & $5 T 0$ & 0.500 & 0973 & 3520 & 2717 & 0010101 & 0607 & 1497 & 79 & & & & & & & \\
\hline & 175 & & D85 & 10522 & 0933 & 35151 & 2720 & & & 1496 & 67 & & & & & & & \\
\hline & & & 510 & 0.600 & 0752 & 3499 & 2736 & 0000311 & 0699 & 1490 & 09 & & & & & & & \\
\hline & 175 & & D.85 & 0680 & 0620 & 34906 & 2747 & & & 1407 & 70 & & & & & & & \\
\hline & & & STD & 0700 & 0606 & 3491 & 2749 & 0006965 & 0775 & 1488 & 867 & & & & & & & \\
\hline & & & $5 T 0$ & 0800 & 0545 & 3495 & 2760 & 0005981 & 0840 & 1486 & 160 & & & & & & & \\
\hline & 175 & & D 85 & 10847 & 0520 & 34962 & 2764 & & & 1485 & 58 & & & & & & & \\
\hline & & & 510 & 0900 & 0495 & 3496 & 2767 & 0005379 & 0897 & 1485 & 156 & & & & & & & \\
\hline & & & STO & 1000 & 0456 & 3496 & 2771 & 0005017 & 0949 & 1485 & 157 & & & & & & & \\
\hline & & & STO & 1100 & 0429 & 3495 & 2774 & 0004812 & 0998 & $14 \mathrm{Bt}$ & 162 & & & & & & & \\
\hline & & & $5 \mathrm{TO}$ & 1200 & 0413 & 3495 & 2775 & 0004742 & 1040 & 1487 & 72 & & & & & & & \\
\hline & 175 & & D8 5 & T1290 & 0408 & 34940 & 2775 & & & 1488 & 85 & & & & & & & \\
\hline
\end{tabular}

\begin{tabular}{|c|c|c|c|c|c|c|c|c|c|c|c|c|c|c|c|c|c|c|}
\hline \multirow{2}{*}{\begin{tabular}{|c|c|}
\multicolumn{2}{|c|}{ REFEAENCE } \\
Crer & 10 \\
Coo & No. \\
\end{tabular}} & \multirow{2}{*}{$\begin{array}{c}5 \mathrm{HII} \\
\mathrm{COOE}\end{array}$} & \multirow{2}{*}{\multicolumn{2}{|c|}{$\begin{array}{c}\text { MIIIUOE } \\
1 / 10\end{array}$}} & \multirow{2}{*}{ LONGITUDE } & \multirow{2}{*}{$\begin{array}{l}\text { MARSOIN } \\
\text { SOUARE }\end{array}$} & \multirow{2}{*}{\multicolumn{2}{|c|}{$\begin{array}{l}\text { STA TION BIMA } \\
\text { IGMHI }\end{array}$}} & \multicolumn{2}{|c|}{ ORGINATOR'S } & \multirow{2}{*}{$\begin{array}{c}\text { OEPIH } \\
10 \\
\text { 1OTROM } \\
\end{array}$} & \multirow{2}{*}{ 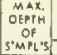 } & \multirow{2}{*}{\multicolumn{2}{|c|}{$\begin{array}{l}\text { WAVE } \\
\text { OUSËAYATONS }\end{array}$}} & \multirow{2}{*}{$\mid \begin{array}{l}\text { WEA- } \\
\text { HHA } \\
\text { CODE }\end{array}$} & \multirow{2}{*}{\begin{tabular}{|c|}
$\begin{array}{c}\text { cloug } \\
\text { coots }\end{array}$ \\
Thi 1 Am?
\end{tabular}} & & & \multirow{2}{*}{$\begin{array}{l}\text { NODC } \\
\text { SAPION } \\
\text { NUMAER }\end{array}$} \\
\hline & & & & & & & YEAR & $\begin{array}{c}\text { EnUise } \\
\text { NOS. } \\
\end{array}$ & $\begin{array}{l}\text { STA TION } \\
\text { NUMBEE }\end{array}$ & & & & & & & & & \\
\hline \multirow[t]{42}{*}{311265} & DG & 4400 & $\mathrm{~N}$ & $04051 W$ & 149,40 & 05,07 & \begin{tabular}{l|l|}
26 & 1968 \\
\end{tabular} & \begin{tabular}{|l|l|}
018 & 002 \\
\end{tabular} & & & 17 & 20 & $\begin{array}{lll}2 & 2\end{array}$ & & \begin{tabular}{l|l|l|}
3 & 4 \\
\end{tabular} & & & 0002 \\
\hline & & & & & WAI & IfER & WINO IAMO & o. & & & & & & & & & & \\
\hline & & & & & $\begin{array}{c}\mathrm{COLOR} \\
\mathrm{COOE}\end{array}$ & $\begin{array}{c}\substack{\text { IRank } \\
\text { tani }} \\
\end{array}$ & 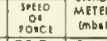 & \begin{tabular}{l|l} 
oar & w \\
nuts & it \\
\end{tabular} & $\begin{array}{lll}\text { WET } & \text { vit } \\
\text { ULLA } & \\
\end{array}$ & Oons. & OBSERYA & A IONS & & & & & & \\
\hline & & & & & & 19 & 515 & 172 & 567 & 14 & & & & & & & & \\
\hline & $\begin{array}{l}\text { MESSENGE } \\
\text { HiMM } \\
\text { ME } 1 / 10\end{array}$ & 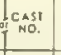 & $\underset{\text { Irpe }}{\text { Cano }}$ & $D E P(H)(m)$ & $r \tau$ & $s \%$ & SIGMA-1 & 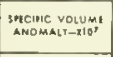 & 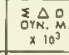 & $\begin{array}{l}\text { Soun } \\
\text { Yeloc }\end{array}$ & $\begin{array}{l}\text { JNO } \\
\text { JCITY }\end{array}$ & $0 . \mathrm{mlat}$ & 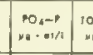 & 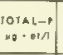 & $\mid \begin{array}{l}\mathrm{NO}_{2}-\mathrm{N} \\
\mathrm{NO}-\mathrm{N} / 1\end{array}$ & $\begin{array}{l}\mathrm{NO}_{3}-\mathrm{N} \\
\mathrm{No}=\mathrm{NO/1}\end{array}$ & $\begin{array}{l}310,-50 \\
40=0+1 / 4\end{array}$ & $\begin{array}{l}-5, \\
14\end{array}$ \\
\hline & 125 & & $\begin{array}{l}510 \\
085\end{array}$ & $\begin{array}{l}0000 \\
0000\end{array}$ & $\begin{array}{l}1523 \\
1523\end{array}$ & $\begin{array}{l}3585 \\
35853\end{array}$ & $\begin{array}{l}2660 \\
2660\end{array}$ & 0014505 & 0000 & $\begin{array}{l}150^{\circ} \\
150^{\circ}\end{array}$ & & & & & & & 1 & 1 \\
\hline & & & $5 T 0$ & 0010 & 1520 & 3595 & 2668 & 0013764 & 0014 & 150 & 94 & & & & & & & \\
\hline & & & STD & 0020 & 1506 & 3600 & 2675 & 0013134 & 0028 & 130 & 91 & & & & & & & \\
\hline & 126 & & 085 & 0027 & 1489 & 36002 & 2679 & & & 1508 & 87 & & & & & & & \\
\hline & & & 510 & 0030 & 1470 & 3596 & 2680 & 0012702 & 0041 & 150 & 181 & & & & & & & \\
\hline & & & $5 T 0$ & 0050 & 1386 & 3578 & 2684 & 0012361 & 0066 & 1505 & 55 & & & & & & & \\
\hline & 125 & & 085 & 0053 & 1380 & 35769 & 2684 & & & 1505 & 53 & & & & & & & \\
\hline & & & STL & 0075 & 1398 & 3585 & 2667 & 0012164 & 0096 & 150 & 64 & & & & & & & \\
\hline & 126 & & DBS & 0081 & 1359 & 35856 & 2687 & & & 1500 & 165 & & & & & & & \\
\hline & & & 510 & 0100 & 1389 & 3584 & 2688 & 0012128 & 0127 & $150 \mathrm{t}$ & 65 & & & & & & & \\
\hline & 126 & & 085 & 0109 & 1385 & 35837 & 2688 & & & 1506 & 65 & & & & & & & \\
\hline & & & STo & 0125 & 1378 & 3582 & 2688 & 0012125 & 0157 & 1506 & 65 & & & & & & & \\
\hline & & & 510 & 0150 & 1366 & 3579 & 2689 & 0012175 & 0187 & 1506 & 165 & & & & & & & \\
\hline & 126 & & OBS & 0164 & 1359 & 35778 & 2089 & & & 1506 & 165 & & & & & & & \\
\hline & & & $5 T 0$ & 0200 & 1343 & 3573 & 2689 & 0012296 & 0248 & 1506 & 65 & & & & & & & \\
\hline & 126 & & Des & 10220 & 1331 & 35708 & 2690 & & & 1506 & & & & & & & & \\
\hline & & & SIO & 0250 & 1305 & 3566 & 2691 & 0012195 & 0310 & 1506 & & & & & & & & \\
\hline & & & STO & 0300 & 1253 & $355 d$ & 2695 & 0011904 & 0370 & 1504 & & & & & & & & \\
\hline & 126 & & DES & 0335 & 1211 & 35525 & 2699 & & & 1504 & & & & & & & & \\
\hline & & & STO & 0400 & 1122 & 3540 & 2707 & 0011024 & 0485 & 1501 & & & & & & & & \\
\hline & 126 & & DBS & I 0453 & $10 \geq 6$ & 35297 & 2714 & & & 1493 & & & & & & & & \\
\hline & & & $5 \mathrm{TO}$ & 0500 & 0932 & 3518 & 2723 & 0009562 & 0587 & 1426 & & & & & & & & \\
\hline & 126 & & oBs & 0502 & OB 19 & 35065 & 2732 & & & 1493 & & & & & & & & \\
\hline & & & STO & 0600 & $07 \in 9$ & 3504 & 2737 & 0008199 & 9676 & 1491 & & & & & & & & \\
\hline & 126 & & D85 & 10673 & 0083 & 35006 & 2747 & & & 1489 & & & & & & & & \\
\hline & & & 510 & 0700 & 0557 & 3501 & 2750 & 0006975 & 0752 & 1480 & & & & & & & & \\
\hline & & & STD & 0800 & 0574 & 3500 & 2761 & 0005986 & 0817 & 1407 & & & & & & & & \\
\hline & 120 & & OBS & 0836 & 0514 & 34398 & 2768 & & & 1486 & & & & & & & & \\
\hline & & & $5 \mathrm{TO}$ & 0900 & 0513 & 3500 & 2708 & 0005326 & 0874 & 1486 & & & & & & & & \\
\hline & & & $5 T 0$ & 1000 & 0403 & 3497 & 2771 & 0005114 & 0926 & 1486 & & & & & & & & \\
\hline & & & 510 & 1100 & 0457 & 3498 & 2773 & 0004943 & 0976 & 1487 & & & & & & & & \\
\hline & 126 & & 085 & T1130 & 0450 & 34978 & 2774 & & & 1487 & & & & & & & & \\
\hline & & & STD & 1200 & 0435 & 3457 & 2775 & 0004841 & 1325 & 1488 & & & & & & & & \\
\hline & & & $5 \mathrm{TO}$ & 1300 & 0416 & 3497 & 2777 & 0004692 & $: 073$ & 1467 & & & & & & & & \\
\hline & & & $5 \pi 0$ & 1400 & 0401 & 3496 & 2777 & 0004663 & 1119 & 1490 & & & & & & & & \\
\hline & & & $5 \mathrm{IO}$ & 1500 & 0390 & 3495 & 2778 & 0004682 & 1160 & 1491 & 13 & & & & & & & \\
\hline & 126 & & D 85 & 12737 & 0379 & 34940 & 2778 & & & 1494 & & & & & & & & \\
\hline
\end{tabular}




\section{TABLE IX.-Continued}

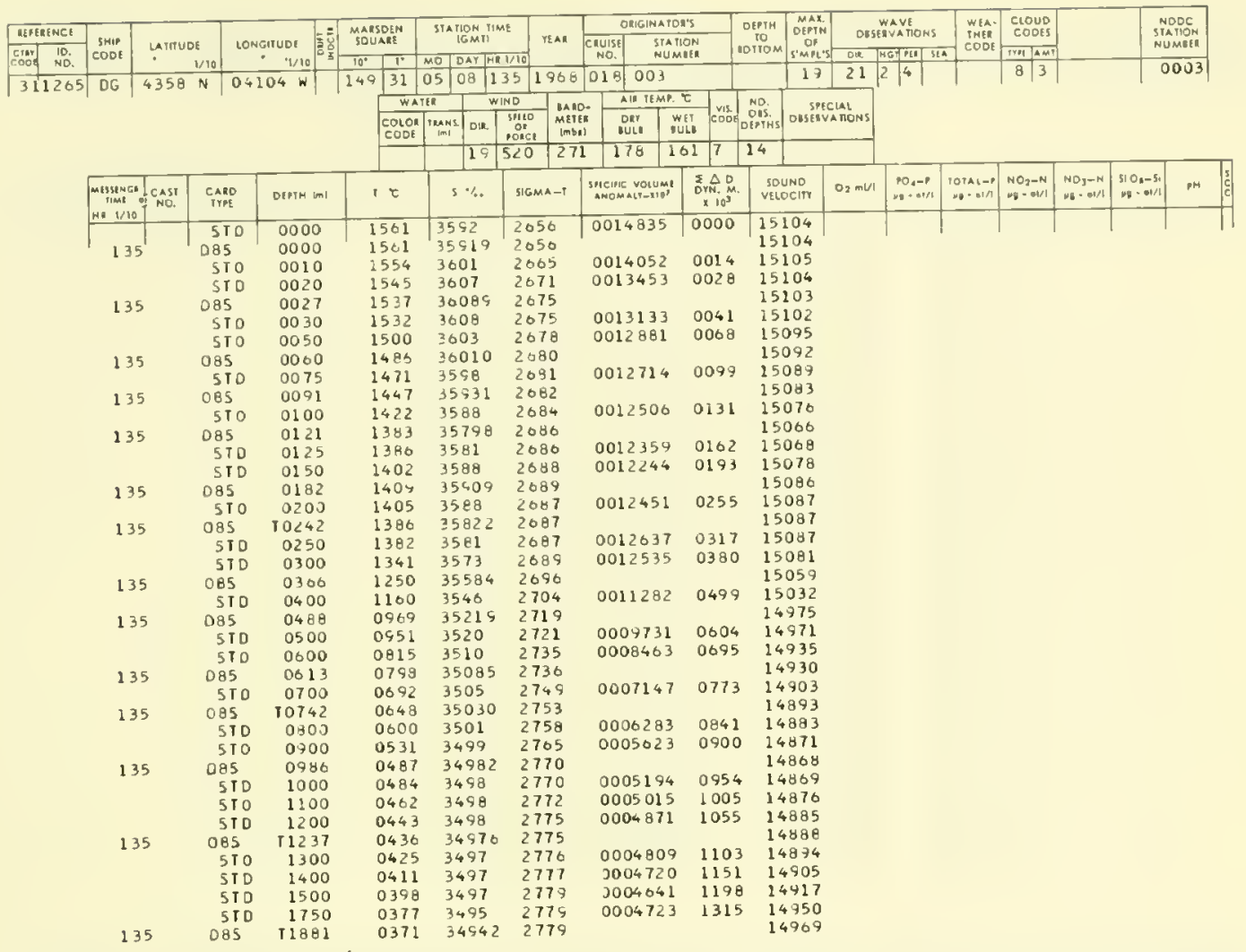

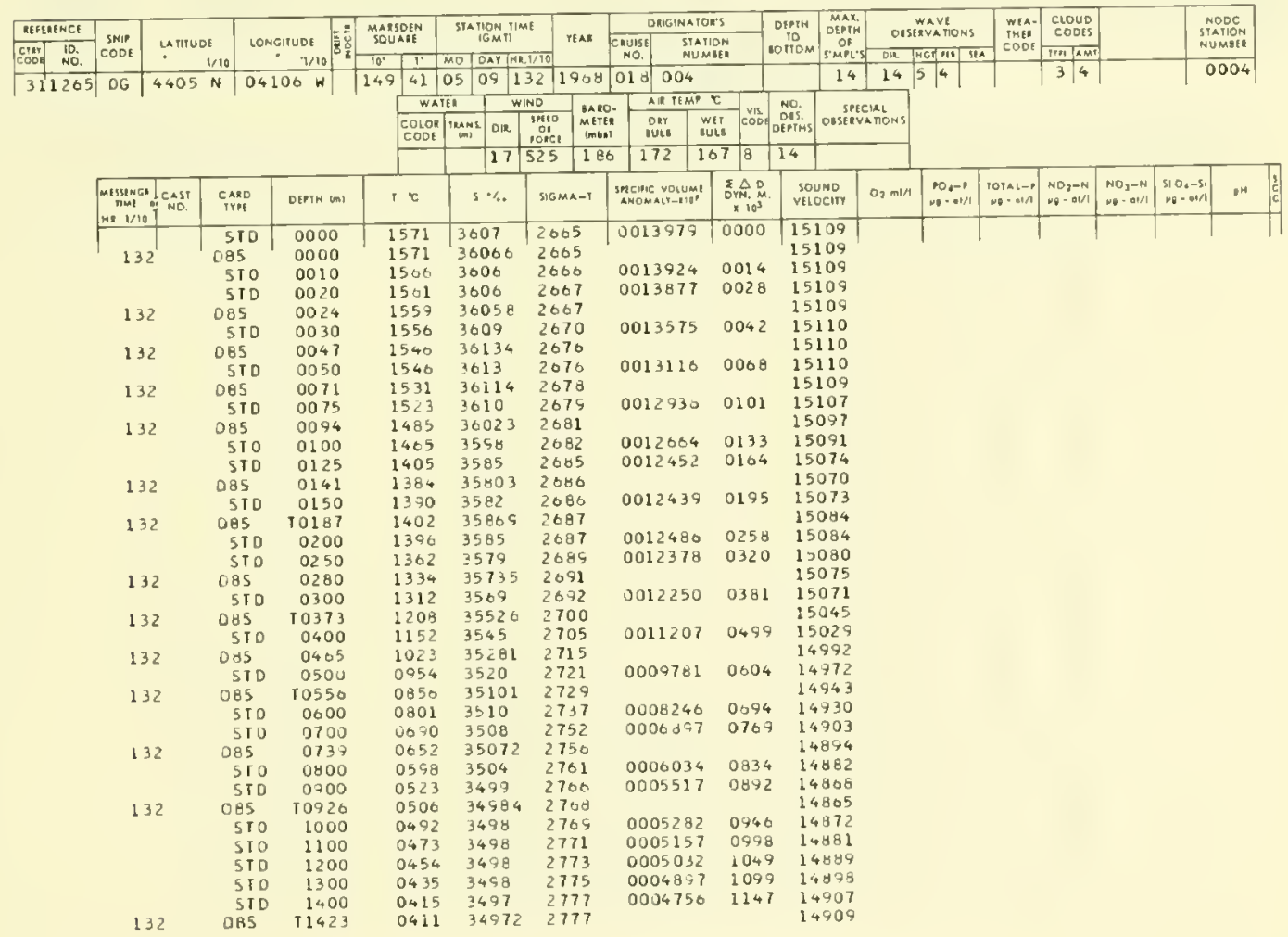


TABLe IX.-Continued

\begin{tabular}{|c|c|c|c|c|c|c|c|c|c|c|c|c|c|c|c|c|c|c|}
\hline \multirow{2}{*}{ 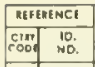 } & \multirow{2}{*}{ 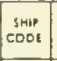 } & \multirow{2}{*}{\multicolumn{2}{|c|}{$\begin{array}{c}\text { tantudet } \\
-\quad 1 / 10 \\
\end{array}$}} & \multirow{2}{*}{ 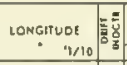 } & \multirow{2}{*}{$\begin{array}{c}\text { MARSDEN } \\
\text { SQUAKE }\end{array}$} & \multirow{2}{*}{\begin{tabular}{|c|} 
SIAFION IIMAI \\
IGMTI
\end{tabular}} & \multirow[b]{2}{*}{ TEAR } & \multicolumn{2}{|c|}{ ONGINAIOK'S } & \multirow{2}{*}{\begin{tabular}{|c|} 
Ofmin \\
Io \\
IOTTOM
\end{tabular}} & \multirow{2}{*}{\begin{tabular}{|c|} 
MAR, \\
OEPTH \\
Of \\
S'MPL'S
\end{tabular}} & & \multirow{2}{*}{$\begin{array}{l}\text { WEA- } \\
\text { INEI } \\
\text { CODI } \\
\end{array}$} & \multirow{2}{*}{$\begin{array}{l}\text { CLOUD } \\
\text { COO85 } \\
\end{array}$} & & & \multirow{2}{*}{$\begin{array}{l}\text { NODC } \\
\text { SHAFON } \\
\text { NUMIE }\end{array}$} \\
\hline & & & & & & & & $\begin{array}{c}\text { CRUise } \\
\text { NO. } \\
\end{array}$ & $\begin{array}{l}\text { STATHON } \\
\text { NU MABS }\end{array}$ & & & \multicolumn{2}{|c|}{ 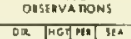 } & & & & & \\
\hline \multirow[t]{40}{*}{$|311265|$} & \multirow[t]{4}{*}{$\overline{D G}$} & 4357 & $N$ & $04050 \mathrm{~W}$ & \begin{tabular}{l|l|}
49 & 30 \\
\end{tabular} & \begin{tabular}{l|l|l}
05 & 10 & 1
\end{tabular} & $133 \mid 1968$ & \begin{tabular}{|l|l|}
018 & 005 \\
\end{tabular} & & & 14 & 275 & \begin{tabular}{|l|l|}
5 & 2 \\
\end{tabular} & & \begin{tabular}{l|l|}
0 & 3
\end{tabular} & & & 0005 \\
\hline & & & & & WAI & 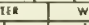 & VINO & AIR IIMT. & $\tau$ & & & & & & & & & \\
\hline & & & & & $\begin{array}{l}\text { Colon } \\
\text { CoOI }\end{array}$ & 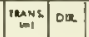 & $\begin{array}{l}\operatorname{lng} \\
\text { of } \\
\text { oncl }\end{array}$ & \begin{tabular}{l|l} 
DRY \\
ouLi
\end{tabular} & $\begin{array}{l}\text { WET } \\
\text { WUL }\end{array}$ & OPS. & oustev & Anons: & & & & & & \\
\hline & & & & & & 17 & 517 & 183 & \begin{tabular}{l|l}
61 & 8
\end{tabular} & 14 & & & & & & & & \\
\hline & 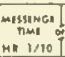 & ocAs: & $\begin{array}{l}\text { CAND } \\
\text { ImEE }\end{array}$ & OERTH $(\mathrm{m}$ ) & $\tau \tau$ & $s \%$ & SIGMA-1 & 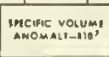 & 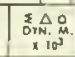 & $\begin{array}{l}\text { soun } \\
\text { veioc }\end{array}$ & S & $0, \mathrm{~min}$ & 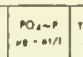 & Potal-B & 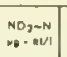 & $\begin{array}{l}\mathrm{NO}_{3} \sim \mathrm{N} \\
\mathrm{HO}\end{array}$ & $\begin{array}{l}510,-51 \\
10,-1 / 1,1\end{array}$ & DN \\
\hline & 133 & & $\begin{array}{l}\text { STO } \\
\text { DQS }\end{array}$ & $\begin{array}{l}0000 \\
0000\end{array}$ & $\begin{array}{l}1571 \\
1571\end{array}$ & $\begin{array}{l}3600 \\
36003\end{array}$ & $\begin{array}{l}2660 \\
2660\end{array}$ & 0014438 & 0000 & $\begin{array}{l}151 \\
151\end{array}$ & $\begin{array}{l}08 \\
08\end{array}$ & & & & & & & \\
\hline & 131 & & STO & 0010 & $\begin{array}{l}1301 \\
1505\end{array}$ & $\begin{array}{l}36003 \\
3600\end{array}$ & 20001 & 0014367 & 0014 & $\begin{array}{l}1510 \\
1510\end{array}$ & 08 & & & & & & & \\
\hline & & & STo & 0020 & 1557 & 3600 & 2663 & 0014250 & 0029 & 151 & .07 & & & & & & & \\
\hline & 133 & & 085 & 0025 & 1553 & 35994 & 2664 & & & 151 & 07 & & & & & & & \\
\hline & & & S10 & 0030 & 1550 & 3602 & 2666 & 0013956 & 0043 & 151 & 07 & & & & & & & \\
\hline & & & STO & 0050 & 1528 & 3007 & 2075 & 0013220 & 0070 & 151 & .04 & & & & & & & \\
\hline & 133 & & 085 & 0050 & 1528 & 36065 & 2675 & & & 151 & .04 & & & & & & & \\
\hline & & & SIO & 0075 & 1474 & 3596 & 2678 & 0012952 & 0103 & 150 & 90 & & & & & & & \\
\hline & 133 & & 085 & 0075 & 1474 & 35956 & 2678 & & & 150 & 90 & & & & & & & \\
\hline & 133 & & 085 & 0090 & 1434 & 35855 & 2679 & & & 150 & 79 & & & & & & & \\
\hline & & & STO & 0100 & 1423 & 3583 & 2080 & 0012892 & 0135 & 150 & 76 & & & & & & & \\
\hline & & & STO & 0125 & 1371 & 3571 & 2681 & 0012788 & 0167 & 150 & 162 & & & & & & & \\
\hline & 133 & & DBS & 0144 & 1346 & 35600 & 2683 & & & 150 & 56 & & & & & & & \\
\hline & & & STD & 0150 & 1345 & 3567 & 2684 & 0012636 & 0199 & 150 & 57 & & & & & & & \\
\hline & 133 & & Des & 10189 & 1339 & 35673 & 2685 & & & 150 & 61 & & & & & & & \\
\hline & & & STO & 0200 & 1319 & 3563 & 2686 & 0012552 & 0262 & 150 & 56 & & & & & & & \\
\hline & & & STO & 0250 & 1246 & 3549 & 2670 & 0012296 & 0324 & 150 & 38 & & & & & & & \\
\hline & 133 & & 085 & 0280 & 1215 & 35442 & 2692 & & & 150 & 31 & & & & & & & \\
\hline & & & STO & 0300 & 1207 & 3547 & 2696 & 0011840 & 0384 & 150 & 32 & & & & & & & \\
\hline & 133 & & 085 & 0366 & 1282 & 35479 & 2701 & & & 150 & & & & & & & & \\
\hline & & & STO & 0400 & 1119 & 3540 & 2707 & 0010909 & 0498 & 1501 & 17 & & & & & & & \\
\hline & 133 & & 085 & 0450 & 1027 & 35286 & 2715 & & & $149^{\circ}$ & 91 & & & & & & & \\
\hline & & & STO & 0500 & 0936 & 3519 & 2723 & 0009554 & 0601 & 149 & 65 & & & & & & & \\
\hline & 133 & & DBS & 10536 & 0873 & 35131 & 2728 & & & 149 & 147 & & & & & & & \\
\hline & & & STO & 0600 & 0752 & 3503 & 2739 & 0008017 & 0689 & 149 & 10 & & & & & & & \\
\hline & & & STO & 0700 & 0811 & 3494 & 2751 & 0006805 & 0763 & $148^{\circ}$ & & & & & & & & \\
\hline & 133 & & D8S & 0700 & 0611 & 34941 & 2751 & & & 148 & 70 & & & & & & & \\
\hline & & & STO & 0800 & 0548 & 3456 & 2761 & 0005947 & 0827 & 1486 & & & & & & & & \\
\hline & 133 & & D8S & Т 0879 & 0507 & 34963 & 2766 & & & 148 & & & & & & & & \\
\hline & & & $5 T 0$ & 0900 & 0503 & 3496 & 2766 & 0005455 & 0884 & 1485 & 159 & & & & & & & \\
\hline & & & STO & 1000 & 0482 & 3496 & 2708 & 0005323 & 0938 & 148 & 167 & & & & & & & \\
\hline & & & STO & 1100 & 0460 & 3495 & 2770 & 0005193 & 0990 & 148 & 175 & & & & & & & \\
\hline & & & STD & 1200 & 0439 & 3495 & 2772 & 0005058 & 1041 & 148 & 83 & & & & & & & \\
\hline & & & STO & 1300 & 0418 & 3494 & 2774 & 0004911 & 1091 & 148 & & & & & & & & \\
\hline & 133 & & 085 & T1358 & 0406 & 34941 & 2775 & & & 148 & 96 & & & & & & & \\
\hline
\end{tabular}

\begin{tabular}{|c|c|c|c|c|c|c|c|c|c|c|c|c|c|c|c|c|c|c|}
\hline \multirow{2}{*}{\begin{tabular}{|c|c|} 
REFUINCE \\
Cran & 10. \\
COOH & HO. \\
\end{tabular}} & \multirow{2}{*}{ swip } & \multirow{2}{*}{\multicolumn{2}{|c|}{ InTtude }} & \multirow{2}{*}{ LONGITUAE } & \multirow{2}{*}{$\begin{array}{l}\text { MAnsoEN } \\
\text { SQUAAR }\end{array}$} & \multirow{2}{*}{$\begin{array}{l}\text { STATION TIME } \\
\text { IG MII }\end{array}$} & \multirow{2}{*}{ TEAT } & \multicolumn{2}{|c|}{ TONGINATOR's } & \multirow{2}{*}{\begin{tabular}{|c|} 
DIPIN \\
IO \\
nOTIOM
\end{tabular}} & \multirow{2}{*}{\begin{tabular}{|l|}
$M A X$ \\
$O E P I N$ \\
OF \\
SMMPLES
\end{tabular}} & & \multirow{2}{*}{$\begin{array}{l}\text { WEA. } \\
\text { BHEL } \\
\text { COOI }\end{array}$} & \multirow{2}{*}{\begin{tabular}{|l|} 
Clovo \\
CODIS \\
T3M
\end{tabular}} & & & \multirow{2}{*}{$\begin{array}{l}\text { NODC } \\
\text { STANION } \\
\text { NUMHER }\end{array}$} \\
\hline & & & & & & & & $\begin{array}{l}\text { CRUIS: } \\
\text { No. }\end{array}$ & $\begin{array}{l}\text { SIRTION } \\
\text { NUMHEI }\end{array}$ & & & 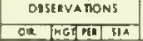 & & & & & & \\
\hline & OG & 4358 & $N \quad 0$ & $04056 W$ & \begin{tabular}{|l|l|}
149 & 30 \\
\end{tabular} & \begin{tabular}{|l|l|l}
05 & 11 & 1
\end{tabular} & \begin{tabular}{l|l}
183 & 1968
\end{tabular} & 018006 & & & 19 & 17 & 94 & & \begin{tabular}{|l|l|l|}
8 & 4
\end{tabular} & & & 0006 \\
\hline & & & & & WAT & \begin{tabular}{|l|l|l|} 
& 1 \\
\end{tabular} & WINO & 10. AIIIMP. & & & & & & & & & & \\
\hline & & & & & $\begin{array}{l}\text { Colon } \\
\text { COOB }\end{array}$ & 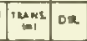 & 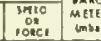 & \begin{tabular}{c|c} 
ons & on \\
out: & "n
\end{tabular} & uil $=000$ & ous. & ousing & $\begin{array}{l}\text { CIAL } \\
\text { CAnONS }\end{array}$ & & & & & & \\
\hline & & & & & & \begin{tabular}{|l|l|}
23 \\
\end{tabular} & \begin{tabular}{|l|l|}
535 & 07
\end{tabular} & 139 & $2 2 \longdiv { 8 }$ & 14 & & & & & & & & \\
\hline & 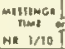 & ICAs! & $\begin{array}{l}\text { eAlo } \\
\text { irfi }\end{array}$ & D[PTH $(m)$ & $T t$ & $5 \%$ & SIGMA-1 & 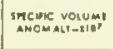 & 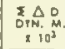 & $\begin{array}{l}\text { SOU } \\
\text { vito }\end{array}$ & $\begin{array}{l}\text { JND } \\
\text { SCMT }\end{array}$ & $0, \mathrm{~m} / \mathrm{M}$ & $\left|\begin{array}{c}m_{1}=1 \\
\ldots a, A\end{array}\right|$ & 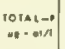 & 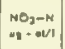 & $\mid \begin{array}{l}n+\mathrm{N} / 1 \\
\mathrm{HO}^{2}-\mathrm{N}\end{array}$ & 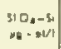 & nN \\
\hline & & & STO & 0000 & 1562 & 3603 & 2004 & 0014054 & 0000 & 151 & 106 & & & & & & & \\
\hline & $183^{\prime}$ & & DBS & 0000 & 1562 & 36029 & 2664 & & & 151 & 100 & & & & & & & \\
\hline & & & STD & 0010 & 1560 & 3603 & 2665 & 0014010 & 0014 & $15 i$ & 107 & & & & & & & \\
\hline & & & STO & 0020 & 1557 & 3604 & 2060 & 0013906 & 0028 & 251 & 108 & & & & & & & \\
\hline & & & $5 T 0$ & 0030 & 1555 & 3604 & 2667 & 0013922 & 0042 & 251 & 109 & & & & & & & \\
\hline & 183 & & 085 & 0032 & 1554 & 36039 & 2067 & & & 151 & 109 & & & & & & & \\
\hline & & & STO & 0053 & 1503 & 3597 & 2675 & 0013236 & 0069 & 150 & 295 & & & & & & & \\
\hline & 183 & & 085 & 0063 & 1474 & 35952 & 2678 & & & 150 & 88 & & & & & & & \\
\hline & & & $5 T 0$ & 0075 & 1453 & $35 \$ 1$ & 2679 & 0012851 & 0102 & 150 & 382 & & & & & & & \\
\hline & 183 & & 085 & 0095 & 1434 & 35872 & 2081 & & & 150 & 77 & & & & & & & \\
\hline & & & STO & 0100 & 1436 & 3589 & 2682 & 0012714 & 0134 & 150 & 81 & & & & & & & \\
\hline & & & STO & 0125 & 1444 & 3592 & 2082 & 0012747 & 0106 & 150 & 88 & & & & & & & \\
\hline & 183 & & DBS & 0127 & 1445 & 35525 & 2682 & & & 150 & 89 & & & & & & & \\
\hline & & & STO & 0150 & 2427 & 3550 & 2084 & 0012610 & 0197 & 150 & 86 & & & & & & & \\
\hline & 183 & & 085 & 0190 & 1380 & 35803 & 2687 & & & 150 & 76 & & & & & & & \\
\hline & & & sio & 0200 & 1360 & 3575 & 2687 & 0012489 & 0260 & 150 & 71 & & & & & & & \\
\hline & & & sro & 0250 & 1272 & 3553 & 2688 & 0012503 & 0322 & 150 & 97 & & & & & & & \\
\hline & 183 & & DOS & 0252 & 1209 & 35522 & 2688 & & & 150 & 940 & & & & & & & \\
\hline & & & STD & 0300 & 1217 & 3546 & 2093 & 0012096 & 0384 & 150 & 30 & & & & & & & \\
\hline & 183 & & 085 & 0379 & 1125 & 35357 & 2703 & & & 150 & 15 & & & & & & & \\
\hline & & & STO & 0400 & 1101 & 3534 & 2706 & 0011084 & 0500 & 150 & 10 & & & & & & & \\
\hline & & & STD & 0500 & 0966 & 3522 & 2720 & 0009836 & $\mathrm{O}_{5} \mathrm{O}_{4}$ & 149 & 70 & & & & & & & \\
\hline & 183 & & DBS & 10508 & 0554 & 35207 & 2721 & & & 147 & 973 & & & & & & & \\
\hline & & & STO & 0600 & $07 \varepsilon 5$ & 3506 & 2736 & 0008295 & 0695 & 149 & 223 & & & & & & & \\
\hline & 183 & & DES & 0031 & 0735 & 35028 & $274 \mathrm{~L}$ & & & 149 & & & & & & & & \\
\hline & & & STD & 0700 & 0642 & 3499 & 2751 & 000687 & 0771 & 248 & 83 & & & & & & & \\
\hline & 283 & & DAS & 10754 & 0582 & 34560 & 2757 & & & 1400 & 807 & & & & & & & \\
\hline & & & SID & 0800 & 0555 & 3496 & 2700 & 0006018 & 0835 & 148 & 304 & & & & & & & \\
\hline & & & STO & 0900 & 0504 & 3490 & 2706 & 0005510 & 0893 & 148 & 100 & & & & & & & \\
\hline & & & STO & 1000 & 0465 & 3455 & 2770 & 0005161 & 0746 & $14 d t$ & 860 & & & & & & & \\
\hline & 183 & & D85 & 2007 & 0403 & 34950 & 2770 & & & 148 & 101 & & & & & & & \\
\hline & & & STo & 1100 & 0447 & 3455 & 2772 & 0005035 & 0997 & 146 & 370 & & & & & & & \\
\hline & & & $5 r 0$ & 1200 & 0431 & 3495 & 2774 & 0004921 & 1047 & 148 & & & & & & & & \\
\hline & 183 & & Des & 11264 & 0422 & 34953 & 2775 & & & 1488 & & & & & & & & \\
\hline & & & STO & 2300 & 0417 & 3455 & 2775 & 0004852 & 1096 & 148 & 391 & & & & & & & \\
\hline & & & STO & 1403 & 0405 & 3495 & 2770 & 0004783 & 2144 & 1490 & 302 & & & & & & & \\
\hline & & & STD & 1500 & 0395 & 3495 & 2777 & 0004747 & 1192 & 2471 & 15 & & & & & & & \\
\hline & & & 510 & 1750 & 0376 & 3455 & 2779 & 0004709 & 1310 & 149 & & & & & & & & \\
\hline & 103 & & 085 & 11914 & 0370 & 34939 & 2779 & & & $14 y$ & & & & & & & & \\
\hline
\end{tabular}


TABLE IX.-Continued

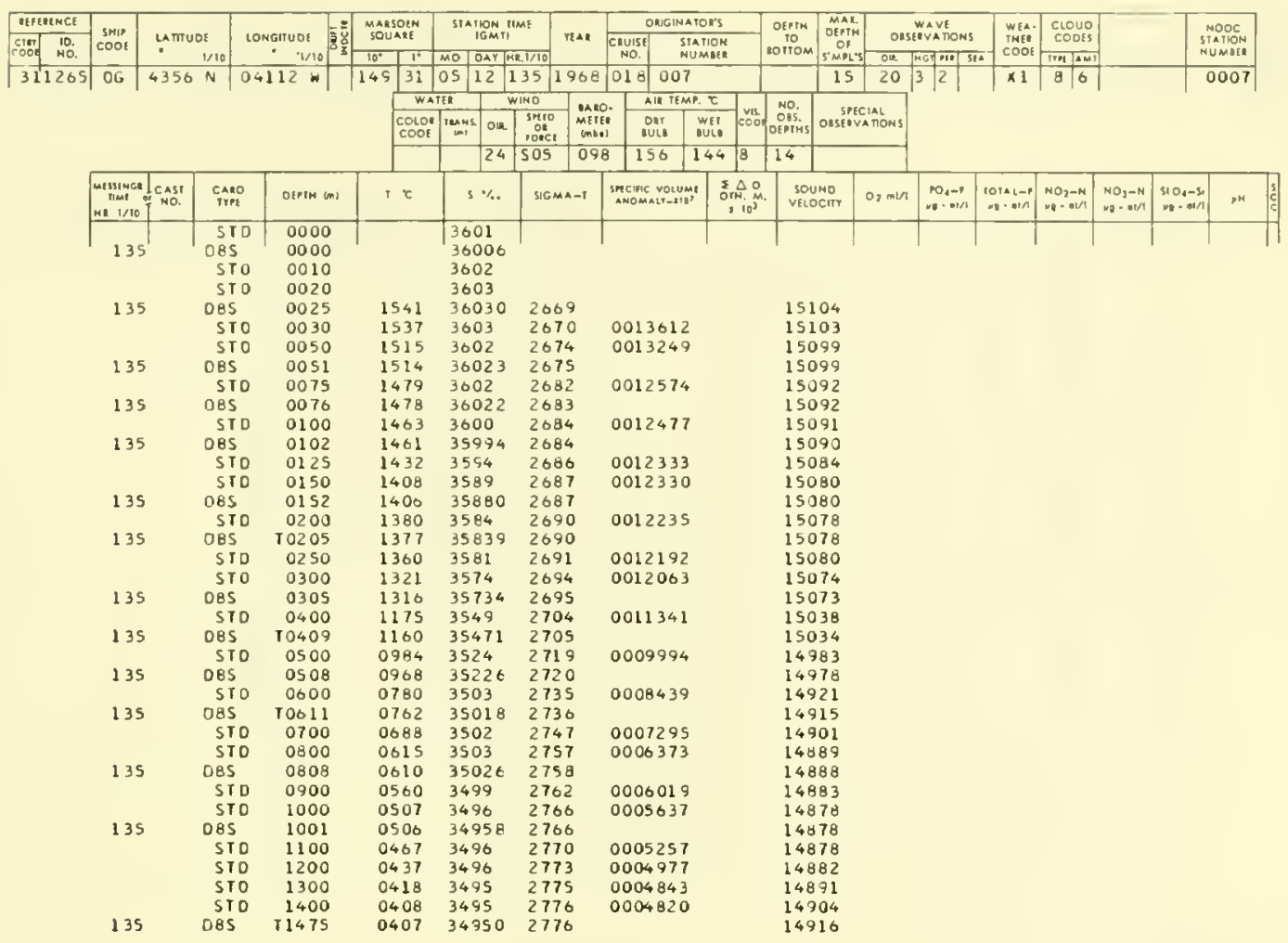

\begin{tabular}{|c|c|c|c|c|c|c|c|c|c|c|c|c|c|c|c|c|c|c|}
\hline BEFERENCE & \multirow{2}{*}{ coot } & \multirow{2}{*}{\multicolumn{2}{|c|}{$\begin{array}{c}4 \text { umvor } \\
\quad 1 / 10\end{array}$}} & \multirow{2}{*}{ LONGITUOE } & $\begin{array}{l}\text { MAASOEN } \\
\text { SOUAIE }\end{array}$ & \multirow{2}{*}{\begin{tabular}{|c|} 
STA TION TiME \\
IGMNI
\end{tabular}} & \multirow[b]{2}{*}{ VAR } & \multicolumn{2}{|c|}{\begin{tabular}{|l} 
ONGINATOR'S \\
\end{tabular}} & \multirow{2}{*}{$\begin{array}{c}\text { Oepit } \\
\text { fo } \\
\text { LOTOM }\end{array}$} & \multirow{2}{*}{\begin{tabular}{|} 
MAR \\
OEFTH \\
OF \\
S'MP'S
\end{tabular}} & \multirow{2}{*}{\multicolumn{2}{|c|}{$\begin{array}{l}\text { WAVE } \\
\text { ONSEVANONS }\end{array}$}} & \multirow{2}{*}{$\begin{array}{l}\text { NEA } \\
\text { IMEE } \\
\text { COOE }\end{array}$} & \multirow{2}{*}{ 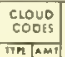 } & & & \multirow{2}{*}{$\begin{array}{l}\text { NOOC } \\
\text { STIINON } \\
\text { NUMAE* }\end{array}$} \\
\hline \multirow{2}{*}{$\frac{\operatorname{cin}_{\text {coos }} \begin{array}{c}10 . \\
\text { No. }\end{array}}{311265}$} & & & & & $10^{\circ} 1^{\circ}$ & & & 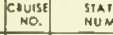 & HON & & & 02 & & & & & & \\
\hline & \multirow[t]{4}{*}{ DG } & \multirow[t]{4}{*}{4358} & \multirow[t]{4}{*}{$\mathrm{N}$} & $04100 \mathrm{~W}$ & 14931 & \begin{tabular}{l|l|}
05 & 13 \\
\end{tabular} & \begin{tabular}{|l|l|}
137 & 1968 \\
\end{tabular} & \begin{tabular}{|l|l|}
018 & 008 \\
\end{tabular} & & & 16 & 17 & $\mid$\begin{tabular}{|l|l|}
3 & 2
\end{tabular} & $\times 2$ & \begin{tabular}{|l|l|}
6 &
\end{tabular} & & & 0008 \\
\hline & & & & & WAT & \begin{tabular}{|l|l|l|} 
& \\
\end{tabular} & WINO & - ATR TEMP. & & & & & & & & & & \\
\hline & & & & & \begin{tabular}{|l|}
$\begin{array}{c}\text { COLOR } \\
\text { COOE }\end{array}$ \\
\end{tabular} & $\begin{array}{c}\text { tennis } \\
\text { Tal }\end{array}$ & \begin{tabular}{c|c|c|} 
SFEto & METE \\
Conc1 & ImD \\
\end{tabular} & 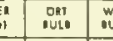 & $\begin{array}{ll}819 \\
\text { ut coos }\end{array}$ & Ons. & onserve & A Tlons & & & & & & \\
\hline & & & & & & 25 & 524 & 111 & \begin{tabular}{l|l}
00 & 7
\end{tabular} & 14 & & & & & & & & \\
\hline & 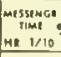 & $\begin{array}{l}\text { ICASI } \\
\text { I NO. }\end{array}$ & $\begin{array}{l}\text { caspo } \\
\text { irpt }\end{array}$ & OEPTH $\mathrm{mI}$ & $1 t$ & $5 \%$ & SIGMA-1 & 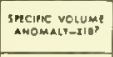 & 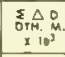 & $\begin{array}{l}\text { Sout } \\
\text { viloo }\end{array}$ & $\begin{array}{l}\text { UNO } \\
\text { OCITY }\end{array}$ & $0, \mathrm{~m} / \mathrm{Al}$ & 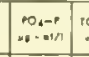 & 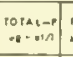 & $\left|\begin{array}{l}\mathrm{NO}_{2}-\mathrm{N} \\
\mathrm{ZZ}-\mathrm{N} / 1\end{array}\right|$ & $\begin{array}{l}N O_{1}-N \\
M-M V / 1\end{array}$ & $\begin{array}{c}310 .-51 \\
48-01 / 1\end{array}$ & $\mathrm{OH}$ \\
\hline & & & ST0 & 0000 & 1547 & 3607 & 2671 & 0013425 & 0000 & 151 & 102 & & & & & & & \\
\hline & 137 & & OBS & 0000 & 1547 & 36071 & 2671 & & & 151 & 102 & & & & & & & \\
\hline & & & $\begin{array}{l}\text { STO } \\
\text { STO }\end{array}$ & $\begin{array}{l}0010 \\
0020\end{array}$ & 1545 & 3607 & 2071 & 0013421 & 0013 & 151 & 103 & & & & & & & \\
\hline & 137 & & OBS & $\begin{array}{l}0020 \\
0027\end{array}$ & $\begin{array}{l}1543 \\
1542\end{array}$ & $\begin{array}{l}3607 \\
30069\end{array}$ & $\begin{array}{l}2672 \\
2672\end{array}$ & 0013410 & 0027 & $\begin{array}{l}151 \\
151\end{array}$ & $\begin{array}{l}104 \\
105\end{array}$ & & & & & & & \\
\hline & & & STO & 0030 & 1542 & 3607 & 2672 & 0013425 & 0040 & isi & 105 & & & & & & & \\
\hline & & & STO & 0050 & 1541 & 3607 & 2072 & 0013492 & 0007 & 151 & 108 & & & & & & & \\
\hline & 137 & & 085 & 0053 & 1541 & 36065 & 2672 & & & 151 & 109 & & & & & & & \\
\hline & & & STO & 0075 & 1532 & 3605 & 2073 & 0013492 & 0101 & 151 & 109 & & & & & & & \\
\hline & 137 & & ORS & 0379 & 1527 & 36045 & 2673 & & & 151 & 108 & & & & & & & \\
\hline & & & STO & 0100 & 1482 & 3601 & 2081 & 0012801 & 0134 & 150 & 097 & & & & & & & \\
\hline & 137 & & 085 & 0105 & 1473 & 35998 & 2082 & & & 150 & 095 & & & & & & & \\
\hline & & & STO & 0125 & 1456 & 3597 & 2683 & 3012625 & 0166 & 150 & 092 & & & & & & & \\
\hline & & & STO & 0150 & 1435 & 3554 & 2686 & 0012483 & 0197 & 150 & 089 & & & & & & & \\
\hline & 137 & & 085 & 0159 & 1428 & 35925 & 2086 & & & 150 & 088 & & & & & & & \\
\hline & & & SIO & 0200 & 1398 & 3587 & 2698 & 0012301 & 0259 & 150 & 085 & & & & & & & \\
\hline & 137 & & 0R5 & 0212 & 2386 & 15858 & 2067 & & & 150 & 084 & & & & & & & \\
\hline & & & STO & 0250 & 1361 & 3580 & 2090 & 0012285 & 0321 & 150 & 080 & & & & & & & \\
\hline & & & STD & 0300 & 1313 & 3571 & 2693 & 0012124 & 4382 & 150 & 71 & & & & & & & \\
\hline & 137 & & 085 & 0319 & 1292 & 35678 & 2695 & & & 150 & 067 & & & & & & & \\
\hline & & & STO & 0400 & 1181 & 3550 & 2703 & 0011380 & 0499 & 150 & 040 & & & & & & & \\
\hline & 137 & & 085 & 10427 & 1140 & 35430 & 2705 & & & 150 & 029 & & & & & & & \\
\hline & & & STO & 0500 & 1023 & 3521 & 2710 & 0010835 & 0611 & 143 & 397 & & & & & & & \\
\hline & 137 & & 085 & $0>35$ & 0459 & 35137 & 2715 & & & 147 & 979 & & & & & & & \\
\hline & & & STO & 0600 & 0808 & 3500 & 2733 & 0008649 & 0708 & 149 & 932 & & & & & & & \\
\hline & 137 & & 085 & T0644 & 0725 & 35015 & 2742 & & & 1490 & 906 & & & & & & & \\
\hline & & & STO & 0700 & 0606 & 3500 & 2749 & 0907140 & 0797 & 145 & 372 & & & & & & & \\
\hline & & & sto & 0800 & 0578 & 3498 & 2759 & 0000202 & 0354 & 148 & 874 & & & & & & & \\
\hline & 137 & & 085 & 0863 & 0533 & 34973 & 2704 & & & 148 & 806 & & & & & & & \\
\hline & & & STO & 0900 & 0515 & 3457 & 2700 & 0005559 & 0713 & 148 & 865 & & & & & & & \\
\hline & & & STD & 1000 & 0473 & 3450 & 2770 & 0005198 & 0967 & 148 & 354 & & & & & & & \\
\hline & 137 & & 085 & 71076 & 0445 & 34948 & 2772 & & & 148 & 365 & & & & & & & \\
\hline & & & STO & 1100 & 0443 & 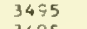 & 2772 & 0004959 & 1018 & 148 & 808 & & & & & & & \\
\hline & & & STO & 1200 & 0431 & 3495 & 2773 & 0004972 & 1067 & 143 & 380 & & & & & & & \\
\hline & & & SIO & 1300 & 0419 & 3494 & 2774 & 0004933 & 1117 & 148 & 392 & & & & & & & \\
\hline & & & STO & 1400 & 0408 & 3494 & 2775 & 0004897 & 1166 & 147 & 203 & & & & & & & \\
\hline & & & STO & 1500 & $03>6$ & 3434 & 2770 & 0004850 & 1215 & 1491 & 115 & & & & & & & \\
\hline & 137 & & 085 & 11639 & 0380 & 34934 & 2778 & & & 149 & 332 & & & & & & & \\
\hline
\end{tabular}


TABle IX.-Continued

\begin{tabular}{|c|c|c|c|c|c|c|c|c|c|c|c|c|c|c|c|c|c|c|}
\hline REFERENCE & \multirow[b]{2}{*}{$\begin{array}{l}\text { SHIF } \\
\text { COOE }\end{array}$} & \multirow{2}{*}{\multicolumn{2}{|c|}{$\begin{array}{c}\text { ustivues } \\
1 / 10 \\
\end{array}$}} & \multirow{2}{*}{$\begin{array}{c}\text { LONGITUOE } \\
-1 / 10 \\
\end{array}$} & $\begin{array}{l}\text { MARSOEN } \\
\text { SOUARE }\end{array}$ & \multirow{2}{*}{\multicolumn{2}{|c|}{$\begin{array}{l}\text { STA TION FIME } \\
\text { IGM II }\end{array}$}} & \multicolumn{2}{|c|}{\begin{tabular}{|l} 
ORIGSNATOKSS \\
\end{tabular}} & \multirow{2}{*}{$\begin{array}{c}\text { OEPTH } \\
\text { TO } \\
\text { 10TIOM }\end{array}$} & \multirow{2}{*}{\begin{tabular}{|l|} 
MAX. \\
DEPIH \\
OF \\
SMPL'S
\end{tabular}} & \multirow{2}{*}{\multicolumn{2}{|c|}{$\begin{array}{c}\text { WAVE } \\
\text { OBSEAVATONS }\end{array}$}} & \multirow{2}{*}{$\begin{array}{l}\text { WEA- } \\
\text { THER } \\
\text { COOE }\end{array}$} & \multirow{2}{*}{ 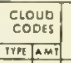 } & & & \multirow{2}{*}{$\begin{array}{l}\text { NOOC } \\
\text { STATON } \\
\text { NUMBER } \\
\end{array}$} \\
\hline 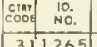 & & & & & \begin{tabular}{|l|l|}
\multicolumn{2}{|c|}{ SOU ARE } \\
$10^{\circ}$ & 1. \\
\end{tabular} & & reAR & \begin{tabular}{|c|c|} 
CRUISE & STA \\
NOE. & NUN \\
\end{tabular} & $\begin{array}{l}\text { ATION } \\
\text { UMBER }\end{array}$ & & & & & & & & & \\
\hline & \multirow[t]{4}{*}{ OG } & \multirow[t]{4}{*}{4400} & \multirow[t]{4}{*}{$\mathrm{N}$} & \multirow[t]{4}{*}{$04058 w T$} & \multirow[t]{2}{*}{149} & \begin{tabular}{l|l|}
05 & 14 \\
\end{tabular} & 13012900 & \begin{tabular}{|l|l|}
018 & 009 \\
\end{tabular} & & & 13 & 132 & \begin{tabular}{|l|l|l|}
2 & 2 & \\
\end{tabular} & $\times 5$ & \begin{tabular}{|l|l|}
6 & 6 \\
\end{tabular} & & & 0009 \\
\hline & & & & & & \begin{tabular}{l|l} 
TEN & $y$ \\
\end{tabular} & WINO & - AII TEMt. & 1. $x$ & & & & & & & & & \\
\hline & & & & & \begin{tabular}{|l|} 
COLOR \\
CODE \\
\end{tabular} & Thens OiR & 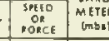 & \begin{tabular}{l|l} 
on & put \\
ovit
\end{tabular} & With coof & ORS & $\begin{array}{l}\text { SPECI } \\
\text { OESEAVA }\end{array}$ & A NONS & & & & & & \\
\hline & & & & & & 18 & 1515 & 133 & \begin{tabular}{l|l}
122 & 7 \\
\end{tabular} & 14 & & & & & & & & \\
\hline & $\begin{array}{l}\text { Messing: } \\
\text { timat } \\
\text { He } 1 / 100\end{array}$ & 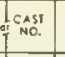 & $\begin{array}{l}\text { CARO } \\
\text { TIVt }\end{array}$ & OEPTH $(m)$ & $i \tau$ & $s \%$ & SIGMA-T & $\begin{array}{l}\text { SPECIRC VOLUA, } \\
\text { ANOMALT-110' }\end{array}$ & 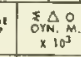 & $\begin{array}{l}\text { SOUN } \\
\text { vetoo }\end{array}$ & $\begin{array}{l}\text { IND } \\
\text { SIIYY }\end{array}$ & $0_{3} \mathrm{ml} / \mathrm{l}$ & 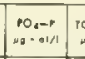 & 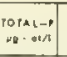 & 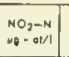 & $\begin{array}{l}\mathrm{NO}_{2}-\mathrm{N} \\
\mathrm{V}-\mathrm{at} / 1\end{array}$ & $\begin{array}{l}510,-51 \\
80=81 / 1\end{array}$ & PN \\
\hline & & & 510 & 0000 & 1513 & 3584 & 2601 & 0014381 & 0000 & 150 & 88 & & & & & & & \\
\hline & 130 & & 085 & 0000 & 1513 & 35841 & 2661 & & & 150 & & & & & & & & \\
\hline & & & $5 T 0$ & $\begin{array}{l}0010 \\
0020\end{array}$ & $\begin{array}{l}1505 \\
1497\end{array}$ & $\begin{array}{l}3589 \\
3593\end{array}$ & $\begin{array}{l}2660 \\
2671\end{array}$ & $\begin{array}{l}0013910 \\
0013460\end{array}$ & $\begin{array}{l}0014 \\
0028\end{array}$ & $\begin{array}{l}150 \\
150\end{array}$ & $\begin{array}{l}88 \\
88\end{array}$ & & & & & & & \\
\hline & 130 & & OES & 0024 & 1494 & 35948 & 2673 & & 0000 & 150 & 38 & & & & & & & \\
\hline & & & SIO & 0030 & 1457 & 3550 & 2678 & 0012871 & 0041 & 150 & 76 & & & & & & & \\
\hline & 130 & & 085 & 0047 & 1381 & 35808 & 2687 & & & 150 & 153 & & & & & & & \\
\hline & & & SIO & 0050 & 1376 & 3580 & 2687 & 0012015 & 0066 & 150 & 152 & & & & & & & \\
\hline & 130 & & 085 & 0072 & 1346 & 35753 & 2690 & & & 150 & 145 & & & & & & & \\
\hline & & & STO & 0075 & 1342 & 3574 & 2690 & 0011851 & 0096 & 150 & 144 & & & & & & & \\
\hline & 130 & & 085 & 0094 & 1320 & 35697 & 2691 & & & 2503 & 139 & & & & & & & \\
\hline & & & STO & 0100 & 1313 & 3569 & 2092 & 0011719 & 0125 & 1503 & 38 & & & & & & & \\
\hline & & & 510 & 0125 & 1286 & 3564 & 2693 & 0011631 & 0154 & 1503 & 132 & & & & & & & \\
\hline & 130 & & 085 & 0142 & 1272 & 35020 & 2695 & & & 1503 & 30 & & & & & & & \\
\hline & & & & 0150 & 1270 & 3561 & 2694 & 0011611 & 0183 & 1503 & 31 & & & & & & & \\
\hline & 130 & & 085 & 10187 & 1250 & 35571 & 2695 & & & 1503 & 30 & & & & & & & \\
\hline & & & STO & 0200 & 1236 & 3554 & 2696 & 0011610 & 0241 & 1502 & 27 & & & & & & & \\
\hline & & & STO & 0250 & 1177 & 3544 & 2699 & 0011369 & 0299 & 1501 & 13 & & & & & & & \\
\hline & 130 & & 085 & 0272 & 1150 & 35397 & 2701 & & & 1500 & 07 & & & & & & & \\
\hline & & & $5 T 0$ & $\begin{array}{r}0300 \\
\end{array}$ & 1109 & 3533 & 2703 & 0011063 & 0355 & 1499 & 96 & & & & & & & \\
\hline & 130 & & $\begin{array}{l}0.85 \\
\text { STO }\end{array}$ & $\begin{array}{r}10352 \\
0400\end{array}$ & $\begin{array}{l}1044 \\
1008\end{array}$ & $\begin{array}{l}35240 \\
3521\end{array}$ & $\begin{array}{l}2708 \\
2712\end{array}$ & 0010407 & 0462 & $\begin{array}{l}1498 \\
1477\end{array}$ & $\begin{array}{l}81 \\
75\end{array}$ & & & & & & & \\
\hline & 130 & & 085 & 0433 & 0975 & 35189 & 2716 & & & 1496 & & & & & & & & \\
\hline & & & $\$ \mathbf{T} 0$ & 0500 & 0883 & 3512 & 2726 & 0009204 & 0560 & 1494 & & & & & & & & \\
\hline & 130 & & 085 & 0513 & 0865 & 35110 & 2728 & & & 1494 & & & & & & & & \\
\hline & & & $5 T 0$ & 0600 & 0734 & 3497 & 2737 & 0008191 & 0647 & 1490 & & & & & & & & \\
\hline & 130 & & 085 & 0673 & 0649 & 34928 & 2745 & & & 1488 & & & & & & & & \\
\hline & & & STO & 0700 & 0529 & 3495 & 2750 & 0006987 & 0723 & 1487 & & & & & & & & \\
\hline & & & STO & 0800 & 0504 & 3502 & 2764 & 0005718 & 0787 & 1486 & & & & & & & & \\
\hline & 130 & & OBS & ro836 & 0543 & 35042 & 2768 & & & 1486 & & & & & & & & \\
\hline & & & STO & 0900 & 0510 & 3503 & 2771 & 0005051 & 0841 & 1486 & & & & & & & & \\
\hline & & & 510 & 1000 & 0469 & 3501 & 2774 & 0004763 & 0890 & 1486 & & & & & & & & \\
\hline & & & STO & 1100 & 0439 & 3499 & 2776 & 0004623 & 0937 & 1486 & & & & & & & & \\
\hline & & & STO & 1200 & 0421 & 3497 & 2776 & 0004631 & 0983 & 1487 & & & & & & & & \\
\hline & 130 & & OBS & 11276 & 0416 & 34960 & 2776 & & & 1488 & & & & & & & & \\
\hline
\end{tabular}

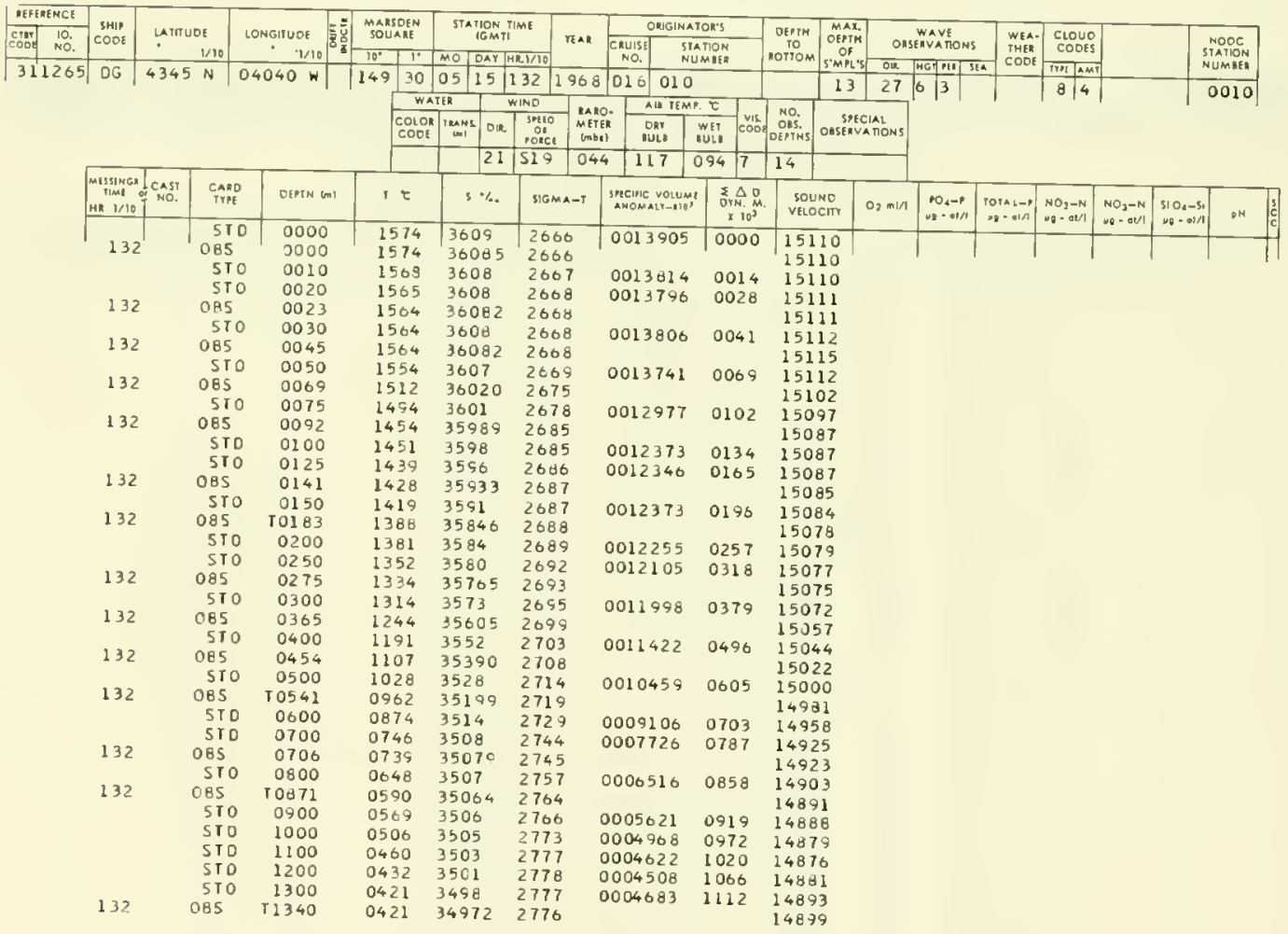


TABLE IX.-Continued

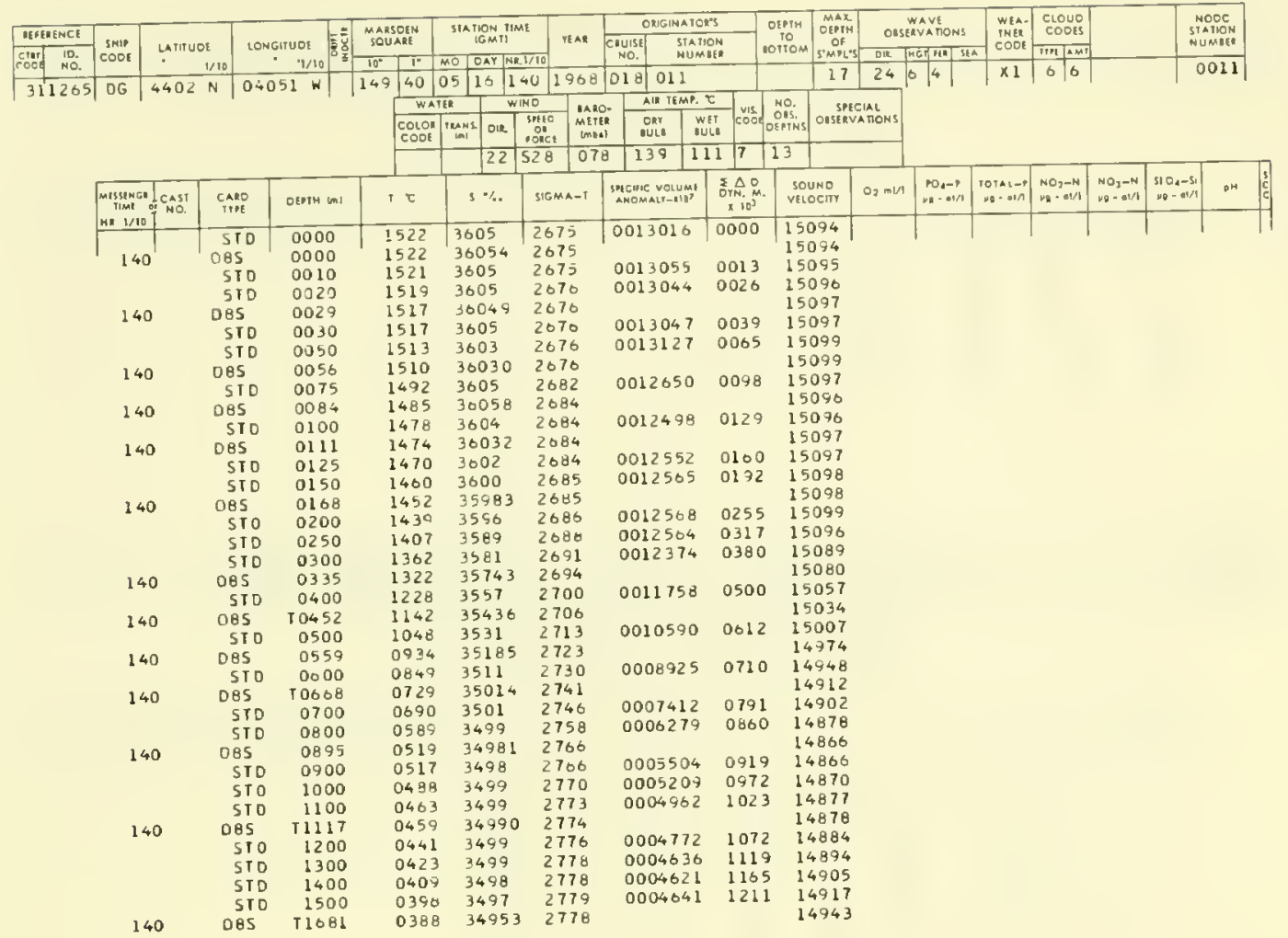

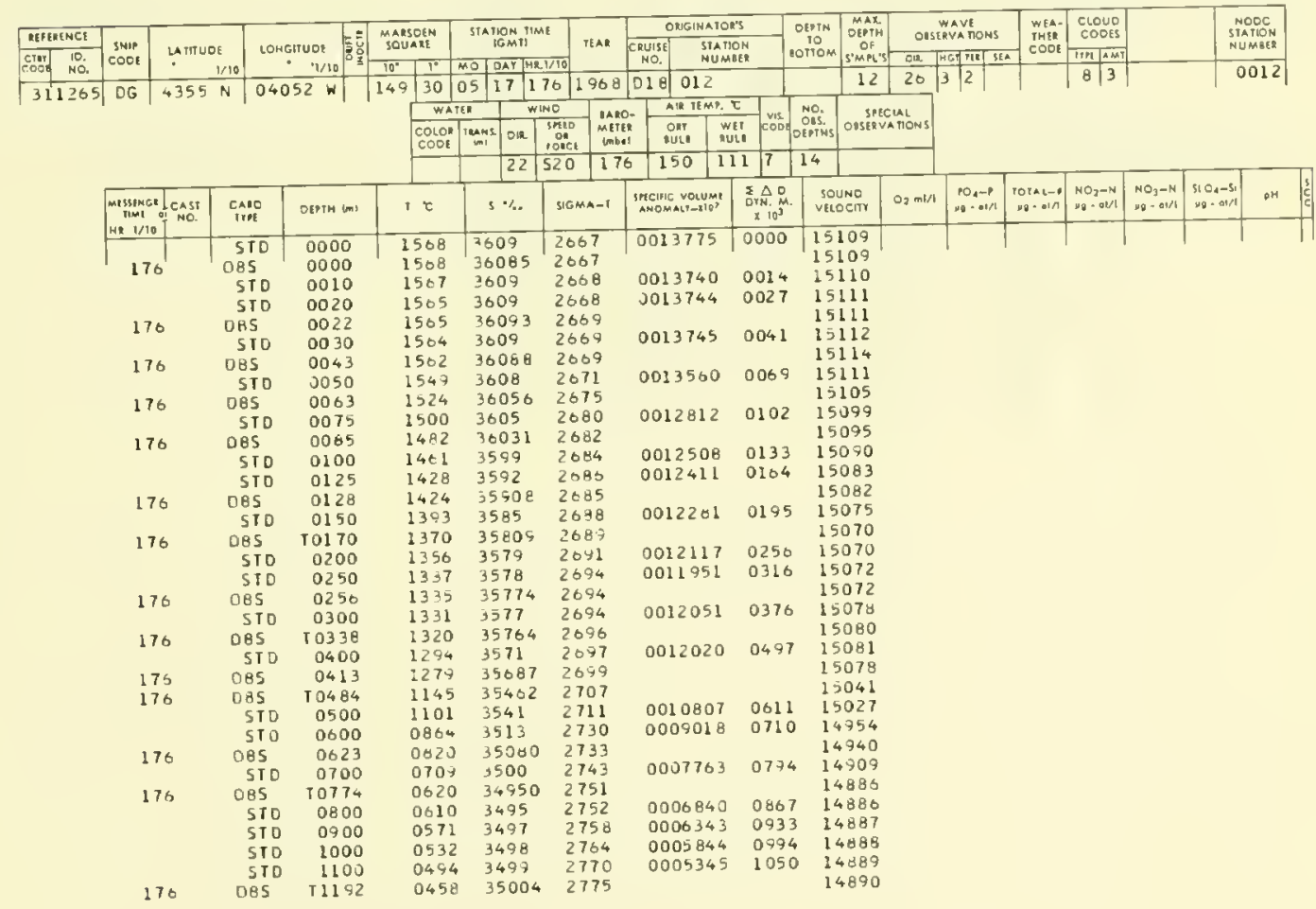


TABle IX.-Continued

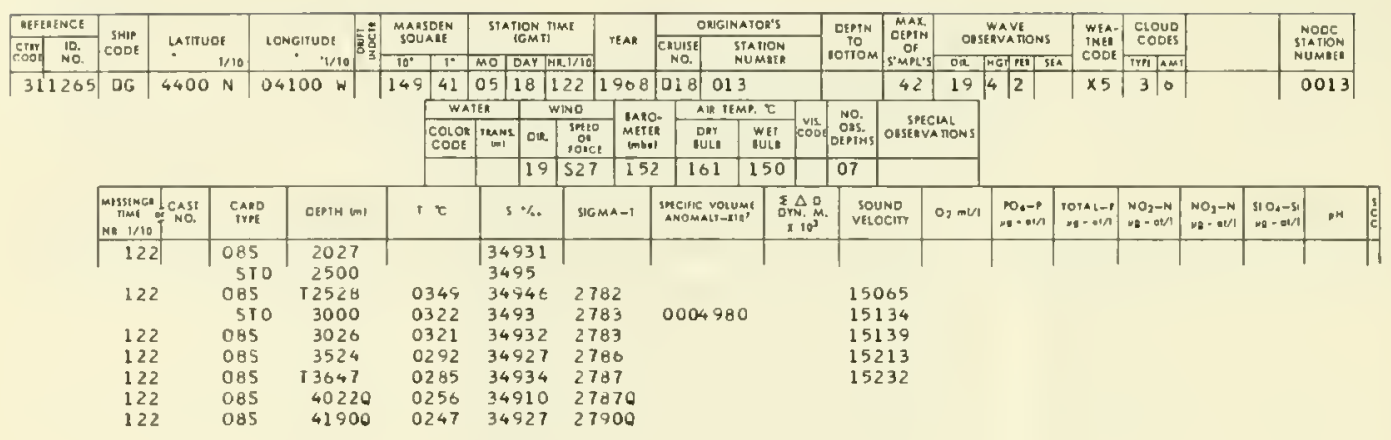

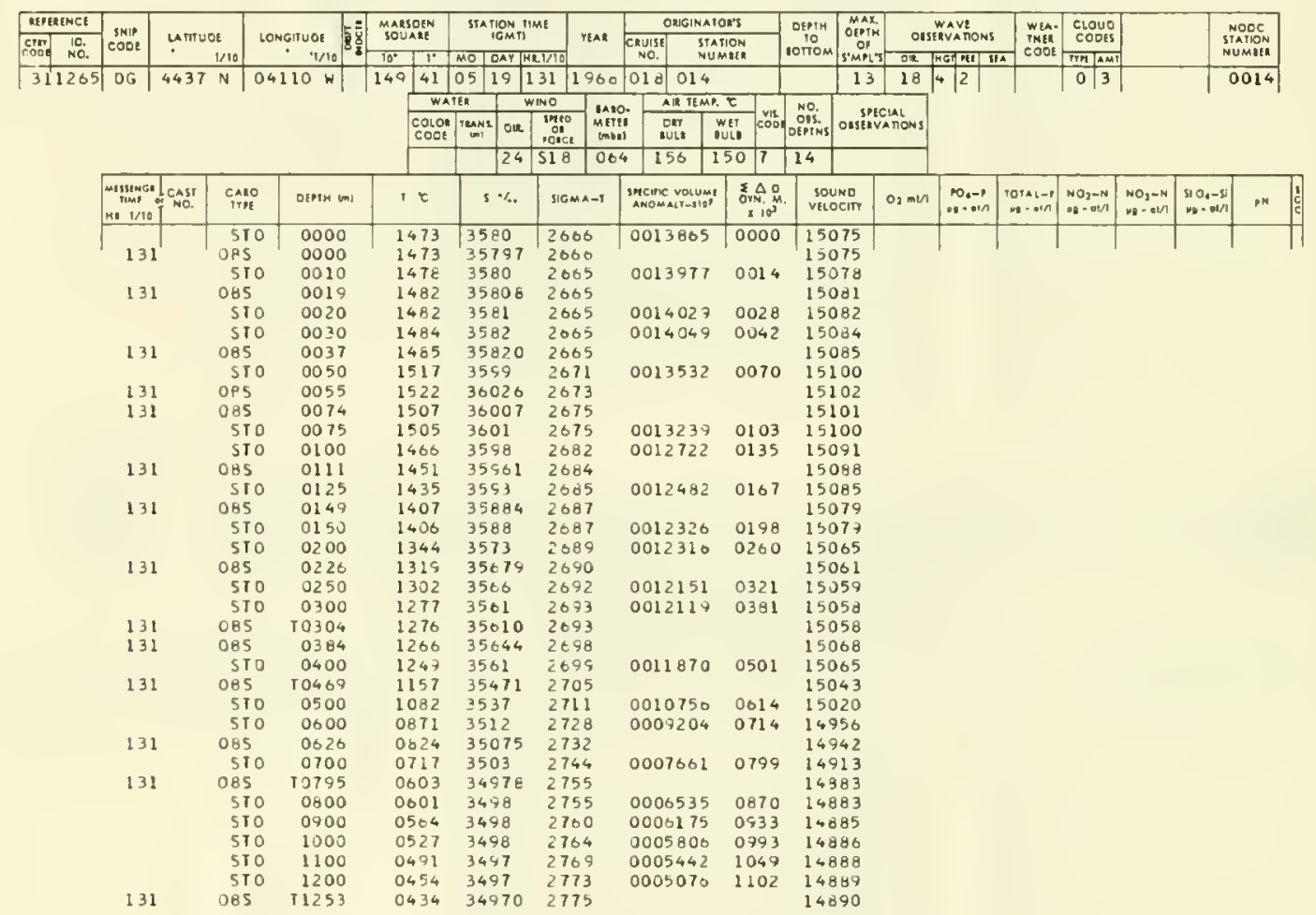


TABLE IX.-Continued

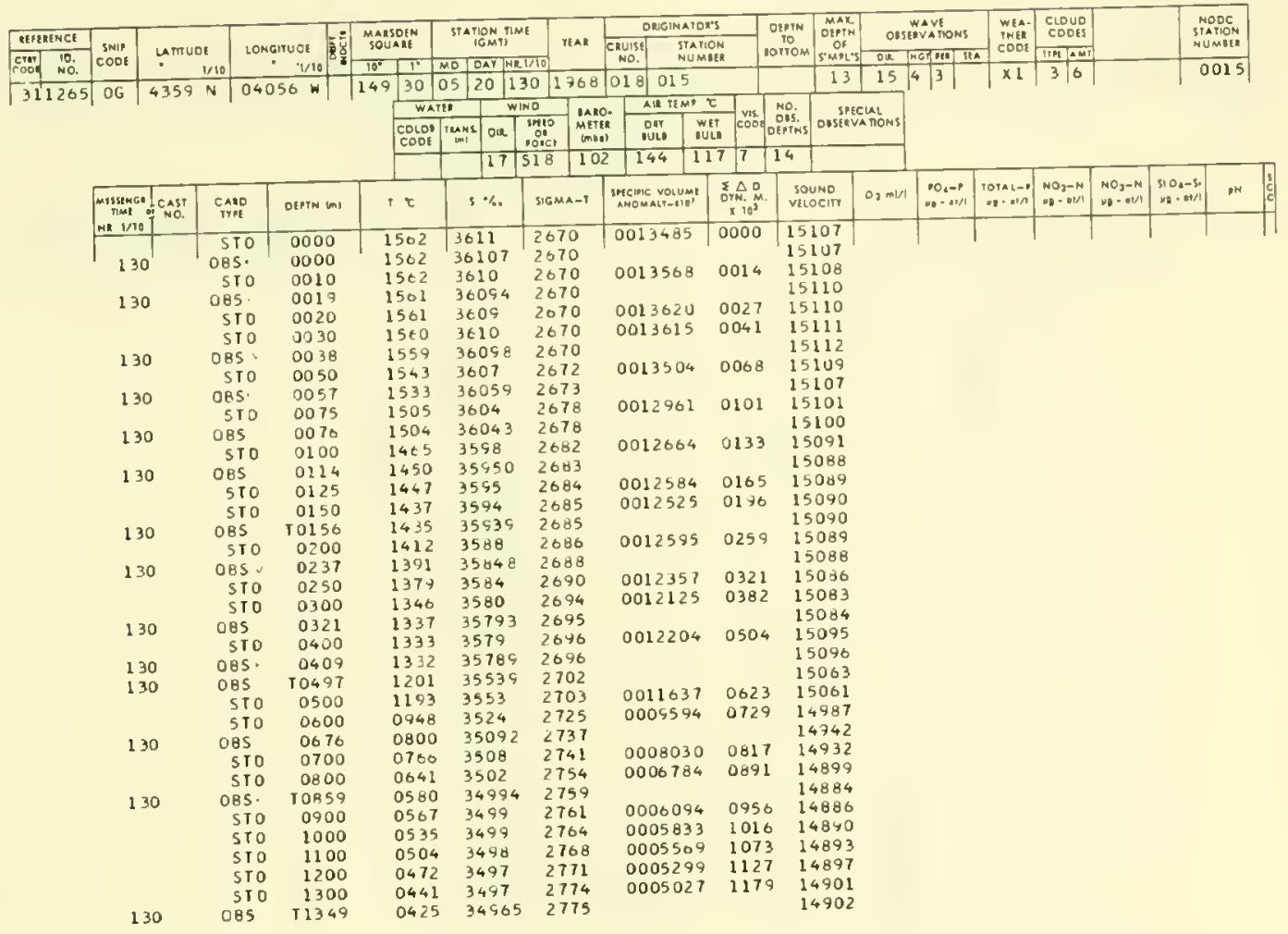

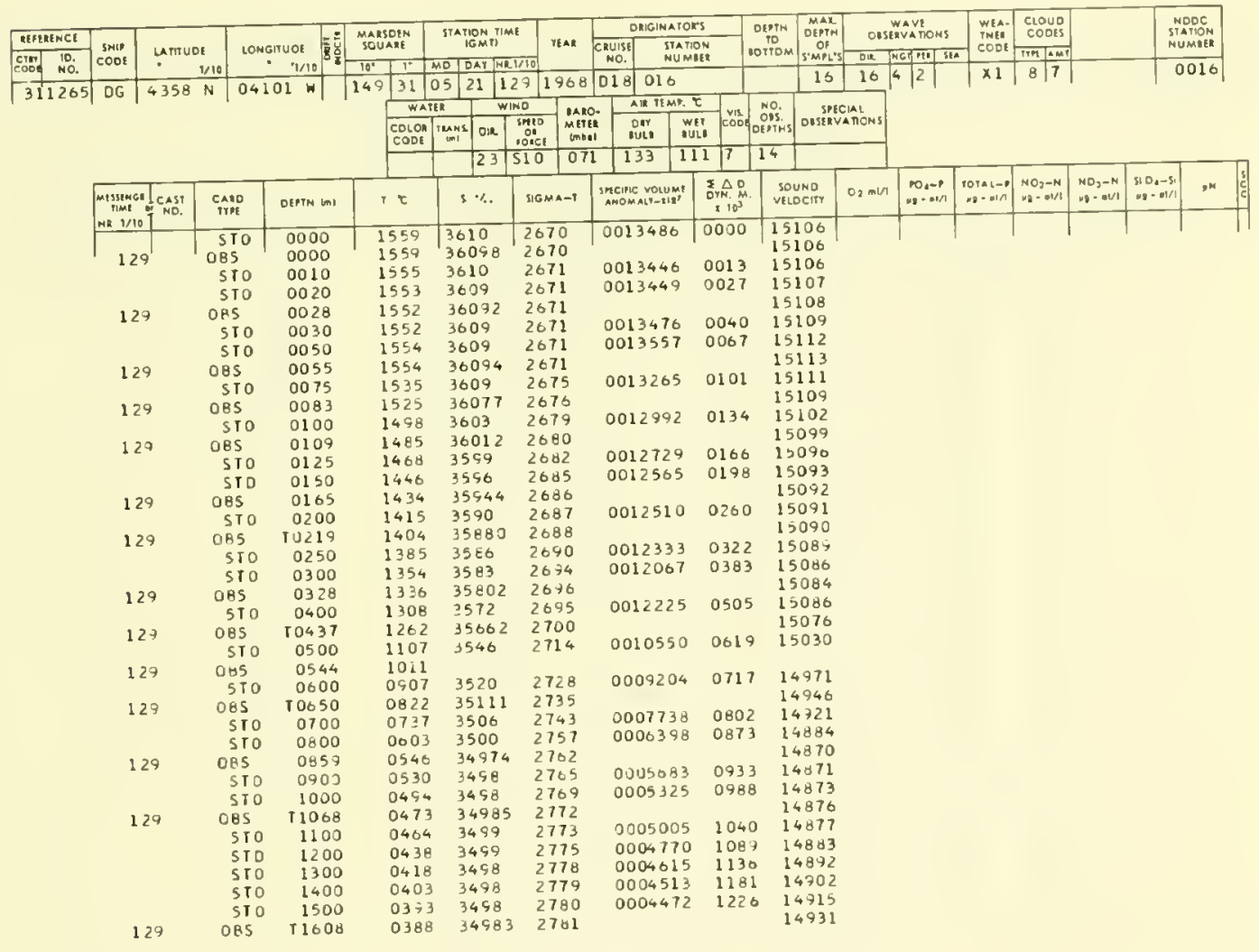


TABLE IX.-Continued

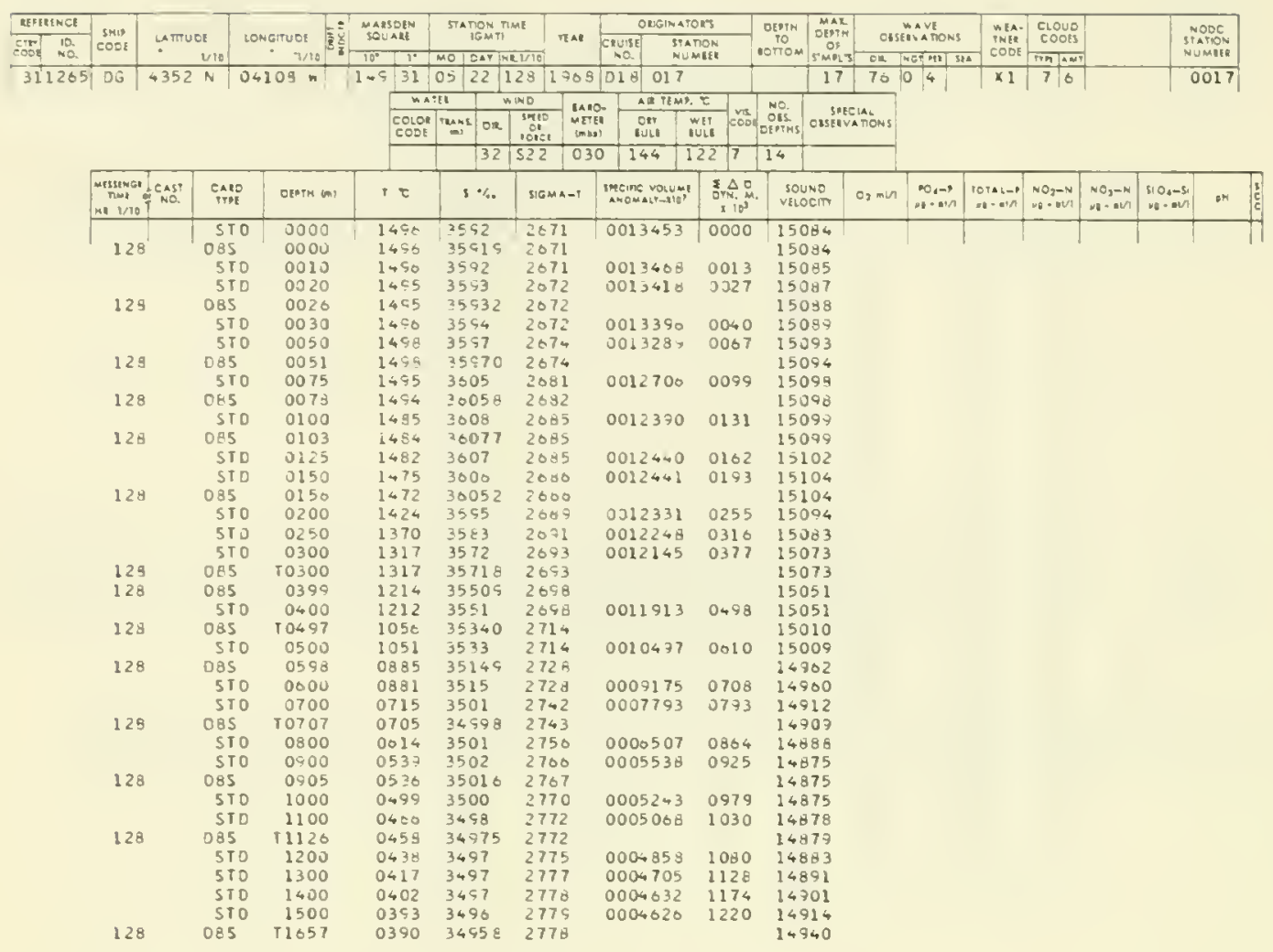

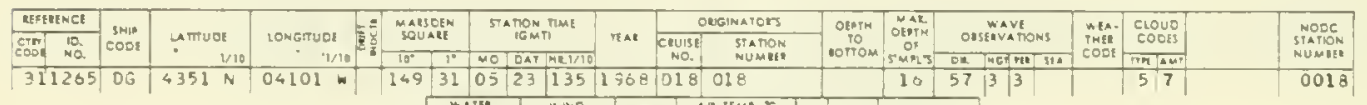

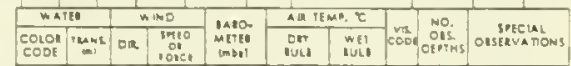

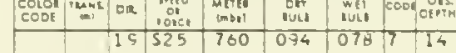

\begin{tabular}{|c|c|c|c|c|c|c|c|c|c|c|c|c|c|c|c|}
\hline 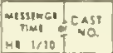 & $\begin{array}{l}\text { cano } \\
\text { fipli }\end{array}$ & DEPT un & $+\varepsilon$ & $s \%$ & DEMA-I & 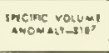 & 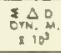 & $\begin{array}{l}\text { Soung } \\
\text { vetoem }\end{array}$ & $0, m i n$ & $\begin{array}{c}x_{1}, \infty \\
n \ldots+n\end{array}$ & 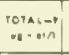 & $\begin{array}{l}\mathrm{NO}_{2}-\mathrm{N} \\
\mathrm{Na} \cdot \mathrm{GU}\end{array}$ & $\begin{array}{l}\mathrm{NO}_{3}-\mathrm{NA} \\
\mathrm{H} \\
\mathrm{H}\end{array}$ & 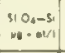 & M \\
\hline \multirow{4}{*}{135} & 510 & 0000 & 1470 & 3552 & 2075 & 0012999 & 0000 & 15078 & & & & & & & \\
\hline & 085 & 0000 & 1470 & 35924 & 2075 & & & 15078 & & & & & & & \\
\hline & 570 & 0010 & 1470 & 3552 & 2875 & 0013050 & 0013 & 13075 & & & & & & & \\
\hline & Sto & 0020 & 1475 & 3592 & 2675 & 0013072 & 0028 & 15081 & & & & & & & \\
\hline \multirow[t]{2}{*}{135} & 085 & 0024 & 1475 & 35 SIE & 2675 & & & 15081 & & & & & & & \\
\hline & 510 & 0030 & 1475 & 3552 & 2075 & 0013132 & 0039 & 15082 & & & & & & & \\
\hline \multirow[t]{2}{*}{135} & DES & $00<9$ & 1470 & 35917 & 2073 & & & 15085 & & & & & & & \\
\hline & STO & 0050 & $1 \rightarrow 7 t$ & 3592 & 2075 & 0013209 & OOBS & 15080 & & & & & & & \\
\hline \multirow[t]{2}{*}{135} & 085 & 0073 & 1474 & 35505 & 2874 & & & 15030 & & & & & & & \\
\hline & STO & 0075 & 1474 & 3551 & 2074 & 0013324 & 0099 & 15004 & & & & & & & \\
\hline \multirow[t]{3}{*}{135} & 085 & 0050 & 1458 & 35502 & 2670 & & & 15087 & & & & & & & \\
\hline & 510 & 0100 & 1444 & 3589 & 2080 & 0012835 & 0131 & 15083 & & & & & & & \\
\hline & 510 & 0125 & 1374 & 3583 & 2650 & 0011971 & 0103 & 15084 & & & & & & & \\
\hline \multirow[t]{2}{*}{135} & 045 & 0145 & 1341 & 35804 & 2695 & & & 15050 & & & & & & & \\
\hline & SIO & 0150 & 1343 & 3562 & $2 \in 55$ & 0011570 & 0192 & 15058 & & & & & & & \\
\hline \multirow[t]{3}{*}{135} & OAS & Tol os & 1351 & 35840 & $20 \times 8$ & & & 15303 & & & & & & & \\
\hline & 510 & 0200 & $13<0$ & $35 \in 4$ & 2650 & 0011032 & 0250 & 15009 & & & & & & & \\
\hline & 510 & 0250 & 1340 & $35 \varepsilon 2$ & 2050 & 0011719 & 0308 & 15073 & & & & & & & \\
\hline \multirow[t]{3}{*}{135} & 065 & 0297 & 1325 & 35792 & 2697 & & & 15070 & & & & & & & \\
\hline & STO & 0300 & 1325 & $3577^{\circ}$ & 2007 & 0011778 & 0367 & 15070 & & & & & & & \\
\hline & STO & 0400 & 127. & 3505 & 2700 & 0011773 & $0<45$ & 12074 & & & & & & & \\
\hline \multirow[t]{2}{*}{135} & $0 B 5$ & $\mathrm{TO}_{4} \mathrm{O}_{4}$ & 1270 & 3538 ? & 2700 & & & 15073 & & & & & & & \\
\hline & 5T0 & 0500 & 1130 & 3543 & 2707 & 0011190 & 0000 & 15038 & & & & & & & \\
\hline \multirow[t]{2}{*}{135} & 085 & 0505 & i 121 & $35-20$ & 2708 & & & 15035 & & & & & & & \\
\hline & 510 & 0000 & $0+11$ & 3517 & 2725 & 0008490 & 0703 & 14972 & & & & & & & \\
\hline \multirow[t]{3}{*}{135} & 085 & T $360 \%$ & 0953 & 35153 & 2727 & & & 14907 & & & & & & & \\
\hline & 510 & 0700 & $074-$ & 3510 & 2745 & 0007025 & 0789 & 14920 & & & & & & & \\
\hline & 570 & 0800 & 0024 & 3500 & 2759 & 0003249 & 085 y & 14853 & & & & & & & \\
\hline \multirow[t]{3}{*}{135} & OAS & 3019 & 0004 & 35048 & 2701 & & & 14868 & & & & & & & \\
\hline & 510 & 0700 & $05-5$ & 3502 & 2760 & 0005590 & $0 \rightarrow 17$ & 14877 & & & & & & & \\
\hline & 510 & 1000 & 0484 & 3499 & 2771 & $00051<0$ & 0971 & 14809 & & & & & & & \\
\hline \multirow[t]{6}{*}{135} & 085 & $T 10 \geq 1$ & 0408 & S४ढ?E & 2772 & & & 14907 & & & & & & & \\
\hline & 510 & $\$ 100$ & 0459 & 3458 & 2773 & 000.792 & 1021 & $1 \rightarrow 975$ & & & & & & & \\
\hline & 510 & 1200 & $04+5$ & 3498 & 2774 & 0004922 & 071 & .4480 & & & & & & & \\
\hline & $5 T 0$ & 1300 & 0.31 & $34 c 7$ & 2775 & 0004853 & 1120 & 14897 & & & & & & & \\
\hline & $\sin$ & 1400 & 0417 & 3697 & 2777 & 0006777 & $1 ; 68$ & $\therefore \angle ; 06$ & & & & & & & \\
\hline & $5 T 0$ & 1500 & 0603 & 3457 & 2770 & 0004701 & 1215 & 14919 & & & & & & & \\
\hline 135 & 085 & 11500 & 0356 & 3.970 & $277 \%$ & & & 14920 & & & & & & & \\
\hline
\end{tabular}


Table IX.-Continued

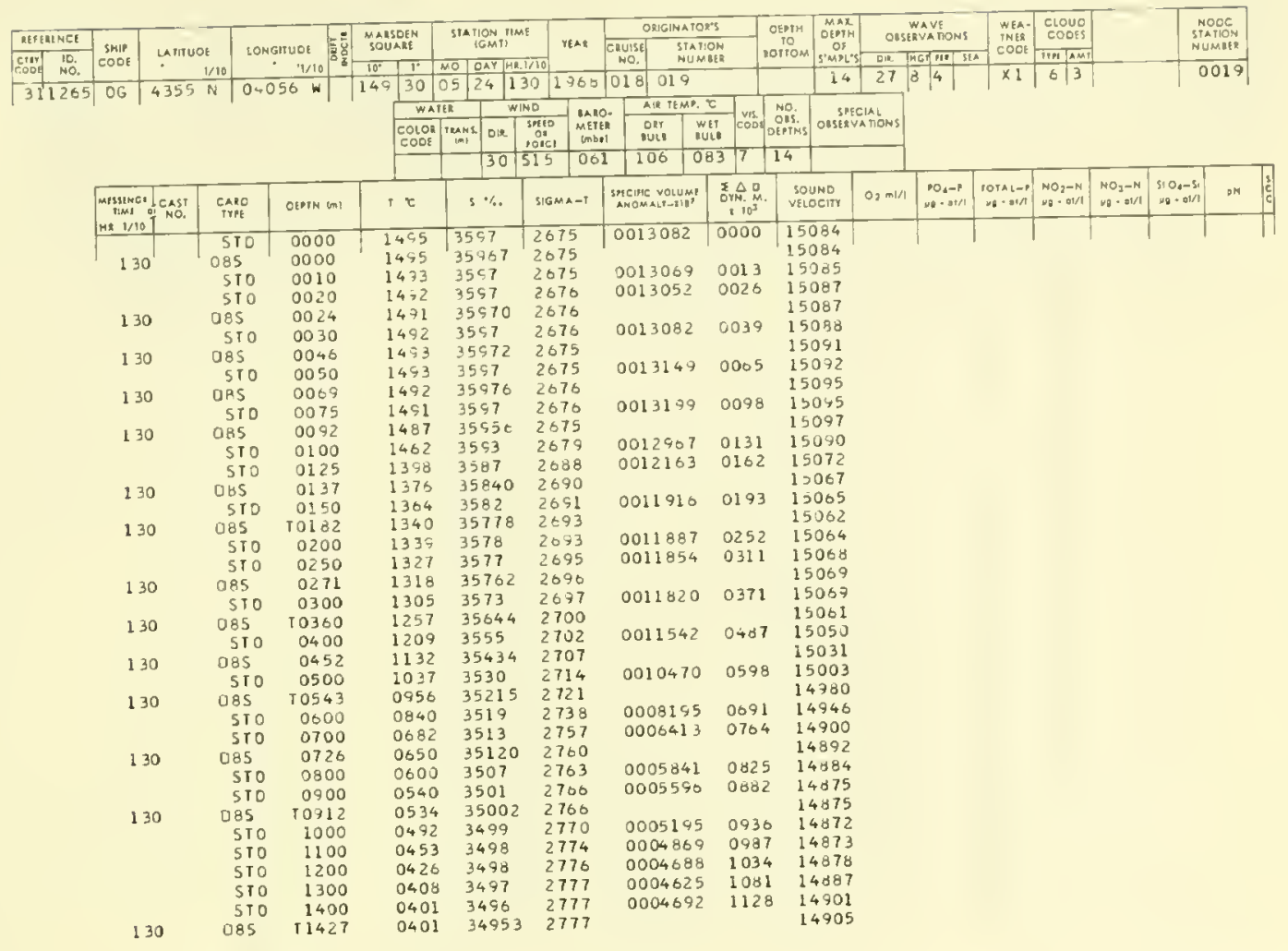

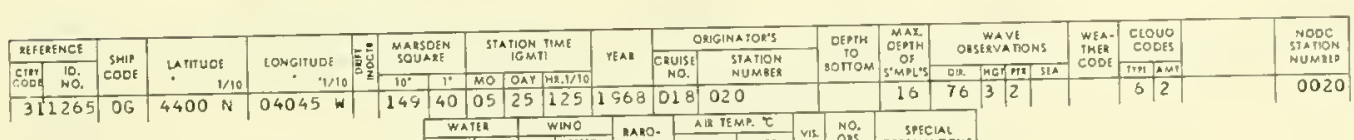

\begin{tabular}{|c|c|c|c|c|c|c|c|c|c|c|c|c|c|c|c|}
\hline & & & WATER & \multicolumn{2}{|c|}{ WINO } & AIS TEMP. $\tau$ & \multirow{2}{*}{\multicolumn{2}{|c|}{ vik No. }} & & \multirow[b]{4}{*}{$\begin{array}{c}10_{4}-1 \\
\mu R=3+11\end{array}$} & \multirow[b]{4}{*}{ 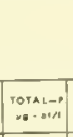 } & \multirow[b]{4}{*}{$\begin{array}{l}\mathrm{NO}_{3}-\mathrm{N} \\
\mathrm{NQ}-\mathrm{OL} / 1\end{array}$} & \multirow[b]{4}{*}{$\begin{array}{l}\mathrm{MO}_{3}-\mathrm{N} \\
\mathrm{N}_{0}=\mathrm{O} / \mathrm{O} / \mathrm{I}\end{array}$} & \multirow[b]{4}{*}{ 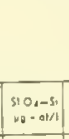 } & \multirow[b]{4}{*}{ on } \\
\hline & & & \multirow{3}{*}{\begin{tabular}{|l|l|}
$\begin{array}{c}\text { colon } \\
\text { coot }\end{array}$ & \\
& \\
$\tau$
\end{tabular}} & \multirow{2}{*}{$\frac{\text { Dir. }}{27}$} & 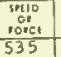 & \multirow{2}{*}{\begin{tabular}{|c|} 
OAr \\
BUL \\
156 \\
\end{tabular}} & & & & & & & & & \\
\hline & & & & & \begin{tabular}{|l|l|}
535 & 09 \\
\end{tabular} & & & \multicolumn{2}{|c|}{ ogsezvanons } & & & & & & \\
\hline 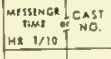 & $\begin{array}{l}\text { CARD } \\
\text { TYPE }\end{array}$ & OEPTH Un & & $s \cdot \%$ & SIGMA-T & 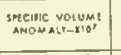 & 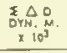 & $\begin{array}{l}\text { SOUNO } \\
\text { VELOCITY }\end{array}$ & $0, m^{1 / 1}$ & & & & & & \\
\hline $125^{\prime}$ & $\begin{array}{r}510 \\
085\end{array}$ & $\begin{array}{l}0000 \\
0000\end{array}$ & $\begin{array}{l}1462 \\
1462\end{array}$ & 554 & 2680 & 0012562 & 0000 & 15073 & & & & & & & \\
\hline & STO & $\begin{array}{l}0000 \\
0010\end{array}$ & $\begin{array}{l}1462 \\
1462\end{array}$ & $\begin{array}{l}5 \subseteq 44 \\
594\end{array}$ & $\begin{array}{l}2080 \\
2680\end{array}$ & & & $\begin{array}{l}15073 \\
15075\end{array}$ & & & & & & & \\
\hline & 510 & 0020 & 1402 & 595 & $=2080$ & $\begin{array}{l}0012593 \\
0012616\end{array}$ & $\begin{array}{l}0013 \\
0025\end{array}$ & $\begin{array}{l}15075 \\
15077\end{array}$ & & & & & & & \\
\hline 125 & 085 & 0026 & 1462 & 5945 & 2680 & & & i 5078 & & & & & & & \\
\hline & $\begin{array}{l}\text { STO } \\
\text { STO }\end{array}$ & $\begin{array}{l}0030 \\
0050\end{array}$ & 1462 & 555 & 2680 & 0012050 & 0038 & 15078 & & & & & & & \\
\hline 125 & ORS & $\begin{array}{l}0050 \\
0051\end{array}$ & $\begin{array}{l}1463 \\
1463\end{array}$ & 555 & 2680 & 0012720 & 0003 & 15082 & & & & & & & \\
\hline & $5 T 0$ & 0073 & $\begin{array}{l}1463 \\
1462\end{array}$ & $\begin{array}{l}5546 \\
595\end{array}$ & $\begin{array}{l}2600 \\
2680\end{array}$ & & & $\begin{array}{l}15082 \\
15086\end{array}$ & & & & & & & \\
\hline 125 & DBS & 0077 & 1462 & 5946 & $\begin{array}{l}2680 \\
2000\end{array}$ & 0012777 & 0095 & 15036 & & & & & & & \\
\hline 125 & $\begin{array}{l}510 \\
085\end{array}$ & $\begin{array}{l}0100 \\
0102\end{array}$ & 1455 & 553 & $26 d 0$ & 0012821 & 0127 & 15087 & & & & & & & \\
\hline & STO & $\begin{array}{l}0102 \\
01125\end{array}$ & $\begin{array}{l}1454 \\
1429\end{array}$ & 5926 & 2680 & & & 15087 & & & & & & & \\
\hline & STo & $\begin{array}{l}0125 \\
0150\end{array}$ & $\begin{array}{l}1429 \\
1405\end{array}$ & 590 & 2084 & 0012577 & 0159 & 15003 & & & & & & & \\
\hline 125 & 085 & 0153 & $\begin{array}{l}1405 \\
1406\end{array}$ & & $\begin{array}{l}2687 \\
2087\end{array}$ & 0012367 & 0190 & 15080 & & & & & & & \\
\hline & 510 & 0200 & 1306 & $\begin{array}{l}5874 \\
584\end{array}$ & $\begin{array}{l}2087 \\
2088\end{array}$ & & & $\begin{array}{l}15080 \\
15080\end{array}$ & & & & & & & \\
\hline 125 & 065 & 0204 & 1384 & 5833 & 2688 & 0012350 & 0252 & $\begin{array}{l}15080 \\
15080\end{array}$ & & & & & & & \\
\hline 125 & 085 & $\begin{array}{l}0300 \\
0306\end{array}$ & 1320 & 3573 & 2694 & 0012117 & 0374 & 13074 & & & & & & & \\
\hline & STO & $\begin{array}{l}0306 \\
0400\end{array}$ & $\begin{array}{l}1314 \\
1201\end{array}$ & 15723 & 2694 & & & 15073 & & & & & & & \\
\hline 125 & 085 & $\mathrm{r} 0405$ & $\begin{array}{l}1201 \\
1189\end{array}$ & $\begin{array}{l}552 \\
550 t\end{array}$ & $\begin{array}{l}2701 \\
2702\end{array}$ & 0011610 & 0493 & $\begin{array}{l}15047 \\
15044\end{array}$ & & & & & & & \\
\hline & STO & 0500 & 1022 & 527 & 2710 & 0010232 & $0 \in 02$ & $\begin{array}{l}15044 \\
149=8\end{array}$ & & & & & & & \\
\hline 125 & 085 & 0515 & 0990 & 5255 & 2718 & & & $\begin{array}{l}149=8 \\
14+90\end{array}$ & & & & & & & \\
\hline & 510 & 0000 & 0850 & 510 & 2730 & 0007014 & $0 \circ 99$ & 14748 & & & & & & & \\
\hline 125 & 085 & 10820 & 0820 & 5077 & 2732 & & & 14740 & & & & & & & \\
\hline & $\begin{array}{l}\text { SIO } \\
\text { STO }\end{array}$ & 0700 & 0727 & 507 & 2740 & 0007515 & 0721 & $14 ; 17$ & & & & & & & \\
\hline 125 & $\begin{array}{l}510 \\
085\end{array}$ & $\begin{array}{l}0800 \\
0835\end{array}$ & $\begin{array}{l}0623 \\
0589\end{array}$ & 3505 & 2758 & 0006309 & 0351 & 14893 & & & & & & & \\
\hline & $5 \mathrm{TO}$ & $\begin{array}{l}0835 \\
0900\end{array}$ & $\begin{array}{l}0587 \\
0527\end{array}$ & $\begin{array}{l}35042 \\
3502\end{array}$ & $\begin{array}{l}2702 \\
\angle 768\end{array}$ & & & 14885 & & & & & & & \\
\hline & $5 T 0$ & $\begin{array}{l}9900 \\
1000\end{array}$ & 0447 & 3497 & $\begin{array}{l}\angle 768 \\
2775\end{array}$ & $\begin{array}{l}0005343 \\
0004645\end{array}$ & $\begin{array}{l}0509 \\
0959\end{array}$ & $\begin{array}{l}14870 \\
14854\end{array}$ & & & & & & & \\
\hline 125 & 085 & 1048 & 0416 & 34583 & 2778 & 0004645 & 0959 & $\begin{array}{l}148349 \\
14849\end{array}$ & & & & & & & \\
\hline & 510 & 1100 & 0413 & 3497 & 2777 & 0004402 & 1004 & 14856 & & & & & & & \\
\hline & STO & 1200 & 0407 & 3496 & 2777 & 0004550 & 1049 & 14870 & & & & & & & \\
\hline & 510 & 1300 & 0401 & 3494 & 2776 & 0004717 & 1096 & 14384 & & & & & & & \\
\hline & 510 & 1400 & 0395 & $34 \$ 4$ & 2777 & 0004733 & 1143 & 14638 & & & & & & & \\
\hline 125 & $\begin{array}{l}510 \\
085\end{array}$ & $\begin{array}{r}1500 \\
1580\end{array}$ & 0389 & 3494 & $\begin{array}{l}2777 \\
2778\end{array}$ & 0004743 & 1190 & 14913 & & & & & & & \\
\hline & 085 & T1586 & 0384 & 34535 & 2778 & & & 14925 & & & & & & & \\
\hline
\end{tabular}


TABLE IX.-Continued

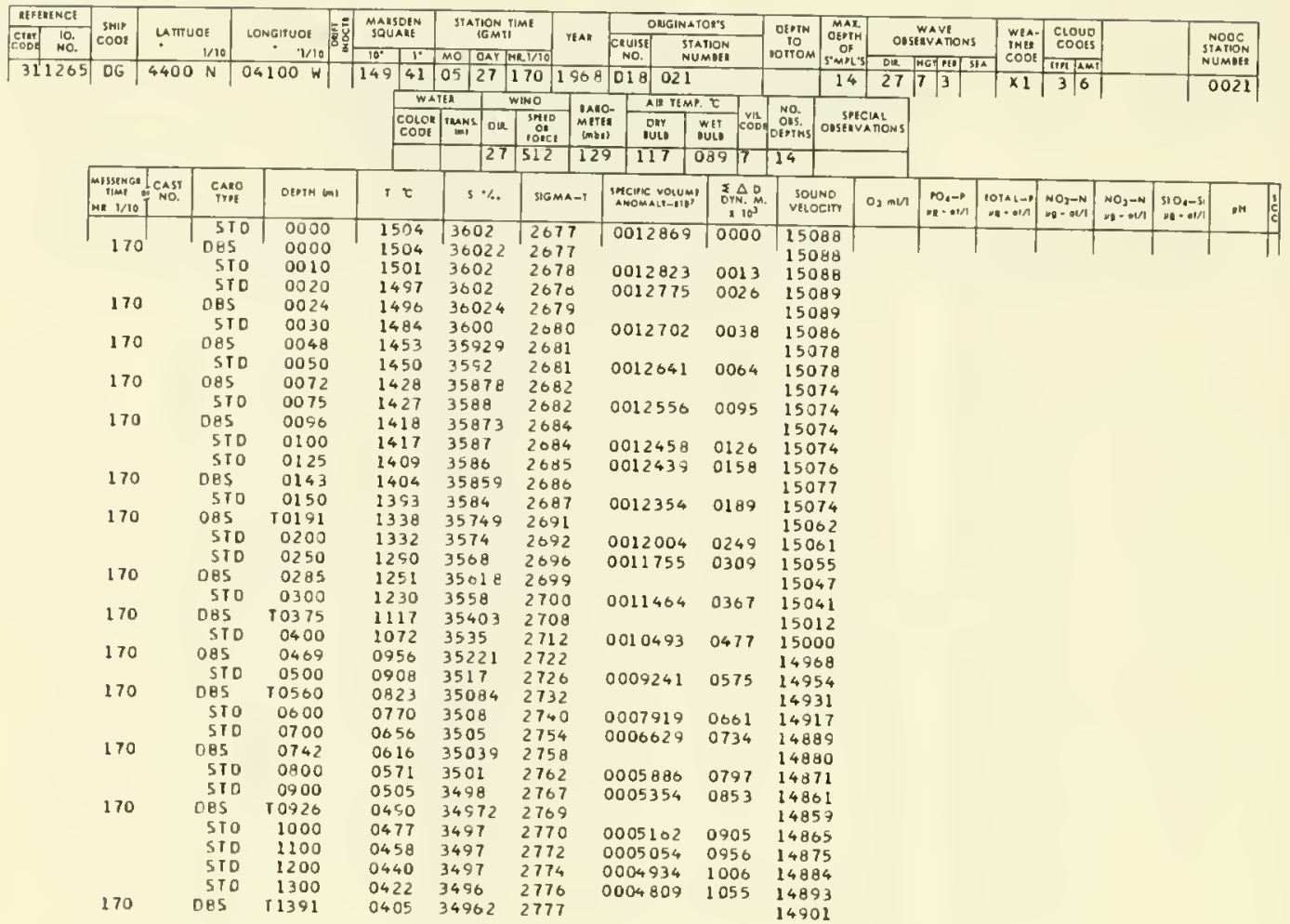

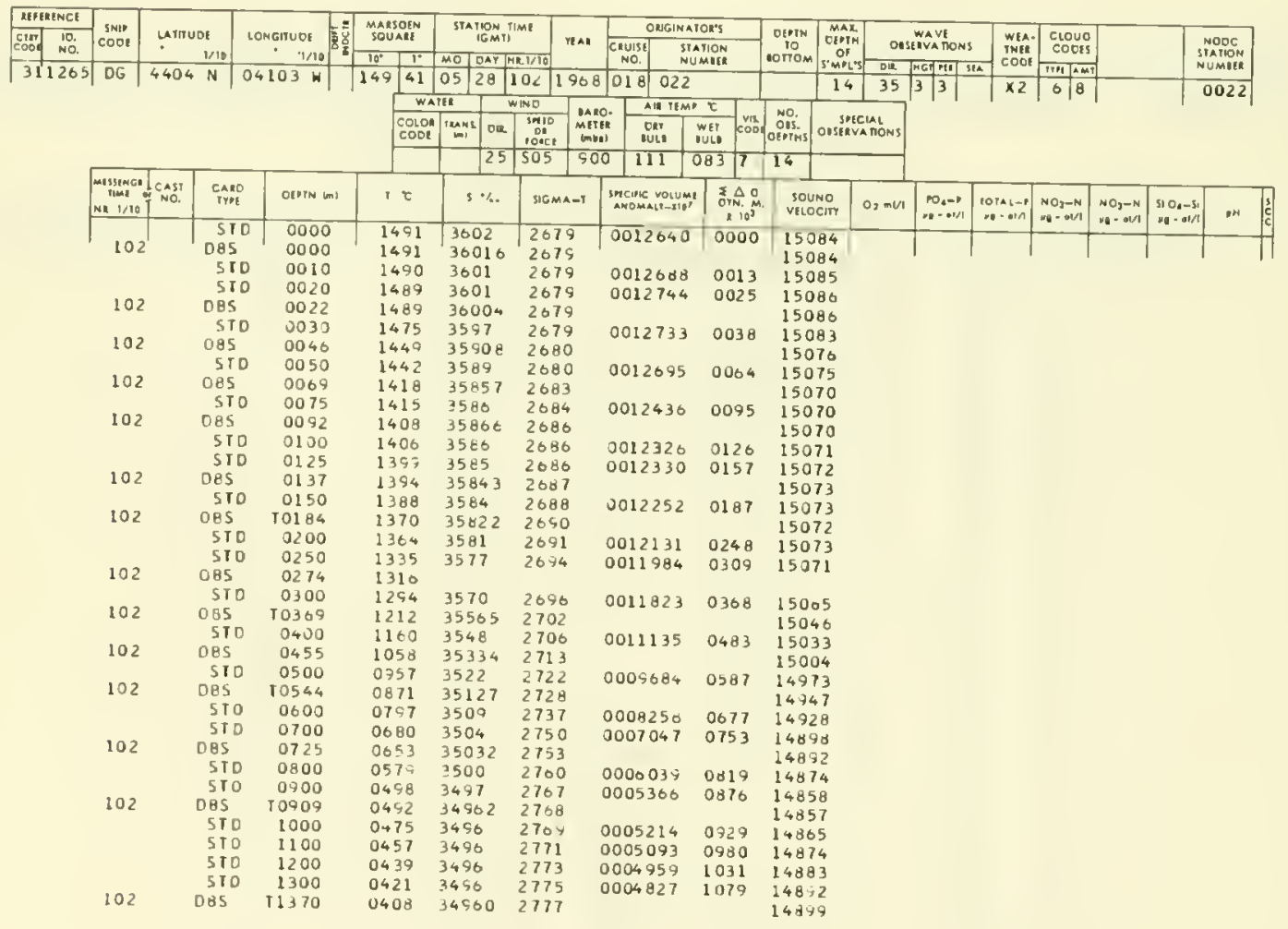



TABLE X.-Observed and interpolated oceanographic data from stations taken by USCGC CASTLE
ROCK at Ocean Station DELTA, 31 May-19 June 1968, prepared from NODC listing No. 31-1269

CK.

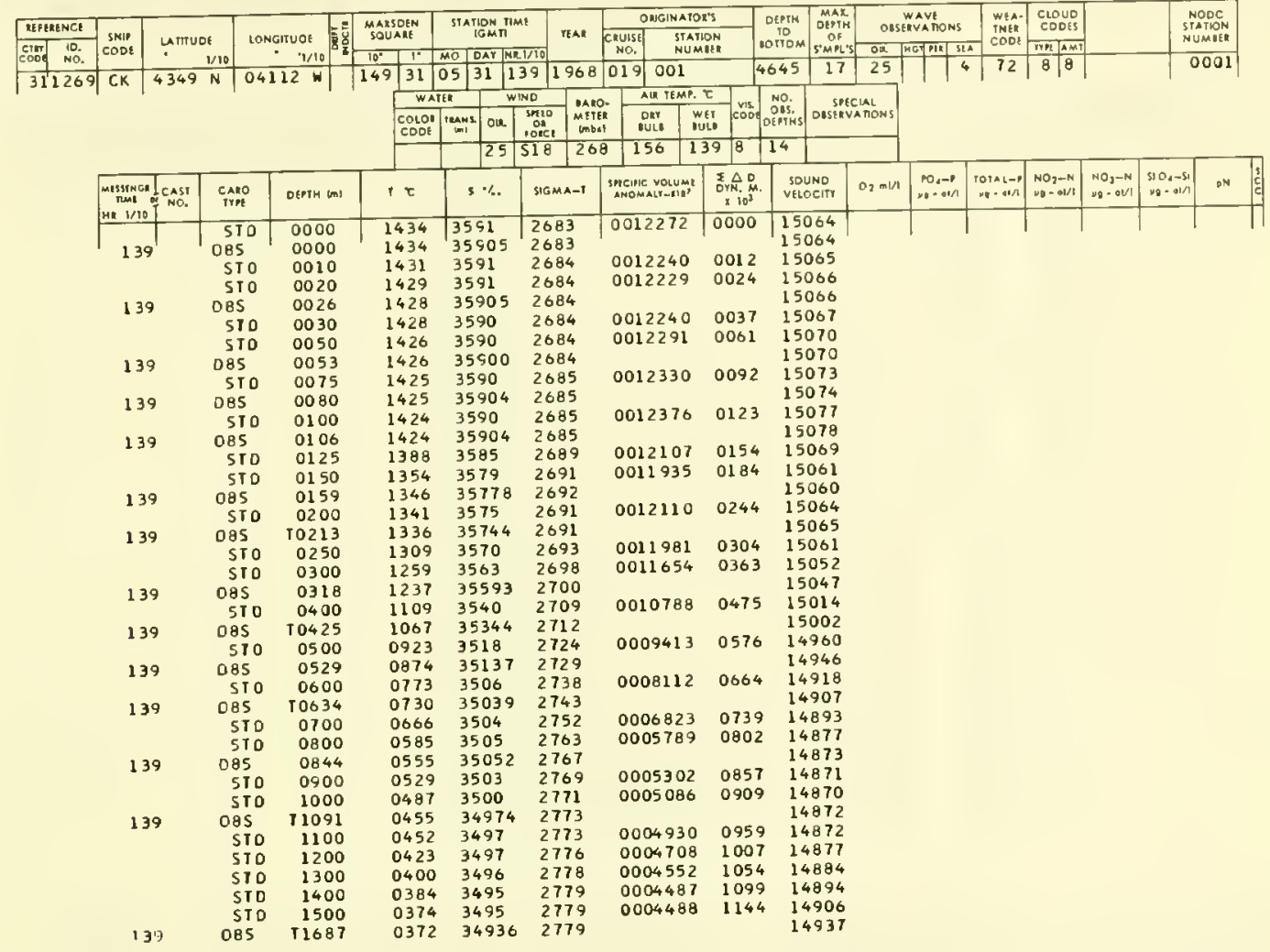

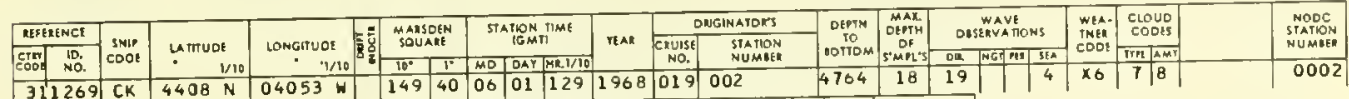

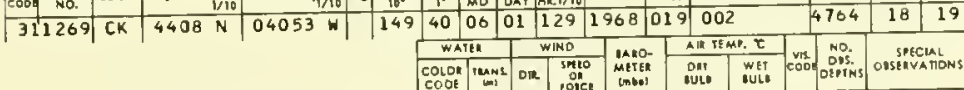

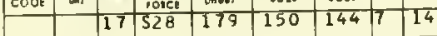

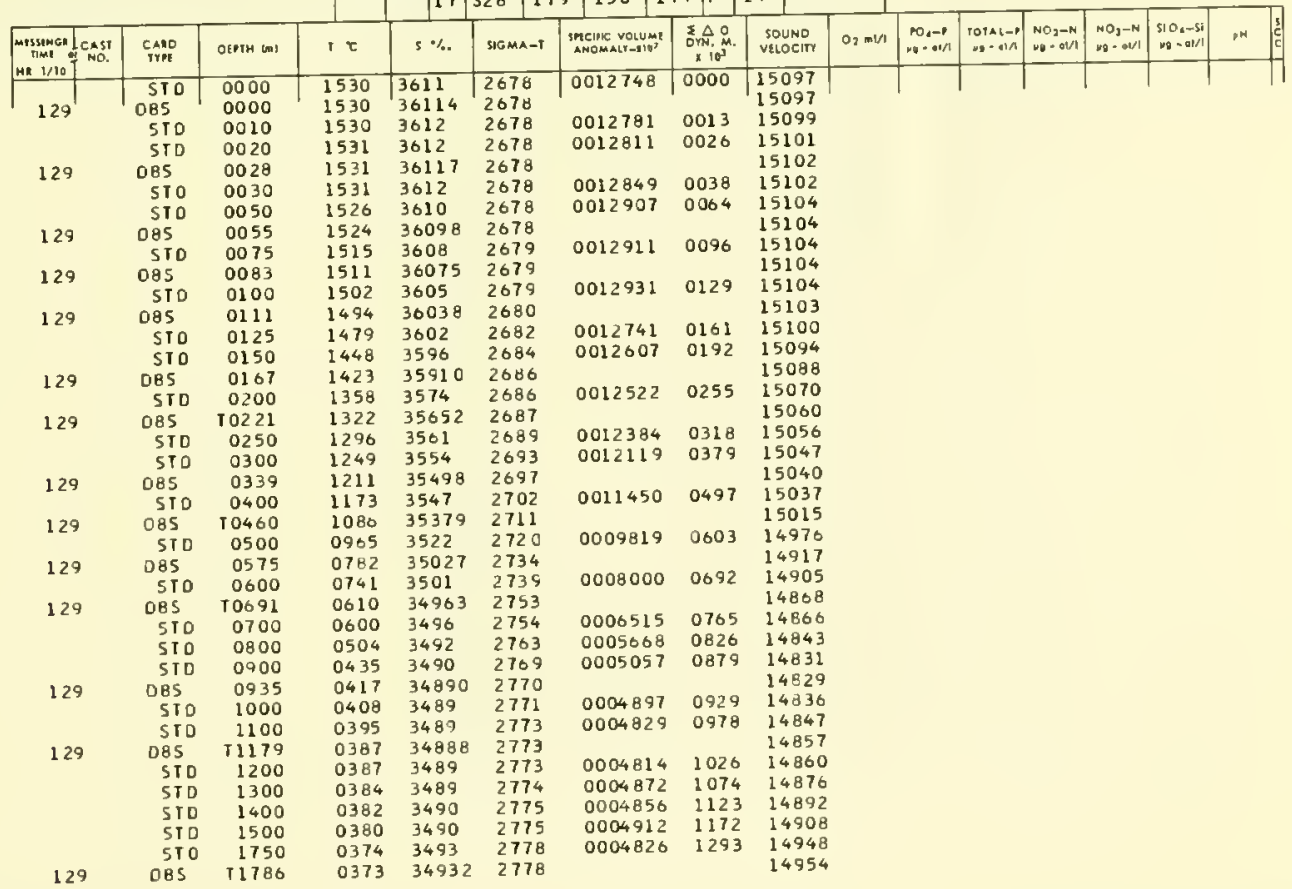


TABLE X.-Continued

\begin{tabular}{|c|c|c|c|c|c|c|c|c|c|c|c|c|c|c|c|c|c|c|}
\hline & \multirow{2}{*}{$\begin{array}{l}3 M 10 \\
\operatorname{coD}\end{array}$} & \multirow{2}{*}{\multicolumn{2}{|c|}{$\begin{array}{c}\text { Lafituoe } \\
1 / 10 \\
\end{array}$}} & \multirow{2}{*}{$\begin{array}{c}\text { LONGITUOE } \\
1 / 10 \\
\end{array}$} & \multirow{2}{*}{$\begin{array}{l}\text { MARSDEN } \\
\text { SOUABE }\end{array}$} & \multirow{2}{*}{\multicolumn{2}{|c|}{$\begin{array}{l}\text { STATION TIME } \\
\text { IGMT! }\end{array}$}} & \multicolumn{2}{|c|}{ DRGINATORS } & \multirow{2}{*}{$\begin{array}{c}\text { DEPTH } \\
\text { TO } \\
\text { IOTOM }\end{array}$} & \multirow{2}{*}{\begin{tabular}{|c|} 
MAX \\
OEPH \\
Of \\
SMPL'S \\
\end{tabular}} & \multirow{2}{*}{\multicolumn{2}{|c|}{$\begin{array}{l}\text { WAVE } \\
\text { onsenVATIONS }\end{array}$}} & \multirow{3}{*}{$\begin{array}{c}\text { WEA- } \\
\text { PHEA } \\
\text { CODE } \\
\times 6\end{array}$} & \multirow{2}{*}{\begin{tabular}{|c|}
$\begin{array}{c}\text { CIOU日O } \\
\text { COOES }\end{array}$ \\
TPA AMT \\
\end{tabular}} & & & \multirow{2}{*}{$\begin{array}{l}\text { NDDE } \\
\text { STANIIN } \\
\text { NUMAER }\end{array}$} \\
\hline \begin{tabular}{|c|c|}
$\begin{array}{c}C B E \\
C 006\end{array}$ & $\begin{array}{c}10 . \\
\text { NO. }\end{array}$ \\
311269 \\
3126
\end{tabular} & & & & & & & YEAR & $\begin{array}{l}\text { ERUISE: } \\
\text { NO. }\end{array}$ & $\begin{array}{l}\text { STATARS } \\
\text { SUMANER } \\
\text { NUMER }\end{array}$ & & & & & & & & & \\
\hline & $C K$ & 4353 & $\mathrm{~N}$ & $04107 \mathrm{W1}$ & \begin{tabular}{|l|l|}
149 & 31 \\
\end{tabular} & \begin{tabular}{|l|l|l}
06 & 02 & 1 \\
\end{tabular} & \begin{tabular}{l|l}
153 & 1908
\end{tabular} & \begin{tabular}{|l|l|}
019 & 003
\end{tabular} & & 4663 & 15 & 19 & 4 & & $6 / 8$ & & & 0003 \\
\hline & & & & & WA & TeR & WIND D IAK & 0. & 2 & & & & & & & & & \\
\hline & & & & & $\begin{array}{l}\text { ceron } \\
\text { coot }\end{array}$ & THNAL DIR & $\begin{array}{l}\operatorname{sen} 10 \\
00 \\
0.08\end{array}$ & \begin{tabular}{c|c} 
ORY & W \\
OULA & Wu
\end{tabular} & \begin{tabular}{l|l} 
WEET \\
WULB
\end{tabular} & of ons & Onserva & 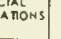 & & & & & & \\
\hline & & & & & & 19 & 518 & 156 & \begin{tabular}{l|l}
50 & 6 \\
\end{tabular} & 14 & & & & & & & & \\
\hline & 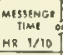 & $\begin{array}{l}\text { IeAst } \\
\text { I NO. }\end{array}$ & $\begin{array}{l}\text { CARD } \\
\text { TYRE }\end{array}$ & DERTA (m) & $T " \tau$ & $s=1 \%$ & $\operatorname{sig} m a-1$ & 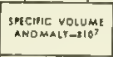 & 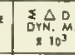 & & $\begin{array}{l}\text { UNO } \\
\text { OCITY }\end{array}$ & $0_{3} \mathrm{~m} / \mathrm{s} /$ & 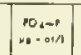 & 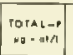 & $\mid \begin{array}{l}\mathrm{NO}_{2}-\mathrm{N} \\
\mathrm{W}=\mathrm{O} / \mathrm{N}\end{array}$ & 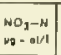 & $\left|\begin{array}{l}310_{1}-51 \\
y_{0}=0.1 / 1\end{array}\right|$ & $\mathrm{OH}$ \\
\hline & & & STO & 0000 & $\$ 451$ & 3589 & 2677 & 0012840 & 0000 & 150 & 069 & & & & & & & \\
\hline & 153 & & D8S & 0000 & 1451 & 35875 & 2677 & & & 150 & 069 & & & & & & & \\
\hline & & & $\begin{array}{l}\text { STD } \\
\text { STD }\end{array}$ & $\begin{array}{l}0010 \\
0020\end{array}$ & $\begin{array}{l}1448 \\
1447\end{array}$ & 3589 & 2679 & 0012698 & 0013 & 150 & 070 & & & & & & & \\
\hline & 153 & & 085 & 0024 & $\begin{array}{l}1448 \\
1446\end{array}$ & $\begin{array}{l}3590 \\
35902\end{array}$ & $\begin{array}{l}2680 \\
2680\end{array}$ & 0012635 & 0025 & $\begin{array}{l}150 \\
150\end{array}$ & 971 & & & & & & & \\
\hline & & & STD & 0030 & 1446 & 3591 & 2081 & 0012571 & 0038 & 150 & 973 & & & & & & & \\
\hline & 153 & & 085 & 0047 & 1445 & 35914 & 2081 & & & 150 & 275 & & & & & & & \\
\hline & & & STD & 0050 & 1444 & 3591 & 2082 & 0012501 & 0063 & 150 & 76 & & & & & & & \\
\hline & 153 & & 085 & 0072 & 1439 & 35916 & 2683 & & & 150 & 78 & & & & & & & \\
\hline & & & STO & 0075 & 1439 & 3592 & 2683 & 0012518 & 0095 & 150 & 78 & & & & & & & \\
\hline & 153 & & 085 & 0095 & 1435 & 35512 & 2083 & & & 150 & 80 & & & & & & & \\
\hline & & & STo & 0100 & 1434 & $359 !$ & 2083 & 0012533 & 0126 & 150 & 80 & & & & & & & \\
\hline & & & STD & 0125 & 1429 & 3590 & 2684 & 0012561 & 0157 & 150 & 83 & & & & & & & \\
\hline & 153 & & 085 & 0144 & 1425 & 35896 & 2684 & & & 1508 & 85 & & & & & & & \\
\hline & & & STD & 0150 & 1412 & 3588 & 2680 & 0012449 & 0188 & 150 & 381 & & & & & & & \\
\hline & 153 & & 085 & 0192 & 1340 & $3577 \mathrm{e}$ & 2693 & & & 150 & 063 & & & & & & & \\
\hline & & & 510 & 0200 & 1337 & 3577 & 2693 & 0011884 & 0249 & 150 & 03 & & & & & & & \\
\hline & & & $5 T 0$ & 0250 & 1309 & 3573 & 2696 & 0011702 & 0308 & 1500 & 162 & & & & & & & \\
\hline & 153 & & DBS & 0285 & 1277 & 35680 & 2698 & & & 1505 & 156 & & & & & & & \\
\hline & & & STD & 0300 & 1255 & 3564 & 2700 & 0011504 & 0367 & 1505 & 51 & & & & & & & \\
\hline & 153 & & ORS & 10376 & 1140 & 35450 & 2707 & & & 1502 & 122 & & & & & & & \\
\hline & & & $5 T 0$ & 0400 & 1110 & 3540 & 2709 & 0010806 & 0478 & 1501 & 14 & & & & & & & \\
\hline & 153 & & 085 & 0470 & $09 \$ 9$ & 35265 & 2718 & & & 1498 & 184 & & & & & & & \\
\hline & & & STO & 0500 & 0934 & 3520 & 2724 & 0009448 & 0579 & 1496 & 164 & & & & & & & \\
\hline & 153 & & 085 & ro559 & 0822 & 35103 & 2734 & & & 1493 & 31 & & & & & & & \\
\hline & & & STD & 0600 & 0764 & 3509 & 2742 & 0007755 & 0665 & 1491 & & & & & & & & \\
\hline & & & STO & 0700 & 0640 & 3505 & 2755 & 0006488 & 0737 & $14 \mathrm{Bg}$ & & & & & & & & \\
\hline & 153 & & 085 & 0740 & 0603 & 35039 & 2700 & & & 1487 & & & & & & & & \\
\hline & & & STD & 0800 & $057 i$ & 3503 & 2763 & 0005737 & 0798 & 1487 & & & & & & & & \\
\hline & & & STO & 0900 & 0520 & 3501 & 2768 & 0005330 & 0853 & 1486 & & & & & & & & \\
\hline & 153 & & 085 & T0950 & 0497 & 35004 & 2770 & & & 1430 & & & & & & & & \\
\hline & & & 510 & 1000 & 0477 & 3500 & 2772 & 0004955 & 0905 & 1480 & & & & & & & & \\
\hline & & & STD & 1100 & 0443 & 3498 & 2775 & 0004770 & 0953 & 1486 & & & & & & & & \\
\hline & & & STD & 1200 & 0417 & 3497 & 2777 & 0004609 & 1000 & 1487 & & & & & & & & \\
\hline & & & STO & 1300 & 0401 & 3496 & 2777 & 0004572 & 1046 & 1488 & & & & & & & & \\
\hline & & & STD & 1400 & 0393 & 3496 & 2778 & 0004559 & 1092 & 1489 & & & & & & & & \\
\hline & 153 & & D8S & 11457 & 0393 & 34954 & 2779 & & & 1430 & & & & & & & & \\
\hline
\end{tabular}

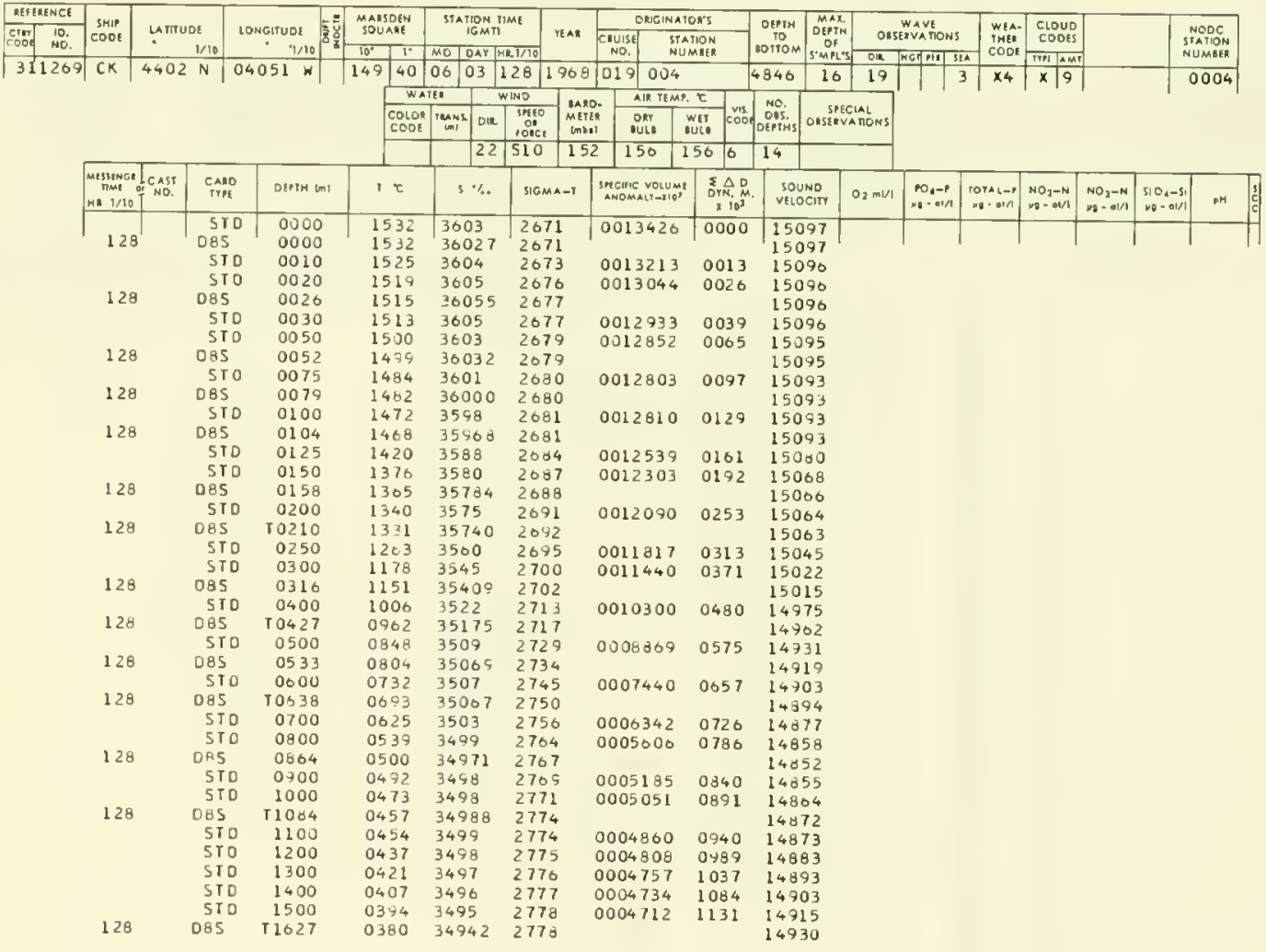


TABLE X.-Continued

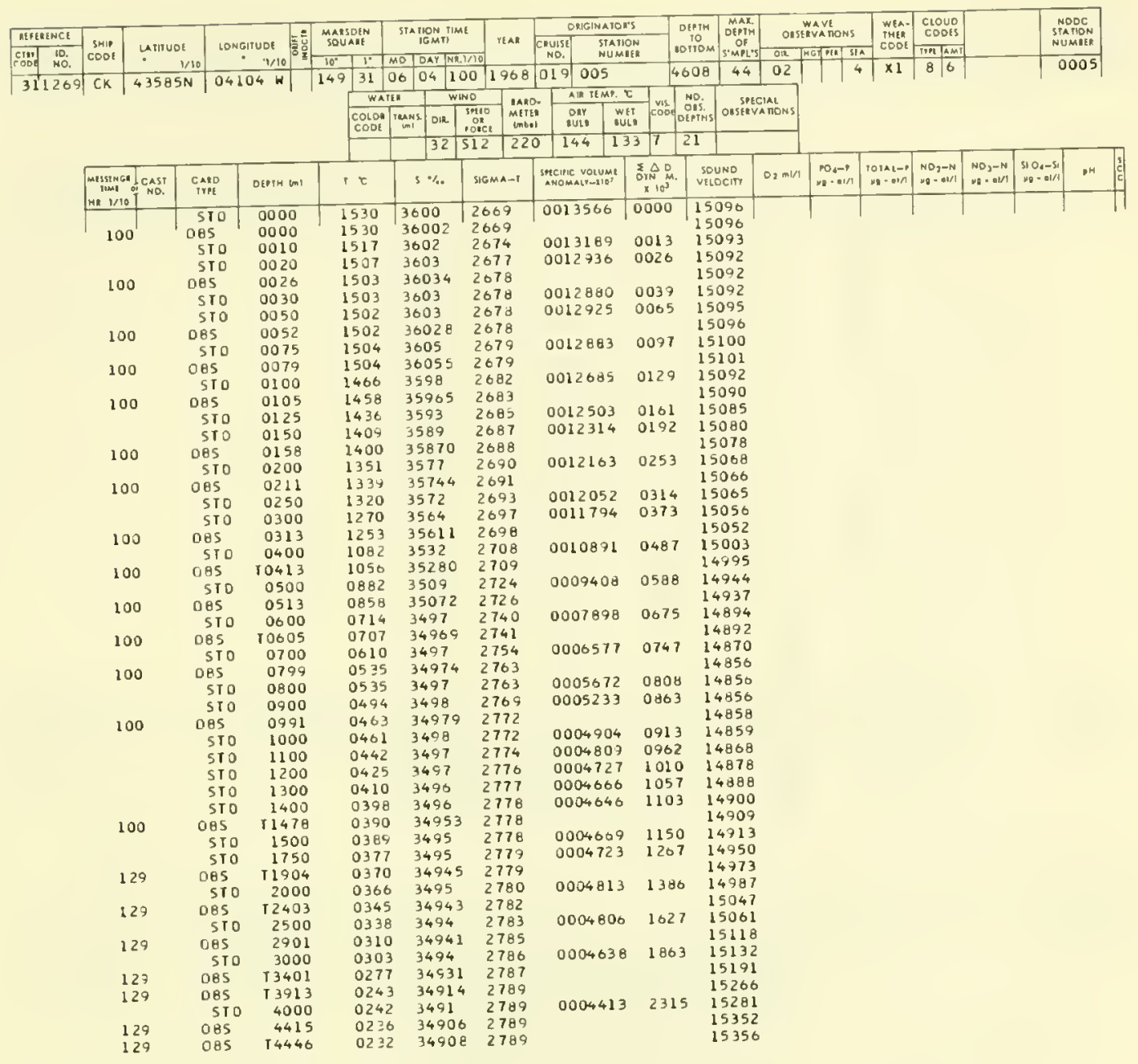


TABLE X.-Continued

\begin{tabular}{|c|c|c|c|c|c|c|c|c|c|c|c|c|c|c|c|c|c|c|}
\hline REFEREACE & \multirow{2}{*}{$\begin{array}{c}\text { SHIP } \\
\text { CODE }\end{array}$} & \multirow{2}{*}{\multicolumn{2}{|c|}{$\begin{array}{c}0 \text { umuder } \\
1 / 10 \\
\end{array}$}} & \multirow{2}{*}{$\begin{array}{c}\text { LONGIIUDE } \\
- \text { W/10 }\end{array}$} & \multirow{2}{*}{$\begin{array}{l}\text { MARSDEN } \\
\text { SOUAKE }\end{array}$} & \multirow{2}{*}{\multicolumn{2}{|c|}{$\begin{array}{c}\text { STA TION NIME } \\
\text { IGM III }\end{array}$}} & \multicolumn{2}{|c|}{ DNGINATOR'S } & \multirow{2}{*}{$\mid \begin{array}{c}\text { DEPTH } \\
\text { PO } \\
\text { BOTOM }\end{array}$} & \multirow{2}{*}{\begin{tabular}{|c|} 
MAX \\
OESIK \\
Of \\
S.MPL'S \\
\end{tabular}} & \multirow{2}{*}{\multicolumn{2}{|c|}{$\begin{array}{l}\text { WAVE } \\
\text { OSERVA TONS }\end{array}$}} & \multirow{3}{*}{\begin{tabular}{|c|c|} 
WEA- \\
THER \\
COOE \\
\end{tabular}} & \multirow{2}{*}{ 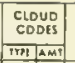 } & & & \multirow{2}{*}{$\begin{array}{l}\text { NODC } \\
\text { STATON } \\
\text { NUMBII }\end{array}$} \\
\hline \multirow{2}{*}{ 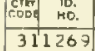 } & & & & & & & reak & \begin{tabular}{c|c} 
chuist & STAT \\
NO.
\end{tabular} & $\begin{array}{l}\text { ATION } \\
\text { UMIER }\end{array}$ & & & & & & & & & \\
\hline & \multirow[t]{4}{*}{ CK } & \multirow[t]{4}{*}{4403} & \multirow[t]{4}{*}{$\mathrm{N}$} & $04057 \mathrm{~W}$ & \begin{tabular}{l|l|}
149 & 40 \\
\end{tabular} & 0605 & 1291968 & \begin{tabular}{|l|l|}
019 & 006 \\
\end{tabular} & & 4709 & 14 & 24 & \begin{tabular}{l|l|l} 
& & 4
\end{tabular} & & \begin{tabular}{|l|l|}
6 & 0 \\
\end{tabular} & & & 0006 \\
\hline & & & & & WAT & \begin{tabular}{l|l|} 
TER & 1 \\
\end{tabular} & WINO & a. AlQ TIM\%. & 7.5 & 40. & & & & & & & & \\
\hline & & & & & $\begin{array}{l}\text { COLOR } \\
\text { COOE }\end{array}$ & 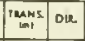 & $\begin{array}{l}\text { sher } \\
\text { ong } \\
\text { ronci }\end{array}$ & \begin{tabular}{c|c} 
Dar & w \\
OuL & Bu \\
\end{tabular} & 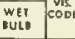 & 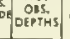 & ouserva & CALDNS & & & & & & \\
\hline & & & & & & 21 & 512 & 156 & \begin{tabular}{l|l}
133 & 7
\end{tabular} & 14 & & & & & & & & \\
\hline & 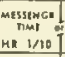 & $\begin{array}{l}\text { Lessi } \\
\text { I No. } \\
\end{array}$ & $\begin{array}{l}\text { CAlOo } \\
\text { IYYE }\end{array}$ & DERTH (m) & it & $s \%$ & SIGMA-1 & $\begin{array}{l}\text { Smeiric volum, } \\
\text { AMOMALY }\end{array}$ & 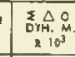 & $\begin{array}{l}\text { sou } \\
\text { velo }\end{array}$ & & $02 \mathrm{~m} / \pi$ & $\left|\begin{array}{l}0 \\
n 0, \cdots+1\end{array}\right|$ & $\left|\begin{array}{c}\text { POTAL- } \\
n \cdots \cdots\end{array}\right|$ & $\begin{array}{l}\mathrm{NO}_{2}-\mathrm{N} \\
\mathrm{NO}_{0}=\mathrm{a} / 1\end{array}$ & $\begin{array}{l}N_{3}-N \\
N D_{0}=-N=1\end{array}$ & $\begin{array}{l}510_{4}-5 \\
28-01 / 1\end{array}$ & $\mathrm{HH}$ \\
\hline & 129 & & $\begin{array}{l}510 \\
085\end{array}$ & $\begin{array}{l}0000 \\
0000\end{array}$ & $\begin{array}{l}1546 \\
1546\end{array}$ & $\begin{array}{l}3600 \\
36002\end{array}$ & 2660 & 0013907 & 0000 & 151 & & & & & & & & \\
\hline & 129 & & STO & 0010 & $\begin{array}{l}1546 \\
1528\end{array}$ & $\begin{array}{l}36002 \\
3600\end{array}$ & $\begin{array}{l}2066 \\
2670\end{array}$ & 0013540 & 0014 & $\begin{array}{l}151 \\
150\end{array}$ & 01 & & & & & & & \\
\hline & & & STO & 0020 & 1514 & 3601 & 2673 & 0013259 & 0027 & 150 & 96 & & & & & & & \\
\hline & 129 & & 085 & 0324 & 2510 & 36007 & 2674 & & & 150 & 93 & & & & & & & \\
\hline & & & $5 T 0$ & 0030 & 1508 & 3002 & 2676 & 0013001 & 0040 & 150 & 194 & & & & & & & \\
\hline & 129 & & Des & 0048 & 1498 & 36038 & 2679 & & & 150 & 94 & & & & & & & \\
\hline & & & STD & 0050 & 1496 & 3603 & 2679 & 0012790 & 0066 & 150 & 194 & & & & & & & \\
\hline & 129 & & DBS & 0071 & 1480 & $\begin{array}{l}36007 \\
3600\end{array}$ & 2681 & & & 150 & 92 & & & & & & & \\
\hline & & & $\begin{array}{l}\text { STO } \\
\text { OBS }\end{array}$ & $\begin{array}{l}0075 \\
0096\end{array}$ & $\begin{array}{l}1480 \\
1473\end{array}$ & $\begin{array}{l}3600 \\
35990\end{array}$ & $\begin{array}{l}2681 \\
2681\end{array}$ & 0012727 & 0098 & $\begin{array}{l}150 \\
150\end{array}$ & $\begin{array}{l}92 \\
93\end{array}$ & & & & & & & \\
\hline & 129 & & $\begin{array}{l}\text { DES } \\
\text { STO }\end{array}$ & $\begin{array}{l}0096 \\
0100\end{array}$ & $\begin{array}{l}1473 \\
1469\end{array}$ & 3598 & 2681 & 0012748 & 0130 & 150 & 193 & & & & & & & \\
\hline & & & 510 & 0125 & 1440 & 3593 & 2684 & 0012585 & 0162 & 150 & & & & & & & & \\
\hline & 129 & & 085 & 0142 & 1419 & 35893 & 2685 & & & 1508 & 182 & & & & & & & \\
\hline & & & STO & 0150 & 1407 & 3587 & 2680 & 0012419 & 0193 & $150^{7}$ & & & & & & & & \\
\hline & 129 & & 085 & 10189 & 1352 & 35782 & 2691 & & & 1500 & & & & & & & & \\
\hline & & & 510 & 0200 & 1343 & 3577 & 2692 & 0012003 & 0254 & 1500 & & & & & & & & \\
\hline & & & 510 & 0250 & 1294 & 3569 & 2696 & 0011760 & 0313 & 1505 & 56 & & & & & & & \\
\hline & 129 & & 085 & 0284 & 1254 & 35622 & 2698 & & & 150 & & & & & & & & \\
\hline & & & 510 & 0300 & 1228 & 3558 & 2700 & 0011426 & 0371 & 1504 & & & & & & & & \\
\hline & 129 & & OBS & $\mathrm{T} 0382$ & 1107 & 35399 & 2709 & & & 1501 & & & & & & & & \\
\hline & & & SIO & 0400 & 1089 & 3538 & 2711 & 0010576 & 0481 & 1500 & & & & & & & & \\
\hline & 129 & & 085 & 0472 & 0992 & 35275 & 2720 & & & 1498 & & & & & & & & \\
\hline & & & STO & 0500 & 0935 & 3522 & 2725 & 0009318 & 0581 & 1490 & & & & & & & & \\
\hline & 127 & & 085 & 10561 & 0820 & 35121 & 2736 & & & 1493 & & & & & & & & \\
\hline & & & 510 & 0600 & 0749 & 3510 & 2745 & 0007457 & 0665 & 1491 & & & & & & & & \\
\hline & & & $5 T 0$ & 0700 & 0606 & 3504 & 2760 & 0006006 & 0732 & 1486 & & & & & & & & \\
\hline & 129 & & 085 & 0756 & 0552 & 35016 & 2765 & & & 1485 & & & & & & & & \\
\hline & & & STO & 0800 & 0541 & 3500 & 2765 & 0005559 & 0790 & 1485 & & & & & & & & \\
\hline & & & STO & 0900 & 0516 & 3497 & 2765 & 0005572 & 0845 & 1486 & & & & & & & & \\
\hline & 129 & & 085 & 10952 & 0504 & 34958 & 2766 & & & 1486 & & & & & & & & \\
\hline & & & sro & 1000 & 0493 & 3496 & 2767 & 0005459 & 0901 & 1487 & & & & & & & & \\
\hline & & & STO & 1100 & 0471 & 3496 & 2770 & 0005258 & 0954 & 1468 & & & & & & & & \\
\hline & & & sto & 1200 & 0450 & 3457 & 2773 & 0005066 & 1006 & 1488 & & & & & & & & \\
\hline & & & STD & 1300 & 0430 & 3457 & 2775 & 0004874 & 1055 & 1489 & & & & & & & & \\
\hline & & & SIO & 1400 & 0411 & 3497 & 2777 & 0004698 & 1103 & 1490 & & & & & & & & \\
\hline & 129 & & OBS & 11430 & 0406 & 34974 & 2778 & & & 1490 & & & & & & & & \\
\hline
\end{tabular}

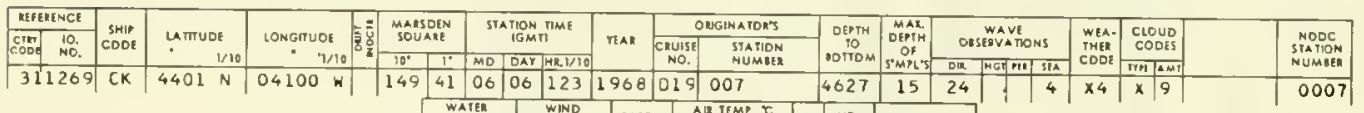

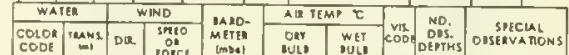

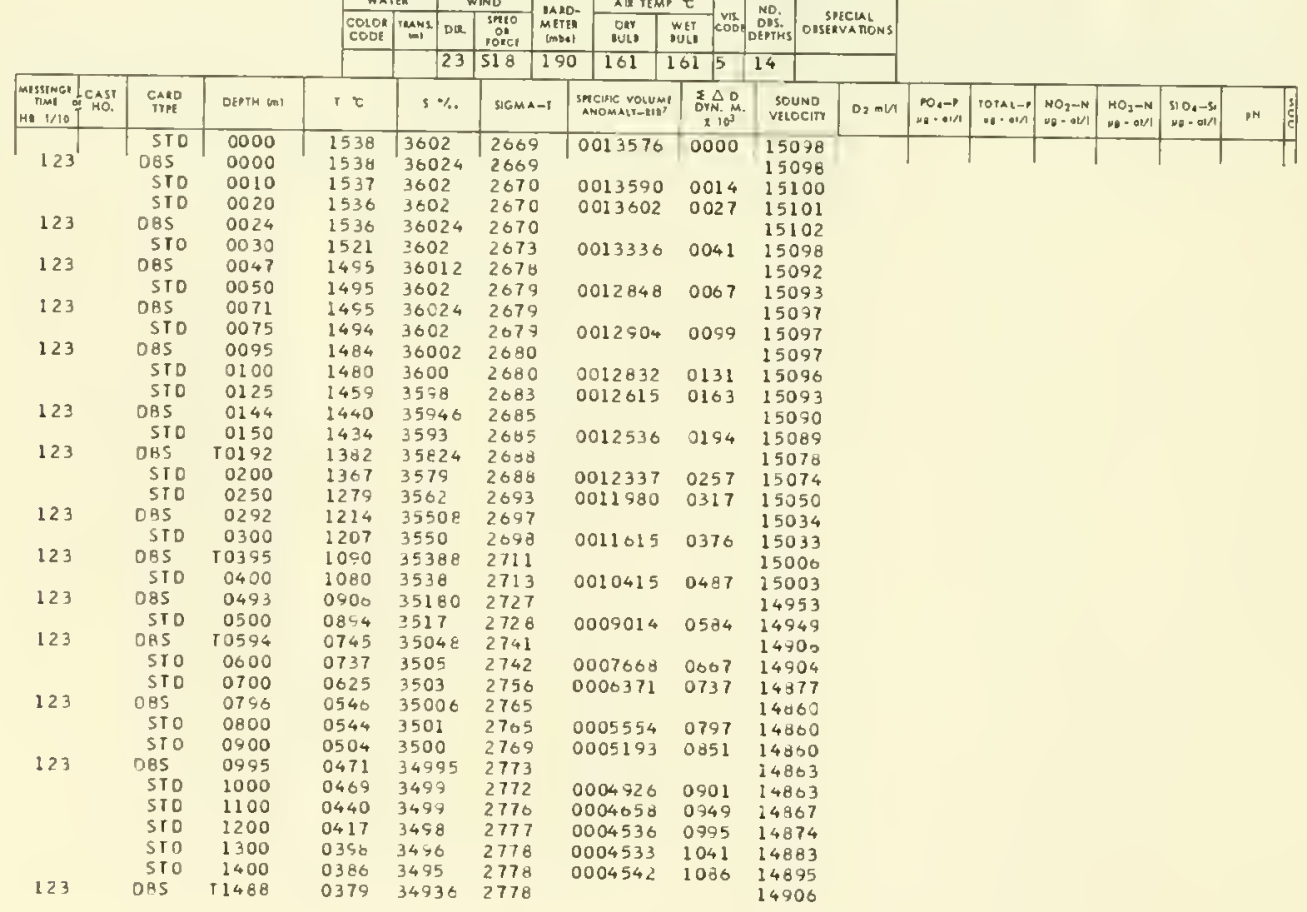




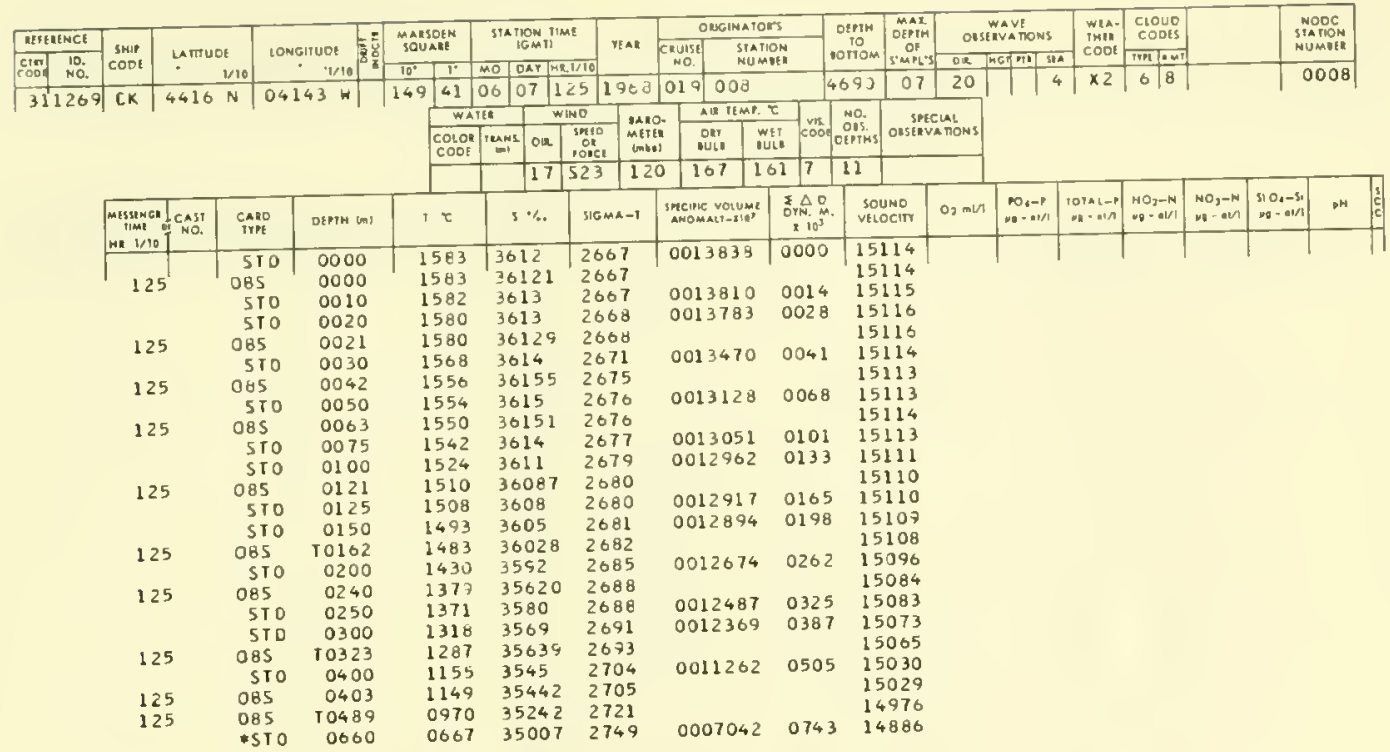

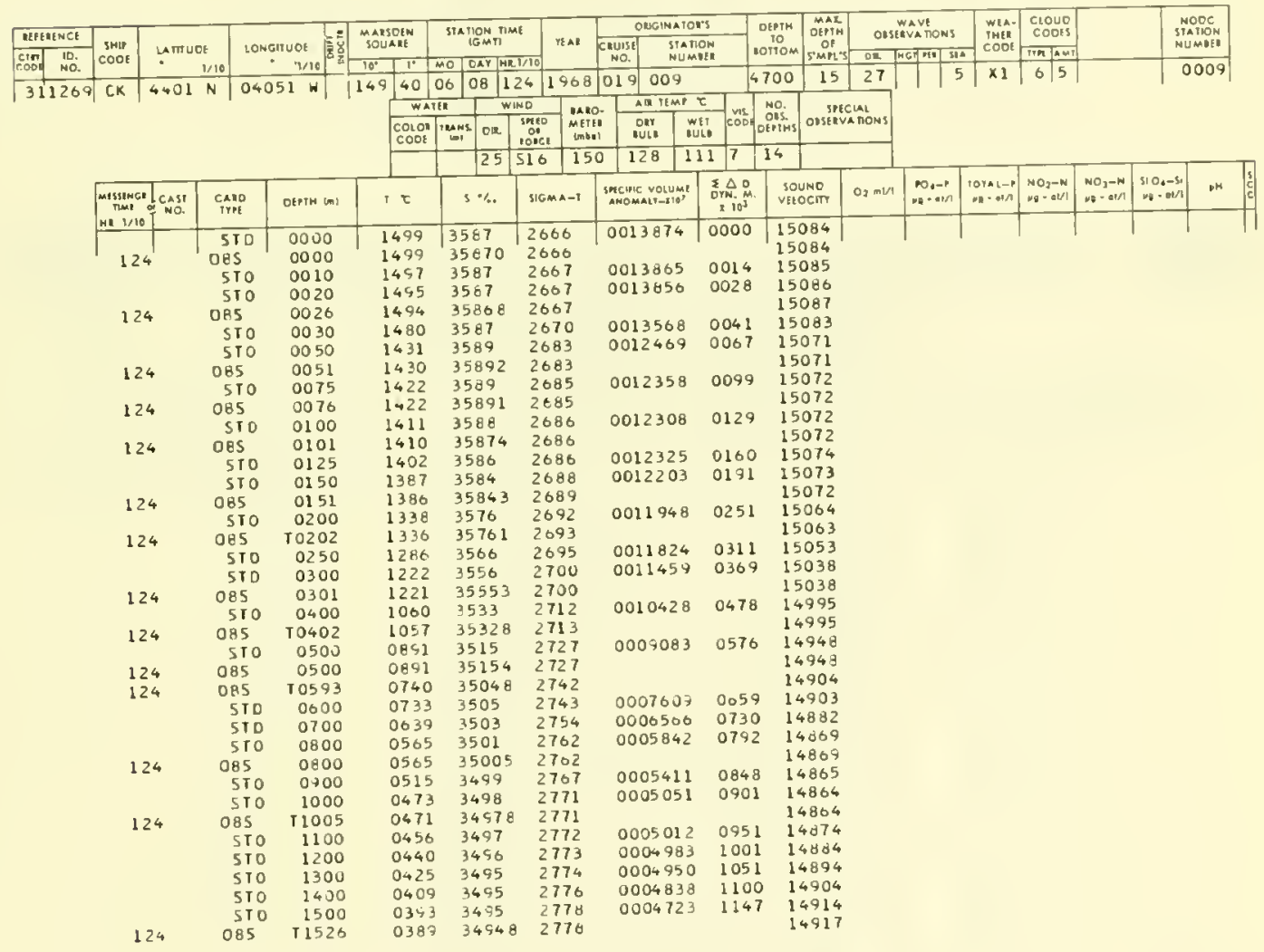


TABLE X.-Continued

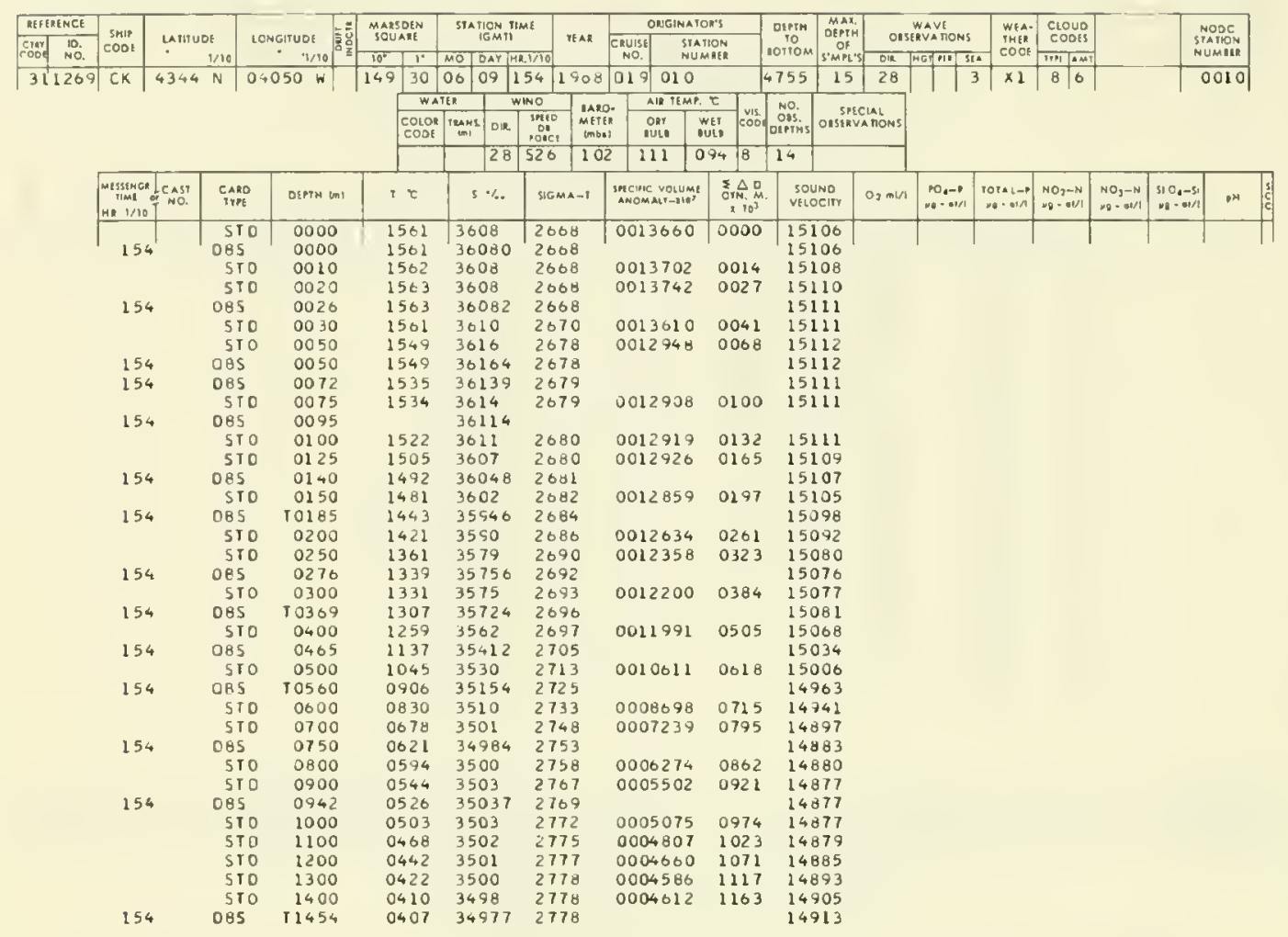

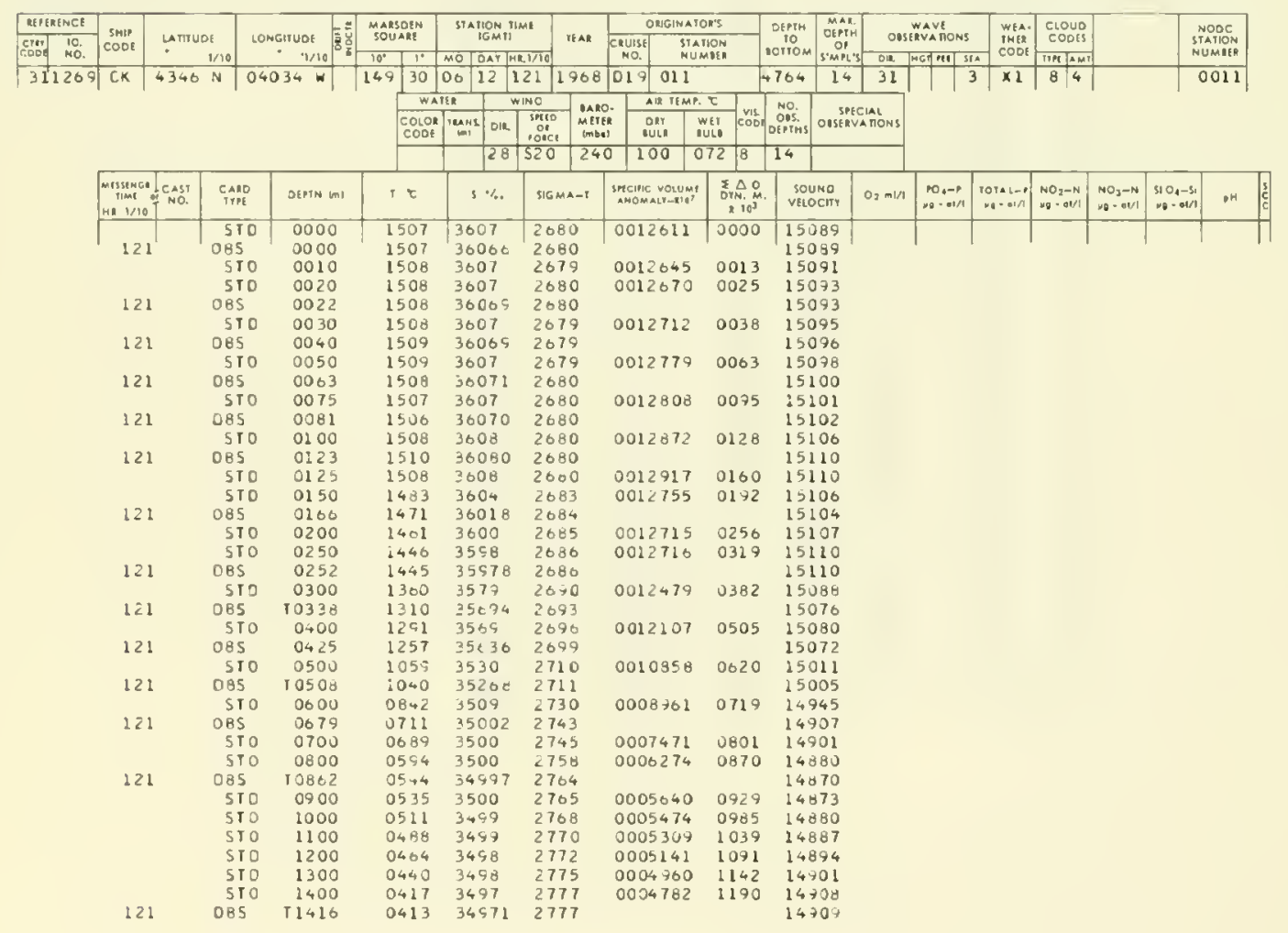




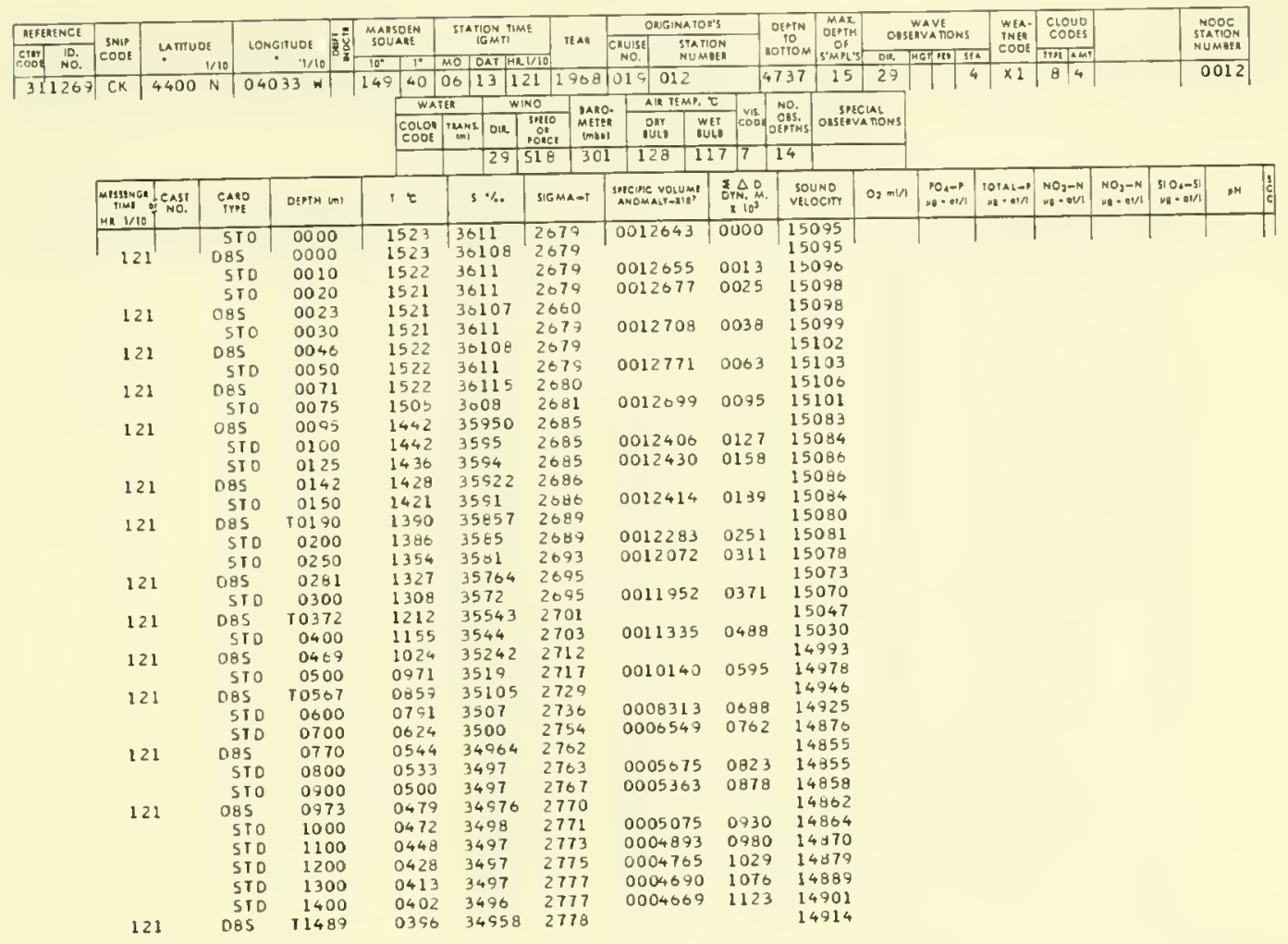

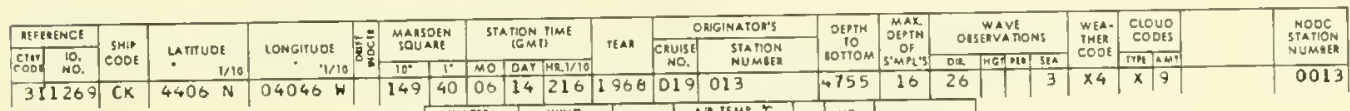

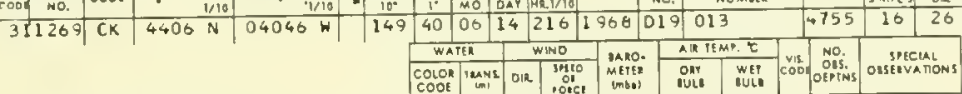

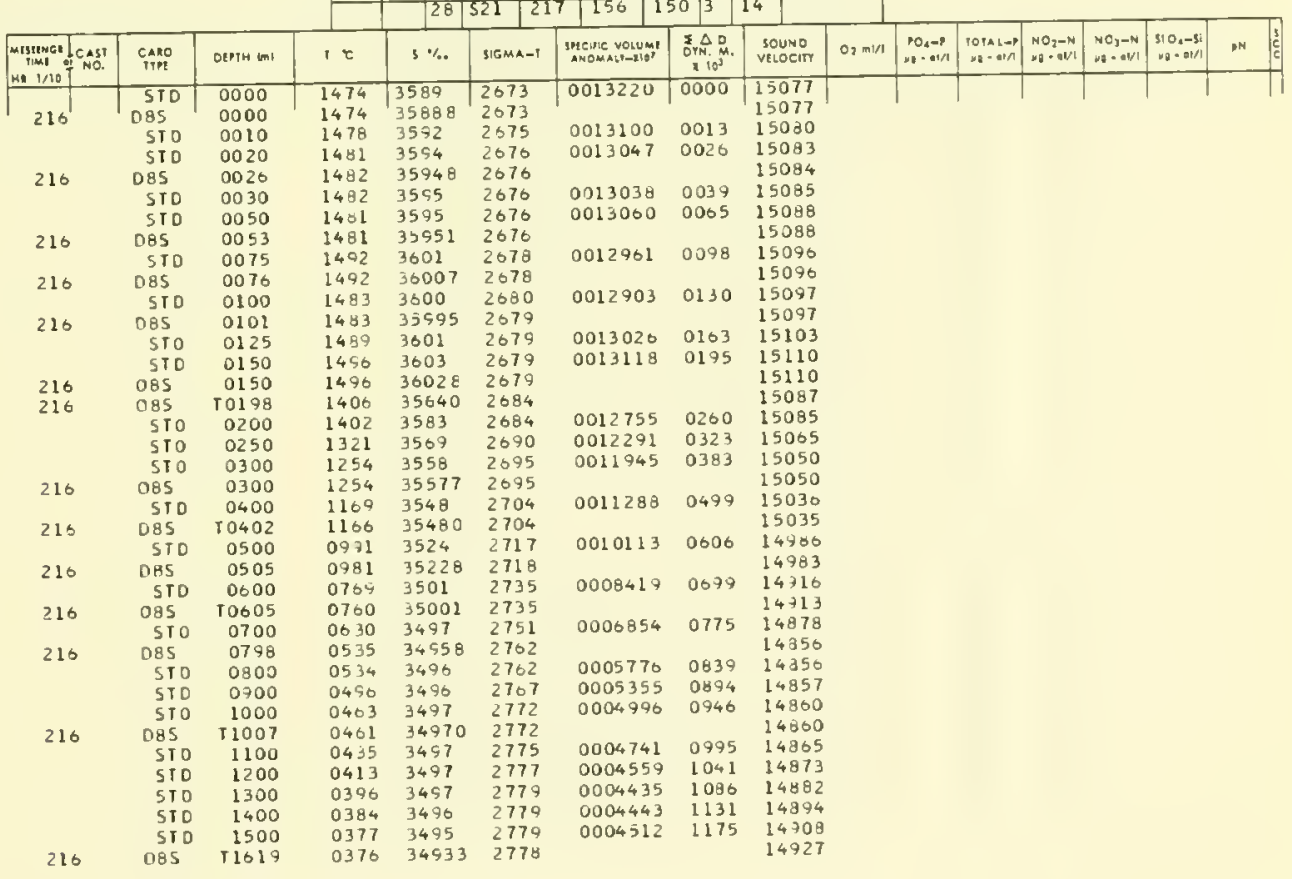


TABLE X-Continued

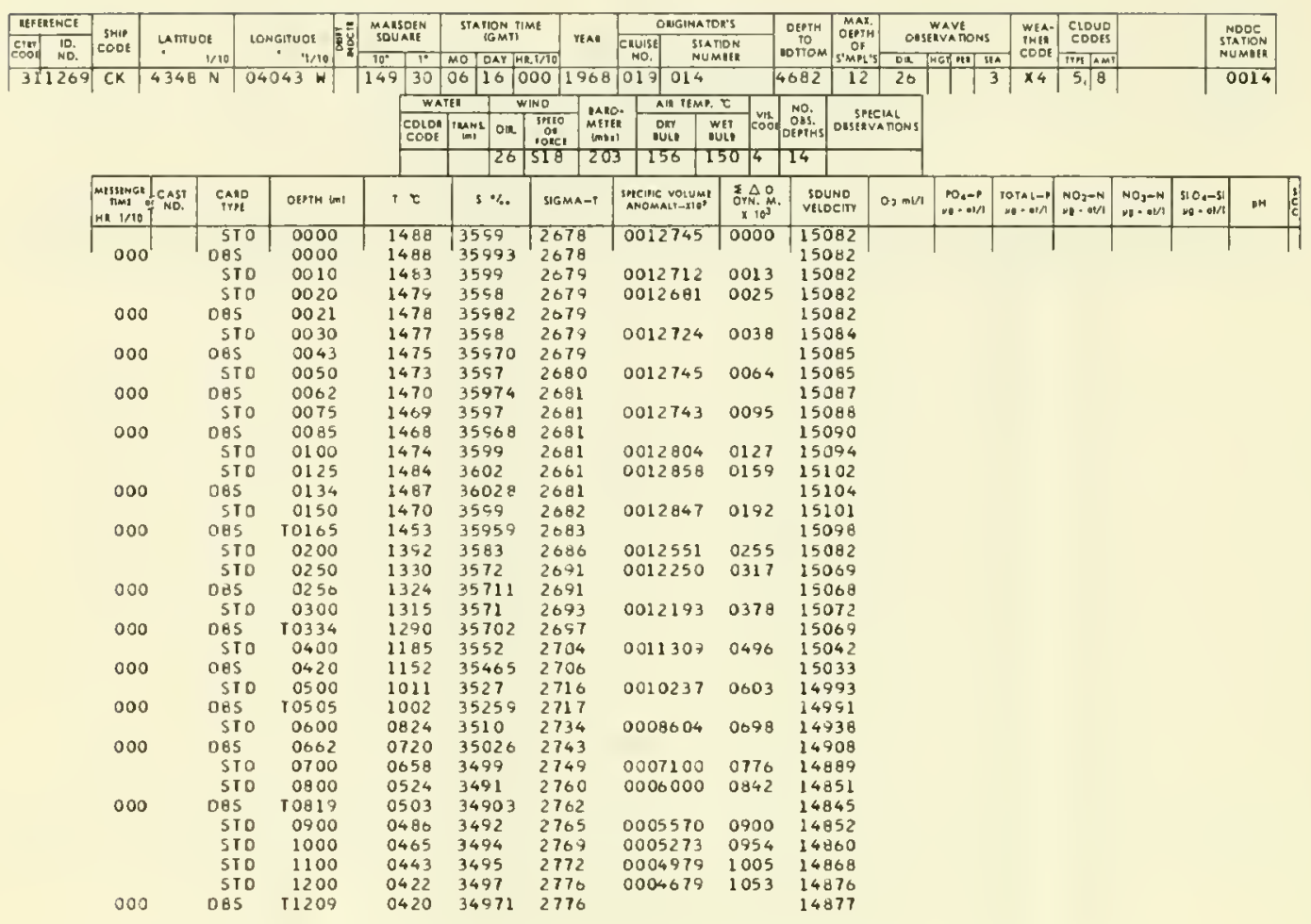

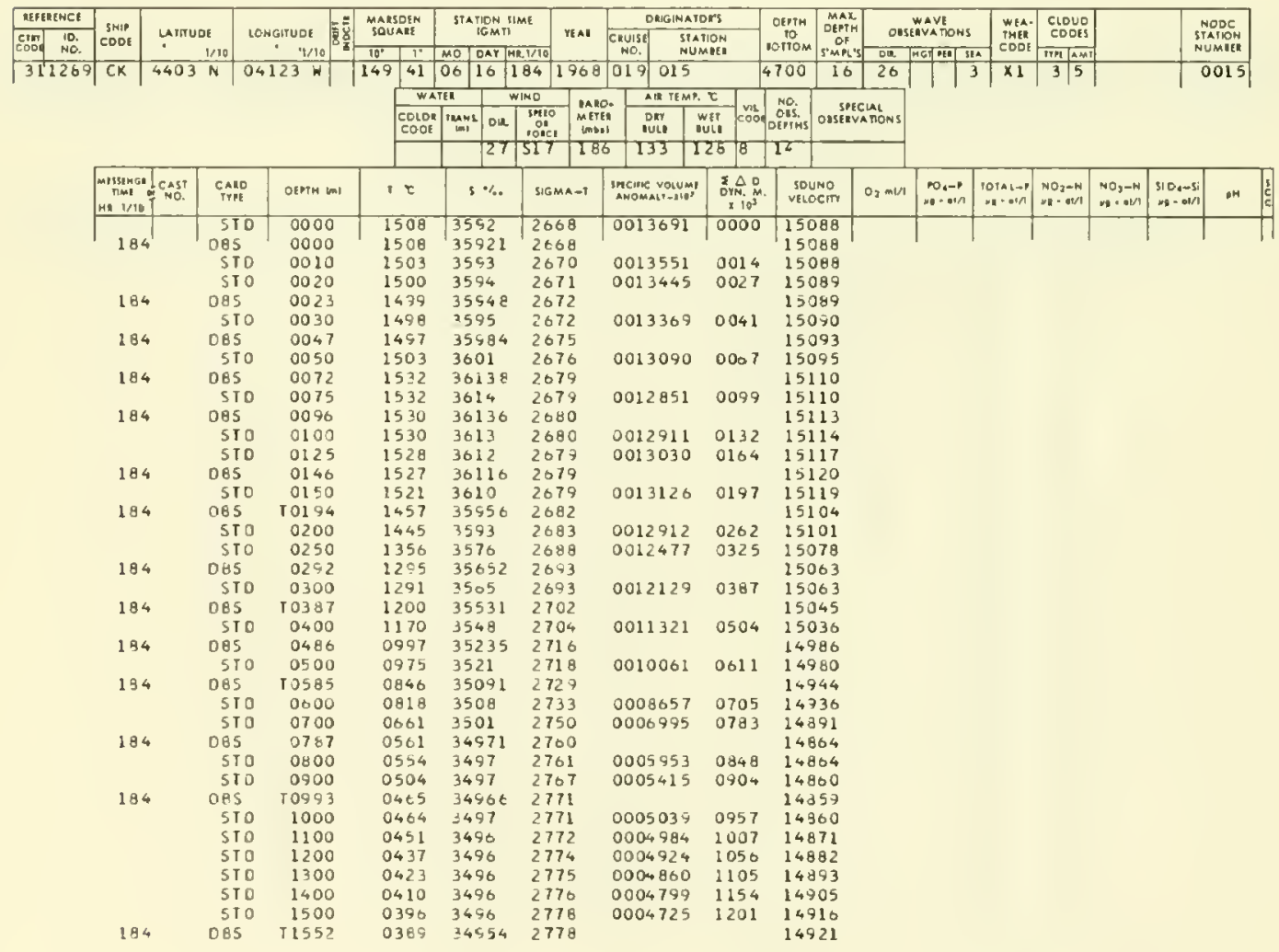




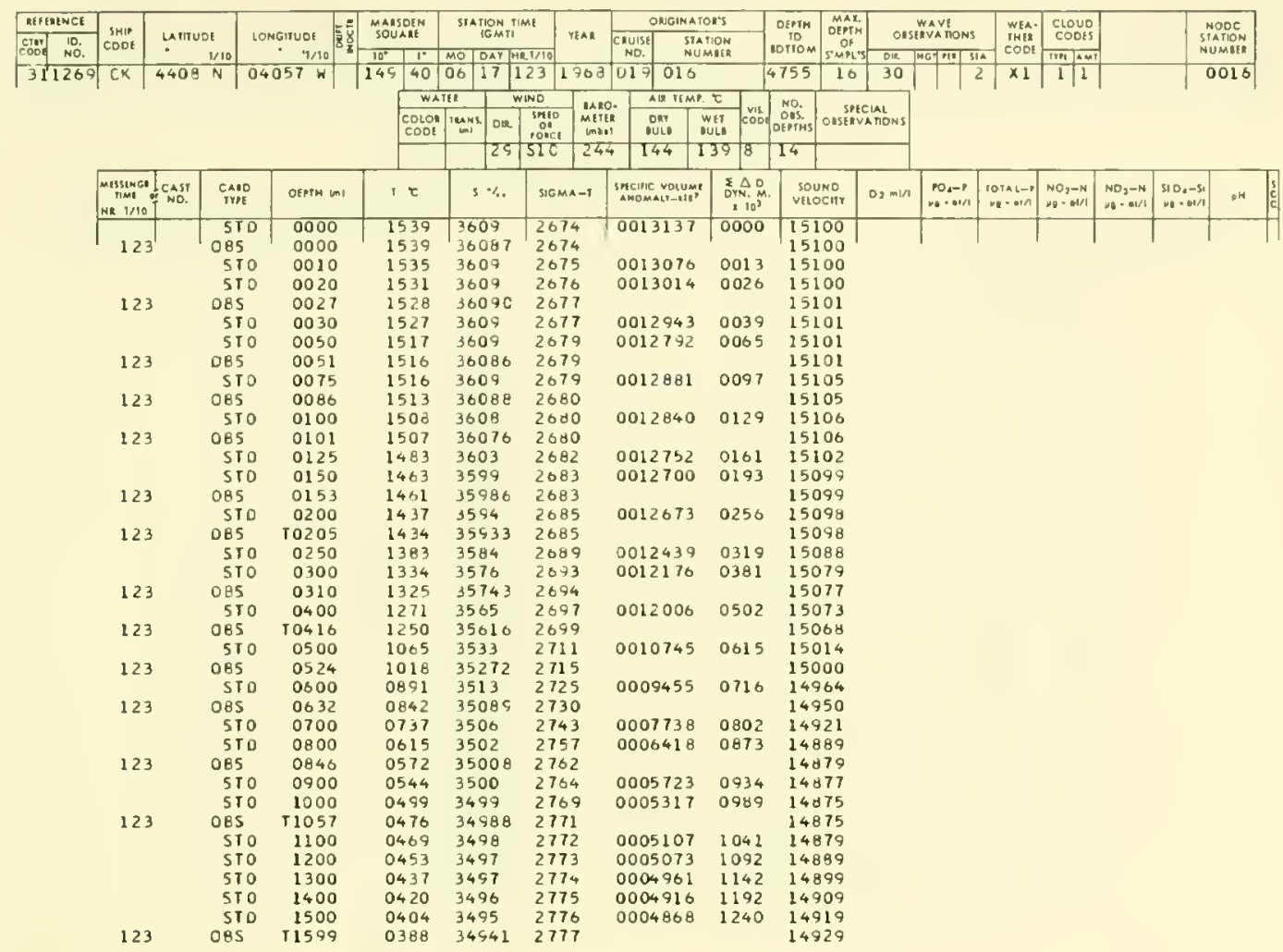

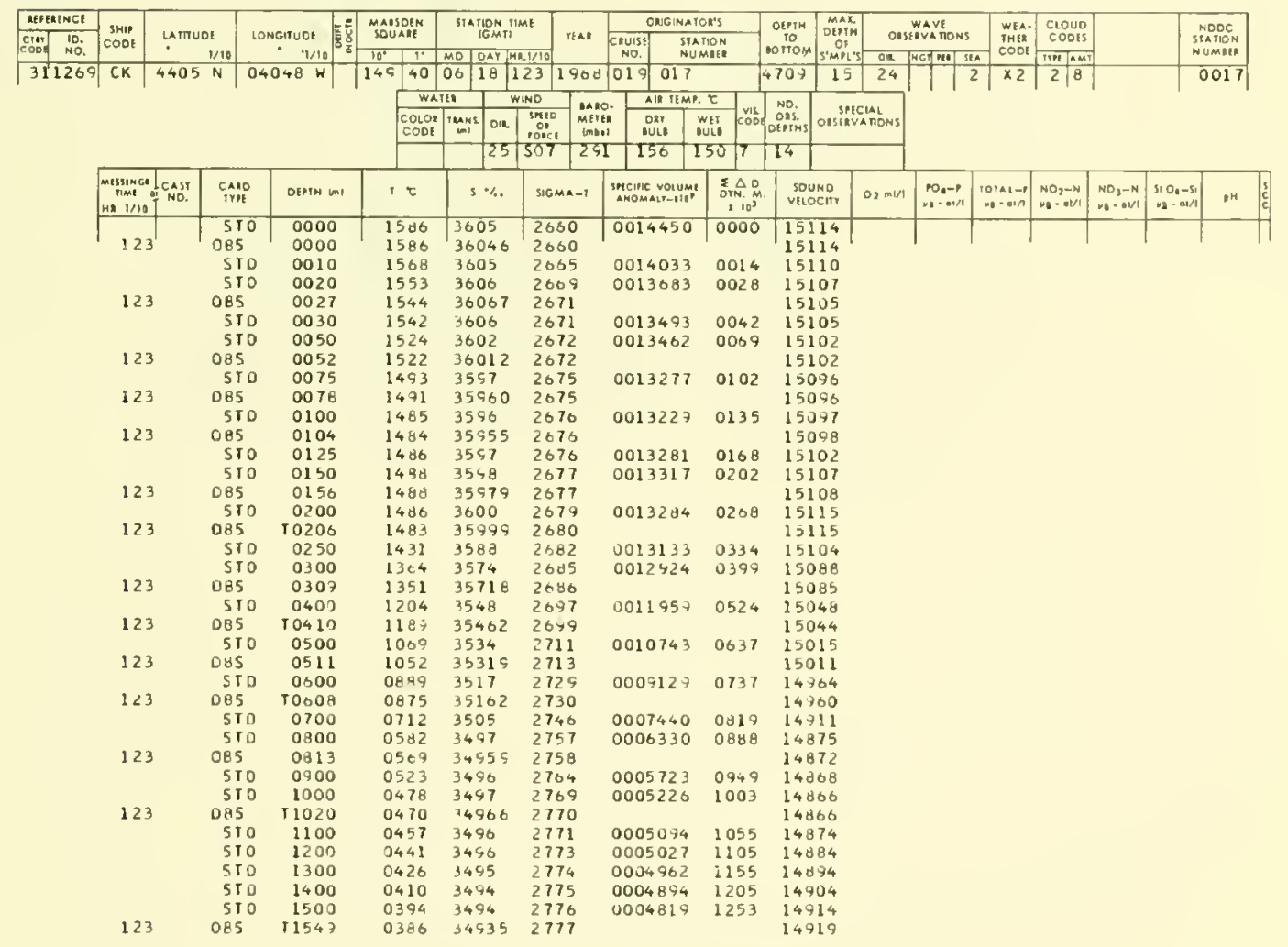


TABLE X.-Continued

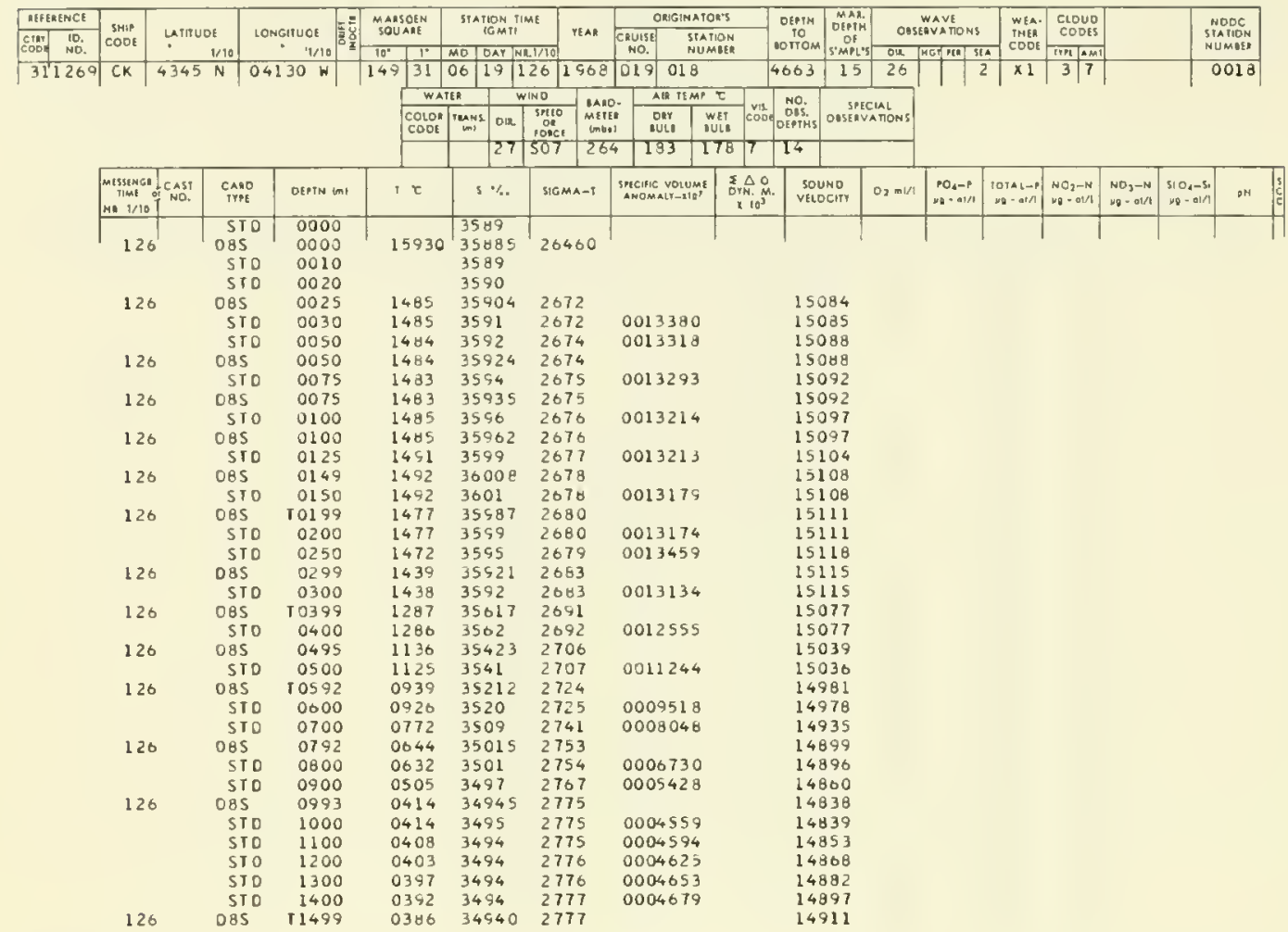


TABLE XI.-Observed and interpolated oceanographic data for stations taken by USCGC HAMILTON at Ocean Station DELTA, 6 July-9 July 1968, prepared from NODC listing No. 31-8042 HT.

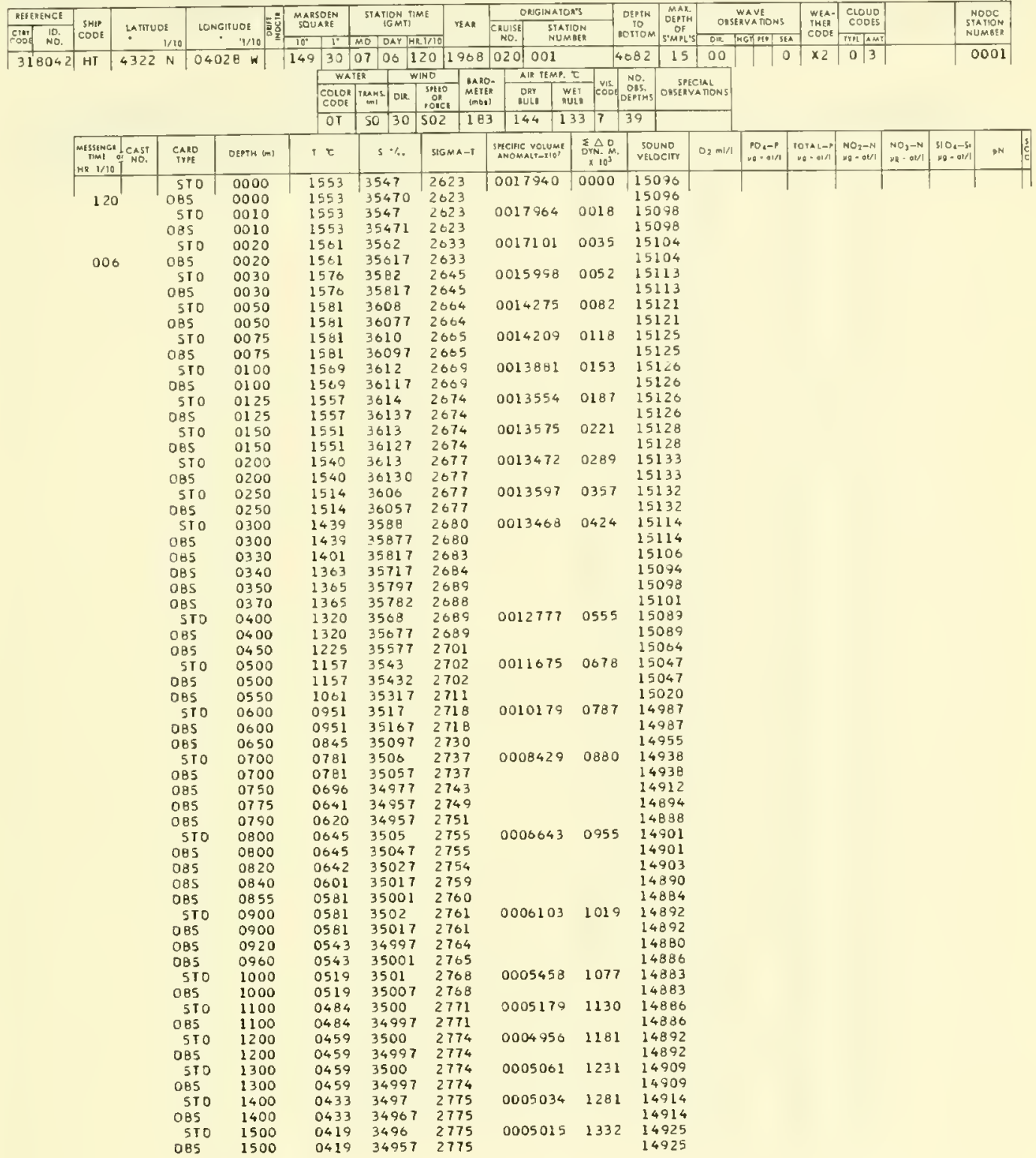


TABLE XI.-Continued

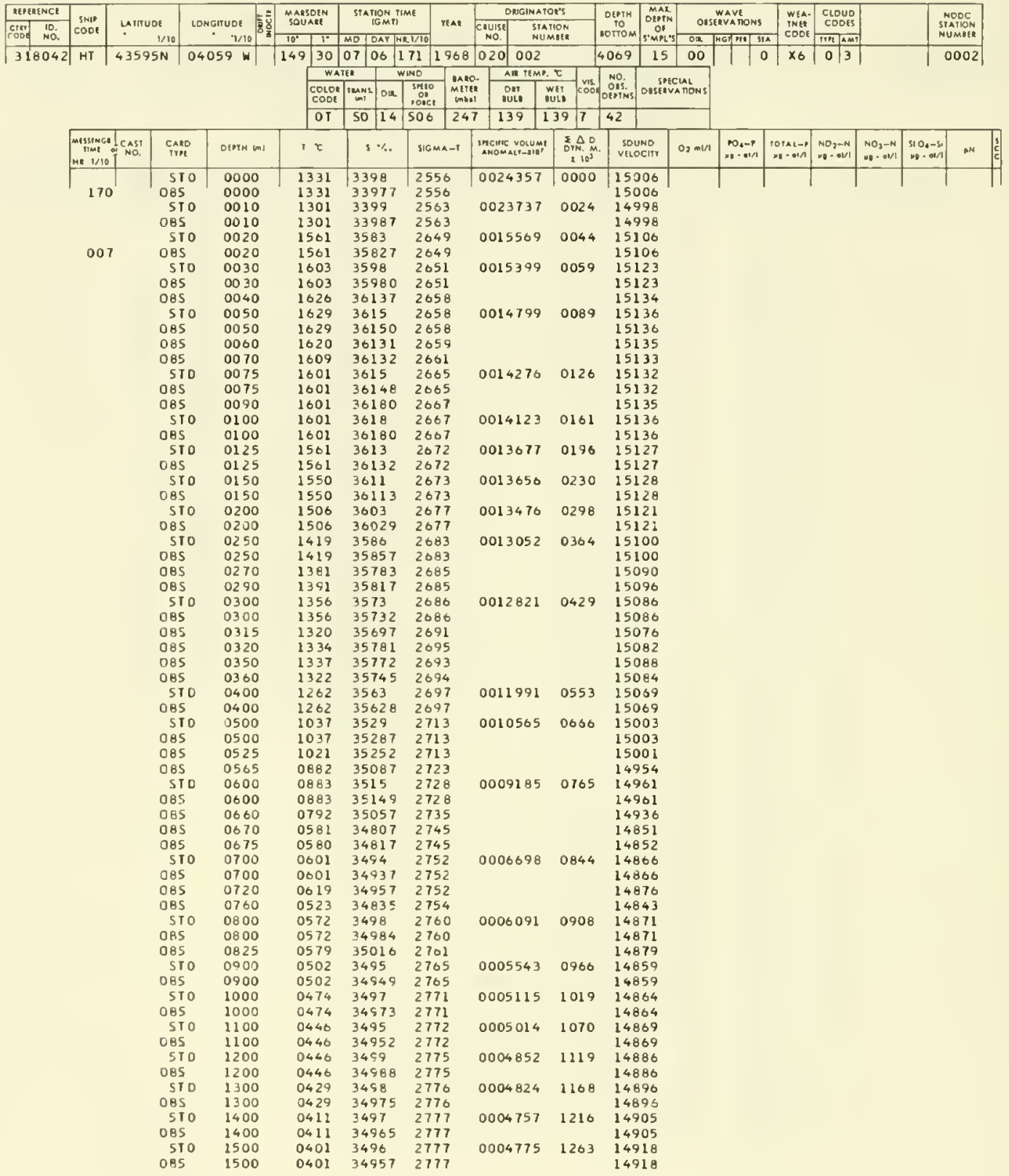


TABLE XI-_Continued

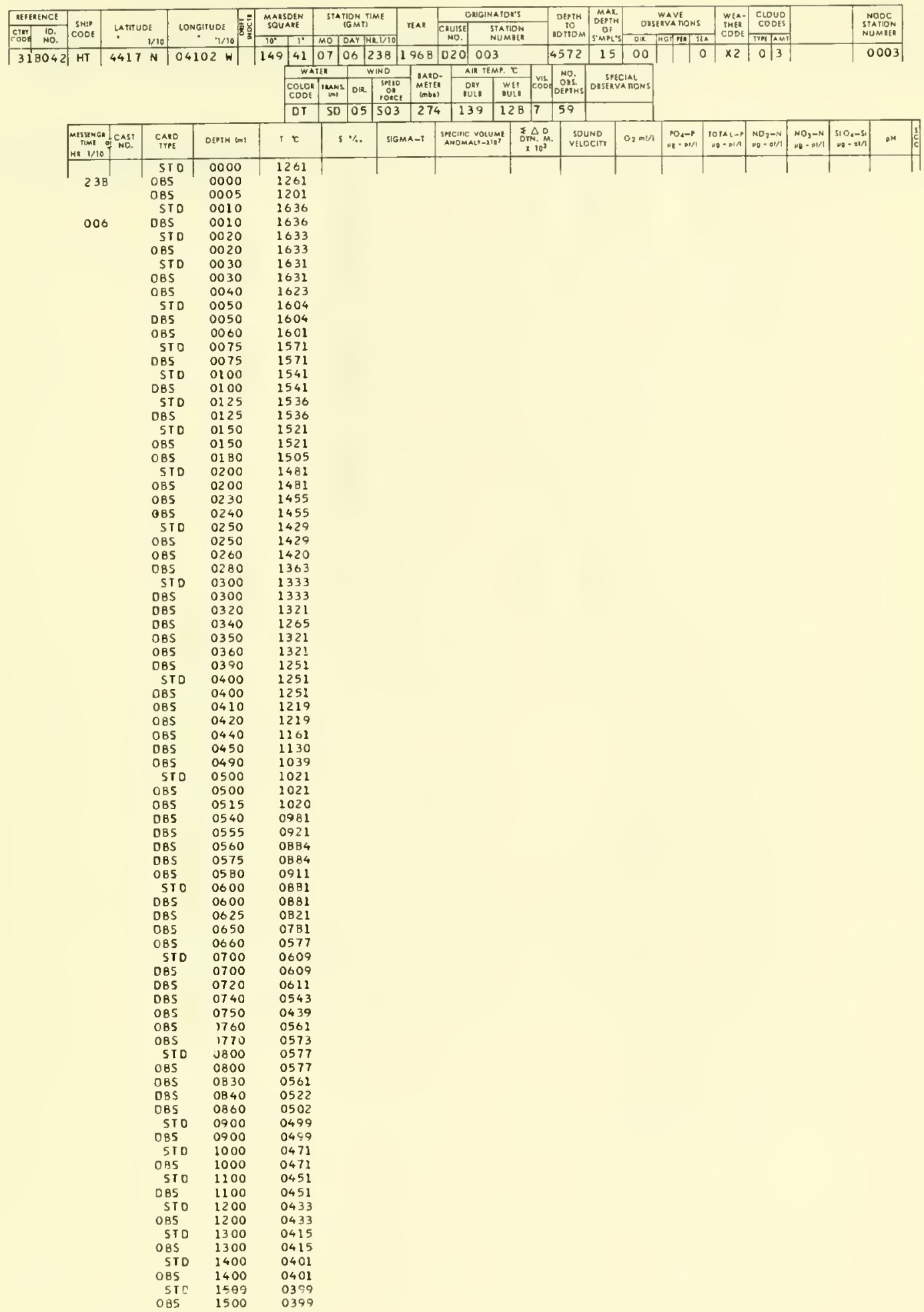


TABle XI-_Continued

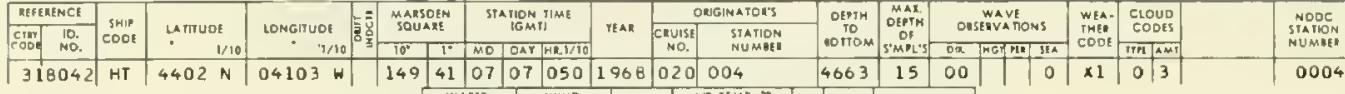

\begin{tabular}{|c|c|c|c|c|c|c|c|c|c|}
\hline \multicolumn{2}{|c|}{ WATER } & \multicolumn{2}{|c|}{ WIND } & \multirow{2}{*}{ 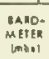 } & \multicolumn{2}{|c|}{ AIR TEMV $\tau$} & \multirow{2}{*}{ vis } & \multirow{2}{*}{$\begin{array}{l}\text { no. } \\
\text { ons. } \\
\text { oirtims }\end{array}$} & \multirow{2}{*}{$\begin{array}{c}\text { Specul } \\
\text { ouseavanow }\end{array}$} \\
\hline $\begin{array}{l}\text { COLOA } \\
\text { coos }\end{array}$ & 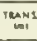 & on. & 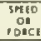 & & $\begin{array}{l}\text { ONy } \\
\text { nuts }\end{array}$ & $\begin{array}{l}\text { WIT } \\
\text { BULI }\end{array}$ & & & \\
\hline DT & 50 & 06 & 508 & 281 & 144 & 133 & & 49 & \\
\hline
\end{tabular}

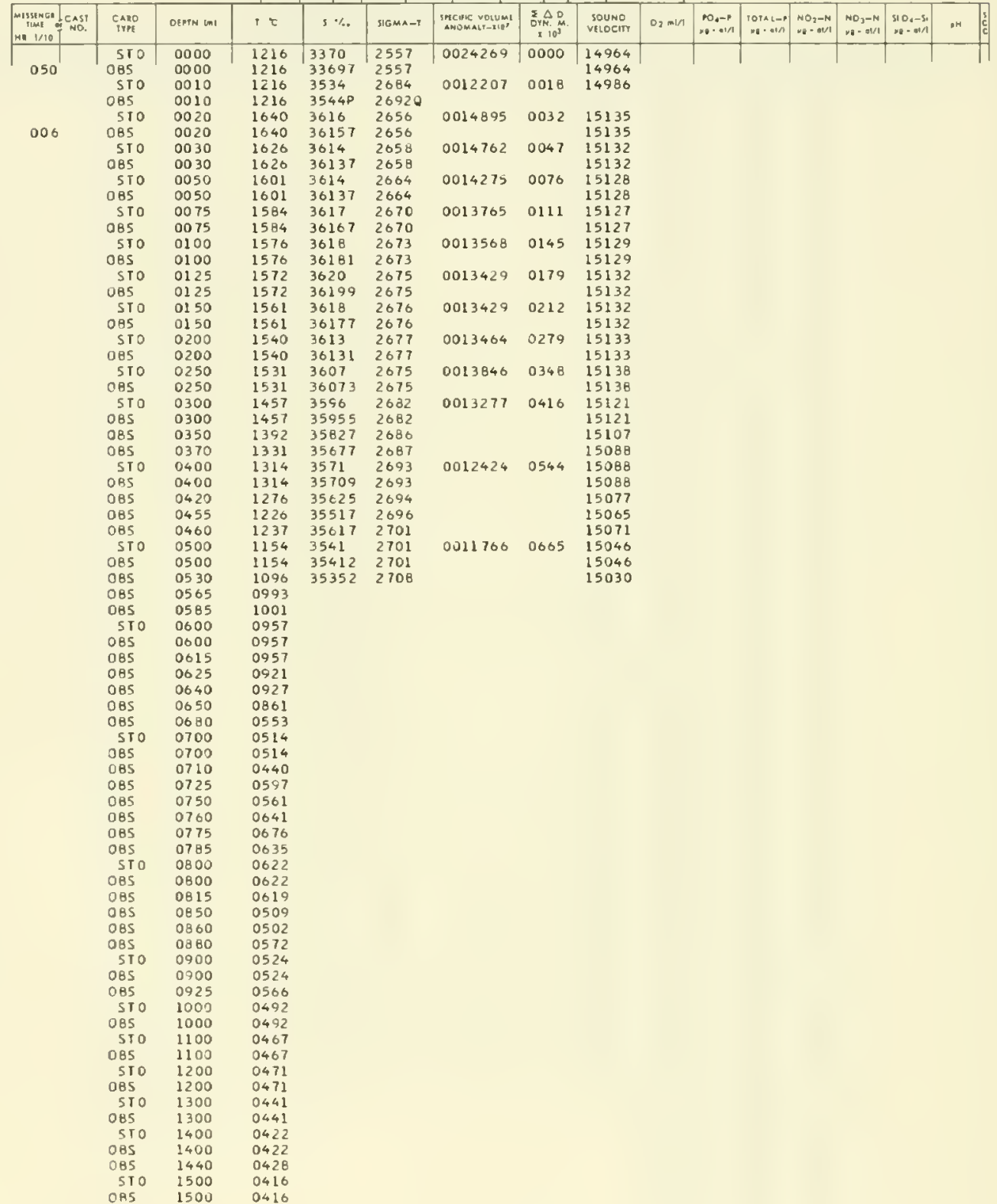


TABLE XI.-Continued

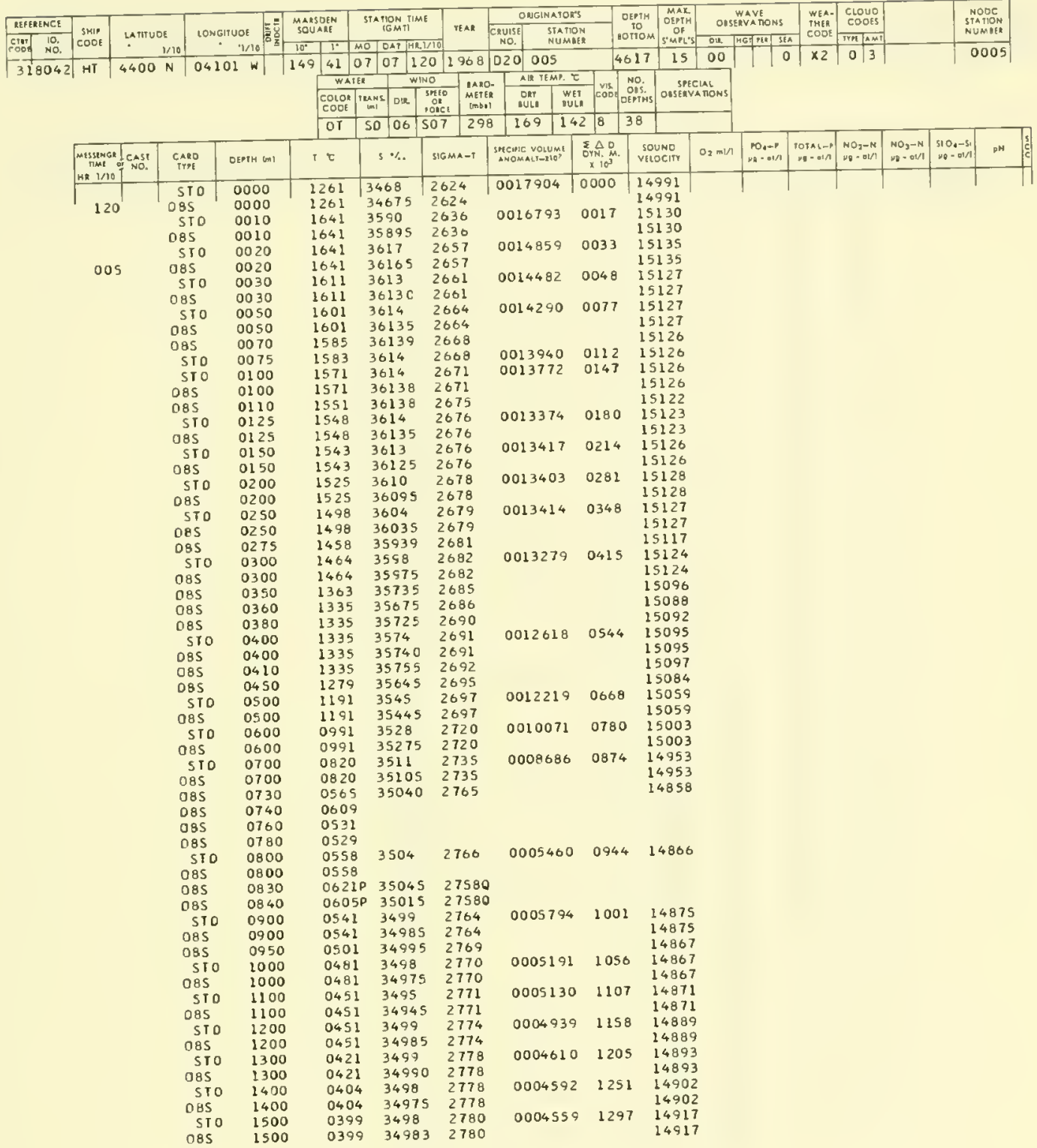


TABLE XI.-Continued

\begin{tabular}{|c|c|c|c|c|c|c|c|c|c|c|c|c|c|c|c|c|c|}
\hline \multirow{2}{*}{ 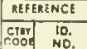 } & \multirow{2}{*}{$\begin{array}{l}\text { SNIP } \\
\text { CODE }\end{array}$} & \multirow{2}{*}{$\begin{array}{l}\text { Lamude } \\
\quad \quad 1 / 10 \\
\end{array}$} & \multirow{2}{*}{$\begin{array}{c}\text { IONGIFUDR } \\
\cdot 1 / 10\end{array}$} & \multirow{2}{*}{\multicolumn{2}{|c|}{$\begin{array}{l}\text { MAASOEM } \\
\text { SOUAIE }\end{array}$}} & \multicolumn{3}{|c|}{$\begin{array}{l}\text { STATION TIME } \\
\text { TGMTI }\end{array}$} & \multirow[b]{2}{*}{ YEAR } & \multicolumn{2}{|c|}{ ONGINATOR'S } & \multirow{2}{*}{\begin{tabular}{|c|} 
OEPTN \\
TO \\
DOTTOM
\end{tabular}} & \multirow{2}{*}{\begin{tabular}{|l|}
$\operatorname{MAX}$ \\
OEPTM \\
Of \\
S'MPL'S
\end{tabular}} & \multirow{2}{*}{$\begin{array}{c}\text { WAVE } \\
\text { OESEAVAIOKS }\end{array}$} & \multirow{2}{*}{$\begin{array}{c}\text { WEA } \\
\text { WHAR } \\
\text { COOE }\end{array}$} & \multirow{2}{*}{$\begin{array}{l}\text { clouo } \\
\text { costs } \\
\text { TWTAMT }\end{array}$} & \multirow[b]{2}{*}{$\begin{array}{l}\text { NOOC } \\
\text { SPATION } \\
\text { NUMBER }\end{array}$} \\
\hline & & & & & & & OAY & (1) & & $\begin{array}{c}\text { CAuise } \\
\text { NO. }\end{array}$ & $\begin{array}{l}\text { STATION } \\
\text { WUMBER } \\
\end{array}$ & & & & & & \\
\hline 318042 & HT & $4352 \mathrm{~N}$ & $04101 \mathrm{~W}$ & 149 & 31 & 07 & 07 & 177 & 1968 & 020 & 006 & 4572 & 15 & 0310,1 & $x 1$ & \begin{tabular}{|l|l|}
0 & 3 \\
\end{tabular} & 0006 \\
\hline
\end{tabular}

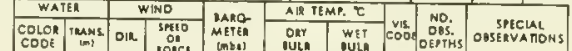

\begin{tabular}{|l|l|l|l|l|l|l|l|l|}
\hline OT & 50 & 07 & 505 & 305 & 161 & 144 & 7 & 39 \\
\hline
\end{tabular}

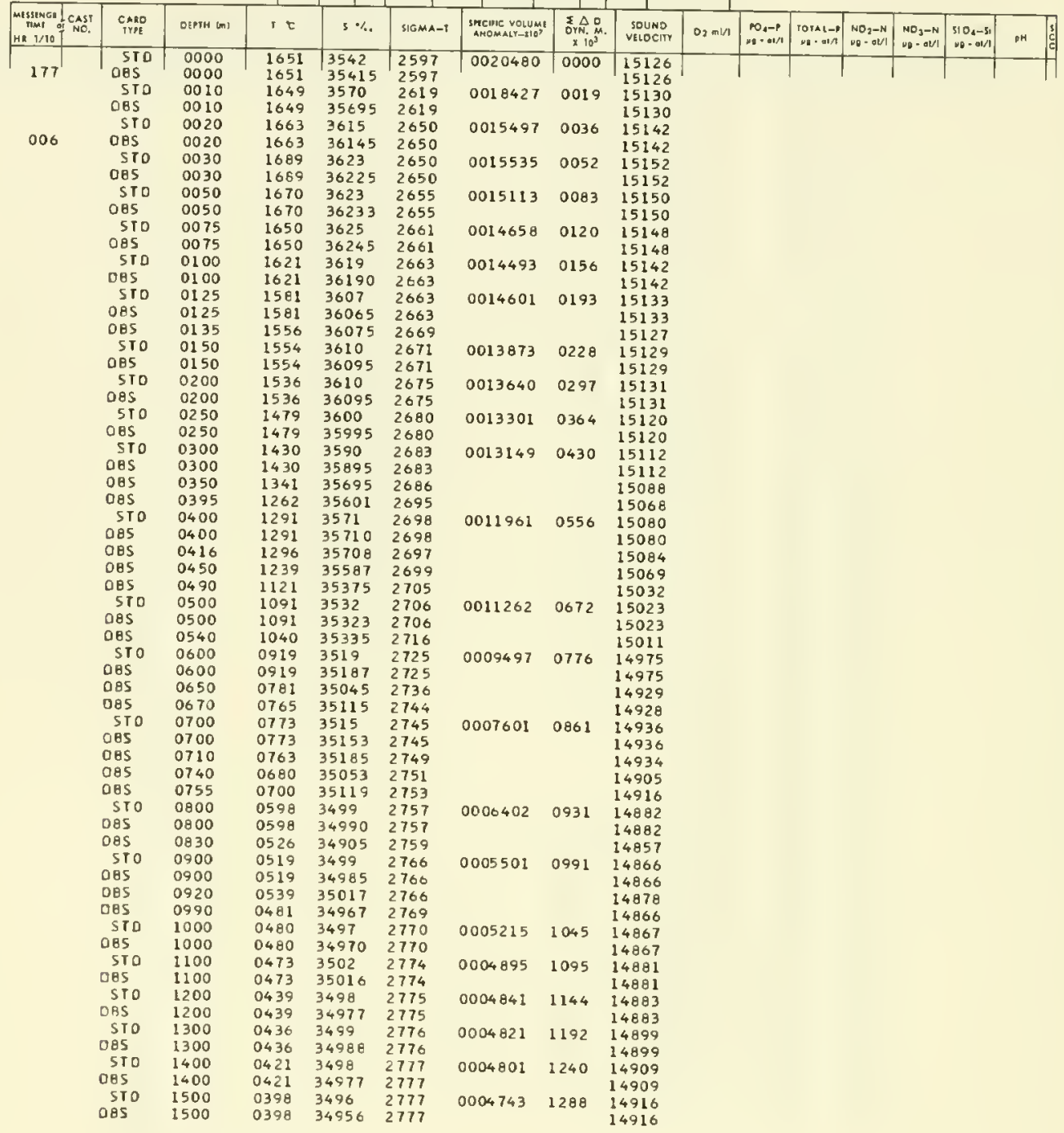


TABLE XI.-Continued

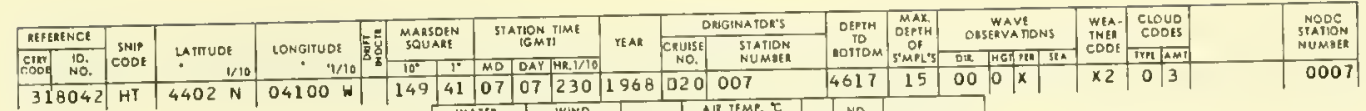

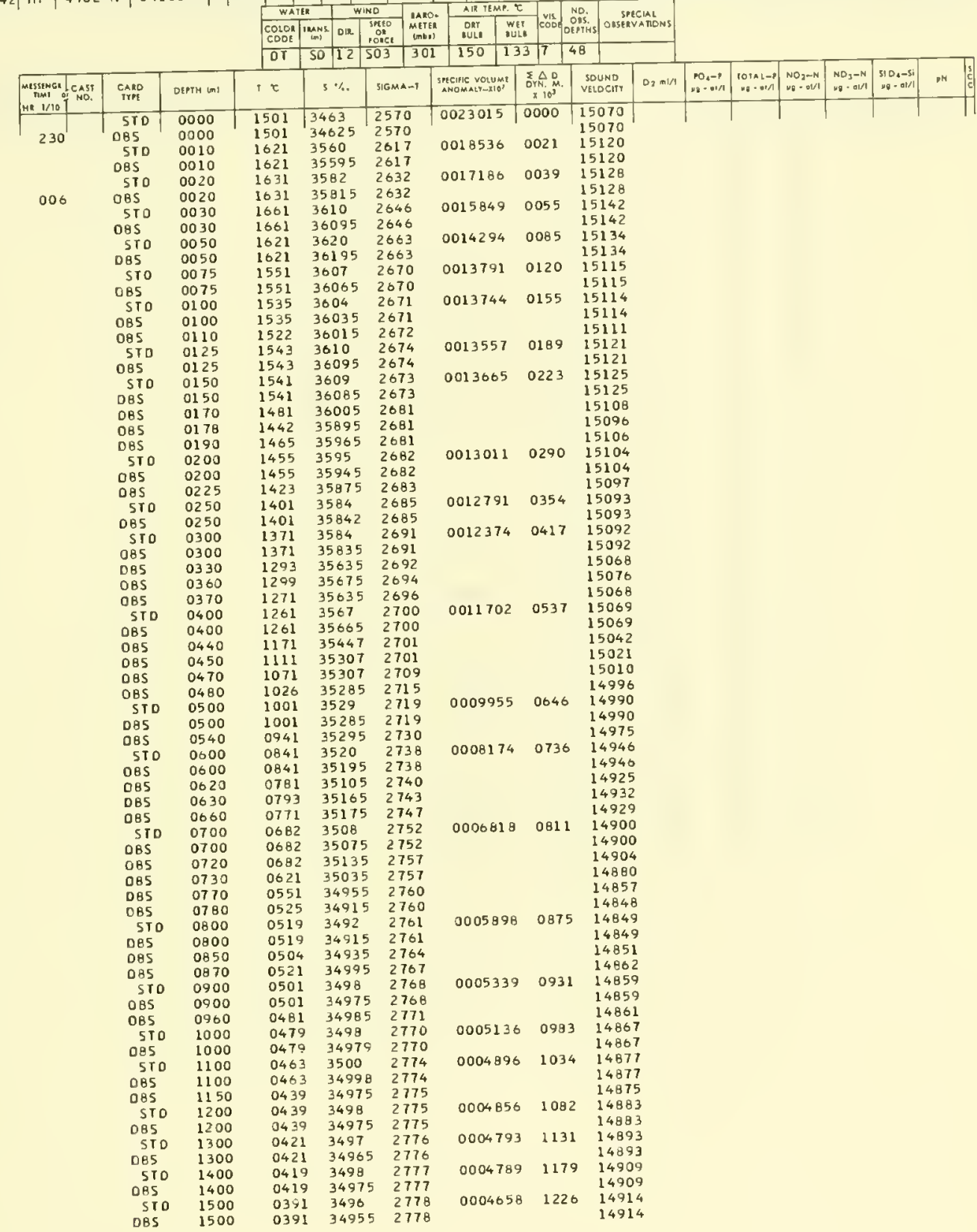




\section{TABLE XI.—Continued}

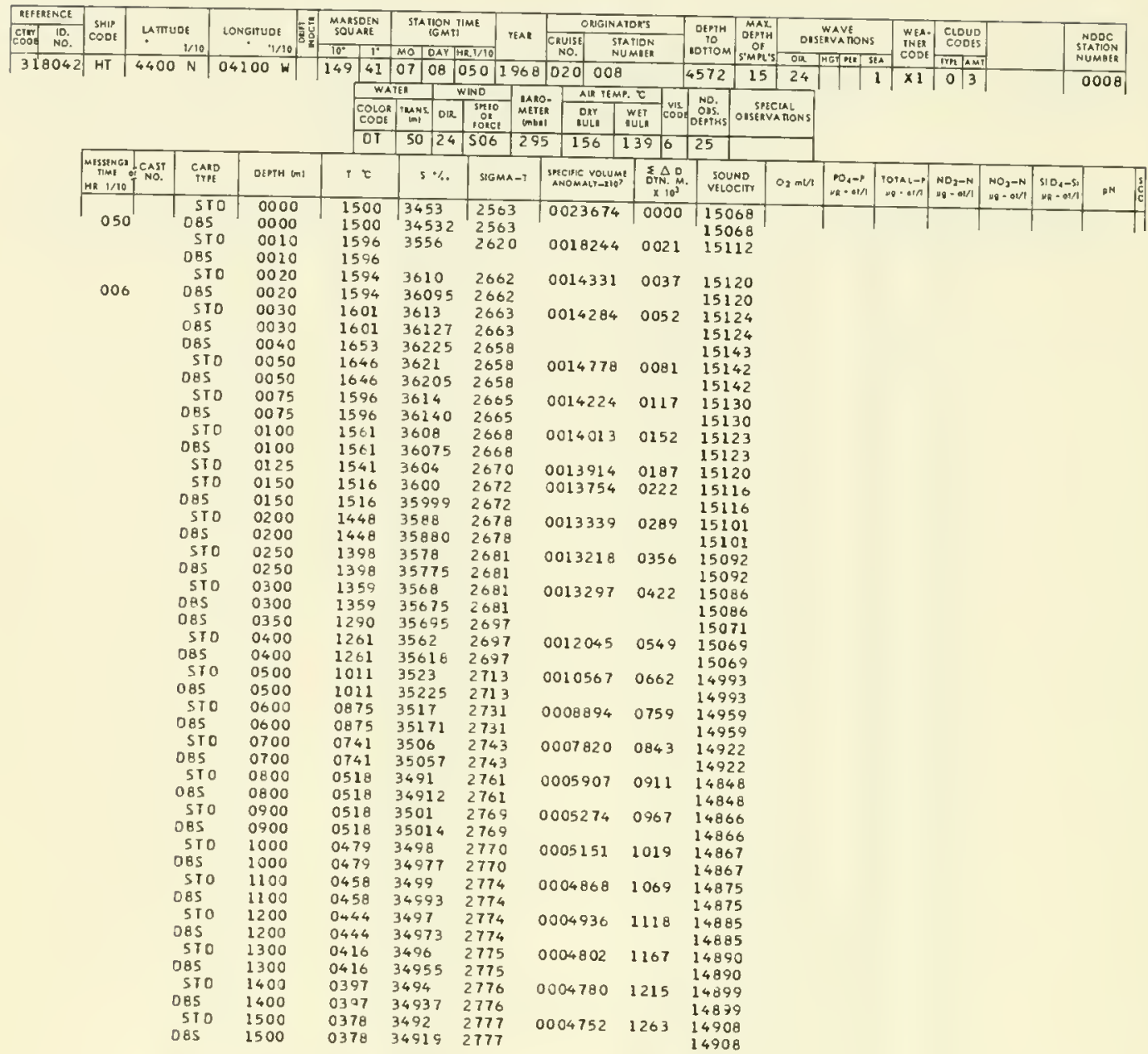




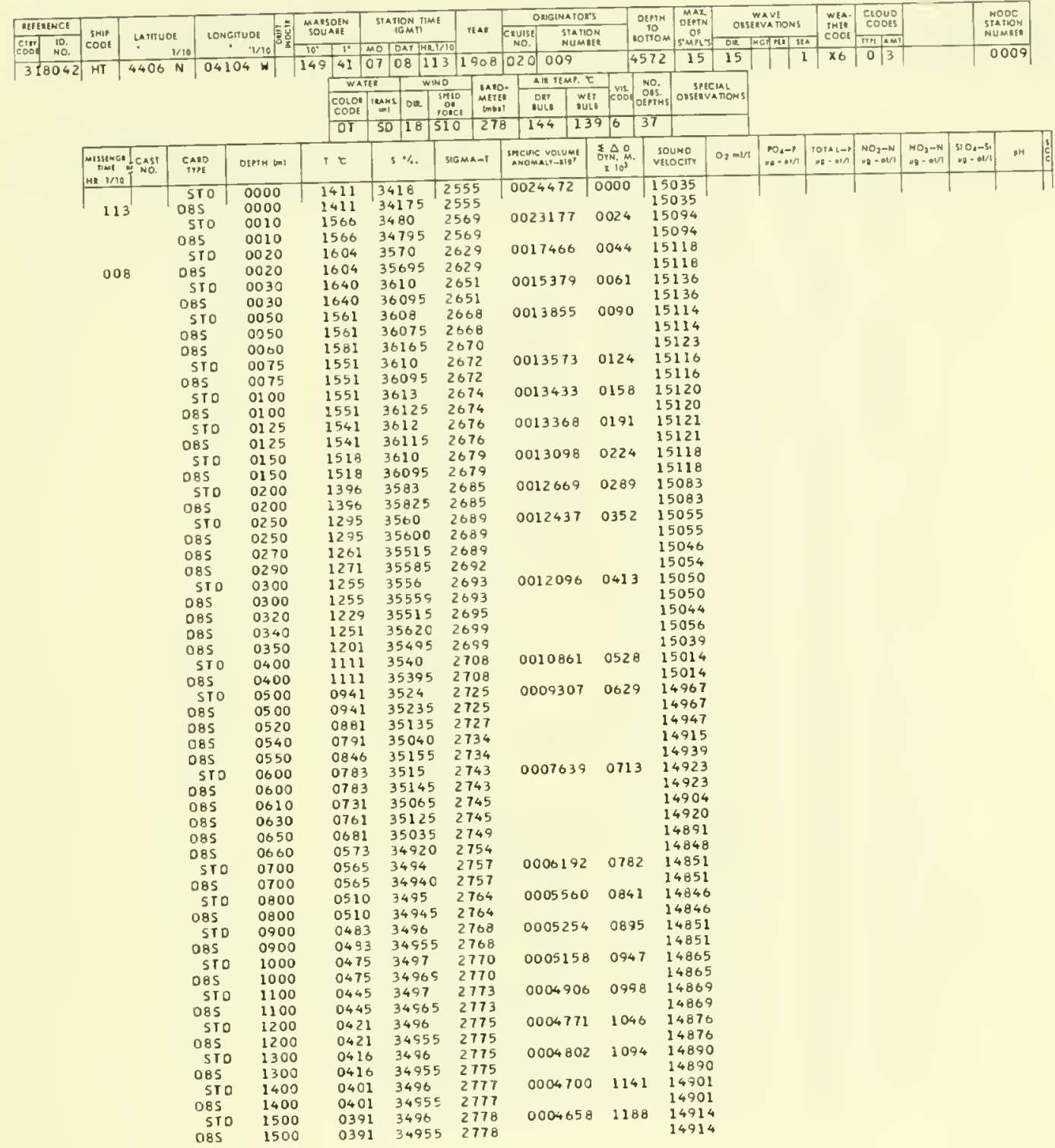


Table XI.-Continued

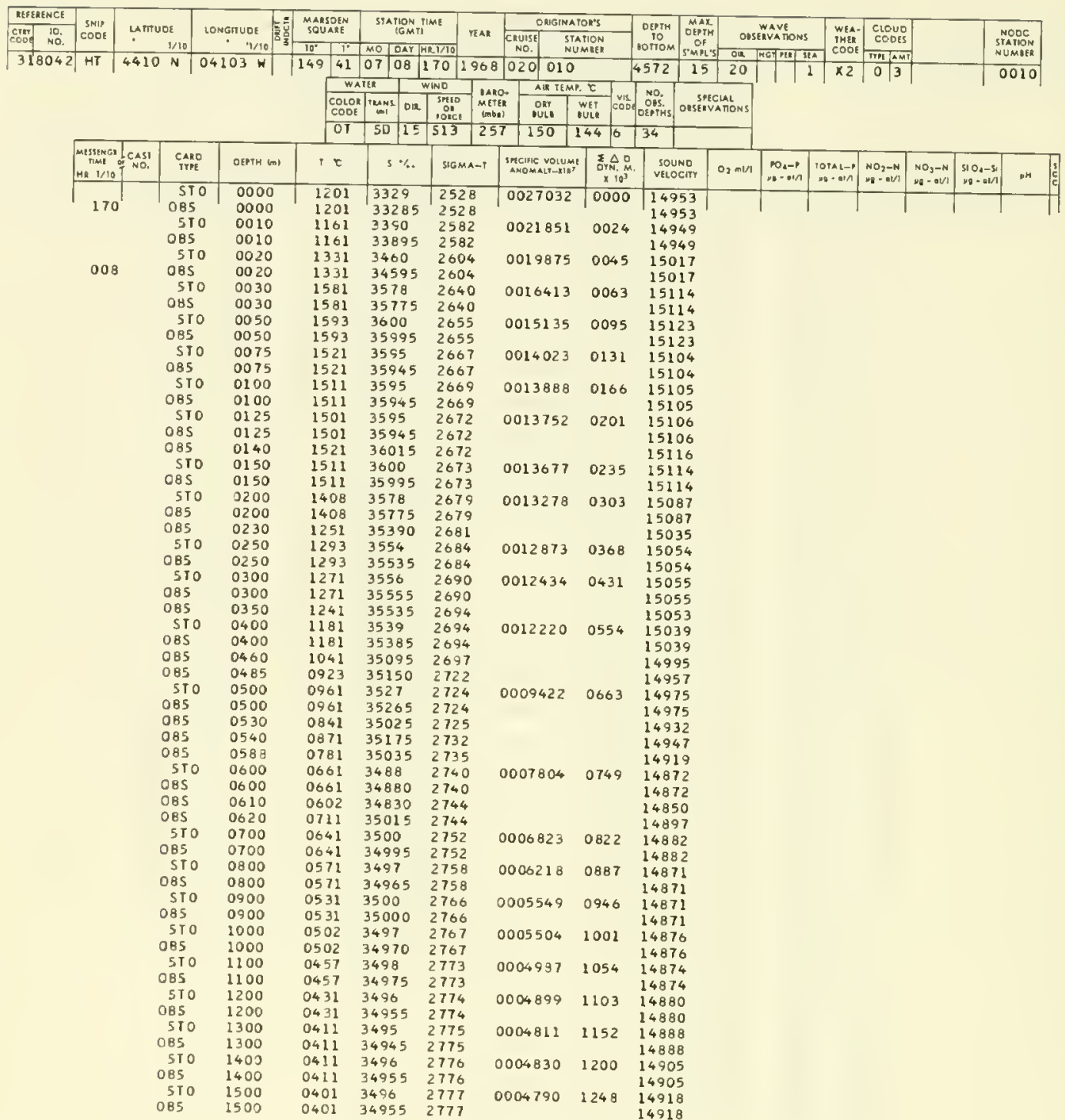


TABLE XI.-Continued

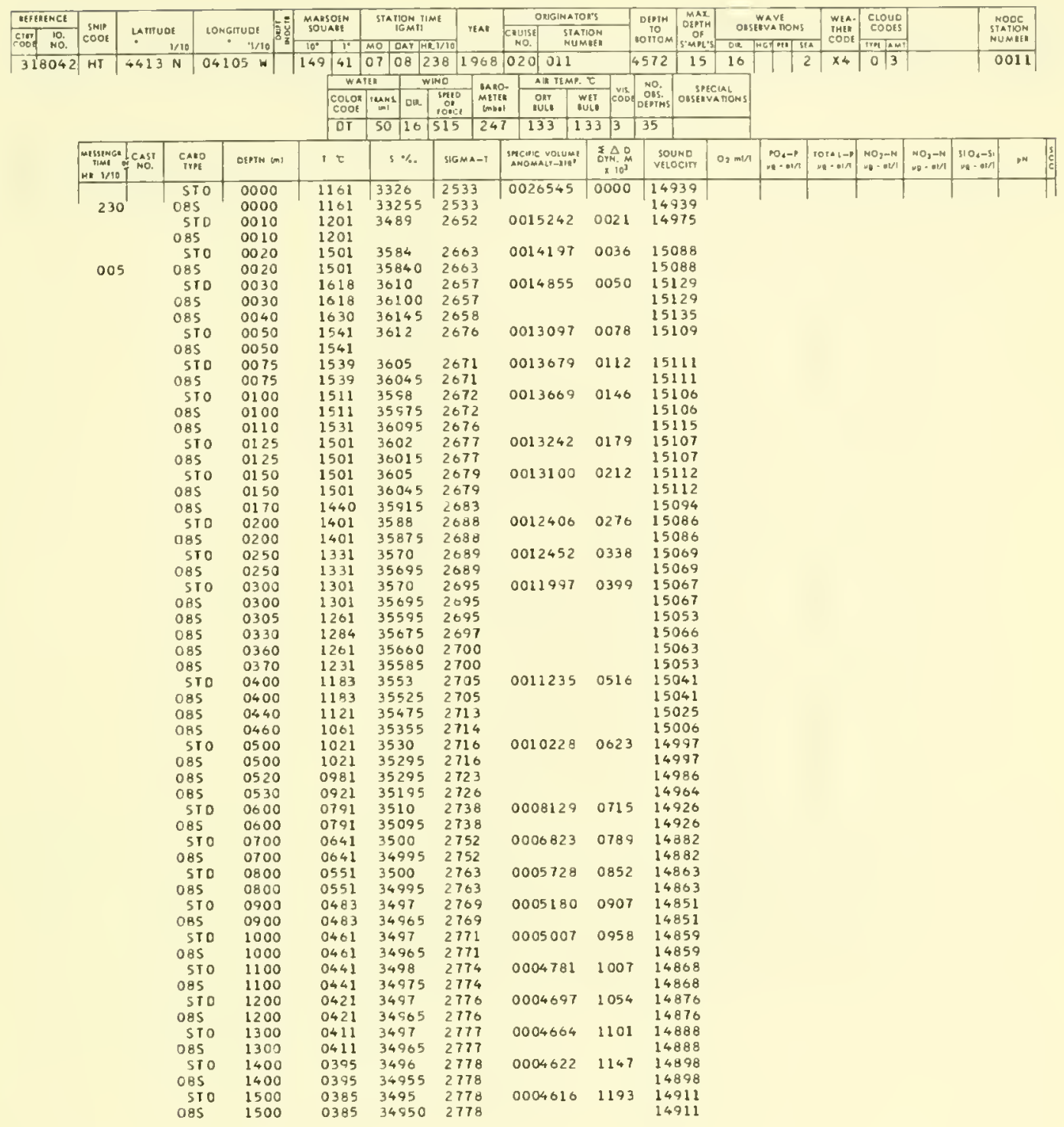


TABle XI.-Continued

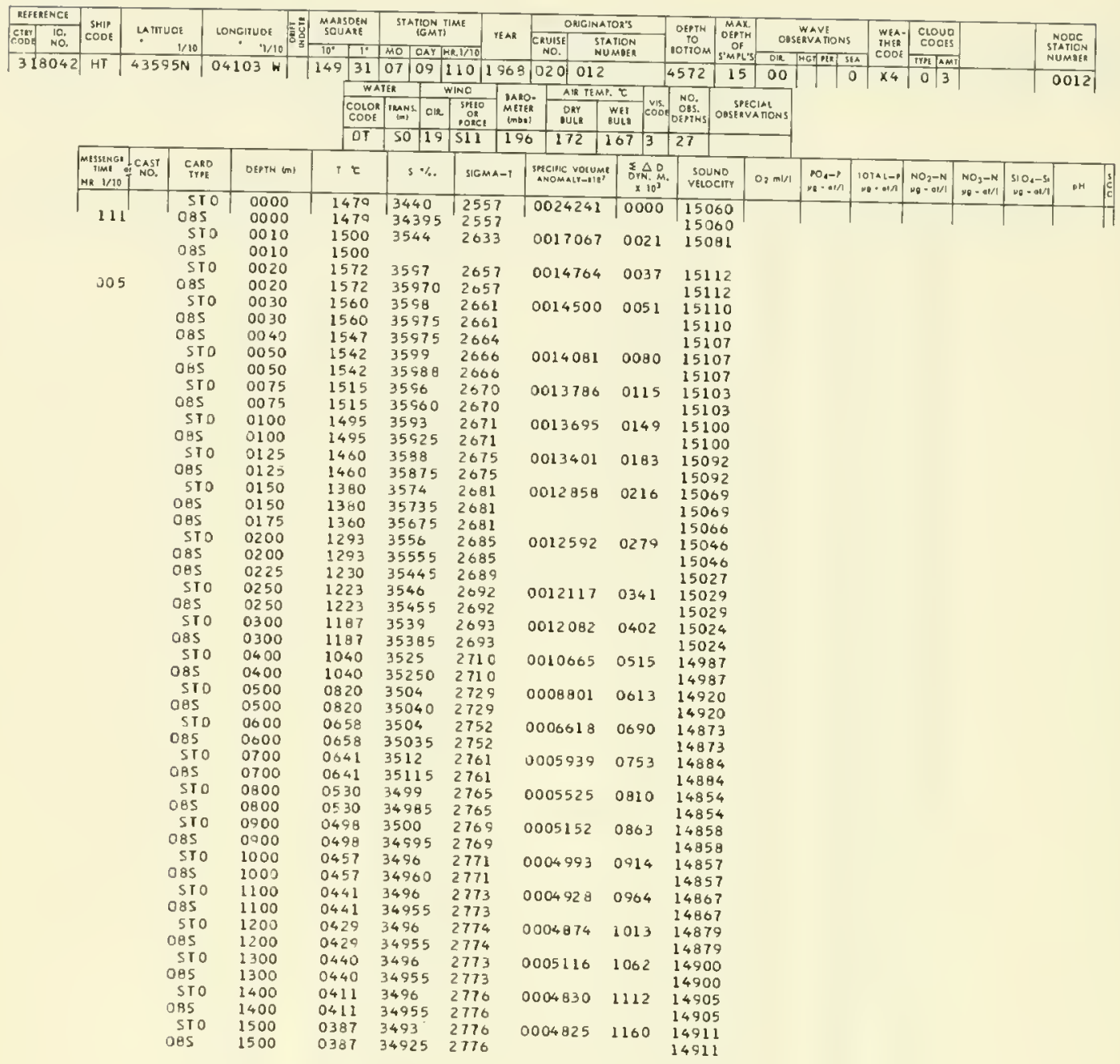


Table XI.-Continued

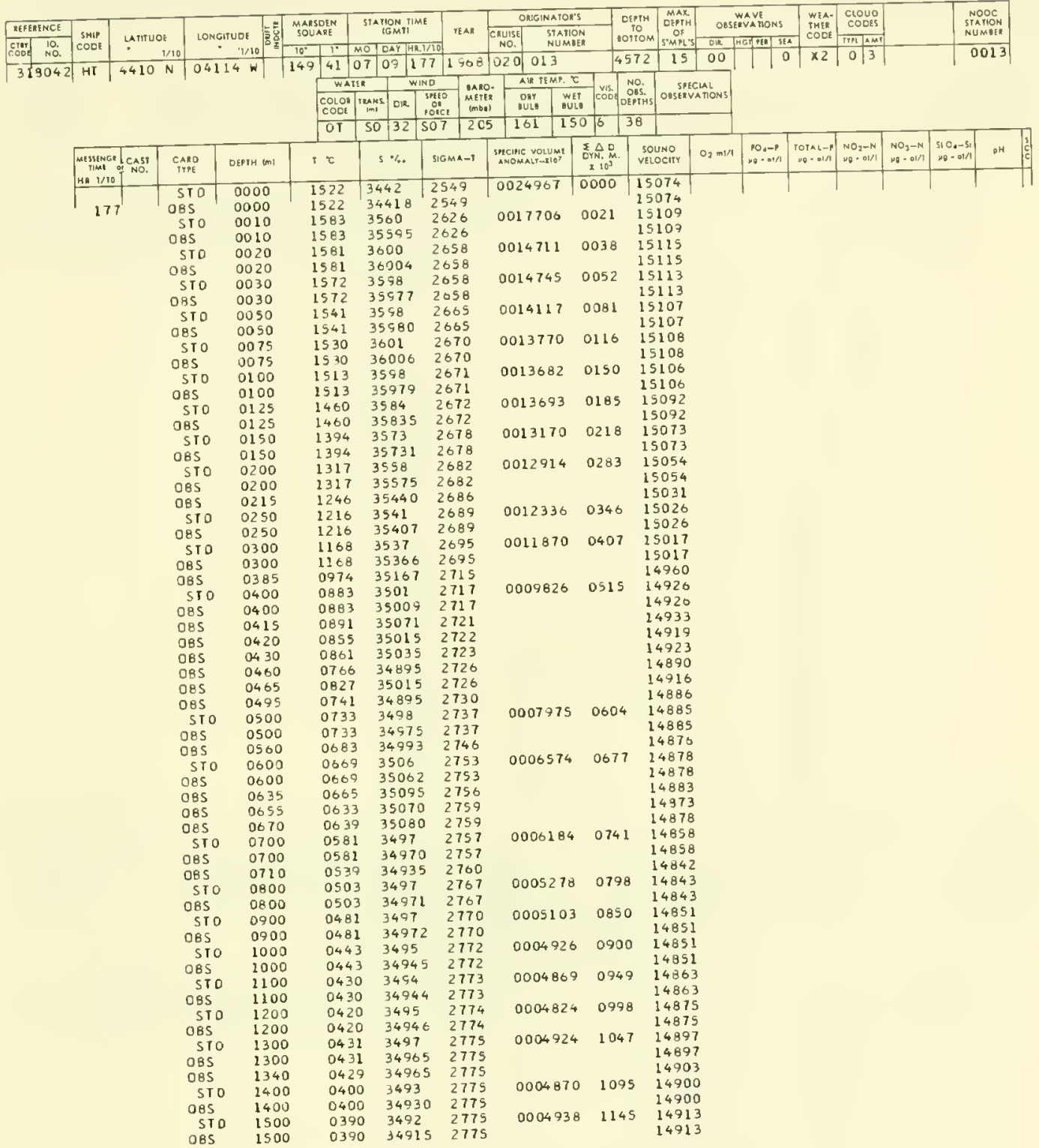


TABLE XII.-Observed and interpolated oceanographic data for stations taken by USCGC DALLAS at Ocean Station DELTA, 13 July-4 August 1968, prepared from NODC listing No. 31-1339 DG.

\begin{tabular}{|c|c|c|c|c|c|c|c|c|c|c|c|c|c|c|c|c|c|}
\hline REP EAF HCE & SNIP & & & & $\begin{array}{l}\text { MAISDEN } \\
\text { SOUAEE }\end{array}$ & STATION & PIME & ORginato & ros's & & & & & & & & \\
\hline 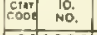 & $\cos \varepsilon$ & Antioc & & 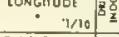 & \begin{tabular}{l|l|}
\multicolumn{2}{|c|}{ Souvalt } \\
$10^{\circ}$ & $1^{\circ}$ \\
\end{tabular} & $\begin{array}{ll} & 16 \mathrm{MTH} \\
M O & \text { OAY T } \\
\end{array}$ & 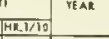 & \begin{tabular}{|c|c|} 
ERUISE: & STA \\
No. & MUN \\
\end{tabular} & $\begin{array}{l}\text { ATHON } \\
\text { AMAER }\end{array}$ & 10 & 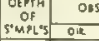 & $\begin{array}{l}\text { SERVABONS } \\
\text { HaGT DI IIA }\end{array}$ & $\begin{array}{l}\text { INEA } \\
\text { COOR }\end{array}$ & \begin{tabular}{|l|} 
CODES \\
TPP \\
APAS
\end{tabular} & & & $\begin{array}{l}\text { STAC } \\
\text { AUMSER }\end{array}$ \\
\hline 311339 & $O G$ & 4359 & $N$ & $04105 \mathrm{~W}$ & 149 & \begin{tabular}{l|l|}
07 & 13 \\
\end{tabular} & \begin{tabular}{|l|l|}
145 & 1968
\end{tabular} & \begin{tabular}{l|l|l|}
8 & 021 & 001 \\
\end{tabular} & & 4663 & 1504 & 12 & $\overline{x 1}$ & \begin{tabular}{|l|l|}
86 \\
\end{tabular} & & & $000 \mathrm{l}$ \\
\hline & & & & & $\mathrm{WA}$ & ATES & WINO & 1. AII TEMP. & & & & & & & & & \\
\hline & & & & & $\begin{array}{l}\text { COLOR } \\
\text { COOE }\end{array}$ & 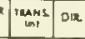 & $\mid \begin{array}{c}\sin 60 \\
\text { on } \\
\text { orcs }\end{array}$ & 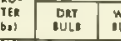 & $\begin{array}{lll}\text { WET } & \text { VIS } \\
\text { OULA } & \\
\end{array}$ & OBS & $\begin{array}{l}\text { SECLAL } \\
\text { OUSEMVATONS }\end{array}$ & & & & & & \\
\hline & & & & & & 00 & 500 & 144 & 1287 & 14 & & & & & & & \\
\hline & 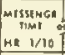 & $\begin{array}{l}\text { CASI } \\
\text { CAOI } \\
\text { NOT. }\end{array}$ & $\underset{\text { GeNO }}{\text { Tref }}$ & OEPTH (m) & $1 \tau$ & $s \%$ & $51 G \mathrm{MAA}-1$ & 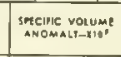 & 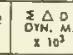 & $\begin{array}{l}\text { SOUNO } \\
\text { VELOCIT }\end{array}$ & $O_{2} m: 1$ & 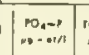 & 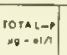 & $\left|\begin{array}{l}\mathrm{NO}_{2}-\mathrm{N} \\
\mathrm{HO}-\mathrm{OUV}^{\prime}\end{array}\right|$ & $\begin{array}{l}\mathrm{NO}_{3}-\mathrm{K} \\
\mathrm{HO}=\mathrm{O} / 1 / 1\end{array}$ & $\left|\begin{array}{c|c}51 O_{4}-51 \\
\mu_{\theta}=01 / 21\end{array}\right|$ & Dh \\
\hline & & & STO & 0000 & 1558 & 3360 & 2479 & 0031712 & 0000 & 15075 & & & & & & & \\
\hline & 145 & & 085 & 0000 & 1558 & 33599 & 2479 & & & 15075 & & & 1 & & & & \\
\hline & & & $\begin{array}{l}\text { STO } \\
\text { STO }\end{array}$ & 0010 & 1639 & 3488 & 2558 & 0024145 & 0028 & 15117 & & & & & & & \\
\hline & 145 & & $\begin{array}{l}510 \\
085\end{array}$ & $\begin{array}{l}0020 \\
0025\end{array}$ & $\begin{array}{l}1664 \\
1694\end{array}$ & $\begin{array}{l}3578 \\
36093\end{array}$ & 2617 & 0018626 & 0049 & 15143 & & & & & & & \\
\hline & & & 510 & 0030 & 1671 & $\begin{array}{l}36093 \\
3611\end{array}$ & $\begin{array}{l}2638 \\
2645\end{array}$ & 0015957 & 0007 & $\begin{array}{l}15151 \\
15145\end{array}$ & & & & & & & \\
\hline & & & STO & 0050 & 1006 & 3619 & 2600 & 0014036 & 0097 & 15130 & & & & & & & \\
\hline & 145 & & 085 & 0051 & 1604 & 36189 & 2667 & & & 15129 & & & & & & & \\
\hline & & & STO & 0075 & 1585 & 3619 & 2671 & 0013648 & 0131 & 15127 & & & & & & & \\
\hline & 145 & & 085 & 0076 & 1584 & 30186 & 2671 & & & 15127 & & & & & & & \\
\hline & & & STD & 0100 & 1509 & 3616 & 2673 & 0013554 & 0165 & 15126 & & & & & & & \\
\hline & 145 & & 085. & 0102 & 1568 & 36160 & 2673 & & & 15126 & & & & & & & \\
\hline & & & STO & 0125 & 1558 & 3616 & 2675 & 0013437 & 0199 & 15127 & & & & & & & \\
\hline & & & STO & 0150 & 1549 & 3615 & 2677 & 0013357 & 0232 & 15128 & & & & & & & \\
\hline & 145 & & 085 & 0155 & 1548 & 36150 & 2677 & & & 15128 & & & & & & & \\
\hline & & & $5 T 0$ & 0200 & 1540 & 3615 & 2679 & 0013326 & 0299 & 15133 & & & & & & & \\
\hline & 145 & & 085 & 10204 & 1538 & 36146 & 2679 & & & 15133 & & & & & & & \\
\hline & & & $\begin{array}{l}\text { STO } \\
\text { STO }\end{array}$ & 0250 & 1467 & 3598 & $\begin{array}{l}2682 \\
2687\end{array}$ & $\begin{array}{l}0013157 \\
0012809\end{array}$ & $\begin{array}{l}0365 \\
0430\end{array}$ & $\begin{array}{l}15116 \\
15097\end{array}$ & & & & & & & \\
\hline & 145 & & 085. & $\begin{array}{l}0300 \\
0306\end{array}$ & $\begin{array}{l}1387 \\
1377\end{array}$ & $\begin{array}{l}3582 \\
35800\end{array}$ & $\begin{array}{l}2687 \\
2687\end{array}$ & & & 15094 & & & & & & & \\
\hline & & & 510 & 0400 & 1223 & 3559 & 2702 & 0011517 & 0552 & 15056 & & & & & & & \\
\hline & 145 & & 085 & $T 0408$ & 1207 & 35570 & 2704 & & & 15051 & & & & & & & \\
\hline & & & 510 & 0500 & 0991 & 3527 & 2720 & 0009894 & 0059 & 14986 & & & & & & & \\
\hline & 145 & & 085. & 0504 & 0979 & 35253 & 2720 & & & 14982 & & & & & & & \\
\hline & 145 & & 085 & T0591 & 0682 & 34942 & 2742 & & & 14880 & & & & & & & \\
\hline & & & 510 & 0600 & 0668 & 3494 & 2744 & 0007453 & 0746 & 14876 & & & & & & & \\
\hline & & & STO & 0700 & 0540 & 3493 & 2759 & 0005960 & 0813 & 14841 & & & & & & & \\
\hline & 145 & & 085 & 0783 & 0476 & 34915 & 2760 & & & 14828 & & & & & & & \\
\hline & & & 570 & 0800 & 0476 & 3493 & 2767 & 0005239 & 0869 & 14831 & & & & & & & \\
\hline & 145 & & $\begin{array}{l}\text { STO } \\
\text { O8S }\end{array}$ & $\begin{array}{r}0900 \\
10972\end{array}$ & $\begin{array}{l}0473 \\
0469\end{array}$ & $\begin{array}{l}3498 \\
35005\end{array}$ & $\begin{array}{l}2771 \\
2774\end{array}$ & 0004942 & 0920 & 14847 & & & & & & & \\
\hline & & & STO & $\begin{array}{r}10972 \\
1000\end{array}$ & $\begin{array}{l}0469 \\
0467\end{array}$ & $\begin{array}{l}35005 \\
3501\end{array}$ & $\begin{array}{l}2774 \\
2774\end{array}$ & 0004789 & & $\begin{array}{l}14858 \\
14862\end{array}$ & & & & & & & \\
\hline & & & $5 T 0$ & 1100 & 0458 & 3500 & 2775 & 0004787 & $\begin{array}{l}0968 \\
1016\end{array}$ & $\begin{array}{l}14862 \\
14875\end{array}$ & & & & & & & \\
\hline & & & STO & 1200 & 0447 & 3500 & 2776 & 0004755 & 1064 & 14887 & & & & & & & \\
\hline & & & STO & 1300 & 0432 & 3500 & 2778 & 0004666 & 1111 & 14898 & & & & & & & \\
\hline & & & 5TD & 1400 & 0415 & 3500 & 2779 & 0004539 & 1157 & 14907 & & & & & & & \\
\hline & 145 & & 085 & 1455 & 0404 & 35001 & 2780 & & & & & & & & & & \\
\hline
\end{tabular}

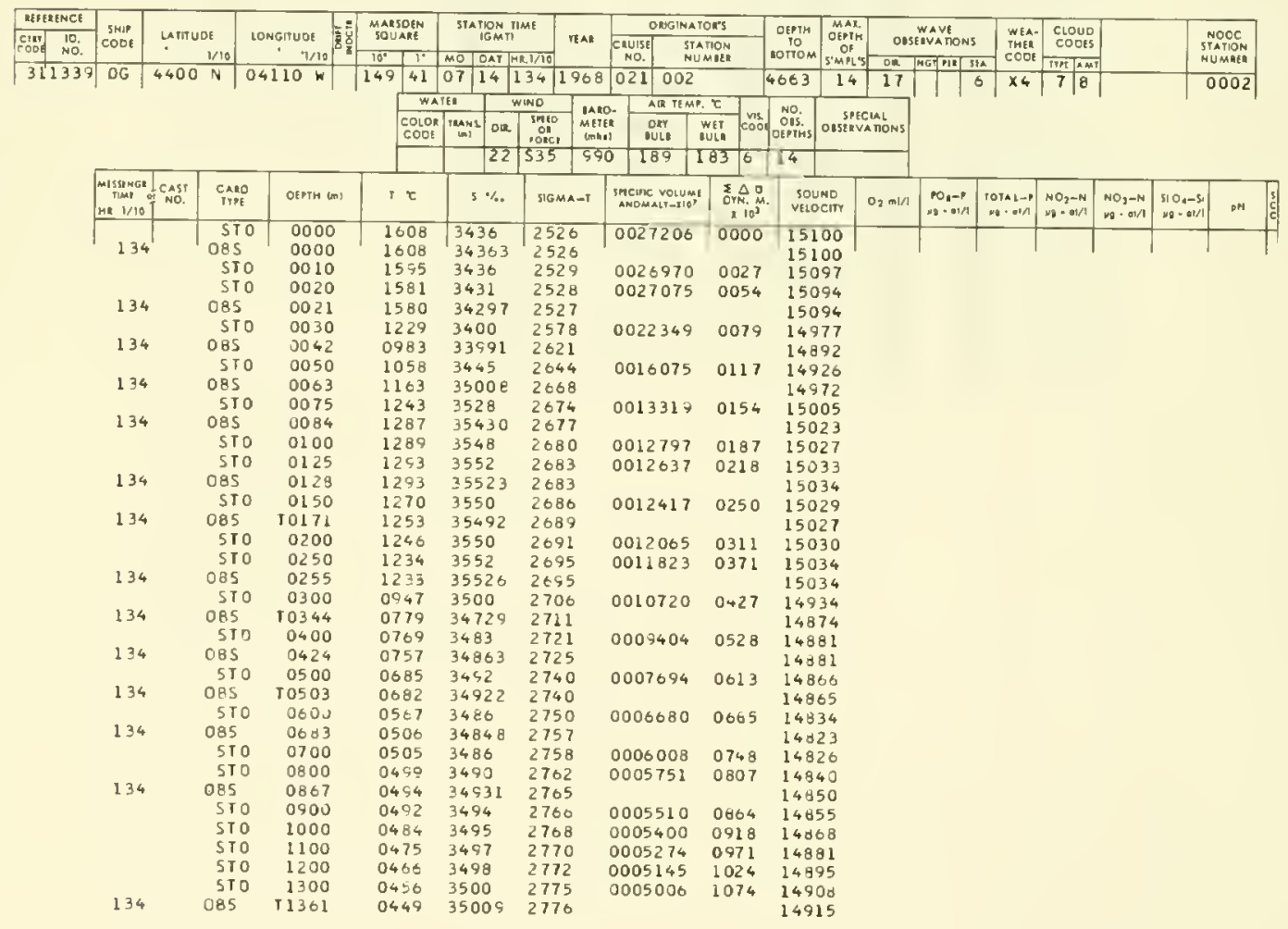


TABLE XII.-Continued

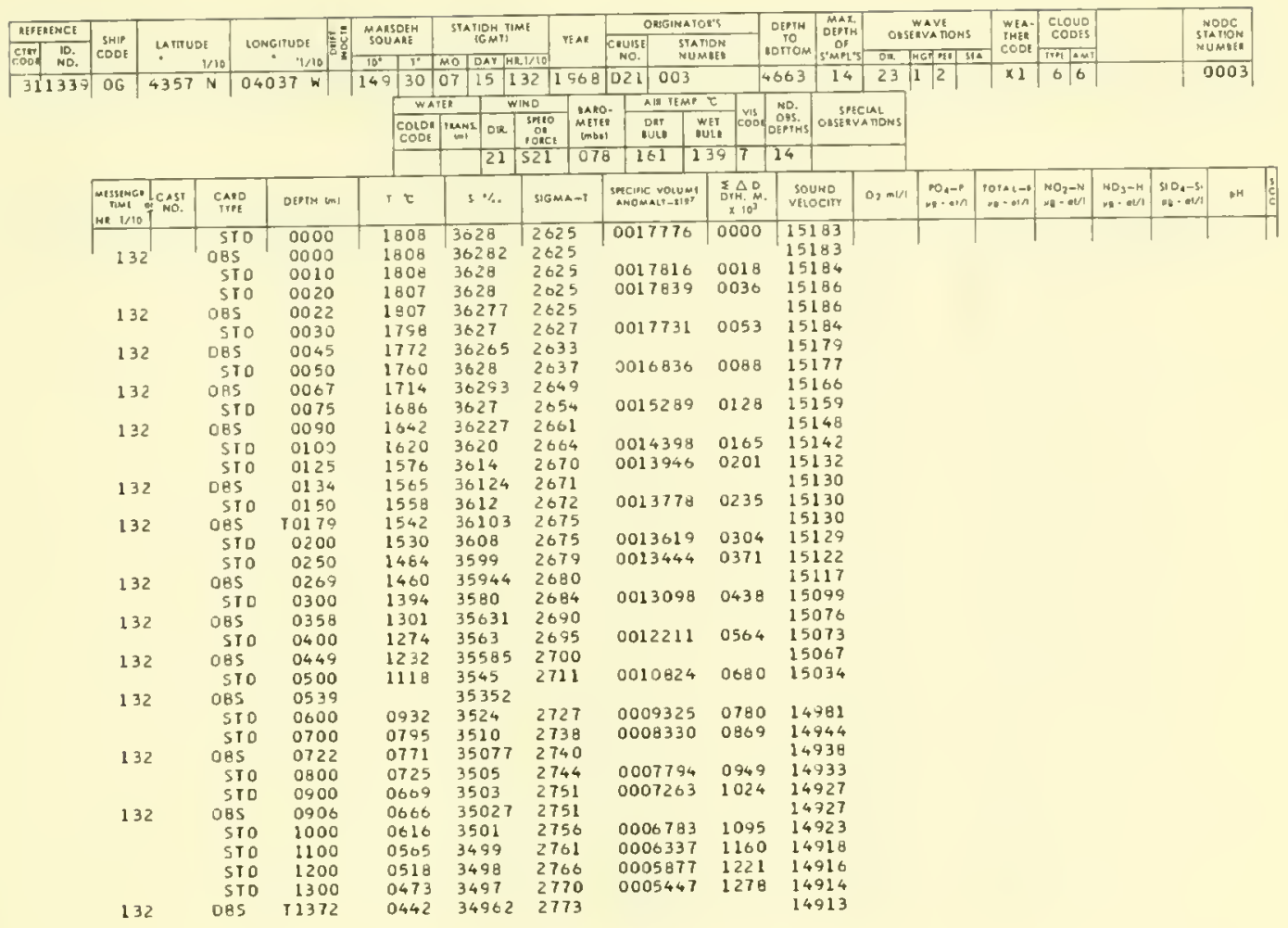

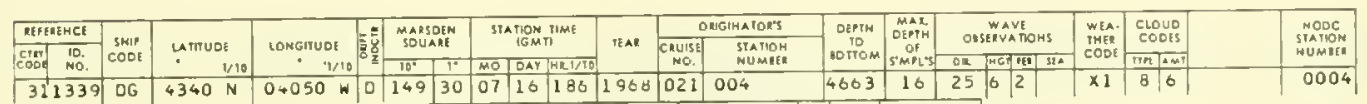

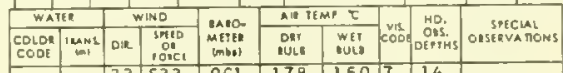

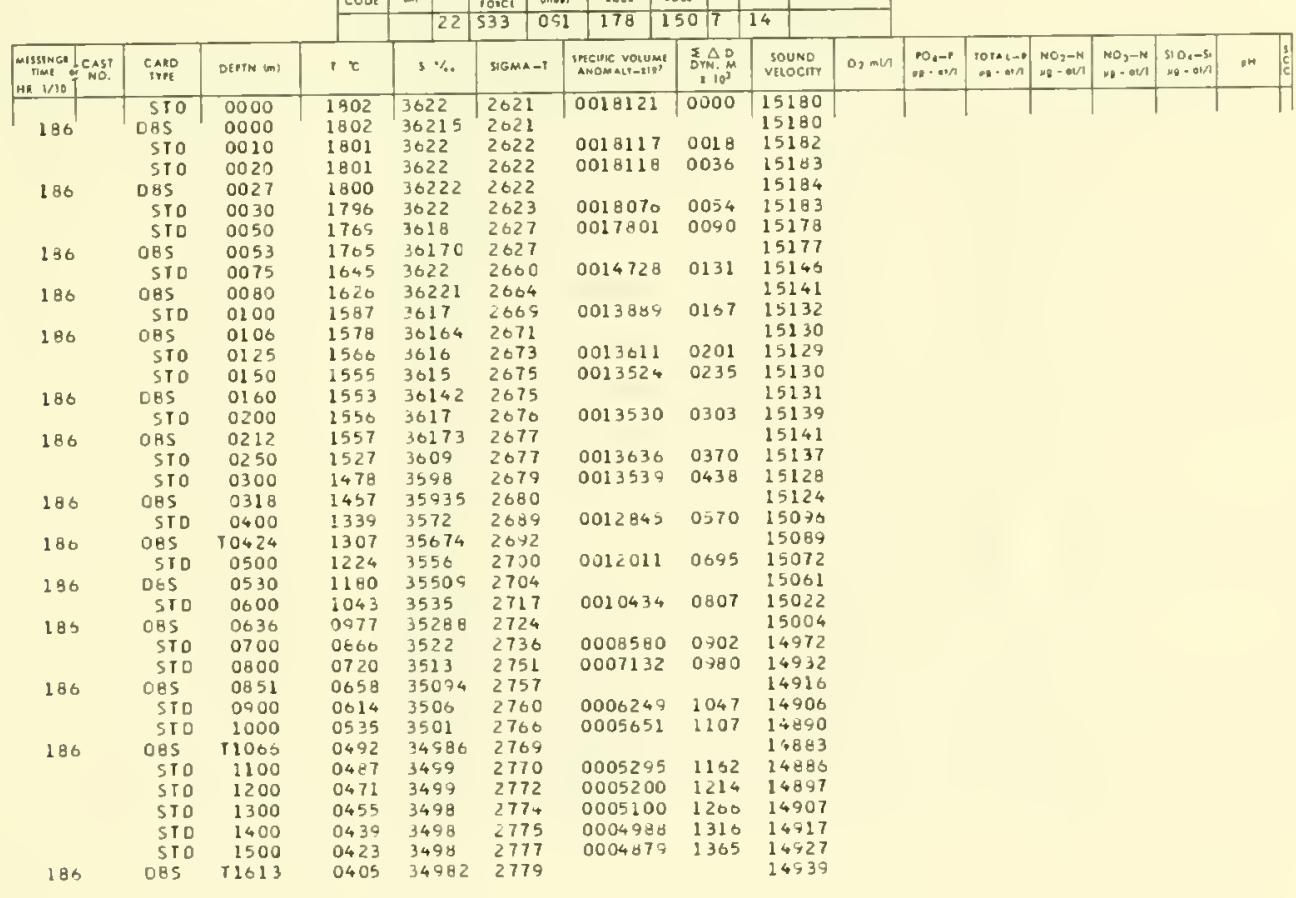


TABLE XII.-Continued

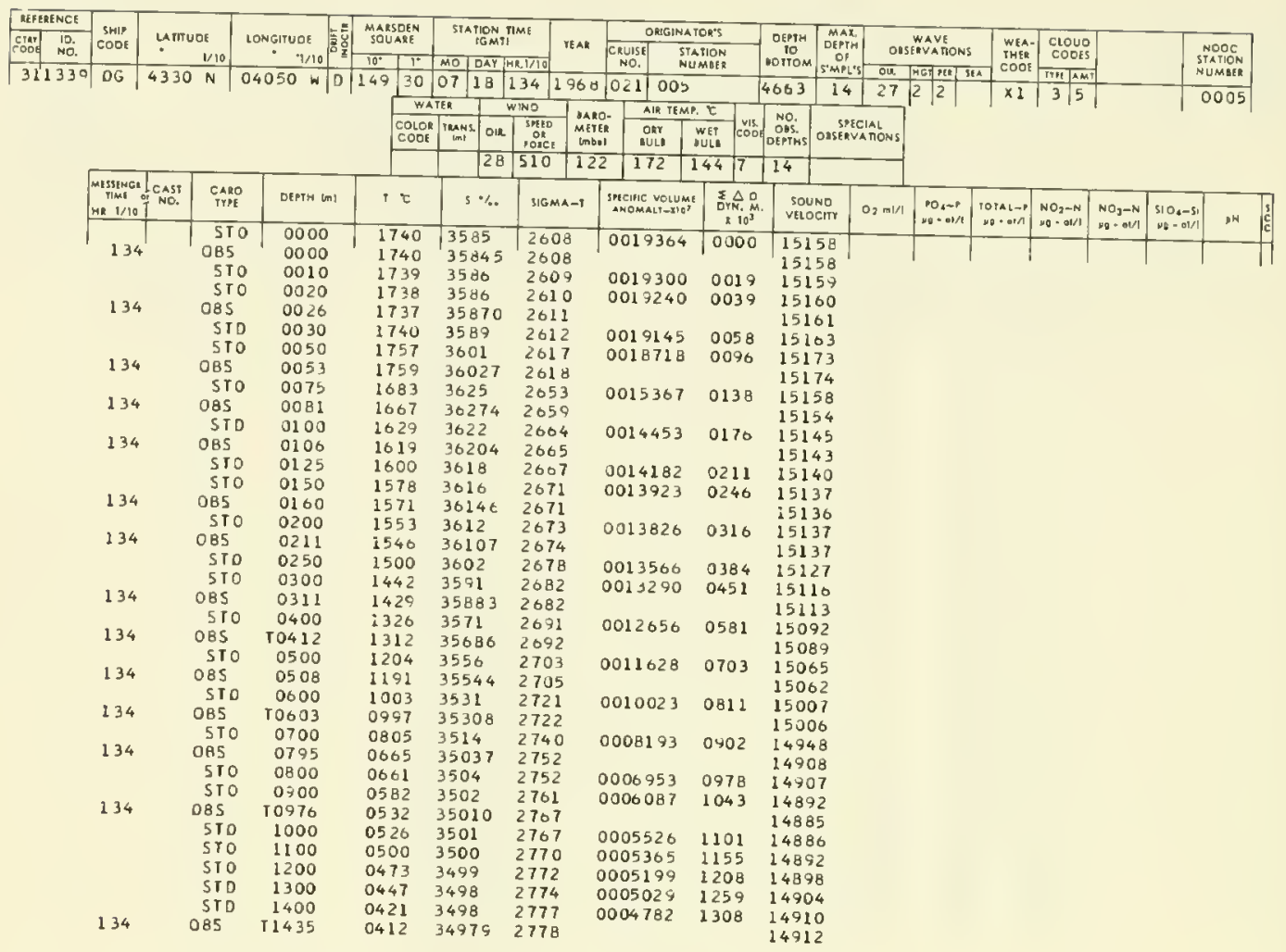

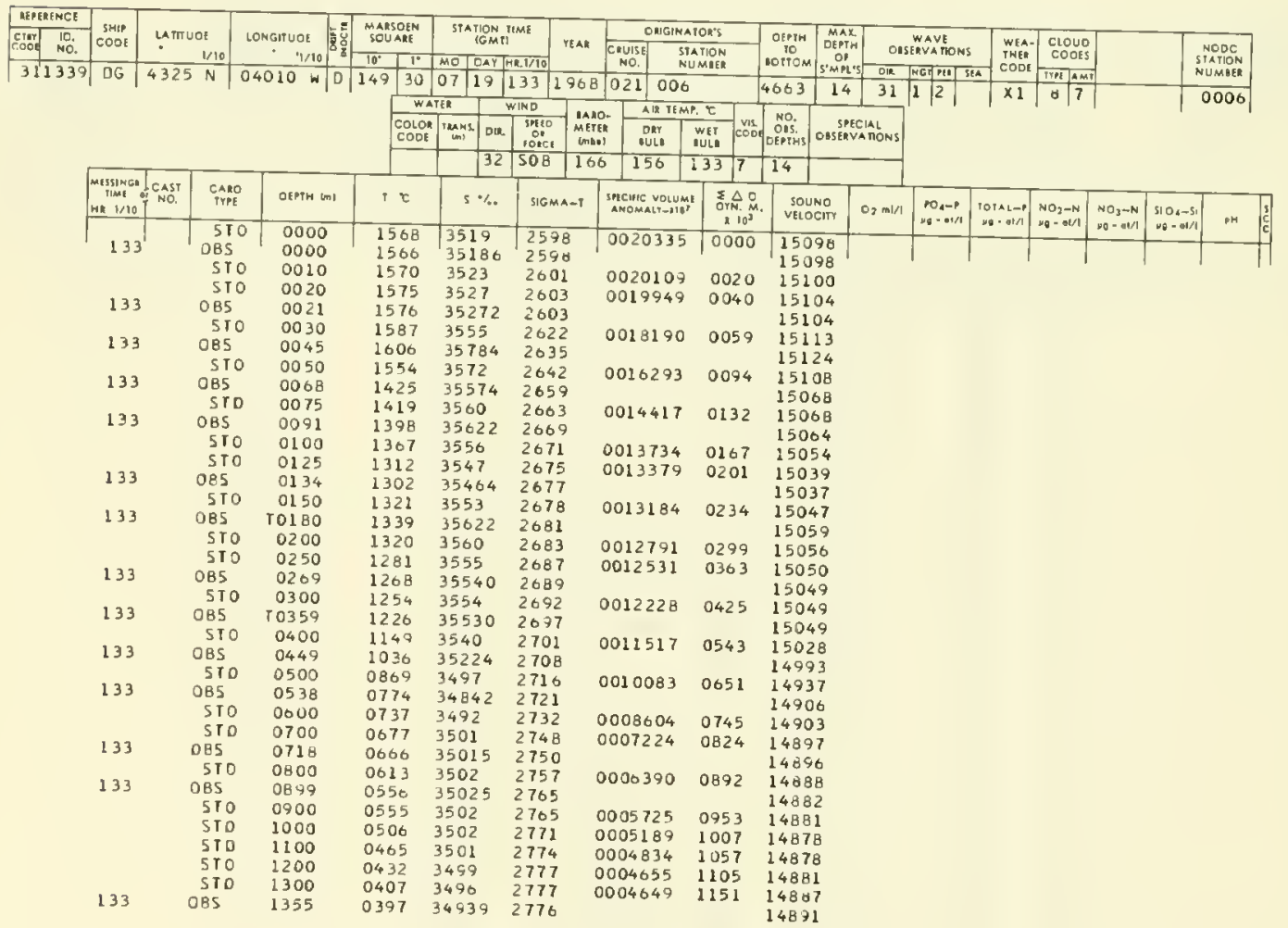


TABLE XII.-Continued
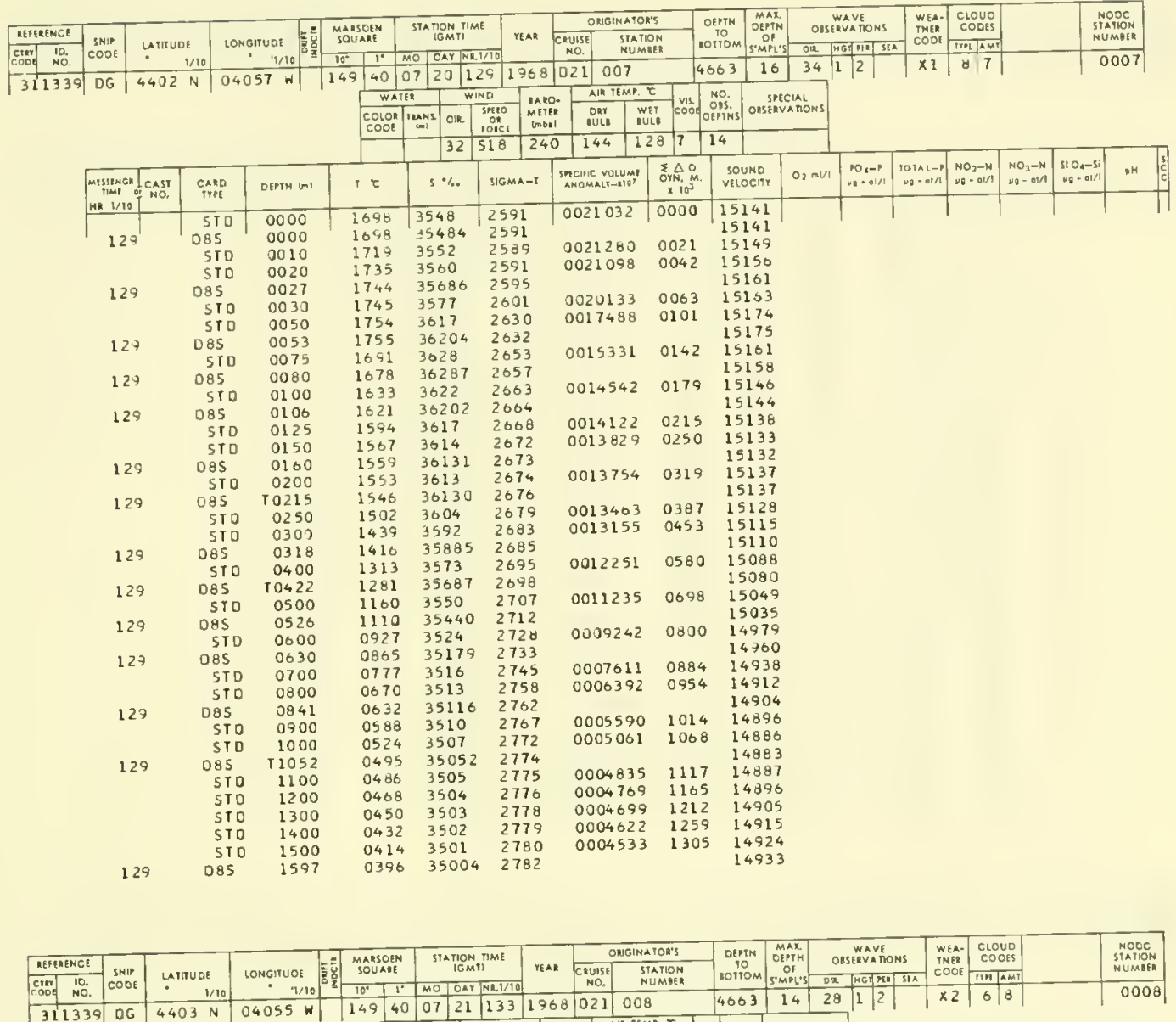

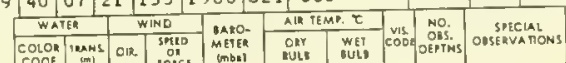

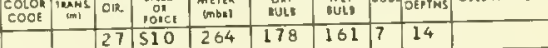

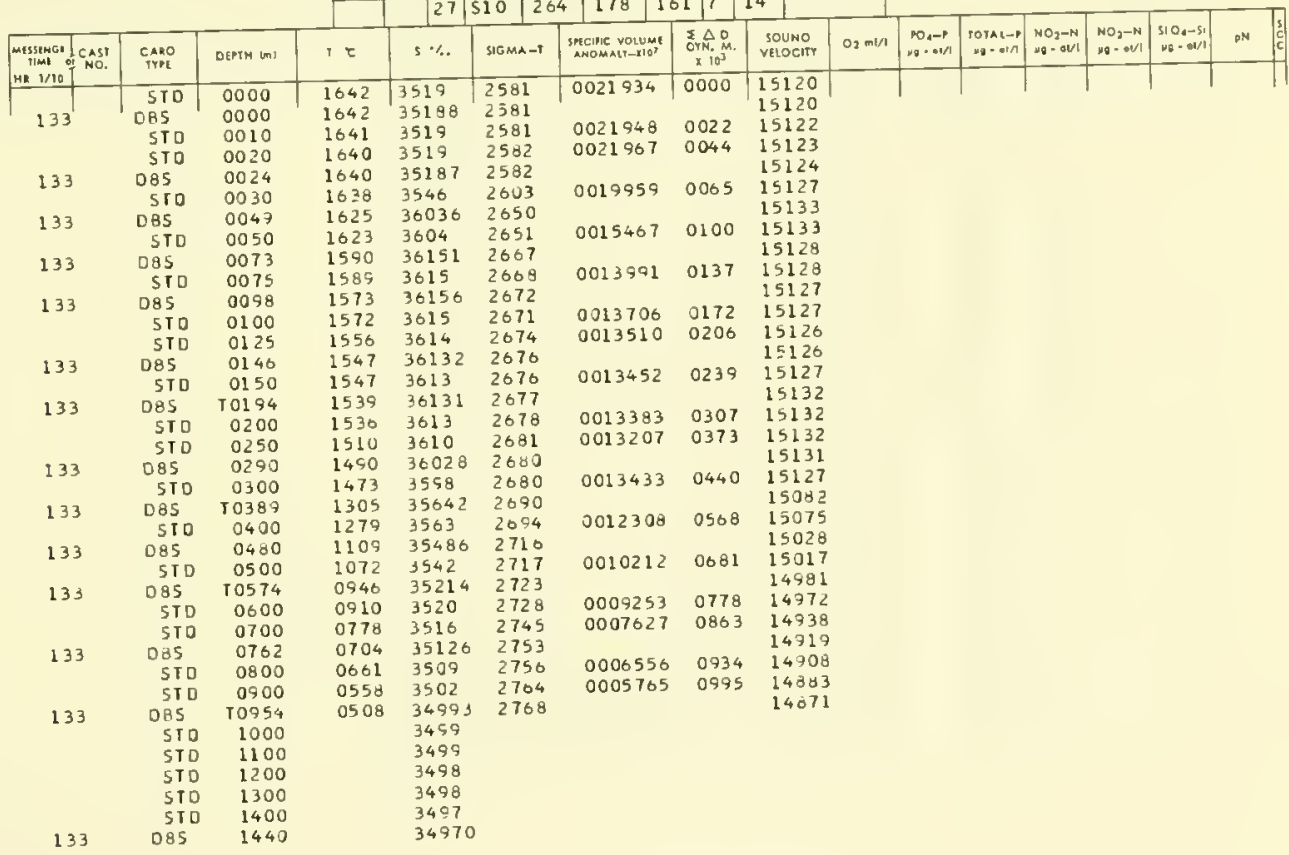


TABLE XII.-Continued

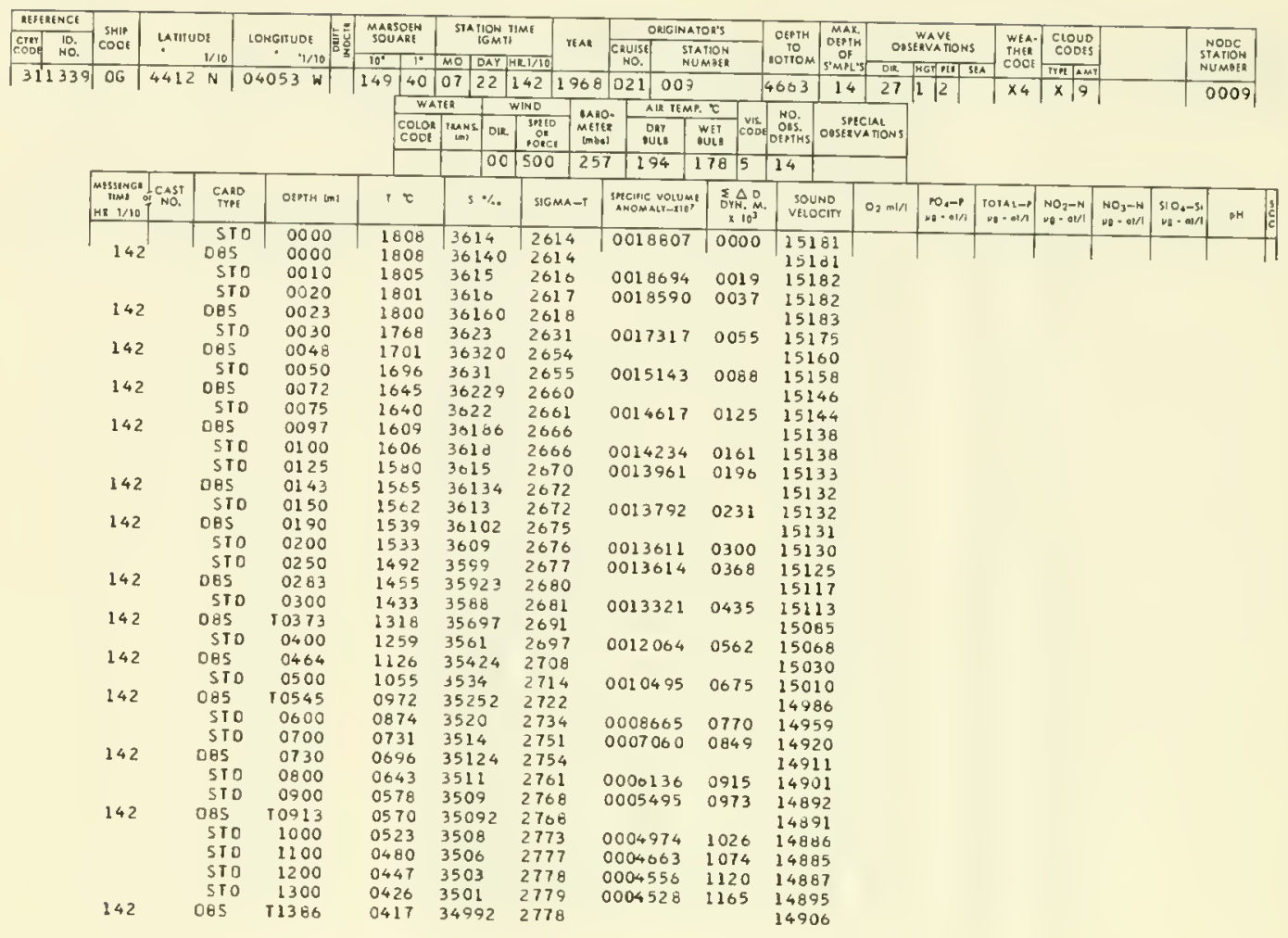

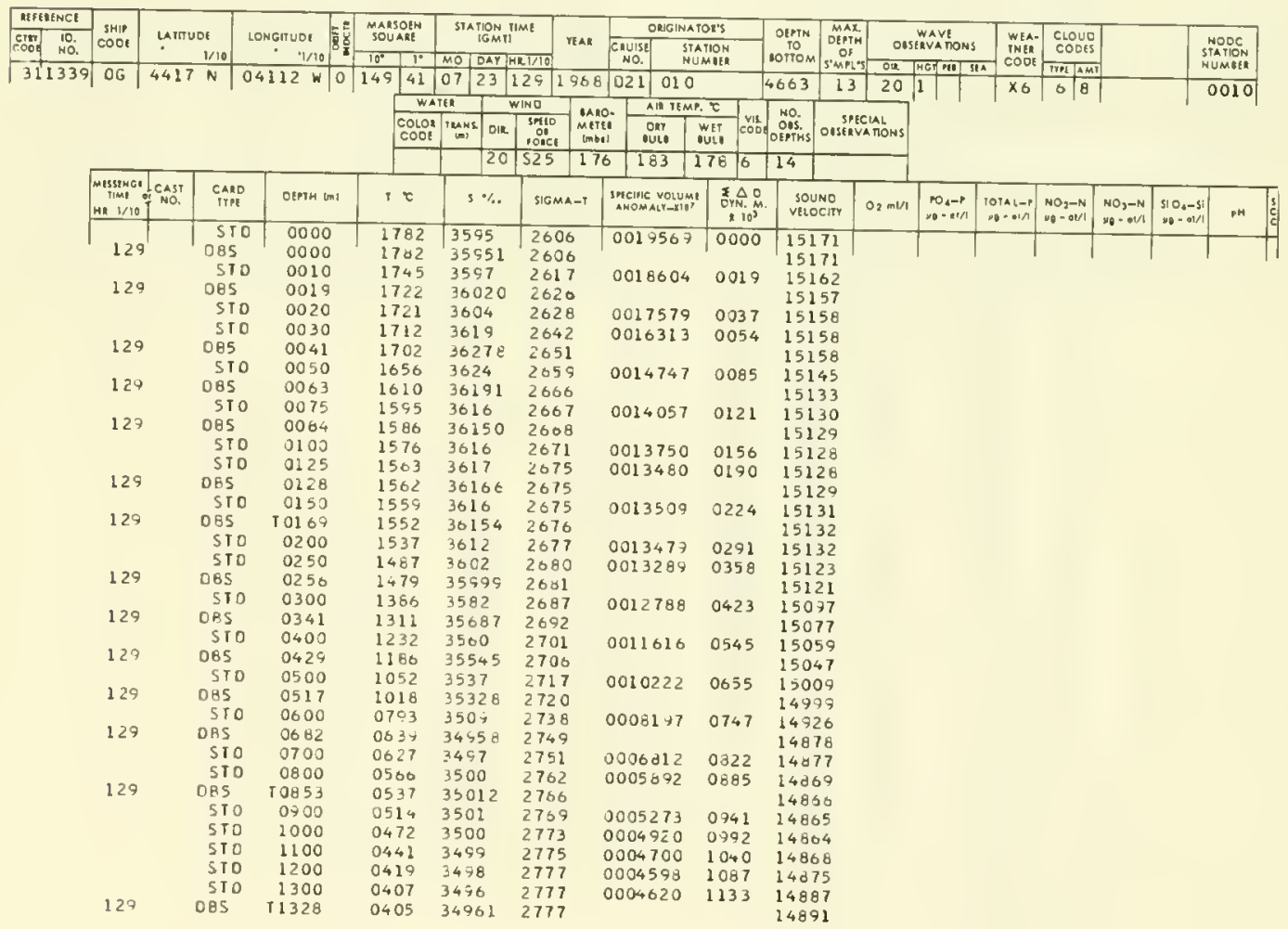


TABLE XII.-Continued

\begin{tabular}{|c|c|c|c|c|c|c|c|c|c|c|c|c|c|c|c|c|c|c|}
\hline ALFERENE: & \multirow{2}{*}{$\begin{array}{c}3 N 10 \\
\operatorname{coo!}\end{array}$} & \multirow{2}{*}{\multicolumn{2}{|c|}{ yamuot }} & \multirow{2}{*}{ 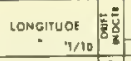 } & \multirow{2}{*}{$\begin{array}{l}\text { MAASOEN } \\
\text { SOUAIE }\end{array}$} & \multirow{2}{*}{$\begin{array}{c}\text { STATION TIME } \\
\text { IGMTI }\end{array}$} & \multirow{2}{*}{1900} & \multicolumn{2}{|c|}{ ONGINATOR's } & & \multirow{2}{*}{ 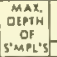 } & \multirow{2}{*}{\multicolumn{2}{|c|}{$\begin{array}{c}\text { Wave } \\
\text { ogsenYATONS }\end{array}$}} & \multirow{2}{*}{$\begin{array}{l}\text { WIA. } \\
\text { INER } \\
\text { COOE }\end{array}$} & \multirow{2}{*}{ 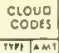 } & & & \multirow{2}{*}{$\begin{array}{c}\text { NOOC } \\
\text { STATILN } \\
\text { NUMGEA }\end{array}$} \\
\hline $\begin{array}{l}\mathrm{Crom} \\
\text { Noo. }\end{array}$ & & & & & & & & \multirow{2}{*}{ 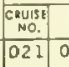 } & $\begin{array}{l}\text { STAFION } \\
\text { NUMBE }\end{array}$ & & & & & & & & & \\
\hline 311339 & OG & 4402 & $\mathbf{N}$ & $04047 \times|0|$ & \begin{tabular}{l|l}
149 & 40 \\
\end{tabular} & \begin{tabular}{|l|l|l|}
07 & 24 \\
\end{tabular} & $121 / 1900$ & & & 4063 & 14 & 3512 & 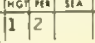 & $\overline{x 1}$ & \begin{tabular}{|l|l|}
6 & 5 \\
\end{tabular} & & & 0011 \\
\hline & & & & & WA & \begin{tabular}{|l|l|} 
TEI & $y$ \\
\end{tabular} & WINO & 1. Al गIMP & $\varepsilon$ & No. & & & & & & & & \\
\hline & & & & & $\begin{array}{l}\text { cotol } \\
\cos t\end{array}$ & 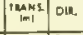 & 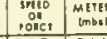 & Ont & $\begin{array}{ll}\text { wet co } \\
\text { outs }\end{array}$ & \{oestins & Ousiev & IONS & & & & & & \\
\hline & & & & & & 36 & 505 & 167 & 1397 & 14 & & & & & & & & \\
\hline & $\begin{array}{l}\text { messenat } \\
\text { thet } \\
\text { He } 1 / 10 \\
\end{array}$ & TSOAST & $\begin{array}{l}\text { CARD } \\
\text { Trre }\end{array}$ & OEPTM $\mathrm{mI}$ & $1 t$ & $s \%$ & SiGMA-t & 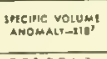 & 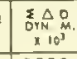 & & $\begin{array}{l}\text { UUND } \\
\text { OCITY }\end{array}$ & $0, \mathrm{~m} / \mathrm{l}$ & 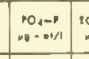 & 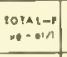 & $\begin{array}{l}\mathrm{NO}_{2}-\mathrm{N} \\
\mathrm{NO} \rightarrow \mathrm{ON} / 1\end{array}$ & $\begin{array}{l}\mathrm{NO}_{1}-\mathrm{N} \\
\mathrm{H}-\mathrm{Am} / \mathrm{I}\end{array}$ & $\begin{array}{l}510_{4}-5 i \\
38-a \mid 1 / 1\end{array}$ & en \\
\hline & 121 & & $\begin{array}{l}\text { STO } \\
\text { OAs }\end{array}$ & $\begin{array}{l}0000 \\
0000\end{array}$ & 1841 & 3615 & 2606 & 0019548 & 0000 & & 130 & & & & & & & \\
\hline & 121 & & $\begin{array}{l}\text { COS } \\
\text { STO }\end{array}$ & $\begin{array}{l}0000 \\
0010\end{array}$ & $\begin{array}{l}1841 \\
1839\end{array}$ & $\begin{array}{l}3614 t \\
3015\end{array}$ & $\begin{array}{l}2006 \\
2007\end{array}$ & 0019530 & 0020 & $\begin{array}{l}15 \\
15\end{array}$ & $\begin{array}{l}190 \\
191\end{array}$ & & & & & & & \\
\hline & & & STO & on 20 & $\begin{array}{l}1839 \\
1836\end{array}$ & $\begin{array}{l}3015 \\
3614\end{array}$ & $\begin{array}{l}2007 \\
2008\end{array}$ & 0019513 & 0039 & & 192 & & & & & & & \\
\hline & 121 & & 085 & 0024 & 1835 & 30144 & 2608 & & & & 193 & & & & & & & \\
\hline & & & STO & 0030 & $1 \forall 02$ & 3622 & 2622 & 0018188 & 0058 & & 185 & & & & & & & \\
\hline & 121 & & $\begin{array}{l}\text { CAS } \\
\text { STO }\end{array}$ & $\begin{array}{l}0049 \\
0050\end{array}$ & 1722 & 36345 & 2651 & & & & 166 & & & & & & & \\
\hline & 121 & & 085 & $\begin{array}{l}0050 \\
0072\end{array}$ & 1716 & $\begin{array}{l}3634 \\
36250\end{array}$ & $\begin{array}{l}2652 \\
2659\end{array}$ & 0015382 & 0091 & & 165 & & & & & & & \\
\hline & & & STO & $\begin{array}{l}0072 \\
0075\end{array}$ & $\begin{array}{l}1660 \\
1655\end{array}$ & $\begin{array}{l}36220 \\
3624\end{array}$ & $\begin{array}{l}2659 \\
2059\end{array}$ & 0014807 & 0129 & $\begin{array}{l}15 \\
15\end{array}$ & $\begin{array}{l}150 \\
149\end{array}$ & & & & & & & \\
\hline & 121 & & 085 & 0097 & 1620 & 36201 & 2664 & & 0129 & & $\begin{array}{l}149 \\
142\end{array}$ & & & & & & & \\
\hline & & & SIO & 0100 & 1613 & 3619 & 2065 & 0014316 & 0106 & & 140 & & & & & & & \\
\hline & & & STO & 0125 & 1569 & 3613 & 2070 & 0013866 & 0201 & & 130 & & & & & & & \\
\hline & 121 & & 085 & 0144 & 1548 & 36106 & 2673 & & & & 126 & & & & & & & \\
\hline & & & 510 & 0150 & 1548 & 3611 & 2674 & 0013630 & 0235 & & 127 & & & & & & & \\
\hline & 121 & & 065 & 10191 & 1546 & 36133 & 2670 & & & & 134 & & & & & & & \\
\hline & & & STO & 0200 & 1539 & 3612 & 2677 & 0013523 & 0303 & & 133 & & & & & & & \\
\hline & 121 & & STo & 0250 & 1493 & $\begin{array}{l}3603 \\
35963\end{array}$ & 2680 & 0013344 & 0370 & & 125 & & & & & & & \\
\hline & & & $\begin{array}{l}085 \\
510\end{array}$ & $\begin{array}{l}0287 \\
0300\end{array}$ & $\begin{array}{l}1455 \\
1441\end{array}$ & $\begin{array}{l}35963 \\
3594\end{array}$ & 2683 & & & & 118 & & & & & & & \\
\hline & 121 & & 085 & $\begin{array}{r}0300 \\
T 0382\end{array}$ & $\begin{array}{l}1441 \\
1338\end{array}$ & $\begin{array}{l}3594 \\
35754\end{array}$ & 2644 & 0013051 & 0436 & 15 & 116 & & & & & & & \\
\hline & & & STO & $\begin{array}{r}T 0382 \\
0400\end{array}$ & $\begin{array}{l}1338 \\
1316\end{array}$ & $\begin{array}{l}35754 \\
3572\end{array}$ & $\begin{array}{l}2692 \\
2694\end{array}$ & & & & 093 & & & & & & & \\
\hline & 121 & & 005 & 0478 & 1185 & 35525 & 2704 & 0012384 & 0563 & $\begin{array}{l}15 \\
15\end{array}$ & $\begin{array}{l}089 \\
055\end{array}$ & & & & & & & \\
\hline & & & STO & 0500 & 1125 & 3544 & 2709 & 0011025 & 0680 & 15 & 036 & & & & & & & \\
\hline & 122 & & 085 & $\operatorname{Tos} 73$ & 095.3 & 35230 & 2723 & & & 14 & 984 & & & & & & & \\
\hline & & & STO & 0600 & 0912 & 3521 & 2728 & 0009213 & 0782 & & 973 & & & & & & & \\
\hline & & & STO & 0700 & 0773 & 3515 & 2745 & 0007623 & 0866 & & 936 & & & & & & & \\
\hline & 121 & & 085 & 0764 & 0696 & 35112 & 2753 & & & & 916 & & & & & & & \\
\hline & & & STO & 0800 & 0657 & 3509 & 2757 & 0006498 & 0936 & & 907 & & & & & & & \\
\hline & & & sTo & 0900 & 0503 & 3503 & 2764 & 0005760 & 0998 & 14 & .885 & & & & & & & \\
\hline & 121 & & 085 & Т0958 & 0518 & 35000 & 2768 & & & 14 & 876 & & & & & & & \\
\hline & & & STO & 1000 & 0508 & 3500 & 2768 & 0005383 & 1053 & & 879 & & & & & & & \\
\hline & & & STO & 1100 & 0484 & 3499 & 2771 & 0005230 & 2107 & 14 & 885 & & & & & & & \\
\hline & & & STo & 1200 & 0460 & 3490 & 2773 & 0005070 & 1158 & 14 & 892 & & & & & & & \\
\hline & & & 510 & 1300 & 0436 & 3498 & 2775 & 0004899 & 1208 & 14 & 899 & & & & & & & \\
\hline & & & STO & 1400 & 0412 & 3497 & 2777 & 0004731 & 1256 & & 906 & & & & & & & \\
\hline & 121 & & 085 & $T 1424$ & 0400 & 34968 & 2778 & & & & 907 & & & & & & & \\
\hline
\end{tabular}


TABLE XII.-Continued

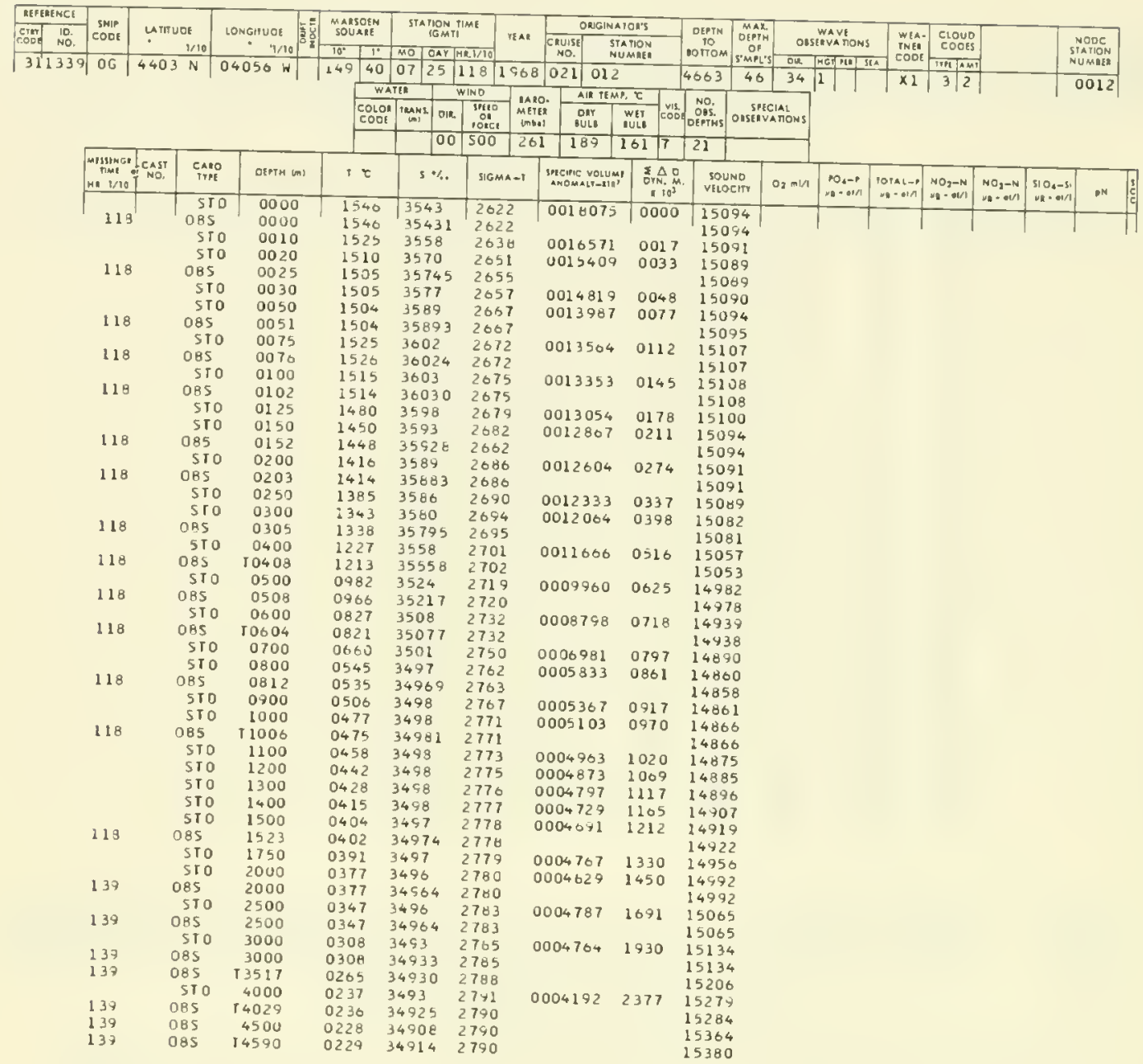



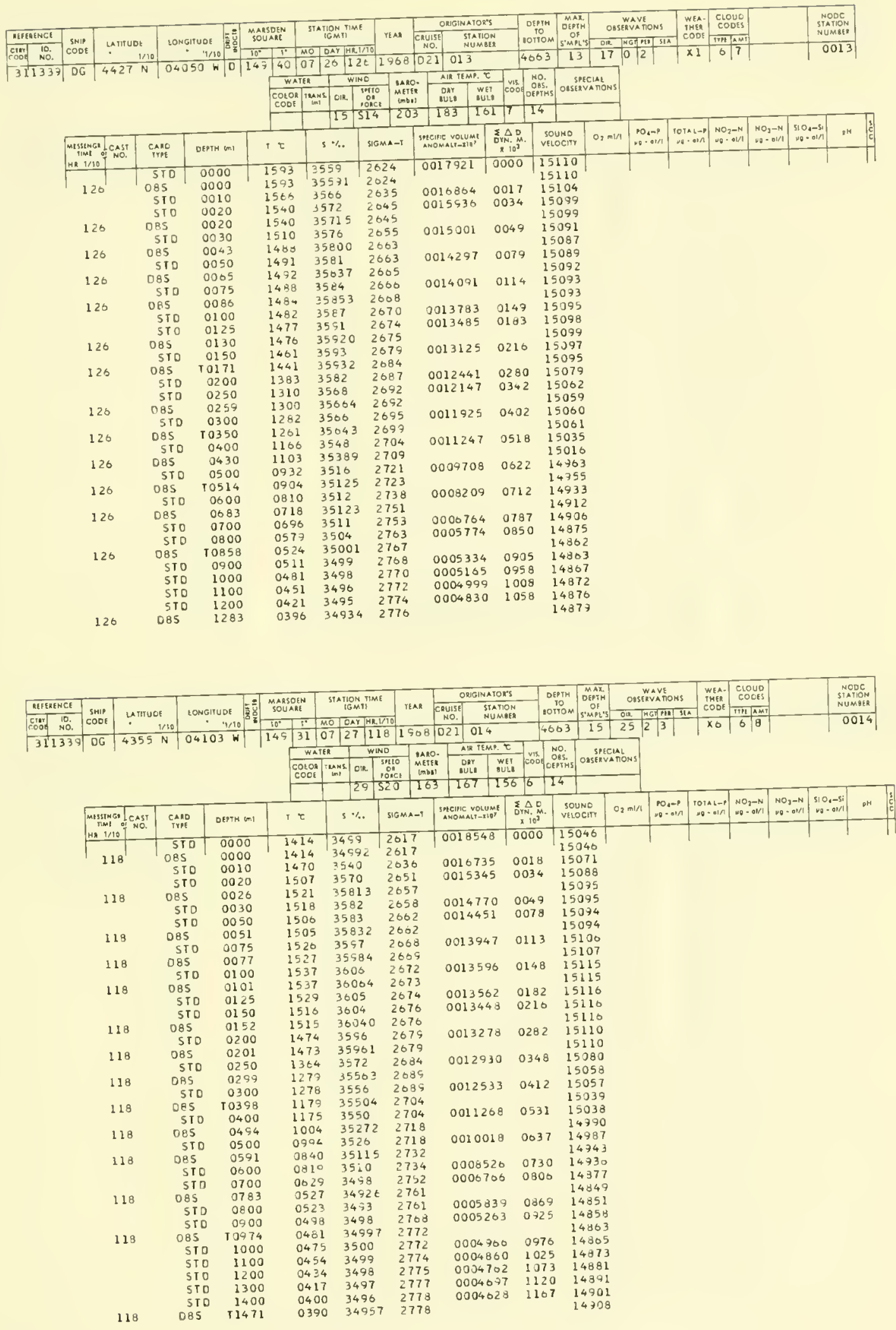
TABLE XII.-Continued

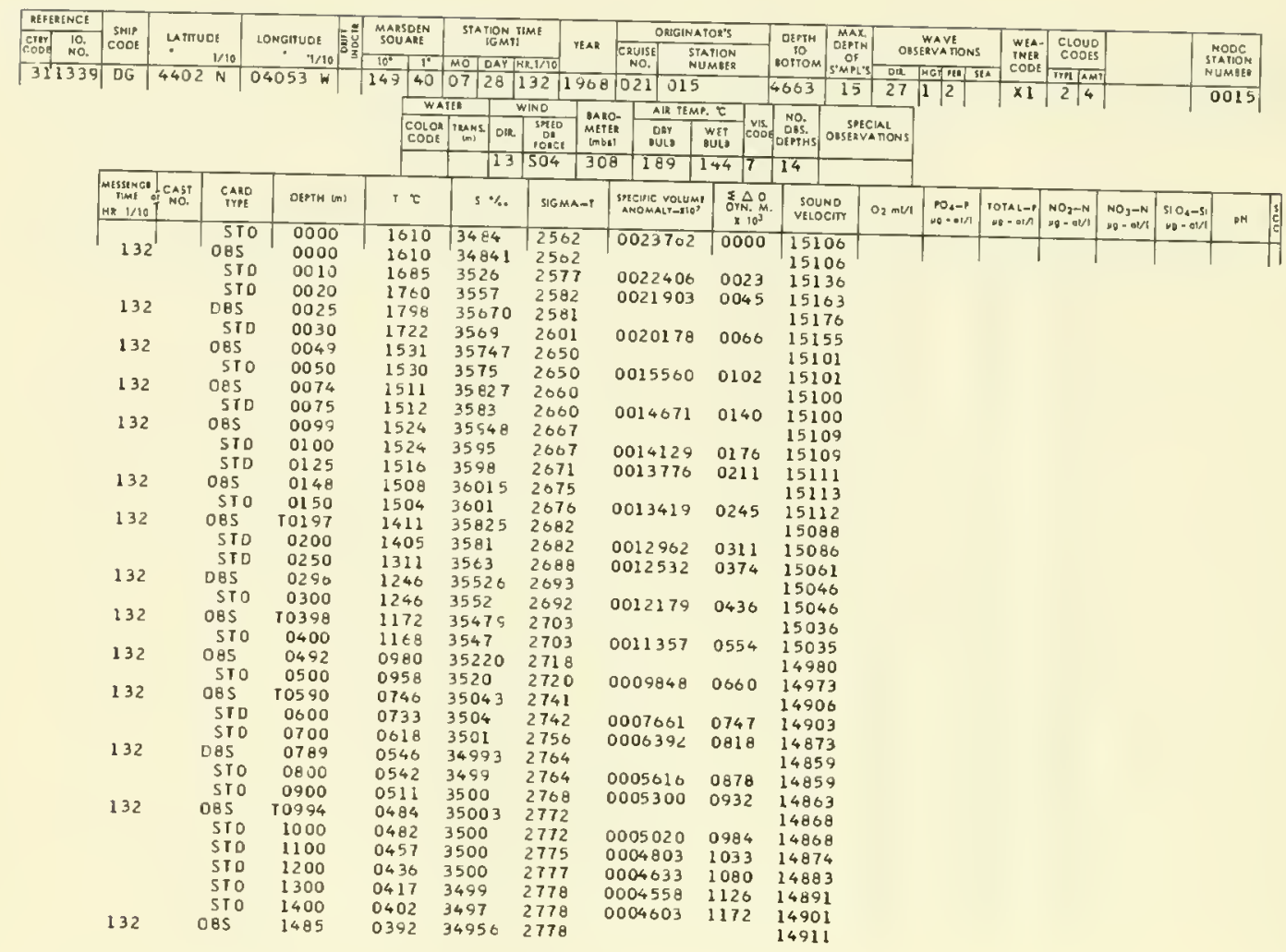

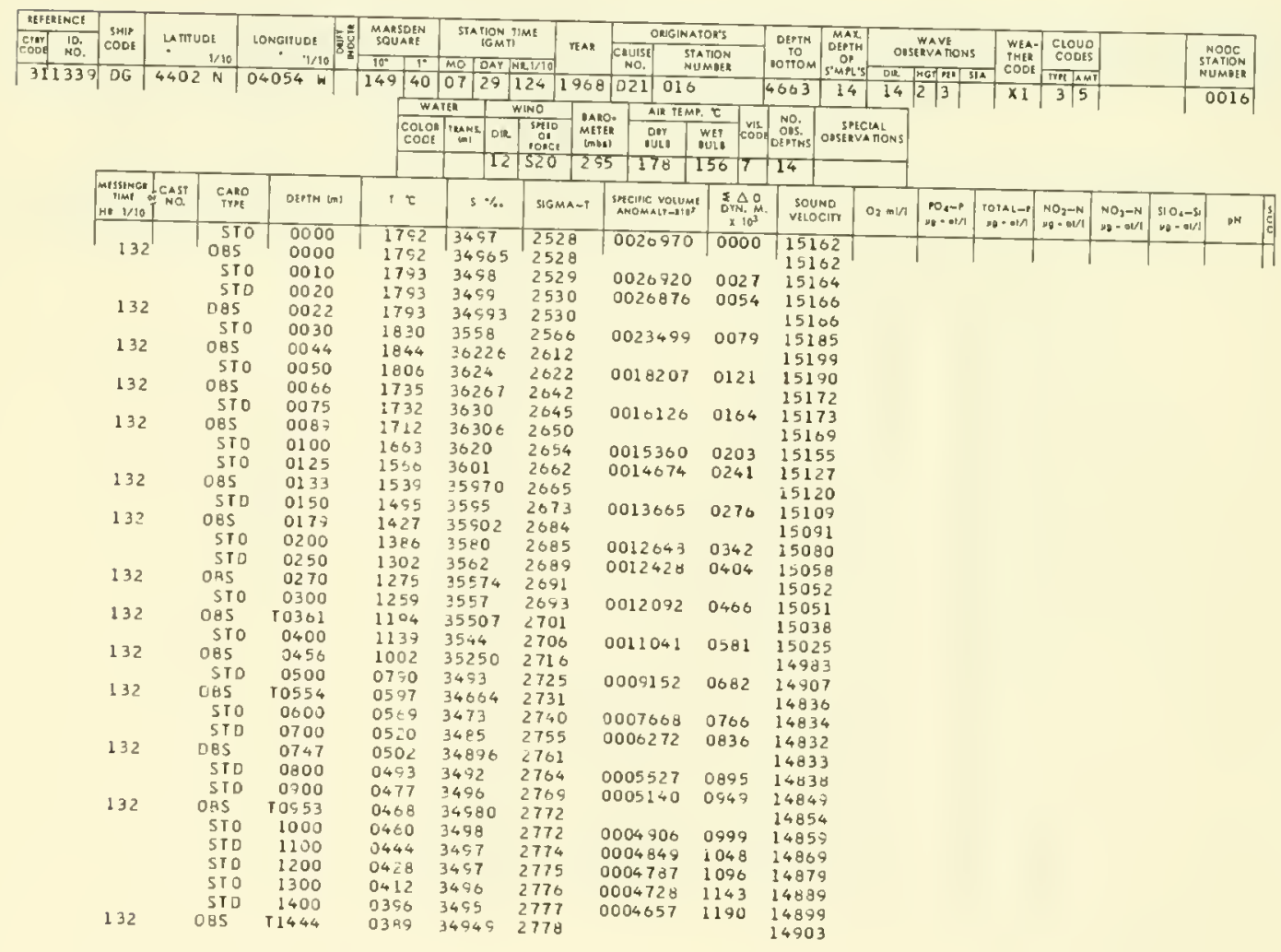


TABLE XII.-Continued

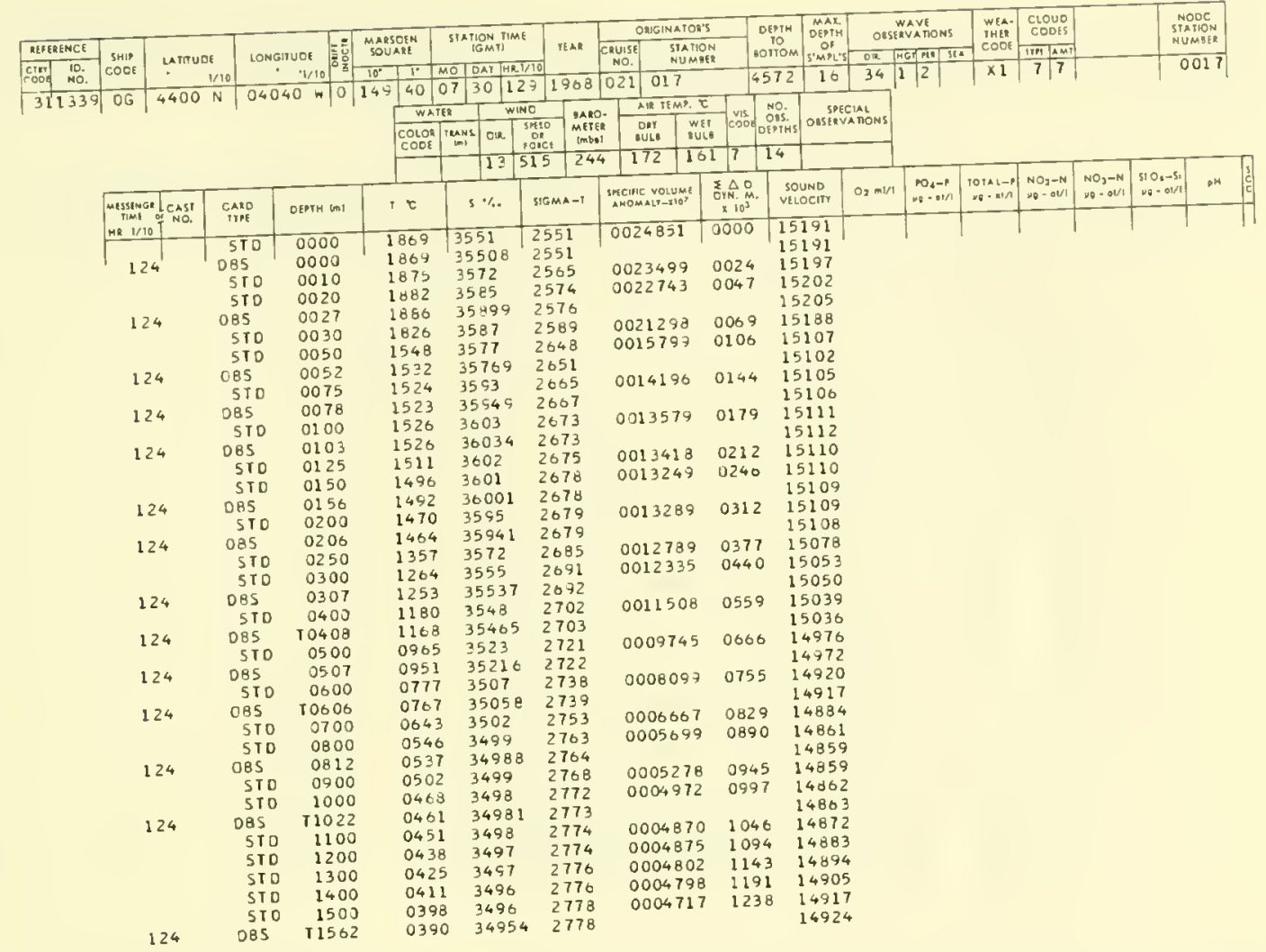

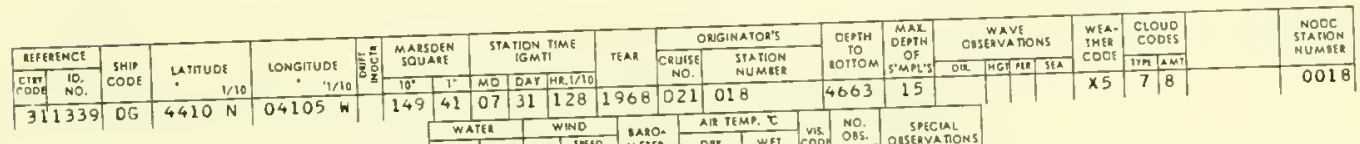

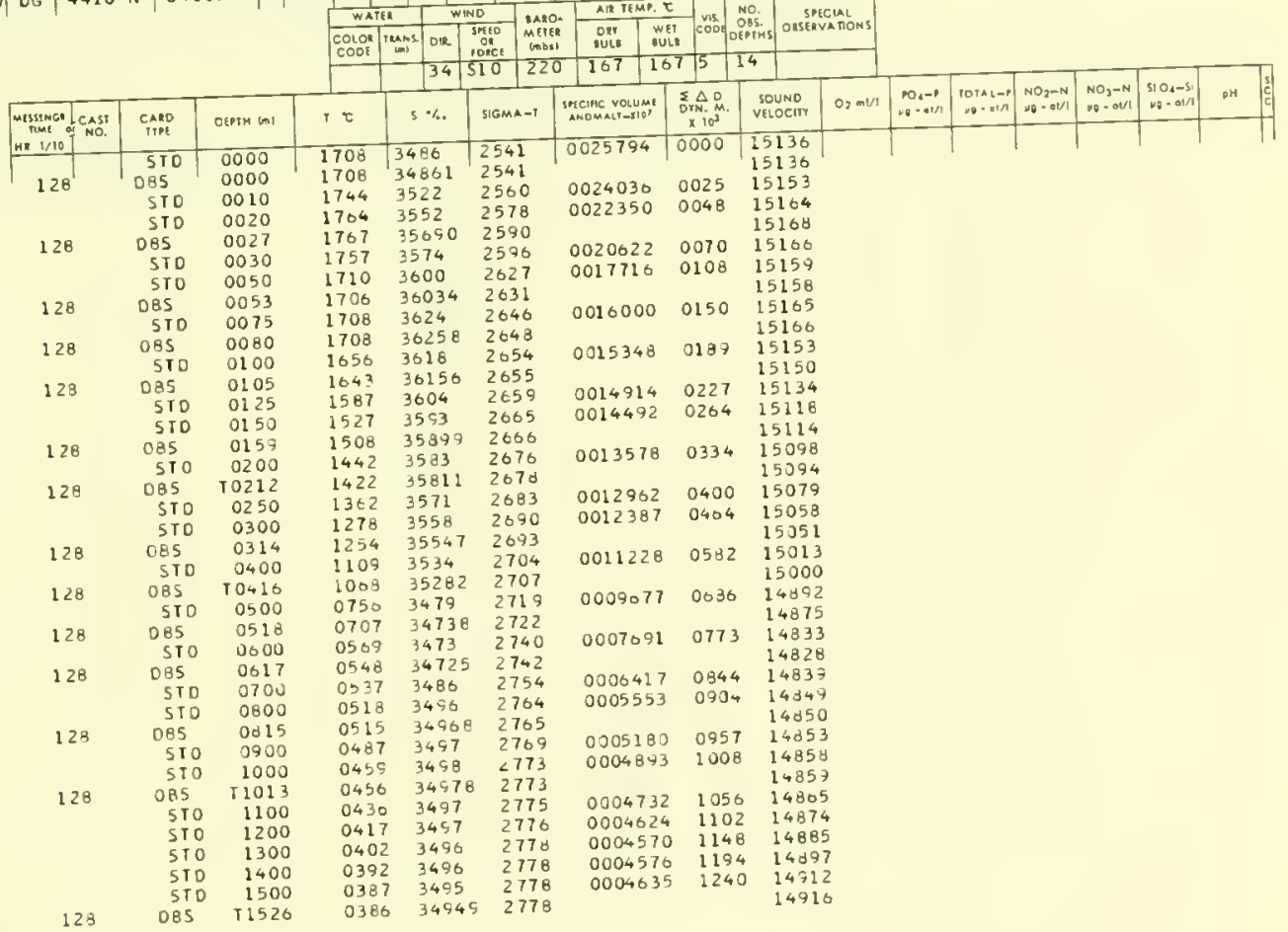


TABLE XII.-Continued

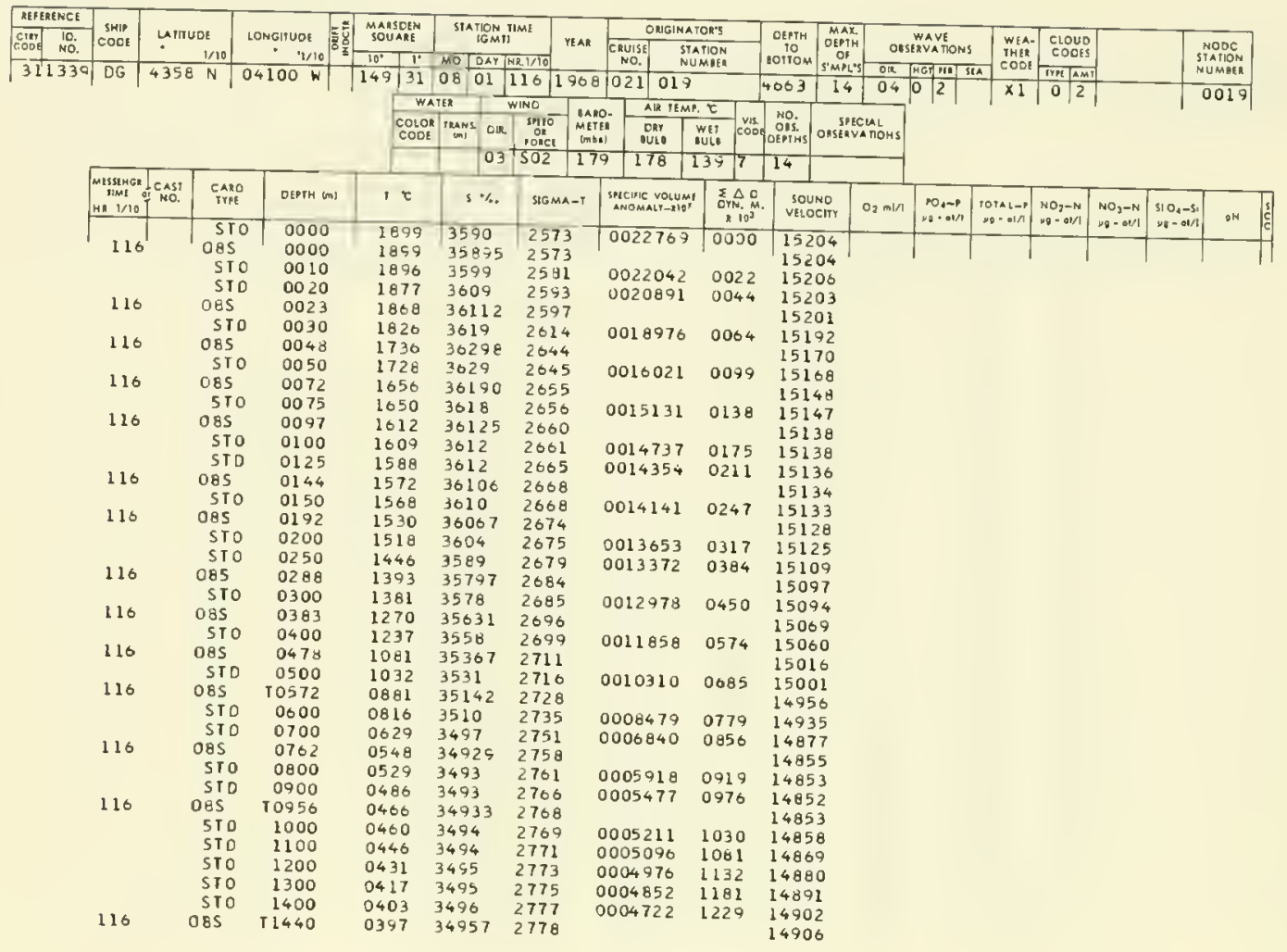

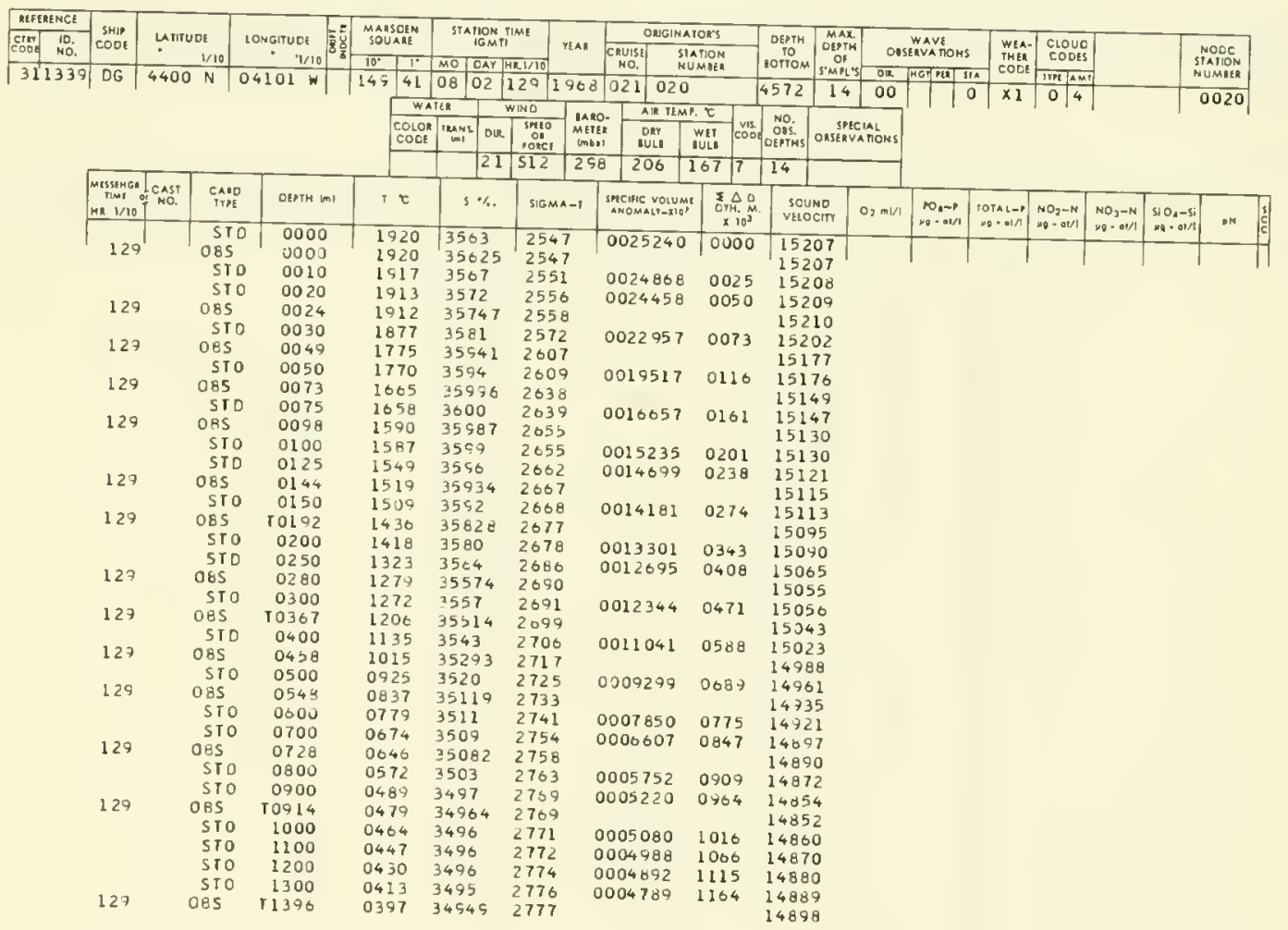




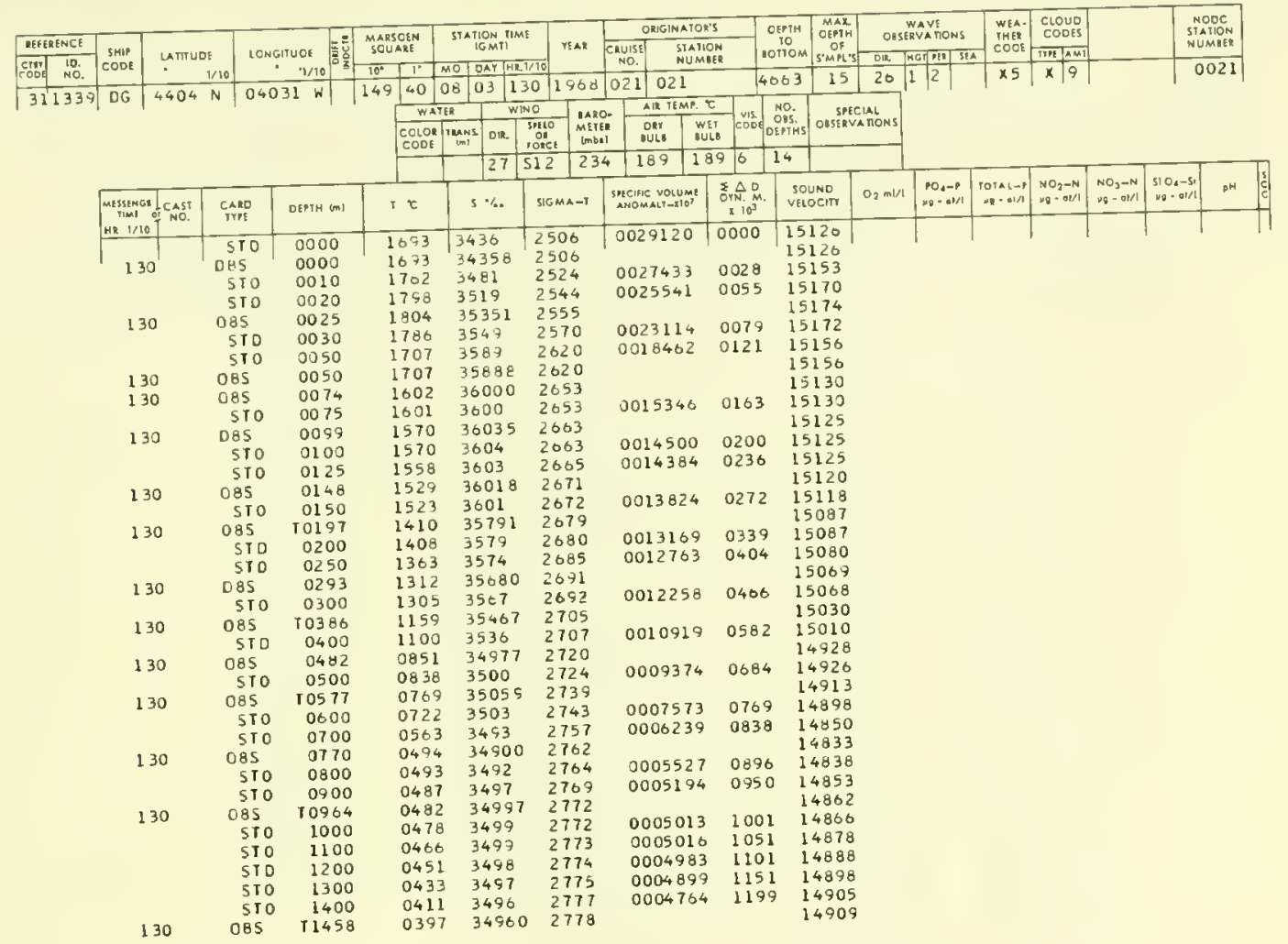

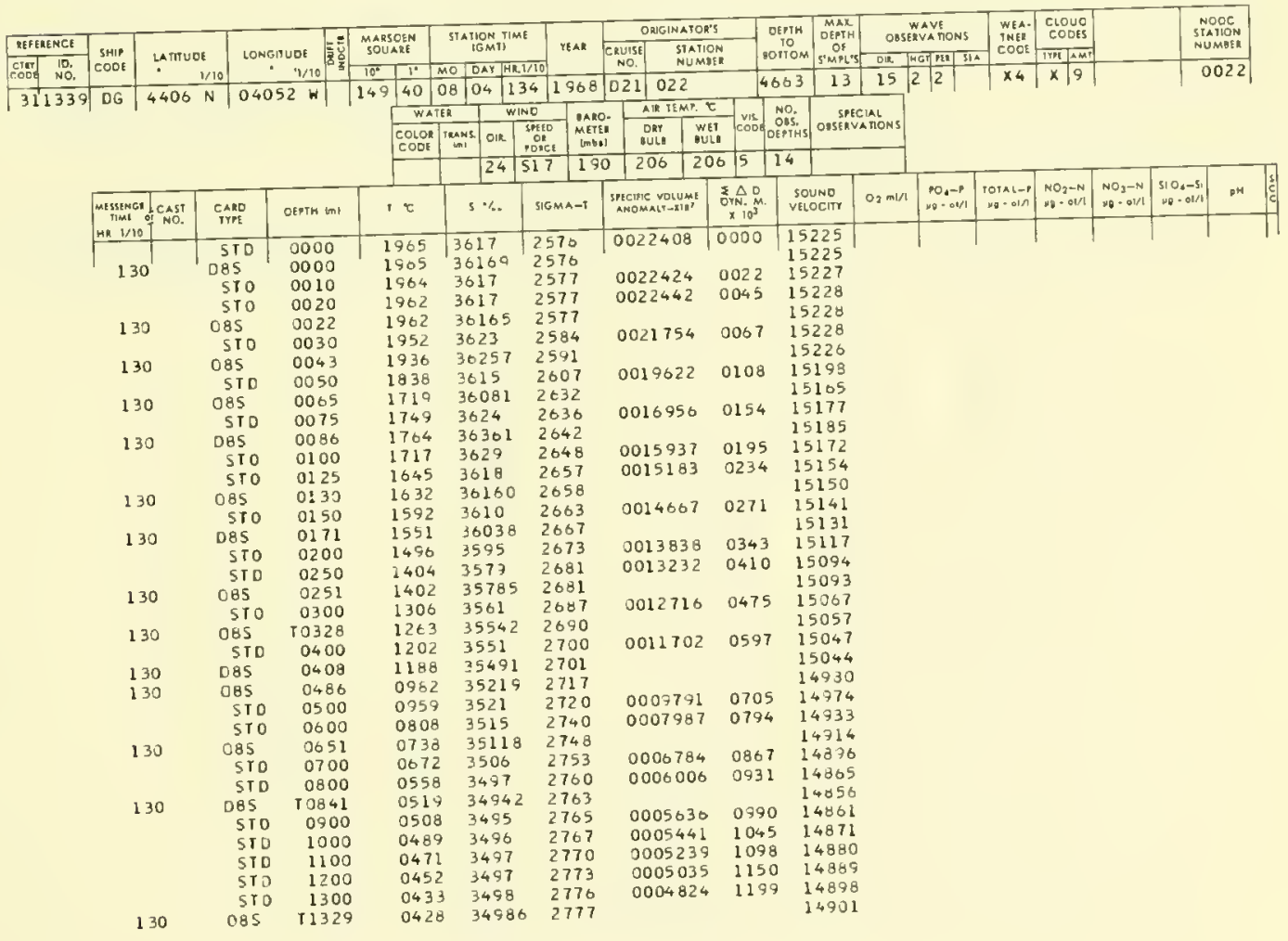


TABLE XIII.-Observed and interpolated oceanographic data for stations taken by USCGC COOK INLET at Ocean Station DELTA, 5 August-25 August 1968, prepared from NODC listing No. $31-1316 \mathrm{CO}$.

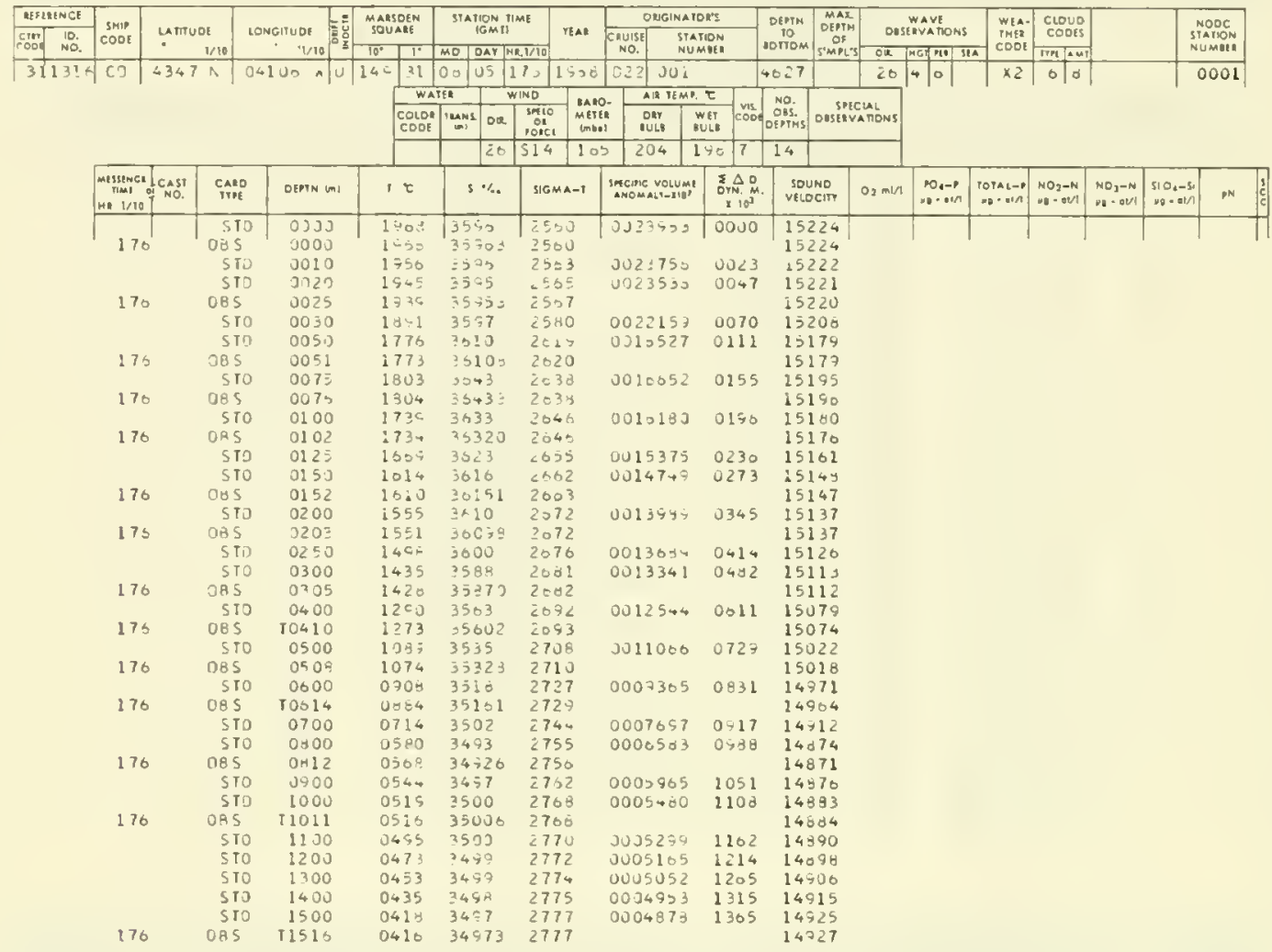

\begin{tabular}{|c|c|c|c|c|c|c|c|c|c|c|c|c|c|c|c|c|}
\hline \multirow{2}{*}{\begin{tabular}{|c|c|} 
BEFGENCE \\
Crnd & 10. \\
\end{tabular}} & \multirow[b]{2}{*}{ soot } & \multirow[b]{2}{*}{$\begin{array}{l}\text { YTITUDE } \\
\text { V/10 }\end{array}$} & \multirow{2}{*}{ LONGIIUOE } & \multirow{2}{*}{\multicolumn{2}{|c|}{$\begin{array}{l}\text { MAASDEN } \\
\text { SOUAEE }\end{array}$}} & \multirow{2}{*}{\multicolumn{2}{|c|}{ 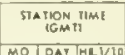 }} & \multirow[b]{2}{*}{ PEAE } & \multicolumn{2}{|c|}{ ONGINATONS } & \multirow{2}{*}{$\begin{array}{c}\text { OLPPH } \\
\text { PO } \\
\text { COITOM }\end{array}$} & \multirow{2}{*}{\begin{tabular}{|c|}
$\operatorname{MAx}$ \\
OEV? \\
of \\
$\sin$ \\
\end{tabular}} & \multirow{2}{*}{ WAVE } & \multirow{2}{*}{$\begin{array}{l}\text { WEA- } \\
\text { THEA } \\
\text { CODE }\end{array}$} & \multirow{2}{*}{$\begin{array}{l}\text { Clouo } \\
\text { COOLS } \\
\text { TYPI }\end{array}$} & \multirow{2}{*}{$\begin{array}{l}\text { NODC } \\
\text { STALON } \\
\text { NUMBUI }\end{array}$} \\
\hline & & & & & & & & & $\begin{array}{l}\text { Cruise } \\
\text { No. } \\
\end{array}$ & $\begin{array}{l}\text { SIATION } \\
\text { NUUMER }\end{array}$ & & & & & & \\
\hline 311310 & co & $4407 N$ & $0.053 \mathrm{n}$ & 149 & $\angle 0$ & 05 & \begin{tabular}{|l|l|} 
OS 133 \\
\end{tabular} & ty25 & 022 & 002 & 4345 & & \begin{tabular}{l|l|l|l}
03 & 6 & 3 &
\end{tabular} & $x_{1}$ & \begin{tabular}{l|l}
6 \\
\end{tabular} & 0002 \\
\hline
\end{tabular}

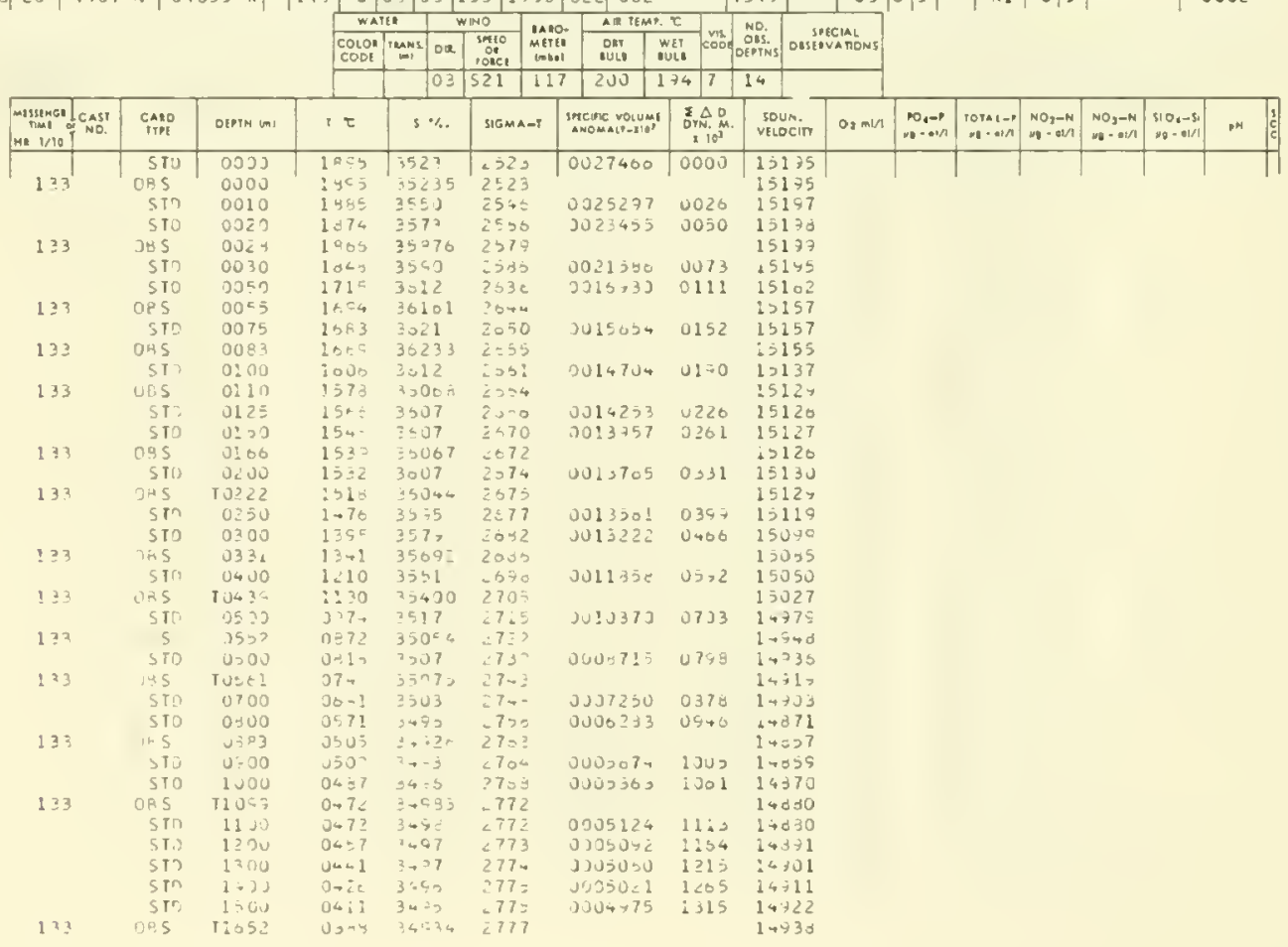


TABLE XIII.-Continued

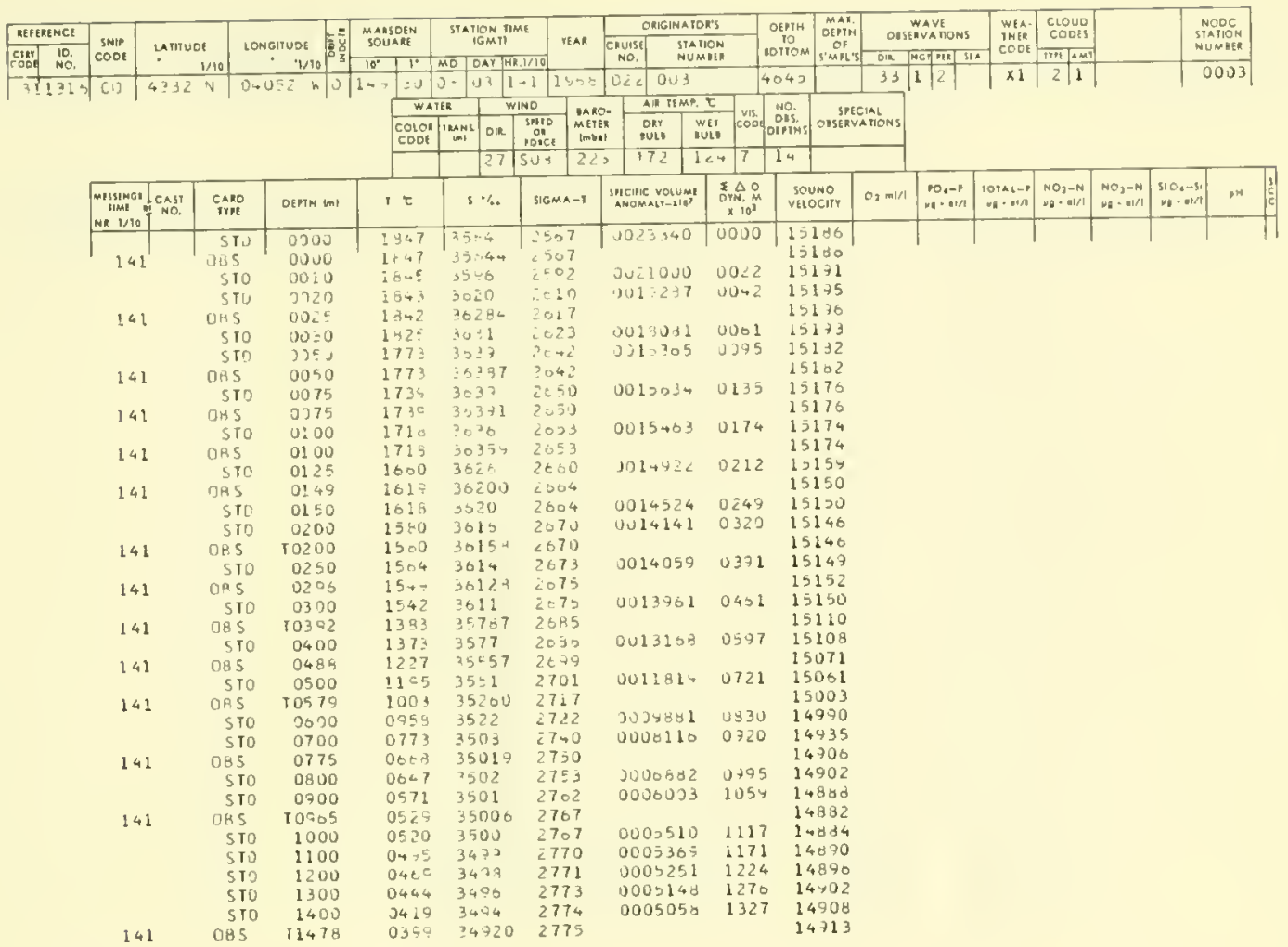

\begin{tabular}{|c|c|c|c|c|c|c|c|c|c|c|c|c|c|c|c|c|c|c|}
\hline REFTENCE & \multirow{2}{*}{$\begin{array}{c}3 N B \\
C O D E\end{array}$} & \multirow{2}{*}{\multicolumn{2}{|c|}{$\begin{array}{c}\text { LATIU } \\
\quad 1 / 10\end{array}$}} & \multirow{2}{*}{ LONGITUDE } & \multirow{2}{*}{$\begin{array}{l}\text { MARSDEN } \\
\text { SOUARE }\end{array}$} & \multirow{2}{*}{ STA TION MIME } & \multirow{2}{*}{ YEAR } & \multicolumn{2}{|c|}{ ORGINATOR'S } & & \multirow{2}{*}{$\begin{array}{c}\text { MAX } \\
\text { OEPT } \\
\text { Of } \\
\text { SMRLs }\end{array}$} & \multicolumn{2}{|c|}{$\begin{array}{l}\text { WAVE } \\
\text { OUSEMATIONS }\end{array}$} & \multirow{2}{*}{$\begin{array}{l}\text { WEA- } \\
\text { IMER } \\
\text { COOE }\end{array}$} & \multirow{2}{*}{$\begin{array}{l}\text { Clouo } \\
\text { COOL15 } \\
\end{array}$} & & & \multirow{2}{*}{ 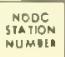 } \\
\hline \begin{tabular}{l|l}
$\mathrm{CnN}$ & 10. \\
$\mathrm{CoOa}$ & $\mathrm{NO}$.
\end{tabular} & & & & & & & & $\begin{array}{c}\text { Conist: } \\
\text { NO. }\end{array}$ & $\begin{array}{l}\text { SFA PION } \\
\text { NUMUER }\end{array}$ & & & of & \multirow{2}{*}{\begin{tabular}{|l|l|l|} 
MGG & PI: \\
3 & 3 & SIA \\
\end{tabular}} & & & & & \\
\hline उ11316 & $\mathrm{CO}$ & 4401 & $N$ & 041046 & \begin{tabular}{|l|l|}
44 & 410
\end{tabular} & $0+0 \div$ & \begin{tabular}{|l|l|}
215 & 1958 \\
\end{tabular} & 0223004 & & $\frac{10}{1010 \mathrm{M}}$ & & 25 & & $x_{6}$ & \begin{tabular}{|l|l|}
7 & 7 \\
\end{tabular} & & & 0004 \\
\hline & & & & & WAT & TER $1 \frac{1}{x}$ & WIND & - AII TEM. & & No. & & & & & & & & \\
\hline & & & & & \begin{tabular}{ll|} 
COLOK \\
COOE \\
\end{tabular} & 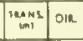 & $\begin{array}{l}\text { spicio } \\
\text { on } \\
\text { IOnCE }\end{array}$ & \begin{tabular}{l|l} 
ORV & $W$ \\
IULI & D \\
\end{tabular} & $\begin{array}{l}\text { WET } \\
\text { UII }\end{array}$ & $\begin{array}{l}\text { OES. } \\
\text { OEMT: }\end{array}$ & OLSERVA & A MONS & & & & & & \\
\hline & & & & & & 28 & 514 & 171 & \begin{tabular}{l|l}
67 & 7
\end{tabular} & 14 & & & & & & & & \\
\hline & $\begin{array}{l}\text { MessimG } \\
\text { Niw1 } \\
\text { Ha } 1 / 10\end{array}$ & leAST & $\underset{\text { TWRE }}{\text { CARO }}$ & DEPT (m) & I & $s \%$ & SIGMA-T & 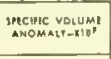 & 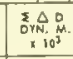 & $\begin{array}{c}\text { so } \\
\text { vet }\end{array}$ & $\begin{array}{l}\text { DUNO } \\
\text { LOCISY }\end{array}$ & O) $m / 1 / 1$ & 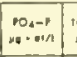 & 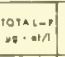 & 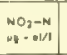 & $\begin{array}{l}\mathrm{NO}_{3}-\mathrm{N} \\
\mathrm{NO}-\mathrm{MI}\end{array}$ & $\begin{array}{l}510,-5 \\
40.0+1 / 1\end{array}$ & .M \\
\hline & & & $\begin{array}{l}5 \text { TO } \\
\text { Sh }\end{array}$ & $\begin{array}{l}0000 \\
0070\end{array}$ & 1 구 3 & 3547 & $25<7$ & $30 \bar{c}>1 \equiv 3$ & 303 & & 5185 & & & & & & & \\
\hline & 21 & & $\begin{array}{l}\text { JH5 } \\
\text { STO }\end{array}$ & $\begin{array}{l}0000 \\
0010\end{array}$ & In? & $3540=$ & 2547 & & & & 5185 & & & & & & & \\
\hline & & & STD & $\begin{array}{l}0010 \\
0020\end{array}$ & 1 करण & $38 \cdot 8$ & $=5.31$ & $\begin{array}{l}00-\angle 01+ \\
00 i>529\end{array}$ & $\begin{array}{l}0023 \\
0044\end{array}$ & $1=$ & $\begin{array}{l}175 \\
135\end{array}$ & & & & & & & \\
\hline & 21 & & OHS & $002 x$ & 1723 & 35057 & 2020 & & & & 5100 & & & & & & & \\
\hline & & & STD & 0030 & 1712 & $35: 0$ & 2024 & $0017+35$ & $0 \cup 53$ & & 158 & & & & & & & \\
\hline & & & $\begin{array}{l}\text { STR } \\
\text { OPS }\end{array}$ & 0050 & $1 . .5$ & $32+9$ & $2=02$ & 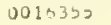 & DU:7 & & 5139 & & & & & & & \\
\hline & 21 & & $\begin{array}{l}\text { nPS } \\
\text { STr }\end{array}$ & $\begin{array}{l}13052 \\
0075\end{array}$ & $10^{2}=$ & , 50 म: & $20+3$ & & & & 130 & & & & & & & \\
\hline & $\geq 1$ & & oes & $\begin{array}{l}0075 \\
0075\end{array}$ & $15 \mathrm{se}$ & 10 & 2004 & $00 ! 4305$ & $0: 35$ & & 120 & & & & & & & \\
\hline & & & STo & $\begin{array}{l}0073 \\
2130\end{array}$ & $\begin{array}{l}15+1 \\
\text { ise? }\end{array}$ & 34107 & $2 \pm 00$ & 3134 & 0170 & & 126 & & & & & & & \\
\hline & 21 & & TAS & 0104 & $\begin{array}{l}i 5 c 2 \\
1 \leq j ;\end{array}$ & $\begin{array}{l}1=11 \\
00102\end{array}$ & $\begin{array}{l}2579 \\
2071\end{array}$ & 3313794 & 0170 & & $\begin{array}{l}123 \\
123\end{array}$ & & & & & & & \\
\hline & & & STD & 0125 & 1255 & 301 ? & 2072 & $001 \div 707$ & $0<05$ & & 5125 & & & & & & & \\
\hline & & & ST & 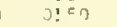 & $15=0$ & 3011 & $=74$ & 3023051 & 0239 & & 5128 & & & & & & & \\
\hline & 21 & & Dos & 0155 & $154 ?$ & 30115 & ¿374 & & & & $512 d$ & & & & & & & \\
\hline & & & STO & 0200 & 1534 & 1010 & -075 & $00: 2041$ & $0: 07$ & & 5133 & & & & & & & \\
\hline & 21 & & UIS & $10 \geq 12$ & $15:=$ & 10044 & 2070 & & & & 2132 & & & & & & & \\
\hline & & & STO & $02 \equiv 0$ & 14,2 & $s 00^{2}$ & $257 \mathrm{c}$ & 0013530 & 0375 & & $51<7$ & & & & & & & \\
\hline & & & STR & 0300 & $14+0$ & アシフル & $20+4$ & 0013317 & 0442 & & $5: 15$ & & & & & & & \\
\hline & ? & & ans 5 & 0311 & $14<t$ & 35075 & $\angle 0+2$ & & & & 5112 & & & & & & & \\
\hline & 21 & & STO & $\begin{array}{r}104100 \\
0500\end{array}$ & $\begin{array}{l}1<>4 \\
1 \leqslant 14\end{array}$ & $\begin{array}{l}35 \leqslant 70 \\
3=65\end{array}$ & 2314 & & & 15 & 5070 & & & & & & & \\
\hline & 21 & & $n+5$ & $051:$ & 1070 & $\begin{array}{l}3=43 \\
25347\end{array}$ & $\begin{array}{l}2707 \\
2711\end{array}$ & $w 0: 1152$ & $00+0$ & $\begin{array}{l}12 \\
15\end{array}$ & $\begin{array}{l}50 \leq 2 \\
5021\end{array}$ & & & & & & & \\
\hline & & & sius & 0,00 & $07\llcorner 2$ & 3410 & $27=7$ & $000 \div 157$ & (3) $7+2$ & & 62,4 & & & & & & & \\
\hline & 21 & & OAS & 10620 & 07.3 & $? \div 01$ & 2730 & & & & +906 & & & & & & & \\
\hline & & & sin & $070 \mathrm{~L}$ & 6700 & 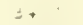 & 2744 & J.) 7074 & 0,70 & !4 & $=706$ & & & & & & & \\
\hline & & & STก & OAOJ & $063 n$ & 3504 & $>750$ & QUU0 507 & 0947 & & 4878 & & & & & & & \\
\hline & 21 & & TAS & $0 \times 1:$ & $0,2:$ & $2502 !$ & 2758 & & & & $4+3+0$ & & & & & & & \\
\hline & & & ST) & 3907 & 0547 & $34 \div 7$ & -763 & 0.00534 & .009 & & 4078 & & & & & & & \\
\hline & & & STO & $: 000$ & 0472 & $34 \div 3$ & 2700 & $000>390$ & 1005 & & 4003 & & & & & & & \\
\hline & 21 & & OHS & T1019 & 0400 & 749.23 & 2709 & & & & $48>1$ & & & & & & & \\
\hline & & & 570 & 1100 & 0422 & 3403 & 2772 & $000 \div 200$ & 1113 & & 4072 & & & & & & & \\
\hline & & & $5\left(T_{1}\right)$ & 1200 & $04 \cdot 3$ & 3474 & 2771 & $1000=1 \leq 5$ & 1.70 & & .354 & & & & & & & \\
\hline & & & $5 \pi 0$ & 1300 & 0433 & 3.24 & 2773 & $000>115$ & $12=2$ & & $+3 \geqslant 7$ & & & & & & & \\
\hline & & & STo & 1400 & $0+24$ & 3.45 & 2774 & D.J13504J & 1273 & & 920 & & & & & & & \\
\hline & & & STII & 1500 & $04: 4$ & 3450 & 2776 & 000.001 & $1 \geq 23$ & & 423 & & & & & & & \\
\hline & 21 & & uns & 11525 & 0412 & 34457 & $=773$ & & & & +725 & & & & & & & \\
\hline
\end{tabular}


TABLE XIII.-Continued

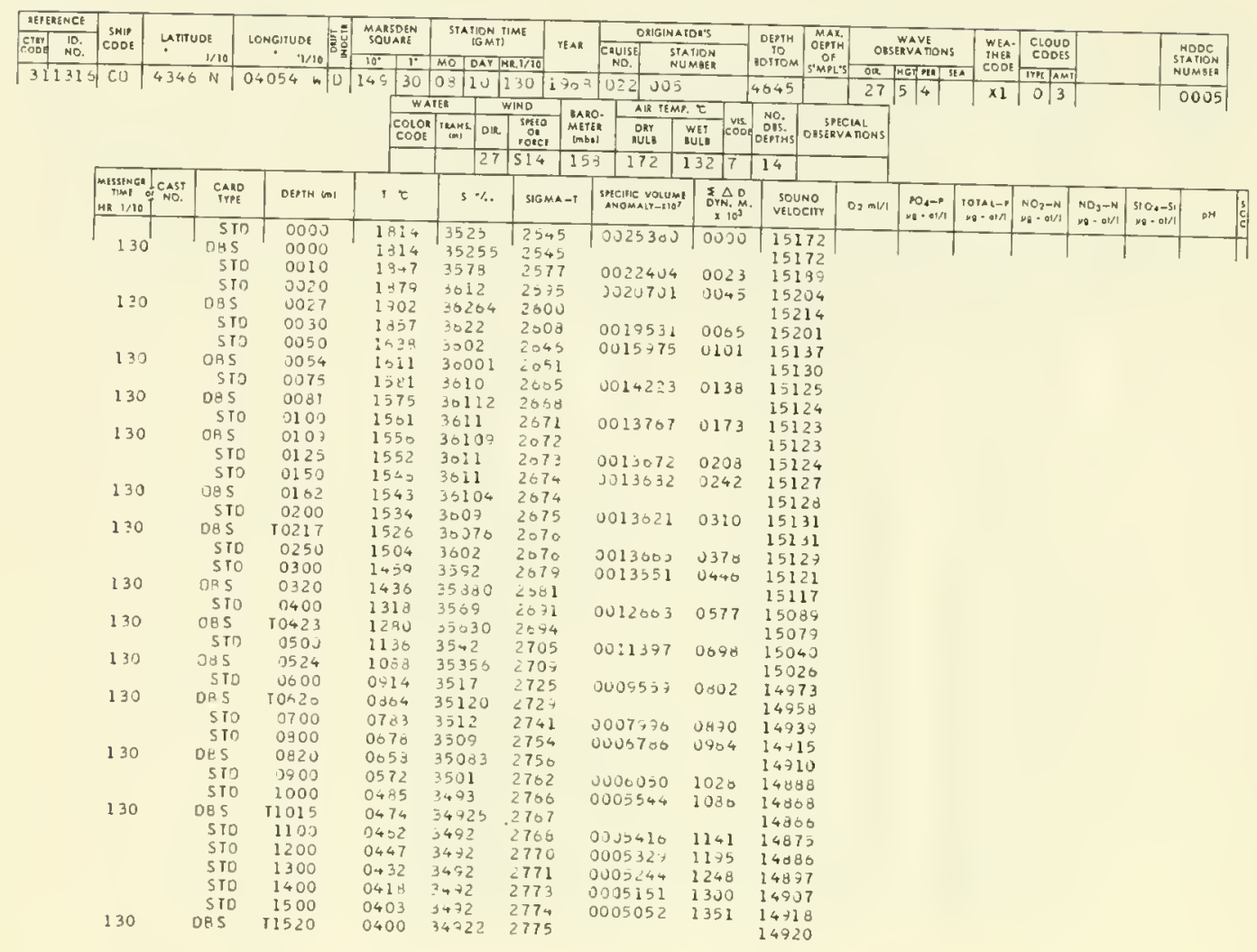

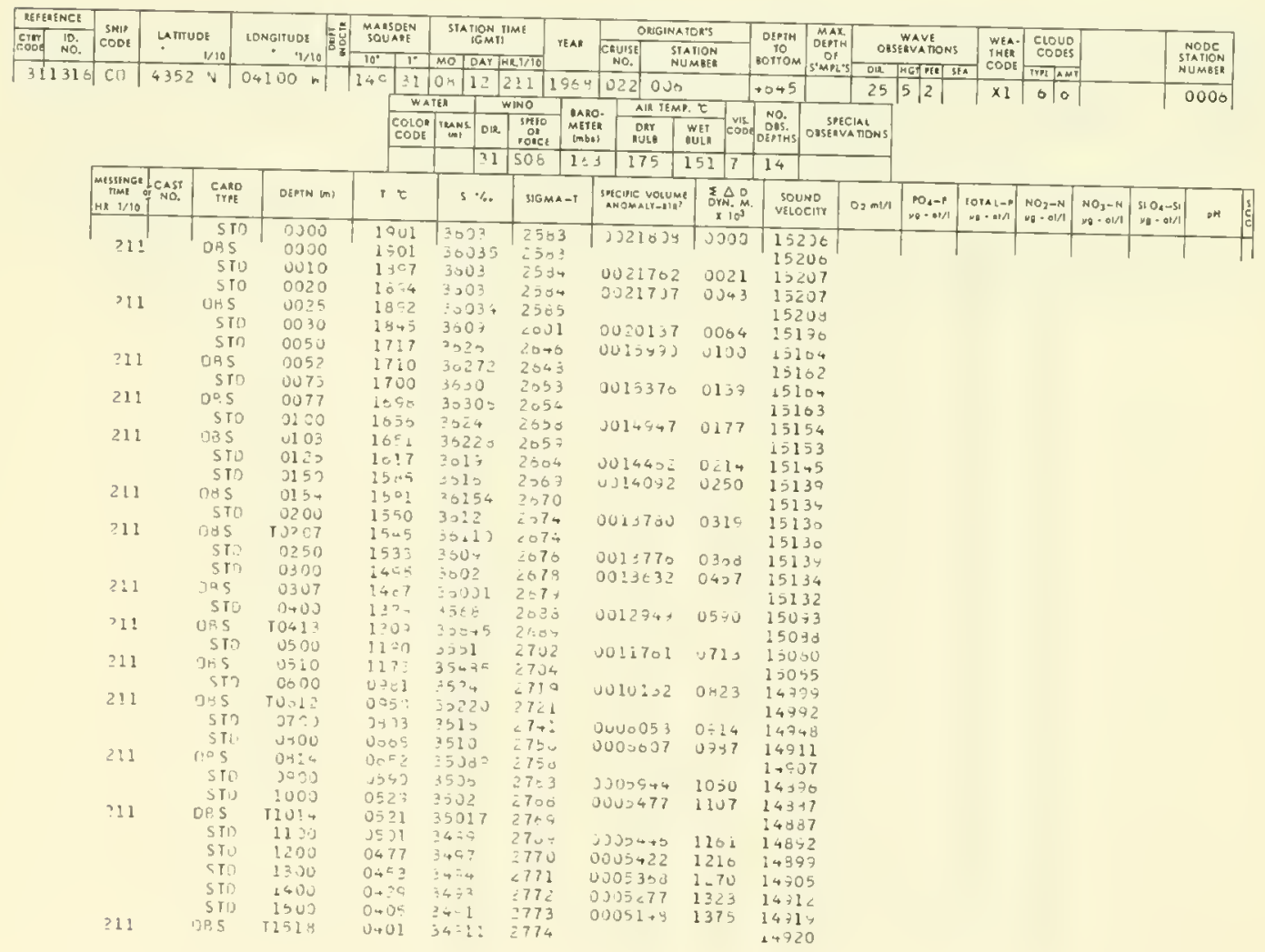


TABLE XIII.-Continued
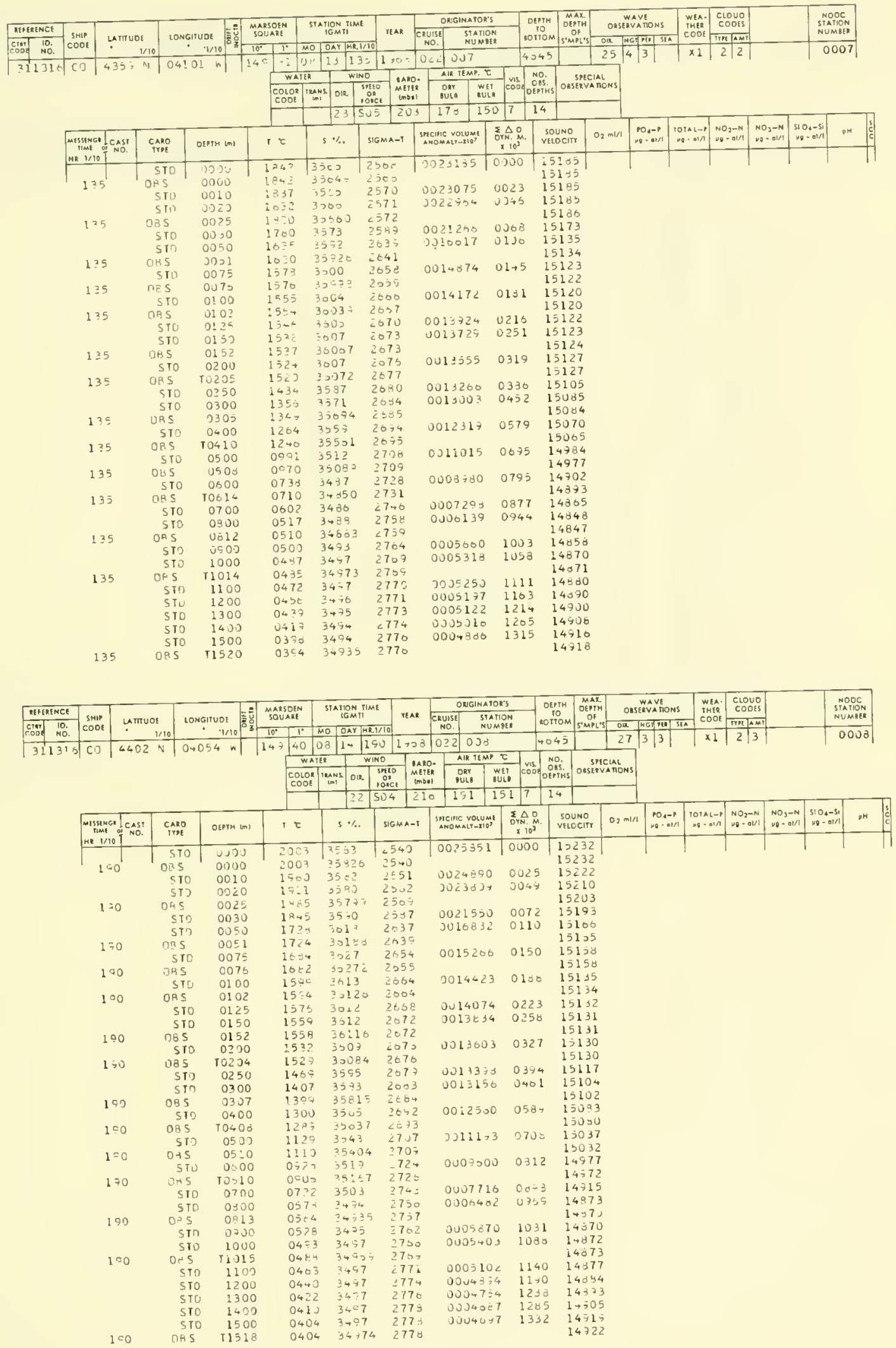
TABLE XIII.—Continued

\begin{tabular}{|c|c|c|c|c|c|c|c|c|c|c|c|c|c|c|c|c|c|c|}
\hline \multirow{2}{*}{ 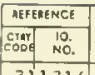 } & \multirow{2}{*}{ cols } & \multirow{2}{*}{\multicolumn{2}{|c|}{$\begin{array}{c}\text { untrude } \\
1 / 10\end{array}$}} & \multirow{2}{*}{$\begin{array}{c}\text { LANGITUDE } \\
\text { 1/10 }\end{array}$} & $\begin{array}{c}\text { MARSDEN } \\
\text { SOUARE }\end{array}$ & \multicolumn{2}{|c|}{$\begin{array}{c}\text { STATION TIME } \\
\text { IG MII }\end{array}$} & \multirow{2}{*}{ reAt } & \multicolumn{2}{|c|}{ OULINATOR'S } & \multirow{2}{*}{\begin{tabular}{|c|} 
DEPYN \\
TO \\
10 HIO \\
\end{tabular}} & \multirow{2}{*}[\begin{array}{c}{\operatorname{MAX}}\\
{\operatorname{DEPTM}}\\
{\text{OF}}\\
{\operatorname{SinP},3}\end{array}]{} & \multirow{2}{*}{ 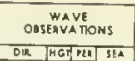 } & \multirow{2}{*}{$\begin{array}{l}\text { WEA. } \\
\text { TNEA } \\
\text { COOE }\end{array}$} & \multirow{2}{*}{ 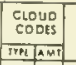 } & & & \\
\hline & & & & & $10^{\circ} 1^{\circ}$ & MO OAY & Maci/10 ${ }^{r e}$ & & $\begin{array}{ll}\text { CRUISE } & \text { STAT } \\
\text { NO. } & \text { NUSM }\end{array}$ & $\begin{array}{l}\text { ATION } \\
\text { UMIER }\end{array}$ & & & & & & & & $\begin{array}{l}\text { NODC } \\
\text { STAPION } \\
\text { NUMBER }\end{array}$ \\
\hline & $\mathrm{CD}$ & 4414 & $N$ & \begin{tabular}{ll|l|l}
$04118 \mathrm{~W}$ & 0 & 1
\end{tabular} & \begin{tabular}{l|l|}
149 & 41 \\
\end{tabular} & \begin{tabular}{l|l|}
03 & 15 \\
\end{tabular} & 134119 & 908 & \begin{tabular}{|l|l|}
022 & 000 \\
\end{tabular} & & 4699 & 19 & \begin{tabular}{|l|l|}
5 & 3
\end{tabular} & $x_{1}$ & 36 & & & 0009 \\
\hline & & & & & WAI & & WINO & Mano. & - ATR TEMP. & $c$ & & & & & & & & \\
\hline & & & & & \begin{tabular}{ll|}
$\mathrm{COLOR}$ \\
$\mathrm{CODE}$ \\
\end{tabular} & 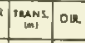 & 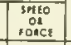 & $\begin{array}{l}\text { MetEk } \\
\text { Smbol }\end{array}$ & \begin{tabular}{l|l} 
ORY \\
ouid & ou \\
\end{tabular} & 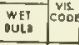 & oestivs a & $\begin{array}{c}\text { SPECIRL } \\
\text { OISERYANENS }\end{array}$ & & & & & & \\
\hline & & & & & & 05 & 510 & 173 & 104 & \begin{tabular}{l|l|}
172 & 7 \\
\end{tabular} & 21 & & & & & & & \\
\hline & 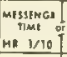 & $\begin{array}{l}\text { lcast } \\
\text { of }\end{array}$ & $\begin{array}{l}\text { CARO } \\
\text { TYPE }\end{array}$ & OETH $(m)$ & T & $3 \%$ & SIGMA- & & 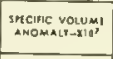 & $\begin{array}{l}\text { E. } \Delta \mathrm{D} \\
\text { DrN } \\
\times 10^{3}\end{array}$ & $\begin{array}{l}\text { Sound } \\
\text { VELOCII }\end{array}$ & $O_{2} \mathrm{~mL} / \mathrm{t}$ & 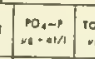 & 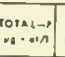 & $\begin{array}{l}\mathrm{NO}_{2}-\mathrm{N} \\
\mathrm{NO}-\mathrm{O}, \mathrm{OU1}\end{array}$ & $\left|\begin{array}{l}\mathrm{NO}_{1}-\mathrm{N} \\
\mathrm{NO}-\mathrm{O} / \mathrm{A}\end{array}\right|$ & $\begin{array}{l}310,-51 \\
20+0 t / 1\end{array} \mid$ & r \\
\hline & & & Sio & 0000 & 1875 & 3504 & 2513 & & 0028392 & 0000 & $151 \mathrm{~d}$ & $d 7$ & & & & & & \\
\hline & 134 & & 085 & $\begin{array}{l}0000 \\
0010\end{array}$ & $\begin{array}{l}1875 \\
1875\end{array}$ & $\begin{array}{l}35041 \\
35 ! 3\end{array}$ & 2513 & & & & 1518 & 87 & & & & & & \\
\hline & & & $\begin{array}{l}510 \\
5 T 0\end{array}$ & $\begin{array}{l}0010 \\
0020\end{array}$ & $\begin{array}{l}1877 \\
1803\end{array}$ & $\begin{array}{l}35 ! 3 \\
3525\end{array}$ & $\begin{array}{l}2517 \\
2528\end{array}$ & & $\begin{array}{l}0027851 \\
002711\end{array}$ & 0028 & 1219 & 171 & & & & & & \\
\hline & 134 & & 085 & 0024 & 1885 & $\begin{array}{l}3525 \\
35314\end{array}$ & $\begin{array}{l}2528 \\
2532\end{array}$ & & 0027113 & 0055 & $\begin{array}{l}1519 \\
1519\end{array}$ & 97 & & & & & & \\
\hline & & & STD & 0030 & 1756 & 3343 & 2567 & & 0023441 & 0030 & 1517 & 75 & & & & & & \\
\hline & & & STO & 0050 & $15 \div 5$ & 3585 & 2643 & & 0015201 & 0120 & 1512 & 22 & & & & & & \\
\hline & 134 & & DBS & 0050 & 1575 & 35.47 & 2043 & & & & 1512 & 22 & & & & & & \\
\hline & 134 & & DBS & 0074 & 1544 & 35922 & 2600 & & & & 1511 & & & & & & & \\
\hline & & & STO & 0075 & 1541 & 3592 & 2001 & & ju14625 & 0153 & $151 \mathrm{i}$ & 10 & & & & & & \\
\hline & & & STO & 0100 & 1477 & 3502 & 2675 & & 0013350 & 0194 & 1509 & 94 & & & & & & \\
\hline & 134 & & Des & 0100 & 1477 & 35920 & 2075 & & & & 15094 & & & & & & & \\
\hline & & & STD & 0125 & 1430 & 3592 & 2681 & & $001287 \bar{z}$ & 0227 & 15090 & & & & & & & \\
\hline & & & SIO & 0150 & 1434 & 3592 & 3684 & & 0012039 & 0258 & $1503 ?$ & & & & & & & \\
\hline & 134 & & OR 5 & 0150 & 1434 & 35917 & 2684 & & & & 15089 & & & & & & & \\
\hline & & & $5 T 0$ & 0200 & 1438 & 3576 & 2600 & & 0012502 & 0321 & 1509 & & & & & & & \\
\hline & 134 & & DRS & 10200 & 1433 & 35558 & 2680 & & & & 1209 & & & & & & & \\
\hline & & & STO & 0250 & 1385 & 3584 & 2659 & & 0012480 & 0384 & 15088 & & & & & & & \\
\hline & 134 & & $5 T 0$ & 0300 & $\begin{array}{l}1347 \\
1346\end{array}$ & $\begin{array}{l}3577 \\
35764\end{array}$ & 2091 & & 0012376 & 0446 & $\begin{array}{l}15083 \\
15083\end{array}$ & $\begin{array}{l}83 \\
83\end{array}$ & & & & & & \\
\hline & & & $\begin{array}{l}\text { D8 S } \\
5 \text { TO }\end{array}$ & $\begin{array}{l}0301 \\
0400\end{array}$ & $\begin{array}{l}1346 \\
1318\end{array}$ & $\begin{array}{l}35764 \\
3575\end{array}$ & $\begin{array}{l}2091 \\
2636\end{array}$ & & 0012204 & 0569 & 15050 & & & & & & & \\
\hline & 134 & & DRS & $\begin{array}{r}0400 \\
\mathrm{~T} 0401\end{array}$ & 1317 & 35749 & $26 \geqslant 5$ & & & & 15090 & & & & & & & \\
\hline & & & STD & 0500 & 1224 & 3557 & 2730 & & 0011944 & 0690 & 15072 & & & & & & & \\
\hline & $1 \geq 4$ & & DBS & 0502 & 1221 & 35565 & 2701 & & & & 15071 & & & & & & & \\
\hline & & & STD & 0600 & 1032 & 3528 & 2713 & & 0010774 & 0804 & 15018 & & & & & & & \\
\hline & 134 & & $\begin{array}{l}\text { DBS } \\
\text { STO }\end{array}$ & $\begin{array}{r}10500 \\
0700\end{array}$ & 1032 & 35277 & $\begin{array}{l}2713 \\
2754\end{array}$ & & 0006725 & 0891 & $\begin{array}{l}15010 \\
14919\end{array}$ & & & & & & & \\
\hline & & & SID & $\begin{array}{l}0700 \\
0000\end{array}$ & $\begin{array}{l}0727 \\
0533\end{array}$ & $\begin{array}{l}3518 \\
3509\end{array}$ & $\begin{array}{l}2754 \\
2773\end{array}$ & & 0004815 & 0949 & 14859 & & & & & & & \\
\hline & 134 & & DES & 0802 & 0535 & & & & & & & & & & & & & \\
\hline & & & sro & 0900 & 0523 & 3503 & 2769 & & 0005228 & 0999 & 14800 & & & & & & & \\
\hline & & & STO & 1000 & 0507 & 3495 & 2767 & & 0005534 & 1053 & 14879 & & & & & & & \\
\hline & 234 & & DAS & 11002 & 0509 & 34973 & 2707 & & & & 14879 & & & & & & & \\
\hline & & & STO & 110.1 & $04=4$ & 3419 & $\bar{C} 709$ & & 0005446 & 1108 & 14829 & & & & & & & \\
\hline & & & STO & 1200 & 0478 & 3493 & 2771 & & 0005337 & $1 \mathrm{ib} 2$ & 14900 & & & & & & & \\
\hline & & & STO & 1300 & $046 ?$ & 2498 & 2772 & & 0005227 & 1214 & 14910 & & & & & & & \\
\hline & & & STO & 1400 & 0446 & 3498 & 2774 & & 0005103 & 1260 & 14920 & & & & & & & \\
\hline & & & STD & 1500 & 0429 & 3498 & 2776 & & 0004978 & 1317 & 14330 & & & & & & & \\
\hline & 134 & & 085 & T1503 & 0428 & 24781 & 2776 & & & & 14931 & & & & & & & \\
\hline & 168 & & DBS & T173 & 0389 & 34554 & 2778 & & & & 14953 & & & & & & & \\
\hline & & & SID & 1750 & OSAC & 3435 & 2778 & & 0004848 & 1439 & 14955 & & & & & & & \\
\hline & & & STO & 2000 & 0377 & 3476 & 2780 & & 0004875 & 1561 & 14792 & & & & & & & \\
\hline & $I \in \theta$ & & OBS & 12249 & 0362 & 34560 & 2701 & & & & 15028 & & & & & & & \\
\hline & & & STO & 2500 & 0341 & $340_{4}$ & $27=2$ & & 0004840 & 1804 & 15002 & & & & & & & \\
\hline & $1 \in d$ & & $\begin{array}{l}\text { DAS } \\
\text { STO }\end{array}$ & $\begin{array}{l}2754 \\
3000\end{array}$ & 0321 & $34 ; 32$ & 2733 & & & & 15097 & & & & & & & \\
\hline & 168 & & 085 & $\begin{array}{r}3000 \\
T 3260\end{array}$ & $\begin{array}{l}0301 \\
0252\end{array}$ & $\begin{array}{l}3493 \\
34 \rightarrow 24\end{array}$ & 2735 & & 0004073 & $20 \div 2$ & $\begin{array}{l}15132 \\
15100\end{array}$ & & & & & & & \\
\hline & 168 & & OBS & 3769 & 0249 & $\begin{array}{l}34+24 \\
3+999\end{array}$ & $\begin{array}{l}2733 \\
2787\end{array}$ & & & & 15243 & & & & & & & \\
\hline & & & STD & 4900 & 0237 & 3480 & 2750 & & 0004400 & $24+9$ & 15279 & & & & & & & \\
\hline & 168 & & 085 & $4 ! 05$ & 0230 & $348>1$ & 2780 & & & & 15277 & & & & & & & \\
\hline & $1 \in 9$ & & Dos 5 & T4211 & 0234 & 34894 & 2758 & & & & 15315 & & & & & & & \\
\hline
\end{tabular}


TABLE XIII.-Continued
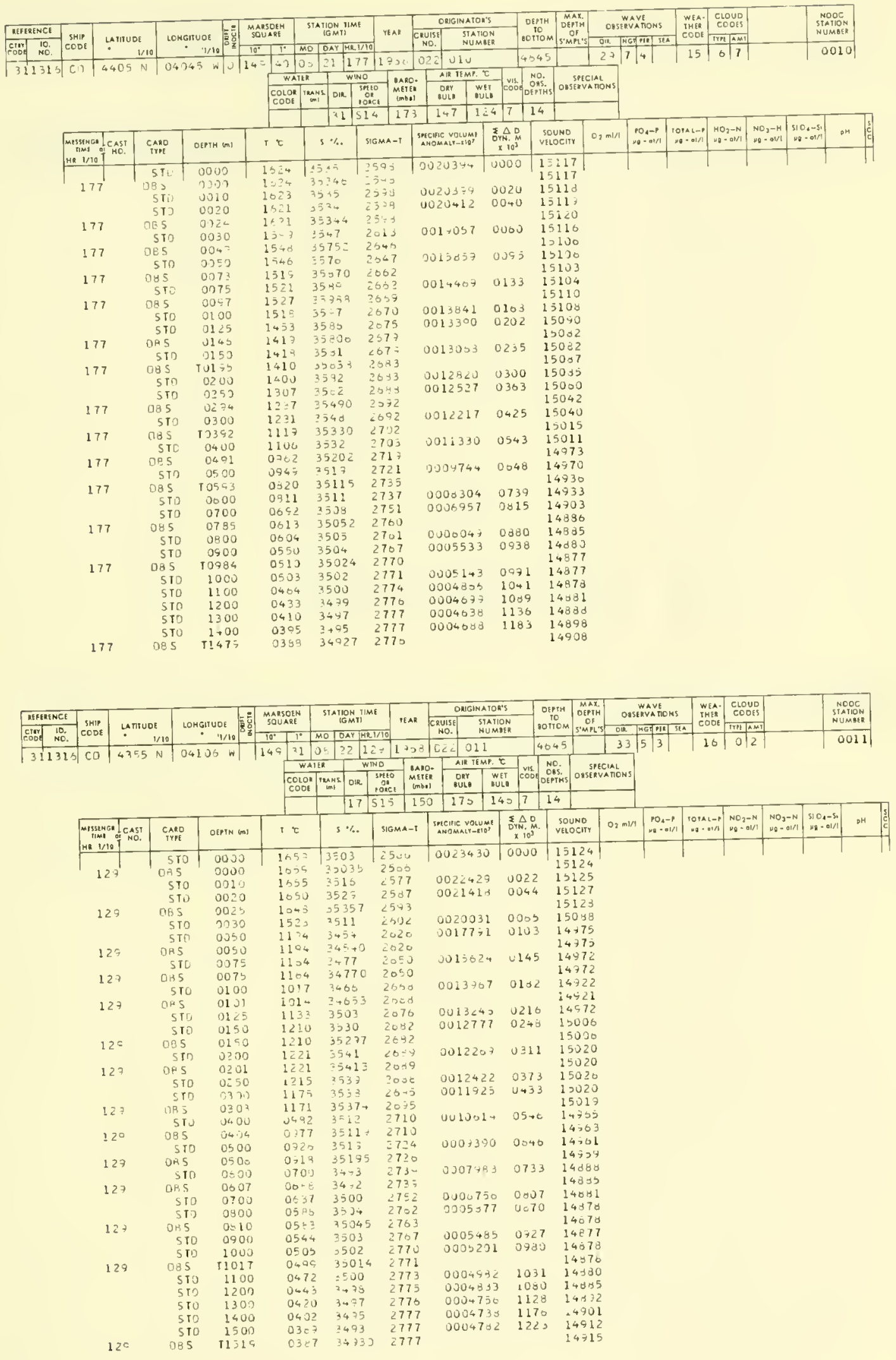
TABLE XIII.-Continued

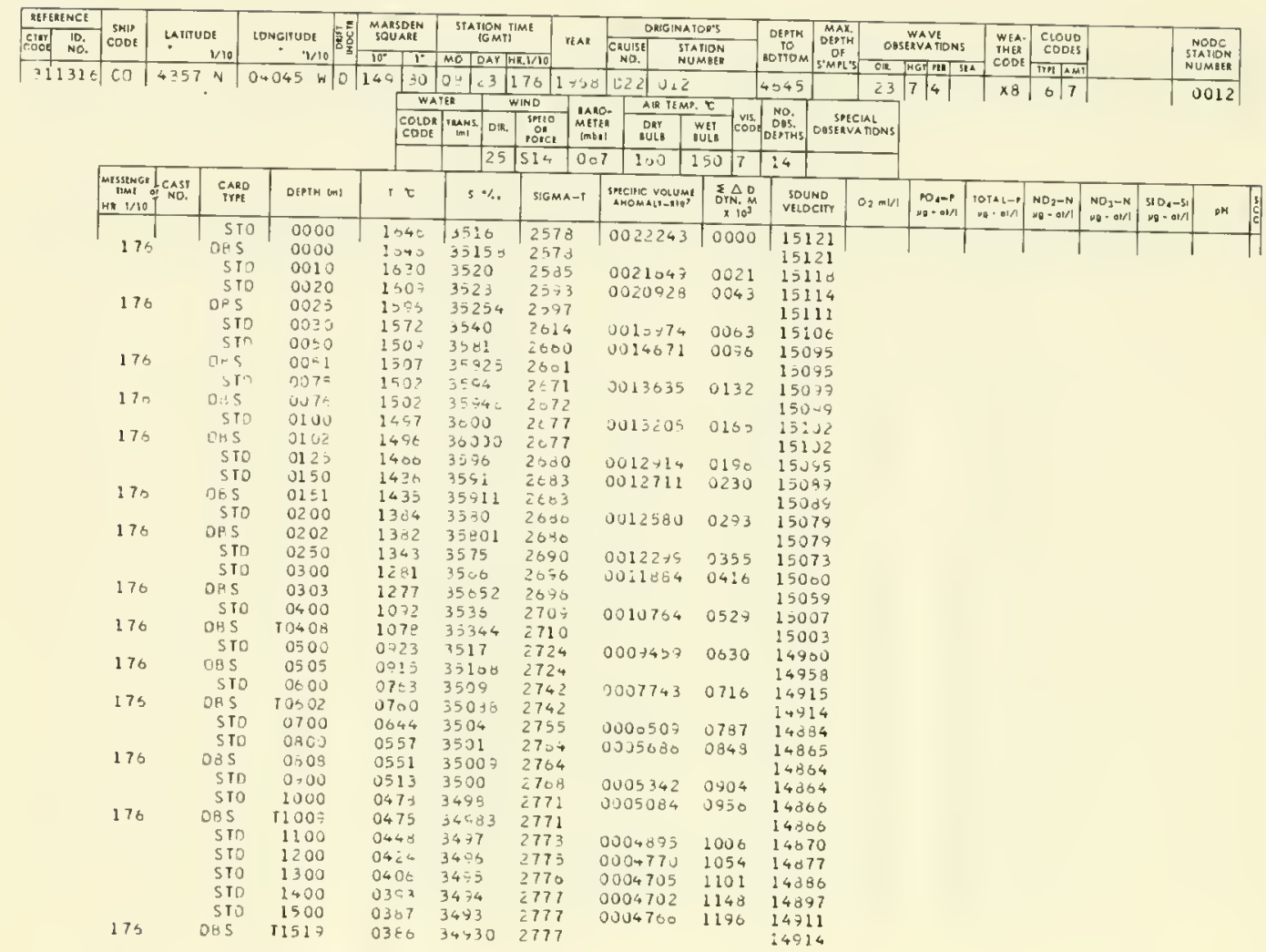

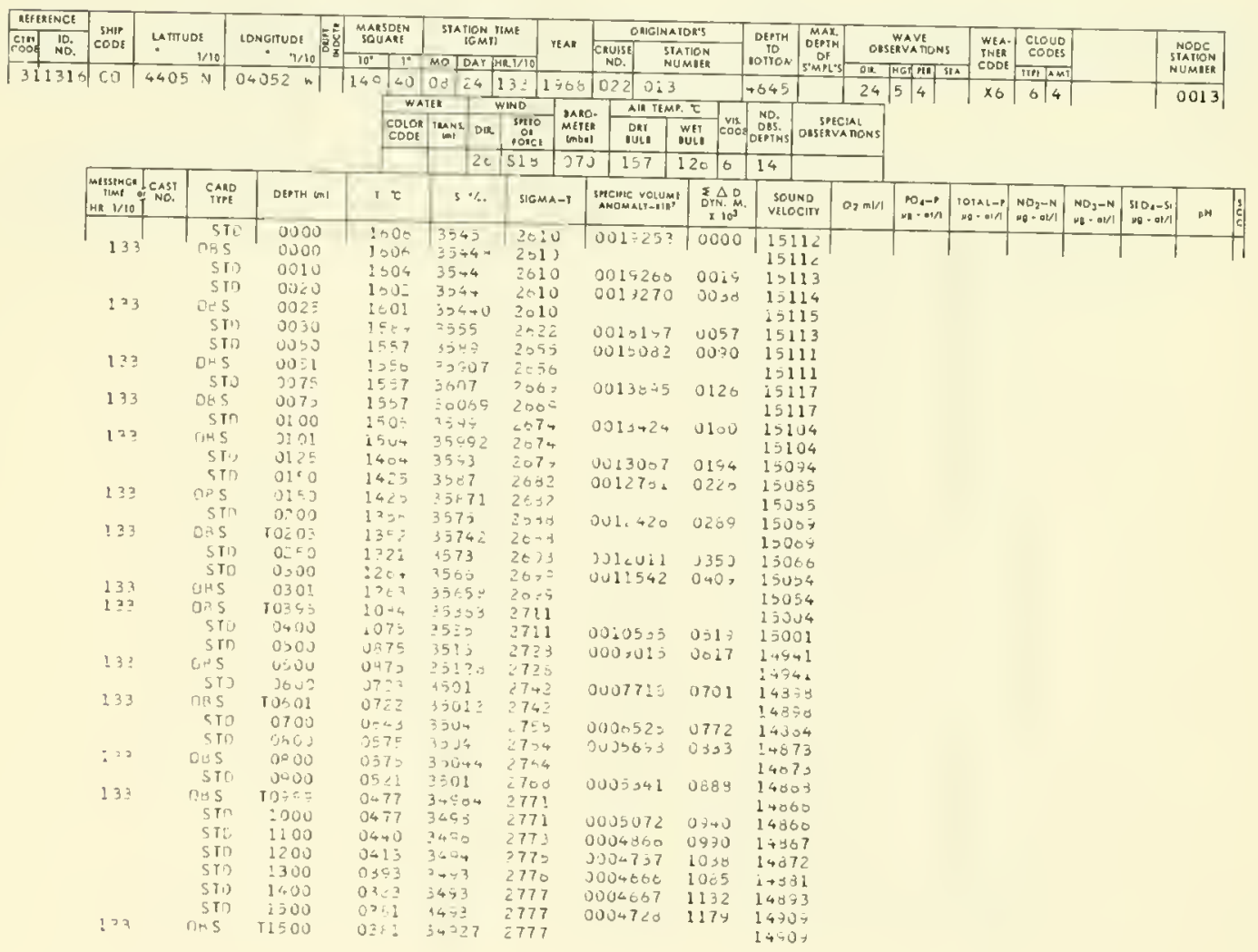


TABLE XIII.-Continued

\begin{tabular}{|c|c|c|c|c|c|c|c|c|c|c|c|c|c|c|c|c|c|c|c|c|c|}
\hline \multirow{3}{*}{\begin{tabular}{|c|c|} 
REFERENCE \\
Crang & 10. \\
Cood & NO. \\
711316
\end{tabular}} & \multirow{2}{*}{ 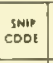 } & \multirow{2}{*}{\multicolumn{2}{|c|}{ umitrot }} & \multirow{2}{*}{ LONGIUDI } & \multirow{2}{*}{\multicolumn{2}{|c|}{$\begin{array}{l}\text { MARSOLN } \\
\text { SOUAIE }\end{array}$}} & \multirow{2}{*}{\multicolumn{3}{|c|}{ 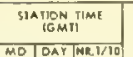 }} & \multirow{2}{*}{ rian } & \multicolumn{2}{|c|}{ OMIGINABOA's } & \multirow{2}{*}{\begin{tabular}{|c|} 
OHIN \\
ID \\
LOTIOM \\
\end{tabular}} & \multirow{2}{*}{ 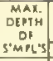 } & \multicolumn{2}{|c|}{$\begin{array}{l}\text { WAVE } \\
\text { OUSEIYAMONS }\end{array}$} & \multirow{2}{*}{$\begin{array}{l}\text { WEA- } \\
\text { TWEI } \\
\text { COOE }\end{array}$} & \multirow{2}{*}{ 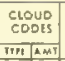 } & & \multirow{2}{*}{\multicolumn{2}{|c|}{$\begin{array}{c}\text { NODC } \\
\text { SATKON } \\
\text { NUMHEB }\end{array}$}} \\
\hline & & & & & & IUAAE & & & & & $\begin{array}{l}\text { CRUISE } \\
\text { No. } \\
\end{array}$ & $\begin{array}{l}\text { STAOHOS } \\
\text { SUMMEI } \\
\text { NUMUI }\end{array}$ & & & ore 14 & सGG DII ISA & & & & & \\
\hline & $C_{7}$ & 4353 & $\mathrm{~N}$ & $04100 \mathrm{~W}$ & 14 & $3:$ & $u=[3$ & 2511 & 134 & 2907 & \begin{tabular}{l|l}
$U<2$ & 014 \\
\end{tabular} & & 4645 & $:$ & 285 & \begin{tabular}{|l|l|}
5 & 3
\end{tabular} & $\overline{x 1}$ & 06 & & & 0014 \\
\hline & & & & & & WAT & TEA & & HWO & & AII TEMP. &.$\tau$ & NQ. & & & & & & & & \\
\hline & & & & & & $\begin{array}{l}\mathrm{COOOO} \\
\mathrm{COOB} \\
\end{array}$ & Imans: & Din & $\begin{array}{l}\text { Spio } \\
\text { OOACt }\end{array}$ & $\begin{array}{l}\text { MERth } \\
\text { (ntmes) }\end{array}$ & \begin{tabular}{l|l} 
Our & nut \\
nut & in
\end{tabular} & $\begin{array}{ll}\text { WE1 } \\
\text { wULE }\end{array}$ & oist & onsteve & A IONS. & & & & & & \\
\hline & & & & & & & & 3 & 503 & 183 & $1+4$ & \begin{tabular}{l|l|}
120 & 7 \\
\end{tabular} & 14 & & & & & & & & \\
\hline & $\begin{array}{l}\text { Missinet } \\
\text { Mut: } \\
\text { Mn } 1 / 10 \\
\end{array}$ & $\begin{array}{l}\text { ICast } \\
\text { I cos. }\end{array}$ & $\underset{\text { TYRP }}{\text { CARP }}$ & DEPTN m & & $1 \%$ & & $\because$ & SLGM & $\operatorname{ma}-1$ & $\begin{array}{l}\text { "Freiple volumi } \\
\text { ANOMALT-nII" }\end{array}$ & 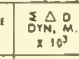 & $\begin{array}{l}\text { sou } \\
\text { VELo }\end{array}$ & $\begin{array}{l}\text { wo } \\
\text { cirr }\end{array}$ & $0, \mathrm{ml} / \mathrm{l}$ & 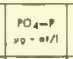 & 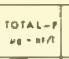 & 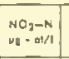 & $\begin{array}{ll}\mathrm{NO}_{2}-\mathrm{N} \\
\mathrm{H}=\mathrm{N} / \mathrm{N}\end{array}$ & 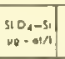 & DH \\
\hline & & & STD & 0003 & & 1014 & 334 & & 201 & 10 & 0013235 & 0000 & 151 & 110 & & & & & & & \\
\hline & 134 & & 735 & 0000 & & $1<1=$ & $3 \equiv 4$ & (4) & $\mathrm{col}$ & 10 & & & 151 & 116 & & & & & & & \\
\hline & & & 510 & 0010 & & $102 t$ & $3=5$ & & 26! & 14 & 0013541 & J01; & & 122 & & & & & & & \\
\hline & & & STD & 0020 & & 1639 & $35=$ & $20^{\circ}$ & 201 & 10 & $001 d>11$ & 0037 & 151 & $1<8$ & & & & & & & \\
\hline & $1 \geq 4$ & & DAS & 0025 & & 2345 & 320 & 079 & 202 & 20 & & & 151 & 131 & & & & & & & \\
\hline & & & STO & 0030 & & 1617 & 357 & 74 & $\angle 02$ & 2; & 3017465 & 0055 & 151 & 124 & & & & & & & \\
\hline & & & 510 & 0050 & & $153 t$ & 358 & $\leqslant 7$ & $2 \leqslant 5$ & 58 & 0014830 & 0008 & 151 & 104 & & & & & & & \\
\hline & 134 & & $0+5$ & $00 \gg 1$ & & 1533 & 355 & 57. & 205 & 55 & & & 132 & 203 & & & & & & & \\
\hline & & & S10 & 0075 & & 1504 & $35=$ & $=5$ & $2+7$ & & 001361 त & 0123 & 150 & 34 & & & & & & & \\
\hline & 134 & & nBS & 0075 & & 1504 & 359 & 952 & 207 & $7 !$ & & & 150 & 099 & & & & & & & \\
\hline & & & STO & 0100 & & 14 मेट & 359 & $\varsigma_{3}$ & 267 & 78 & 0013077 & 4150 & 150 & 948 & & & & & & & \\
\hline & 134 & & 045 & 0121 & & $14 \times 5$ & $35 y$ & Y64 & 267 & 70 & & & 150 & $j+\dot{3}$ & & & & & & & \\
\hline & & & sto & 0125 & & 1452 & 359 & 92 & 203 & 3. & 0012873 & 0189 & 150 & $u>0$ & & & & & & & \\
\hline & & & 5ro & 0150 & & 1420 & 358 & 87 & 258 & 83 & 0012707 & 0221 & $1>0$ & 084 & & & & & & & \\
\hline & 134 & & 085 & 3153 & & 1420 & $35 t$ & $80 R$ & 203 & & & & 150 & 084 & & & & & & & \\
\hline & & & 510 & 0200 & & $136 ?$ & 357 & & 260 & & 0012430 & 0234 & 150 & 73 & & & & & & & \\
\hline & 134 & & 085 & $10 ? 0^{\circ}$ & & 1305 & 357 & 775 & 268 & & & & 150 & $07 ?$ & & & & & & & \\
\hline & & & SID & $02 \leq 0$ & & 1317 & 357 & & 207 & & 3012118 & 0345 & & 005 & & & & & & & \\
\hline & & & ST & $0^{2} 00$ & & 1265 & 350 & & 209 & & 0011777 & 0405 & 150 & 954 & & & & & & & \\
\hline & 134 & & OBS & 0300 & & $12 n 5$ & $35 \%$ & 420 & 20, & & & & 150 & 054 & & & & & & & \\
\hline & 134 & & DAS & 10390 & & 1137 & 354 & 429 & 270 & & & & 150 & 024 & & & & & & & \\
\hline & & & STO & 0400 & & 1235 & 354 & & 270 & & 0011074 & 0517 & 150 & 023 & & & & & & & \\
\hline & 134 & & OBS & $045:$ & & 0743 & 351 & 172 & $=72$ & & & & $1+5$ & 807 & & & & & & & \\
\hline & & & STO & 0500 & & $09 \div 1$ & $35:$ & & 272 & & $00097 \$ 2$ & 0623 & 149 & 907 & & & & & & & \\
\hline & 134 & & 0 as & 135 , & & $07<3$ & 350 & 036 & 273 & & & & 145 & 926 & & & & & & & \\
\hline & & & STO & Osou & & $07 C_{2}$ & 350 & & 273 & & 0008207 & 0713 & 149 & 926 & & & & & & & \\
\hline & & & STU & 0700 & & $0 b a$ & 350 & & 270 & & vous 701 & 0789 & 147 & 900 & & & & & & & \\
\hline & 134 & & OBS & 0755 & & 0547 & 251 & 042 & $27 \mathrm{~s}$ & & & & 143 & 81 & & & & & & & \\
\hline & & & STO & 0800 & & $05>3$ & 350 & & 276 & & 0005960 & 0031 & 140 & 880 & & & & & & & \\
\hline & & & STI & $0>00$ & & 0517 & $3+4$ & & 270 & & 0005443 & 0510 & 141 & 167 & & & & & & & \\
\hline & 134 & & OAS & TugC? & & $04+5$ & $3+c$ & Soj & 677 & & & & 148 & 359 & & & & & & & \\
\hline & & & sTn & 1000 & & 0404 & 349 & & 277 & & 0005036 & 0703 & 140 & 800 & & & & & & & \\
\hline & & & $5 T 0$ & 1100 & & 0443 & $3+2$ & & 277 & & 0005037 & 1014 & 140 & $\$ 70$ & & & & & & & \\
\hline & & & STO & 1200 & & 0433 & $34 c$ & & 277 & & 0004980 & 1004 & 148 & 880 & & & & & & & \\
\hline & & & STD & 1300 & & 0417 & $34=$ & & 277 & & 0004932 & 1113 & 140 & 890 & & & & & & & \\
\hline & & & STO & 1400 & & 0401 & 340 & & 277 & & $000400>$ & 1102 & $14{ }^{\circ}$ & 301 & & & & & & & \\
\hline & 134 & & 085 & 11470 & & 0385 & 34 & 327 & 277 & & & & 149 & 909 & & & & & & & \\
\hline
\end{tabular}







$$
\text { 532-AA }
$$



Woods liole Gceanographic Institution
AILAS - GAZETEER COLECTION 